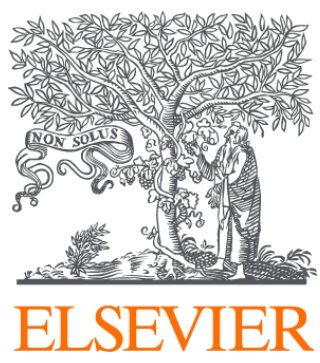

Since January 2020 Elsevier has created a COVID-19 resource centre with free information in English and Mandarin on the novel coronavirus COVID-

19. The COVID-19 resource centre is hosted on Elsevier Connect, the company's public news and information website.

Elsevier hereby grants permission to make all its COVID-19-related research that is available on the COVID-19 resource centre - including this research content - immediately available in PubMed Central and other publicly funded repositories, such as the WHO COVID database with rights for unrestricted research re-use and analyses in any form or by any means with acknowledgement of the original source. These permissions are granted for free by Elsevier for as long as the COVID-19 resource centre remains active. 
Abstracts

\section{European Congress on Biotechnology}

\section{Keynote Lectures}

2

White biotechnology: Science, fiction and reality Dr. Oskar Zelder BASF Aktiengesellschaft

In the last 20 years biotechnology has made tremendous progress in its different application fields: Red Biotechnology, the use of biological methods for medical purposes, is firmly established in the development of new drugs. The use of plant or green biotechnology is under controversial discussion in politics and public. Nevertheless, genetically modified herbicide and insect resistant crops are cultivated to a large extent. Industrial Biotechnology, now often named White Biotechnology, seems widely underestimated in the public perception. It includes all industrial processes for the production of chemical products and enzymes, which fully or partly rely on the biological toolbox of nature. White Biotechnology processes are carried out in a contained environment, typically in a bioreactor in a dedicated industrial plant. Well-known examples are the fermentative productions of antibiotics, amino acids, vitamins and enzymes, products related to medical, food and feed applications. Many products like the amino acids glutamic acid, lysine, threonine and tryptophane are exclusively produced using microbes in large scale industrial processes. In other cases, like the water soluble vitamin B2, biotechnological processes successfully replaced chemical productions, due to lower costs and improved ecoefficiency. In contrast to this, most industrial chemicals and polymers are produced by chemical synthesis based on oil and gas. However, there are some examples for bioproducts among industrial chemicals. The solvents acetone and butanol, for instance, were manufactured by fermentation for several decades in the last century. Since the 1950s these fermentations have been replaced by more efficient and cheaper chemical synthesis. Recently, new pilot and production processes for biopolymers like PHA or biomonomers like 1,3-propanediol or lactic acid were announced by different companies. Currently, ethanol is by far the largest White Biotech product by volume. In Brazil, where Bioethanol is used as liquid transportation fuel, the annual production is in the range of $15 \mathrm{Mio}^{3}$. Bioethanol is of growing importance also in the United States. Business consultants predict a tremendous growth of biotechnological products within the chemical industry. High prices for crude oil, dropping prices for renewable resources, and scientific progresses nourish the expectation that Industrial Biotechnology will replace many bulk chemicals. Is this realistic? Will we switch from a petrochemical industry to a biobased chemistry within the next years? Based on economic considerations it can be stated that this is a long term goal. To achieve this it remains a scientific challenge to make renewable raw materials available for competitive bioproduction of bulk chemicals at low costs. Conversion of lignocellulosic material to fermentation sugar may be a solution. Also green biotechnology can contribute to the supply of cheap fermentation raw materials. Innovative ideas for downstream processing or further chemical conversion of fermentation products are required to enter the chemical value chains. Furthermore, the identification of new higher value bioproducts is a chance for short term successes in White Biotechnology. Enzyme and protein engineering has the potential to create new biomolecules, metabolic engineering can contribute to develop new metabolic pathways, may be even for unnatural compounds.

\section{3}

Systems biology: Transforming biology and medicine Leroy Hood President, Institute for Systems Biology, Seattle, Washington, USA

The advent of the Human Genome Project has transformed biology by providing a genetics parts list of all genes and proteins, by fueling the contention biology is an informational science, and by catalyzing the emergence of biological information (e.g., rapid DNA sequencing or DNA chips). From this has emerged a new approach to biology termed systems biology — centered on the idea one can study biological systems by delineating the relationships of all of their component elements - and, hence, come to understand the resulting systems properties. I will discuss these important points, give several examples of systems approaches, and conclude by discussing the profound change systems biology will engender in medicine-moving us toward a predictive, preventive, and personalized medicine.

4

Interpreting the human and $C$. elegans genomes Robert H. Waterston, Zhirong Bao, John I. Murray, The Chimpanzee Sequencing Consortium Department of Genome Sciences, University of Washington, Seattle, WA 98195, USA 
By continuously increasing the efficiency and throughput of DNA sequencing we, together with colleagues, have sequenced the human genome and the genomes of all the major model organisms. The challenge now centers on understanding these vast instruction sets. Our ability to read these instructions must be enhanced through collection of key additional data sets. One productive path for delineating the functional sequences and inferring their function is comparative sequence. The mouse genome sequence, for example, led to estimates that only $5 \%$ of the human genome is functional. Sequencing of an extensive set of additional mammalian genomes promises to define these functional sequences with a resolution of less than 10 base pairs. On a different course, we have sequenced the chimpanzee genome to learn what has changed in the evolution of humans. Beyond providing for the first time a catalog of the differences between the two genomes, the comparison of the chimpanzee and human genomes reveals the patterns of neutral mutation and regions that deviate from that. The talk will summarize these and related findings. The sequence of additional primate genomes will help delineate what has changed specifically in humans and add power to the analysis. Ultimately, capturing human sequence variation and correlating with phenotypic variation will be required to understand function. But learning what these functional elements do requires new sets of experimental data. For this, we have turned to the nematode $C$. elegans. In this simple system most of the $\sim 20 \mathrm{k}$ genes have been defined and experimentally confirmed. Beyond the hundreds of genes with already known mutants, two centers are systematically producing gene knockouts or RNAi can be used to inhibit any gene temporarily. Sequences of three Caenorhabditis species are already available, and two more are underway. Expression data has been collected for all the genes under many conditions and time points through development. To enhance the resolution of expression data and to simplify phenotypic analysis of embryonic mutants, we are developing a system that will automatically trace the cell lineage and assign gene expression to precise cells with high temporal resolution. The latest results with the system will be described. In the longer term, this and similar datasets should provide an understanding of how the genome specifies the form and behavior of the worm.

5

A human protein atlas for normal and disease tissue Mathias Uhlen Department of Biotechnlogy, AlbaNova University Center, Royal Institute of Technology (KTH), Stockholm, Sweden

Here, we present a new protein atlas database (www.proteinatlas.org) showing the expression and localization of human protein in normal and cancer tissues. The atlas is based on the use of antibodies (Agaton et al., 2003) to generate high-resolution immunohistochemistry images representing 48 normal tissues and 20 different cancer types (Uhlen and Ponten, 2005). Each antibody is used to generate more than 500 individual images and each image has been annotated by a pathologist (Kampf et al., in press). The database has been created by the Swedish Human Proteome Resource (HPR) and the program has been set-up to allow the exploration of the human proteome with Antibody-based Proteomics (Nilsson et al., in press). The basic concept is to generate, in a systematic and high-throughput manner (Uhlen and Ponten, 2005), specific antibodies to all human proteins, and subsequently used these for functional analysis of the corresponding proteins in a wide range of assay platforms, including (i) a protein atlas for tissue profiles (Kampf et al., in press), (ii) specific probes to evaluate the functional role of individual proteins, and (iii) affinity reagents for purification of the specific proteins and their associated complexes for structural and biochemical analyses.

\section{References}

Agaton, C., et al., 2003. Affinity proteomics for systematic protein profiling of chromosome 21 gene products in human tissues. Mol. Cell Proteomics 2 (6), 405-414.

Kampf, C., et al., in press. Antibody-based tissue profiling as a tool in clinical proteomics. Clin. Proteomics.

Nilsson, P., et al., in press. Towards a human protein atlas - monospecific antibodies for tissue profiling, Proteomics.

Uhlen, P., 2005. Antibody-based proteomics for human tissue profiling. Mol. Cell Proteomics 4 (4), 384-393.

\section{Genomics and Systems Biology}

\section{Systems Biology}

\section{2}

New 'Dimensions' in genome annotation Bernhard $O$. Palsson Department of Bioengineering, UCSD, 9500 Gilman Drive, La Jolla, CA 92093-0412, USA. E-mail: palsson@ucsd.edu

Traditional genome annotation involves the enumeration of open reading frames and their functional assignment. Currently, there are on-going efforts to identify all the interactions between these components. The resulting map of interactions effectively represents a two-dimensional annotation. It takes the form of a stoichiometric matrix, if the interactions are described with chemical equations. The formulation and properties of this matrix are detailed and how it can be used as the basis for computing allowable phenotypic functions. The issues associated with the packing of the bacterial genome and the function of the interaction map in three-dimensions will also be discussed. Finally, we will go over the issue of genomes changing in space and time through adaptive evolution.

\section{3}

Understanding role and function of regulatory networks in microorganisms Krist V. Gernaey ${ }^{1,2}$, Morten Lind ${ }^{3}$, Sten B. Jorgensen ${ }^{2}:{ }^{1}$ Department of Industrial Electrical Engineering and Automation, Lund University, Sweden; ${ }^{2}$ CAPEC, Department of Chemical Engineering, Technical University of Denmark, Lyngby, Denmark; ${ }^{3} \emptyset$ rsted•DTU, Automation, Technical University of Denmark, Lyngby, Denmark. E-mail: krist.gernaey@iea.lth.se (K.V. Gernaey)

Microorganisms have to maintain a constant monitoring of extracellular physicochemical conditions in order to respond and modify their gene expression patterns accordingly. Modelling plays an essential role in developing an understanding of the regulatory networks in microorganisms. A structured representation of complex biological systems is to consider their functional organisation. The idea is to describe the biological system as a goal directed system, and to decompose the system into subsystems so that each subsystem serves the needs or provides the means for its super-ordinate system. Meansend analysis, also called functional modelling, is therefore proposed as a methodology to model regulatory networks. Functional modelling is based upon the formal concept of action (function) proposed 
by von Wright (1963). A modelling example for the Escherichia coli lac operon illustrates that the methodology allows breakdown of complex regulatory networks into elementary building blocks. For this example, functional modelling demonstrates to enable modelling changes in qualitative behaviour of microorganisms, and is as such able to summarise available process knowledge. Quantitative dynamic models can be developed within each region of qualitative behaviour, using the logic in the functional model to support in the generation of detailed mathematical descriptions. A key aspect of the selected modelling paradigm is that it supports formal reasoning about causal and functional relations in regulatory networks. Moreover, the proposed modelling formalism enables a straightforward communication between systems engineers and biologists on the mechanisms underlying complex regulatory functions.

Model-based identification of regulation patterns controlling metabolic redundancy in central carbon metabolism Johannes Kluge, Anne Kümmel, Sven Panke, Matthias Heinemann Institute of Process Engineering, ETH Zurich, 8092 Zurich, Switzerland

The topology of metabolic networks is characterized by a high degree of redundancy, realized by isoenzymes and alternative pathways. The utilization of such functional backups upon environmental or genetic perturbations is coordinated by complex biochemical regulation mechanisms. Here, we introduce a new model-based methodology for deciphering such patterns of metabolic regulation. For this, no more than a genome-scale reconstruction of the metabolic network as well as simple experimental data on the (in-)viability of knock-out mutants are required. For the proposed method, flux balance analysis is used to identify in silico the complete set of possible bypasses for every single knock-out mutant. The model predictions are then mapped with in vivo data on (in-)viability of the mutants in order to find out which of the alternative pathways are actually applicable in vivo or not. By this mapping of in silico and in vivo data, regulatory control patterns are identified. The power of the proposed methodology is demonstrated with the Escherichia coli central carbon metabolism, which is, due to the wealth of regulatory information available for this organism, a suitable test case to bench-mark the new method. The metabolic redundancy of central metabolism was analyzed for typical substrates, namely glucose, glycerol, succinate and acetate. Redundancy was found to be higher for substrates using glycolysis than for substrates requiring gluconeogenic activity. Consequently, the number of alternative in silico pathways is highest for glucose, and lowest for acetate. It was found that under the same experimental conditions, an in silico bypass that does not support mutant growth in vivo cannot be employed by any other mutant either. Thus, with this finding experimental efforts can be directed efficiently, such that only a minimal number of knockout mutants have to be constructed and tested, while reliable conclusions about flux rerouting and viability of other mutants can still be drawn. A critical comparison of the obtained results with experimental data on flux rerouting upon gene knock-out (determined by ${ }^{13} \mathrm{C}$ flux analysis) revealed an excellent agreement. The developed methodology may be helpful for a quick and comprehensive identification of regulatory patterns controlling flux rerouting in organisms of interest. Besides the mere identification of the regulation patterns, the regulatory knowledge derived can subsequently also be used to obtain improved internal flux predictions by FBA.

\section{5}

Escherichia coli central and trimethylammonium metabolism model for L-carnitine production optimisation A. Sevilla ${ }^{1}$, J.W. Schmid ${ }^{2}$, K. Mauch ${ }^{2}$, V. Bernal ${ }^{1}$, J.L. Iborra ${ }^{1}$, M. Reuss ${ }^{2}$, M. Canovas $^{1}$ : $^{1}$ Department Bioquímica y Biol. Molec. B, Universidad de Murcia, 30100 Murcia, Spain; ${ }^{2}$ Institute of Biochemical Engineering, University of Stuttgart, Allmandring 31, D-70569 Stuttgart, Germany. E-mail: mcanovas@um.es (M. Canovas)

The aim of this work was to understand the steps controlling the process of the biotransformation of trimethylamonium compounds into L(-)-carnitine by Escherichia coli. Here we present the design and experimental validation of a model of the central carbon metabolism linked to the secondary carnitine metabolism of $E$. coli, which, for the first time, links the carnitine metabolism with the reactions of glycolysis, CAT and the pentose-phosphate pathway. Experimental observations of intracellular concentrations of metabolites and co-metabolites at steady state are used to validate the model's structure and to estimate the kinetic parameters. Thus, the enzyme activities involved in the biotransformation process of crotonobetaine into $\mathrm{L}(-)$-carnitine and in the distribution of metabolites for the tricarboxylic acid cycle were included in high-cell density membrane reactors during continuous cell growth or resting cell processes. In addition, carnitine, crotonobetaine, $\gamma$-butyrobetaine and ATP levels, together with the NADH/NAD+ ratio were measured to ascertain how metabolic fluxes were distributed in the catabolic system. The results provided the first evidence that high levels of ATP are needed to maintain the biotransformation substrate transport and its biotransformation. It was also demonstrated that the higher the number of ATP molecules produced, the higher the number of fumarate molecules required as an electron acceptor to cancel the reducing power generated during the biotransformation. A higher YL-carnitine/glycerol was obtained when glycerol, as the carbon source, was transformed into $\mathrm{CO}_{2}$ or acetate than when lactate was the anaerobic product. Moreover, results demonstrate that there is a futile cycle for ATP, since both trimethylammonium compound carriers, CaiT and ProU, were operated simultaneously. Further, in order to improve the biotransformation process, resting processes as well as CaiT or ProU knock out mutants would yield a more efficient system for L-carnitine production from crotonobetaine or D-carnitine.

\section{Integrated Analysis}

1

Top-down and bottom-up approaches to yeast Systems Biology Steve Oliver Faculty of Life Sciences, The University of Manchester, Michael Smith Building, Oxford Road, Manchester M13 9PT, UK

Metabolic Control Analysis (MCA) is a conceptual and mathematical formalism that models the relative contributions of individual effectors in a pathway to both the flux through the pathway and the concentrations of individual intermediates within it. To exploit MCA in an initial Systems Biology analysis of the eukaryotic cell, two categories of experiments are required. In category 1 experiments, flux is changed and the impact on the levels of the direct and indirect products of gene action is measured. We have measured the impact of changing the flux on the transcriptome, proteome, and metabolome of Saccharomyces cerevisiae. In this whole-cell analysis, flux equates to growth rate. In category 2 experiments, the levels of individual gene products are altered, and the impact on the flux is measured. 
We have used competition analyses between the complete set of heterozygous yeast deletion mutants to reveal genes encoding proteins with high flux control coefficients. These genes may be exploited, in a top-down analysis, to build a coarse-grained model of the yeast cell. More detailed modelling requires that 'natural' biological systems be identified. The combination of flux balance analysis with both genetics and metabolomics in the definition of metabolic systems will be discussed.

2

Wiring and rewiring gene-metabolic networks James C. Liao Department of Chemical Engineering, University of California, Los Angeles, USA

This talk will address two aspects in integrated analysis in genemetabolic networks: a bottom-up approach for deducing regulatory networks and a top-down design approach for re-wiring the genemetabolic approach for a desired function. The bottom-up approach is based on a method recently developed in our laboratory, called network component analysis (NCA). This method uncovers transcription factor activities from gene expression and DNA binding data. The method is applied to deduce (i) cell cycle regulators, (ii) mutation effects on global regulation, (iii) signal transduction pathways, and (iv) interactions among signaling pathways. The top-down approach is used to design and construct novel gene-metabolic circuits for a desired cellular behavior. Success examples include (i) a designer feedback controller for non-native metabolite production, (ii) an artificial cell-cell communication system for synchronization, and (iii) a synthetic gene-metabolic oscillator that mimics features of circadian regulation. In each case, the desired phenotype predicted by the design model was achieved experimentally.

3

A systematic study of the transcriptional regulation on the metabolic fluxes in Saccharomyces cerevisiae T. Grotkjar ${ }^{1,2}, B$. Papp $^{2}$, S. G. Oliver ${ }^{2}$, J. Villadsen ${ }^{1}$, J. Nielsen ${ }^{1}:{ }^{1}$ Center for Microbial Biotechnology, Technical University of Denmark, Denmark; ${ }^{2}$ Faculty of Life Sciences, University of Manchester, UK

Metabolic engineering is defined as the rational approach to strain improvement. A major challenge in metabolic engineering is to interpret and successfully integrate data from high-throughput experimental tools such as DNA microarrays. Cellular metabolic phenotypes can be described by intracellular fluxes, which are determined by both enzyme activities and metabolite concentrations. However, it is still largely unexplored how transcription is correlated with metabolic fluxes. In the present study we quantify the role of transcriptional regulation in the metabolic network of Saccharomyces cerevisiae by studying the relationship between mRNA and flux levels at different specific growth rates. For most enzymes we observe that flux levels increase more steeply with specific growth rate than mRNA concentrations, suggesting that most regulation takes place at the post-transcriptional level. As further evidence, we show that enzymes reported to be modified post-translationally, catalyse reactions that are less likely to be regulated by transcription. Thus, our results suggest that in many cases simple up- or down-regulation at the expression level is insufficient to predict a change in flux.

\section{4}

Microarray analysis of the Aspergillus niger revealed that $X \ln R$ plays an important role in the regulation of different pathways Luisa Trindade, Leo de Graaff Wageningen University, Fungal Genomics Section, Laboratory of Microbiology, Dreijenlaan 2, 6703 HA, Wageningen, The Netherlands. E-mail: leo.degraaff@wur.nl (L.d. Graaff)

The $X \operatorname{lnR}$ protein was initially identified as a transcription activator of different genes encoding cellulolytic and xylanolytic enzymes in Aspergillus niger. A number of experiments have been performed to elucidate the regulation mechanism of $x \ln R$ and the results suggest that the transcription of the $x \ln R$ gene is induced by the presence of $D$-xylose in the culturing media and repressed by D-glucose. As the mRNA levels of XlnR gene are very low, and they can hardly be detected by Northern blot analysis, further research is necessary to confirm these results. To study the $\mathrm{X} \operatorname{lnR}$ regulon, $\mathrm{X} \operatorname{lnR}$ mutants were grown under inducing (D-xylose and xylan), repressing (D-glucose) and neutral (sorbitol) culturing conditions. The whole transcriptome was examined by microarray analysis. The XlnR mutants used in these experiments are an $x \ln R$ knock out mutant, a mutant where $\mathrm{X} \ln \mathrm{R}$ is constitutively expressed, and the wild type phenotype. Comparison of the transcriptome of different XlnR strains under inducing and repressing conditions showed that XlnR regulates several genes that are involved in different pathways. Among these are genes encoding proteins involved in signal transduction, in the regulation of transcription, in sugar transport but also genes encoding enzymes. Previous work suggested that the CreA transcription factor might play a role in the transcriptional regulation of the $\mathrm{X} \operatorname{lnR}$ regulon. This has been shown for the enzyme encoding genes of the regulon, but whether CreA regulates the transcription of the $x \ln R$ gene still was uncertain. In order to investigate this the transcriptome of different single and double CreA derepressed and XlnR mutants were compared using both microarray and qPCR analysis. The results of these experiments lead to a new model for the transcription regulation of $\mathrm{X} \ln R$.

\section{5}

Transcriptome analysis of a shikimic acid producing strain of Escherichia coli W3110 at carbon- and phosphate-limited conditions Louise Johansson, Gunnar Lidén Department of Chemical Engineering, Lund University, Lund SE-22100, Sweden

Shikimic acid, which is produced in the aromatic amino acid pathway in plants and micro-organisms, is an industrially interesting chiral starting material for the synthesis of many chemical substances, e.g. the influenza medicine Tamiflu ${ }^{\circledR}$. When produced by genetically modified Escherichia coli it has previously been found that carbon-rich conditions (e.g. phosphate-limitation) favors production of shikimic acid over shikimate pathway by-products, whereas the situation is the opposite in glucose-limited conditions. In the present study, genetic expression patterns of the shikimate producing strain W3110.shik1 (W3110 with aroL deletion and plasmidoverexpressed aroF) and the wild-type strain W3110 grown at carbon- and phosphate-limited (carbon-rich) chemostat conditions $\left(D=0.23 \mathrm{~h}^{-1}\right)$ were analyzed. The study suggests that the by-product formation under carbon-limitation is explained by a set of upregulated genes coupled to the shikimate pathway. The genes, $y$ diB, aroD and $y d i N$, were only expressed in carbon-limited W3110.shik1 
(W3110.shik1/W3110 under carbon-limitation, $\lg _{2}$ fold changes (fc): $6.25(y d i B), 3.93(\operatorname{aroD})$ and $8.18(y d i N)$; phosphate-/carbonlimitation of W3110.shik1 fc: -5.65 (ydiB), -4.31 (aroD), -6.85 $(y \operatorname{diN}))$.

\section{Genomics and Metabolism}

1

Metabolomics, machine learning and modelling in systems biology: Towards an understanding of the language of cells Douglas B. Kell School of Chemistry, The University of Manchester, Faraday Building, Sackville St, PO Box 88, Manchester M60 1QD, UK. E-mail: dbk@manchester.ac.uk; http://dbk.ch.umist.ac.uk; http://www.mib.ac.uk/

Progress in Systems Biology - or in "understanding complex systems" - depends on new technology (Kell, 2004; Goodacre et al., 2004; O'Hagan et al., 2005; Kell et al., in press), computational assistance (Mendes and Kell, 1998; Ihekwaba, 2004; Brown et al., 2005) and new philosophy (Kell and Oliver, 2004), but probably not in that order (Brenner, 1980). Some developments include all three (King et al., 2004; Nelson et al., 2004). My lecture will present some of the recent developments and challenges in each of these areas. Particular focus will be laid on the uses of evolutionary computation for the analysis and traversal of complex search spaces.

\section{References}

Brenner, S., 1980. Nature, June 5 issue.

Brown, M., Dunn, W.B., Ellis, D.I., Goodacre, R., Handl, J., Knowles, J.D., O’Hagan, S., Spasic, I., Kell, D.B., 2005. A metabolome pipeline: from concept to data to knowledge. Metabolomics 1, 35-46.

Goodacre, R., Vaidyanathan, S., Dunn, W.B., Harrigan, G.G., Kell, D.B., 2004. Metabolomics by numbers: acquiring and understanding global metabolite data. Trends Biotechnol. 22, 245252.

Ihekwaba, A., Broomhead, D.S., Grimley, R., Benson, N., Kell, D.B., 2004. Sensitivity analysis of parameters controlling oscillatory signalling in the NF-kB pathway: the roles of IKK and IkBa. Syst. Biol. 1, 93-103.

Kell, D.B., 2004. Metabolomics and systems biology: making sense of the soup. Curr. Opin. Microbiol. 7, 296-307.

Kell, D.B., Oliver, S.G., 2004. Here is the evidence, now what is the hypothesis? The complementary roles of inductive and hypothesisdriven science in the post-genomic era. Bioessays 26, 99-105.

Kell, D.B., Brown, M., Davey, H.M., Dunn, W.B., Spasic, I., Oliver, S.G., in press. Metabolic footprinting and Systems Biology: the medium is the message. Nat. Rev. Microbiol.

King, R.D., Whelan, K.E., Jones, F.M., Reiser, P.G.K., Bryant, C.H., Muggleton, S.H., Kell, D.B., Oliver, S.G., 2004. Functional genomic hypothesis generation and experimentation by a robot scientist. Nature 427, 247-252.

Mendes, P., Kell, D.B., 1998. Non-linear optimization of biochemical pathways: applications to metabolic engineering and parameter estimation. Bioinformatics 14, 869-883.

Nelson, D.E., Ihekwaba, A.E.C., Elliott, M., Gibney, C.A., Foreman, B.E., Nelson, G., See, V., Horton, C.A., Spiller, D.G., Edwards, S.W., McDowell, H.P., Unitt, J.F., Sullivan, E., Grimley, R., Benson, N., Broomhead, D. S., Kell, D.B., White, M.R.H., 2004.
Oscillations in NF-kB signalling control the dynamics of target gene expression. Science 306, 704-708.

O’Hagan, S., Dunn, W.B., Brown, M., Knowles, J.D., Kell, D.B., 2005. Closed-loop, multiobjective optimisation of analytical instrumentation: gas-chromatography-time-of-flight mass spectrometry of the metabolomes of human serum and of yeast fermentations. Anal. Chem. 77, 290-303.

\section{2}

Design principles of metabolic network operation Eliane Fischer, Lars Blank, Lars Kuepfer, Nicola Zamboni, Uwe Sauer Institute of Molecular Systems Biology, ETH Zürich, Zürich 8093, Switzerland

Data on intracellular mRNA, protein, or metabolite concentrations reveal the composition of metabolic networks. In contrast to such compositional information, molecular fluxes through intact metabolic networks link genes and proteins to higher, system-level functions that result from interactions between the components (Hellerstein, 2003). Thus, fluxes are the functional output of the integrated biochemical and regulatory interactions within such networks, and are key data for metabolic systems biology. Since such system-level in vivo activities cannot be measured directly, they must be inferred indirectly from measurable quantities. The currently most reliable approach is metabolic flux analysis based on ${ }^{13}$ C-labeling experiments (Sauer, 2004). Despite the demonstrated relevance, analysis of metabolic fluxes has long remained a relatively complicated and tedious methodology. Recent developments (Fischer et al., 2004), however, have paved the road for routine fluxome analysis. On the basis of large-scale flux data from Bacillus subtilis mutants (Fischer and Sauer, 2005), we discuss design principles of carbon traffic that include network rigidity and robustness. By integrating the available genomic information into a genomescale stoichiometric model of metabolism (Förster et al., 2003), we then address the question whether or not such flux responses to gene deletions can be predicted with any certainty for the example of $S$. cerevisiae (Blank et al., 2005).

\section{References}

Blank, L.M., Kuepfer, L., Sauer, U., 2005. Large-scale ${ }^{13}$ C-flux analysis reveals mechanistic principles of metabolic network robustness to null mutations in yeast. Genome Biol. 6, R49.

Fischer, E., Zamboni, N., Sauer, U., 2004. High-throughput metabolic flux analysis based on gas chromatography-mass spectrometry derived ${ }^{13} \mathrm{C}$ constraints. Anal. Biochem. 325, 308-316.

Fischer, E., Sauer, U., 2005. Large-scale in vivo flux analysis reveals rigidity and sub-optimal performance of Bacillus subtilis metabolism. Nat. Genet. 37, 636-640.

Förster, J., Famili, I., Fu, P., Palsson, B.O., Nielsen, J., 2003. Genomescale reconstruction of the Saccharomyces cerevisiae metabolic network. Genome Res. 13, 244-253.

Hellerstein, M.K., 2003. In vivo measurement of fluxes through metabolic pathways: the missing link in functional genomics and pharmaceutical research. Annu. Rev. Nutr. 23, 379-402.

Sauer, U., 2004. High-throughput phenomics: experimental methods for mapping fluxomes. Curr. Opin. Biotechnol. 15, 58-63. 
3

Integration of transcriptomic, proteomic, and metabolic flux information for understanding and redesigning metabolism Liqing Wang, Vassily Hatzimanikatis Chemical Engineering, Northwestern University, Evanston, IL 60626, USA. Website: systemsbiology.northwestern.edu

Current knowledge of biological systems is composed by a vast set of data that accumulate with an increasing rate. Advances in analytical methods and development of sophisticated techniques and instrumentation have provided the tools that allow us to know more than we can understand. However, it is well understood that living organisms are characterized by high complexity. This complexity increases from unicellular organisms to isolated tissue cells and multicellular structures, such as tissues and organs. The development of tools and frameworks that will organize the available biological knowledge and will help in the analysis, understanding, and redesign of biological systems is of immediate importance. We will consider here the problem of understanding and redesigning metabolic networks. Understanding of the properties of metabolic networks is crucial, yet unaccomplished due to its high complexity, the different environmental conditions, and the uncertainty in the kinetic parameters of the enzymes in these networks. Knowledge of these kinetic parameters will enable understanding of the function of the network and quantitative prediction of the network responses to genetic and environmental perturbations. While information about mRNA and protein expression, and metabolic flux distribution is available, information about the enzyme kinetics in a metabolic network is significantly limited and, when available, is subject to high degree of uncertainty. We borrowed concepts and methods from risk analysis and decision-making under uncertainty, and we have developed decision-making methods based transcriptomic, proteomic, and metabolic flux information that are tailored to metabolic networks. These methods have been applied on the central carbon pathways of Escherichia coli metabolism. We have studied the distribution of the metabolic rate limiting steps in E. coli grown in a chemostat. We will discuss the distribution of the control of the enzymes on the rates of the substrate uptake and cell growth, and what are the practical implications of these findings.

4

Integration of metabolome and transcriptome profiles from perturbed chemostats of $S$. cerevisiae Pınar Pir ${ }^{1,2}$, Betül Kırdar ${ }^{1,2}$, Z.illsen Önsan ${ }^{1}$, Kutlu Ö. Ülgen ${ }^{1,2}, \overline{\text { Andrew Hayes }}{ }^{2}$, Stephen $G$. Oliver $^{2}$ : ${ }^{1}$ Department of Chemical Engineering, Boğaziçi University, Bebek 34342, İstanbul, Turkey; ${ }^{2}$ The Michael Smith Building, University of Manchester, Oxford Road, Manchester M13 9PT, UK. E-mail: pinarpir@boun.edu.tr (P. Pir)

Analysis of samples produced in perturbation experiments are regarded as the emerging experimental tool of functional genomics. In this study, three types of perturbations which are frequently used in functional genomics studies were investigated: gene deletion, limiting nutrient and dilution (growth) rate. S. cerevisiae cells were grown in chemostat reactors and samples were collected at steady state. mRNA expression levels were detected by using microarrays, and metabolome concentrations were measured by using enzymatic methods. Analysis of variance and PCA were applied to detect the sources of variation in transcriptome data. In these particular experi- ments, most effective source of variation was perturbation in growth medium, followed by perturbation in growth rate. Effect of gene deletion on data variation was found to be less apparent when compared to other perturbations. A significant similarity in variation of metabolome and mRNA data was observed, which may be used as the key point for integration of these two sets of data in functional analysis of genes. Projection to latent structures (partial least squares, PLS) is used for integration of transcriptome and metabolome data. Comparison of PCA and PLS shows that linear model constructed via PLS to predict the metabolome data does not make use of all the variation in transcriptome data. Thus, PLS allows the discrimination between the portion of gene expression change that affects the metabolome profile and the portion that is not directly effective on metabolome. Both PCA and PLS can be used to detect the open reading frames (ORFs) which are the main sources of variation in transcriptome data and/or effective on metabolome profile.

5

Extracellular metabolomics to accelerate the discovery of key genes involved in fibre degradation Silas G. Villas-Bôas, Geoffrey Lane, Graeme Attwood, Adrian Cookson AgResearch Limited, Grasslands Research Centre, Tennent Drive, Private Bag 11008, Palmerston North, New Zealand. E-mail: silas.villasboas@agresearch.co.nz (S.G. Villas-Bôas)

The genome of the hemicellulose-degrading microbe Clostridium proteoclasticum is been sequenced and an array of candidate genes with diverse activity relevant to fibre degradation have been identified by automated gene annotation methods. C. proteoclasticum falls within the Butyrivibrio-Pseudobutyrivibrio assemblage of rumen bacteria which are though to play an important role in the degradation of plant hemicellulose-lignin complexes which limit fibre degradation in the rumen. For New Zealand it makes strategic sense to invest in microbial genomics efforts applied to agriculture where the country holds a strong competitive advantage and where ruminants constitute the vast majority of farmed animals. In conjunction with DNA sequencing, proteomics and transcriptomics (micro-array analysis) we are using metabolomics as an additional Functional Genomics tool for gene discovery. We have established a footprinting approach for microbial metabolome analysis focused mainly on metabolic intermediates of polysaccharide degradation to provide quantitative information on end products of fibre-degrading enzymes. A GC-MS method has been developed that is able to resolve complex biological mixtures containing mono-, di, and oligosaccharides, in addition to a series of organic acids. We are currently phenotyping a series of $C$. proteoclasticum mutants to validate our analytical methodology and we are going to fully characterize the fibrolytic ability of $C$. proteoclasticum to be compared with other fibre-degrading microbes. We believe that our metabolomics data will complement current proteomic analysis of fibre-degrading enzymes and micro-array analysis of gene expression from a series of mutants by providing direct evidence of the metabolic function of key genes involved in fibre-degradation processes. 


\section{Genomics in Microbial Ecology and Medical Microbiology}

The microbial metagenome as a source of new biocatalysts Bert van Loo, Esther Gabor, Dick B. Janssen Biochemical Laboratory, Groningen Biomolecular Sciences and Biotechnology Institute, University of Groningen, 9747 AG, Groningen, The Netherlands. Email: d.b.janssen@chem.rug.nl (D.B. Janssen)

The microbial metagenome of selected environmental samples is a useful source for discovering new enzymes, either by highthroughput screening or by massive DNA sequencing. Especially with the first approach, many new enzymes have been identified for applications in biodegradation and biocatalysis, including nitrilases, epoxide hydrolases and amidases. Sequencing indicates that the vast majority of the biocatalysts that are obtained in this way are different from the enzymes that have been obtained by classical screening and gene cloning, showing that different segments of sequence space are explored by metagenomics approaches and classical enrichment. This is exemplified by several new amidases and a penicillin acylase that we have discovered. On the other hand, screening of environmental gene libraries may fail to retrieve rare enzymes that can only be obtained after enrichment, provided that the gene of interest is present in a selectable metabolic pathway. We have explored different genomic approaches for obtaining new epoxide hydrolases. Expression of putative epoxide hydrolase genes that were detected by analysis of sequenced microbial genomes was routinely possible after fusion at the gene level to maltose binding protein. The latter assisted in expression, folding and purification. It appeared that the putative epoxide hydrolases produced in this way indeed corresponded to active protein, and new enzymes with promising biocatalytic properties were discovered, such as an unusual $(S)$-enantiopreference for styrene epoxide and a highly enantioselective conversion of the meso-compound cyclohexene epoxide. The function of most of these epoxide hydrolases in microorganisms is unknown at this moment. The same holds for the structurally related haloalkane dehalogenases. Both groups of enzymes occur widespread in environmental DNA and in sequenced microbial genomes. We propose that many of the encoded proteins have a broad substrate range and that the corresponding DNA can serve as pool from which organisms acquire specific catabolic genes during genetic adaptation to xenobiotic carbon substrates.

\section{References}

Gabor, E.M., Alkena, W.B.L., Janssen, D.B., 2004. Quantifying the accessibility of the metagenome by random expression cloning techniques. Environ. Microbiol. 6, 879-886.

Gabor, E.M., de Vries, E.J., Janssen, D.B., 2004. A novel penicillin acylase from the environmental gene pool with improved synthetic properties. Enzyme Microb. Technol. 36, 182-190.

Janssen, D.B., 2004. Evolving haloalkane dehalogenases. Curr. Opin. Chem. Biol. 8, 150-259.

2

Mining quorum sensing regulated proteins - role of bacterial cell-to-cell communication in global gene regulation as assessed by proteomics Leo Eberl, Catalina Arevalo-Ferro, Paula Carranza, Kathrin Riedel Department of Microbiology, Technical University of Munich, Am Hochanger 4, D-85350 Freising, Germany
Many Gram-negative bacteria utilize cell-to-cell communication systems that rely on diffusible $N$-acyl homoserine lactone (AHL) signal molecules to monitor the size of the population in a process known as quorum sensing (QS). In human pathogens this form of gene regulation ensures that the cells remain invisible to the immune system of the host until the pathogen has reached a critical population density sufficient to overwhelm host defenses and to establish the infection. The QS regulon of Pseudomonas aeruginosa and Burkholderia cepacia, two important pathogens for patients suffering from cystic fibrosis, has been studied by proteome analyses. Comparative twodimensional gelelectrophoresis of pre-fractionated protein mixtures (extra-, surface-, and intracellular proteins) coupled to mass spectrometry analysis or $\mathrm{N}$-terminal sequencing has been employed to recognize and identify QS-controlled proteins. Our findings strongly support the importance of AHL-mediated cell-cell-communication as a global regulatory system and suggest that QS control also operates via post-translational mechanisms. As QS has been proven to be a central regulator for the expression of pathogenic traits and biofilm formation in opportunistic human pathogens it represents a highly attractive target for the development of novel anti-infective compounds. Functional genomics technologies (transcriptomics and proteomics) have been exploited to validate the target specificity of natural and synthetic QS inhibitors, thus having a great potential as alternative therapeutics for the treatment of bacterial infections.

3

Salmonella isolates with different behavior in adhesion to surfaces studied by DNA micro array assisted analysis Kim Holmstrøm ${ }^{1}$, Katja Nyholm Olsen ${ }^{2}$, Helle Meldgaard ${ }^{3}$, Dorthe Bagge-Ravn $^{3 *}$, Dorte Frees ${ }^{2}$, Dorte Lauritsen ${ }^{1}$, Lone Gram ${ }^{3}$, John Elmerdahl Olsen ${ }^{2}:{ }^{1}$ Bioneer A/S, Hørsholm DK-2970 Denmark; ${ }^{2}$ Department Veterinary Pathobiology, RVAU, Frederiksberg C. DK1870, Denmark; ${ }^{3}$ Department Seafood Research, Danish Institute for Fisheries Research, Kgs. Lyngby DK-2800, Denmark; *Present address: Chr. Hansen A/S, Roskilde DK-4000, Denmark

A number of Salmonella enterica strains representing serotypes Enteritidis PT4, Typhimurium DT104, 4,12,b:, and Liverpool were collected and screened for adhesion to plastic as well as steel surfaces and for the ability to grow and survive under different stress conditions. Strains were identified having different phenotypes from other strains of the same serotype. To elucidate the differences in adhesion and growth properties we employed DNA micro array technology using a gene array containing 166 selected genes from the fully sequenced $S$. enterica serotype Typhimurium These genes represent only a small subset of the entire genome, but are believed to contribute in fundamental functions of the bacteria involved in persistence, virulence and stress adaptation. To compare different strains a strategy was devised based on comparing normalized expression profiles of the strains under different physiological conditions, e.g. exponential growth, heat and osmotic stress. Results of these analyses will be presented.

\section{Tools in Bioinformatics}

1

Temporal interactome for the eukaryotic cell cycle by data integration Søren Brunak Center for Biological Sequence Analysis, Technical University of Denmark. E-mail: brunak@cbs.dtu.dk 
Improved understanding of many complex, cellular mechanisms, such as the eukaryotic cell cycle, necessitates the identification of genes and proteins that belong to broad "systemic" categories, where the diversity in terms of molecular functionality is high. This also holds true for most cellular compartments, for example, the nucleolus or the mitochondrion. Similarly, proteins that are transported, for example secreted, share properties no matter whether they are translocated by the classical secretory pathway, or by alternative, non-classical pathways. For this kind of functional classification, we have constructed a new approach where putative posttranslational modifications, sorting signals, predicted structural features, and calculated features such as chain length, amino acid composition, isoelectric point, hydrophobicity are integrated and used to infer the functional class, which may be broad, like "cell cycle regulated", "secreted", "nucleolar", or "stress response", but also narrow as for the conventional Gene Ontology categories, like ligand-gated ion channel, etc. The approach predicts functional role categories in the "feature" space of the proteome, rather than using the "sequence" space of the genome. One remarkable result from the work is that many proteins seem to display conservation in feature space rather than in sequence space, and the method will therefore be able to transfer functional information from one species to another in new ways. This type of prediction can be integrated with experimental data, such as gene expression data and protein-protein interaction data, and interaction networks can be extracted and characterized. The talk will focus on a protein feature analysis of cell cycle regulated proteins in yeast and man, in addition to a compartment-specific analysis of the human nucleolus.

\section{References}

de Lichtenberg, U., Jensen, T.S., Jensen, L.J., Brunak, S., 2003. Protein feature-based identification of cell cycle regulated proteins in yeast, J. Mol. Biol. 329, 663-674.

de Lichtenberg, U., Jensen, L.J., Brunak, S., Bork, P., 2005. Dynamic protein complex formation during the cell cycle, Science, 307, 724-727.

Dyrlov Bendtsen, J., Jensen, L.J., Blom, N., von Heijne, G., Brunak, S., 2004. Feature based prediction of non-classical protein secretion, Protein Eng. 17, 349-356.

2

Bioinformatics database infrastructure for biotechnology research RolfApweiler The European Bioinformatics Institute, Wellcome Trust Genome Campus, UK

The increasing sophistication to store, manipulate and communicate information has transformed the way life scientists work. The information of life - DNA coding for complex proteins involved in intricate biological processes - has become accessible during the last decades; fortuitously an era when computer hardware and methodology has seen a comparable revolution. The way scientists deal with data has been completely transformed. It is possible to collect, analyse, communicate and share huge amounts of information rapidly and accurately. Molecular biology, driven by the need to deal with large volumes of information, was quick to build large collections of shared scientific information. Substantial international efforts now support databases of nucleotide sequences, protein sequences and protein structures. Aside from these major projects, numerous other shared information repositories developed, and the research in pro- teomics will lead to additional databases storing large amounts of biological data in different, often quite specialised databases. However, while there is a vast amount of valuable information, it often exists as islands, with little interconnection, it can be ill defined and difficult to use, and there is little to help the user distinguish between high quality and low quality information. Improvements in exploiting this information depends on database integration as well as rigorous usage of controlled vocabularies and common standards to describe the data, and it is on such efforts like InterPro, UniProt, GeneOntology, and Integr8 projects that my talk will concentrate.

\section{3}

Towards system-level understanding of microbial metabolism Kiran R. Patil, Jens Nielsen Center for Microbial Biotechnology, BioCentrum-DTU, Technical University of Denmark, DK-2800 Kgs. Lyngby, Denmark. E-mail: krp@ bocentrum.dtu.dk (K.R. Patil)

Metabolism plays a key role in providing Gibbs free energy and precursors for biosynthesis and cellular functionality. Consequently, cellular response to genetic and environmental perturbations is often reflected and/or mediated through changes in the metabolism. One of the challenges in understanding operation of complex metabolic networks is to uncover transcriptional regulatory machinery coordinating activities of different pathways. It is difficult to map such global transcriptional responses by using traditional methods, as many genes in the metabolic network have relatively small changes at their transcription level. We therefore developed a novel algorithm that is based on systems view of metabolism as a whole, to uncover the transcriptional regulatory architecture of metabolic networks. By using information on the metabolic network topology from genome-scale metabolic reconstruction, we show that it is possible to reveal patterns in the metabolic network that follow a common transcriptional response. Thus, the algorithm enables identification of so-called reporter metabolites (metabolites around which the most significant transcriptional changes occur) and a set of connected genes with significant and coordinated response to genetic or environmental perturbations. We identified the reporter metabolites for several transcriptional datasets for the yeast $S$. cerevisiae. We also show that the proposed algorithm is robust towards the missing information in the metabolic models. We conclude that cells respond to perturbations by changing the expression pattern of several genes involved in the specific part(s) of the metabolism where a perturbation is introduced. These changes are then propagated through the metabolic network and the essence of these changes is reflected in, and quantified by, reporter metabolites and sub-networks identified.

\section{4}

STRING: Modeling of biological systems through cross-species data integration Lars Juhl Jensen European Molecular Biology Laboratory, Meyerhofstrasse 1, 69117 Heidelberg, Germany. E-mail: Jensen@embl.de

To model a biological system, the interactions among its constituents must be known. From a functional perspective, "interaction"can mean anything from direct physical binding to indirect functional association such as participation in the same metabolic pathway or cellular process. Such interactions can be inferred from a wide variety of large-scale data types, which are unfortunately currently scattered over numerous resources and model organisms. STRING aims to simplify the access to this information by providing 
a comprehensive, quality-controlled collection of both known and predicted protein interactions for a large number of organisms. The interactions are derived by re-analyzing high- and low-throughput experimental data, by mining biological databases and literature, and by genomic context analysis of 180 fully sequenced genomes. STRING assigns quality scores to each of these interactions, which allow the very different evidence types to be combined, and automatically transfers interactions to orthologous protein pairs in other organisms. This consistent cross-species integration of diverse data types enables the extraction of high-confidence protein interaction networks for many biological processes in various model organisms, e.g. the yeast mitochondria system. STRING is available via a web interface at http://string.embl.de/.

5

3D-MCSIS BUILDER: A new generation of molecular class specific information systems Henk-Jan Joosten ${ }^{1}$, Simon Folkertsma ${ }^{2}$, Frank van Zimmeren ${ }^{1}$, Remko Kuipers ${ }^{1}$, Erik Ittmann ${ }^{1}$, Peter Schaap ${ }^{1}$, Gert Vriend ${ }^{2}$ : ${ }^{1}$ Fungal Genomics, department of Micro Biology, Wageningen University and Research Center, Wageningen, The Netherlands; ${ }^{2}$ Centre for Molecular and Biomolecular Informatics (CMBI), Radboud University of Nijmegen, Nijmegen, The Netherlands

A major difficulty for scientists that want to gain new biological insights in a protein (family) is correlating the sheer amount of data available on the Internet and in the literature. This data is often heterogeneous and divided over multiple databases, making it difficult and time consuming to make correlations. Moreover, protein sequences from different databases or homologues sequences from different organisms have an incongruent amino acid numbering. To cope with these problems, protein superfamily-specific databases (Horn et al., 2001) are needed, that organize data around a single class of molecules using a common numbering scheme for structurally equivalent amino acids. Here we describe a system (3DMCSIS Builder) that can automatically build protein superfamily databases in which every amino acid is individually stored and connected via a structure based multiple sequence alignment (3D-MSA). The system starts with building a large accurate 3D-MSA of a class of proteins (superfamily). Such alignments can be built by making separate subfamily alignments starting from structures belonging to the different subfamilies. These separate subfamily alignments can be aligned to each other by determining the structurally equivalent amino acid positions using a superpositioning of the starting structures. In the first step structure files are automatically superimposed and a common core of structurally conserved amino acid positions is defined to which a $3 \mathrm{D}$ numbering scheme is applied. Then profiles derived from the starting structures, with high gap-penalty scores at structurally conserved positions, are used to build accurate subfamily multiple sequence alignments. 3D-MCSIS Builder stores every amino acid from the resulting 3D-MSA in the database together with its corresponding 3D number. Storing separate amino acids with their 3D number has a number of advantages. Firstly, it solves the problem of inconsistent numbering. The numbering used in the database of origin is also stored, enabling easy navigation between the different numbering schemes. Secondly, it enables storage of amino acid associated data, like mutational information (Van Durme et al., 2003), ligand- and substrate contacts, protein interaction sites, phospho- rylation sites, hydrogen-bonding status, solvent accessibility, etc. in relation to the amino acids. Thirdly, evolutionary information, such as correlated mutations or conservation of amino acid positions, intrinsically present in the alignment is directly coupled to all other amino acid related information stored in the database (Folkertsma et al., 2004). In addition, all this information can easily be mapped in a 3D environment because the system automatically makes homology models of all sequences in the alignment and it also incorporates the 3D numbering scheme in all models as well as in all starting structures. The alignment and all associated data are visualized and can be retrieved via interactive HTML pages (www.systemsbiology.nl/3DMCSIS/). By clicking on the amino acid in the alignment the user can retrieve all data related to that specific amino acid.

\section{References}

Folkertsma, S., van Noort, P., Van Durme, J., Joosten, H.J., Bettler, E., Fleuren, W., Oliveira, L., Horn, F., de Vlieg, J., Vriend, G., 2004. A family-based approach reveals the function of residues in the nuclear receptor ligand-binding domain. J Mol Biol. 341 (2), 321-335.

Horn, F., Vriend, G., Cohen, F.E., 2001. Collecting and harvesting biological data: the GPCRDB and NucleaRDB information systems. Nucleic Acids Res. 29 (1), 346-349.

Van Durme, J.J., Bettler, E., Folkertsma, S., Horn, F., Vriend, G., 2003. NRMD: nuclear receptor mutation database. Nucleic Acids Res. 31 (1), 331-333.

www.systemsbiology.n1/3D-MCSIS/.

\section{Poster Abstracts Genomics and Systems Biology (GSB)}

\section{GSB1}

Evolutionary engineering of osmotolerant Saccharomyces cerevisiae T. Aloğlu ${ }^{1,2}$, C. Tamerler $^{1,2}$, U. Sauer ${ }^{1,2}$, Z.P. Çakar ${ }^{1,2}$ : ${ }^{1}$ Istanbul Technical University, TR-34469 Istanbul, Turkey; ${ }^{2}$ Institute of Biotechnology, ETH Zurich, CH-8093 Zurich, Switzerland. E-mail: aloglu@itu.edu.tr (T. Aloğlu)

In industrial yeast bioprocesses, yeast cells frequently encounter osmotic stress. As the principles of osmoadaptation are conserved across eukaryotes, cellular response to hyperosmolarity in the baker yeast as a model organism has attracted attention. An evolutionary engineering approach was applied in this study to select for osmotolerant Saccharomyces cerevisiae mutants. For this purpose, batch selection protocols were applied to a chemically mutagenized, diverse population. Each new generation of mutants was obtained either at the same constant, mild osmotic stress level, or by gradually increasing the osmotic stress level (i.e. increasing the concentration of $\mathrm{NaCl}$ ). To determine the stress resistance levels and the relative survival level compared to wild type $S$. cerevisiae, a high throughput procedure in 96-well plates was employed based on the most probable number (MPN) method. Mutant individuals selected randomly from final mutant populations were tested for their resistance and survival levels. Additionally, they were also tested for their cross resistance to other common stresses that yeast cells encounter during industrial bioprocesses. The different stress resistances of the mutants are compared and discussed in this study. 


\section{GSB2}

Integrated approach to reconstruct signal transduction networks K.Y. Arga ${ }^{1}$, B. Kirdar ${ }^{1}, K . \ddot{\text { Ulgen }}{ }^{1}$, J. Nielsen ${ }^{2}$ : ${ }^{1}$ Department of Chemical Engineering, Boğaziçi University, 34342 Istanbul, Turkey; ${ }^{2}$ Center of Microbial Biotechnology, Technical University of Denmark, Lyngby DK-2800, Denmark. E-mail: argakazi@boun.edu.tr (K.Y. Arga)

As interaction networks become increasingly large and complex, there is a growing need to break them down into more manageable subnetworks representing groups of proteins that together contribute to the same cellular function. The approach we have presented allows one to simultaneously browse protein-protein interaction data, gene expression data, gene ontology annotations by means of structural analysis tools in order to extract a group of highly-connected and -correlated proteins from global data and to isolate a subnetwork of particular interest without knowledge of pathway intermediates. We showed that this approach enables the reconstruction of the glucose induction signaling pathway successfully, also predicting "candidate" proteins to be tested for further analysis. The integrated approach can be used to (i) predict new signaling pathways, (ii) identify unknown members of documented pathways, (iii) represent potential drug targets, (iv) identify network modules consisting of a group of related components that often incorporate the same functional mechanism, or (v) identify smaller correlated clusters of interacting proteins.

\section{GSB3}

Structural robustness of protein interaction networks are due to modularity K.Y. Arga ${ }^{1}$, Z.I. Önsan ${ }^{1}$, B. Kırdar ${ }^{1}$, J. Nielsen $^{2}, K$. Ülgen ${ }^{1}:{ }^{1}$ Department of Chemical Engineering, Boğaziçi University, Istanbul 34342, Turkey; ${ }^{2}$ Center of Microbial Biotechnology, Technical University of Denmark, Lyngby DK-2800, Denmark. E-mail: argakazi@ boun.edu.tr (K.Y. Arga)

One of the major reasons for the increased complexity of biological networks is to improve stability and robustness. Robustness provides in-depth understanding on how the system maintains its functional properties against various disturbances (mutations, variations in protein levels, aging, etc.). Several biological systems have been found to be robust at a variety of levels from genetic switches to physiological reactions. Such properties are achieved through feedback mechanisms, modularity, redundancy and structural stability. Not all biological systems are robust and it is important to know which parts of the systems are not robust and why. In this study, we examined the robustness of protein interaction networks of several species (C. elegance, S. cerevisiae, and D. melanogaster) from different origin. Topological and pathway analysis is used in determination of network robustness. In order to test whether the protein interaction network is robust against errors, we perturbed the network by knockout of a protein. The average robustness parameter of each network was varying between 0.88 and 0.98 , indicating very high robustness independent of species and origin. We are also concerned with how protein interaction networks achieve robust performance despite facing significant fluctuations in their internal conditions. For each species, the proteins that have low robustness parameters are investigated and a local part of the network that is not robust is deducted. The results come with the conclusion that the robustness is maintained by modular design, which pre- vents the spread of damage in one part of the system to the entire system.

\section{GSB4}

Bacterial NER and the genetic stability of the CTG/CAG repeats Marta J. Majchrzak ${ }^{1,2}$, Pawel Parniewski ${ }^{1}$ : ${ }^{1}$ Centre for Medical $\overline{\text { Biology PAS, } 106}$ Lodowa Str., 93-232 Lodz, Poland; ${ }^{2}$ Faculty of Biotechnology and Food Sciences, Technical University of Lodz, Stefanowskiego 4/10 Str., 90-924 Lodz, Poland. E-mail: martinam@cbm.pan.pl (M.J. Majchrzak)

Neurogenetic diseases including myotonic dystrophy (DM), fragile X syndrome, Huntington's disease, spinobulbar muscular atrophy, spinocerebellar ataxias, and Friedreich's ataxia result from expanded triplet repeat sequences (TRS) CTG/CAG, CGG/CCG, or GAA/TTC within their genes. Long tracts of TRS are unstable and show repeat size polymorphism in successive generations and in different tissues. In addition to observations in humans, TRS instabilities have been demonstrated in Escherichia coli, yeast, transgenic mice, and cultured cells from patients and are influenced by factors including host strain genotypes, DNA replication, methyldirected mismatch repair (MMR), growth conditions, transcription, and nucleotide excision repair (NER). This study demonstrates further analyses on effect of mutations of different NER genes on the genetic stability of long CTG/CAG motifs. Also, the influence of length (ranging from 80 to 175 repeats), composition (presence of $\mathrm{G}$ to A interruptions) and orientation of the TRS will be discussed.

\section{Acknowledgement}

This work was partially funded by the KBN grant 2 P05A 00926.

\section{GSB5}

Identification of potential virulence factors in the genomes of pathogenic fungi $J$. Varga ${ }^{1}, S$. Kocsubé ${ }^{1}$, B. Tóth ${ }^{2}$, T. Papp $^{1}, C$. Vágvölgyi ${ }^{1}:{ }^{1}$ Department of Microbiology, Faculty of Sciences, University of Szeged, P.O. Box 533, H-6701 Szeged, Hungary; ${ }^{2}$ Cereal Research non-Profit Company, P.O. Box 391, H-6701 Szeged, Hungary. E-mail: pappt@bio.u-szeged.hu (T. Papp)

Fungal polyketide synthases (PKSs) and nonribosomal peptide synthetases (NRPSs) are responsible for the biosynthesis of several mycotoxins and other secondary metabolites which serve as virulence factors in several plant-pathogen interactions. We searched the public genomic databases of Fusarium graminearum, Aspergillus fumigatus and A. nidulans using PKS and NRPS genes of other fungi, and identified several homologues by bioinformatic techniques. Our search identified 16, 14 and 27 putative PKS gene sequences in the genomes of $F$. graminearum, A. fumigatus and A. nidulans. None of the putative $F$. graminearum PKS genes was found to belong to the MSAS type of fungal PKSs. One putative PKS exhibited more than $90 \%$ homology to a PKS identified in Gibberella fujikuroi indicating that these PKSs are possibly responsible for the biosynthesis of the same metabolite. Clustering of the ketosynthase domain sequences of the identified PKS genes correlated well with the type of metabolites produced by the corresponding PKSs. For NRPS genes, 15, 14 and 14 were found in the genomes of F. graminearum, A. fumigatus and A. nidulans. One of these NRPS genes was found to be closely related to peptide synthetases of various fungi taking part in ferrichrome biosynthesis, while another NRPS gene was similar to that identified in A. oryzae which is possibly 
responsible for the biosynthesis of fusarinine, an extracellular ironchelating siderophore. To our knowledge, this is the first report on the identification of a putative NRPS gene possibly responsible for the biosynthesis of fusarinine-type siderophores. Further studies are in progress to clarify the role of some of the identified PKS and NRPS in pathogenesis. This work was supported by OTKA grants (D38486, T037217)

\section{GSB6}

(CGG) $n$ microsatelite as a useful tool for diagnosis of Tuberculosis using a PCR assay Pawel Parniewski, Jaroslaw Dziadek Centre for Medical Biology PAS, 106 Lodowa Str., 93-232 Lodz, Poland. E-mail: parniew@cbm.pan.pl (P. Parniewski)

Trinucleotide sequences (TRS) are very often found in bacterial genomes. It has been demonstrated that (CGG)n repeat is overrepresented in Mycobacterium tuberculosis and may be used as a hybridization probe in diagnosis and epidemiology of this pathogen. The (CGG)5 repeat is a unique sequence for some mycobacterial species, and (CGG)5 fingerprinting can be used as an epidemiologic method for these species as well as IS6110 fingerprinting can. (CGG)5 fingerprinting patterns were relatively more stable than IS6110 patterns. I this work we demonstrate that (N)4(CGG)4 sequence may be successfully used as a single oligonucleotide starter for PCR analyses of various Mycobacterium species.

\section{Acknowledgement}

This work was partially funded by the KBN grants 2 P05A 00926 and 2 P05A 01927.

\section{GSB7}

Analysis of gene expression profiles at chromosomal level Andrea Callegaro, Silvio Bicciato Department of Chemical Engineering Processes, University of Padova, Padova, Italy. E-mail: silvio.bicciato@unipd.it (S. Bicciato)

Transcriptional profiling of whole genomes using $c \mathrm{DNA}$ or oligonucleotide high-density arrays is becoming increasingly popular among the biomedical research community. Although advances in technology and the rapid rise in microarray data availability are leading to new insight into fundamental biological problems, investigators are still confronted with the major problem of upgrading the information content of regulated gene lists obtained from microarray experiments. Indeed, the efficient exploitation of gene expression databases requires not only computational tools for management, analysis, and functional annotation of primary data, but also integrating lists of modulated genes with of other sources of genomic information, such as gene sequence, locus or structural characteristics. In particular, integration between expression profiles and chromosomal localizations could be effective in detecting gene structural abnormalities such as genomic gains and losses and/or translocations. The aim of the present study is to apply computational tools for mapping transcriptional data at chromosomal level and detecting clusters of regionally modulated genes in cancer specimens. Statistical tests and signal processing procedures are used to integrate expression profiles and gene sequence information and identify peculiar regions of modulated expression. In particular, the method is based on the application of a smoothing, coordinate-dependent function (e.g., cubic splines) to a standard transcriptional specificity statistic (e.g., standard $F$-statistic), commonly used to detect differentially expressed genes. This computational tool has been tested on different microar- ray data sets obtained from various human tumor samples (e.g., solid tumors and hematological disorders). In particular, the application of chromosomal level analysis to the transcriptional database presented by Bhattacharjee et al. (2001), Armstrong et al. (2002), and Ross et al. (2003) allowed the detection of regional signals corresponding to known as well as putative loci with high frequent genomic losses and gains or marking translocation events.

\section{References}

Armstrong, S.A., et al., 2002. Nat Genet 30 (1), 41-47.

Bhattacharjee, A., et al., 2001. Proc. Natl. Acad. Sci. U.S.A. 98 (24), 13790-13795.

Ross, M.E., et al., 2003. Blood 102 (8), 2951-2959.

\section{GSB8}

Metabolic fingerprinting of the deer's antler using nuclear magnetic resonance spectrometry Hyun-Jung Kim ${ }^{1}$, Kyoung-Hee Kim $^{2}$, Whan-Kyun Hwang ${ }^{1}$, Min-Won Lee ${ }^{1}$, Hyung-Kyoon Choi ${ }^{1}$ : ${ }^{1}$ College of Pharmacy, Chungang University, Seoul 156-756, South Korea; ${ }^{2}$ Korea Drug Test Laboratory, Seoul 130-060, South Korea. E-mail: hykychoi@cau.ac.kr (H.-K. Choi)

Metabolic fingerprinting of various species of deer's antlers was tried using nuclear magnetic resonance spectrometry (NMR). Simple and reproducible preparation method was developed by $50 \%$ methanol and chloroform solvent system. Tentative assignment of the peaks was performed and the major peaks assigned in the 50\% methanol fraction were various kinds of amino acids and organic acids. The peaks in the chloroform fraction were proton signals derived from fatty acids or lipids. It was possible to differentiate each sample according to the species of various deer's antlers by visual inspection of the NMR spectra, and it means that we can differentiate deer's antlers from different species by NMR based technique. The multivariate statistical analysis of the chemical shift and signal intensity will be conducted to clearly show the difference of each sample's spectra and to elucidate the compounds contributing to the differentiation of each samples.

\section{GSB9}

Stoichiometric modeling of the human red blood cells Saliha Durmuş, Tunahan Çakır, Kutlu Ö. Ülgen Department of Chemical Engineering, Boğaziçi University, Bebek, Istanbul 34342, Turkey. Email: saliha.durmus@boun.edu.tr (S. Durmuş)

The human red blood cell (RBC) metabolism is investigated by calculating steady state fluxes using stoichiometric modeling approaches; flux balance analysis (FBA), method of minimization of metabolic adjustment (MOMA) and regulatory on-off minimization (ROOM). For the wild type RBC metabolism, FBA is performed via optimization of various objective functions and the maximization of the production of ATP and NADPH is found to be the primary objective of the RBC metabolism since the optimization results have a good correlation with the kinetic models. To predict the flux distribution of the network in enzyme deficiencies, FBA and two novel approaches, MOMA and ROOM, which can describe the behavior of the metabolic networks in the case of enzymopathies, are applied. The deficiencies in G6PDH, TPI, PGI, DPGM and PGK are investigated and the flux distributions are compared with the wild type case to observe the changes in response to enzyme deficiencies. The stoichiometric modeling results are found to be in accordance with the 
experimental findings in the literature related to metabolic behavior of the human red blood cells.

\section{GSB10}

An experimental screen for genes that have undergone a period of accelerated evolution Antoine Le Quéré ${ }^{1}$, Kasper Astrup Eriksen ${ }^{2}$,

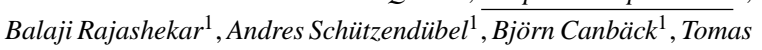
Johansson ${ }^{1}$, Anders Tunlid ${ }^{1}$ : ${ }^{1}$ Department of Microbial Ecology, Lund University, Ecology Building, SE-223 62 Lund, Sweden; ${ }^{2}$ Department of Theoretical Physics, Lund University, Sölvegatan 14A, SE-22362 Lund, Sweden. E-mail: kasper@thep.lu.se (K.A. Eriksen)

Many closely related organisms are adapted to slightly different environments. It is of interest to identify the genes that primarily are involved in these adaptive shifts. During the shift in phenotype these genes are expected to experience a period of accelerated evolution. Standard tools to detect accelerated evolution include $\mathrm{dn} / \mathrm{ds}$ analysis and comparing the branch lengths in three-species trees. Unfortunately these methodologies require whole genome sequence information, something that typically not is available for all the strains with an interesting phenotype. Three-species tree comparison. $\mathrm{B}$ and $\mathrm{C}$ are two closely related species or strains that display different phenotypes. A is a distantly related outgroup. The exemplified gene has undergone accelerated evolution in the C-branch. We have modified the three-species comparison technique such that it can be applied on CGH data. In the CGH technique genomic DNA from the relevant strains (B and C) is hybridized on a standard microarray. Thus, CGH data are easily obtainable when a micro-array is available for one of the strains. The method has been used to study the genomic basis for host preference in symbiotic fungi (Paxillus sp.).

\section{GSB11}

Modeling cell cycle complex formation from high-throughput data sets Lars Juhl Jensen European Molecular Biology Laboratory, Meyerhofstrasse 1, 69117 Heidelberg, Germany. E-mail: Jensen@embl.de

To analyze the dynamics of protein complexes during the mitotic cell cycle, we integrated data on protein interactions and gene expression. The resulting time-dependent interaction network for the first time places both periodically and constitutively expressed proteins in a temporal cell cycle context, thereby revealing novel components and modules. We discover that most complexes consist of both periodically and constitutively expressed subunits, suggesting that the former control complex activity by a mechanism of just-in-time assembly. Consistent with this, we show that additional regulation through targeted degradation and phosphorylation by Cdk1 (Cdc28p) specifically affects the periodically expressed proteins.

\section{GSB12}

Analysis of un-replicated time-course microarray experiments Alessandra Luchini, Andrea Callegaro, Silvio Bicciato Department of Chemical Engineering Processes, University of Padova, Padova, Italy. E-mail: alessandra.luchini@unipd.it (A. Luchini)

Since transcriptional control is the result of complex networks, analyzing dynamical states of gene expression is of paramount importance to detect the multivariate nature of biological mechanisms. Although hundreds of studies fully demonstrated the relevancy of microarrays in describing different physiological conditions, to reconstruct complex interaction pathways it is necessary to analyze the temporal evolution of transcriptional states. However, a robust experimental design for identifying differentially expressed genes over a temporal window would require large amounts of microarrays. Unfortunately, replicates for each time point and experimental condition are not always available, because of cost limitations and/or biological samples scarcity. In addition, common data analysis tools, like ANOVA, require replicates and disregard correlation structure among times. We present a method for the identification of differentially expressed genes in un-replicated time-course experiments. The procedure does not assume any model or distribution function, takes into account the correlation of data, and does not require sample replicates at the various time points, other than the presence of an initial time point for all analyzed conditions. The identification of differentially expressed genes as the result of a system perturbation is formally stated as a hypothesis testing problem in which a defined statistic is used to rank transcripts in order of evidence against the null hypothesis. Specifically, (i) data are structured so that measurements are correlated in time, within the same biological condition; (ii) the null hypothesis is formulated so that changes in expression levels at different time points are equivalent; (iii) time point t0 represents the system before the perturbation. Therefore, modulated genes are detected testing the statistical significance of expression differences between physiological states at each time point, once corrected by the variability at $\mathrm{t} 0$, and given an empirical null distribution constructed using permutations. Statistical significance is assessed by the $q$-value. The method has been tested on time-course microarray experiments aimed at studying the temporal changes of gene expression in: (i) skeletal muscle cells treated with a histone deacetylase inhibitor (Iezzi et al., 2004) and (ii) immature mouse dendritic cells (DC) exposed to larval and egg stages of S. mansoni (Trottein et al., 2004). Differentially expressed genes, identified using the proposed algorithm, have been compared with results obtained from ANOVA model and SAM paired test. The biological significance and soundness of selected transcripts was also verified using global functional profiling by means of OntoTools. Results demonstrate that this novel procedure allows the identification of biologically relevant genes using half of the replicates required by standard model-based approaches.

\section{References}

Iezzi, et al., 2004. Dev. Cell. 6 (5), 673-684.

Trottein, et al., 2004. J. Immunol. 172, 3011-3017.

\section{GSB13}

Gene expression profiling of rat liver after in utero exposure to the fungicide prochloraz using GeneChip ${ }^{\circledR}$ Rat Genome Array Stine B. Metzdorff, Peter Laier, Majken Dalgaard, Ulla Hass, Anne Marie Vinggaard Danish Institute for Food and Veterinary Research, Denmark. E-mail: metz@dfvf.dk (S.B. Metzdorff)

Exposure to environmental agents during foetal life may possibly be associated with the declining male reproductive health observed in industrial countries. Prochloraz, a commonly used imidazole fungicide, has recently been identified as a novel endocrine disruptor that acts as an antiandrogen by blocking the androgen receptor and inhibiting fetal steroidogenesis, though the precise mechanistic action remains unknown. Imidazole fungicides have the potential to 
inhibit activities of a broad spectrum of cytochrome P450-dependent enzymes, and as most P450s are located in liver cells, reproductive toxic effects in liver from male rat fetuses were examined at the transcriptional level after in utero exposure to prochloraz in order to reveal molecular targets affected by this endocrine disruptor. Dams were exposed from GD7 with two doses of prochloraz; 50 and $150 \mathrm{mg} / \mathrm{kg} /$ day. Dams were taken for caesarean sections at GD21 and different organs and blood were taken from male rat fetuses. Testosterone and progesterone levels in testis and plasma revealed that prochloraz significantly reduced plasma and testicular testosterone levels, whereas testicular progesterone was increased, indicating steroidogenesis inhibition in GD21 male fetuses. Total RNA from liver was extracted and used for hybridisation on Affymetrix GeneChip ${ }^{\circledR}$ Rat Genome 2302.0 Arrays with three slides per dose. Preliminary results from comparative analysis of control and exposed animals indicate deregulation of several genes including Сур $3 a 3$.

\section{GSB14}

Construction of a genome-wide defined mutation library of Aspergillus nidulans Jakob Blaesbjerg Nielsen, Michael Lynge Nielsen, Jens Nielsen, Uffe Hasbro Mortensen Center for Microbial Biotechnology, BioCentrum-DTU, Denmark. E-mail: jbn@biocentrum.dtu.dk (J.B. Nielsen)

In recent years, advancements in high throughput sequencing and bioinformatics have resulted in the accumulation of numerous complete genome sequences. Although this sequence information has already proved useful, a tremendous potential exists to further advance biotechnology, provided that novel molecular techniques are developed, to rapidly and reliably allow precise site-directed genomic modifications. Unfortunately, development of gene targeting techniques in filamentous fungi and many other higher eukaryotes, have been hampered by the fact that foreign DNA is predominantly integrated randomly into the genome and not at the desired sites. Since efficient gene targeting relies on the homologous recombination we have focused our research on developing targeting methods that use substrates that are preferred by the homologous recombination pathway. We have found that the targeting efficiency can be improved by transforming protoplasts with DNA substrates where the marker is divided into two parts. These "bi-partite" substrates have to be processed by homologous recombination in order to constitute a functional marker. It seems that selecting for homologous recombination of the marker increases the likelihood that the rest of the fragment will integrate by the same pathway. Since the bi-partite targeting fragments are halved in size, their generation by PCR is facilitated. We intend to use this method for high throughput generation of knockouts in A. nidulans, and thus initiate a genome-wide mutant library. The mutant library will primarily contain full gene deletions; however the method can easily be adapted for the introduction of specific point mutations by allele replacements, promoter swaps, and tagging with fluorescent markers. In an effort to improve targeting efficiency further, constructions of null strains, defective in some of the NHEJ pathway genes is currently under way.

\section{GSB15}

Genetic stability of direct and inverted repeats in Aspergillus nidulans Michael Lynge Nielsen, Line Albertsen, Uffe Mortensen Center for Microbial Biotechnology, BioCentrum-DTU, Technical University of Denmark, 2800 Kgs. Lyngby, Denmark. E-mail: mln@biocentrum.dtu.dk (M.L. Nielsen)

Homologous recombination facilitates error free repair of DNA double strand breaks because it uses a homologous sequence as a template for the repair. However, most genomes contain repetitive sequences such as transposable elements, rDNA, centromeric repeats, etc., which in some cases cause inadvertent homologous recombination leading to chromosomal translocations or genomic instabilities. In many filamentous fungi, including Aspergillus nidulans, transformation of recombinant DNA often leads to the integration of multiple copies of this DNA either as inverted or tandem repeats. For many industrial applications transformants with multiple gene copies are obtained from screening because such strains often have high gene expression levels, but consequently, the repeated sequences result in genetic instability. For filamentous fungi there are no accurate methods for assessing this type of genetic instability. Therefore, we have developed a sensitive conidiospore color assay in A. nidulans that allows the detection of as little as a single spontaneous or induced recombination event within a fungal colony and also determines whether the event proceeded as a loop-out or a gene conversion event. The assay provides a unique way to measure the recombinogenic effects of specific direct or inverted repeats, and can effectively be used to measure the genotoxicity of compounds or the effect of various gene mutations on recombination.

\section{GSB16}

An integrative and probabilistic approach for discovering regulatory modules in molecular interaction networks Ana Paula Oliveira, Kiran Raosaheb Patil, Jens Nielsen CMB, BioCentrum-DTU, Technical University of Denmark, DK-2800 Kgs. Lyngby, Denmark. E-mail: apo@ biocentrum.dtu.dk (A.P. Oliveira)

In metabolic engineering and cellular physiology it is desirable to predict metabolic responses to genetic and environmental perturbations, and this requires an understanding of cellular organization and regulatory mechanisms prevailing in the living system under study. A cell can be seen as a complex system of interacting molecules working in a coordinated way and leading to energetically favorable actions. Different types of cellular interactions include protein-protein interactions (PPI), protein-DNA interactions (PDI) and enzyme-metabolite interactions. The set of all PPI and PDI in a cell is often called interactome. High-throughput methods such as yeast two-hybrid systems and mass spectrometry of purified complexes have been applied to analyze protein-protein interactions at the whole-cell level. This has resulted in identification of around 80,000 PPI for the yeast Saccharomyces cerevisiae. However, the overlap between these different methods resulted in less than $10 \%$ common interactions. This should imply a careful utilization of the generated information. Genome-wide gene expression data is also vastly available, representing the simultaneous measurement of mRNA levels from all genes of a cell. This type of data offers the opportunity to identify regulatory structures and to explore corregulated modules changing in response to a perturbation. In this work 
we describe a probabilistic method to integrate interactome and transcriptome data, which is an extended approach to the one described by Ideker et al. (2002). The developed method allows us to look for high scoring and highly probable sub-networks, decreasing the false discovery rate of other approaches.

\section{GSB17}

Constructing THO1 allelic ladder by molecular cloning method for DNA typing purpose M. Tavallaei $^{1}$, A. Sajjadian ${ }^{1}$, A. Khosravi $^{1}$, H. Namazy $^{2}$, Z. Lashgari ${ }^{2}$, S. Zeinali ${ }^{3}$ : ${ }^{1}$ Imam Hossein University, Faculty of science Tehran, Iran; ${ }^{2}$ Forensic Medical Organization of Tehran, Iran; ${ }^{3}$ Pasteur institute of Tehran, Iran. E-mail: mtavalae@ihu.ac.ir (M. Tavallaei)

We have constructed a standard THO1 allelic ladder in order to solve the problem of the accuracy and standardization for using this STR marker for DNA typing purpose. Six different PCR amplified THO1 allelic fragments were isolated from the gel, eluted into TE buffer and reamplified by PCR. The purified allelic fragments were cloned using TA-cloning vector PGEM $^{\circledR}$-T Easy Vector System and transected into competent Escherichia coli top $10 F^{\prime}$ cells. Each clone was sequenced to determine the number of repeats. The recombinant plasmids DNA with six inserts were then used as templates for reamplification to generate THO1 standard ladder. A large quantity of standard THO1 allelic ladder was obtained, with which the genetic polymorphisms of THO1 locus in Iranian population were studied. The STR standard ladder constructed by this method has high value in the forensic application, and the THO1 locus is robust for forensic analysis.

\section{GSB18}

Large conformational change on giant DNA induced by ascorbic acid: A nobel scheme on its antioxidative activity Yuko Yoshikawa, Emi Sakai, Yoshiko Oda Department of Food and Nutrition, Nagoya Bunri College, Nagoya 451-0077, Japan

Ascorbic acid is often regarded as an antioxidant in vivo, where it protects against DNA damage by scavenging reactive oxygen species. In the present, we will show another potent scenario on the protective effect of ascorbic acid through a significant structural change of giant DNA. Recently, we examined the effect of ascorbic acid on the higher order structure of DNA through single molecular observation with fluorescence microscopy, and found that ascorbic acid generates a pearling structure in giant DNA molecules, where elongated and compact parts coexist along a molecular chain. The results of observations with atomic force microscopy indicate that the compact parts assume a loosely packed conformation. As the extension, here we study the protective effect against doublestrand breaks by reactive oxygen at different concentrations of ascorbic acid, in relation to the change of the higher order structure of giant DNA. We have performed a real time observation on the double-strand breaks on individual DNA molecules by use of fluorescence microscopy. We have found that the double-strand break is markedly protected when ascorbic acid exists over millimolar concentrations. It is found that such a protective effect of ascorbic acid corresponds well to the above mentioned change on the higher order structure of DNA. It has been reported that human circulating immune cells, such as neutrophils, monocytes and lymphocytes, accumulate ascorbic acid in millimolar concentrations. Therefore, it is expected that the ascorbic acid concentration that induces the large conformational change on DNA may be of physiological significance.

\section{References}

Yoshikawa, Y., et al., 2003. Eur. J. Biochem., 270, 3101-3106. Yoshikawa, Y., et al., 2004. FEBS Lett. 566, 39-42.

\section{GSB19}

On/off switching of transcription caused by folding transition in giant DNA: Novel scenario on genetic control $\mathrm{K}$. Yoshikawa $^{1}, F$. Luckel $^{1}$, A. Yamada ${ }^{1}$, Y. Yoshikawa ${ }^{2}{ }^{1}$ Department of Physics, Kyoto University, Kyoto 606-8502, Japan; ${ }^{2}$ Nagoya Bunri College, Nagoya 451-0077, Japan

During the last decade, it has been established that giant DNA molecules undergo large discrete transition between elongated state and folded compact state (Yoshikawa, 2002; Yoshikawa et al., 2002). It has become clear that such on/off switching of the conformation is induced by nonspecific environmental chemicals, such as polyamines, hydrophilic polymer, cationic surfactant, etc. In the present paper, we will show that on/off switching (Tsumoto et al., 2003) on the transcriptional activity of giant DNA is caused by the discrete transition, which is induced by environmental chemical parameters. The on/off switching of the transcriptional activity is the intrinsic property only for giant DNA molecules over several tens of kbp. It is noted that the on/off change accompanied with the folding transition is rather robust and is effective for number of genes over the regions of several tens kbp, whereas key-lock specific interaction is fuzzy in individual cellular system because of the "noisy" circumstance and is incorporated on single or small number of genes. Based on these results, we would like to propose novel hypothesis on the self-regulation of activity of tens of thousands genes.

\section{References}

Tsumoto, K., et al., 2003. Giant DNA molecules exhibit on/off switching of transcriptional activity through conformational transition. Biophys. Chem. 106, 23.

Yoshikawa, K., 2002. Field Hypothesis on the self-regulation of gene expression. J. Biol. Phys. 28, 701.

Yoshikawa, K., et al., 2002. All-or-none folding transition in giant mammalian DNA. Chem. Phys. Lett. 354, 354.

\section{GSB20}

Molecular evolution of envelope-class retrotransposons in Gossypium Elayed Hafez, Essam A. Zaki Nucleic Acids Research Department, Genetic Engineering \& Biotechnology Research Institute (GEBRI), Mubarak City for Research, Alexandria, Egypt

Retrotransposons are ubiquitous and abundant in plants and retroviruses are known in animals. Retrotransposons and retroviruses share similar intracellular life cycles and major encoded proteins. Retrotransposons, however, lack the envelope (env) critical for infectivity. The aim of the current study is to investigate the molecular evolution of env-containing retrotransposons in an allopolyploid and diploid species of cotton (Gossypium L). This allows env-containing retrotransposons evolution to be studied in two levels: first, in the diploids, subsequent to their divergence from a common ancestor, and second, in the polyploid, after retrotransposons have been reunited in a common genome. The detection of env-containing retrotransposons in cotton suggests that enveloped retroviruses are not limited 
to animals. In addition, their ubiquitous nature and the potential for horizontal transfer implicate these endogenous retroviruses as important vehicles for plant genome evolution.

\section{GSB21}

Population variation of human mitochondrial DNA hypervariable regions I and II in 100 Egyptian individuals demonstrated by DNA sequencing analysis Essam A. Zaki Department of Nucleic Acids Research, Genetic Engineering \& Biotechnology Research Institute (GEBRI), Mubarak City for Scientific Research, Research Area, Borg El Arab, Post Code 21934, Alexandria, Egypt

Human mtDNA is a non-recombining molecule with maternal inheritance and practically haploid genetics. Differences between mtDNA sequences are only due to mutations. As time passes, mutations accumulate sequentially along less and less related molecules that constitute independent lineages known as haplotypes. Relationships among lineages can be estimated by phylogenetic networks, whereas mutations are classified in hierarchical levels. The geographic distribution of these human haplotypes has shed light on crucial aspects of human history, such as the probable origin and approximate dating of migrations into the New World and Polynesia, and quantitative estimations of the relative Paleolithic and Neolithic contributions to the extant European mtDNA diversity. African populations have a rich and important history from which we can learn about our origins as a species and the way in which genetic variation affects human phenotypes, including diseases. Africa is thought to be the ancestral homeland for all Modern Humans. The study of the levels and patterns of genetic diversity among the multitude of ethnically diverse African populations will shed the light on many imperative questions concerning human evolutionary history and the genetic basis of phenotypic variation. However, despite the important contributions that studies of African populations can make, these populations have been understudied compared with nonAfrican populations. This has promoted the initiative to determine the mtDNA sequence diversity in the Egyptian populations. Here, we report sequence variation detection from 100 Egyptian individuals using DNA sequence analysis.

\section{GSB22}

Genome-wide scan, comparison and evolutionary history of envelope-containing retrotransposons in diverse plant genomes Essam A. Zaki Genetic Engineering \& Biotechnology Research Institute (GEBRI), Mubarak City for Scientific Research, Research Area, Borg El Arab, Post Code 21934, Alexandria, Egypt. E-mail: eazaki@link.net

Genome sequencing efforts indicate that a large portion of most eukaryotic genomes consist of retroelements. Retroelements include retroviruses, retrotransposons, and retroposons. Genome sequencing projects have enhanced our understanding of diversity and evolutionary trends among retroelements. For example, retrotransposon insertions are 20 times more numerous per unit length of DNA in the large human genome compared to the small Drosophila genome. In this regard, the recent molecular isolation and characterization of env-like gene in cotton Gossypium, has promoted the initiative to conduct a genome-wide scan for the occurrence of env-like coding sequences and their evolutionary history in the genomes of different plant species. This approach was carried out using data published in GenBank, as well as the complete genome sequence of Arabidop- sis thaliana and Orzya sativa. Our results revealed that envelopecontaining retrotransposons represent a standard component of plant genomes. Moreover, phylogenetic analysis demonstrated that plant $e n v$-like sequences group together, suggesting their monophyletic origin.

\section{GSB23}

Polymorphism at the mitochondrial DNA level among saprophytic Trichoderma viride isolates Z. Antal ${ }^{1}$, L. Kredics ${ }^{1}$, A. Szekeres $^{2}$, L. Manczinger $^{2}$, L. Hatvani $^{2}$, C. Vágvölgyi ${ }^{2}$, E. Nagy $^{1,3}$ : ${ }^{1}$ Hungarian Academy of Sciences, University of Szeged, Microbiological Research Group, P.O. Box 533, H-6701 Szeged, Hungary; ${ }^{2}$ Department of Microbiology, University of Szeged, Szeged, Hungary; ${ }^{3}$ Department of Clinical Microbiology, University of Szeged, Szeged, Hungary. E-mail: kredics@ bio.u-szeged.hu (L. Kredics)

Trichoderma species are common soil-borne fungi, some of them known as biocontrol agents of fungal plant pathogens. Certain species produce enzymes used in the paper-, fermentation-, and animal food industries as well. However, green mold epidemics of commercially grown mushrooms caused by certain Trichoderma species are also of increasing importance. The analysis of the mitochondrial DNA, due to its relatively small size, became widespread in the comparative analysis of fungi. The aim of the present study was to investigate the restriction length polymorphism of the mtDNA of Trichoderma viride strains derived from Hungarian soil samples. After the purification of total DNA from the mycelia of 29 isolates, the samples were digested with BsuRI or Hin6I restriction enzymes, respectively. After $B s u$ RI digestion, the isolates could be divided into five mtDNA types on the basis of the variations appearing in their mtDNA RFLP patterns. Restriction profiles obtained from the Hin6I digested samples gave the same result: classifications based on the two different enzymes proved to be identical. All of these results indicate very extensive mtDNA polymorphism even among $T$. viride isolates derived from a small territory. Further strains will be examined in order to gain more information about the mtDNA variability within the species $T$. viride. This work was supported by the grant F037663 of the Hungarian Scientific Research Found.

\section{GSB24}

Quality control of microarray experiments Tara Hill, Agilent Technologies Ltd., UK. E-mail: tara_hill@agilent.com

DNA microarray technology is a powerful research tool that enables the global measurement of transcriptional changes between paired RNA samples. While strong biological inferences can be made from microarray transcriptional data, they must be made within the correct biological and experimental context. This is because DNA microarrays capture a static view of a dynamic molecular event. This static view challenges researchers to tease out meaningful biological changes from the associated noise (due to sample acquisition, target labeling, microarray processing, etc.). A major issue in gene expression work using microarrays is having enough good quality RNA. Here we provide an overview of solutions that enable quality control, labelling and hybridization procedures that require less RNA input. 


\section{GSB25}

In vivo analysis of Arabidopsis High Mobility Group proteins Dorte Launholt $^{1}$, Klaus D Grasser ${ }^{1}$, Thomas Merkle ${ }^{2}{ }^{1}$ Section of Biotechnology, Department of Life Sciences, Aalborg University, DK-9000 Aalborg, Denmark; ${ }^{2}$ Department of Genome Research Center of Biotechnology, University of Bielefeld, D-33594 Bielefeld, Germany. E-mail: dl@bio.aau.dk (D. Launholt)

The High Mobility Group (HMG) proteins are a heterogeneous group of small and abundant non-histone proteins present in the cell nuclei of all eukaryotes. The HMG proteins interact with the eukaryotic DNA and upon binding the proteins modulate the structure of the DNA. They thereby facilitate the binding of, e.g. transcription factors to the DNA that then initiate the transcription of genes. The HMG proteins are therefore very important for the function of the organism. Although there have been reports indicating an involvement of HMG proteins in replication, transcription and nucleosome assembly, the exact cellular function of the proteins is still largely unknown in plants. The main focus of the project is therefore to use various methods to analyse the protein functions using Arabidopsis thaliana as the model plant. The study is based on three primary approaches: (1) analyses of $\mathrm{hmg}$ gene promoter activity using $\mathrm{hmg}$ promoter-reporter gene fusion, (2) examination of the developmental differences between plants lacking the HMG protein, plants having excess amount of the HMG protein and wild-type plants and (3) determination of the cellular localisation, nuclear localisation sequence and protein dynamics using HMG-GFP fusion proteins.

\section{GSB26}

Use of metabolome data and metabolic network structure to identify reaction significances $T$. Çakır ${ }^{1}, K . R$. Patil $^{2}, B . K_{\text {Irdar }}{ }^{1}$, Z.I. Önsan ${ }^{1}$, K. Ö. Ülgen ${ }^{1}$, J. Nielsen ${ }^{2}{ }^{1}$ Department of Chemical Engineering, Boğaziçi University, 34342 Bebek, Istanbul, Turkey; ${ }^{2}$ Center for Microbial Biotechnology, Biocentrum-DTU, Technical University of Denmark, Kgs. Lyngby DK-2800, Denmark. E-mail: cakirtun@boun.edu.tr (T. Çakır)

In a recent study quantitative metabolome data were obtained for the yeast Saccharomyces cerevisiae (Villas-Bôas et al., 2005). Such metabolomics data represent an integrated response of cellular metabolism towards genetic/environmental perturbations and gives a snapshot of metabolic activity of cell under given conditions. Here we report on a hypothesis-driven method to integrate quantitative metabolomics data with genome-scale metabolic models for understanding metabolic basis of changes in intracellular metabolite levels. The algorithm uses the metabolome data to infer the relative reaction significance information following a metabolic perturbation, and thereby enables the identification of so-called reporter enzymes, which represent hot spots in terms of metabolic regulation. We also address and test different ways to overcome several problems arising due to the lack of measurements for large number of metabolites. Moreover, we also provide detailed statistical analysis of the data to enrich information extracted. Combining results from several methods we show that the metabolomics data can be used not only for characterization and classification, but also for inferring information about metabolic regulation leading to different metabolic phenotypes.

\section{GSB27}

Systems biology study of stringent response in Streptomyces coelicolor A3(2) Irina Borodina, Jens Nielsen Center for Microbial Biotechnology, Technical University of Denmark, BioCentrum-DTU Building 223, Kgs Lyngby DK-2800, Denmark

Streptomyces coelicolor A3(2) is by far the best genetically studied Streptomyces strain and has become a model organism for Streptomyces species. Release of the S. coelicolor A3(2) genome sequence has further expanded the knowledge of this organism and enabled the application of genome-wide analysis techniques like DNA arrays for transcriptome analysis and proteomics studies. We believe that integration of the genome-wide data through the use of mathematical models will enhance the extraction of information about the molecular mechanisms governing different processes in S. coelicolor A3(2). To assist with the analysis of the data, we have recently reconstructed genome-scale metabolic model based on annotated genes, physiological and biochemical information (Borodina et al., in press). The developmental processes in S. coelicolor A3(2) reference strain are compared with a congenic $\triangle$ relA mutant. relA is responsible for the biosynthesis of ppGpp, a global signalling molecule that induces stringent response in starving cells. The cultivations are performed in triplicates in a batch mode in well-controlled fermentors on nitrogenlimited defined minimal medium supplemented with casamino acids. The samples taken in the course of cultivation are analysed for gene expression, protein and metabolites concentrations. Additionally, the central carbon metabolism fluxes are estimated with the help of ${ }^{13} \mathrm{C}$ labeling experiments. The results of several analysis types will be presented.

\section{Reference}

Borodina, I., Krabben, P., Nielsen, J., in press. Genome-scale analysis of Streptomyces coelicolor A3(2) metabolism. Genome Res.

\section{GSB29}

Expression profile of the sty and paa genes in Pseudomonas sp. Y2 by means of DNA microarrays David Bartolomé-Martín ${ }^{1}$, David Juck ${ }^{2}, M^{a}$ Teresa del Peso-Santos ${ }^{1}$, Charles W. Greer ${ }^{2}$, Julián Perera ${ }^{1}$ : ${ }^{1}$ Departamento de Bioquímica y Biología Molecular I, Facultad de Ciencias Biológicas, Universidad Complutense de Madrid, 28040 Madrid, Spain; ${ }^{2}$ Environmental Microbiology Group, Biotechnology Research Institute, National Research Council Canada, Montréal, Que., Canada H4P 2R2. E-mail: perera@bio.ucm.es (J. Perera)

DNA microarrays are a new and powerful tool to study gene expression in very diverse systems. Environmental biotechnology and biodegradation are some of the fields of research where this technology may be very promising. Pseudomonas sp. Y2 is a bacterium able to grow in minimal medium plus either styrene (STY) or phenylacetic acid (PAA) as the sole carbon and energy sources. This bacterium is the only organism where the genes that code for both the upper (sty genes) and the lower ( $p a a$ genes) catabolic pathways for the styrene degradation have been described till now. It is unique in having two active copies of the genes encoding the lower pathway (paa1 andpaa 2 gene clusters). We have designed a DNA microarray with the sty and paa genes in order to analyse their expression in the wild type Pseudomonas sp. Y2, in P. sp. Y2 T2 (apaa2 deletion mutant) and in $P$. sp. Y2 C1 (a crc gene mutant). This analysis has been performed on bacterial cultures grown in media with different 
carbon sources. Interesting data on the expression profile of the sty and paa genes in Pseudomonas sp. Y2 have been obtained, and have raised new questions on styrene and PAA degradation by this bacterium.

\section{GSB30}

Mutasynthesis in Streptomyces for production of novel calciumdependent antibiotics

The calcium-dependent antibiotic (CDA) is a lipopeptide synthesised non-ribosomally and produced by Streptomyces coelicolor A3(2). CDA contains several non-proteinogenic amino acid residues. Hydroxyphenylglycine (4-HPG) is one of the unusual amino acids in the structure of the CDA and vancomycin groups of antibiotics. For the members of the vancomycin group of antibiotics, the 4HPG residue plays crucial roles in the structure and function of the final glycopeptide antibiotic. To reveal the putative biosynthetic pathway of this amino acid in CDA, a standard "double crossover replacement strategy" was used to delete 4-hydroxymandelic acid synthase (4-HMAS, encoded by $h p d$ ) from S. coelicolor MT1110 and 2377, using the delivery plasmid pZMH3. There was no CDA production in the disrupted strains. Plates containing a gradient of hydroxymandelic acid were used to restore CDA production in both S. coelicolor MT1110 $\Delta h p d$ and $2377 \Delta h p d$. Exogenous supply of 4- hydroxyl phenylglyoxylate and 4-hydroxyphenylglycine reestablished CDA production by the hpd mutant. Feeding analogs of these precursors to the mutant resulted in the directed biosynthesis of novel lipopeptides with modified arylglycine residues (mutasynthesis).

\section{GSB31}

A CXCL2 tandem repeat promoter polymorphism is associated with susceptibility to severe sepsis in the Spanish population N. Maca-Meyer ${ }^{1}, C$. Flores ${ }^{1}$, L. Pérez-Méndez ${ }^{1}, R$. Sangüesa ${ }^{2}, E$. Espinosa ${ }^{2}, J$. Villar ${ }^{1}$ : ${ }^{1}$ Research Institute, Hospital Universitario N.S. de Candelaria, S/C Tenerife 38010, Spain; ${ }^{2}$ Department of Anesthesiology, Hospital Universitario N. S. de Candelaria, S/C Tenerife 38010, Spain. E-mail: nmacame@ull.es (N. Maca-Meyer)

Sepsis describes a complex clinical syndrome resulting from a systemic inflammatory response to bacteria, and remains an important cause of mortality in the intensive care unit. CXCL2 chemokine (or $M I P-2$ ) exhibits a pivotal role in the immune response, and several functional studies in animal models of sepsis have catalogued CXCL2 as a candidate gene for the development of sepsis. We have performed a case-control association study of $C X C L 2$ gene variants and susceptibility to severe sepsis in 179 hospitalised patients and 364 healthy individuals. After the examination of linkage disequilibrium in the region, we analysed whether two promoter polymorphisms (SNP rs3806792 and a newly described polymorphic short tandem repeat D4S3454) were associated with the syndrome. We found a significant association of common variants at D4S3454 with the development of severe sepsis (heterozygote carriers OR 2.82; 95\% CI 1.10-7.24, and homozygote carriers OR 3.65; 95\% CI 1.41-9.43; Mantel-Haenszel $\chi^{2}$ test for linear trend $\left.P=0.0006\right)$. The risks remained significant even after a genomic control adjustment, based on 20 additional genotyped polymorphisms not linked to the candidate gene. These preliminary results suggest that $C X C L 2$ gene variants may contribute to the development of severe sepsis.

\section{GSB32}

Induction of gene expression by glucose in Saccharomyces kluyveri Kasper Møller ${ }^{1}$, Ana Paula Oliveira ${ }^{1}$, Jens Nielsen ${ }^{2}$, Mark Johnston ${ }^{1:}{ }^{1}$ Center for Microbial Biotechnology, BioCentrum, Technical University of Denmark, Denmark; ${ }^{2}$ Department of Genetics, School of Medicine, Washington University, St. Louis, USA

Glucose is the preferred carbon and energy source for most cells. In Saccharomyces cerevisiae, a complex regulatory network ensures that $S$. cerevisiae ferments glucose to ethanol even in the presence of oxygen. To obtain a better understanding of this Crabtree effect and the logic of the glucose signalling network in S. cerevisiae, we are analyzing glucose sensing and signalling in the related species Saccharomyces kluyveri, which exhibits much less of a Crabtree effect (it prefers not to ferment glucose when oxygen is available). We show that there are only two major glucose transporters in S. kluyveri, and that these are regulated in response to the availability of glucose via a glucose sensor and a signalling pathway similar to the glucose induction (RGT2/SNF3-RGT1) pathway in $S$. cerevisiae. We have used DNA-microarrays for $S$. kluyveri to find targets of the $S$. kluyveri glucose induction pathway, as well as to evaluate the global response to a change in environment from growth on ethanol to growth on glucose. This study identifies a number of differences in the regulation of glucose uptake and global responses to glucose between $S$. kluyveri and S. cerevisiae, which may contribute to their different glucose metabolism.

\section{GSB33}

Detection and analysis of microRNA using LNA probes Nana Jacobsen, Christian Lomholt, Peter Mouritzen, Peter Stein Nielsen, Mikkel Noerholm Exiqon A/S, Bygstubben 9, DK-2950 Vedbæk, Denmark. E-mail: mouritzen@exiqon.com (P. Mouritzen)

MicroRNAs are a class of short endogenous RNAs that act as post-transcriptional modulators of gene expression. Growing evidence suggest that microRNAs exhibit a wide variety of regulatory functions and exert significant effects on cell growth, development, and differentiation. Recent studies have shown that human microRNA genes are frequently located in cancer associated genomic regions and perturbed microRNA expression patterns have been observed in many malignant tumors. We have exploited the significantly improved hybridization properties of LNA oligonucleotides against RNA targets to design LNA-modified DNA probes for detection of different microRNAs in animal and plants by northern blot analysis, microarray hybridization and in situ hybridization. We will describe the results obtained from detection and analysis of different microRNAs in C. elegans, zebrafish, mouse, and plants. In addition, we will describe a novel LNA-based method for expression profiling of mature microRNAs by quantitative RT-PCR.

\section{GSB34}

Expression profile of the sty and paa genes in Pseudomonas sp. Y2 by means of DNA microarrays David Bartolomé-Martín ${ }^{1}$, David Juck ${ }^{2}, M^{a}$ Teresa del Peso-Santos ${ }^{1}$, Charles W. Greer ${ }^{2}$, Julián Perera ${ }^{1}$ : ${ }^{1}$ Departamento de Bioquímica y Biología Molecular I, Facultad de Ciencias Biológicas, Universidad Complutense de Madrid, 28040 Madrid, Spain; ${ }^{2}$ Environmental Microbiology Group, Biotechnology Research Institute, National Research Council Canada, Montréal, Que., Canada H4P 2R2. E-mail: perera@bio.ucm.es (J. Perera) 
DNA microarrays are a new and powerful tool to study gene expression in very diverse systems. Environmental biotechnology and biodegradation are some of the fields of research where this technology may be very promising. Pseudomonas sp. Y2 is a bacterium able to grow in minimal medium plus either styrene (STY) or phenylacetic acid (PAA) as the sole carbon and energy sources. This bacterium is the only organism where the genes that code for both the upper (sty genes) and the lower (paa genes) catabolic pathways for the styrene degradation have been described till now. It is unique in having two active copies of the genes encoding the lower pathway (paal and paa 2 gene clusters). We have designed a DNA microarray with the sty and paa genes in order to analyse their expression in the wild type Pseudomonas sp. Y2, in P. sp. Y2 T2 (a paa2 deletion mutant) and in $P$. sp. Y2 C1 (a $c r c$ gene mutant). This analysis has been performed on bacterial cultures grown in media with different carbon sources. Interesting data on the expression profile of the sty and paa genes in Pseudomonas sp. Y2 have been obtained, and have raised new questions on styrene and PAA degradation by this bacterium.

\section{GSB35}

Dynamics in induced repression of phosphomannose isomerase PMI40 gene of Saccharomyces cerevisiae Anssi Törmä ${ }^{1,2}$, Juha-Pekka Pitkänen $^{1,2}$, Laura Huopaniemi ${ }^{2}$, Risto Renkonen ${ }^{2}$ : ${ }^{1}$ MediCel Ltd., Haartmaninkatu 8, 00290 Helsinki, Finland; ${ }^{2}$ Rational Drug Design Program, Department of Bacteriology and Immunology, Haartman Institute and Biomedicum, University of Helsinki, P.O. Box 63, 00014 Helsinki, Finland. E-mail: juhapekka.pitkanen@medicel.com (J.-P. Pitkänen)

GDP-mannose is the precursor of cell wall biosynthesis in $S$. cerevisiae. To understand the system level role of GDP-mannose, we studied a conditional knock-out strain of the key enzyme in its synthesis; PMI40. The experimental procedure allowed us to study the order of mechanisms the cells launch in order to adjust to a sudden malfunction in the metabolic machinery. We collected 100 samples from continuous cultivations over $80 \mathrm{~h}$ and measured genome-wide gene expression levels, 10 enzyme activities, and concentrations of 30 intracellular metabolites. For sampling we have built a sample robot, which automatically takes and preserves the samples. In order to carry out this magnitude of experimentations and generated data, we have constructed a proprietary software platform to handle all the phases from project management in wet-lab to workflow and pathway management in in silico. After normalization and clustering, significantly changed genes and metabolites were searched for enrichment in biological processes and molecular complexes. Further, gene expression levels, metabolite concentrations, and enzyme activities were searched against each other for causality over time. Overall, we focused on thorough analysis of our own data and known database data in order to reward our efforts with knowledge. At the transcriptome level, repression of PMI4O led to two major types of activation profiles, one peaking at the time when Pmi40p activity and GDP-mannose were depleted and the other later during recovery from the perturbation. The primary response was most enriched with genes known to play roles in mating and filamentous growth and associated with the transcription factors Ste12p, Tec1p, Dig1p, and $\mathrm{Mcm} 1 \mathrm{p}$, whereas the secondary response consisted of genes involved in carbon metabolism and associated with the general stress response regulators Msn2p and Msn4p. Skn7p, a high-level transcription factor was associated with both the primary and the secondary response, consistent with its suggested role of coordinating environmental responses and developmental processes.

\section{GSB36}

Transcriptome of pig ovarian cells: Discriminant genes involved in follicular development Bonnet A., Le Cao K.A., Low-So G., San Cristobal M., Tosser-Klopp G., Hatey F. Laboratoire de Génétique Cellulaire, Centre INRA de Toulouse, Castanet-Tolosan 31326, France

In order to identify genes and gene networks involved in pig ovarian follicular development, we built subtractive suppressive hybridization libraries (SSH) from granulosa cells of healthy follicles (small, medium or large). The RNA isolated from these cells was used to hybridize cDNA nylon micro-arrays. Data analysis using a gaussian linear mixed model showed that $83 \%$ of the variability is due to the genes. Two hundred fifty one regulated genes (from the 956 expressed) were identified and clustered into three groups according to the follicle size. Moreover, we found previously identified genes such as aromatase, IGFBP2 which supported the validity of our experimental model. Ramdom forest analysis put forward the most discriminant 11 genes between the three follicle classes. This study put forward gene sets such as those involved in cell modeling, regulation of transcription, apoptosis during follicle growth. The next step will be to describe more precisely the spatio-temporal expression patterns at the mRNA levels of the genes identified by these experiments.

\section{GSB37}

Comparative genomics of the red microalgae Porphyridium sp. and Dixoniella grisea (Rhodoplantae) R.P. Shrestha, M. Lapidot, V. Chalifa-Caspi, S. (Malis) Arad, Y. Weinstein Ben-Gurion University, P.O. Box 653, Beer-Sheva 84105, Israel. E-mail: roshan@bgu.ac.il (R.P. Shrestha)

Microalgae constitute a significant source of valuable natural products, e.g. sulfated polysaccharides, polyunsaturated fatty acids, and phycobiliproteins that find applications in wide range of industries, including food, pharmaceutical, agricultural and cosmetics. However, genomic and molecular genetic studies of microalgae lag far behind those of higher plants. In order to accelerate red microalgal genomic studies by taking advantage of current genomics and post-genomic technologies, we have generated expressed sequence tag (EST) databases of two red microalgae Porphyridium sp. and Dixoniella grisea grown under various physiological conditions. To date we have sequenced 7210 and 6231 ESTs of Porphyridium sp. and D. grisea, respectively. The sequence assembly resulted into, ca. 2000 non-redundant unigenes for each microalga, only $40 \%$ of which were identified by similarity to sequences in the public databases. Porphyridium sp. and D. grisea unigenes were compared with the whole-genome predicted proteomes of three microalgae and those of representative eukaryotic and prokaryotic organisms. Both microalgae have highest similarity to the red microalga Cyanidioschyzon merolae. The order of sequence similarity to other organisms examined was Arabidopsis thaliana, Oryza sativa, Chlamydomonas reinhardtii, Thalassiosira pseudonana (diatom), Saccharomyces cerevisiae, Caenorhabditis elegans, archaea and cyanobacteria. Although red microalgae are considered as phylogenetic bridge 
between prokaryotes and eukaryotes, our data show that the red microalgae have strong similarity to eukaryotes and only distant similarity to prokaryotes. Gene expression profiles were studied by analyzing cDNA and subtraction libraries constructed from algae grown under various physiological conditions. We observed that top three most abundant ESTs in the stationary phase of Porphyridium sp. were ADP ribosylation factor like-1, flavohemoglobin and ADP ribosylation factor-1. In addition, we have identified several genes which were specific to nitrate- and sulfate starvation.

\section{Proteomics and Protein Engineering}

\section{Protein Structure and Function}

Understanding the structure and function of the calcium pump Poul Nissen Department of Molecular Biology, University of Aarhus, Denmark

The sarco(endo)plasmic reticulum $\mathrm{Ca}^{2+}$-ATPase (SERCA, "the calcium pump"), is responsible for pumping the $\mathrm{Ca}^{2+}$ released into the cytoplasm during muscle contraction back into the sarcoplasmic reticulum store while proton are pumped the opposite way as counter-cations. These transport processes go against the concentration gradients and are therefore energy consuming. The energy is derived from ATP hydrolysis via formation and break-down of a phospho-enzyme intermediate. Over the last year a number of new crystal structures have been published which have added to our understanding of how this task is accomplished, which provides an impressive insight to the mechanism of a molecular pump.

\section{2}

Mechanism and function of the intramembrane-cleaving protease rhomboid Marius Lemberg ${ }^{1}$, Javier Menendez ${ }^{2}$, Christopher Koth $^{2}$, Matthew Freeman ${ }^{1}:{ }^{1}$ MRC Laboratory of Molecular Biology, Cambridge, UK; ${ }^{2}$ Ontario Center for Structural Proteomics, Toronto, Canada

Rhomboids are a family of intramembrane serine proteases that are widely conserved throughout evolution. Among diverse functions discovered so far, rhomboids participate in intercellular signalling, parasite invasion, membrane dynamics and bacterial quorum sensing, making them potentially valuable therapeutic targets. The identification of physiological substrates and of selective inhibitors will be key towards their evaluation as drug targets. We have developed an in vitro cleavage assay to monitor rhomboid activity in the detergent solubilised state, enabling the first isolation of a highly pure rhomboid with catalytic activity. Analysis of purified mutant proteins suggests that rhomboids use a serine protease catalytic dyad instead of the previously proposed triad, and gives insights into subsidiary functions like ligand binding and water supply.

\section{Acknowledgement}

This work was supported principally by EMBO and the MRC.

\section{3}

Structure and target-specificity of thioredoxin h Kenji Maeda ${ }^{1}$, Anette Henriksen ${ }^{2}$, Per Hägglund ${ }^{1}$, Christine Finnie $^{1}$, Birte Svensson ${ }^{1}{ }^{1}$ Biochemistry and Nutrition Group, BioCentrum-DTU, Technical University of Denmark, DK-2800 Kgs. Lyngby, Denmark; ${ }^{2}$ Biostructure Group, Carlsberg Laboratory, DK-2500 Valby, Denmark. E-mail: kenji@biocentrum.dtu.dk (K. Maeda)

Thioredoxins are ubiquitous small proteins with protein disulphide reductase activity. Thioredoxins can alter the structures and the activities of various target proteins by reducing their disulphide bonds. Seeds of several plants are abundant in cytosolic thioredoxins referred as h-type. In barley, two thioredoxin h isoforms, HvTrxh1 and HvTrxh2 that share 51\% sequence identity but differ in temporal and spatial distributions were previously identified and characterised. In the present study, the relationship between structures and targetspecificities of h-type thioredoxins are analysed. The 3D-structures of HvTrxh1 and HvTrxh2 are determined by X-ray crystallography as the first crystal structures of thioredoxin $h$. Comparison of the structures shows that the majority of solvent exposed residues near the active sites are conserved between the two isoforms. This is in agreement with previously observed similarity in target-specificity of the two isoforms. Thioredoxins from organisms distantly related to barley, such as E. coli, have highly similar folds but different surface charge distributions compared to barley thioredoxins. A comparison of the target-specificities of HvTrxh1, HvTrxh2, E. coli thioredoxin and several thioredoxin mutants will be attempted to reveal the structural features that influence specificity of barley thioredoxin $h$ isoforms.

\section{4}

Localization and structural analysis of the O-linked glycosylation sites of ENBREL ${ }^{\circledR}$ (etanercept) by Tandem Mass Spectrometry A. Balland, J. Boyce, P. Jones, C. Koffley, A. Goetze, W. Wang Amgen, 1201 Amgen Court West, Seattle, WA, USA

ENBREL is a dimeric fusion protein consisting of the extracellular ligand binding portion of the human $75 \mathrm{kDa}$ (p75) tumor necrosis factor receptor (TNFR) linked to the Fc portion of human IgG1. The CHO-expressed molecule contains both $\mathrm{N}$ - and O-linked oligosaccharides with a total carbohydrate content of $20 \%$ by mass. The O-linked oligosaccharides were released by hydrazinolysis and their structure determined by exoglycosidase sequencing and MALDITOF mass spectrometry. To locate precisely the O-linked sites, the glycosylation heterogeneity of TNFR:Fc was simplified by treatment with $\mathrm{N}$-acetyl neuraminidase and $\mathrm{N}$-glycanase. The remaining molecule, which only carries core O-linked glycan structures, was cleaved by trypsin and analyzed by LC-MS. Precise localization of O-glycosylation sites was determined based on the concept of a specific modification of the O-glycosylated serine into 2aminopropenoic acid and O-glycosylated threonine into 2-amino-2butenoic acid. The deficit in mass resulting from this transformation was the marker used to localize the modified residues on the peptides by tandem mass spectrometry sequencing (MS-MS). MS-MS spectrum of ENBREL glycopeptides were interpreted based on the presence of 2-aminopropenoic acid and 2-amino-2-butenoic acid, resulting in a complete map of O-linked glycans precisely located at 10 different sites. 
Wnts as Tools and Tools for Wnts Anu Mursula, Beatrix Fahnert, Sari Krapu, Eija-Riitta Hämäläinen, Ritva Isomäki, Peter Neubauer Bioprocess Engineering Laboratory and Biocenter Oulu, University of Oulu, Oulu, Finland. E-mail: anu.mursula@oulu.fi (A. Mursula)

Wnt proteins form a highly conserved family of secreted glycoproteins important in cell-cell signaling events during embryogenesis and adult tissue maintenance. Impairments within this complex signaling pathway can lead for example to developmental defects in embryos, degenerative diseases and cancer. Respectively, Wnt proteins can be used as tools in basic research concerning Wnt function, developmental biology, screening for interacting compounds, and for medical applications (e.g. therapeutics, stem cells). Hence, recombinant Wnts provide a valuable basis for these purposes. However, production of recombinant Wnt proteins is challenging, because they contain multiple disulfide bonds making the folding very difficult. A process for production of murine Wnt-1 in E. coli has been developed in our laboratory. The knowledge obtained from this research has also been applied to the expression of other Wnts, namely Wnt-4 and Wnt-6, and can be used to approach other cysteine-rich proteins as well. Since the expression level of Wnt proteins is rather low so far, tools for monitoring and optimizing the production process have been established. By means of this sandwich hybridization method the level of target (Wnt) mRNA can be measured. The technique has already been applied to analyzing Wnt-1 mRNA levels. Probes also for Wnt-4 and Wnt-6 have been generated. Thus, transcription of Wnt genes in all kind of cells (e.g. tissue, recombinant hosts) in general as well as kinetics of transcription can be studied using these tools.

\section{Proteomics}

\section{1}

Quantitative proteomics to study global cell signalling Blagoy Blagoev, Irina Kratchmarova, Shao-En Ong, Jesper V. Olsen, Matthias Mann Center for Experimental BioInformatics (CEBI), University of Southern Denmark, 5230 Odense M, Denmark

In growth factor signaling, stimulation of cell-surface receptors first triggers activation of the receptor itself and then of a large number of intracellular effector molecules. The stimulus is integrated with a host of other cellular processes, leading to cytoskeletal changes, activating transcriptional programs in the nucleus and ultimately resulting in cell proliferation, differentiation or motility. Classical signaling pathways and networks depict potential protein-protein interactions only in a static form. In the cell, these interactions are dynamic and occur in an ordered fashion. Here, we apply a mass spectrometric method that converts temporal changes to differences in peptide isotopic abundance in order to study the global dynamics of signaling events. Briefly, three cell populations are metabolically labeled with either normal arginine or a ${ }^{13} \mathrm{C}$ substituted form, or a ${ }^{13} \mathrm{C}{ }^{15} \mathrm{~N}$ variant (Stable Isotope Labeling by Amino acids in Cell culture, SILAC). Each population was then stimulated with EGF for a different time period and tyrosine phosphorylated proteins were affinity purified with anti-phosphotyrosine antibodies. The proteins from the precipitated complexes were quantitatively analyzed and identified using LC-MS/MS. Arginine containing peptides occurred in three forms, directly indicating protein activation at the corre- sponding time point. Combination of two experiments sharing one common time point of activation then generated five-point dynamic profiles. From the 202 proteins quantified, we identified 81 signaling proteins, including virtually all known EGFR substrates and 31 novel effectors, and the time course of their activation upon EGF stimulation. Discriminating proteins involved in the signaling network from unspecific binders was straightforward as these presented an activation profile. We have now further extended this study by directly measuring in vivo phosphorylation sites in response to growth factor stimulation and monitoring the time evolution of the phosphorylation events. Finally, we determined and quantitatively compared the global EGF and PDGF tyrosine phosphoproteomes in human mesenchymal stem cells and revealed a control point in their differentiation into bone-forming cells. Such global activation profiles provide a novel perspective in cell signaling and will be crucial to model the highly dynamic signaling networks in a systems biology approach.

2

Proteomics-a powerful tool in kinase signalling studies $\underline{\text { Klaus Schneider }}^{1}$, Dave G Smith ${ }^{2}$, Steven Skaper ${ }^{2}$, Alastair D. Reith ${ }^{1}:{ }^{1}$ Discovery Research, GlaxoSmithKline, Coldharbour Road, Harlow, Essex, UK; ${ }^{2}$ Neurology \& Gastrointestinal CEDD, GlaxoSmithKline, Coldharbour Road, Harlow, Essex, UK

Over the last 15 years, progress in signal transduction research has revealed an astonishing degree of complexity in cell signalling which is manifested in positive and negative regulations and feedback loops within signalling pathways and by cross-talks between pathways, all of which are highly cell-type dependent. It has become evident that protein phosphorylation by protein kinases plays a major role in this complex regulation of cell signalling (Hunter, 2000). Due to the importance of signal transduction in disease processes, many protein kinases may constitute key targets for disease intervention. Yet, the lack of a full understanding of the regulation, the activation and, importantly, of downstream substrates of particular protein kinases requires often more detailed studies before initiation of resource-intensive efforts to find disease-modifying molecules. Technologies for the study of protein kinase signalling include ${ }^{32} \mathrm{P}$ labelling, mutational and knock-out studies and more recently RNA interference. These tools are complemented by approaches that are based on proteomic technologies developed over the course of the last 10 years. In this presentation, the scope of proteomics technologies to contribute to an understanding of kinase signalling will be discussed. An overview of available technologies will be given and results will be presented from a proteomic study of Glycogen-Synthase Kinase 3 (GSK3) signalling (Coghlan et al., 2000). Novel findings will be presented on a study of GSK3 inhibition in a primary neuronal cell line by differential 2D gel electrophoresis, which resulted in the identification of more than 40 proteins that were significantly regulated.

\section{References}

Coghlan, M.P. et al., 2000. Chem. Biol. 7, 793-803.

Hunter, T., 2000. Cell 100, 113-127. 
Comparison of proteome analysis by HPLC-Chip/MS versus nanospray LC/MS Martin Vollmer, Christine Miller, Georges L. Gauthier Agilent Technologies R\&D GmbH, Hewlett-Packard Str.8 Waldbronn 76337, Germany. E-mail: georges_gauthier@agilent.com (G.L. Gauthier)

Proteome analysis is typically done by nanospray LC/MS in order to achieve higher sensitivity and thus a greater number of protein identifications. However, nano-scale LC systems can be more challenging to use and maintain. To obtain the best chromatographic performance, connections must be made carefully to minimize band broadening. Improved chromatographic performance can enhance the mass spectrometric results by tandem MS as a greater number of peptides can be detected. A microfluidic chip-based system has been developed (Yin et al., 2004) that minimizes the number of connections and the delay volumes. This work evaluates the performance of this device against the traditional nanospray approach. A yeast extract sample was separated by SDS-PAGE and bands were excised from the gel for further analysis. After in-gel digestion, the sample was analyzed by both traditional nanospray and the microfluidicbased chip device. After protein database searching, the identified proteins and the protein sequence coverage's were compared for the two approaches. The microfluidic device was demonstrated to be equivalent or better compared to the traditional approach.

\section{Reference}

Yin, H., Killeen, K., Brennen, R., et al., 2004. Anal. Chem. 77, 527-533.

\section{Protein Engineering and Protein Structure}

2

Functional screening of a library of yeast-expressed plant cytochromes P450: A toolkit for agronomical, pharmaceutical and other industrial applications Florence Schneider, Danièle Werck-Reichhart Department of Plant Metabolic Response, CNRS-UPR 2357, Université Louis Pasteur, 28 rue Goethe, 67000 Strasbourg

Plant cytochromes P450 (P450s) play key roles in the biosynthesis of most bioactive compounds with agronomic and therapeutic applications. A collection of about 120 plant P450s was expressed in yeast. The cDNAs were isolated from the model plant with a sequenced genome Arabidopsis thaliana, some others from wheat, Helianthus tuberosus or Vicia sativa. They were expressed under the control of a galactose-inducible promoter in an engineered strain of Saccharomyces cerevisiae in which the gene of the native $\mathrm{P} 450$ reductase was replaced with the gene of a $\mathrm{P} 450$ reductase from $A$. thaliana under the control of the same galactose-inducible promoter, in order to provide an optimal context for plant P450 expression and activity (Pompon et al., 1996). An original procedure was designed for the high-throughput functional screening of this enzyme collection. It is based on the detection of oxygen consumed during the catalytic reaction by a fluorochrome embedded in the bottom of the microwell plates. This method was validated using several recombinant P450s of known activity. It also allows for a very efficient screening for enzyme inhibitors. The advantages and limits of the method will be discussed.

\section{Acknowledgement}

This work was carried out with the support of Génoplante Programme (NO 2001004).

\section{Reference}

Pompon, D., Louerat, B., Bronine, A., Urban, P., 1996. Methods Enzymol 272, 51-64.

\section{3}

Folding of a bacterial membrane protein studied by protein engineering Daniel E.Otzen, Pankaj Sehgal, Peter A. Christensen Department of Life Sciences, Aalborg University, Sohngaardsholmsvej 49, DK - 9000 Aalborg. E-mail: dao@bio.aau.dk (D.E. Otzen)

We have carried out a kinetic analysis of the folding of the 4-helix transmembrane protein DsbB in a mixed micelle system consisting of varying molar ratios of sodium dodecyl sulfate and dodecyl maltoside. This analysis incorporates both folding and unfolding rates, making it possible to determine both the stability of the native state and the process by which the protein folds. The analysis also takes into account the composition of the mixed micelles, which is different from the bulk detergent composition. Refolding and unfolding are consistent with a three-state folding scheme involving the SDSdenatured state, the native state and an unfolding intermediate that accumulates only under unfolding conditions at high mole fractions of SDS. The temperature-dependence of the folding reaction displays an unusual decrease in heat capacity accompanying unfolding, which probably reflects the amphiphilic environment of the membrane protein. Destabilization of DsbB by different short-chain alcohols correlates very well with the alcohols' respective hydrophobicities. Data from a series of Ala-scanning mutants tentatively identify a nucleus for folding, which is relatively diffuse and involves all four helices. We are currently complementing this work with studies of the association of peptides corresponding to individual transmembrane segments of DsbB

\section{4}

Polarized light spectroscopy on RecA: Studying the active nucleoprotein filament Karolin Frykholm, Katsumi Morimatsu, Bengt Nordén Department of Chemical and Biological Engineering, Chalmers University of Technology, Gothenburg, Sweden. E-mail: frykholm@chalmers.se (K. Frykholm)

The RecA protein of E. coli plays a crucial role in homologous recombination and DNA repair. The recombination process takes place in a filamentous complex, in which the protein monomers are arranged in a helical manner around a single-stranded DNA (ssDNA). In the presence of ATP the filament can accommodate a second, double-stranded DNA (ds-DNA) and the strand exchange reaction can occur. The three-dimensional structure of RecA itself and its complex with ADP have been determined by X-ray crystallography. The active nucleoprotein filament, however, has only been studied at low resolution. Both electron microscopy (EM) and small-angle neutron scattering (SANS) indicate significant differences between the structures of the active nucleoprotein filament and the compressed, inactive filament of only RecA. We have presented a structural model of the RecA protein in its active filament with ssDNA, using data obtained by linear dichroism (LD) polarized-light spectroscopy, based on a technique, we call "Site-Specific Linear 
Dichroism", which allows the orientation of a set of amino acids to be determined from LD data by systematic modification of the protein. Here, we show that LD data of the nucleoprotein filament with ds-DNA is over all similar to the data of the complex with ss-DNA, indicating that the orientation as well as internal structure of RecA in the active filament is not significantly altered when the bound DNA is changed from single-stranded to double-stranded. This result supports the idea that the strand exchange reaction occurs without large conformational change of the RecA protein.

5

The choline-binding modules: A powerful biotechnological tool Jesús M. Sanz Instituto de Biología Molecular y Celular, Universidad Miguel Hernández, Elche, Spain

Choline-binding modules (ChBMs) are present in some virulence factors of Streptococcus pneumoniae (pneumococcus). The most extensively studied ChBM is C-LytA, the carboxy-terminal domain of the pneumococcal cell-wall amidase LytA. The three-dimensional structure of choline-ligated C-LytA is built up from six loop-hairpin structures ("choline binding repeats", ChBRs) forming a left-handed $\beta$-solenoid with four choline binding sites. Although the structure of the ligand-free form is not yet known, our folding studies suggest that it is more loosely packed, with a partially unfolded amino-terminal region and a stable carboxy-terminal moiety that is extremely resistant to chemical denaturation (Maestro and Sanz, 2005). The affinity of C-LytA for choline and other structural analogues allows its use as an efficient affinity tag for overexpression, immobilization and single-step purification of proteins of biomedical interest (CLYTAG fusion protein purification system). This system presents many advantages when compared to current commercial methods, namely simplicity, compatibility with buffers and robustness. The availability of multiple supports that specifically bind ChBMs (such as multiwell plates) has recently allowed the development of a new procedure for the immobilization of C-LytA-containing hybrid proteins that may be used in proteomics, diagnostics and peptide display. In this communication, we present our last results about the stability, folding and engineering of C-LytA, together with a compendium of the current biotechnological potential of this protein, and highlight the productive link between basic molecular studies and their application.

\section{References}

C-LYTAG fusion protein purification system: User's Manual. http://www.biomedal.es/clytag_purification.htm.

Maestro, B., Sanz, J.M., 2005. Biochem. J. 387, 479-488.

\section{Applied Proteomics}

\section{1}

Threading peptides and proteins into disease surveillance strategies György Marko-Varga AstraZeneca R\&D Lund, Respiratory Biological Sciences, 22187 Lund, Sweden. E-mail: gyorgy.markovarga@strazeneca.com

Many of the modern approaches for studying disease compare steady state functions, such as repair, growth, and regulated gene expression within the various biological compartments organised by specialized function, be it mitochondria or blood vessels. The assignment of protein identities, which are linked to key biological mechanisms, which are associated with disease processes and disease progressions are an important area of this work (MarkoVarga and Fehniger, 2004). Today, the technology available for studying proteome expression and resolving exact protein and peptide identities in complex mixtures of biological samples allows global protein expression within cells, fluids, and tissue to be approached with confidence. This confidence is due in part to reproducible repetitive sampling and analysis technologies including robotics data acquisition and high level mass spectrometry including both laser-desorbtion and electro spray ionisation. The precision in defining differences between normal and diseased steady states is aided by the creation of compiled reference and master data sets and by new methods for multiplexing the analysis of samples in groups. The establishment of key representative reference proteome systems representing the dynamic changes in protein expression during disease will be vital to the interpretation of changes observed in specific samplings of disease states and specific cells obtained from these samples. The creation of reference databases of proteins linked to disease pathways will play an important role in furthering our understanding of the "proteome of disease". Examples will be given where protein expression patterns have been generated from compartments within tissue sections.

\section{Reference}

Marko-Varga, G., Fehniger, T.E., 2004. J. Proteome Res. 3, 167178.

2

Adaptation of the Saccharomyces cerevisiae proteome to nutrient limitations studied by metabolic stable isotope labeling and mass spectrometry Albert J.R. Heck Netherlands Proteomics Centre and Utrecht University, The Netherlands. E-mail: heck@npc.genomics.nl. Url: www.netherlandsproteomicscentre.nl

One of the major aims of proteomics is to provide quantitative data on differential protein expression levels. Recently, mass spectrometry-based methods have been introduced that can provide quantitative data on differential protein expression, mostly using stable isotope labeling (Goshe and Smith, 2003). We opted for metabolic labeling as this provides efficient means to quantify differential protein expression, and has the advantage that all proteins are labeled universally (Romijn et al., 2003). In their natural habitat microorganisms encounter non-optimal growth conditions and often growth is limited by one nutrient. Microorganisms need to respond rapidly to changes in the environment in order to survive. In the present study, we investigate the proteome response of chemostat cultivated wildtype Saccharomyces cerevisiae to two different nutrient limitations, namely carbon and nitrogen limitation. Yeast was metabolically labeled in well-controlled chemostat cultures. ${ }^{14} \mathrm{~N}$ and ${ }^{15} \mathrm{~N}$ labeled proteins were separated using $1 \mathrm{D}$ gel electrophoresis followed by RP-LC-ESI-MS on a LC-Q. Relative quantification was performed by using Relex software (MacCoss et al., 2003). We quantified 759 proteins, using on average 8 peptide peak pairs per protein. This analysis revealed that 419 proteins showed a significant increase/decrease in expression level. The functional annotation of these proteins revealed that the yeast cells change expression levels of enzymes involved in metabolism of the growth-limiting compound. The protein expression ratios were compared with corresponding transcript levels. Moreover, we compared the accuracy of quantifica- 
tion by stable isotope labeling with that achieved by DIGE analysis of the same yeast samples (Kolkman et al., 2005).

\section{References}

Goshe, M.B., Smith, R.D., 2003. Curr. Opin. Biotechnol. 14, 101-109.

Kolkman, A., Dirksen, E.H., Slijper, M., Heck, A.J., 2005. Mol. Cell. Proteomics 4, 255-266.

MacCoss, M.J., Wu, C.C., Liu, H., Sadygov, R., Yates, J.R., 3rd, 2003. Anal. Chem. 75, 6912-6921.

Romijn, E.P., Krijgsveld, J., Heck, A.J., 2003. J. Chromatogr. A 1000, 589-608.

3

Proteome analysis of the xylose-fermenting Saccharomyces cerevisiae strain TMB 3400 Kaisa Karhumaa $^{1 *}{ }^{*}$, Anna-Karin Påhlman ${ }^{2}$, Halfdan Grage ${ }^{1}$, Bärbel Hahn-Hägerdal ${ }^{1}$, Fredrik Levander ${ }^{2}$, Marie-F. Gorwa-Grauslund ${ }^{1}$ : ${ }^{1}$ Lund University, Applied Microbiology, P.O. Box 124, 22100 Lund, Sweden; ${ }^{2}$ Lund University, Protein Technology, P.O. Box 7031, 22007 Lund, Sweden. E-mail: kaisa.karhumaa@tmb.lth.se (K. Karhumaa)

Saccharomyces cerevisiae is unable to utilize the pentose sugar xylose, which is abundant in lignocellulose hydrolysates used for biofuel ethanol production. S. cerevisiae strains carrying heterologous genes for xylose reductase (XR) and xylitol dehydrogenase $(\mathrm{XDH})$ are capable of slow rates of xylose utilisation. Random mutagenesis was used to improve xylose utilisation in the industrial $S$. cerevisiae strain TMB 3399, resulting in xylose fermenting and growing strain TMB 3400 (Wahlbom et al., 2003. FEMS Yeast Res. 3: 319-326). To identify the changes induced by the mutagenesis, transcriptome (Wahlbom et al., 2003. Appl. Env. Microbiol. 69: 740-746) and proteome (this work) analyses were performed on both strains grown in continuous chemostat cultivations. The 2D-electrophoresis for proteome analysis was performed using the differential in-gel method. The gel images were analysed with the DeCyder 6.0 software (Amersham Biosciences) and protein spots that was found to be differentially expressed in the two strains were analysed by mass spectrometry. Results of the proteome analysis were compared with the transcription data.

\section{4}

Proteomics of the regenerating rat peripheral nerve after injury Connie R. Jiménez ${ }^{1}$, Floor J. Stam ${ }^{1}, \mathrm{Ka}$ Wan Li ${ }^{1}$, Fred De Winter ${ }^{2}$, Martin P. Hornshaw ${ }^{3}$, Roel van der Schors ${ }^{1}$, Joost Verhaagen ${ }^{2}$, August B. Smit ${ }^{1}$ : ${ }^{1}$ Department of Molecular and Cellular Neurobiology, Research Institute Neurosciences Amsterdam, Faculty of Earth and Life Sciences, Vrije Universiteit Amsterdam, The Netherlands; ${ }^{2}$ Netherlands Institute for Brain Research, Amsterdam, The Netherlands; ${ }^{3}$ Applied Biosystems, Lingley House, 120 Birchwood Boulevard, Warrington, Cheshire, WA3 7QH, Great Britain, UK

Using proteomics, we investigated the temporal expression profiles of proteins in rat sciatic nerve after experimental crush. Extracts of sciatic nerves collected at 5, 10 and 35 days after injury were analyzed by two-dimensional gel electrophoresis and quantitative image analysis. Of the $\sim 1500$ protein spots resolved on each gel, 121 showed significant regulation during at least one time point. Using cluster analysis these proteins were grouped into two expression profiles of down-regulation and four of up regulation. These profiles mainly reflected differences in cellular origins in addition to different functional roles. Mass spectrometric analysis identified 82 proteins pertaining to several functional classes, i.e. acute phase proteins, antioxidant proteins and proteins involved in protein synthesis/maturation/degradation, cytoskeletal (re)organization and in lipid metabolism. Several proteins not previously implicated in nerve regeneration were identified, e.g. translationally-controlled tumor protein, annexin A9/31, Vitamin D-binding protein, $\alpha$-crystallin B, $\alpha$-synuclein, dimethylargininases and reticulocalbin. Real-time PCR analysis of selected genes showed which were expressed in the nerve versus the dorsal root ganglion neurons. In conclusion, this study highlights the complexity and temporal aspect of the molecular process underlying nerve regeneration and points to the importance of glial and inflammatory determinants.

5

Biomarker research with SELDI-TOF mass spectrometry Jakob Albrethsen $^{1}$, Rikke Bøgebo ${ }^{1}$, Jesper Olsen ${ }^{2}$, Hans Raskov ${ }^{3}$, Steen Gammeltoft ${ }^{1}$ : ${ }^{1}$ Department of Clinical Biochemistry, Glostrup Hospital, 2600 Glostrup, Denmark; ${ }^{2}$ Department of Gastrosurgery, Glostrup Hospital 2600 Glostrup, Denmark; ${ }^{3}$ Colotech ltd., Copenhagen Science Park Symbion, Fruebjergvej 3, 2100 Copenhagen $\emptyset$, Denmark

SELDI-TOF MS was evaluated with respect to mass accuracy, intra- and inter-assay reproducibility of peak intensity and sensitivity with analysis of the plasma proteome. Sample processing was analysed with respect to biological variation (fasting, age, gender and diurnal variation), sample preparation (coagulation time, freeze/thaw cycles, storage on ice and storage at room temperature) and sample analysis (instrument settings, matrix solutions and MALDI-TOF MS on the SELDI instrument (PBSII)). The average coefficient of variance (CV) for the $\mathrm{m} / \mathrm{z}$ value was $0.02 \%$ and the $\mathrm{CV}$ for the peak intensity was $13 \%$, for 34 reproducible peaks in the range from 2 to $30 \mathrm{kDa}$. Significant inter-assay variation of the peak intensity was observed with SELDI-TOF MS. Individually, but homogenously, prepared matrix solutions contributed significantly to the inter-assay variation. Pre-fractionation of serum improved the sensitivity of the plasma proteome. The serum protein profile with SELDI-TOF MS varied with fasting, coagulation time, storage on ice for several hours and storage at room temperature. A preliminary biomarker for colorectal cancer was identified, consisting of a combination of six protein peaks identified solely by their $\mathrm{m} / \mathrm{z}$ values ( $m / z: 3982,5909,6886,8932,13759,28065)$. The biomarker pattern was developed by artificial neural network analysis of the spectral output from 110 serum samples from patients with colorectal cancer (Dukes' A-D) and 88 serum samples from healthy individuals. The samples were analysed on seven individual days over a period of 5 weeks. The biomarker pattern accurately classified cancer versus healthy serum from an individual and blinded validation experiment. 
Poster Abstracts Proteomics and Protein Engineering (PPE)

\section{PPE1}

Yeasts plasma membrane macromolecular components involved in stress resistance Paola Branduardi, Paola Paganoni, Danilo Porro Dipartimento di Biotecnologie e Bioscienze, Università degli Studi di Milano-Bicocca, Piazza della Scienza, 2-20126 Milano, Italy. E-mail: paola.branduardi@unimib.it (P. Branduardi)

The plasma membrane is a universal structure of living cells constituting an essential barrier dividing and defining the intracellular from the extracellular environment. It is consequently easy to deduce the crucial role played by said structure for any cell of any living organism, and especially for unicellular organisms, since all the information deriving from the external environment as well as many of the consequent cellular responses have to pass through this barrier. Unicellular organisms, thanks to easy manipulation and cultivation techniques, can represent a very useful model for studying the plasma membrane function and response. In addition microorganisms, and among them yeasts, can be considered advantageous cell factories for recombinant productions. In this contest, the implementation of any process of production has to take into account, among others, the response and the tolerance of the host to the external environment. From these considerations derives the interest of our group to analyse the main macromolecular components of yeasts plasma membranes (proteins, lipoproteins and lipids), isolated from cells grown under different stress conditions, with particular attention to acidic environments. Here, we present our recent data about separation and identification (by sequencing analyses) of lipoproteins isolated from the conventional yeast Saccharomyces cerevisiae as well as from the non-conventional and acid tolerant yeast Zygosaccharomyces bailii cell cultures grown in different conditions. In parallel, the protein fraction is under evaluation through a differential 2D proteomic approach and consequent analyses.

\section{PPE2}

Effect of fungal polysaccharides on the expression of pancreatic proteins in streptozotocin-induced diabetic rats Sang Woo $\mathrm{Kim}^{1}$, Hye Jin Hwang ${ }^{1}$, Kwang Bon Koo ${ }^{2}$, Jang Won Choi ${ }^{2}$, Jong Won Yun ${ }^{1 *}:{ }^{1}$ Department of Biotechnology; ${ }^{2}$ Department of Bioindustry, Daegu University, Kyungsan, Kyungbuk 712-714, Korea. E-mail: jwyun@daegu.ac.kr (J.W. Yun)

In an attempt to search novel biomarkers for monitoring diabetes prognosis, we examined the influence of the hypoglycemic fungal extracellular polysaccharides (EPS) on the differential expression of pancreatic proteins in streptozotocin-induced diabetic rats. The results of diabetic study revealed that orally administrated EPS exhibited excellent hypoglycemic effect, lowering the average plasma glucose level of the diabetic rats to $52.3 \%$. Pancreatic proteome were analyzed by 2-DE system, which separated more than 2000 individual spots. The 2-DE analysis demonstrated that thirty-four proteins from a total of about 500 matched spots were differentially expressed, of which 26 spots were identified as the proteins whose expression has previously been associated with diabetes. Twenty-two overexpressed and twelve underexpressed proteins were significant $(p<0.05)$ between the healthy and diabetic rats, and the altered proteins were restored $(p<0.05)$ upon EPS treatment. It was first found that carbonyl reductase (18.6-fold, $p<0.001)$ and MAWDBP (31.4-fold, $p<0.01$ ) were surprisingly upregulated upon diabetes induction, and then those two protein concentrations were completely restored by EPS treatment. Moreover, we obtained eight unidentified proteins that have not been reported to be related with diabetes mellitus. These results evidenced the effect of fungal EPS on searching potential markers for diagnosis and therapeutic manipulation of diabetes mellitus. Although molecular basis of protein modulation after EPS administration in diabetic rats was not verified in this study, the results of the proteomic analysis provide impetus for further studies of mining the biomarkers for diabetic therapy. The model established in our experiment is expected to mimic human diabetic status, which will help us to interpret the roles of biomarkers in diabetic state.

\section{PPE3}

The use of polyol-responsive monoclonal antibodies in immunoaffinity chromatography and as a probe for unfolding of wild-type and altered (T103I) amidase from Pseudomonas aeruginosa S.Martins ${ }^{1}$, J. Andrade ${ }^{1}$, A. Karmali ${ }^{1}$, A.I. Custódio $^{2}$, M.L. Serralheiro ${ }^{3}:{ }^{1}$ Centro de Investigação de Engenharia Química e Biotecnologia, Instituto Superior de Engenharia de Lisboa, Rua Conselheiro Emídio Navarro, 1, 1949-014 Lisboa, Portugal; ${ }^{2}$ Unidade de Tecnologia de Proteínas e Anticorpos Monoclonais, Instituto Nacional de Engenharia e Tecnologia Industrial, Estrada do Paço do Lumiar, 1649-038 Lisboa, Portugal; ${ }^{3}$ Centro de Química e Bioquímica, Faculdade de Ciências da Universidade de Lisboa, Edifício C8, Campo Grande, Lisboa, Portugal. E-mail: smartins@deq.isel.ipl.pt (S. Martins)

Since immunoaffinity chromatography is a powerful protein purification technique of interest in proteomics, monoclonal antibodies (Mabs) against mutant (T103I) amidase from P. aeruginosa were raised by hybridoma technology. In order to identify Mabs that bind T103I amidase tightly but release under gentle conditions, hybridoma clones secreting polyol-responsive Mabs (PR-Mabs) were previously screened. Nearly $10 \%$ of ELISA assay-positive hybridoma produced clones secreting PR-Mabs with potential application as ligands for immunoaffinity chromatography. To select the optimal conditions for amidase elution, an ELISA-elution assay was carried out, with two of these clones (F6G7; E2A6). The dissociation of ag-ab complex required $10 \%$ of propylene glycol and either $0.25 \mathrm{M}\left(\mathrm{NH}_{4}\right)_{2} \mathrm{SO}_{4}$ or $0.25 \mathrm{M} \mathrm{NaCl}$. The binding of purified Mab of IgM class (E2A6) to wild-type and mutant amidases was investigate by direct ELISA, which revealed that it recognised specifically a common epitope on both amidases. Conformational changes on antigen molecule were studied. Mab E2A6 showed a higher affinity for heat denatured forms than for native forms as revealed by affinity constants suggesting that the Mab recognizes a cryptic epitope. The effect of Mab E2A6 on amidase activity was also investigated. The binding of Mab to wild-type and mutant amidases exhibited an inhibition and activation of $60 \%$ as a function of time, respectively. This PR-Mab is useful as a probe to detect conformational changes in native and denatured amidases as well as a ligand in immunoaffinity chromatography, which is of great interest in protein purification and proteomics. 


\section{PPE4}

Fragility and solubility of non-classical inclusion bodies $\breve{S}_{\text {. Peternel }}^{1}$, A. Ristič ${ }^{1,2}$, V. Gaberc-Porekar ${ }^{1}$, V. Menart $^{1,2}$ : ${ }^{1}$ National Institute of Chemistry, Ljubljana, SI-1000; ${ }^{2}$ Lek Pharmaceuticals d.d., Ljubljana, SI-1000. E-mail: spela.peternel@ki.si (Š. Peternel)

Human granulocyte colony stimulating factor (G-CSF) is a pharmaceutically important cytokine. When overexpressed in Escherichia coli, it is usually accumulated in the form of inclusion bodies (IBs). When produced at $42^{\circ} \mathrm{C}$ classical insoluble IBs are formed while at $25^{\circ} \mathrm{C}$ non-classical IBs containing a high amount of correctly folded G-CSF are formed. As higher fragility and solubility of non-classical IBs were noticed, we decided to check whether bacterial cell disruption method has any influence on their mechanical stability and solubility. Enzymatic lysis, sonication and homogenization, methods often used for disruption of bacterial cells during the isolation of IBs were compared. Lysozyme treatment of bacterial cells appears to be mild enough disruption method not influencing the integrity of IBs. Homogenization of bacterial cells at high pressure (100.000-120.000 kPa) shows no impact on classical IBs while some loss of target protein from the non-classical IBs is observed. Sonication seems to be most harmful as even at rather low sonication altitudes, noticeable disassembling and solubilization of non-classical IBs occurs while no effect on classical IBs is perceived. Our studies show that non-classical IBs are much more fragile and soluble than classical ones. Therefore, one should extremely carefully choose the method for cell disruption to avoid undesirable loss of the target protein.

\section{PPE5}

Identification of the blood hosts of $I$. ricinus by ELISA J. Vennestroem, J.M. Jensen Department of Ecology, Section for Genetics and Microbiology, KVL, Frederiksberg, Denmark

The Danish tick Ixodes ricinus parasitize three different hosts both mammals and birds during the 3-year life cycle. The aim of this study was to identify the last blood host being the host, which the nymph had parasitized before molting to the adult instar. The reason for the study was to reveal the origin of the host contributing the most to the life cycle of the tick and thereby the maintenance of tick-borne diseases in Denmark. The most common tick-borne diseases are Lyme Borreliosis and Tick-borne encephalitis (TBE) causing illness in both animals and humans. We analyzed adult ticks, which were collected from known hosts. The analysis was performed at different heat stable proteins, which could be detected during the off host period by ELISA. We found that heat stable proteins could be used as identification markers for host recognition.

\section{PPE6}

Mushroom polysaccharides alter the expression of diabetesassociated proteins in the liver of streptozotocin-induced diabetic rats Hye-Jin Hwang ${ }^{1}$, Sang-Woo Kim ${ }^{1}$, Kwang-Bon Koo ${ }^{2}$, JangWon $\overline{\text { Choi }^{2} \text {, Jong-Won Yun }}{ }^{1 *}:{ }^{1}$ Department of Biotechnology, Daegu University, Kyungsan, Kyungbuk 712-714, Korea; ${ }^{2}$ Department of Bioindustry, Daegu University, Kyungsan, Kyungbuk 712-714, Korea. E-mail: jwyun@ daegu.ac.kr (J.-W. Yun)

In the present study, we investigated the influence of the hypoglycemic fungal extracellular polysaccharides (EPS) on the differential expression of liver proteins in streptozotocin (STZ)-induced diabetic rats. The results of diabetic study revealed that orally administrated EPS exhibited an excellent hypoglycemic effect, lowering the average plasma glucose level in EPS-fed rats to $52.3 \%$. In the next step, we analyzed the differential expression patterns of rat liver proteins from each group, to discover potent candidates for diabetesassociated proteins. A total of 69 proteins of the 2-DE gel were expressed differentially between diabetic and healthy rats. Among them, 34 proteins were upregulated and 35 proteins were downregulated upon diabetes induction. Many of these changes were in accordance with observations in previously published studies. Surprisingly, the altered levels of most proteins in diabetic group were fully or partially restored to those of non-diabetic control group by EPS treatment. Moreover, we obtained 13 unidentified proteins that have not been reported to be related with diabetes mellitus. Although molecular basis of protein modulation after EPS administration in diabetic rats was not verified in this study, the results of the proteomic analysis provide impetus for further studies of mining the biomarkers for diabetic therapy.

\section{PPE7}

Recombinant major cat allergen FeldI for diagnosis of cat allergy Marlena Szalata ${ }^{1,2}$, Daniel Lipinski ${ }^{2}$, Robert Kalak ${ }^{1}$, Paulina Tobola ${ }^{3}$, Joanna Lehmann ${ }^{1}$, Marta Pawlik ${ }^{1}$, Wojciech Juzwa ${ }^{1}$, Karolina Wielgus ${ }^{1}$, Marek Pienkowski ${ }^{4}$, Ryszard Slomski ${ }^{1,2,3,4}$, ${ }^{1}$ Department of Biochemistry and Biotechnology, Agricultural University, Poznan, 60-637, Poland; ${ }^{2}$ Institute of Human Genetics, Polish Academy of Sciences, Poznan, 60-479, Poland; ${ }^{3}$ Delta Pharma BV, Hengelo, 7554 TR, the Netherlands; ${ }^{4}$ Allergic Diseases, Asthma and Immunology Clinic, P.C., Knoxville, TN 37917, USA. E-mail: marsz@au.poznan.pl (M. Szalata)

Current diagnostics and therapeutics for cat allergy are based on cat epithelial extracts. Natural allergen extracts contain a mixture of allergenic and non-allergenic components that are difficult to standardize. Recombinant allergens can improve diagnosis and de-sensitization against single component. Major cat allergen is a heterodimer composed of disulfide linked 7.8 and $10.1 \mathrm{kDa}$ polypeptide chains. Both chains of FeldI protein were obtained in E. coli system. Purification of recombinant proteins was performed in denaturing conditions using immobilized metal affinity chromatography specific for proteins with histidine tag. From $1000 \mathrm{ml}$ culture approximately $13 \mathrm{mg}$ protein for FeldI chain 1 and $43 \mathrm{mg}$ of FeldI chain 2 were obtained. After purification histidine tag was removed by hydrolysis with thrombin. Immunological activity of FeldI against serum of patients allergic to cat was narrowed to subgroup of patients allergic to FeldI protein by surface plasmon resonance. Immunological activity of each chain and renatured heterodimer was also tested using immunoprecipitation techniques against serum of population group.

\section{PPE8}

Expression of human $\beta 1,4$-galactosyltransferase to "humanize" $N$-glycans in alfalfa Estelle Marquet-Blouin ${ }^{1,2}$, Martial Séveno ${ }^{1,2}$, Muriel Bardor ${ }^{2}$, Claude Saint-Jore-Dupas ${ }^{2}$, Marc-André D'Aoust ${ }^{1}$, Patrice Lerouge ${ }^{2}$, Louis Vézina ${ }^{1}$, Loïc Faye ${ }^{2}$, Véronique Gomord ${ }^{2}$ : ${ }^{1}$ MEDICAGO Inc., 1020 route de l'Eglise Sainte Foy, Qué., Canada G1V 3V9; ${ }^{2}$ CNRS UMR 6037-IFRMP23-GDR2590, Université de Rouen 76821 Mont Saint Aignan Cedex, France

Plants are considered as a promising system for the production of recombinant therapeutic proteins. However, the use of this 
potential is still limited by the differences observed in the structure of plant and mammalian $\mathrm{N}$-glycans. Indeed, theses differences and particularly the presence of $\beta 1,2$-xylose and $\alpha 1$,3-fucose glycoepitopes are responsible for the immunogenicity of plant $\mathrm{N}$-glycans. In order to reduce the structural differences between plant and mammalian $\mathrm{N}$-glycans, current strategies are to knock out plant-specific glycosyltransferases or to humanize plant $\mathrm{N}$-glycans by expression of mammalian glycosyltransferases in plants. In the present study, we have expressed a human $\beta 1,4$-galactosyltransferase in alfalfa. In order to further increase the efficiency of the human $\beta 1,4$-galactosyltransferase in the plant Golgi apparatus, we have exchanged the endogenous targeting signal of this human glycosyltransferase for the ones from plant glycosyltransferases recently characterized in our laboratory. We will illustrate this approach of targeted expression with the results obtained by fusion of the catalytic domain of human $\beta 1$,4-galactosyltransferase with the $\mathrm{N}$-terminal sequence of a plant glycosyltransferase that targets the fusion to the very early compartments of the Golgi apparatus. The efficiency of natural versus targeted expression of human $\beta 1,4$ galactosyltransferase in alfalfa will be compared in term of $\mathrm{N}$-glycan humanization. Altogether, our results clearly illustrate that we are now on the way to get perfect copy of mammalian glycoproteins in alfalfa plants.

\section{PPE9}

Construction of $\operatorname{recB}$-recD gene fusion and analysis of fusion enzyme activities Oytun Portakal ${ }^{1}$, Gerald R. Smith ${ }^{2}$, Pakize Doğan ${ }^{1}{ }^{1}$ Department of Biochemistry, Hacettepe University Medical School, 06100, Ankara, Turkey; ${ }^{2}$ Divisions of Basic Sciences, Fred Hutchinson Cancer Research Center, Seattle, WA 98109-1024, USA. E-mail: oytun@ hacettepe.edu.tr (O. Portakal)

Protein folding is a fundamental process to gain protein function. In an oligomeric protein, the interaction between polypeptides affects the folding process and assembly to the holoenzyme. RecBCD is a heterotrimeric and multifunctional enzyme that plays an essential role for major pathway of homologous recombination in $E$. coli. It is composed of $r e c \mathrm{~B}, r e c \mathrm{C}$ and $r e c \mathrm{D}$ gene products. RecD is the fast motor unit of the RecBCD enzyme. RecD also plays a role for high affinity dsDNA binding, nuclease activity and Chidependent regulation. This study was designed to test the hypothesis that RecD polypeptide regulates the essential RecA loading activity. The approaching of the study was to fuse $r e c \mathrm{D}$ gene to subsequent $r e c \mathrm{~B}$ gene and to observe the changes in enzyme activity and structure. For these purpose two genetic fusion mutations, two-nucleotide deletion and three-codon substitution were created at the overlap sites (TA) of $r e c \mathrm{~B}$ and $r e c \mathrm{D}$ genes. Fusion mutations were constructed by phage-mediated recombination system, which is called Recombineering. This technology requires $\lambda$ Red function but not host RecA protein function. Here, we showed the RecBD fusion polypeptides in crude extracts. Genetic characterization tests were revealed that both fusion enzymes are recombination proficient and have wild-type phenotype. Biochemical assays demonstrated that RecBDC fusion heterotrimers have dsDNA exonuclease, unwinding and Chi cutting activities. They were also resistant to DNA damaging agents. Western blot analysis also detected a wild type length RecD polypeptide together with RecBD fusion polypeptides and a decreased heterotrimer compared to wild type. Our findings suggest that $r e c \mathrm{~B}-r e c \mathrm{D}$ genetic fusions may affect RecD assembling to the heterotrimer, but not affect it's native folding.

\section{PPE10}

Sandwich immunoassay-a simple strategy for enhancement of the sensitivity and the specificity in prostate specific antigen detection based on surface plasmon resonance Cuong Cao, Sang Jun Sim Department of Chemical Engineering Sungkyunkwan University, 300 Chunchun-Dong, Jangan-Gu Suwon, Kyunggi-Do 440-746, Korea. E-mail: simsj@skku.edu (S.J. Sim)

Prostate cancer is a deadly disease in men. Prostate specific antigen (PSA) has been proved to be the most reliable and specific biomarker in preoperative diagnosis, monitoring and followup of patients with prostate cancer. In this study, a biochip based on surface plasmon resonance was fabricated to detect PSA at concentrations ranging from 1 to $1000 \mathrm{ng} / \mathrm{ml}$. To reduce nonspecific binding, the chemical surface of sensor was constructed by using various ethyleneglycol mixtures of different molar ratios of $\mathrm{HS}\left(\mathrm{CH}_{2}\right)_{11}\left(\mathrm{OCH}_{2} \mathrm{CH}_{2}\right)_{6} \mathrm{COOH}$ and $\mathrm{HS}\left(\mathrm{CH}_{2}\right)_{11}\left(\mathrm{OCH}_{2} \mathrm{CH}_{2}\right)_{3} \mathrm{OH}$. We also biotinylated the SAMs surface to enhance the orientation of protein immobilization. By using this surface, SPR-based PSA detection gave a positive $\mathrm{RU}$ value at the fist response in the whole range of PSA concentrations. However, this RU value could get better and more reliable by simply applying a secondary interactant, the PSA polyclonal antibody, in sandwich immunoassay. The results shown this approach could satisfy our purpose without modify the secondary interactant, which has usually been done by the other report.

\section{PPE11}

Expression of epitopic domains of human coagulation factor VIII in Escherichia coli Amir Amiri Yekta ${ }^{1,2}$, Naser Amirizadeh $^{3}$, Alireza Zomorodipour ${ }^{1 *}$, Fariba Ataei ${ }^{1}{ }^{1}{ }^{1}$ Department of Mol Genet. National Institute for Genet Eng \& Biotechnol Tehran-Iran P.O. Box: 14155-634, Tehran, Iran; ${ }^{2}$ Islamic Azad University of Jahrom, Jahrom, Iran; ${ }^{3}$ Department of Hematol, Faculty of Med, Tarbiat Modarres University, Tehran, Iran. E-mails: amir_amiriyekta@yahoo.com (A.A. Yekta), *zomorodi@nrcgeb.ac.ir (A. Zomorodipour)

Human factor VIII (hFVIII) plays major role in the intrinsic pathway of blood coagulation and is used to treat individuals with hemophilia A for bleeding episodes. Many researches have been focused on the molecular aspects of this protein. In this regard, epitopes of hFVIII as well as their corresponding antibodies have many important applications. Bacterially produced FVIII-epitopes are capable to neutralize the alloantibodies that inhibit hFVIII activity. The purpose of present study was to over-express two epitope-containing fragments of FVIII in E. coli under T7 promoter (Novagen). Two DNA fragments from light- and heavy-chains of hFVIII (942bp-C1C2 and 1644bp-A1A2, respectively) were subcloned in the expression vector. The use of $\mathrm{His}_{6}$-tagged tail was also considered for detection and purification purposes. In each of the examined clones, a protein of expected size was detectable. In the $\mathrm{C} 1 \mathrm{C} 2$-expressing clone the specificity of the over-expressed protein was confirmed by its reaction with the rabbit serum directed against native hFVIII as well as anti-His-tag antibody. In the heavy chain-related-expressing clone, the expression level was low, but it was detectable by immunoblotting experiments. Manipulations of the growth as well as induction may be required. The over- 
expression of the other epitopes reported in the heavy chain may be achievable by the expression of (a) sub-fragment(s) of this region. The over-expressed His-tagged C1C2-related protein was appeared to be trapped in the cell as non-soluble inclusion bodies. Therefore, after homogenizing of the induced recombinant cells, the nonsoluble fraction was dissolved in a solution of denaturant (Guanidine hydrochloride) and subjected for the purification, using a Ni-NTA resin (Qiagen) followed by protein measurement. Accordingly, an expression level of $5 \mathrm{mg} / \mathrm{l}$ (of culture) of the purified C1C2-related peptide was obtained. The recombinant hFVIII C1C2-derived peptide has provided useful mean for further experimental and medical applications.

\section{PPE12}

Microfluidic isotachophoretic analysis of milk proteins in epoxy(poly)dimethylacrylamide coated glass chips $C$.-H Brogren ${ }^{1,2}, J$. Vial $^{2}$, J.-M. Busnel ${ }^{2}$, S. Descroix ${ }^{2}$, M. Cretich $^{3}, M$. Chiari $^{3}, G$. Peltre ${ }^{2}:{ }^{1}$ Department of Medical Biochemistry and Genetics, University of Copenhagen, DK-2200, Denmark; ${ }^{2}$ Laboratoire Environnement et Chimie Analytique, Ecole Superieure de Physique et de Chimie Industrielles, Paris F-75005, France; ${ }^{3}$ Institute of Biocatalysis and Molecular Recognition, Consiglio Nazionale delle Ricerche3, Milano I-20131, Italy

Isotachophoresis has almost exclusively been applied for contracting and stacking samples ions before zone electrophoretic separation of proteins. This study attempts to apply microfluidic isotachophoresis (ITP) as a high resolution analytical method for proteins. Beta-lactoglobulin and other milk proteins with slightly different $\mathrm{pI}$ were labelled with Fluorescent Red 646 and analysed by the Micralyne $\mu \mathrm{TK}$ system using microfluidic glass chips, either with simple cross (SC) or double cross (TT) injection or designed 2D-ITP-CZE chips with double TT injection and SC for transfer to the second dimension CZE channel, efficiently non-covalently coated with $0.6 \%$ w/v epoxy-polydimethylacrylamide to lower electroendoosmosis. Capillary zone electrophoresis (CZE) in borate or phosphate buffer was reproducibly perform for more than 75 consecutive runs using Upchurch $^{\mathrm{TM}}$ reservoirs glued to the wells to enable larger buffer volumes and greater run-to-run stability. Finally, isotachophoretic anionic separation of the proteins were done using phosphate $(\mathrm{pH}$ $8.1)$ or chloride $(\mathrm{pH} 8.5)$ as leading ion and $\varepsilon$-amino-caproic acid $(\mathrm{pH} 8.9)$ as terminating ion. The effect of narrow cut ampholytes as spacers needs further investigations. The perspective aim is to combine the migrating ITP separated zones with second dimension capillary zone electrophoresis as a new microfluidic proteomic 2Danalysis.

\section{PPE13}

Development of strategies for heterologous expression of glucose dehydrogenase from the halophilic archaeon Halobacterium sp. NRC-1 Juan Carlos Cruz-Jiménez ${ }^{1}$, Lorenzo Saliceti-Piazza ${ }^{1}$, Rafael Montalvo ${ }^{2}:{ }^{1}$ Chemical Engineering, University of Puerto Rico-Mayagüez Campus, Mayagüez 00680, Puerto Rico; ${ }^{2}$ Biology, University of Puerto Rico-Mayagüez Campus, Mayagüez 00680, Puerto Rico. E-mail: juancruzj@ hotmail.com (J.C. Cruz-Jiménez)

Halophilic archaea are excellent model organisms and valuable for biotechnology applications; they are easy to culture in the lab, genetically tractable, and exhibit a variety of interesting and useful characteristics. Most halophilic archaea require $1.5 \mathrm{M} \mathrm{NaCl}$ to sustain growth and structural integrity. Among the 2.630 genes in Halobacterium, we are studying the gene encoding a glucose dehydrogenase, gene ID is 446, located between the 345205 and 346272 bases (Halobacterium sp. NRC-1 genome project). This extremozyme is bioengineerable, and its use as a model for studying biocatalysis in aqueous/organic and nonaqueous media has not been explored to date. The utilization of enzymes in organic solvents has several potential advantages over aqueous systems. A major benefit is the increased solubility of many substrates, resulting in higher concentrations of reactant and products, hence reducing and purification costs and simplifying recovery protocols. Cells were grown aerobically during seven days at $37^{\circ} \mathrm{C}$ in a complex medium, harvested by centrifugation and their genomic DNA extracted. For cloning of the gene, primers were designed based on the sequence recently published by the Halobacterium sp. NRC-1 genome project. The forward (5'-CCGCATGCGCC CACAGTCCC- $\left.3^{\prime}\right)$ and reverse (5'CCGGCCTCTAGAACGGCCTGG- $3^{\prime}$ ) primers were designed to incorporate restriction sites for Sph I and Xba I, respectively (in bold). We are PCR amplifying the genomic DNA and developing methods for the heterologous expression using the mesophilic Escherichia coli, as well as purifying the enzyme. The purification procedure will be carried out using high resolution methods based on the protein's halophilicity. Bioinformatics methods will be used to facilitate conforming of protein function and for comparison with a native enzyme.

\section{PPE14}

Preliminary investigation: Development of a two component feeding strategy with the help of proteome analysis Johannes Bader $^{1}$, Boris Neumann ${ }^{2}$, Karima Schwab ${ }^{1}$, Milan K. Popovic ${ }^{1}$, Christian Scheler ${ }^{2}$, Rakesh Bajpai ${ }^{3}$ : ${ }^{1}$ Studiengang Biotechnologie, Fachbereich V, TFH-Berlin, Seestr. 64, 13347, Berlin, Germany; ${ }^{2}$ Proteome Factory AG, Dorotheenstr. 94, 10117, Berlin, Germany; ${ }^{3}$ Department of Chemical Engineering, University of Missouri-Columbia, W2061 EBE, Columbia, MO, USA. E-mail: popovic@tfh-berlin.de (M.K. Popovic)

The objective of this study was to investigate the conditions that would result in increased production of thermostable $\alpha$-Amylase from Bacillus caldolyticus DSM 405. Preliminary experiments in batch shake flasks led to an optimised initial cultivation medium. Shake flask experiments in extended-batch and in fed-batch mode of operation indicated that the a-Amylase production was enhanced with continuous feeding of starch. The activity of the $\alpha$-Amylase with optimised initial medium in batch-operated shake flasks was $5.7 \mathrm{U} / \mathrm{mL}$ compared to $15.4 \mathrm{U} / \mathrm{mL}$ in the extended-batch culture and $21 \mathrm{U} / \mathrm{mL}$ in fed-batch culture. The improvements were achieved by avoiding any excess of starch in medium that led to accumulation of glucose followed by acetate formation. Adding casitone as the second component of the feeding solution in a fed-batch aerated and agitated 7-1 bioreactor led to an increased $\alpha$-Amylase activity of up to $163.7 \mathrm{U} / \mathrm{mL}$. All phases of cultivation were analysed using 2D-gel electrophoresis in combination with ESI-MS/MS. Pyruvate kinase, 6-phosphofructokinase, GltC, anti-sigma F factor, glycogen synthase and several important variable proteins were detected. With help of these results, possible improvements of the two-component feeding strategies are discussed. 


\section{PPE15}

Monitoring of the therapeutic protein, alpha galactosidase A, in human serum using liquid chromatography coupled with electrospray mass spectrometry M. Kennedy ${ }^{1}$, J.P.C. Vissers ${ }^{1}, I$. Campuzano $^{2}$, T. McKenna ${ }^{2}$, J. Langridge ${ }^{2}$, J.M.F.G. Aerts ${ }^{3}:{ }^{1}$ Waters Corporation, European Centre for Mass Spectrometry, Almere, The Netherlands; ${ }^{2}$ Waters Corporation, MS Technologies Centre, Manchester, UK; ${ }^{3}$ Academic Medical Center, University of Amsterdam, Department of Biochemistry, Amsterdam, The Netherlands

Fabry disease is an $\mathrm{X}$-linked recessive glycolipid storage disorder that is caused by deficient activity of the lysosomal enzyme $\alpha$-galactosidase A ( $\alpha$ gal A). In affected patients progressive accumulation of the glycolipid substrate for this enzyme, globotriaosylceramide, occurs within vulnerable cells and tissue. Death results from renal failure, cardiac or cerebral complications of hypertension or other vascular disease. Administration of recombinant alpha-gal A has been shown to alleviate symptoms of the disease and has been used as a long-term enzyme replacement therapy in patients with a confirmed diagnosis of Fabry's disease. Investigation and monitoring of circulating levels of $\alpha$ gal A can provide information upon the uptake and metabolism of the replacement enzyme and could potentially allow the therapeutic level of the circulating drug to be determined in patient samples. This would allow the dose of administered drug to be adjusted accordingly, to maintain safe, cost effective levels of the treatment. Here, we detail a preliminary investigation into the potential of LC-MS for analysing tryptically digested samples of human serum, containing spiked levels of recombinant $\alpha$ gal A. In this work, we have determined the limit of detection of the enzyme, present in the complex serum background, and have established the linear dynamic range over which measurements can be made. Finally, we have investigated, and will present, the potential for relative quantification of $\alpha \mathrm{gal} \mathrm{A}$ in human serum across patient sample sets.

\section{PPE16}

Quantitative measurements by mass spectrometry of hundreds of proteins simultaneously using the new proteinchip systemseries 4000 P. Iversen, E. Fernvik Ciphergen Biosystems Inc., Symbion Research Park, Fruebjergvej 3, DK-2100 Copenhagen, Denmark. E-mail: piversen@ ciphergen.com (P. Iversen)

Most mass spectrometry methods used in proteomics allow for the identification of multiple proteins in a limited number of complex samples, but lack the ability to assess the quantity of the proteins and their modifications. However, mounting evidence shows specific cleavage of well-known proteins as being strong candidates for specific biomarkers, and in order to discover these biomarkers one has to be able to monitor the quantity and mass of hundreds of proteins from hundreds of complex samples reproducibly. The new series 4000 instrument in connection with ProteinChip Arrays ${ }^{\circledR}$ from Ciphergen Biosystems enables this. The new 4000 series instrument is optimized for sensitivity, reproducibility and quantitation. New ion optics allows the use of higher acceleration voltages thus increasing the sensitivity, but without lowering the resolution. A new method of blanking the detector in connection with a non-linear gain of the detector also increases the sensitivity to the effect that IgG can be detected down to $0.2 \mathrm{fmol}$. Furthermore, the unique design of the instrument permits the detection of proteins with great variation in both mass and concentration and thus making it ideal for proteomics studies. A unique feature of the 4000 series instrument is the possibility to normalize the output by controlling the laser and detector so that results can be read with equal precision on different instruments, which is not often possible in mass spectrometry where individual instruments yield different results. This feature is vital in the validation of research results beyond individual laboratories.

\section{PPE17}

Liquid chromatography coupled with electrospray mass spectrometry for the global monitoring of protein expression levels A. Wallace, Chris Hughes, T. McKenna, J. Langridge Waters Corporation, Micromass MS Technologies Centre, Manchester, UK

The coupling of liquid chromatography with mass spectrometry is now firmly established as a routine method for the identification of proteins that have been subjected to enzymatic digestion. In an on-line LC-MS experiment, the column eluent is coupled to the electrospray source via an emitter and any tryptic peptides present in the mixture are mass analyses as they elute from the HPLC column. Should there be any co-eluting species in the eluent, these will be separated in the mass analyser by their mass-to-charge ratio. It has become increasingly clear that relative quantification of protein expression changes is important in modern biology and medicine. Several current approaches have been developed that utilise stable isotope labelling of samples in combination with separation and subsequent analysis by mass spectrometry. However, we have recently described an LC-MS strategy where quantification is achieved via normalisation of the MS datasets and comparison of the peptide intensities (of the observed tryptic peptides) across samples is performed. In this case, it is desirable to perform replicate injections and hence reduce statistical errors. This approach places a requirement upon good chromatography, especially in terms of retention time reproducibility. In addition exact mass measurement of the eluting ions is required as well as the ability to generate reproducible and reliable peak intensity, or area, calculations for the eluting tryptic peptides. The ability to measure the mass to charge ratios of ions accurately, across injections and across samples, increases confidence that the same ions have been matched from each sample injection. In this presentation our current strategy for the relative quantification of proteins will be discussed using, as examples, complex protein mixtures from Salmonella enterica, Eschericia coli and Human serum.

\section{PPE18}

Proteomic analysis for the production of rhCTLA4Ig in transgenic rice cell cultures using DIGE Ji-Suk Cho, Song-Jae Lee, Dong-Il Kim Department of Biological Engineering, Inha University, Incheon 402-751, Korea. E-mail: kimdi@ inha.ac.kr (D.-I. Kim)

Difference in gel electrophoresis (DIGE) technology using fluorescent dyes is a novel method, which simplifies the process of detecting and matching proteins between multiple gels by allowing the separation of up to three separate protein samples in the same gel. It provides accurate quantitative and reproducible differential expression values for proteins in several samples. Recombinant human cytotoxic $\mathrm{T}$ lymphocyte-associated antigen 4-immunoglobulin (rhCTLA4Ig) was produced in the transgenic rice suspension cell cultures using $\alpha$-amylase promoter system. This system is efficient for the production of recombinant proteins, as it secretes target proteins into 
culture medium under sugar-depleting condition. In this study, the intracellular proteins expressed at both growth and induction stages of culture were separated and analyzed using 2-D DIGE. Each sample from different conditions and internal standard were labeled with $\mathrm{N}$-hydroxy succinimidyl ester-derivatives of Cy2, Cy3 and Cy5 dyes and run within a single DIGE gel. Using DeCyder ${ }^{\mathrm{TM}}$ software, 2218 spots were detected with two-fold thresholds with $95 \%$ confidence and it was found that 60 proteins underwent significant change during the production of rhCTLA4Ig with normalization method improving data distribution. A pooled sample mixture for the picking gel was prepared with Deep Purple staining and analyzed with mass spectrometry. In addition, the intracellular rhCTLA4Ig spots were identified with Western blot analysis using goat anti-human $\operatorname{IgG}(\mathrm{Fc})$ antibody after DIGE gel was transferred to PVDF membrane.

\section{PPE19}

Study of substrate specificity of RNR-exoribonucelases using hybrid proteins Ana Barbas, Mónica Amblar, Cecília M. Arraiano Instituto de Tecnologia Química e Biológica, EAN, 2784-505 Oeiras, Portugal. E-mail: ab@itqb.unl.pt (A. Barbas)

The ribonucleases are essential enzymes responsible for the regulation of gene expression and have shown to be important for biotechnology purposes. For instance, commercial mutants deficient in ribonucleases have been quite relevant for the over-production of recombinant proteins. Escherichia coli RNase II is a processive $3^{\prime}-5^{\prime}$ exoribonuclease prototype of the RNR family that has homologues widespread in the majority of the sequenced genomes. By sequence alignment it has been proposed for the RNR type proteins the existence of three different domains: an N-terminal cold shock nucleotide binding domain (CSD), a RNB catalytic domain, and a C-terminal $\mathrm{S} 1$ nucleotide binding domain. We have constructed several RNase II deletion mutants to enable the characterization of each domain. These studies have allowed us to determine that both CSD and S1 are involved in the binding of the enzyme to the RNA substrate, being the $\mathrm{S} 1$ domain the most important. In RNA-binding proteins it has been shown that the $\mathrm{S} 1$ domain's conformation is highly conserved. However, it is not known whether the substrate specificity is S1-dependent. In order to characterize the S1 domain and verify if it is directly related to substrate specificity, we have constructed RNase II hybrid proteins in which the S1 domain was substituted by the $\mathrm{S} 1$ of two other exoribonucleases, RNase R (RNII-RNR) and PNPase (RNII-PNP). Preliminary results have demonstrated that both quimeric proteins are capable of binding and degrading various RNA substrates. In addition, studies are currently being carried out to verify the possibility that $\mathrm{S} 1$ domain of PNP in the hybrid protein might be involved in multimerization and/or interaction with other proteins.

\section{PPE20}

Studies of thermostability of monoclonal antibody against digoxin Soheila Kashanian

The murine monoclonal antibody $\mathrm{IgG} 1, \kappa$ anti-digoxin was produced in a rolling bottle fermentor. Purification was performed on a protein G column. CD spectra were recorded on a JASCO-810 spectropolarimeter. Protein concentrations of $20-50 \mu \mathrm{g} / \mathrm{ml}$ and path length of $1 \mathrm{~cm}$ were used for measurements in a far UV region. All measurements were performed in a cell holder thermostand with an accuracy of \pm 0.2 at $25^{\circ} \mathrm{C}$. At this temperature the predominance of $\beta$-strands is indicated. Large conformational changes occur at $78^{\circ} \mathrm{C}$. At this temperature the spectra tense to irregular $\beta$-strands and unordered structures. These evidences confirming temperaturedependent conformational changes of protein and also high thermal stability of mentioned monoclonal antibody.

\section{PPE21}

Generation of monoclonal antibodies for the assessment of protein purification by recombinant ribosomal coupling Janni Kristensen, Kim Kusk Mortensen, Hans Peter Sфrensen ${ }^{1}$ Laboratory of BioDesign, Department of Molecular Biology, Aarhus University, Gustav Wieds Vej 10 C, DK-8000 Aarhus C, Denmark. E-mail: hans.peter.sorensen@teknologisk.dk (H.P. Sørensen)

We recently described a conceptually novel method for the purification of recombinant proteins with a propensity to form inclusion bodies in the cytoplasm of Escherichia coli. Recombinant proteins were covalently coupled to the E. coli ribosome by fusing them to ribosomal protein 23 (rpL23) followed by expression in an rpL23 deficient strain of $E$. coli. This allowed for the isolation of ribsomes with covalently coupled target proteins which could be efficiently purified by centrifugation after in vitro proteolysis at a specific site incorporated between rpL23 and the target protein. To assess the efficiency of separation of target protein from ribosomes, by site specific proteolysis, we required monoclonal antibodies directed against rpL23 and GFP. We therefore purified rpL23-GFP-His, rpL23-His and GFP from E. coli recombinants using affinity, ion-exchange and hydrophobic interaction chromatography. These proteins could be purified with yields of 150,150 and $1500 \mu \mathrm{g}$ per gram cellular wet weight, respectively. However, rpL23-GFP-His could only be expressed in a soluble form and subsequently purified, when cells were cultivated at reduced temperatures. The purified rpL23GFP-His fusion protein was used to immunize balb/c mice and the hybridoma cell lines resulting from in vitro cell fusion were screened by ELISA using rpL23-His and GFP to select for monoclonal antibodies specific for each protein. This resulted in 20 antibodies directed against rpL23 and 3 antibodies directed against GFP. Antibodies were screened for isotypes and their efficiency in western immunoblots. The most efficient antibody against rpL23 and GFP were purified by Protein G Sepharose affinity chromatography. The purified antibodies were used to evaluate the separation of ribosomes from GFP, streptavidin, murine interleukin-6, a phagedisplay antibody and yeast elongation factor $1 \mathrm{~A}$ by centrifugation, when ribosomes with covalently coupled target protein were cleaved at specific proteolytic cleavage sites.

\section{PPE22}

Proteomic analysis for the production of rhCTLA4Ig in transgenic rice cell cultures using DIGE Ji-Suk Cho, Song-Jae Lee, Dong-Il Kim Department of Biological Engineering, Inha University, Incheon 402-751, Korea. E-mail: kimdi@inha.ac.kr (D.-I. Kim)

Difference in gel electrophoresis (DIGE) technology using fluorescent dyes is a novel method, which simplifies the process of detecting and matching proteins between multiple gels by allowing the separation of up to three separate protein samples in the same gel. It provides accurate quantitative and reproducible differential expression values for proteins in several samples. Recombinant human cytotoxic T lymphocyte-associated antigen 4-immunoglobulin (rhCTLA4Ig) was produced in the transgenic rice suspension cell cultures using 
$\alpha$-amylase promoter system. This system is efficient for the production of recombinant proteins, as it secretes target proteins into culture medium under sugar-depleting condition. In this study, the intracellular proteins expressed at both growth and induction stages of culture were separated and analyzed using 2-D DIGE. Each sample from different conditions and internal standard were labeled with $\mathrm{N}$-hydroxy succinimidyl ester-derivatives of Cy2, Cy3 and Cy5 dyes and run within a single DIGE gel. Using DeCyderTM software, 2218 spots were detected with two-fold thresholds with $95 \%$ confidence and it was found that 60 proteins underwent significant change during the production of rhCTLA4Ig with normalization method improving data distribution. A pooled sample mixture for the picking gel was prepared with Deep Purple staining and analyzed with mass spectrometry. In addition, the intracellular rhCTLA4Ig spots were identified with Western blot analysis using goat anti-human $\operatorname{IgG}(\mathrm{Fc})$ antibody after DIGE gel was transferred to PVDF membrane.

\section{PPE22}

Similarity searches and multiple alignment of $S_{1}$ and $S_{2}$ protein of SARS-CoV for modeling 3D structure and its evolution (origin) Mohammad Soltany Rezaee Rad, Iman Tavassoly, Negar Mottaghi, Banafsheh Rezaee. E-mail: mohammad.soltany@gmail.com (M.S.R. Rad)

Aims: The exact origin of the cause of Severe Acute Syndrome (SARS) is still an open question. Nowadays 8 recombinant origins for this virus have been found. $S_{1}$ and $S_{2}$ subunit of spike protein of this virus are the most important proteins responsible for severe acute respiratory syndrome. In fact they are glycoproteins of this virus exist on its surface. They are responsible for mediating fusion of viral and cellular membrane. The classification and modeling 3D structure of this virus can help us to suggest new ideas about its charististics and function, which may lead to new therapeutic and preventing modalities.

Methods: We used Nucleotide Sequence of $\mathrm{S}_{1}$ and $\mathrm{S}_{2}$ subunit of $\mathrm{S}$ (spike) protein for multiple alignments. We have done multiple alignments with different bioinformatics software (ClusterX, Entrez) for comparing the sequence with the other viruses and, we used WebLab View software for modeling and Identifying 3D structure of these proteins.

Findings: The similarity searches on Nucleotide Sequence of this protein with the 30 single strands RNA (ssRNA) shows the virus belong to a known classification named Coronaviridae. These 3D structures show the responsibility of $\mathrm{S}$ protein in this syndrome. Another findings based on these alignments is an important similarity between these subunits and genome of HIV-1 showing they have familiar mechanism in pathogenesis.

Discussion: Multiple alignments are powerful tool in classification of new recombinational virus and emerging infection. 3D structure model of this virus is an important guide to understand the mechanism of this virus. The shape of glycoprotein that modeled with bioinformatics software can help us in understanding mechanism of binding this virus to human cells. This fact can be used in designing drug and vaccine to cure and prevent the SARS. Blocking these origins and sites leads to inhibiting the virus attachment. Also the similarity between this virus and HIV-1 shows us that both of them have similar proteins that cause pathogenesis of these viruses.

\section{Biochemical Engineering}

\section{Downstream Processing I - Recovery of Biological Products}

Simulated Moving Bed technology for bio-separations Galatea Paredes $^{1}$, Marco Mazzotti ${ }^{1 *}$, Joachim Stadler ${ }^{2}$ : ${ }^{1}$ ETH Swiss Federal Institute of Technology Zurich, Zurich, Switzerland; ${ }^{2} \mathrm{GE}$ Healthcare, Freiburg, Germany. ${ }^{*}$ E-mail: marco.mazzotti@ipe.mavt.ethz.ch (M. Mazzotti)

The simulated moving bed (SMB) technology is a continuous countercurrent chromatographic separation technique that has been applied successfully in the last years to a number of significant problems. An SMB consists of a series of fixed bed chromatographic columns connected in a loop, and outperforms column chromatography in terms of productivity and solvent consumption. The use of SMB instead of batch processes for bioseparations, i.e. separations involving large and rather complex molecules with multiple 3D configurations depending on parameters such as $\mathrm{pH}$, temperature, etc., is becoming of greater and greater interest. Examples of these are therapeutic proteins, antibodies and plasmid DNA among others. For all chromatographic processes in this field, one of the most crucial issues is the cleaning of the chromatographic media with a special solvent system, an operation usually referred to as cleaning in place (CIP). In single column chromatography this is easily done off-line, but this is not compatible with standard SMB operation. In order to overcome this limitation, the standard SMB configuration has been modified by adding a dedicated section plus an additional section for the re-equilibration of the freshly cleaned column with the working solvent before it is re-inserted into the SMB loop. In such a way, CIP according to GMP criteria can be incorporated into the SMB unit and operation, which is then called CIP-SMB. This new SMB configuration is also related to the three fraction separation unit called 3 F-SMB that has been recently introduced and applied to the separation of nucleosides. In this work we apply CIP-SMB using a size exclusion stationary phase to the separation of plasmid DNA from the filtered cell lysate solution. Plasmid purification has become a key issue in the last years as a result of the advances in gene therapy, whereas traditional laboratory methods are not always suitable for therapeutic purposes. We report about separation performances, which are then discussed in the light of SMB design criteria and compared to column chromatography performance.

2

Computer guided optimization of adsorptive bioseparation processes Bernt Nilsson Department of Chemical Engineering, Lund University, 22100 Lund, Sweden. E-mail: bernt.nilsson@chemeng.lth.se

Separation processes like chromatography can be highly nonlinear and the behavior can sometimes be hard to predict. Optimization of preparative chromatography is often done experimentally, which is both time consuming and expensive. A model-based approach to optimization is therefore an attractive and challenging way to overcome some drawbacks in the traditional working procedure in biotechnical industry. Efficient model-based optimization for industrial needs requires three parts; models, methods and tools. Model-based methodology requires a set of chromatography column model structures, which can capture the phenomenon of interest. For 
instance they have to capture column load variations, elution profile changes, operation condition disturbances, column configurations and stationary phase properties. To derive a reliable model for optimization it has to be calibrated and validated to experimental data, which requires an efficient calibration procedure. Different calibration procedures are discussed and compared. After validation the model can be used in the design of a separation step. To do a robust design a set of requirements have to be fulfilled. The column size and operation conditions are used to optimize the performance of the step, which requires a constraint nonlinear optimization method. The choice of objective function for optimization and corresponding constraints are not obvious and the resulting operation conditions are often not robust. Therefore there have to be additional methods available for analysis of the performance, like sensitivity and robustness analysis. Optimization of the purification of antibodies is discussed and exemplified. The work with mathematical models and numerical methods has to be supported by a set of computer tools of different kinds in order to solve industrial problems effective. There is a need for different kinds of tools; customized tool to solve specific problems by a non skilled user and general toolbox for the expert. An example of a toolbox is presented.

Chromatographic properties of plasmids and nanoparticles on monoliths and chromatography particles with giga pores Tina Tarmann, Alois Jungbauer Department of Biotechnology, University of Natural Resources and Applied Life Sciences, Vienna, Austria

Plasmids and viruses are the contemporary vehicles for genetherapy and genetic vaccination. Extremely promising results have been reported from in-vitro, in-vivo and clinical studies. Currently a lot of these compounds are manufactured with a technology which has been directly transferred from laboratory to pilot scale without further engineering. Membrane based separations, chromatographic separations and precipitation have been employed for separation of plasmids and viruses. Chromatographic separation have been designed with aim of protein separation. Thus such processes suffer from either mass transfer limitations or low capacity. Monoliths without intraskeleton mesopores and chromatography particles with giga pores are excellently suited for adsorption and separation of plasmids and viruses. Low mass transfer resistance and high capacity compared to conventional beaded materials can be observed. Adsorption kinetics were derived from infinite and finite bath methods and isotherms were constructed. These data also suggest that a conformational change of the plasmids takes place upon adsorption. Discussion of the mass transfer properties and an example of scale up of a chromatographic separation process using these novel materials will be shown and discussed in respect to already existing processes.

\section{4}

Separation of supercoiled and open circular plasmid DNA isoforms by chromatography with a histidine-agarose support F. Sousa ${ }^{1}$, C.T. Tomaz ${ }^{1}$, D.M.F. Prazeres ${ }^{2}$, J.A. Queiroz ${ }^{1}:{ }^{1}$ Centro de Investigação em Ciências da Saúde, Universidade da Beira Interior, 6201-001 Covilhã, Portugal; ${ }^{2}$ Centro de Engenharia Biológica e Química, Instituto Superior Técnico, 1049-001 Lisboa, Portugal. E-mail: fanidesousa@yahoo.com (F. Sousa)
The recent developments in molecular therapies such as non-viral gene therapy and DNA vaccination have fostered the development of efficient plasmid DNA (pDNA) purification processes. The separation of supercoiled (sc) and open circular (oc) isoforms is one of the key steps in the large scale purification of pDNA vectors intended for a therapeutic use. Although Escherichia coli produces mainly the more compact sc pDNA isoform, oc, linear and denatured pDNA isoforms are usually present and are likely to be less efficient in transferring gene expression. For this reason, regulatory agencies specify that more than $90 \%$ of pDNA in a therapeutic product is in the sc isoform. In this work histidine-base recognition is explored as a mean to separate pDNA isoforms. The agarose gel used here combines the mild hydrophobic characteristics of an epoxy spacer arm with a pseudo-affinity histidine ligand. Chromatographic profiles were obtained by injection of native plasmid $(\mathrm{sc}+\mathrm{oc})$ samples in the histidine-agarose support showing an efficient and baseline separation of both isoforms. The high resolution obtained with this support indicates that the method is potentially applicable to the separation of pDNA at preparative and analytical scale.

\section{5}

Affinity ligand development with a novel encoded bead screening technology Ib Johannsen, VersaMatrix A/S, Gamle Carlsberg Vej 10, DK-2500 Valby, Denmark. E-mail: www.versamatrix.com

The presentation describes a new invention for fast development of affinity ligands, where up to 20,000 ligands can be screened onbead and identified in a few hours. Combinatorial synthesis by the split and mix procedure is a powerful technique for generating vast numbers of diverse chemical compounds on polymer beads with relatively little effort. Traditionally, the technique is hampered by the laborious spectroscopic and chemical analysis, needed to determine the exact structures of the ligand on selected beads. In this way, 6-12 month analysis time could easily be spent just to analyze a tiny fraction of the library. In the Versaffin ${ }^{\mathrm{TM}}$ Technology each bead is encoded, individually tracked, and identified during the synthesis and screening. This decreases the whole ligand development time from months to weeks and increases the amount of information significantly. The bead code further enables evaluation of the ligandprotein binding under varying binding and elution conditions. The instrument for reading the encoded beads and for quantifying the amount of bound protein is presented. The encoded beads we use are based on functional cross-linked polyethylenglycol (PEG), which is compatible with water as well as most organic solvents. Thus, the combinatorial synthesis can be carried out in organic solvents and the resulting compounds can be evaluated, still bound to the parent beads, under aqueous conditions. A further advantage of using PEG based beads for on-bead screening is the fact that PEG is biologically inert and therefore does not interfere in a bioassay.

\section{Downstream Processing II - Industrial Applications and Scale- Up}

1

Speeding up process development for biopharmaceuticals Klaus Graumann, Microbial Development, Biopharmaceutical Operations, Sandoz GmbH, 6250 Kundl, Austria. E-mail: klaus.graumann@sandoz.comwww.sandoz.com 
In the biopharmaceutical industry, pressure is mounting to shorten development times and thereby time to market, e.g. in the field of monoclonal antibodies, generic processes have been established which allow for more rapid development from gene to production of pre-clinical and proof of concept/phase I material. For non-mAb products from various expression systems, productspecific approaches still prevail. However, for most product types similar issues like clearance of process- and product-related impurities, overall purity and yield, or manufacturing issues have to be dealt with in downstream process development. Integrated and timely approaches based on process science and developed orthogonal analytical tools are often hampered by tight time frames and limited resources available. On the other hand, thorough understanding and analytical characterization of product characteristics but also of (process-related) impurities are pre-requisites for fully exploiting separation power and for achieving final purities way above $95 \%$ in a robust and cost-effective manner. From primary separation to polishing steps, we have made several attempts to improve the efficiencies of process steps themselves but also of ways to develop them. The strategies applied comprise implementation of innovative processing tools, rational streamlining and optimization of a sequence of unit operations, tech transfer and scale up considerations. Also in this context, the applicability of scale down and ultra scale down models for process development and optimization purposes, their potential for speeding up and their limitations will be discussed.

\section{2}

Chromatographic monolithic columns: A versatile support for purification of large biomolecules Aleš Podgornik, Miloš Barut, Aleš Štrancar BIA Separations d.o.o., Teslova 30, 1000 Ljubljana, Slovenia

Chromatographic monoliths are rather new chromatographic stationary phases. They consist of a single piece of a highly porous material. The pores are interconnected forming a network of channels. Since the transport mechanism is predominantly based on convection, mass transfer between mobile and stationary phase is significantly enhanced resulting in short separation times. Because of that they seem to be an ideal support for separation and purification of extremely large molecules like proteins, DNA or even viruses. In this talk, various features of the monoliths like high porosity, fast mass transfer, surface accessibility and dynamic binding capacity will be described. Effect of each feature on the separation and purification efficiency will be discussed in terms of molecular size and properties. While chromatographic monoliths are already widely accepted in microchip fluid devices, capillary columns as well as analytical columns, very few reports about preparative monolithic columns can be found. Reasons for lack of preparative chromatographic columns will be elucidated and preparation strategy for construction of several liter volume methacrylate based monoliths will be presented. Finally, several examples of biomolecule purification like large proteins, plasmid and genomic DNA and viruses on CIM Convective Interaction Media ${ }^{\circledR}$ monolithic columns will be given. Further, their application as bioreactors and supports for solid state synthesis will be demonstrated.
3

Intraparticle diffusion measurement of proteins in process chromatographic media Magnus Schroeder, Eric von Lieres, Juergen Hubbuch Institute of Biotechnology, Forschungszentrum Juelich, 52425 Juelich, Germany. E-mail: m.schroeder@fz-juelich.de (M. Schroeder)

The intraparticle diffusion coefficient is an important parameter for modeling of chromatographic separation processes. A new method based on dynamic measurements of intraparticle concentration profiles of proteins in process chromatographic media with a confocal laser scanning microscope is presented. The diffusion coefficient is determined by fitting experimental data to a spherical diffusion model. Excellent agreement of experimental data with simulation results is obtained. The diffusion coefficient is measured for seven proteins in Sepharose 6 FF, spanning molecular weights from 14.3 to $160 \mathrm{kDa}$. In addition, multicomponent diffusion processes for combination of differently sized proteins are analyzed and the influence of adsorbed proteins on the diffusion coefficient is measured in SP or Q Sepharose FF. Taken together the presented method allows measuring the diffusion coefficient of proteins in process chromatographic media in a packed column.

\section{4}

Use of automated docking for predicting chromatographic behavior of proteins in hydrophobic interaction chromatography Andrea Mahn, M. Elena. Lienqueo Contreras University of Chile, Santiago, Chile

In the present work, we have extended and automated the methodology proposed by Mahn et al., 2005 for predicting protein behavior in hydrophobic interaction chromatography (HIC). This methodology is based on the good correlation level between the average surface hydrophobicity of the interfacial zone (local hydrophobicity LH) and protein retention time in HIC, for only three different ribonucleases. For determining the LH it is necessary to select the most probable protein-ligand conformation. In this work, we have determined the most probable conformation, of more than 12 proteins, using (i) first, the module Insight II Affinity by Accelrys for providing automated docking (Grid Method) of ligands (Phenyl) to the proteins (100 conformations); (ii) then, the different probable docked protein-ligand conformations were automatically scored using the module Insight II LUDI by Accelrys; (iii) after that, each conformation was clustered and each cluster was scored by using the average score of each cluster (iv) finally, the most probable conformation was selected using a function based on the number of cluster components, and the average score value. Then, when the most probable conformation was selected, the local hydrophobicity (LH) was calculated using the graphical representation and analysis of structural properties (GRASP) program. The results have shown an acceptable correlation level $(r>0.90)$ between LH and the experimental dimensionless retention time (DRT). In view of these results, we consider that this methodology could be used to adequately represent the chromatographic behavior in HIC for all kinds of proteins (with a heterogeneous and homogeneous surface hydrophobicity distribution) and without a large number of tedious experiments, but only using computational simulation and adequate score criterion. 


\section{Reference}

Mahn, A., Zapata-Torres, G., Asenjo, J.A., 2005. J. Chromatogr. A 1066,81 .

5

Food-grade protein from industrial potato starch effluent recovered by an expanded bed adsorption process Knut O. Stratkvern $^{1}$, Sissel L $\phi k r{ }^{1}$, Morten Aa Olander ${ }^{2}$, Allan Lihme ${ }^{2}:{ }^{1}$ Department of Natural Science \& Technology, Hedmark University College, N2317 Hamar, Norway; ${ }^{2}$ UpFront Chromatography A/S, DK-2100 Copenhagen, Denmark. E-mail: knutos@hihm.no (K.O. Strætkvern)

Potato tuber proteins are nutritious and show potential as functional ingredient in food systems. However, the present bulk processing technology can only recover byproduct protein for animal feed use. An expanded bed adsorption (EBA) process for isolating functional food-grade protein from crude potato starch effluent was previously developed. Moderate capture efficiency (20-25\%) of the total crude protein was most likely caused by diffusion limitations and aggregated protein, inaccessible for adsorption. We employed the same adsorption ligand attached to agarose-tungsten carbide beads to create stable beds of 2.0-2.7 $\times$ expansion using flow rates at $400-750 \mathrm{~cm} \mathrm{~h}^{-1}$. A pilot scale EBA process was run in a commercial processing plant over a three month campaign of starch production from potatoes of mixed variety. Fresh crude effluent $(150-300 \mathrm{~L} /$ cycle $)$ was applied to a column $(20 \mathrm{~cm} \times 2 \mathrm{~m})$ containing $20 \mathrm{~L}$ of resin. Protein capture by EBA was reliable in operation, producing a refined protein material, which after dewatering and gentle drying, showed improved functionality over heat-coagulated protein produced at the same plant. Overall productivity increased. However, finding a robust operating window of predictable productivity is challenging since the potato fruit water is complex and deteriorates easily. From breakthrough curves, it is observed that the major bulk protein, patatin, displays non-Langmuir adsorption behavior. This may indicate a range of interactions for different species of the same protein. Chlorogenic acid (CA), the main polyphenolic substance in potato tuber, causes enzymatic browning and undesirable flavor changes, but polyphenols can also react with protein. Assessing the effects of interacting cell components therefore applies to the bioprocessing of plant material.

\section{Nano- and Microbiotechnology}

Applications of fully electrical micro arrays Rainer Hintsche Fraunhofer Institut für Siliziumtechnologie/eBiochip Systems $\mathrm{GmbH}$, Fraunhoferstr. 1, 25524 Itzehoe. E-mail: hintsche@isit.fhg.de

At present the acceptance of biochip technology for on site use, e.g. diagnosis or environmental control is hindered by rather expensive and complex instrumental systems. There is a need to provide reliable and cost-effective systems that can be operated with minimal training. The construction of electronic biochip microarrays using semiconductor technology enables the construction of compact systems with high integration at acceptable production costs. The key feature of the fully electrical biochip technology are micro arrays made in advanced Si-technology and carrying several array positions with interdigitated nanometer gold electrodes on its surface. The chips are fabricated by standard silicon fabrication methods allow- ing high volume production and to minimise the cost per chip. The advantage of fully electronic microarrays is the intrinsic high spatial resolution and direct signal coupling of the biosensing element and the transducer. The function of fully electronic biochips is also based on the electrochemical transduction and quantification of the formation of affinity complexes on the chip surface. A portable device for field applications and point of care diagnosis have been designed and manufactured. The amperometric device enables the recognition of biomolecular interactions by measuring the redox recycling products of ELISA enzyme labels. The highly sensitive signal transduction is achieved with a 16-channel interdigitated ultramicroelectrode array. One major advantage of fully electronic microarrays is the direct signal coupling of the biosensing element and the resulting robustness and opportunity for miniaturisation. Those electrical biochip arrays have been adapted for the detection of all types of affinity complexes, such as for DNA, RNA, proteins and haptens. Self assembling of capture oligonucleotides via thiol-gold coupling have been used to construct the array chip nucleic acid interface. Thus e.g. pathogenic micro organisms have been identified and quantified via their genomic DNA or ribosomal RNA respectively. Another application based on immobilized antibodies is shown to sense extreme low concentration of bioagent toxins. For processing the assay formats and the electrical read out of the detection of affinity complexes a modular fully automated measurement system has been developed. It is manufactured in industrial lines and available at market.

\section{2}

Dynamics and self-assembly of organic molecules on surfaces revealed by high-resolution, fast-scanning STM Flemming Besenbacher Interdisciplinary Nanoscience Center (iNANO), University of Aarhus, DK-8000 Aarhus C, Denmark. E-mail: fbe@inano.dk

In the interdisciplinary area of nanoscience and nanotechnology, the adsorption and self-assembly of organic molecules on singlecrystal surfaces have attracted much attention lately due to the potential applications in fields ranging from molecular electronics to biocompatible interfaces. The supramolecular structures formed upon deposition of molecular species on solid surfaces depend on the molecular architecture and the distribution of functional groups on one hand, which determines the thermodynamically stable molecular arrangement, and on the other hand, on kinetic factors like thermal diffusion, spontaneous rotations and conformational dynamics. I will show how the unique aspect of our Aarhus STM and the time-resolved, high-resolution STM imaging can be used to obtain important new insight into the dynamics, and can provide very important new information on the atomic-scale realm and on the dynamics of molecular nanostructures. The time-resolved STM data are visualized in the form of STM movies (see www.inano.dk/spm) which can subsequently be analyzed in order to extract quantitative information on the activation energy, the prefactors and the adsorbate-promoted diffusion. I will specifically discuss: (i) the self-assembly of guanine quartets on $\mathrm{Au}\left(\begin{array}{lll}1 & 1 & 1\end{array}\right)$ and the influence of cooperative hydrogen bonds, and (ii) the molecular recognition in binary mixtures of DNA bases. G molecules are found to self-assemble into a hydrogen-bonded network of G-quartets, whose structure corresponds perfectly with the quartet structure of telomeric DNA determined by X-ray crystallography. The strong preference of $\mathrm{G}$ molecules to form quartets can be explained by a 
cooperative effect that strengthens the hydrogen bonds within the G-quartet network over the hydrogen bonds in isolated dimers. By means of a combination of STM experiments and DFT calculations we compare the 2D molecular networks formed on deposition of the binary mixtures G-C (purine-pyrimidine pair of complementary bases) and A-C (purine-pyrimidine pair of non-complementary bases). We find that the non-complementary bases segregate into islands of pure A and a network of pure $\mathrm{C}$, whereas the complementary bases $\mathrm{G}$ and $\mathrm{C}$ form a network that cannot be separated by annealing up to the desorption temperature for C. High-resolution STM images allow us to identify the structures for the enhanced thermal stability as structures that contain $\mathrm{G}-\mathrm{C}$ bonds, possibly with the same structure as the Watson-Crick pairs in DNA molecules.

\section{References}

Kühnle, R., et al., 2002. Nature 415, 891.

Otero, R., et al., 2004. Angewandte Chemie Int. Ed. 43, 2092.

Otero, R., et al., 2004. Nat. Mater. 3, 779.

Otero, R., et al., 2005. Angewandte Chemie Int. Ed. 44, 2.

Rosei, F., et al., 2002. Science 296, 328.

Schunack, M., et al., 2002. Phys. Rev. Lett. 88, 156102.

\section{3}

Chitosan-based nanoparticles for biomedicine M. Johnsen, $O$. Hansen, K.A. Howard, X. Liu, J. Kjems, F. Besenbacher Chitosan Innovation Consortium, Bioneer a/s and iNANO (Aarhus University), Denmark. E-mail: mgj@bioneer.dk (M. Johnsen)

Biomedical and pharmaceutical companies are using an increasing number of carbohydrate polymers in the formulation of drugs. One such polymer with highly attractive features is chitosan that can be produced from crustacean shells. Chitosan is non-toxic, biocompatible and biodegradable. Chitosan can be formulated as nanoparticles or membranes and have enhanced several bioprocesses. Among the well-documented features are enhanced drug uptake by tight junction relaxation and enhanced in vivo uptake and protection of nucleic acid formulations. Chitosan research has increased throughout this decade. Research programs are addressing the potential of chitosan applications but preparations with variable molecular size and charge are not easily available. Specifically companies working with the development of new drugs and enhancement of drug functionality are in need of formulation technology that provides well characterized biocompatible material. In the Chitosan Innovation Consortium the Danish companies, Coloplast, Novozymes Biopolymers, Pipeline Biotech, and ZGene, together with the research center iNANO (Aarhus University) and Bioneer a/s have developed a series of chitosan preparations suitable for research of biopharmaceutical applications (www.chitosan.dk). The ability to obtain functional formulations is currently being tested in both in vitro and in vivo experiments. The consortium participants have established a platform from which chitosan processing, characterization and formulation technology can be extracted. By providing specified chitosan preparations the polymer feature can be adjusted to fit specialized biopharmaceutical applications.

\section{New Concepts in Bioreactor Design}

1

High throughput bioprocessing Govind Rao Center for Advanced Sensor Technology, UMBC, Baltimore, MD 21250, USA
The post genome era holds a great deal of promise. An enormous number of new proteins await study. These will require sophisticated culture techniques, as cells will have to be grown under large numbers of environmental conditions to elucidate expression triggers. Unfortunately, unlike molecular biology, bioreactor technology is little changed since its inception. The primary reason has been a lack of sensor technology that can be readily employed to monitor the cellular environment. We will take a look at the current status of the technology and report on promising optical sensor technology that permits low-cost high throughput cell culture and fermentation. Noninvasive sensors that monitor $\mathrm{pH}, \mathrm{pO}_{2}$ and $p \mathrm{CO}_{2}$ and high sensitivity solutions for glucose and glutamine measurements will be presented. In addition, the mixing characteristics that determine bioreactor performance will be examined at the small scale and their relevance to the large scale will be demonstrated.

2

On-line monitoring and fed-batch operation in shake flask and micro titre plate cultures Jochen Büchs ${ }^{1}$, Frank Kensy ${ }^{1}$, Markus Jeude ${ }^{1}$, Tibor Anderlei ${ }^{2}$, Barbara Dittrich ${ }^{3}$, Doris Klee ${ }^{3}$ : ${ }^{1}$ Biochemical Engineering, RWTH Aachen University, Aachen, Germany; ${ }^{2}$ AC Biotec, Jülich, Germany; ${ }^{3}$ Textile Chemistry and Macromolecular Chemistry, RWTH Aachen University, Aachen, Germany

Although methods of molecular biology has led to rational design of micro-organisms to suit our requirements, screening of large numbers of strains and media is still one of the most important tasks in biotechnology. Batch operation of shaken bioreactors and absence of on-line monitoring is still the general state of the art for that purpose. It is also a very common practice in screening projects that only the final product titre is measured at the end of the culture for the evaluation of the "best performers". In the recent years several approaches were introduced to follow microbial cultures also in shaken bioreactors like shake flasks or micro titre plates (MTP's). It became obvious that the cultures can behave quite unexpected and most relevant and essential information is lost, if only the final product titre at the end of the cultures is utilised for evaluation. As a result, the screening may be directed to an unknown and non-intended direction or may even fail. This is demonstrated with some examples in this contribution. New methods and techniques are introduced to measure the oxygen and carbon dioxide transfer rate and the respiratory quotient in shake flasks and the optical density, $\mathrm{NADH}$ fluorescence, $\mathrm{pH}$ and $\mathrm{DO}_{2}$ in MTP's. If the desired product can be fused to a fluorescence protein, like GFP or YFP, also the product formation can be monitored on-line in MTP's. It is of utmost importance that the operating conditions of the applied shaking bioreactors are suitable and shaking motion is not stopped during the measurement. Otherwise, e.g. power input, mixing and oxygen supply is interrupted and the micro-organisms will ongoingly rearrange their metabolism to cope with these disturbances of their environmental conditions. Another problem of screening is the commonly applied batch operation mode. A lot of microbial systems display an overflow metabolism, substrate or osmotic inhibition or are characterised by a catabolite repressed product formation. In all these cases, batch operation is not the preferred operation mode and, therefore, these cultures are run in fed-batch in larger scales. In particular, it is nearly impossible to screen systems, which are catabolite repressed by the carbon source, in batch mode in defined 
mineral media. After initial growth has led to a nearly complete consumption of the carbon source, the product formation is derepressed. But then no more carbon source is available to continue with production. It is quite questionable whether in batch operation mode suitable strains can be selected for later fed-batch operation or not. This consideration has resulted in the development of a new technique which allows to run the screening in fed-batch operation mode. This technique is applicable in shake flasks as well as in MTP's. Dramatic increases in product titre between 4- and 400-folds were observed under these conditions compared to conventional batch screenings.

\section{3}

Applications of rotary jet heads for mixing and mass transfer Mikkel Nordkvist, John Villadsen Center for Microbial Biotechnology, Technical University of Denmark, DK-2800 Lyngby. E-mail: mnq@biocentrum.dtu.dk (M. Nordkvist)

Efficient mixing and mass transfer are highly important in the chemical industry and in the fermentation industry. Poor mixing can result in low yield and variable product quality in a number of cultivation processes, and mass transfer can easily become the limiting step in aerobic cultivations, especially at high cell density. We have tested a new tank reactor system, where liquid is withdrawn from the bottom of a tank, rapidly circulated, and injected back into the bulk liquid through the nozzles of rotary jet heads. Liquid feed as well as gas is added in the recirculation loop and thereby distributed via the rotary jet heads. Solid feed in powder form can also be added in the loop with advantage, and heat is efficiently removed in a plate-type heat exchanger, which is part of the loop. The system has a very simple design with no internal baffles or heat exchange area, and between batches the rotary jet heads are used for cleaning in place. A number of applications ranging from dispersion of liquid and powder to mass transfer will be presented. Mass transfer applications include baker's yeast cultivation and oxidation of lactose to lactobionic acid by a carbohydrate oxidase.

\section{4}

Miniaturized bioreactor platforms for high-throughput bioprocess development and gene expression analysis Nicolas Szita ${ }^{1, *}$, Paolo Boccazzi ${ }^{2}$, Zhiyu Zhang ${ }^{1}$, Andrea Zanzotto ${ }^{1}$, Anthony J. Sinskey ${ }^{2}$, Klavs F. Jensen ${ }^{1}$ : ${ }^{1}$ Department of Chemical Engineering, Massachusetts Institute of Technology, Cambridge, MA 02139, USA; ${ }^{2}$ Department of Biology and Health Sciences and Technology, Massachusetts Institute of Technology, Cambridge, MA 02139, USA; *Present address: Department of Micro and Nanotechnology, Technical University of Denmark, DK-2800 Kongens Lyngby, Denmark. E-mail: szita@mic.dtu.dk (N. Szita)

Fast and accurate analytical information that can be used to rapidly evaluate the interactions between biological systems and bioprocess operations is essential for optimization of biological production processes. We have researched and developed a multiplexed microbioreactor system for the parallel operation of multiple microbial fermentations. The microbioreactors have working volumes from 5 to $150 \mu \mathrm{L}$, and are instrumented for real-time monitoring of dissolved oxygen, $\mathrm{pH}$ and optical density. The growth profiles obtained with Escherichia coli compare favorably to results obtained from conventional $500 \mathrm{~mL}$ batch bioreactors. We also demonstrate the use of our microbioreactors coupled to DNA microarray analy- sis, as a tool for accelerated discovery and elucidation of metabolic pathways and gene expression profiles. The multiplexed system represents a significant step towards high-throughput data acquisition and has the potential to replace current instrumented bioreactors, which are bulky, expensive to run, and require many mechanical manipulations.

\section{5}

Design of a laboratory scale bioreactor to study solid-state tobacco fermentation M. Di Giacomo, L. Nappi, D. Silvestro, M. Paolino, D. Parente R\&D Biology Department, British American Tobacco Italia, Naples 80126, Italy

Italian Toscano cigar production is based on the fermentation of dark fire-cured tobacco. The process starts with the rise of leaf moisture to levels of water activities assuring development of the wild phylloplane microflora in the absence of free water. The intense growth of microorganisms modifies leaf characteristics ( $\mathrm{pH}$ rise from acidic to alkaline condition) contributing to define the Toscano typical smoke profile. Tobacco fermentation takes place in great bulks of $500 \mathrm{~kg}$ which cause considerable amount of heat evolution as a function of the metabolic activities of the microorganisms. This heat accumulates and temperature can rise to as high as $70^{\circ} \mathrm{C}$. A laboratory cylindrical packed-bed bioreactor was designed to work under isothermal conditions. The reactor was ideal to ferment small quantities of tobacco $(200 \mathrm{~g})$ and was made up of a column aerated from the bottom with humidified air and placed in a thermoregulated room. Experiments were conducted with constant temperature and air flow. Moreover, bioenrichment experiments were conducted in the presence of different microbial starter cultures. Fermentation courses were monitored by measuring microbial counts and chemi$\mathrm{cal} /$ physical modification of the substrate. With this laboratory scale system we obtained different kinds of information on the role and dynamics of the microorganisms involved in the fermentation process and on the influence of different environmental conditions. For the future, the design of an adiabatic device for tobacco fermentation is planned.

\section{Advances in Bioprocess Control}

1

On-line liquid chromatography as a process analytical technology for monitoring and control of biotech processes Rick E. Cooley ${ }^{1,2}$ : ${ }^{1}$ Process Analytics Center of Excellence, Dionex Corporation, Sunnyvale, CA, USA; ${ }^{2}$ Eli Lilly and Company, Indianapolis, IN, USA (retired)

Biotech processes, used to produce an active pharmaceutical ingredient (API), generally differ from small molecule API manufacturing processes in that the starting materials tend to be more variable, more complex, and the product of interest in lower concentration due to the fact that they originate from a biological rather than a chemical process. This complexity and low starting concentration has generated a high level of interest in developing technologies that can be utilized to increase yield and reduce variability in the initial bioreactor phase, as well as, downstream isolation and purification operations. The use of process analytics, or on-line analytical measurements, is a technology approach that can contribute to increased process understanding and control. This presentation will provide examples of how various analytical measurement technologies have 
been utilized to monitor typical bioprocess unit operations leading to increased automation and control. Examples of the use on-line liquid chromatography to monitor bioreactors, process scale chromatography columns, and enzymatic reactions will be presented in more detail.

2

Application of advanced monitoring strategies for recombinant protein production Karl Bayer Department of Biotechnology, University of Natural Resources and Applied Life Sciences, Vienna A-1190, Austria

High yield in combination with the required quality are the key objectives of large-scale production of recombinant proteins using different host/vector systems. However, in the past the efficient production of recombinant proteins was frequently limited due to inadequate exploitation of the cell factory and deficiencies in process design. To achieve optimal exploitation of microbial cell factories the key requirement is to enhance the monitoring capabilities to improve the insight into the host metabolism dynamics and to cope with limited understanding of the interaction of recombinant protein synthesis with host cell metabolism. Since each protein exerts an individual influence on the host/vector system, the selection of appropriate analytical methods is even more important. In order to overcome these problems high throughput technology platforms, such as DNA microarrays for transcriptome and differential 2-D electrophoresis (DIGE) for proteome analysis provide extensive data to screen for significant analytes and provide an appropriate basis for in silico modelling and reverse engineering of regulatory networks. Taking advantage from such an iterative process experimental design will be improved and further aid to increase the performance of modelling. In addition, transcriptome data are used to screen fast stress responsive promoters to set up GFP based reporter gene fusions for in-situ monitoring of the metabolic load due to recombinant gene expression. Moreover chemometric methods are frequently applied to model complex data from easily obtainable on-line data sets to overcome the limited monitoring capabilities due to the high complexity and nonlinearity of biological reactions and reaction networks. This strategy has been successfully applied to model BDM (bacterial dry matter), PCN (plasmid copy number) and the amount of recombinant protein using data sets acquired from off-gas analysis $\left(\mathrm{O}_{2}\right.$ consumption, $\mathrm{CO}_{2}$ evolution), alkaline consumption rate, in-situ capacitance and multi-wavelength fluorescence measurements.

\section{3}

At-line monitoring of bioprocess-relevant marker genes using an electric DNA-chip Britta Jürgen ${ }^{1}$, Daniel Pioch ${ }^{1}$, Le Thi $H_{\text {Hoi }}{ }^{1}$, Jörg Albers ${ }^{2}$, Rainer Hintsche ${ }^{2}$, Stefan Evers ${ }^{3}$, KarlHeinz-Maurer ${ }^{2}$, Michael Hecker ${ }^{4}$, Thomas Schweder ${ }^{1}$ : ${ }^{1}$ Institut für Pharmazie, Ernst-Moritz-Arndt-Universität, 17487 Greifswald, Germany; ${ }^{2}$ eBiochipSystems, 25524 Itzehoe, Germany; ${ }^{3}$ VTBEnzymtechnologie, Henkel KGaA, 40191 Düsseldorf, Germany; ${ }^{4}$ Institut für Mikrobiologie, Ernst-Moritz-Arndt-Universität, 17487 Greifswald, Germany. E-mail: britta.juergen@uni-greifswald.de (B. Jürgen)

The Gram-positive bacteria Bacillus licheniformis and Bacillus subtilis represent important industrial hosts for the production of enzymes (e.g., proteases and amylases) or antibiotics (e.g., Bacitracin). Both organisms are attractive for this purpose because of their apathogenity and their classification as GRAS organisms (generally regarded as save). Moreover, their easy cultivation and their high natural capacity to secrete proteins into the growth medium qualify them for the industrial overproduction of homologous or heterologous proteins (Simonen and Palva, 1993). For the control of the physiological state and the productivity of the production cells efficient analysis tools are of great interest. A prerequisite for the evaluation of the physiological state of cells during industrial fermentation processes is the analysis of so-called process-relevant marker genes, the expression of which indicates unfavorable growth and production conditions (Schweder and Hecker, 2004). By means of proteome and transcriptome analyses we have identified critical process-relevant genes of B. licheniformis and B. subtilis cells under different nutrient limitation conditions and during industrialclose bioprocesses. DNA-chips with probes for such process-relevant marker genes could be valuable diagnostic tools for the monitoring of the cellular physiology during microbial bioprocesses. In order to provide reliable tools for the monitoring of the cell physiology during microbial bioprocesses, we have developed a fast mRNA analytical approach, which allows an at-line monitoring of the transcriptional activity of selected marker genes during bioprocesses. This approach is based on an easy, fast and reliable RNA isolation procedure and the measurement of specific mRNAs by means of an electric DNA-chip (Gabig-Ciminska et al., 2004; Barken et al., 2004). The measurement of selected marker genes of B. subtilis and B. licheniformis model fermentation processes by the electric DNA-chip was validated by the real time RT-PCR technique

\section{References}

Barken, K.B., Gabig-Ciminska, M., Holmgren, A., Molin, S., 2004. Biotechniques 36 (1), 124-132.

Gabig-Ciminska, M., Holmgren, A., Andresen, H., Bundvig Barken, K., Wümpelmann, M., Albers, J., Hintsche, R., Breitenstein, A., Neubauer, P., Los, M., Czyz, A., Wegrzyn, G., Silfversparre, G., Jürgen, B., Schweder, T., Enfors, S.O., 2004. Biosens. Bioelectron. 19, 537-546.

Simonen, M., Palva, I., 1993. Microbiol. Rev. 57 (1), 109-137.

Schweder, T., Hecker, M., 2004. Adv. Biochem. Eng. Biotechnol. $89,47-71$.

4

A new approach to quantify the robustness of bioprocess performance Hyunkee Kim, Josh M.P. King, Nigel J. Titchener-Hooker, Yuhong Zhou Department of Biochemical Engineering, University College London, Torrington Place, London WC1E 7JE, UK

A robust bioprocess is crucial to ensure the consistent process performance and provide the high quality of product for drug manufacturing in biopharmaceutical industries. Existing methodologies for bioprocess design do not involve establishing mechanisms to achieve the desirable robust bioprocesses and have low capacity in handling uncertainty in the product manufacturing. Also, the solutions are often obtained step wise and do not account for interactions between the steps. Despite its importance, the robustness of a bioprocess has not been properly defined and studies carried out in statistic sense are often retrospective. In addition, the computational cost is expensive due to using a line search algorithm for finding an optimal operating solution. Finally, the existing methodologies are difficult to apply to the whole bioprocess in biopharmaceutical industries. This 
paper attempts to define rigorously a measure for process robustness and presents a new methodology for evaluating the robustness of bioprocess operations and their performance. The methodology is based on the concept of 'windows of operation' which shows the whole range of possible operating regions. The methodology also establishes a lower bound for the largest variation of a design variable to ensure the performance. These bounds are achieved by min-max optimization techniques. A direct search algorithm has been developed and its computational cost is much lower than the line search algorithm. Results include visualization of robust operating regions and a set of indices which compare the performance of different operating strategies. The capabilities and efficiency of this methodology are illustrated by applying it to the centrifuge selection for the clarification of high solids density cell broths. The research work will impact considerably upon robust bioprocess operation.

\section{5}

Simple and quick in-situ calibration of a FTIR instrument to control fed-batch fermentations of Pichia pastoris J. Schenk, M. Dabros, I.W. Marison, U. von Stockar Laboratory of Chemical and Biochemical Engineering, Ecole Polytechnique Fédérale de Lausanne (EPFL), Lausanne, Switzerland, 1014. E-mail: jonas.schenk@epfl.ch (J. Schenk)

When grown on methanol, Pichia pastoris is able to synthesize proteins to high titres as well as secreting and glycosylation, thereby making this organism a very interesting host for the production of recombinant drugs at large scale. The methanol residual concentration has been reported to strongly influence the specific productivity, the optimum concentration being around $3 \mathrm{~g} / \mathrm{L}$. A suitable monitoring and control technique is therefore necessary to study and improve the productivity of $P$. pastoris fermentations. The current research aims at showing how a mid-infrared spectrometer (ATR-FTIR) can be calibrated in-situ in order to monitor and control P. pastoris fermentations. This method is simple and fast, and eliminates the need of both standards preparation and off-line calibration. It is based on the observation that during fed-batch processes, only substrate and biomass concentrations vary significantly. The method therefore consists in adding a known amount of methanol at the beginning of the process, just after inoculation, and subsequently calibrating the instrument.

\section{Acknowledgement}

Financial support for the Swiss National Science Foundation is gratefully acknowledged.

\section{Poster Abstracts Biochemical Engineering (BE)}

\section{BE1}

Leukocyte adhesion and activation on PEGylated polymer surfaces Z. Ademovic ${ }^{1}$, B. Holst ${ }^{1}$, X.Hou ${ }^{2}$, B. Winter-Jensen ${ }^{1}$, R.A. $\mathrm{Kahn}^{3}$, T. Brevig ${ }^{3,4}$, P. Kingshott ${ }^{1}{ }^{1}$ Danish Polymer Centre, Ris $\varnothing$ National Laboratory, Frederiksborgvej 399, 4000 Roskilde, Denmark; ${ }^{2}$ Radiation Research Department, Ris $\varnothing$ National Laboratory, Frederiksborgvej 399, 4000 Roskilde, Denmark; ${ }^{3}$ Bioneer A/S, Mammalian Cell Biology, Kogle Alle 2, 2970 Hørsholm, Denmark;

${ }^{4}$ Nunc A/S, Kamstrupvej 90, Roskilde, Denmark

Implantable biomaterials are subjected to inflammatory responses mediated by adherent phagocytes such as monocytes and macrophages. These cellular responses and behavior have been shown to be dependent on the type of protein that adsorbs to the surface. Surface modification is necessary to control and prevent protein adsorption, and thus modulate the inflammatory responses. Hydrophilic surfaces that adsorb minimal amounts of protein are considered useful for minimizing the inflammatory reactions to biomaterials. In this study we have used two routes to modify polyethylene terephthalate (PET) films: (1) a wet-chemical method for attachment of linear polyethylene glycol chains (mPEG); and (2) gas-phase plasma polymerisation of diethylene glycol vinyl ether (DEGVE) to generate PEG-like surfaces. The surface chemistry was assessed by X-ray photoelctron spectroscopy (XPS), Fourier transform infrared spectroscopy (FTIR) and time-flight secondary ion mass spectrometry (ToF-SIMS). The two PEGylated surfaces were compared for their ability to minimise both fibrinogen adsorption and the adhesion and activation of macrophage-like human leukocytes. Adsorbed fibrinogen has been shown to be one of the key proteins in stimulating inflammatory responses to biomaterials. Adsorption was investigated quantitatively using ${ }^{125}$ I-radiolabeled human fibrinogen. In addition, the conformation of the adsorbed protein was tested using an antifibrinogen monoclonal antibody in an enzyme-linked immunosorbent assay. The results showed that PEGylated surfaces adsorbed up to $90 \%$ less fibrinogen, and that unfolding of adsorbed fibrinogen was more pronounced on the linear mPEG layers than on the PEG-like plasma polymer surfaces. Adhesion of in-vitro differentiated macrophage-like U937 cells was reduced on both the PEG-like plasma polymer surfaces and the linear mPEG layers compared to the unmodified PET surface, but cells adhering to the PEG-like plasma polymer surfaces secreted less tumor necrosis factor- $\alpha$ (TNF- $\alpha$ ) than cells adhering to the linear mPEG layers. Thus, the linear mPEG surface is relatively efficient at reducing adhesion of macrophage-like cells, but those cells that do attach are in a more activated and proinflammatory state.

\section{BE2}

Analysis of ceramides from biological samples M. Budvytiene ${ }^{1}$, J. Liesiene ${ }^{1}$, B. Niemeyer ${ }^{2}, N$. Babenko ${ }^{3}:{ }^{1}$ Department of Chemical Technology, Kaunas University of Technology, Radvilenu pl. 19, 50254 Kaunas, Lithuania; ${ }^{2}$ Institute of Thermodynamic, HelmutSchmidt-University/University of the Federal Armed Forces Hamburg, Holstenhofweg 85, D-22043 Hamburg, Germany; ${ }^{3}$ Department of Physiology of Ontogenesis, Institute of Biology, Kharkov, Karazin National University, 4 Svobody pl., 61077 Kharkov, Ukraine

Ceramides in human skin play an important role in the regulation of cell growth, differentiation and apoptosis. Moreover, they are involved in the numerous signaling pathways. The growing interest in the investigations of ceramides physiological functions requires efficient separation methods of ceramides from biological resources and sensitive analytical methods. In this work some sensitive and selective methods, involving thin layer chromatography (TLC), high performance liquid chromatography (HPLC), mass spectrometry (MS) and nuclear magnetic resonance spectroscopy (NMR) were employed for the separation and characterization of ceramides from human foreskin. Epidermal lipids were extracted from human foreskin for $24 \mathrm{~h}$ at room temperature using three solvent mixtures (chloroform/ethanol/water 1:2:0.5, v/v/v); (chloroform/ethanol 1:1, $\mathrm{v} / \mathrm{v}$ ), and (chloroform/ethanol 2:1, v/v). Ceramides retention char- 
acteristics in TLC and HPLC were compared with the retention of commercial standards. The best separation was obtained using normal phase column packed with HILIC silica $3 \mu \mathrm{m}$. The elution was performed using mixture of chloroform and ethanol 50/50 $(\mathrm{v} / \mathrm{v})$ as an eluent. Two commercial standards $N$-stearoyl-sphingosine Cer(NS), $R_{\mathrm{f}}=0.51$, and $N$-palmitoyl-sphingosine Cer(NP), $R_{\mathrm{f}}=0.50$, were selectively separated with HPLC system in above mentioned conditions. The retention time of Cer(NP) and Cer(NS) was 4.31 and $5.19 \mathrm{~min}$, respectively. The same lipids were detected by HPLC in the human foreskin extracts. The structure of the lipids from collected fractions was confirmed by means mass spectrometry and NMR. The physiological functions of the separated ceramides are investigated.

\section{BE3}

Production recombinant human thymosin- $\alpha_{1}$ overexpressed as intein fusion protein in E. coli Roman S. Esipov, Vasily N. Stepanenko, Anatoly I. Miroshnikov, Shemyakin-Ovchinnikov Institute of Bioorganic Chemistry, Russian Academy of Sciences, ul. MiklukhoMaklaya 16/10, Moscow, 117997 Russia. E-mail: esipov@ibch.ru (Roman S. Esipov)

Medicines based on polypeptides consisting of 30 and more amino acid residues are widely spread in pharmaceutical market at the present time. Practically all polypeptide medicines known are prepared by general chemical synthesis that caused high cost of their production. That's why biotechnological way of polypeptide medicines preparation using recombinant gene expression in bacteria seems to be promising. During our work we design hybrid construction allowing solving a problem of specific cleavage of target polypeptide from the hybrid protein using system of protein splicing from New England BioLabs. In case of thymosin- $\alpha_{1}$ production system intein mediated purification with affinity chitin (IMPAC system)-binding tag has been used, where the modified Sce VMA from $S$. cerevisiae has been applied as intein. In contrast to other systems, IMPACT allows the preparation of target protein without using of serine protease and other factors that may cleave the hybrid protein. In the presence of thiol reagents, such as dithiothreitol, mercaptoethanol, or cystein the hybrid protein can be site-specifically cleaved to give intein, the target protein and small fragment of $\mathrm{N}$ extein. Using of this system does not allow to obtain the target protein with Ser, Cys or Thr on N-terminal of protein, because in those cases target protein and $\mathrm{N}$-extein ligation product will be formed. Ser is $\mathrm{N}$-terminal amino-acid in thymosin- $\alpha_{1}$. It was recently found that some metal ions essentially affect the splicing. We tried to use $\mathrm{ZnCl}_{2}$ and we have found that, in case of intein-thymosin- $\boldsymbol{\alpha}_{\mathbf{1}}$, the maximal yield of target polypeptide and the minimal yield of splicing products are observed in the absence of dithithreitol and in the presence of $0.5-1 \mathrm{mM}$ zinc chloride in buffers on all stages of thymosin $\alpha_{1}$ isolation. The structure of recombinant thymosin- $\alpha_{1}$ of human was confirmed by the determination of $\mathrm{N}$ - and $\mathrm{C}$ - terminal amino-acid sequences and by MALDI TOF mass-spectrometry.

\section{BE4}

Sampling in microbioreactors for cell metabolism inactivation Sarah-Maria Fendt, Michael Werner, Oliver Geschke, Nicolas Szita, MIC-Department for Micro- and Nanotechnology, Technical University of Denmark, Building 345 East, DK-2800 Kgs. Lyngby, Denmark. E-mail: smf@mic.dtu.dk (S.M. Fendt)
We present a microbioreactor with thermoelectric cooling to inactivate cellular metabolism by cell culture freezing. Small-scale cultivation methods have gained increased importance and their development has been supported by advances in bioprocess monitoring methods. Yet efficient sampling methods for off-line analysis remain important where in-situ real-time measurements are difficult, for example for intracellular metabolite concentration or enzyme activity measurements, and for all methods which are invasive by nature, such as protein purification. Freeze-stop measurements of metabolite levels are ideal, because they are inert, i.e. do not require the addition of a chemical. In large systems, the chilling time is often the limiting factor, and alternative methods for cell metabolism inactivation are required, such as the spraying of the cell suspension in $60 \%$ methanol at a temperature of $-40^{\circ} \mathrm{C}$, or the use of boiling buffered ethanol. Due to the small thermal mass of microsystems, shorter chilling times can be expected. Sample cooling to $4{ }^{\circ} \mathrm{C}$ in a microbioreactor has been presented previously. In this contribution, we investigate sample freezing to completely inactivate the cell metabolism from microbioreactor working volumes of approximately $100 \mu 1$.

\section{BE5}

The use of multi-parameter flow cytometry for characterisation and monitoring of insect cell-baculovirus fermentations in a mechanically-agitated bioreactor Bojan Isailovic ${ }^{1}$, Alvin W. Nienow $^{1}$, Ian W. Taylor ${ }^{2}$, Ryan Hicks ${ }^{2}$, Christopher J. Hewitt ${ }^{1}$ : ${ }^{1}$ Biochemical Engineering, Centre for Formulation Engineering, School of Engineering (Chemical Engineering), The University of Birmingham, Edgbaston, B15 2TT, UK; ${ }^{2}$ AstraZeneca, Mereside, Alderley Park, Macclesfield, Cheshire SK10 4TG, UK. E-mail: BXI897@bham.ac.uk (B. Isailovic)

Bacteria and mammalian cells have been traditionally used as hosts for commercial recombinant protein production. However, in recent years, the insect cell-baculovirus system has emerged as a potentially attractive recombinant protein expression vehicle. Although flow cytometry has been used widely for analysis of mammalian and microbial cells, there is very little information on applications of this powerful technique in insect cell culture. Here we compared cell ratiometric counts and viability (propidium iodide and calcein AM) of Sf-21 cell cultures using flow cytometry to those determined by more traditional methods using a haemocytometer and the trypan-blue exclusion dye. Flow cytometry has also been used to monitor various parameters during cultures of Sf21 infected with the recombinant Autographa californica nuclear polyhedrosis virus (AcNPV) containing the inserted nucleic acid sequence amFP486 coding for AM-Cyan coral protein, which emits natural green fluorescence.

\section{BE6}

NIR-spectroscopy model development of indicator metabolites in fermentation processes Parveen Kousar ${ }^{1}$, Alvin W. Nienow ${ }^{1}$, Graham Poulter ${ }^{2}$, Reg Wilson ${ }^{3}$,Henri Tapp ${ }^{3}$ and Christopher J. Hewitt ${ }^{1}$ : ${ }^{1}$ Biochemical Engineering, Centre for Formulation Engineering, School of Engineering (Chemical Engineering), The University of Birmingham, Edgbaston, B15 2TT, UK; ${ }^{2}$ Specac Limited, Kent, BR5 $4 \mathrm{HE} ;{ }^{3}$ Institute of Food Research, Norwich Research Park, Norwich NR4 7UA, UK. E-mail: pxk203@bham.ac.uk (P. Kousar) 
The optimization of a fermentation process requires the organism to be cultivated under desirable conditions, which depends on how well the fermentation process is controlled. Inadequate mixing and mass transfer are responsible for the heterogeneous environment at large scale in terms of nutrient concentration and $\mathrm{pH}$ profile, resulting in lower product yields. These have been associated with, inadequate control of $\mathrm{pH}$ and the production of acetate or formate in response to over-feeding of glucose and oxygen deficiency. Rapid analyses of substrates and indicator metabolites in a fermentation process is critical for optimal control. This can be achieved in real time with NIR spectroscopy. In this study, NIR-spectroscopy has been applied to monitor the concentrations of glucose, acetate, formate, ammonium hydroxide and biomass in the cultivation of E. coli (W3110). A comparison of partial least square models built using water standards, synthetic medium standards and fermentation samples has been made.

\section{BE7}

Studies on the impact of mixing during brewing using near and mid-infrared spectroscopy Georgina McLeod ${ }^{1}$, Alvin W. Nienow ${ }^{1}$, Graham Poulter $^{2}$, Reg Wilson ${ }^{3}$, Henri Tapp ${ }^{3}$, Christopher J. Hewitt ${ }^{1}$ : ${ }^{1}$ Biochemical Engineering, Centre for Formulation Engineering, School of Engineering (Chemical Engineering), The University of Birmingham, Edgbaston, B15 2TT, UK; ${ }^{2}$ Specac Limited, Kent, BR5 4HE, UK; ${ }^{3}$ Institute of Food Research, Norwich Research Park, Norwich NR4 7UA, UK. E-mail: GXM828@bham.ac.uk (G. McLeod)

The control of the brewing process is important for improving product quality, and lowering costs. Infrared spectroscopy is a technique that can be used at-line and on-line to rapidly measure component concentrations of unprocessed whole broth samples in real time. In this study, both mid -infrared (MIR) and near-infrared (NIR) spectroscopy have been used and compared for the monitoring of ethanol, flavour components, wort sugars, biomass and specific gravity during the brewing. Partial least-squares regression (PLS) was used to model relationships between component concentrations and spectra. The performance of these models was evaluated in terms of the standard error of prediction (SEP), number of PLS factors and the correlation coefficient $(r)$. Calibration models were constructed using spectra acquired for multi-component mixtures, intended to simulate brewing fermentation conditions, and actual brewing fermentation samples. Chemometric results indicated that NIR is a powerful tool for accurately measuring sugar, ethanol and biomass concentrations.

\section{BE8}

Production of recombinant HIV-1 nef in different host cell systems: A techno-economical comparison Raisa Jokiaho ${ }^{1}$, Jani Koskinen $^{2}$, Katri Salonen ${ }^{2}$, Noora Sirén ${ }^{1}$, Jan Weegar $^{3}$, John Dahlbacka $^{3}$, Niklas von Weymarn ${ }^{1}{ }^{1}$ Department of Chemical Technology, Helsinki University of Technology, Finland; ${ }^{2}$ Biocentrum/Cell and Protein Production Unit, University of Helsinki, Finland; ${ }^{3}$ Faculty of Chemical Engineering, Åbo Akademi University, Finland. E-mail: raisa.jokiaho@tkk.fi (R. Jokiaho)

When choosing an expression host for production of a specific recombinant protein, one can essentially select from a multiplicity of different systems. While E. coli bacterium is usually the starting point for any cloning and expression effort, there is no universal expression host that would work optimally for all proteins. Practical issues to consider include e.g. need for post-translational modifications and protease activity of the host. We have produced recombinant HIV-1 Nef in different host cell systems: E. coli, P. pastoris yeast and stable Drosophila S2 insect cells. Using strain/cell line development, production and purification data from practical experiments, we were able to conduct a techno-economical comparison of the different host cell systems. The annual production goal was set at $100 \mathrm{mg}$ of high-purity Nef. This was supposed to be produced campaign-wise in 1-2 batches using laboratory-scale bioreactors and other equipment. In this study, it was shown that although the production costs of the different systems were in the same range, the production in E. coli was most inexpensive, and the S2 cell system was the most expensive. Regardless of the selected host system, the labour costs incurred the most expenses. When comparing different stages of the work (strain/cell line development, bioreactor production and down-stream processing), the strain development, the most man-hours demanding stage, involved approximately half of the costs of every production system. Although E. coli was the most inexpensive host system for producing Nef, it has some definite disadvantages: e.g. the production of endotoxins and the disability to perform post-translational modifications. If these disadvantages are of importance, the production must be done using more expensive system.

\section{BE9}

Modelling and control of industrial fermentation J.K. Rasmussen, S.B. Jorgensen CAPEC, Department of Chemical engineering, Technical University of Denmark, DK-2800 Lyngby, Denmark. E-mail: jkr@kt.dtu.dk (J.K. Rasmussen)

Fed-batch processes play a very important role in chemical and biochemical industry. Fermentations in biochemical industry are most often carried out as fed-batch processes. Present control schemes do not utilize the full potential of the production facilities and may fail to achieve uniform product quality and optimal productivity. The introduction of model based control strategies is considered difficult because suitable models are not readily available. First principle engineering models can be used but the usually limited knowledge of the regulatory network in the micro-organism makes model development very time consuming. Another strategy is to use a purely data-driven approach where only limited prior knowledge of the process is required. A framework for generation of such blackbox models is used in this project. This framework is called "grid of linear models" (GoLM), it uses a large number of linear models which each describes the behaviour of the process within a certain time interval. The combination of these models results in a model which covers the entire time span of the fermentation and approximates the nonlinear time varying behaviour. A procedure for deriving GoLM models from operational data has been developed and because they consist of a large set of linear models it makes them suitable for model predictive control implementation with iterative learning capabilities from batch to batch. Iterative learning model predictive control (ILMPC) based on a GoLM model is being implemented on a fermentor at Novozymes A/S. The results will be evaluated in terms of the controller's capability to ensure uniform product quality and reject both intra and inter batch process disturbances. The model based approach renders optimization of the process recipe possible by using the ILMPC capability. This opportunity provides a great potential for increase of productivity and reduction of cost. 


\section{BE10}

Recombinant shrimp trypsinogen production in Pichia pastoris J.M. Viader-Salvadó, J.A. Fuentes-Garibay, L.J. Galán-Wong, M. Guerrero-Olazarán Institute of Biotechnology, Biological Science School, Autonomous University of Nuevo León, San Nicolás de los Garza, N.L., México, 66450. E-mail: jviader@fcb.uanl.mx (J.M. Viader-Salvado)

The production of recombinant trypsin and trypsinogen has been reported as difficult due to a probably toxicity on host or its instability. In an effort to attain high-level production of shrimp trypsin for aquaculture applications, we have evaluated shrimp trypsinogen production by recombinant Pichia pastoris strains in 5-L bioreactors. A P. pastoris GS115 Mut+ containing in its genome the Litopenaeus vannamei trypsinogen cDNA fused in frame to Saccharomyces cerevisiae $\alpha$-factor secretion signal, previously constructed in our laboratory, was used. Four three-step fermentations (glycerol batch, glycerol and methanol fed-batch) were carried out. The glycerol batch step ( $2 \mathrm{~L}$ of basal salts medium, $50 \mathrm{~g} / \mathrm{L}$ glycerol, $8.8 \mathrm{~mL}$ biotin $0.02 \%, 8.8 \mathrm{~mL}$ PTM1, $250 \mu \mathrm{L} 289$ antifoam, pH 5 adjusted to with $28 \% \mathrm{NH}_{4} \mathrm{OH}$ ) was carried out until glycerol was completely exhausted $(21 \mathrm{~h})$. The glycerol fed-batch step was carried out feeding with $50 \%$ glycerol (12 $\mathrm{mL} / \mathrm{L}$ biotin $0.02 \%$ and PTM1) at $0.8 \mathrm{~mL} / \mathrm{min}$ by $9 \mathrm{~h}$ and $45 \mathrm{~min}$ of posterior starvation. The methanol fed-batch step was carried out feeding with $100 \%$ methanol $(12 \mathrm{~mL} / \mathrm{L}$ biotin $0.02 \%$ and PTM1) by $133 \mathrm{~h}$ using a methanol concentration on/off feedback control to maintain constant the methanol concentration in the culture medium to $1 \mathrm{~g} / \mathrm{L}$. In all the fermentations the air flow rate and the agitation were set at $5 \mathrm{~L} / \mathrm{min}$ and $800-1000 \mathrm{rpm}$, respectively. With the four fermentation assays, the influence of the $\mathrm{pH}$ and temperature in the production phase to the recombinant shrimp trypsinogen production were evaluated. In the four fermentations, at the end of the second step a biomass of $250 \mathrm{~g} / \mathrm{L}$ wet weight were obtained (OD600 230). The methanol demand in the four fermentations surprisingly was not increasing rather initially it was $0.37 \mathrm{~mL} / \mathrm{min}$, after $32 \mathrm{~h}$ decreased 2.5 times for $29 \mathrm{~h}$, increased to $0.49 \mathrm{~mL} / \mathrm{min}$ for $23 \mathrm{~h}$ and afterwards it was decreased manually to a constant value of $0.3 \mathrm{~mL} / \mathrm{min}$ for that the dissolved oxygen will not decrease to values less than $20 \%$ (last $48 \mathrm{~h}$ ). The total protein amount in the culture medium supernatant increased during the production step until values of $1.6 \mathrm{~g} / \mathrm{L}$ (assay at $\mathrm{pH}$ 6), 6.5 times more than the worst assay ( $\mathrm{pH} 3$ ) Recombinant shrimp trypsinogen production was confirmed by SDS-PAGE (about $500 \mathrm{mg} / \mathrm{L}$ ) and trypsin enzymatic activity was detected using BAPNA as substrate after trypsinogen activation with shrimp hepatopancreas extract.

\section{BE11}

Large conformational change on giant DNA induced by ascorbic acid: A nobel scheme on its antioxidative activity Yuko Yoshikawa, Emi Sakai, Yoshiko Oda Department of Food and Nutrition, Nagoya Bunri College, Nagoya 451-0077, Japan

Ascorbic acid is often regarded as an antioxidant in vivo, where it protects against DNA damage by scavenging reactive oxygen species. In the present, we will show another potent scenario on the protective effect of ascorbic acid through a significant structural change of giant DNA. Recently, we examined the effect of ascorbic acid on the higher order structure of DNA through single molecular observation with fluorescence microscopy, and found that ascorbic acid generates a pearling structure in giant DNA molecules, where elongated and compact parts coexist along a molecular chain. The results of observations with atomic force microscopy indicate that the compact parts assume a loosely packed conformation. As the extension, here we study the protective effect against double-strand breaks by reactive oxygen at different concentrations of ascorbic acid, in relation to the change of the higher order structure of giant DNA. We have performed a real time observation on the doublestrand breaks on individual DNA molecules by use of fluorescence microscopy. We have found that the double-strand break is markedly protected when ascorbic acid exists over millimolar concentrations. It is found that such a protective effect of ascorbic acid corresponds well to the above mentioned change on the higher order structure of DNA. It has been reported that human circulating immune cells, such as neutrophils, monocytes and lymphocytes, accumulate ascorbic acid in millimolar concentrations. Therefore, it is expected that the ascorbic acid concentration that induces the large conformational change on DNA may be of physiological significance.

\section{References}

Yoshikawa, Y., et al., 2004. FEBS Lett. 566, 39-42.

Yoshikawa, Y., et al., 2003. Eur. J. Biochem. 270, 3101-3106.

\section{BE12}

Effect of substrate, enzyme and hydrolysate concentration on corn gluten hydrolysis Dilek Kiliç apar, Belma Kin özbek Yıldız Technical University, Department of Chemical Engineering, Davutpaşa Campus 34210, Esenler/Istanbul, Turkey. E-mail: dkilic@yildiz.edu.tr (D. Kiliç apar)

Plant proteins are increasingly being used as an alternative to proteins from animal sources and substantially contribute to the human diet in several developing countries. There are many process both industrial and food based which employ protein hydrolysis and hydrolytic products have a wide variety of applications from industrial fermentation media to food additives. Traditionally, proteins are hydrolysed by chemical means. Acid hydrolysates of protein are used to produce food ingredients and flavour compounds. However, hydrolysis by chemical reagents produce potentially hazardous byproducts and these non-selective hydrolysis products cannot easily be defined. The use of enzymes allows for selective hydrolysis of protein and thus produces a potentially safer and more defined material. The present investigation describes the effects of substrate, enzyme and hydrolysate concentration on the hydrolysis of corn gluten. The corn gluten was hydrolysed by a commercial protease preparation Neutrase. The protein hydrolysis reactions were carried out in $0.2 \mathrm{~L}$ of aqueous solutions at the temperature of $50^{\circ} \mathrm{C}$ and $\mathrm{pH} 7$ and were monitored by using $\mathrm{pH}$-stat Method. The degree of hydrolysis (\%) and soluble protein concentration depending on time were investigated by using $1,2,4,6,8$ and $10 \%(\mathrm{w} / \mathrm{v})$ substrate concentrations; and $0.25,0.4,0.5,0.6$ and $0.75,1 \%$ (v/v) enzyme concentrations; and 25, 50, 75 and $100 \%$ (v/v) hydrolysate concentrations. For soluble protein determination in the hydrolysates samples, the Folin-Lowry Method (1951) was used. 


\section{BE13}

Medium optimization by orthogonal array designs for urease production by Aspergillus niger PTCC5011 M. R. Bakhtiari ${ }^{1}$, M. G. Faezi ${ }^{1,2}$, M. Fallahpour ${ }^{1}, A$. Noohi ${ }^{3}$, N. Moazami $^{1}, Z$. Amidi ${ }^{1}$ : ${ }^{1}$ Department of Biotechnology, Iranian Research Organization for Science and Technology (IROST), Tehran 15819, Iran; ${ }^{2}$ Department of Microbiology, Lahijan Azad University, Lahijan, Iran; ${ }^{3}$ Department of Microbiology, Faculty of Science, Tehran University, Tehran, Iran. E-mail: bakhtiari@irost.ir (M.R. Bakhtiari)

This paper describes medium optimization for urease production by Aspergillus niger PTCC 5011 by one-factor-at-a-time and orthogonal array design methods. The one-factor-at-a-time method was used to study the effects of carbon and nitrogen sources on urease production. Among various carbon and nitrogen sources used, sucrose and yeast extract were the most suitable for urease production, respectively. Subsequently, the concentration of sucrose, yeast extract and mineral sources were optimized using the orthogonal array method in two stages. The effects of nutritional components for urease production by A. niger PTCC 5011 in the first and second stages were in order of urea $>\mathrm{NiSO} 4>$ sucrose $>\mathrm{KH}_{2} \mathrm{PO}_{4}>$ $\mathrm{K}_{2} \mathrm{HPO}_{4}>\mathrm{CaCl}_{2}>$ yeast extract $>\mathrm{MgSO}_{4}$ and yeast extract $>$ sucrose $>\mathrm{K}_{2} \mathrm{HPO}_{4}>\mathrm{KH}_{2} \mathrm{PO}_{4}>$ urea $>\mathrm{CaCl}_{2}>\mathrm{NiSO}_{4}$, respectively. The optimal concentrations of nutritional components for improved urease production were determined as $20 \mathrm{~g} / \mathrm{L}$ sucrose, $0.85 \mathrm{~g} / \mathrm{L}$ urea, $3.4 \mathrm{~g} / \mathrm{L}$ yeast extract, $0.03 \mathrm{~g} / \mathrm{L} \mathrm{NiSO}_{4} \cdot 6 \mathrm{H}_{2} \mathrm{O}, 0.5 \mathrm{~g} / \mathrm{L} \mathrm{MgSO}{ }_{4} \cdot 7 \mathrm{H}_{2} \mathrm{O}$, $0.04 \mathrm{~g} / \mathrm{L} \mathrm{CaCl} 2,0.35 \mathrm{~g} / \mathrm{L} \mathrm{KH}_{2} \mathrm{PO}_{4}$, and $0.35 \mathrm{~g} / \mathrm{L} \mathrm{K}_{2} \mathrm{HPO}_{4}$. These results showed that urea, $\mathrm{NiSO}_{4}$, yeast extract and sucrose had significant effect on urease production by A. niger PTCC 5011. Tween 80 and $\mathrm{MgSO}_{4}$ had negligible effect on urease production by this strain. The subsequent confirmation experiments determined the validity of the models. Maximum urease activity in optimized media by onefactor-at-a-time and orthogonal array methods were about 1.14 and 2.74 times greater than with the basal medium, respectively.

\section{BE14}

Carbon sources create fingerprint fermentation characteristics Pınar Çalık ${ }^{2}$, Güzide Çalık ${ }^{1}$, Tunçer H. Özdamar ${ }^{1}$ : ${ }^{1}$ BRE Lab, Department of Chemical Engineering, Ankara University, 06100 Ankara, Turkey; ${ }^{2} \mathrm{IB}$ Lab, Department of Chemical Engineering, METU, 06531 Ankara, Turkey. E-mail: calik@eng.ankara.edu.tr (G. Çalık)

This work reports on a systematic investigation of the interactions between the single-carbon sources, i.e. glucose and citric acid, and complex-medium components, i.e., carbon and nitrogen sources, in enzyme fermentation processes, i.e. serine alkaline protease (SAP; EC 3.4.21.62), and $\beta$-lactamase (EC 3.5.2.6), with the oxygentransfer and $\mathrm{pH}$ conditions to demonstrate the influences of carbon sources that create the fingerprint fermentation characteristics, moreover, their influences on the product and by-product formations and the intracellular reaction rates. The influence of the medium composition i.e. citric acid-, glucose-, molasses- and soybean-based media together with the oxygen transfer (OT)- and $\mathrm{pH}$-conditions applied, on the product and by-product distributions and OT characteristics were investigated in batch bioreactors. For SAP, in general, under uncontrolled-pH operations the variation of the medium $\mathrm{pH}$ in SAP fermentation process has a tendency to increase in the SAP production phase; however, depending on the carbon source used, its behaviour changes in the early stages of the fermentation as the consequences of the directed functioning of the intracellular bioreaction network. The loci of the dissolved oxygen (DO) curves also strongly depend on the carbon source(s) utilised, in addition to the applied OT conditions. The complex media profiles are significantly different compared to the defined media as the $\mathrm{pH}$ and $\mathrm{DO}$ profiles are interrelated owing to the bioreactor operation conditions affecting the metabolic reaction network. The highest volumetric oxygen uptake rates were obtained with soybean-based medium that was ca. three-fold higher than the values reported in citrate-based and glucose based media, and ca. 1.5-2-fold higher than the values reported in molasses-based medium. The significant changes, moreover, the drastic change observed with the use of soybean-based complex medium are due to the compositions of the fermentation media used, and its influence on the intracellular bioreaction network. Thus, we conclude that the change in medium composition based on the carbon source changes the fermentation characteristics under the designed bioreactor operation conditions that appear as the fingerprints of the bioprocess.

\section{BE15}

Effects of oxygen transfer on $\boldsymbol{\alpha}$-amylase production by $\boldsymbol{B}$. amyloliquefaciens Nurhan Güngör, Güzide Çalık BRE Lab, Department of Chemical Engineering, Ankara University, 06100 Ankara, Turkey $\alpha$-Amylase (E.C. 3.2.1.1) a commercially important enzyme used in food, textile, detergent, brewing, paper, and animal feed industries; hydrolyses $\alpha-1,4$ glucosydic bonds in amylose, amylopectin, and related polysaccharides. Optimum medium composition and influence of bioreactor operation parameters on $\alpha$-amylase production with high yield and selectivity were determined together with the metabolic flux distributions using $B$. amyloliquefaciens (NRRL B-14396) which is found to be a good producer of the enzyme. Systematic investigation of oxygen transfer in relation with the metabolic fluxes for $\alpha$-amylase is not available in the literature. Shake-flask experiments were conducted in $0.5 \mathrm{dm}^{3}$ airfiltered bioreactors in orbital shakers with agitation and heating controls (B. Braun, Certomat-BS1). Laboratory-scale bioreactors were composed of agitation, heating, foam, dissolved-oxygen and $\mathrm{pH}$-controlled 1.0 and $3.5 \mathrm{dm}^{3}$ systems (B. Braun, Biostat Q; and Chemap). After separation of the cells with a Sorval RC28S ultracentrifuge, $\alpha$-amylase activity was measured by the DNS method (Bernfeld, 1955). Amino acid concentrations were determined with a HPLC (Waters), protein and organic acid concentrations were measured with a HPCE (Waters, Quanta 4000E) (Çalık et al., 1998). Oxygen transfer characteristics in the bioreactor systems were calculated using the Dynamic method (Rainer, 1990). In the mass flux balance-based analyses, a pseudo-steady state approximation for the intracellular metabolites and the accumulation rates of the extracellular metabolites measured throughout the fermentations in considerations of the biochemical feature of the system were used to acquire the flux distributions. In laboratory scale, the effects of different $\mathrm{C}$ sources i.e., glucose, fructose, maltose, lactose and soluble starch; $\mathrm{N}$ sources i.e., $\left(\mathrm{NH}_{4}\right)_{2} \mathrm{HPO}_{4},\left(\mathrm{NH}_{4}\right)_{2} \mathrm{SO}_{4}$, and $\mathrm{NH}_{4} \mathrm{Cl}$ and/or their concentrations; and the operation parameters, $\mathrm{pH}$ and temperature on cell growth, substrate utilization, $\alpha$-amylase and byproduct concentrations, and $\alpha$-amylase activity were investigated. In pilot scale, the fermentation and oxygen transfer characteristics 
of the bioreaction system together with the metabolic fluxes were determined.

\section{References}

Bernfeld, P., 1995. Methods Enzymol. 1, 149-159.

Çalık, P., Çalık, G., Özdamar, T.H., 1998. Enzym. Microb. Technol. 23, 451-461.

Rainer, B.W., 1990. Chem. Biochem. Eng. 4, 185-196.

\section{BE16}

Oxygen transfer regulates benzaldehyde lyase production in E. coli Pınar Çalık IBLab, Department of Chemical Engineering, METU, 06531 Ankara, Turkey. E-mail: pcalik@metu.edu.tr

The effects of oxygen transfer rate on benzaldehyde lyase (BAL) production by pUC18::bal carrying recombinant $E$. coli on a defined medium with $8.0 \mathrm{~kg} / \mathrm{m}^{3}$ glucose were investigated in order to finetune the bioreactor performance, in $V=3 \mathrm{dm}^{3}$ batch bioreactors at five different conditions with the parameters at, i.e. $Q_{0} / V_{\mathrm{R}}=0.5 \mathrm{vvm}$ and $N=250,375,500$ and $750 \mathrm{~min}^{-1}$ and; $Q_{0} / V_{\mathrm{R}}=0.7 \mathrm{vvm}$ and $N=750 \mathrm{~min}^{-1}$. The concentrations of the product and by-products amino acids and organic acids were determined in addition to BAL activities. Medium oxygen transfer rate conditions and uncontrolled $\mathrm{pH}$ operation at $\mathrm{pH}_{0} 7.25$ are optimum for maximum BAL activity, i.e. $860 \mathrm{U} / \mathrm{cm}^{3}$ at $12 \mathrm{~h}$, and productivity and selectivity. On the bases of the data, response of the intracellular bioreaction network of r- $E$. coli to oxygen transfer conditions were analysed using a mass-flux balance based stoichiometric model that contains 102 metabolites and 133 reaction fluxes. The results reveal that metabolic reactions are intimately coupled with the oxygen transfer conditions. Oxygen transfer rate showed diverse effects on the product formation by influencing metabolic pathways and changing metabolic fluxes. Metabolic flux analysis was helpful to describe the interactions between the cell and the bioreactor by predicting the changes in the fluxes and the rate controlling step(s) in the metabolic pathways. Therefore, knowing the distribution of the metabolic fluxes during the growth, and BAL and by-product formations provide new information for understanding physiological characteristics of the r-E.coli, and reveals important features of the regulation of the bioprocess and opens new avenues to successful application of metabolic engineering.

\section{BE17}

Multiple detection of foodborne pathogenic bacteria using 16S rDNA-based signature chip Byeong Hee Hwang, Ho Seop Eom, Hyung Joon Cha Department of Chemical Engineering, Pohang University of Science and Technology, Pohang 790-784, Korea. E-mail: jcha@postech.ac.kr (H.J. Chah)

The detection and diagnosis of pathogenic bacteria causing many diseases to the human body is an area of important research to public welfare. Food is the most important energy source to humans, but it can give rise to disease caused by pathogenic bacteria not performing adequate detection tests. Oligonucleotide-based microarrays are becoming increasingly useful for the analysis of expression profiles and polymorphisms among interested genes. Here, we checked the possibility of development of oligonucleotide-based microarrays for detection and diagnosis of foodborne pathogenic bacteria. The oligonucleotide chip technology was applied to one control strain and seven foodborne pathogenic bacteria strains. It was designed repeated spots of eight hyperspecific and two highly conserved (control) capture probes from $16 \mathrm{~S}$ rDNA sequences. In order to validate experimental quality and to certificate specificities among specific spots at a glance by $2 \mathrm{D}$ and $3 \mathrm{D}$ views, quantitative visualization tool was developed. Using the proposed oligonucleotide chip, we could classify and diagnose species and even subtypes of some pathogens.

\section{BE18}

Induction of in vitro neuro-muscular junctions using neuroblastoma and fibroblast cell lines for facilitating receptor-binding studies with botulinum toxin Arindam Chaudhury, Bal Ram Singh Department of Chemistry and Biochemistry, University of Massachusetts Dartmouth, 285 Old Westport Road, North Dartmouth, MA 02747-2300, USA. E-mail: g_achaudhury@umassd.edu (A. Chaudhury)

Botulinum toxin (BoNT), the most potent biological toxin known, is responsible for botulism, a fatal paralytic disease of the neuromuscular transmission. It blocks the release of acetylcholine at the neurotransmitter junction of the synapse. The objective of the current study was to induce in vitro neuro-muscular junctions through co-culturing of nerve and precursor-muscle cell lines. Presently no known primary cultures or cell lines are available for nerve-muscle co-culture, thus validating the current work. J2-3T3 Fibroblast cell line was first adapted to grow in media conducive for growth of $\mathrm{SH}-$ SY5Y Neuroblastoma cell line. The two cell lines were then splitted and co-cultured and observed for junction formations. Light and fluorescent microscopic studies revealed en plaque (twitch-type) and en grappe (bulbous nerve endings) nerve-muscle junctions. Growth rate of J2-3T3 cells decreased substantially when the media was initially changed. Structurally they were more spindle-shaped than the normal reticular shapes of J2-3T3 cells, when grown in a media tailor-made for them. The formation of nerve-muscle junctions were confirmed using markers specific for each cell type. Future work is focusing on receptor identification for the botulinum toxin in the established in vitro neuro-muscular junctions and also the transcellular translocation of the toxin.

\section{BE19}

Addressing instability issues in FTIR monitoring of bioprocesses M. Dabros, I.W. Marison, U. von Stockar Laboratory of Chemical and Biochemical Engineering (LGCB), Swiss Federal Institute of Technology, CH-1015 Lausanne, Switzerland. E-mail: michal.dabros@epfl.ch (M. Dabros)

Fourier-transform infrared (FTIR) spectrometers have recently enjoyed widespread popularity in bioprocess monitoring applications due to their non-invasiveness and in-situ sterilizability. Their online applicability creates an interesting opportunity for process control and optimization. However, the precision and accuracy of the predicted analyte concentration values directly depend on the quality of the measured signal and the robustness of the calibration model. Instability and time drift in the measured spectra are currently one of the main obstacles in FTIR monitoring. The intensity of the detected signal is influenced both by random noise and structural drifts and offsets. As a result, it is often necessary to scale the measured spectrum with respect to a constant reference spectrum, a technique similar to the internal standard approach used in analytical assays, such as HPLC. Applying this technique has lead to a noticeable decrease in the standard error of prediction in the monitoring of an anaerobic 
fermentation of the Saccharomyces cerevisiae yeast. In order to test the robustness of the calibration model and to increase its resistance to signal instability, random spikes of known amounts of analytes were introduced into the measured medium. This approach can be used to fine-tune the calibration model on-line and is currently one of the aspects investigated in this laboratory.

\section{BE20}

The effect of the stringent response induction on L-valine biosynthesis by Corynebacterium glutamicum Ilze Denina $\stackrel{1,2}{ }$, Longina Paegle $^{2}$, Liga Zala ${ }^{1,2}$, Maija Ruklisha ${ }^{2}:{ }^{1}$ Faculty of Biology, University of Latvia, Kronvalda blvd. 4, Riga LV-1586, Latvia; ${ }^{2}$ Institute of Microbiology and Biotechnology, University of Latvia, Kronvalda blvd. 4, Riga LV-1586, Latvia. E-mail: ilzede@hotmail.com (I. Denina)

The present study was focused on methods of the stringent response induction and on investigation of its effect on valine overproduction by isoleucine auxotrophs of Corynebacterium glutamicum. The intracellular level of guanosine $5^{\prime}$-diphosphate $3^{\prime}$ diphosphate (ppGpp) increased and bacterial growth rate $(\mu)$ decreased during the short-term experiments when the exponentially growing cells were exposed to isoleucine limited conditions. The induction of the cellular stringent response resulted in a drastic increase in the activity of acetoxydroxy acid synthase (AHAS), also by a significant increase in valine production. In contrast, an increase in AHAS activity and valine synthesis by $C$. glutamicum was not achieved when bacterial growth was down-regulated in a ppGpp-independent manner. These results demonstrated that induction of the ppGpp-mediated stringent response might be significant in order to increase valine overproduction by $C$. glutamicum.

\section{BE21}

Studies on isolation of a fusion protein containing the AD-1 domain of the glycoprotein $\mathbf{B}$ of human cytomegalovirus $F$. Sousa, S. Ferreira, J.A. Queiroz, F.C. Domingues Health Sciences Research Centre, University of Beira Interior, $6200-001$ Covilhã, Portugal

Infections with human cytomegalovirus (HCMV) continue to be an important health problem in certain patient populations, such as newborns, graft recipients of solid organs, or bone marrow and AIDS patients. In these groups, HCMV is a major cause of morbidity and mortality. The complex biology of HCMV necessarily begins with an initial interaction between the envelope of the infectious virus and the host cell. Glycoprotein $\mathrm{B}(\mathrm{gB})$ is the major antigen, on the envelope of HCMV, for the induction of neutralizing antibodies. The region between aa 552 and 635 of $\mathrm{HCMV} \mathrm{gB}$ (termed antigenic domain 1, AD-1) has been identified as the immunodominant target for the humoral immune response following natural infection. Screening methods for detection of neutralizing antibodies have not been used because they are costly and labor intensive and thus far are not feasible for use on a large scale. For the development of reliable and inexpensive serodiagnostic tests the AD-1 of HCMV glycoprotein gp58, which are known to bind neutralizing antibodies, was expressed in prokaryotic systems. In this work, one prokaryotically expressed fusion protein which codifies AD- 1 with $\beta$-galactosidase was used. The influence of different process conditions, on the production of the fusion protein containing the AD- 1 as well as sugars addition to the fermentation medium was investigated. In order to analyze the expression of fusion protein, the $\beta$-galactosidase activity was followed throughout the fermentation. Lysis process was also optimized and some final confirmation tests about protein antigenicity were performed.

\section{BE22}

Polyenzymic systems for the preparation of drugs based on modified nucleosides D. Chuvikovsky, R. Esipov, T. Muravyova, A. Miroshnikov Shemyakin-Ovchinnikov Institute of Bioorganic Chemistry, Russian Academy of Sciences, ul. Miklukho-Maklaya 16/10, Moscow 117997, Russia. E-mail: esipov@ibch.ru (R. Esipov)

Considerable progress in the preparation of nucleoside analogues was achieved by combination of chemical and biochemical transformations. Enzyme-catalyzed chemical transformation is now widely recognized as practical alternative to traditional organic synthesis in pharmaceutical and chemical industries. Pentofuranosyltransfer reaction catalyzed by nucleoside phosphorylases was successfully employed for the synthesis of a variety of base- and sugar-modified nucleosides. Enzymes involved in the metabolism of ribose phosphate and deoxyribose phosphate, such as ribokinase and phosphopentomutase, were used for the preparation of sugar-modified nucleosides. Nucleosides phosphorylases (thymidine phosphorylase (TP), uridine phosphorylase (UP) and purine nucleoside phosphorylase (PNP)), ribokinase and phosphopentomutase from $E$. coli have been cloned and overexpressed in $E$. coli. Fast and efficient methods for the purification of nucleosides phosphorylases have been developed. The amount of purified protein was about $140 \mathrm{mg} / \mathrm{L}$ of cell culture, corresponding to $6300,16,800$ and 4200 units, respectively, of the UP, TP and PNP. Synthesis of medicinal drugs ribavirin (1-( $\beta$-D-ribofuranosyl)1,2,4-triazole-3-carboxamide), cladribine (2-chloroadenine-9- $\beta$-D$2^{\prime}$-deoxyribofuranoside) and fludarabine (2-fluoroadenine-9- $\beta$-Darabinofuranoside) with the use of recombinant enzymes were studied. Several important factors affecting the modified nucleosides production ( $\mathrm{pH}$, temperature, enzyme concentration, donor/acceptor ratio) were investigated and optimized. Under optimum conditions ribavirin, cladribine and fludarabine produced in the reaction mixture in yields of 84,85 and $80 \%$, referred to 1,2,4-triazole-3-carboxamide, 2-chloroadenosine and 2-fluoroadenosine, respectively.

BE23

Aggregation and adsorption of fibroin molecules in aqueous solution Won Hur School of Biotechnology and Bioengineering, Kangwon National University, Chunchon 200-701, Korea. E-mail: wonhur@kangwon.ac.kr

Fibroin, the structural protein from Bombyx mori, is composed of heavy chain (generally called 'fibroin') and light chain polypeptides of about 370 and $25 \mathrm{kDa}$, respectively. This study investigated the aggregation of fibroin and the adsorption between fibroin and surfaces. The variations of particle size and zeta potential were investigated by electrophoretic light scattering spectrophotometer (ELS). The adsorption of fibroin on surface was investigated in a continuous flow system by BIAcore applied surface plasmon resonance(SPR) technique. The particle size and zeta potential range of aqueous fibroin were $140 \mathrm{~nm}, \pm 20 \mathrm{mV}$, respectively. Iso-electric point $\left(\mathrm{pH}_{\text {iep }}\right)$ of fibroin was $\mathrm{pH} 4.29$. The amount of fibroin adsorbed on a gold surface was less than $0.1 \mu \mathrm{g} / \mathrm{Ml}$ even in the presence of high concentration of fibroin. The modification of gold surface was accomplished by applying chemicals known to form self-assembled 
monolayer, Those are carrying $\mathrm{NH}_{3}{ }^{+}, \mathrm{COO}^{-}$, benzene ring and peptide that similar structure with fibroin. The adsorbed amount of fibroin on the self-assembly monolayers (SAMs) increased in the following order: $\mathrm{NH}_{3}{ }^{+}>$Benzene ring $>\mathrm{COO}^{-}>$peptide surface. The deposition of fibroin in aqueous solution on non-waven fabric was affected by $\mathrm{NaCl}$ and high temperature.

\section{BE24}

pH influences metabolite profiling of $\beta$-lactamse producing $B$. licheniformis Nazar İleri ${ }^{1}$, Plnar Çalık ${ }^{1}$, Ali Şengül ${ }^{2}:{ }^{1}$ IB Lab, Department of Chemical Engineering, METU, 06531 Ankara, Turkey; ${ }^{2}$ Gülhane Sch Med, Dept Immunol, 06018 Ankara, Turkey. E-mail: e115715@metu.edu.tr (N. İleri)

$\mathrm{pH}$ conditions in the bioreactor affect product and by-product formations by influencing metabolic pathways and changing metabolic fluxes, based on its influence on, i.e. DNA transcription, protein synthesis, transport mechanism, ATP generation and cellular energetics. Whereupon, some fermentation processes favours uncontrolled-pH conditions while others favours controlled-pH conditions. On the bases of the interactions between the cell and the bioreactor through a process, carried out at either uncontrolled- or controlled-pH conditions, intracellular $\mathrm{pH}$ can be widely different and variable during the fermentation process. Consequently, if one aims towards a quantitative understanding of the cell metabolism, one has to take into account the time variations of the intracellular $\mathrm{pH}$ and its effects on the in-vivo kinetics of the metabolic steps involved. Moreover, since the presence of dormant or dead cells in the cultivation medium have negative effect on the synthesis of the production of desired product; the physiological state of the culture has great importance. In this context, the effects of $\mathrm{pH}$ on the regulation of intracellular $\mathrm{pH}$, transport mechanism, and metabolic activity of $B$. licheniformis during production of $\beta$-lactamase (EC 3.5.2.6), an industrial enzyme catalyzing the hydrolysis of beta-lactam ring in beta-lactam antibiotics, was investigated. In addition, the physiological state of the organism and its effect on the production were observed. The optimal controlled-pH operation was found to be $\mathrm{pH} 6.75$ with $54 \mathrm{U} / \mathrm{cm}^{3}$ enzyme activity. The intracellular and extracellular $\mathrm{Na}^{+}, \mathrm{K}^{+}$ion concentrations increased significantly throughout the process with increasing $\mathrm{pH}$. On the other hand, the intracellular $\mathrm{NH}_{4}{ }^{+}$ion concentration was relatively constant.

\section{BE25}

Isolation and characterization of angiotensin-I converting enzyme inhibitory peptides by use of anti-peptide antibody Fida Hasan ${ }^{1}$, Megumi Kitagawa ${ }^{2}$, Yoichi Kumada ${ }^{1}$, Naoya Hashimoto $^{1}$, Masami Shiiba ${ }^{2}$, Shigeo Katoh ${ }^{1}$, Masaaki Terashima ${ }^{2}$ :

${ }^{1}$ Graduate School of Science and Technology, Kobe University, Nada, Kobe 657-8501, Japan; ${ }^{2}$ Department of Human Science, Kobe College, Okadayama, Nishinomiya 662-8505, Japan

Inhibitory peptides against angiotensin-I converting enzyme can be promising bio-functional peptides as natural alternatives for the non-peptide ACE inhibitory drugs. These peptides are inactive within sequences of parent proteins and can be released during enzymatic digestion or food processing. Immunointeraction is very effective for the purification of proteins and peptides with high purity. In this study, ACE inhibitory peptides from hydrolysate of bonito meat were isolated by an anti-peptide antibody column and HPLC, and kinetics of production of these ACE inhibitory peptides was studies. An anti- peptide antibody against an ACE inhibitory peptide, which was found by Kohama et al. from tuna was obtained by immunization of the antigen peptide PC-IACE (KKPTHIKWGD). Water extract of bonito meat was digested at $37^{\circ} \mathrm{C}$ in a modified gastric juice, $1.76 \mathrm{mg} / \mathrm{mL}$ $\mathrm{NaCl}$ containing $312 \mu \mathrm{g} / \mathrm{mL}$ pepsin ( $\mathrm{pH}$ 2). Peptides in hydrolysates were purified by use of an affinity column coupled with the antipeptide antibody and separated by HPLC equipped with a reverse phase column (Cosmosil 5C18- MS-II, $4.6 \mathrm{~cm} \times 150 \mathrm{~cm}$ ). Amino acid sequences and $\mathrm{IC}_{50}$ values of the potent $\mathrm{ACE}$ inhibitory peptides were determined. SDS-PAGE and western blotting experiments clarified that bonito protein contained peptides having similar sequence to the antigen peptide. A fraction of retention time 18-28 min in HPLC purification samples showed high inhibitory activity, and several peptides in this fraction were separated. After $48 \mathrm{~h}$ digestion, two major inhibitory peptides, HERDPTHIKWGD and PTHIKWGD, were found to be relatively stable in the gastric juice.

\section{BE26}

Kluyveromyces marxianus physiology on several levels of carbon, nitrogen sources and oxygenation during inulinase production Silva-Santisteban Yépez, O. Bernardo, Francisco Maugeri Department of Food Eng./Unicamp, 13081-970, Campinas, SP-Brazil. Email: maugeri@fea.unicamp.br (F. Maugeri)

Inulinase produced by yeasts is an interesting alternative compared with the one produced by filamentous molds, as culture conditions can be better controlled. During the assays, it was observed that inulinase production levels varied with nutritional conditions in batch culture. Kluyveromyces marxianus ATCC 16045 culture is described by two main phases, the first one being the growth phase, where substrate consumption and basal inulinase production were performed, and the second one being the phase where some metabolites are uptaken and high inulinase production is observed. The metabolic fluxes analyses were used to describe the cell physiology in the first phase, in a variety of conditions of sucrose and ammonium sulfate concentration and aeration condition. The metabolic network included the main metabolic pathways such as glycolisis, pentose phosphate pathway, Krebs cycle, oxidative phosphorylation and biomass biosynthesis. The physiology in this phase was correlated with high inulinase production in the second phase. It was also noticed that inulinase production diminished when sucrose was in high concentration, leading, additionally, to ethanol production. In these terms, it was unveiled a kind of Crabtree effect performed by this strain.

\section{BE27}

Forward extraction of L-aspartic acid from fermentation broths by reverse micelles and backward extraction by gas hydrate method $\ddot{O}$. Aydoğan ${ }^{1}$, E. Bayraktar ${ }^{1}, \ddot{U}$. Mehmetoğlu ${ }^{1}, M$. Parlaktuna ${ }^{2}$, T. Mehmetoğl $u^{2}$ : ${ }^{1}$ Department of Chemical Engineering, Faculty of Engineering, Ankara University, Tandogan, Ankara 06100, Turkey; ${ }^{2}$ Department of Petroleum and Natural Gas Engineering, Middle East Technical University, Ankara 06531, Turkey. E-mail: mehmet@eng.ankara.edu.tr (Ü. Mehmetoğlu)

Recently gas hydrate method has been applied as a technique for backward extraction of amino acids from reverse micelle systems. In this study, backward extraction of L-aspartic acid was investigated by gas hydrate method. At the first stage, production of L-aspartic acid was carried out using $50 \mathrm{~mL}$ of $0.5 \mathrm{M}$ ammonium fumarate $(\mathrm{pH}$ 
9.5) as substrate at $37 \mathrm{CircC}$ in an orbital shaker at $150 \mathrm{rpm}$ for $4 \mathrm{~h}$. E. coli (ATCC 11303) was used as biocatalyst. At the end of reaction excess fumaric acid was extracted in reverse micelle phase. Then forward extraction of $\mathrm{L}$-aspartic acid was carried out with injection method in reverse micelle phase. For back extraction, $\mathrm{CO}_{2}$ is used to form gas hydrates crystalline structure. Back extraction experiments were carried out between 35-37 bar g pressure and at 2 CircC. At the end of the back extraction L-aspartic acid was obtained in crystalline form. The results indicate that recovery of L-aspartic acid from reverse micelles by forming gas hydrate can be achieved with a yield of 55.3\%. Consequently, gas hydrate method can be used as a new technique for backward extraction of amino acids from reverse micelles.

\section{Acknowledgement}

The authors gratefully acknowledge the financial supports given to this work by Ankara University, Biotechnology Institute (Project No 2001K120240-41).

\section{BE28}

Aerobic and anaerobic cultivations of Aspergillus niger on different nitrogen sources Susan Meijer, Gianni Panagiotou, Lisbeth Olsson and Jens Nielsen Center of Microbial Biotechnology, Biocentrum-DTU, Technical University of Denmark, DK-2800 Lyngby, Denmark

In this study, we aim at creating a succinic acid producing strain of A. niger. A. niger is known to be a strictly aerobic organism, meaning it is not able to use the reductive part of the TCA cycle to produce succinate. During aerobic growth A. niger uses oxygen as electron acceptor in the respiratory chain, thereby reoxidizing the produced $\mathrm{NADH}$ to $\mathrm{NAD}^{+}$. However, under anaerobic conditions other compounds than oxygen, such as $\mathrm{NO}_{3}{ }^{-}$are required as electron acceptor (denitrification). This process consists of $\mathrm{NO}_{3}{ }^{-}$ reduction to $\mathrm{NH}_{4}{ }^{+}$coupled to substrate-level phosphorylation that supports growth under anaerobic conditions. In the present study, our aim was to investigate the effect of different nitrogen sources on the physiology of A. niger during growth under aerobic and anaerobic conditions. Aerobic growth experiments on three different nitrogen sources; ammonium, nitrate and nitrite, showed that ammonium and nitrate could be consumed by the filamentous fungus. Nitrite on the other hand could not facilitate growth, indicating the absence of a nitrite uptake system. However, under anaerobic conditions notable growth was only observed on nitrate. These data support the hypothesis of the existence of an alternative electron acceptor that might facilitate anaerobic growth of A. niger.

BE29

Effects of various environmental factors on Chinese Hamster Ovary cell cultures for production of recombinant antibody D.Y. Kim ${ }^{1,2}$, J.C. Lee ${ }^{3}$, H.N. Chang ${ }^{2}$, Duk Jae Oh ${ }^{1}$ : ${ }^{1}$ Department of Bioscience and Biotechnology, Sejong University, 98 Gunjadong, Gwangjin-gu, Seoul 143-747, South Korea; ${ }^{2}$ Department of Chemical and Biomolecular Engineering, Korea Advanced Institute of Science and Technology, 373-1, Guseong-dong, Yuseong-gu, Daejon 305-701, South Korea; ${ }^{3}$ Korea Biotechnology Commer- cialization Center, Korea Institute of Industrial Technology, 994 Dongchoon-Dong, Yeonsu-Gu, Incheon 406-130, South Korea. Email: djoh@sejong.ac.kr (D.J. Oh)

Among the therapeutic proteins derived from mammalian cells, recombinant antibodies received a great deal of attention as a prominent product through biotech pipelines toward the marketplace. They now occupy about $25 \%$ of the estimated medicines in clinical development and many more antibodies which lead the value of the market going forward are reported. There are various environmental factors affecting rCHO cell cultures such as medium components, temperature, $\mathrm{pH}$, and byproducts (ammonia, lactate, and, etc.). Because most of mammalian cells are very sensitive to their environmental change, appropriate control of environmental parameters is a very important consideration to enhance cell growth and production of target proteins. Balanced addition of limiting medium components plays an essential role on improvement of cell density and product concentration. Temperature and $\mathrm{pH}$ are easily adjustable process parameters, being reported to influence cell growth and recombinant protein production. Ammonia and lactate are well-known byproducts which have an inhibitory effect on cell growth when their concentrations exceed a specific level. In this work, effects of various environmental factors including temperature, $\mathrm{pH}$, amino acids, vitamins, hormones, and metabolic byproducts on cell growth and recombinant antibody production were investigated in the cultivation of recombinant Chinese Hamster Ovary cells. The most suitable condition of each environmental condition was proposed for enhancement of the cell growth and the productivity of recombinant antibody.

BE30

Inhibitory effect of astragali radix on matrix degradation in human articular cartilage and chondrocytes Tae-Ryeon Heo, SooIm Choi, Sun-Mi Jung, Jae-Won Lee, Sang-Min Park Department of Biological Engineering, Inha University, Incheon 402-751, Korea

The present study was carried out in order to assess the protective effects of calycosin-7-O- $\beta$-D-glucopyranoside isolated from Astragali Radix (AR) on hyaluronidase (HAase) and the recombinant human interleukin-1 $\beta$ (IL-1 $\beta$ ) induced matrix degradation in human articular cartilage and chondrocytes. We isolated the active component from the $n$-butanol soluble fraction of AR as HAase inhibitor and structurally identified as calycosin-7-O- $\beta$-D-glucopyranoside by LC-MS, IR, ${ }^{1} \mathrm{H}$ NMR, and ${ }^{13} \mathrm{C}$ NMR analyses. The protective effect of $\mathrm{ARBu}$ on the matrix gene expression of immortalized chondrocyte cell line C-28/I2 treated with HAase was investigated using a reverse transcription polymerase chain reaction (RT-PCR). Its effect on HAase and IL- $1 \beta$-induced matrix degradation in human articular cartilage was determined by using a staining method and calculating the amount of degraded glycosaminoglycan (GAG) from the cultured media. Pretreatment with Calycosin-7- $O$ - $\beta$-D-glucopyranoside effectively protected against matrix degradation of the human chondrocytes and articular cartilage. Therefore, it would appear that Calycosin-7- $O$ - $\beta$-D-glucopyranoside from AR is a potential natural ant-inflammatory or anti-osteoarthritis agent and can be effectively used to protect against proteoglycan (PG) degradation. 


\section{BE31}

Preliminary studies on production and isolation of Human Catechol-O-Methyltransferase from an Escherichia coli strain L. A. Passarinha ${ }^{1}$, M. J. Bonifácio ${ }^{2}$, P. Soares-da-Silva ${ }^{2}$, J. A. Queiroz ${ }^{1}{ }^{1}$ Centro de Investigação em Ciências da Saúde, Universidade da Beira Interior, 6201-001 Covilhã, Portugal; ${ }^{2}$ Departamento de Investigação e Desenvolvimento, BIAL, 4745-457 São Mamede do Coronado, Portugal. E-mail: luis_passarinha@ hotmail.com (L.A. Passarinha)

Catechol- $O$-methyltransferase (COMT) is an enzyme that catalyses a variety of endogenous and exogenous catechol substrates by transferring a methyl group from $S$-adenosylmethionine (SAM) to either the meta- or the para-hydroxyl group of the catechol ring. The enzyme has a physiological role in the metabolism of the catechol estrogens, inactivation of the catecholamine neurotransmitters such as dopamine and epinephrine and detoxification of a variety of xenobiotic catechols. COMT activity has been identified in various tissues; however with the developments in molecular biology and gene technology, the production and purification of large amounts of recombinant COMT is a good option for biochemical, pharmacological and structural studies. In this work, cultures of recombinant $E$. coli harbouring a model plasmid were grown in a $500 \mathrm{~mL}$ shake-flask containing $125 \mathrm{~mL}$ of complex medium. The influence of medium composition and induction time on COMT production, recovery and clarification by sonication, ammonium sulphate precipitation and purification by hydrophobic interaction chromatography onto a butyl-sepharose column will be presented and discussed.

\section{BE32}

Bioactive bacterial exopolysaccharides Corinne Sinquin ${ }^{1}$, Karim Senni $^{2}$, Jacqueline Ratiskol ${ }^{1}$, Farida Guéniche ${ }^{2}$, Jean Guézennec ${ }^{1}$, Gaston Godeau ${ }^{2}$, Sylvia Colliec-Jouault ${ }^{1}:{ }^{1}$ Ifremer, 44311 Nantes Cedex 3, France; ${ }^{2}$ EA2496 Université René Descartes, 92120 Montrouge, France. E-mail: corinne.sinquin@ifremer.fr (C. Sinquin)

Interest in mass culture of microorganisms from the marine environment has increased considerably, representing an innovative approach to the biotechnological use of under-exploited resources. Marine bacteria associated with deep-sea hydrothermal conditions have demonstrated their ability to produce in an aerobic carbohydrate-based medium, unusual extracellular polymers (Guezennec, 2002; Colliec-Jouault et al., 2004). These exopolysaccharides (EPS) present original structural features that can be modified to design innovative bioactive compounds and improve their specificity. With the aim of promoting biological activities, chemical modifications (depolymerization and substitution reactions) of one EPS produced by Vibrio diabolicus have been undertaken (Raguenes et al., 1997). The structure of the native EPS has been described (Rougeaux et al., 1999): $(\rightarrow 3)-\beta-D-G l c p N A c-(1 \rightarrow 4)-$ $\beta$-D-GlcpA- $(1 \rightarrow 4)-\beta-D-G l c p A-(1 \rightarrow 4)-\alpha-D-G a l p N A c-(1 \rightarrow)$. The potential of the EPS derivatives as therapeutical agents will be presented.

\section{References}

Colliec-Jouault, S., et al., 2004. Pathol. Biol. 52, 127-130.

Guezennec, J.J., 2002. Ind. Microbiol. Biotechnol. 29, 204-208.
Raguenes, G., et al., 1997. Int. J. Syst. Bacteriol. 47, 989-995. Rougeaux, H., et al., 1999. Carbohydr. Res. 322, 40-45.

\section{BE33}

Physiological responses of $E$. coli to glucose and oxygen shifts in fed-batch fermentations Jaakko Soini ${ }^{1}$, Christina Saarimaa ${ }^{1}$, Arne Matzen $^{2}$, Peter Neubauer: ${ }^{1}$ Bioprocess Engineering Laboratory, Department of Process and Environmental Engineering, University of Oulu, Oulu FI-90014, Finland; ${ }^{2}$ Sanofi-Aventis, Germany. E-mail: jaakko.soini@oulu.fi (J. Soini)

In high-cell density fermentations $E$. coli cells are often subjects of transient changes in microenvironment around them. These changes can be, for example, medium component gradients or differences in oxygen availability. We have studied the physiological response of $E$. coli $\mathrm{W} 3110$ cells to simultaneous oxygen limitation and overfeeding of glucose. The aim is to obtain more information of physiological changes for better understanding of the bottlenecks in such processes. The response of the cells for glucose and oxygen shifts was studied by analyzing key metabolites and proteins and mRNA transcript levels. The transcript levels were measured using a sandwich hybridization technique (Rautio et al., 2003) Proteomic analysis was carried out by $2 \mathrm{D}$-electrophoresis and the metabolite analysis by HPLC. The main focus of this study is to monitor the expression patterns of marker genes involved in mixed acid fermentation, glycolytic pathway and tricarbonic acid cycle.

\section{Reference}

Rautio et al., 2003. Sandwich hybridisation assay for quantitative detection of yeast RNAs in crude cell lysates. Microb. Cell Fact.

\section{BE34}

Influence of NER on genetic instability of the (CTG/CAG) tracts in bacterial chromosome Sylwia Szwarocka ${ }^{1}$, Pawet Parniewski ${ }^{2}$ : ${ }^{1}$ Department of Microbiology and Immunology, University of Łódź, 90-237 Łódź, Banacha 12/16, Poland; ${ }^{2}$ Centre for Medical Biology, Polish Academy of Sciences, 93-232 Łódź, Lodowa 106, Poland

Many human hereditary neurological diseases, including fragile $\mathrm{X}$ syndrome, myotonic dystrophy and Friedreich's ataxia, are associated with expansions of triplet repeat sequences (TRS) (CGG/CCG, CTG/CAG and GAA/TTC) in or near specific genes. Mechanisms that mediate the expansions and deletions of TRS include DNA replication, repair and recombination. Many investigations suggest that the structural properties of the TRS play a consequential role in their genetic instabilities. Nucleotide excision repair (NER) is the major cellular system in both prokaryotes and eukaryotes and recognises damages due to distortion of the DNA helix. Involvement of NER in the hairpin loop repair that can form within CTG tracts has been reported. The participation of this repair systems in the TRS instability was investigated in E. coli only on multicopy plasmids. The results showed that deficiency of some NER functions dramatically affects the stability of long (CTG/CAG) inserts. In this work we present a chromosomal model to study the instability of the TRS in E. coli. We introduced the (CTG/CAG)n tracts into the chromosome of E. coli and used strains with some deficiency of the NER and investigated genetic stability of these tracts after multiple recultivations. In general, our results show that the (CTG/CAG)n repeats are much more stable in the chromosome than in plasmids. These data may suggest that instability of TRS in plasmids is associated with interaction 
between repetitive tracts on different plasmid molecules inside the cell. However, mutations of NER genes may increase ( $u v r A$ and $u v r B$ mutants) or decrease ( $u v r C$ and $u v r D$ mutants) stability of the TRS in the $E$. coli chromosome.

\section{Acknowledgement}

This study was partially funded by the KBN grant 2 P05A 01927.

\section{BE35}

Performance analyses of a multi-stage integrated fermentation process for lactic acid production Hsun-Tung Lin, Feng-Sheng Wang Department of Chemical Engineering, National Chung Cheng University, Chia-yi 621-02, Taiwan. E-mail: chmfsw@ccu.edu.tw (F.S. Wang)

In this work, we considered a multi-stage integrated continuous fermentation process for producing lactic acid. Each stage consists of a mixing tank, a fermenter, a cell recycle unit and an extractor. The generalized kinetic model is first applied to formulate the integrated process. We have compared the overall productivity and conversion of the integrated process with those of two simplified processes. From the design equations, we obtain that three processes have the identical overall conversion. However, the proposed process has the greatest overall productivity. The specific kinetic model for lactic production (Youssef et al., 2000) was applied to the integrated process in order to find the maximum overall productivity. Two optimization problems are respectively considered to determine the optimal stages, operating conditions and design variables. The first problem supposes that the integrated process has the equal working volume ratio for each fermenter. Such a process requires four stages to yield the maximum overall productivity and the nearly complete overall conversion. However, if the working volume ratio for each stage is considered as the decision variables in the second optimization problem, three stages is enough to achieve the identical overall productivity.

\section{Reference}

Youssef, C.B., Guillou, V., Olmos-Dichara, A., 2000. Contr. Eng. Pract. 8, 1297-1307.

\section{BE36}

Modelling of the binding of ligands to macromolecules J $\phi$ rgen $M$. Mollerup Department of Chemical Engineering, Building 229, DTU, 2800 Lyngby, Denmark

A variety of factors that govern the properties of proteins are utilized in the development of chromatographic processes for the recovery of biological products including the binding and release of protons, the non-covalent association with non-polar groups (often hydrophobic interactions), the association of small ions (ion exchange) and the highly specific antigen-antibody interaction (affinity interactions). The fulcrum point in the understanding and modelling a chromatographic separation is the adsorption isotherm that determines the peak shape at preparative load. To enable an efficient chromatographic process development strategy it is necessary to conduct theoretical and experimental investigations of the adsorptive behaviour of proteins. Thermodynamically consistent models for ion exchange chromatography and hydrophobic interaction chromatography have been developed and can be utilised in the simulation of a chromatographic separation. Besides, measurements on HIC media can be utilised to determine the Cohn salting-out coefficient. The lig- and binding process can frequently be coupled to associated structure changes in the protein, the ligand or both. This gives rise to nonlinear adsorptive behaviour known as cooperativity which cannot be modelled using conventional models which displays convex behaviour. Examples of cooperative behaviour are the reversible binding of oxygen and carbon monoxide to haemoglobins and the binding of NAD ${ }^{+}$ to yeast glyceraldehydes 3-phosphate dehydrogenase. In the paper we discuss the modelling of reversible binding of mobile as well as immobilised ligands to macromolecules and compare modelling to experiment.

\section{BE37}

Comparative analysis of the temperature policy for processes with a deactivating native enzyme $I$. Grubecki, M. Wojcik Department of Chemical and Biochemical Engineering, University of Technology and Agriculture, 85-326 Bydgoszcz, ul. Seminaryjna 3, Poland

A comparative analysis of the temperature policy for an enzymatic reaction with Michaelis-Menten kinetics in a batch reactor has been carried out. Both isothermal and optimal temperature policies for processes with deactivating native enzyme have been considered. In the model, the thermal deactivation was described by a first-order reaction, and the Arrhenius-type dependence between rate parameters and temperature was assumed. As an indicator for a direct comparison between the isothermal and optimal temperature policies the quotient of conversions under identical initial and final condition was used. A method was presented to calculate this indicator, which is based on the analytical and numerical solutions. This method can be of great importance for the industrial practice. Application of changeable temperature policy could result in significant increase in conversion when ratio of activation energy for deactivation and activation energy for reaction is high.

\section{BE38}

Studies on the impact of mixing during brewing using near and mid-infrared spectroscopy Georgina McLeod ${ }^{1}$, Alvin W. Nienow ${ }^{1}$, Graham Poulter $^{2}$, Reg Wilson ${ }^{3}$, Henri Tapp $^{3}$, Christopher J. Hewitt ${ }^{1}$ : ${ }^{1}$ Biochemical Engineering, Centre for Formulation Engineering, School of Engineering (Chemical Engineering), The University of Birmingham, Edgbaston B15 2TT, UK; ${ }^{2}$ Specac Limited, Kent BR5 4HE, UK; ${ }^{3}$ Institute of Food Research, Norwich Research Park, Norwich NR4 7UA, UK. E-mail: gxm828@bham.ac.uk (G. McLeod)

The control of the brewing process is important for improving product quality, and lowering costs. Infrared spectroscopy is a technique that can be used at-line and on-line to rapidly measure component concentrations of unprocessed whole broth samples in real time. In this study, both mid -infrared (MIR) and near-infrared (NIR) spectroscopy have been used and compared for the monitoring of ethanol, flavour components, wort sugars, biomass and specific gravity during the brewing. Partial least-squares regression (PLS) was used to model relationships between component concentrations and spectra. The performance of these models was evaluated in terms of the standard error of prediction (SEP), number of PLS factors and the correlation coefficient $(r)$. Calibration models were constructed using spectra acquired for multi-component mixtures, intended to simulate brewing fermentation conditions, and actual brewing fermentation samples. Chemometric results indicated that 
NIR is a powerful tool for accurately measuring sugar, ethanol and biomass concentrations.

\section{BE39}

NIR-spectroscopy model development of indicator metabolites in fermentation processes Parveen Kousar ${ }^{1}$, Alvin W. Nienow ${ }^{1}$, Graham Poulter ${ }^{2}$, Reg Wilson ${ }^{3}$, Henri Tapp ${ }^{3}$, Christopher J. Hewitt ${ }^{1}$ : ${ }^{1}$ Biochemical Engineering, Centre for Formulation Engineering, School of Engineering (Chemical Engineering), The University of Birmingham, Edgbaston B15 2TT, UK; ${ }^{2}$ Specac Limited, Kent BR5 4HE, UK; ${ }^{3}$ Institute of Food Research, Norwich Research Park, Norwich NR4 7UA, UK. E-mail: pxk203@bham.ac.uk (P. Kousar)

The optimization of a fermentation process requires the organism to be cultivated under desirable conditions, which depends on how well the fermentation process is controlled. Inadequate mixing and mass transfer are responsible for the heterogeneous environment at large scale in terms of nutrient concentration and $\mathrm{pH}$ profile, resulting in lower product yields. These have been associated with, inadequate control of $\mathrm{pH}$ and the production of acetate or formate in response to over-feeding of glucose and oxygen deficiency. Rapid analyses of substrates and indicator metabolites in a fermentation process is critical for optimal control. This can be achieved in real time with NIR spectroscopy. In this study, NIR-spectroscopy has been applied to monitor the concentrations of glucose, acetate, formate, ammonium hydroxide and biomass in the cultivation of E. coli (W3110). A comparison of partial least square models built using water standards, synthetic medium standards and fermentation samples has been made.

\section{BE40}

Template refolding utilizing biospecific interactions Shigeo Katoh ${ }^{1}$, Yoichi Kumada ${ }^{1}$, Nanae Maeshima ${ }^{1}$, Daisuke Nohara ${ }^{2}:{ }^{1}$ Graduate School of Science and TechnologyKobe University, Kobe 657-8501, Japan; ${ }^{2}$ Department of Biomolecular ScienceGifu University, Gifu 501-1193, Japan. E-mail: katoh@kobe-u.ac.jp (S. Katoh)

Recombinant proteins over-expressed in E. coli are often accumulated as insoluble particles called inclusion bodies. Proteins in inclusion bodies must be solubilized by a denaturing agent, such as urea and guanidine hydrochloride, and refolded to recover their native structures having biological activities. In bioprocesses it is important to obtain high refolding efficiencies and high throughputs at high protein concentrations. In refolding operation, a denatured protein solution is usually added batch-wise into a large volume of a refolding buffer in order to start refolding by reducing the concentration of a denaturant and to prevent aggregate formation of renaturing molecules. Thus, a large volume of a stirred tank is required, and the concentration of protein after renaturation becomes low. Biointeractions between a pair of biomolecules, such as enzyme-inhibitor, antigen-antibody and hormone-receptor, are highly specific and have been used for detection and separation of biomolecules. These interactions may be used as templates for refolding of target molecules, which can be captured with the templates and are prevented from aggregate formation and, in the case of proteases, from autoproteolysis. The specific interaction might promote refolding of the target molecules. These might improve the refolding efficiency. The biointeractions between antigen-antibody and enzyme-inhibitor were used for efficient refolding in packed columns, in which template ligands (antibody, inhibitor) were coupled on gel support. Denatured solutions of target molecules (carbonic anhydrase and S. griseus trypsin) were mixed with refolding buffer and supplied to the affinity column coupled with the template ligands for refolding. With refolding in the column, higher refolding efficiencies were obtained than those by the batch dilution method with relatively low concentrations of denaturants. By increasing the adsorption capacity of the column, throughput of refolding can be increased without decrease in the refolding efficiency.

\section{BE41}

The use of multi-parameter flow cytometry for characterisation and monitoring of insect cell-baculovirus fermentations in a mechanically-agitated bioreactor Bojan Isailovic ${ }^{1}$, Alvin $W$. Nienow $^{1}$, Ian W. Taylor ${ }^{2}$, Ryan Hicks ${ }^{2}$, Christopher J. Hewitt ${ }^{1}$ : ${ }^{1}$ Biochemical Engineering, Centre for Formulation Engineering, School of Engineering (Chemical Engineering), The University of Birmingham, Edgbaston, B15 2TT, UK; ${ }^{2}$ AstraZeneca, Mereside, Alderley Park, Macclesfield, Cheshire SK10 4TG, UK. E-mail: bxi897@bham.ac.uk (B. Isailovic)

Bacteria and mammalian cells have been traditionally used as hosts for commercial recombinant protein production. However, in recent years, the insect cell-baculovirus system has emerged as a potentially attractive recombinant protein expression vehicle. Although flow cytometry has been used widely for analysis of mammalian and microbial cells, there is very little information on applications of this powerful technique in insect cell culture. Here we compared cell ratiometric counts and viability (propidium iodide and calcein AM) of Sf- 21 cell cultures using flow cytometry to those determined by more traditional methods using a haemocytometer and the trypan-blue exclusion dye. Flow cytometry has also been used to monitor various parameters during cultures of Sf21 infected with the recombinant Autographa californica Nuclear Polyhedrosis Virus (AcNPV) containing the inserted nucleic acid sequence amFP486 coding for AM-Cyan coral protein, which emits natural green fluorescence.

\section{BE42}

Carbon sources create fingerprint fermentation characteristics Pınar Çalık ${ }^{2}$, Güzide Çalık ${ }^{1}$, Tunçer H. Özdamar ${ }^{1}{ }^{1}$ BRE Lab, Department of Chemical Engineering, Ankara University, 06100 Ankara, Turkey; ${ }^{2}$ IB Lab, Department of Chemical Engineering, Metu, 06531 Ankara, Turkey. E-mail: calik@eng.ankara.edu.tr (G. Çalık)

This work reports on a systematic investigation of the interactions between the single-carbon sources, i.e., glucose and citric acid, and complex-medium components, i.e., carbon and nitrogen sources, in enzyme fermentation processes, i.e., serine alkaline protease (SAP; EC 3.4.21.62), and $\beta$-lactamase (EC 3.5.2.6), with the oxygentransfer and $\mathrm{pH}$ conditions to demonstrate the influences of carbon sources that create the fingerprint fermentation characteristics, moreover, their influences on the product and by-product formations and the intracellular reaction rates. The influence of the medium composition i.e. citric acid-, glucose-, molasses- and soybean-based media together with the oxygen transfer (OT)- and $\mathrm{pH}$ - conditions applied, on the product and by-product distributions and OT characteristics were investigated in batch bioreactors. For SAP, in general, under uncontrolled-pH operations the variation of the medium $\mathrm{pH}$ in SAP 
fermentation process has a tendency to increase in the SAP production phase; however, depending on the carbon source used, its behaviour changes in the early stages of the fermentation as the consequences of the directed functioning of the intracellular bioreaction network. The loci of the dissolved oxygen (DO) curves also strongly depend on the carbon source(s) utilised, in addition to the applied OT conditions. The complex media profiles are significantly different compared to the defined media as the $\mathrm{pH}$ and DO profiles are interrelated owing to the bioreactor operation conditions affecting the metabolic reaction network. The highest volumetric oxygen uptake rates were obtained with soybean-based medium that was ca. three-fold higher than the values reported in citrate-based and glucose based media, and ca. 1.5-2-fold higher than the values reported in molasses-based medium. The significant changes, moreover, the drastic change observed with the use of soybean-based complex medium are due to the compositions of the fermentation media used, and its influence on the intracellular bioreaction network. Thus, we conclude that the change in medium composition based on the carbon source changes the fermentation characteristics under the designed bioreactor operation conditions that appear as the fingerprints of the bioprocess.

\section{BE43}

Kinetic resolution of racemic benzoin with different lyophilized microorganisms Ç. Babaarslan ${ }^{1}, \ddot{U}$. Mehmetoğlu ${ }^{1}$, A.S. Demir ${ }^{2}$ : ${ }^{1}$ Ankara University, Faculty of Engineering, Department of Chemical Engineering, 06100, Ankara, Turkey; ${ }^{2}$ Middle East Technical University, Department of Chemistry, 06531 Ankara, Turkey. E-mail: barslan@eng.ankara.edu.tr (Ç. Babaarslan)

The biocatalytic resolution of racemates is valuable tool for enantioselective synthesis and proved to be a convenient method for obtaining optically enriched compounds from their racemic form. In this work, enantiomerically pure benzoin which is one of the 2-hydroxy ketones was synthesized by kinetic resolution of racemic benzoin using different lyophilized microorganisms as lipase sources. The effect of lyophilized microorganism type, solvent type and acyl donor type on enantioselectivity were studied. In kinetic resolution experiments, lyophilized microorganism was resuspended in the media containing solvent, racemic benzoin and acyl donor at $30^{\circ} \mathrm{C}$ and $150 \mathrm{rpm}$ on orbital shaker. The reaction was followed by TLC during the experiment and the enantiomeric ratio of benzoin was determined by HPLC analysis using Chiral cell OB column. The best enantioselectivity value was obtained with lyophilized Rhizopus orayzae CBS 112-07 as $\mathrm{ee}_{\mathrm{s}}=16 \%$ and $\mathrm{ee}_{\mathrm{p}}=30 \%$ (conversion $=35 \%$ ) using THF as solvent and vinyl acetate as acyl donor.

\section{Acknowledgements}

The authors gratefully acknowledge the financial supports given to this work by Ankara University, Biotechnology Institute (Project No. 2001K120240-40) and the microorganisms given to this work by Prof. Dr. Francesco MOLINARI at University of Milano, and Prof. Dr. Leyla AÇIKEL at Gazi University.

\section{BE44}

Otimizing the fermentation broth for tanase production by a new isolated strain Paecilomyces variotii Vania Battestin, Gláucia Pastore, Gabriela Macedo Department of Food Science, UNICAMP, P.O. Box 6121, Campinas, CEP 13083-862, São Paulo, Brazil

Tannase is an inducible enzyme that catalyses the breakdown of ester linkages in hydrolysable tannins, resulting in gallic acid and glucose. The fermentation broth can use by-products as wheat bran, rice or oats, adding tannic acid. The use of by products or residues rich in carbon source for fermentation purposes an alternative to solve pollution problems that can be caused by an incorrect environmental disposal. In the present study we have optimized the production of an extracellular tannase by a new isolated Paecilomyces variotii using response surface methodology. The first step was identify the variables having a significant effect on enzyme production. The variables evaluated were temperature, residues ratio (coffe: wheat bran), concentration of tannic acid, salt solution during 3, 5 and 7 days of fermentation time. Results showed that temperature, residues ratio (coffe: wheat bran) and tannic acid had significant effects on tannase production. Commercial wheat bran (CWB) and coffe rusk residues $(\mathrm{CR})$ were used as solid substrate. For fermentation the mediun was composed by, CWB:CR were mixed with distilled water and transferred into $250 \mathrm{~mL}$ capacity Erlenmeyers flasks and autoclaved at $120^{\circ} \mathrm{C}$ for $20 \mathrm{~min}$. The medium was then inoculated with spores $\left(5.0 \times 10^{7}\right)$ and the flaks were incubated at $32^{\circ} \mathrm{C}$. Tannase was assayed according to the methodology of Mondal et al. (2001). Acording to the statist analyses, the optimum conditions to produce tannase was the range of temperature $\left(29-34{ }^{\circ} \mathrm{C}\right)$; tannic acid (8.5-14\%); residues \% (coffe: wheat bran) (50:50) and 5 days fermentation time. The enzyme production increased 8.6 times more enzyme production than that was obtained before this optimization.

\section{BE45}

How to cope with FDA's PAT-initiative with respect to fermentation process monitoring and control Marco Jenzsch ${ }^{1}$, Andreas Luebbert ${ }^{1}$, Rimvydas Simutis ${ }^{2}$ : ${ }^{1}$ Institute of Bioengineering, Martin-Luther-University Halle-Wittenberg, Halle (Saale), Germany; ${ }^{2}$ Process Control Department, Kaunas University of Technology, Kaunas, Lithuania

With its PAT initiative, FDA forces drug manufacturers to increase their activities in innovative manufacturing techniques, and, more than previously, to focus on quality assurance. The agency particularly places emphasis on making use of modern process supervision and control techniques such as up-to-date process analytics, multivariate data acquisition and analysis tools in order to improve process monitoring and control. In this contribution we show by means of practical examples how this guidance can be applied to cultivations of genetically modified microorganisms. A comparison of different multivariate state estimation techniques will be presented and compared with more knowledge-based techniques such as the Extended Kalman Filter. The comparison was made for the model system GFP expressed from E. coli bacteria (BL21/DE3/GFP) for which more than 40 full data sets are available. All these techniques have already been used during real protein formation at productionscale fermenters, with the same success. Hence, the requirements expressed in the PAT initiative can immediately be put into practice. Feedback control of the recombinant protein production processes 
based on such estimations is show for several cultivation systems. Simple parameter adaptive controllers are compared with model supported controllers, for instance, generic model controllers and model predictive controllers. The results clearly show that we have at hand a rather extended arsenal of feedback control procedures that can be used successfully to tightly control the processes even along set-point profiles of physiological variables such as the specific growth rate $(\mu)$. Again, FDA's suggestion with respect to "control in the engineering sense" can be applied immediately to reduce batch-to-batch variances and thus to increase process quality.

\section{BE46}

Extending life by alternative respiration? Alexander Kern, Franz Hartner, Anton Glieder Institute of Biotechnology, Graz University of Technology, A- 8010 Graz, Austria. E-mail: a.kern@tugraz.at (A. Kern)

Alternative oxidase transfers electrons directly from the ubiquinol pool in mitochondria to oxygen, allowing cell respiration in presence of complexs III and IV inhibitors like antimycin A or cyanide. Electron transfer by alternative oxidase is not coupled with proton transfer across the mitochondrial membrane, thereby uncoupling the supply of small metabolic intermediates by the central metabolic pathway from energy production in the cell. Alternative oxidase is present in mitochondria of plants, many fungi and a few, mostly Crabtree-negative yeasts, but not in P. angusta (Hansenula polymorpha) and $S$. cerevisiae. Alternative oxidase has multiple functions in different organisms. It is involved in stress answers, in programmed cell death, maintenance of the cellular redox balance, and also citric acid accumulation in A. niger. We isolated the alternative oxidase gene from the methylotrophic yeast $P$. pastoris in order to study its effects on the cellular energy content, respiratory activity, its protective role against oxidative stress. Our results indicate the importance of an exact regulation of the alternative oxidase due to its impact on many cellular functions.

\section{BE47}

Study of liquid mixing in a pilot scale U-loop fermenter $L$. Soland $^{1}$, A.D. Jensen ${ }^{2}$, M. Nordkvist ${ }^{1}$, M. Michelsen ${ }^{2}$, J. Villadsen $^{3}$, T. J. Hobley ${ }^{1}$ : ${ }^{1}$ Centre for Microbial Biotechnology, BioCentrumDTU, Technical University Denmark, 2800 Lyngby, Denmark; ${ }^{2}$ Department of Chemical Engineering, Technical University Denmark, 2800 Lyngby, Denmark; ${ }^{3}$ Centre for Biochemical Engineering, Technical University Denmark, 2800 Lyngby, Denmark. E-mail: th@biocentrum.dtu.dk (T. Hobley)

New types of energy efficient fermenters with better mass transport, mixing and cooling properties than the current crop of Rushton turbine derived tank bioreactors are likely to be required in the future. Such fermenters will be needed in order to meet the increasing pressure on costs for low price commodity type products such as single cell protein or food and technical grade enzymes, and to meet the demands of the new wave of white biotech, in which bio-produced chemicals must be made at prices competitive with those of the traditional chemical industry. With this in mind, a prototype pilot scale $(500 \mathrm{~L})$ U-loop fermenter has recently been commissioned at BioCentrum-DTU. In this fermenter, liquid circulation is driven by a propeller pump through a vertical U-shaped pipe, which is connected at the top with a de-gassing tank. We present here a study of liquid mixing and dispersion in the prototype U-loop fermenter. Sub- sequently we show that the results can be described with the tanks in series model. Mixing was characterised using pulses of $\mathrm{NaCl}$ tracer, which were detected with a conductivity probe in various parts of the fermenter. Bodenstein numbers $(\mathrm{Bo})$ were determined for flow rates corresponding to a linear fluid velocity of $1.05 \mathrm{~m} / \mathrm{s}$ in the 'legs' of the reactor and showed that the majority of the mixing occurred in the top degassing part $(B o=29)$ rather than in the U-loop section $(B o=422)$. It was also observed that the time for mixing to $90 \%$ homogeneity after tracer pulse addition was a function of the number of cycles through the reactor (3-3.5) within the range of flow velocities $(u)$ studied $(u=0.41 \mathrm{~m} / \mathrm{s}$ to $u=1.74 \mathrm{~m} / \mathrm{s})$. The mixing time to $90 \%$ homogeneity was between $44.6 \mathrm{~s}$ (at $u=1.74 \mathrm{~m} / \mathrm{s}$ ) and $181 \mathrm{~s}$ (at $u=0.41 \mathrm{~m} / \mathrm{s}$ ).

\section{BE48}

Multiwavelength fluorescence in combination with chemometry as an on-line tool for monitoring of filamentous fungi cultivations Anna Eliasson Lantz ${ }^{1}$, Martin Haack ${ }^{1}$, Peter P. Mortensen ${ }^{2}$, Lisbeth Olsson ${ }^{1}:{ }^{1}$ Center for Microbial Biotechnology, BioCentrumDTU, DK-2800 Kgs. Lyngby, Denmark; ${ }^{2}$ Novozymes A/S, Process Support Laboratories, DK-4400 Kalundborg, Denmark. E-mail: ael@biocentrum.dtu.dk (A.E. Lantz)

Today many biotechnological processes are operated at suboptimal conditions and according to best practice. However, the current industrial development is towards analyzing more parameters and in particular there is a large interest in analysis of biological/biochemical variables. The quality of products and also the possibility to optimize production in submerged cultivations would be greatly enhanced if more on-line/real-time information were at hand. The present investigation was undertaken with the aim of evaluating the potential in using multi-wavelength fluorescence for monitoring and control of filamentous fungi fed-batch cultivations. A recombinant $A$. oryzae expressing a heterologous lipase was applied as model system. Spectra of multi-wavelength fluorescence were collected every five minutes with the BioView ${ }^{\circledR}$ system (DELTA, Denmark) and both explorative and predictive models, correlating the fluorescence data with the important biological parameters cell mass and lipase activity, were built. The models will be presented, furthermore, advantages and disadvantages of multiwavelength fluorescence for monitoring of cultivation processes will be discussed.

\section{BE49}

Moving from $R \& D$ to pharmaceutical development is a costly process. It is therefore of paramount importance to design a manufacturing process that combines robust and well-documented technological platforms. Therapeutic recombinant proteins designed for human administration should be as close to the authentic product as possible. Here, the use of a scalable process and an economically sound affinity tag can be a relevant choice. The TAGZyme ${ }^{\mathrm{TM}}$ system has been designed to allow for the precise removal of amino terminal affinity tags. The system is based on the use of recombinant aminopeptidases including dipeptidyl peptidase I (DAPase ${ }^{\mathrm{TM}}$ ). $\mathrm{DAPase}^{\mathrm{TM}}$ is currently produced under cGMP providing a suitable strategy for its use in pharmaceutical production. The TAGZyme ${ }^{\mathrm{TM}}$ system is superior to other methods since:

(1) It is based on exopeptidases, precluding, e.g., unspecific protein cleavage reported when using so-called site-specific endoproteases. 
(2) It has been tested for production of more than 200 recombinant proteins.

(3) It is easily scalable from lab scale to $\mathrm{kg}$ of processed protein.

(4) It allows the use of His-tags for commercial production without patent infringment, due to our IPR position.

(5) The commercial use of TAGZyme ${ }^{\mathrm{TM}}$ does not require any licensing, only purchase of the enzyme(s).

(6) The use of aminopeptidases for pharmaceutical production has been extensively documented for approved drugs.

(7) A number of therapeutics is currently being developed using TAGZyme ${ }^{\mathrm{TM}}$.

(8) Unizyme can assist in the optimization of the DSP to enable further cost reduction in the process.

These aspects will be discussed and illustrated in the presented poster.

Website: http://www.unizyme.com

BE50

Metabolic control analysis and metabolic pathway analysis of sphingolipid metabolism for anticancer drug target identification F. Betül Kavun, Kutlu Ö. Ülgen Dept. of Chem. Eng., Bogazici University, 34342 Bebek, Istanbul, Turkey. E-mail: betul.kavun@boun.edu.tr (F. Betül Kavun)

Sphingolipids are biologically active molecules involved in the regulation of a large quantity of biological responses. They function in cell proliferation, survival and death (apoptosis) as messengers. Dysregulation of apoptosis has significance in numerous pathological conditions including cancer. Several anticancer agents act by increasing tumor cell ceramide (a kind of sphingolipid) content. So, a novel approach to cancer therapy would be the pharmacological manipulation of sphingolipid metabolism. In this study, sphingolipid metabolism in baker's yeast $S$. cerevisiae is used as a model system as many of its sphingolipid related genes and proteins have been characterized. Gepasi-biochemical kinetics simulator was used for metabolic control analysis (MCA) of the above-specified system. The concentration control coefficients (CCC), flux control coefficients (FCC) and elasticity coefficients were calculated, and their significance in identification of anticancer drug targets is determined. Elementary flux modes were also identified and metabolic pathway analysis (MPA) was performed. Quantitatively, control effective flux (CEF) values were used for potential drug target identification. The results from MCA and MPA indicate almost the same potential drug targets: serine palmitoyl transferase, ceramide synthase and ceramidase. Drugs against these targets are in preclinical and clinical development. For the identification of new potential drug targets, the CCCs, FCCs, CEFs and elasticity coefficients were examined with an objective function of maximizing the cell ceramide concentrations. It was found that manipulation of inositol-1-phosphate synthase and phosphoinositide kinase activities have considerable effects on ceramide concentrations. If a drug targeting the two enzymes at the same time is designed, it might give a better outcome in terms of cancer therapy.

\section{BE51}

Simulation of gluconic acid fermentation in an airlift bioreactor M. Jurašč í, I.Sikula, J. Markoš Department of Chemical and Biochemical Engineering, Faculty of Chemical and Food Technology, Slovak University of Technology, Bratislava, Radlinského 9, 812 37, Slovakia. E-mail: jozef.markos@stuba.sk (M. Juraščík)

In recent years, there is a growing interest in utilization of airlift reactors (ALRs) to biotechnological processes. Nevertheless, their industrial application still remains limited because of a lack of reliable studies on transfer phenomena and mixing enabling a suggestion of suitable scale-up procedure. The way to more widely utilization of ALRs to biological processes lies in experimental research (on a model medium as well as on a real fermentation medium) followed by mathematical modelling and scaling-up of the processes. This paper deals with a modelling of a glucose-gluconic acid fermentation by A. niger in an internal loop airlift reactor. Knowledge of the stoichiometric relationship in the key reaction provides a good opportunity for estimation of substrate and product concentration. The model is based on material balance equations and has been adjusted to experimental data obtained from three internal loop airlift reactors $(10.5,40$ and $200 \mathrm{~L})$. In the model, the ALR is divided into ideal stirred tanks in series. In each zone (tank) of the ALR the material balance is calculated in two phases (the gas and the liquid phase).

\section{Acknowledgement}

This work was supported by the Slovak Scientific Grand Agency, grant number VEGA 1/0066/03

\section{BE52}

Thermal inactivation of cow's and goat's alkaline phosphatase in milk and buffer solution Alina Wilińska, Jolanta Bryjak, Viera Illeová, Milan Polakovič Department of Chemical and Biochemical Engineering, Slovak University of Technology, Bratislava 812 37, Slovak Republic. E-mail: alina.wilinska@stuba.sk (A. Wilińska)

Alkaline phosphatase (AP, E.C. 3.1.3.1) is a thermolabile enzyme which is indigenous to all dairy products. It has an inactivation temperature slightly above the value that is required to destroy the most resistant pathogenic microorganism likely to be found in milk. Due to that feature, this enzyme is used as an indicator of proper pasteurization. The effect of temperature treatment on the activity of AP was investigated in raw cow's and goat's milk. The stability of alkaline phosphatase in raw milk was compared with the stability of this enzyme in a $0.1 \mathrm{M}$ potassium phosphate buffer with $\mathrm{pH}$ 6.6. The $\mathrm{pH}$ value of the buffer was approximately the same as that of raw milk. The inactivation curves were measured in the temperature range from 54 to $69^{\circ} \mathrm{C}$. AP in cow's milk was completely inactivated at $69{ }^{\circ} \mathrm{C}$ during $60 \mathrm{~s}$ but approximately $30 \%$ of activity remained at $54^{\circ} \mathrm{C}$ after $100 \mathrm{~min}$ of treatment. The time required for a complete inactivation of the enzyme in the raw cow's milk was reduced from 90 to $1 \mathrm{~min}$ as the temperature increased by $11^{\circ} \mathrm{C}$. Heat treatment of goat's milk caused the decrease of activity of the enzyme in the same temperature range as in the case of cow's milk. The increase of temperature from 58 to $68^{\circ} \mathrm{C}$ reduced the inactivation time from $35 \mathrm{~min}$ to $40 \mathrm{~s}$. The study of thermal stability of the alkaline phosphatase in the buffer solution showed that the time required for inactivation of enzyme was significantly shorter than in milk. Milk thus had a protective effect on the activity of alkaline phosphatase. The experi- 
mental curves were fitted simultaneously using kinetic models where the initial heating period was considered.

\section{Acknowledgements \\ This work was supported by a grant of 6th Framework Program of EU, Project FOODPRO, No. SME-2003-1-508374.}

\section{BE53}

Kinetics of binding and adsorption capacity of proteins on the resins designated for separation of monoclonal antibodies Michal Gramblička, Darina Tóthová, Monika Antošová, Milan Polakovič* Department of Chemical and Biochemical Engineering, Slovak University of Technology, Radlinského 9, 812 37 Bratislava, Slovakia. *E-mail: milan.polakovic@stuba.sk (M. Polakovič), michal.gramblicka@stuba.sk (M. Gramblička)

During the process of separation, purification and concentration of monoclonal antibodies (MAbs) at industrial scale, the chromatographic unit operations have an important role. Three different protein-binding modes are employed: ion-exchange, hydrophobic and affinity binding. Two adsorbent properties are of uppermost importance: a high selectivity and adsorption capacity. In the case of ion-exchange/hydrophobic chromatography, the binding of charged proteins can be affected by $\mathrm{pH}$ and ionic strength. In this work, the adsorption capacity of eight commercially available adsorbents designed for separation of MAbs (MabSelect, rProtein A Sepharose FF, Poros 50A, ProSep-vA, FractoGel EMD SE Hicap (M), SP Sepharose FF, MEP HyperCel, S Ceramic HyperD F) was measured as a function of $\mathrm{pH}$. As a model MAb and contaminant proteins, human immunoglobulin ( $\operatorname{IgG})$, human serum albumin (HSA) and horse skeletal muscle myoglobin (MYO) were used. The resin properties were investigated within the range of $\mathrm{pH} 4-8$. The experiments were conducted in a batch-mode, individually for each model protein. The results showed that ion-exchange and hydrophobic resins provided the best selectivity for $\mathrm{IgG}$ at $\mathrm{pH} 6$. The selectivity of affinity adsorbents was essentially unaffected by $\mathrm{pH}$, however, the highest capacity for $\mathrm{IgG}$ was at $\mathrm{pH}$ 7. Another investigated aspect was the dynamics of protein binding. The solution of individual protein in contact with tested adsorbent was circulated through an UV spectrophotometer, what enabled the measurement of time-dependent decrease of protein concentration. The results indicated that affinity adsorbents with a rigid matrix needed approximately four times shorter time to reach the adsorption equilibrium with IgG in comparison with a gel. The gels, however, provided higher adsorption capacity. At ion-exchange resins, the time necessary to adsorb $99 \%$ of total amount of $\mathrm{IgG}$ was about $0.5-2 \mathrm{~h}$. The affinity adsorbents were highly selective and therefore they adsorb very small amount of tested contaminant proteins (HSA, MYO). The adsorption capacity was saturated by $50 \%$ in less than $25 \mathrm{~min}$ in all cases of dynamic adsorption measurements.

\section{Acknowledgement}

This work was supported by a grant of 6th Framework Program of EU, Project AIMs, No. NMP3-CT-2004-500160.

\section{BE54}

Development of a microreactor for enzymatic processes Malene S. Thomsen, Bernd Nidetzky Research Centre Applied Biocatalysis, and Institute of Biotechnology and Biochemical Engineering, Graz University of Technology, Graz A-8010, Austria. E-mail: malene.thomsen@a-b.at (M.S. Thomsen)

Microtechnology has for several years been applied within chemical reaction engineering. The advantages of microtechnology are that it makes it possible to develop light weight and compact systems, and the systems enable large surface-to-volume ratio, which results in low mass-transfer distances. In addition, parameters like pressure, temperature, residence time, and flow rate are more easily controlled. The use of microtechnology is also beginning to find its ways into the field of biotechnology. What we are aiming at is the development of a microreactor that can be applied as a production tool in industry as an alternative to conventional enzymatic reactors. Our strategy is to use a small plate of a suitable material with microchannels fabricated into its surface, and the approach is to covalently couple enzymes into the microchannels. Substrate can then be pumped through the channels and the enzymatic conversion will take place within the channels. As model enzyme in the development of the microreactor we are applying CelB, a thermostable $\beta$-glycosidase from Pyrococcus furiosus.

\section{BE55}

Kinetic resolution of racemic benzoin with different lyophilized microorganisms C. Babaarslan $^{1}, \ddot{U}$. Mehmetoğlu ${ }^{1}$, A.S. Demir ${ }^{2}$ : ${ }^{1}$ Ankara University, Faculty of Engineering, Department of Chemical Engineering, 06100, Ankara, Turkey; ${ }^{2}$ Middle East Technical University, Department of Chemistry, 06531, Ankara, Turkey. Email: barslan@eng.ankara.edu.tr (Ç. Babaarslan)

The biocatalytic resolution of racemates is valuable tool for enantioselective synthesis and proved to be a convenient method for obtaining optically enriched compounds from their racemic form. In this work, enantiomerically pure benzoin which is one of the 2-hydroxy ketones was synthesized by kinetic resolution of racemic benzoin using different lyophilized microorganisms as lipase sources. The effect of lyophilized microorganism type, solvent type and acyl donor type on enantioselectivity were studied. In kinetic resolution experiments, lyophilized microorganism was resuspended in the media containing solvent, racemic benzoin and acyl donor at $30^{\circ} \mathrm{C}$ and $150 \mathrm{rpm}$ on orbital shaker. The reaction was followed by TLC during the experiment and the enantiomeric ratio of benzoin was determined by HPLC analysis using Chiral cell OB column. The best enantioselectivity value was obtained with lyophilized Rhizopus

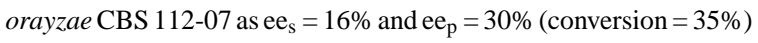
using THF as solvent and vinyl acetate as acyl donor.

\section{Acknowledgements}

The authors gratefully acknowledge the financial supports given to this work by Ankara University, Biotechnology Institute (Project No 2001K120240-40) and the microorganisms given to this work by Prof. Dr. Francesco MOLINARI at University of Milano, and Prof. Dr. Leyla AÇIKEL at Gazi University. 


\section{BE56}

Investigation of pore accessibility of adsorbents for separation of monoclonal antibodies Michal Gramblička, Ivana Tatárová, Monika Antošová, Milan Polakovič* Department of Chemical and Biochemical Engineering, Slovak University of Technology, Radlinského 9, 81237 Bratislava, Slovakia. E-mail: milan.polakovic@stuba.sk (M. Polakovič), michal.gramblicka@stuba.sk (M. Gramblička)

Chromatography is one of the most important unit operations at separation, purification and concentration of monoclonal antibodies (MAbs) at industrial scale. Since these proteins belong to the group of immunoglobulins, their molecular weight (about $150,000 \mathrm{~g} / \mathrm{mol}$ ) or hydrodynamic radius $(10.7 \mathrm{~nm})$, respectively, is relatively large. The adsorbents used in ion-exchange/affinity chromatography of these biomolecules should thus provide a high pore accessibility coupled with a high value of specific surface area in order to ensure a sufficient ligand density and a high binding capacity. In this study, the pore accessibility of eight commercially available adsorbents designed for separation of MAbs (MabSelect, rProtein A Sepharose FF, Poros 50A, ProSep -vA, FractoGel EMD SE Hicap (M), SP Sepharose FF, MEP HyperCel, S Ceramic HyperD F) was investigated via size exclusion of standard-sized molecules (glucose, sucrose and dextrans with molar weight range $1200-40 \times 10^{6} \mathrm{~g} / \mathrm{mol}$ ) at nonbinding conditions. The experiments were conducted in a batch and column-mode. The batch experiments provided absolute partition coefficients, which were calculated from a mass balance and represent the fraction of pore water accessible to a solute. It was found that several adsorbents contained a small fraction of very small pores (less than $1 \mathrm{~nm}$ ), whereas some adsorbents contained a significant fraction of pores larger than $100 \mathrm{~nm}$. The column measurements provided relative partition coefficients, which were calculated from the retention volumes of solutes and represented the relative accessibility of pores, scaled between the accessibility of the smallest and largest solute used. When the absolute partition coefficients were recalculated into the relative form, it was found that the coefficients obtained by both methods correlated very well. The relative partition coefficients of solutes with the hydrodynamic radius of $10 \mathrm{~nm}$ (corresponding to MAbs) was about $0.2-0.4$ at ion-exchange and hydrophobic adsorbents and 0.6-0.8 at affinity adsorbents. The relation between hydrodynamic radius of the solutes and their partition coefficients was successfully described with the Giddings random plane model.

\section{Acknowledgement}

This work was supported by a grant of 6th Framework Program of EU, Project AIMs, No. NMP3-CT-2004-500160.

\section{BE57}

Production of fructosyltransferase in laboratory, semi-pilot stirred-tank and air-lift bioreactors $\underline{M}$. Vandáková, M. Juraščík, M. Polakovič Department of Chemical and Biochemical Engineering, Faculty of Chemical and Food Technology, Slovak University of Technology, Bratislava, Radlinského 9, Slovak Republic 81237. E-mail: marcela.vandakova@ stuba.sk (M. Vandáková)

The transfer of laboratory results to a larger scale is often a critical step in process development to industrial application. The objective of this study was to scale-up the bioreactor for the fructosyltransferase production based on the results obtained in a $3 \mathrm{dm}^{3}$ stirred bioreactor. The investigations made in this bioreactor provided a clear picture of the effect of medium composition on the obtained fructosyltransferase (Ftase) activity but the influence of mixing intensity was unequivocal. The increase of agitation rate had a positive effect up to a certain level where both fructosyltransferase and biomass production increased. The final optimal yield factor of Ftase per dry cell mass obtained in the laboratory bioreactor was $10,300 \mathrm{U} \mathrm{g}^{-1}$. We studied the effect of oxygen transfer on the process of Ftase production at a larger scale, in 12 and $100 \mathrm{dm}^{3}$ mechanically stirred bioreactors and in air-lift bioreactors, 20 and $60 \mathrm{dm}^{3}$ whilst the medium composition was kept constant. The yield factors of Ftase were comparable in both mechanically stirred bioreactors and they were about $7500 \mathrm{Ug}^{-1}$. This decrease compared to that in the laboratory bioreactor could be explained by a slower cell growth. This fact was also confirmed by that glucose was not depleted till the end of fermentation and free fructose concentration was also lower. The yield factor of Ftase was $7400 \mathrm{U} \mathrm{g}^{-1}$ in the $60 \mathrm{dm}^{3}$ air-lift reactor and $6100 \mathrm{U} \mathrm{g}^{-1}$ in the $20 \mathrm{dm}^{3}$ air-lift bioreactor. The lower yield of Ftase in $20 \mathrm{dm}^{3}$ bioreactor was caused by a better biomass growth.

\section{Acknowledgements}

This work was supported by Slovak Scientific Grand Agency, grant numbers VEGA 1/0066/03 and 1/0065/03 and by Science and Technology Assistance Agency, grant number APVT-20-025704.

\section{BE58}

Direct bioproduct isolation from crude feedstocks using magnetic micro adsorbents Matthias Franzreb ${ }^{1}$, Niklas Ebner ${ }^{1}$, Martin Siemann $^{2}$, Josef Altenbuchner ${ }^{2}$, Christina Fritz ${ }^{2}$, Zsuzsana Mayer ${ }^{2}$, Nadja Schultz ${ }^{3}$, Christoph Syldakt ${ }^{3}:{ }^{1}$ Institute of Technical Chemistry, Forschungszentrum Karlsruhe, Hermann-von-Helmholtz-Platz 1, 76344 Eggenstein-Leopoldshafen, Germany; ${ }^{2}$ Institute of Biochemical Engineering and Institute of Industrial Genetics, University of Stuttgart, Allmandring 31, 70569 Stuttgart, Germany; ${ }^{3}$ Chair of Technical Biology, University of Karlsruhe, Engler Bunte Ring 1, 76131 Karlsruhe, Germany. E-mail: matthias.franzreb@itcwgt.fzk.de (M. Franzreb)

The poster gives an overview of the objectives and achieved results of an interdisciplinary project on direct product isolation from crude feedstocks using magnetic micro adsorbents in combination with suitable magnet technology. The project was funded by the Deutsche Bundesstiftung Umwelt and was running between August 2002 and November 2004. In the course of the project several milestones could be met, which can be looked at as critical key points on a route towards an industrial realization of the process. Among these milestones are: (i) the production of magnetic micro adsorbents with high capacity and selectivity in batches up to $50-100 \mathrm{~g}$; (ii) the proof that the micro adsorbents can be reused many times; (iii) generation of a variety of recombinant tagged, active enzymes; and (iv) the design, assembly and operation of a fully automated pilot plant capable of generating approx. $5 \mathrm{~g} / \mathrm{h}(\approx 95 \%$ purity $)$ protein. The process was also simulated by help of the software tool SuperPro Designer and simple mass balance and sorption equilibrium approaches were used to derive rules for estimating optimum process parameters and productivities. Finally an environmental performance evaluation was conducted externally by the German DECHEMA. 


\section{BE59}

Effect of cysteine addition on glutathione metabolism in Saccharomyces cerevisiae MT-32 A.M. De Martín Barry, M.J. Guardia Alba Departamento de Fermentación, Puleva Biotech S.A., 18004, Granada, Spain. E-mails: ademartin@pulevabiotech.es (A.M. De Martín Barry), mjguardia@pulevabiotech.es (M.J. Guardia Alba)

In this study the effect of fed-batch cysteine addition to a culture of a high-GSH-accumulating yeast strain on the metabolism of glutathione was investigated. It is known that cysteine is the rate limiting amino acid in the biosynthesis of GSH. The influence of the consumption rate of cysteine on glutathione metabolism and growth of $S$. cerevisiae MT-32 was determined. The results show that for rates of consumption below a critical value the microorganism growth is similar to a culture without feed of cysteine, but glutathione production is increased two-fold. On the other hand, if cysteine consumption rate is above the critical value the changes of cell metabolism implies ethanol accumulation in the extracellular media which diminishes biomass synthesis. The maximum specific glutathione production in this case is maintained at two-fold; however, gamma-glutamylcysteine accumulation is increased. Cysteine present in culture media directs cell metabolism to a greater synthesis of ammonia and amino acids.

\section{BE60}

Hydrophobic interaction chromatography of proteins: unfolding of proteins upon adsorption Emmerich Haimer, Rainer Hahn, Alois Jungbauer Department of Biotechnology, University of Natural Resources and Applied Life Sciences, Vienna, Austria

Hydrophobic interaction chromatography (HIC) exploits the hydrophobic properties of protein surfaces for separation and purification by performing interactions with chromatographic sorbents of hydrophobic nature. In contrast to reversed phase chromatography this methodology is less detrimental to the protein and is therefore more commonly used in industrial scale as well as in bench scale when the conformational integrity of the protein is important. Hydrophobic interactions are promoted by salt and thus proteins are retained in presence of a cosmotropic salt. When proteins are injected on HIC columns with increasing salt concentrations under isocratic conditions only, a fraction of the applied amount is eluted. The higher the salt concentration the lower is the amount eluted protein. The rest can be desorbed with a buffer of low salt concentration or water. It has been proposed that the stronger retained protein fraction has partially changed the conformation upon adsorption. This has been also corroborated by physicochemical measurements. The retention data of five different model proteins and 10 different stationary phases were evaluated. Partial unfolding of proteins upon adsorption on surfaces of HIC-media were assumed and a model describing the adsorption of native and partial unfolded fraction was developed. Furthermore we hypothesize that the surface acts as catalyst for partial unfolding, since the fraction of partial unfolded protein is increasing with length of the alkyl chain.

\section{BE61}

Stationary phases for bioseparation of glycoproteins $J$. Aniulyte ${ }^{1}$, J. Liesiene ${ }^{1}$, B. Niemeyer ${ }^{2}:{ }^{1}$ Department of Chemical Technology, Kaunas University of Technology (KTU), Radvilenu pl. 19, 50254 Kaunas, Lithuania; ${ }^{2}$ Institute of Thermodynamic, Helmut-SchmidtUniversity/University of the Federal Armed Forces Hamburg, Holstenhofweg 85, Hamburg, Germany D-22043

Nowadays glycomics raise new challenges for affinity chromatography related with an abundance of glycoconjugates in living organisms and with scaling-up of the preparative processes. Economics, efficiency and practicality dictate the search of novel chromatographic biospecific adsorbents that could contribute to enhancing the productivity of the affinity separation process. The purpose of the work was to prepare cellulose- and silica-based biospecific adsorbents with immobilized lectins and to evaluate them for the affinity chromatography of glycoproteins. Cellulose-based matrix Granocel and silica coated with hydrophilic polymers were used as a support. The effect of support characteristics, such as pore size, chemistry of active groups and their density on the support' surface on lectin immobilization and on the efficiency of adsorbents obtained were evaluated.Three different methods were used for the activation of the support: oxidation with sodium periodate, modification with pentaethylenhexamine (spacer arm of 18 atoms) and carbonyldiimidazole activation.ConA and WGA two lectins of different molecular weight and shape were selected to notice differences resulting from the size and diffusion behaviour. Chromatographic performances of the adsorbents were studied applying two different glycoproteins (GOD and fetuin) carrying specific terminal glycomoieties of mannose (GOD), and $N$-acetylglucosamine (fetuin) for specific interaction with ConA and WGA, respectively. The adsorbents demonstrated high affinity to glycoproteins with a sorption capacity in the column up to $7.4 \mathrm{mg}$ per $\mathrm{mL}$ support and a high recovery (up to 93\%). It was shown that spacer arm affected ligand coupling kinetic as well as the chromatographic behavior of the adsorbents obtained. The adsorption isotherms of GOD onto ConA adsorbents reveal an adsorption behavior with high and low affinity binding sites. The dissociation constant $K_{\mathrm{d}}$ of the ligand-sorbate complex is approximately $1 \times 10^{-6} \mathrm{M}$, and $0.4 \times 10^{-5} \mathrm{M}$, respectively. It was supposed that the second step is related to the sorption of solvated GOD onto already adsorbed GOD forming sorbate dimers.

\section{BE62}

Influence of the extent of disruption of $E$. coli on the adsorption of B-galactosidase in expanded bed adsorption chromatography B. Balasundaram, S.T.L. Harrison Department of Chemical Engineering, University of Cape Town, Cape Town, RSA. E-mail: bala@chemeng.uct.ac.za (B. Balasundaram)

Cell disruption and chromatography are key unit operations in the downstream processing of an intracellular product. The cost involved in the extraction and purification of intracellular products can be reduced by selective release of proteins and reduction in the number of steps involved in the purification. The extent of disruption can be varied to provide a selective release, limiting the release of the contaminant proteins. The particle size distribution of the cell debris in the resulting suspension depends on the extent of disruption. Expanded bed adsorption chromatography allows for the direct capture of the proteins from an unclarified suspension. This technique 
allows for the integration of solid-liquid separation, concentration and preliminary purification in one unit operation. A perfectly stable expanded bed can be obtained by choosing the appropriate flow conditions and a suitable adsorbent. The difference in the density between the adsorbent and the cell debris in the suspension, permits the cell debris to flow through the column without blocking, whilst the protein molecules in the suspension are adsorbed onto the adsorbent. After sample application, the bed is washed with buffer and the proteins eluted from the column in the packed bed mode. The presence of the cell debris in the feedstock influences the expansion of the bed and the adsorption of protein molecules. The physical properties of the suspension obtained after cell disruption depends on the extent of disruption. The particle size distribution of the cell debris, the viscosity and the release of soluble proteins and other intracellular components are influenced by the extent of disruption. The influence of the extent of disruption of $E$. coli on the expansion of the bed and the adsorption of B-galactosidase is presented in the current study. E.coli cells were disrupted at different operating pressure using a high pressure homogenizer. The resulting crude homogenate is subjected to expanded bed adsorption chromatography using STREAMLINE DEAE as adsorbent. The disrupted suspension was characterised in terms of viscosity, density, particle size distribution of the cell debris and the extent of protein and B-galactosidase released. The interaction between cell debris and adsorbent was quantified as the cell transmission index (ratio of the amount of cells present in the sample before and after passing through the bed). The expansion of the bed at a constant settled bed height and flow rate was measured. The influence of the cell debris on the extent of adsorption of B-galactosidase has been quantified in terms of dynamic binding capacity (DBC) at $10 \%$ of the inlet concentration. The DBC of $\beta$-galactosidase that was released by disruption at $7500 \mathrm{psi}(5 \%$, w/v, w/w, 1 pass $)$ was found to be $100 \mathrm{U} / \mathrm{mL}$ of adsorbent while DBC of samples disrupted at $2500 \mathrm{psi}(5 \% \mathrm{w} / \mathrm{v}$, w/w, 1 pass) was $67 \mathrm{U} / \mathrm{mL}$ of adsorbent. The extent of disruption of $E$. coli over a wide range and its effect on the expansion and adsorption will be presented.

\section{BE63}

Study of DNA binding during expanded bed adsorption and factors affecting adsorbent aggregation Ayyoob Arpanaei ${ }^{1}$, Niels Mathiasen $^{1}$, Timothy Hobley ${ }^{1}$, Owen RT $\overline{\text { Thomas }^{1,2}:{ }^{1} \text { Center for }}$ Microbial Biotechnology, Building 223, BioCentrum-DTU, Technical University of Denmark, 2800, Lyngby, Denmark; ${ }^{2}$ Department of Chemical Engineering, University of Birmingham, Edgbaston, B15 2TT, UK. E-mail: aa@biocentrum.dtu.dk (A. Arpanaei)

The adsorption of sonicated calf thymus DNA (as a model DNA molecule) to BioSepra Q Hyper Z adsorbents was evaluated in batch and expanded bed modes. Stability of the expanded bed during feedstock loading was also studied. Two batches of prototype Q Hyper $\mathrm{Z}$ (batch 1 and 2) were examined, which had ionic capacities measured to be 122 and $147 \mathrm{mmol} \mathrm{Cl}^{-} / \mathrm{mL}$ support respectively. In all adsorption experiments a $50 \mathrm{mM}$ Tris- $\mathrm{HCl} \mathrm{pH} 8$ buffer was used. Maximum static binding capacities of adsorbent batches 1 and 2 were determined to be 20.9 and $23.8 \mathrm{mg} \mathrm{DNA} / \mathrm{mL}$ particle, respectively. Dynamic binding capacity at $10 \%$ breakthrough $\left(\mathrm{DBC}_{10 \%}\right)$ was measured in a $1-\mathrm{cm}$ diameter EBA column containing $6.7 \pm 0.5 \mathrm{~cm}$ settled bed with a feed of $20 \mu \mathrm{g} / \mathrm{mL}$ DNA. $\mathrm{DBC}_{10 \%}$ of the adsorbents were 7.4 and $12.7 \mathrm{mg}$ DNA/mL support for batches 1 and 2, respectively in buffer containing no salt. However, the maximum $\mathrm{DBC}_{10 \%}$ for batch 1 (10.1 mg DNA/mL support) and 2 (18.9 mg DNA/mL support) were obtained in buffers containing 0.25 and $0.35 \mathrm{M} \mathrm{NaCl}$, respectively. Further increases in salt concentration led to a decrease in $\mathrm{DBC}_{10 \%}$ for both adsorbent batches. The bed compression during loading that was observed in experiments at high conductivities (achieved by adding salt) was less than that seen with low conductivity $(2 \mathrm{mS} / \mathrm{cm})$ solutions. Aggregation of adsorbent particles and channeling of flow were not observed in the presence of salt concentrations more than $0.1 \mathrm{M}$. The effect of different concentrations of DNA during loading in the presence of $0.15 \mathrm{M} \mathrm{NaCl}$ was studied. It was found that increasing DNA concentrations in the feed from 20 to $40 \mu \mathrm{g} / \mathrm{mL}, 60$ to $80 \mu \mathrm{g} / \mathrm{mL}$ resulted in a decrease of $\mathrm{DBC}_{10 \%}$ by 16 , 24 and $30 \%$, respectively. The bed compressed slower during loading of feedstock with low DNA concentrations compared to that for higher concentrations. The expanded bed showed a partly reversible compression behavior during feedstock loading. This is attributed to the electrostatic interaction between DNA adsorbed on the particles surface and rearrangements of DNA strands as the number of free ligands on the adsorbent surfaces decrease during loading.

\section{BE64}

Optimisation of precipitation steps for the purification of plasmid DNA S.S. Freitas, J.L. Santos, D.M.F. Prazeres Centre for Biological and Chemical Engineering, Instituto Superior Técnico, Av. Rovisco Pais, 1049-001 Lisbon, Portugal. E-mail: sindelia@ist.utl.pt (S.S. Freitas)

Large-scale production of plasmid DNA for gene therapy and DNA vaccination applications has become necessary as a result of the increasing number of approved protocols using non-viral vectors for gene delivery. A major challenge of large-scale plasmid production is to establish a robust cGMP manufacturing capable of producing hundreds of milligrams or grams of a pharmaceutical grade product. Alcohol and salt precipitation are operations largely used in the early steps of plasmid downstream processes. However, there are few systematic studies on the influence of these precipitation agents in the final plasmid recovery and purity. In this work, alcohol and salt precipitation steps used in a plasmid purification process developed by our group have been optimized aiming at large-scale production. The optimization of alcohol precipitation indicated that almost $100 \%$ of the pDNA precipitated when 0.6 vol. of isopropanol were used. The studies also indicated that the precipitation profile was strongly influenced by pDNA initial concentration. Finally, the final plasmid recovery and purity after a sequential alcohol and salt precipitation were strongly dependent on the concentrations of these precipitation agents. Thus, a commitment between high recovery and purity level should be made during the development of the downstream processes.

\section{BE65}

Comparison of novel and conventional processes for protein refolding and initial purification $H$. Ferré $e^{1,2}, U$. Jorgensen ${ }^{1}$, L. Mathiasen 1, K. Lamberth $^{2}$, S. Buus ${ }^{2}$, O.R.T. Thomas ${ }^{1,3}$, T.J. Hobley ${ }^{1}$ : ${ }^{1}$ Center for Microbial Biotechnology, BioCentrumDTU, Technical University of Denmark, DK-2800, Lyngby, Denmark; ${ }^{2}$ Institute of Medical Microbiology and Immunology, University of Copenhagen, The Panum Institute, Blegdamsvej 3C, DK2200, Denmark; ${ }^{3}$ Present Address: Department of Chemical Engi- 
neering, School of Engineering, University of Birmingham, Edgbaston, Birmingham, B15 2TT, UK. E-mail: th@ biocentrum.dtu.dk (T. Hobley)

Scale down of downstream processing unit operations is convenient for assessing process alternatives, particularly if feedstock is scarce. In this study it was imperative to use the smallest possible scale for comparison of a new system for continuous protein refolding and direct expanded bed adsorption (EBA) capture with a traditional process composed of discrete operations of batch renaturation, centrifugation, microfiltration and packed bed chromatography (PBC). Minimisation of the scale was restricted by the EBA step: the smallest practical scale being a $1 \mathrm{~cm}$ diameter column with $5-6 \mathrm{~cm}$ of settled bed, expanded two fold. In order to permit a fair comparison a similar column diameter and adsorbent volume was used in the packed bed process. In both alternatives, chelating media charged with $\mathrm{Cu}^{2+}$ was used and a feedstock of denatured HAT-tagged human beta- 2 microglobulin (HAT-hß2m). Following batch refolding and clarification, the performance of the packed column was severely hampered due to fouling of the top adapter. Reducing the protein loaded to the packed bed to $50 \%$ of $\mathrm{DBC}_{\text {working }}$ lead to a recovery of $68.5 \%$ at a purity of $87 \%$ and 4.6 -fold concentration. The EBA-based process performed unimpeded and productivity was calculated to be $8 \%$ higher than for that employing a packed bed. However, due to the severe scale restrictions placed on the EBA process, which limited optimisation, significant productivity improvements of EBA over packed bed are expected at larger scale.

\section{BE66}

Advances in high-gradient magnetic fishing for downstream and bioprocessing T.J. Hobley ${ }^{1}$, H. Ferré , $^{1,2}$, C.S.G. Gomes ${ }^{1}$, D.B. Hansen $^{1}$, T.L. Petersen ${ }^{1}$, S. Buus ${ }^{2}$, O.R.T. Thomas ${ }^{1,3}:{ }^{1}$ Center for Microbial Biotechnology, BioCentrum-DTU, Technical University of Denmark, DK-2800, Kgs. Lyngby, Denmark; ${ }^{2}$ Institute of Medical Microbiology and Immunology, The Panum Institute, University of Copenhagen, Blegdamsvej 3, DK-2200, Copenhagen, Denmark; ${ }^{3}$ School of Engineering, Chemical Engineering, The University of Birmingham, Edgbaston, Birmingham, B15 2TT, UK. E-mail: th@ biocentrum.dtu.dk (T. Hobley)

High gradient magnetic filtration has the potential for rapid processing of large volumes of crude bioprocess liquors when magnetic adsorbents are employed. The binding of a protein to a superparamagnetic solid support provides a unique selective 'handle'. Typically the focus is placed on using the magnetic handle for direct capture of a protein from a fermentation broth. However, magnetic adsorbents may provide solutions to a range of downstream processing problems and in this presentation we illustrate this with a number of case studies. Using whey as a model system, we show that the extent of the tryptic hydrolysis (ca. $0.2 \mathrm{mg} / \mathrm{mL}$ added enzyme) of proteins could be controlled by adding benzamidine-linked magnetic adsorbents after a given period (4-15 $\mathrm{min})$, followed by removal of the loaded adsorbents using a magnetic filter. Hydrolysis was stopped effectively and approx. $50 \%$ of the added trypsin could be recovered. A coupled process was devised for the refolding and purification of inclusion body proteins. Solubilised (in $8 \mathrm{M}$ urea) inclusion bodies of recombinant histidine affinity tagged human beta 2 microglobulin (HAT-h-beta2m), were refolded by dilution in a pipe reactor (14 s), then captured directly on $\mathrm{Cu}$ (II) charged magnetic immobilised metal affinity adsorbents in a second pipe reactor (10 s residence time). Loaded adsorbents were retained in a magnetic filter, then washed and the protein eluted.

\section{BE67}

A generic framework for the prediction of scale-up when using compressible chromatographic packings $\underline{R . \operatorname{Tran}^{1}}{ }^{1}, J$. Joseph ${ }^{1}$, A. Sinclair ${ }^{2}, Y$. Zhou ${ }^{1}$, N. Titchener-Hooker ${ }^{1}:{ }^{1}$ Innovative Manufacturing Research Centre for Bioprocessing, Advanced Centre for Biochemical Engineering, University College London, Torrington Place, London WC1E 7JE, UK; ${ }^{2}$ Biopharm Services UK, Lancer House, East Street, Chesham, Bucks HP5 1DG, UK. E-mail: r.tran@ucl.ac.uk (R. Tran)

Packed bed chromatography is the pre-eminent technique in the downstream purification of many biological products. The aspect ratio of a packed bed has a significant effect on the column pressure drop by virtue of wall support which is reduced at low aspect ratios. This can result in unexpectedly high pressures during manufacturing caused by the compression of the matrix via drag forces due to fluid flow through the bed. The need for an accurate model to predict flow conditions at increasing scale is essential for the scaling-up of chromatographic processes and for avoiding bed compression during operation so that maximum throughput can be achieved. Several studies have generated correlations which allow for the prediction of column pressure drops but have either been mathematically complex, which makes their practical use unfeasible, or they have used highly specific empirical constants and hence require a large amount of experiments to be performed before they can be used. In this study, we have established relationships to link the critical velocity of operation, to bed height $(L)$, column diameter $\left(D_{\mathrm{c}}\right)$, feed viscosity $(\mu)$ and also to the matrix rigidity through the level of agarose cross-linking (a\%). The correlation is straight forward to use and involves very few system-specific constants thus significantly reducing the need for any preceding laboratory-scale experimentation. This paper describes the series of experiments that were performed to establish the correlation, using a range of cross-linked agarose matrices (2-10\%), at various aspect ratios, fluid flow rates and varying viscosities (0.9-1.85 MPas). A mathematical model was developed where parameter estimation for multi-variables was achieved by least squares optimisation. The model can be used to predict the extent of compression in industrial chromatography applications and will be useful in the development of chromatographic operations and for column sizing.

\section{BE68}

Towards the integration of SMB technology and an aldolasecatalysed reaction Stefan Makart, Matthias Bechtold, Sven Panke Institute of Process Engineering, Swiss Federal Institute of Technology, Zurich. E-mail: makart@ ipe.mavt.ethz.ch (S. Makart)

Simulated moving bed (SMB) technology receives increasing attention in biotechnology and in the biopharmaceutical industry as it enables an increase in productivity per unit mass of stationary phase, reduced solvent consumption, and fast and reliable scale-up. Combining continuous chromatographic separation unit and reactor should enable the production of biopharmaceuticals and fine chemicals with high purity and yield at the same time. Due to the increasing demand of enantiopure intermediates in the pharmaceutical industry, biocatalytic processes gain more and more importance 
because of their excellent enantioselectivity. Yet the application of biocatalytic carbon-carbon bond formation on process scale is often hampered by an unfavourable equilibrium position and difficult downstream processing due to substrate/product mixtures. Coupling a continuous separation unit to such a process would improve the feasibility by driving the reaction to completion and thus increasing the overall yield. We will discuss the design of such an integrated biocatalytic/SMB process, taking the formation of L-allo-threonine from glycine and acetaldehyde, catalysed by the glycine-dependent aldolase GlyA from E. coli, as a model reaction. The enzyme exhibits absolute stereoselctivity at the C-alpha atom, whereas selectivity is less strict at C-beta. In situ product removal, by the integration of an SMB unit, would aid to maintain a high diastereomeric excess as it shortens the residence time of the products in the reactor, in addition to shifting the reaction to the product side. The in-line coupling of the chromatographic unit to the enzyme reactor requires the use of the same solvents for reaction and separation, so the choice is limited to aqueous solutions close to physiological $\mathrm{pH}$, limiting in turn the possible stationary phase materials. In a screening of different cation exchangers, Amberlite CG-120 II gave promising results: threonine is more retained than glycine, acetaldehyde is poorly and the cofactor PLP is not retained. Adsorption isotherms were determined by the retention time method and a SMB under process conditions was simulated. By improving the packing of the column, i.e. achieving a more even particle size distribution, we tried to further increase the efficiency of the separation step.

\section{BE69}

Development of an integrated process for the production and isolation of a lipase from Trichosporon beigilie T. M.-D. Phan, E. M. del Amor Villa, R. Wichmann Biochemical Engineering Group, Biochemical and Chemical Engineering Department, University of Dortmund, D-44221 Dortmund, Germany. E-mail: delamor@bci.uni-dortmund.de (E.M. del Amor Villa)

The application of enzymes for the synthesis of optically active substances is nowadays of growing importance in the pharmaceutical industry. This requires a proper cultivation of the microorganism as well as a posterior isolation process yielding a constant catalyst quality at high purity. Goal of this project is the development of an integrated process for the production and isolation of a lipase from Trichosporon beigilie and its posterior application for the enantioselective synthesis of pharmaceutical products. The cultivation of the microorganism is optimised in a laboratory and pilot-scale fermenter in a fed-batch mode. Parameters like media composition, temperature, $\mathrm{pH}$ and aeration rate are set up. Taking advantage of the localisation of the enzyme (covalently attached to the cell membrane) the first step of product isolation consists of a continuous cell disruption. Optimal results are achieved with the continuous bead mill disruption process $(68 \%$ enzyme release with a specific activity of $0.8 \mathrm{U} / \mathrm{mg}$ of protein). The non disrupted cells are recycled as inoculums for a new cultivation, increasing the yield of the overall process (by 5-times in the pilot-scale fermenter). In order to isolate the product two different process sequences are considered. The first one consists of an extraction (PEG and phosphate buffer) coupled to an ion-exchange chromatography (Q-Sepharose FF). The second one applies a precipitation step with ammonia sulphate followed by a hydrophobic interaction chromatography (Sepharose-HIC) provid- ing a lipase yield of $72 \%$ (8-times higher than the one provided by combining extraction-chromatography). An ultrafiltration process is used in order to concentrate the lipase and its final properties (molecular weight, isoelectric point, activity, stability and kinetic data) are studied using p-nitrophenylacetate as model substrate. The relevance of the obtained product for its application in the pharmaceutical industry is proven by transforming $(R, S)$-naproxen-methylester into $(S)$-naproxen acid with an enantiomeric excess of $>99 \%$ (after $24 \mathrm{~h}$ ).

\section{BE70}

Development of an integrated process for the enzymatic synthesis of biotensides E. M. del Amor Villa, R. Wichmann Biochemical and Chemical Engineering Department, University of Dortmund, D44221 Dortmund, Germany. E-mail: delamor@bci.uni-dortmund.de (E.M. del Amor Villa)

Biotensides (sugar fatty acid esters, SFAEs) find nowadays a wide range of applications in pharmaceutical, personal care and food industry because of their biocompatibility, biodegradability and special surfactant properties. Goal of this project is the development and optimisation of an integrated process for the enzymatic synthesis of SFAEs from renewable sources to be used in cosmetic formulations. The following figure shows the scheme of the overall process. Commercial and also new screened lipases are applied in the reaction between sugar and fatty acid. The mixture grade of the initial reaction system is increased by ultrasounds taking into account the influence on the catalyst characteristics and also the necessity of an organic solvent as adjuvant. The reaction takes place in an enzymatic membrane reactor (EMR) equipped with an ultrafiltration membrane which retains the catalyst. The separation of the by-product (water) from the rest of the components can be achieved by means of a pervaporation unit which coupling to the EMR allows the semi-batch process. In order to separate the esters from the fatty acid a stepwise elution chromatography method is developed using silica as adsorbent and ethyl acetate and methanol as eluents. With this system $91 \%$ of the dimer is isolated with purity (HPLC) of $93 \%$. The application of a dialysis membrane technique allows the separation of $80 \%$ of the fatty acid by building ester micelles changing the polarity of the organic solvent used as eluent.

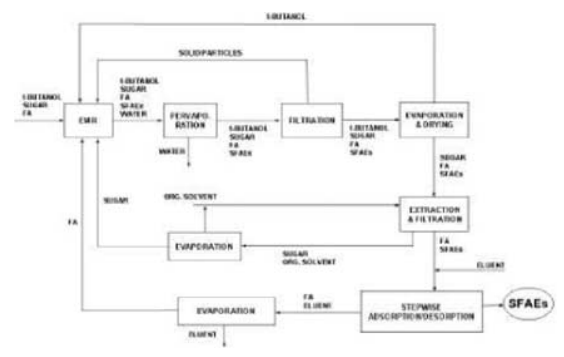

\section{BE71}

Solubility and crystallisation properties of recombinant Bacillus halmapalus $\boldsymbol{\alpha}$-amylase Cornelius Faber Centre for Microbial Biotechnology, Biocentrum DTU, Building 223, 2800 Lyngby, Denmark 
A comprehensive knowledge of solubility properties is a prerequisite for the efficient design and operation of bulk enzyme recovery processes, however, complete phase diagrams are only available for very few proteins, in particular lysozyme of high purity. Here, we present the results of detailed solubility studies in aqueous solutions of an industrially relevant $\alpha$-amylase of technical grade. Experiments were conducted in small scale batch mode (working volume of $1 \mathrm{~mL}$ ). The influence of selected cations and anions from the Hofmeister series on the stability of the $\alpha$-amylase was examined. The Hofmeister series for anions was followed in the correct order at all salt concentrations studied, i.e. from 0 to $1 \mathrm{M}$, whereas the series was reversed for monovalent cations at concentrations up to $0.5 \mathrm{M}$, with the exception of lithium. To further investigate why the position for lithium was different to the Hofmeister series established for lysozyme, the zeta potential of protein solutions at low concentrations of selected salts was determined. The results of these measurements indicate a pronounced effect of lithium on the zeta potential, as compared to other salts. In particular, the $\mathrm{pH}$ of zero zeta potential (i.e. the $\mathrm{p} I$ ) was shifted approximately $0.5 \mathrm{pH}$ units towards alkaline conditions in the presence of lithium, whereas the $\mathrm{p} I$ stayed almost constant for sodium and potassium. Since the solubility exhibits a minimum at $\mathrm{pH}$-values at or near the protein's $\mathrm{p} I$, shifts in $\mathrm{pH}$ caused by salt addition are important to identify and quantify to avoid uncontrolled phase separation. The measurement of the zeta potential of proteins in solution holds significant promise as an attractive tool for understanding and controlling processes that are operated close to the solubility limit and which are often plagued by uncontrolled precipitation or crystallisation and thus rely on carefully chosen operating conditions.

\section{BE72}

Polyphenolic interactions with potato proteins during industrial expanded bed adsorption processing Sissel L $\phi \mathrm{kra}^{1}$, Knut Olav Stratkvern ${ }^{1}$, Bjørg Egelandsdal ${ }^{2}$, Gerd Vegarud ${ }^{2}{ }^{1}$ Department of Natural Science \& Technology, Hedmark University College, N2317 Hamar, Norway; ${ }^{2}$ Norwegian University of Life Sciences, 1432 Ås, Norway. E-mail: sissel.lokra@1nb.hihm.no (S. Løkra)

In plant extracts it has been shown that polyphenols have a tendency to react with proteins, either by covalent or non-covalent interactions. These reactions can induce changes in the surface properties of the proteins, and, e.g. cause proteins to be insoluble and precipitate at $\mathrm{pH}$-values below their isoelectric point. Potato proteins have a high nutritional quality and show interesting functional properties in food systems. Moreover, chlorogenic acid (CA) and caffeic acid constitute about $90 \%$ of the total polyphenol content of potato tuber. We have experienced expanded bed adsorption (EBA) chromatography to be a method well suited for recovering industrial proteins from potato starch effluent. The process separates proteins from polyphenolic pigments, fiber and minerals. During the adsorption step, patatin, the major potato tuber protein shows complex binding kinetics demonstrated by breakthrough curves. In addition to diffusion limitations in the EBA resin, changes in protein structure and surface properties probably are likely to affect this adsorption behavior. Reactions between CA and patatin might result in a range of interactions for different species of the same protein. This project therefore aims to assess the interactions between CA, patatin and other major tuber protein fractions and how these changes affect the protein capture in EBA. Changes in size and charge are screened in 2-D electrophoresis and analyzed further. Samples of different protein fractions are taken from breakthrough curves and dynamic binding capacity experiments in model systems with real feedstock.

\section{BE73}

Sandwich hybridisation assay for analysis of brewery contaminants S. Huhtamella ${ }^{1}$, M. Leinonen ${ }^{1}$, T. Nieminen ${ }^{1}$, A. Breitenstein $^{2}$, P. Neubauer ${ }^{1}$ : ${ }^{1}$ Bioprocess Engineering Laboratory, University of Oulu, Finland; ${ }^{2}$ Scanbec GmbH, Halle, Germany. Email: peter.neubauer@oulu.fi (P. Neubauer)

Here we describe the development of a sensitive, cultivationindependent analytical method for the analysis of brewery contaminants which can be performed within three hours in crude sample extracts. The method is based on $16 \mathrm{~S}$ rRNA detection by a paramagnetic bead based sandwich hybridization assay (SHA) with two oligonucleotide probes designed to either detect the species or a group of contaminants. The signals were read out either by a fluorimeter (Rautio et al., 2003; Leskelä et al., 2005) or potentiometrically with an electric biochip instrument (eBiochip Systems) (Gabig-Ciminska et al., 2004). This assay is advantageous over RTPCR becasue it only detects viable cells and the method can be directly applied to crude cell extracts without prior purification. We describe the principle of designing and evaluating a series of groupspecific Lactobacillus probes and the optimisation towards effective cell lysis and high assay sensitivity. The applicability of the SHA was evaluated with real brewery samples and the results were compared to routine tests. In all steps of the evaluation the reliability and usability of the method was prioritised. The optimised method combined with a $24 \mathrm{~h}$ pre-enrichment period gave reliable results, had a detection limits of about $10^{4}-10^{5}$ cells per assay and was easily applicable in a brewery environment.

\section{References}

Gabig-Ciminska et al., 2004. Electric chips for rapid detection and quantification of nucleic acids. Biosens. Bioelectron. 19, 537-546.

Leskelä et al., 2005. Rapid and sensitive genus specific detection of Legionella by a $16 \mathrm{~S}$ rRNA based SHA. J. Microbiol. Method., (in press).

Rautio et al., 2003. Sandwich hybridisation assay for quantitative detection of yeast RNAs in crude cell lysates. Microb. Cell Fact. $2(4), 1-9$.

\section{BE74}

OUR measurements during $\boldsymbol{R}$. erythropolis IGTS8 desulfurisation biocatalyst production V.E. Santos, C. Galdeano, E. Gómez, A. Alcón, F. García-Ochoa Dpto. Ingeniería Química. Facultad Ciencias Químicas. Universidad Complutense de Madrid. 28040-Madrid, Spain. E-mail: vesantos@quim.ucm.es (V.E. Santos)

Biodesulfurization is one of the possibilities studied by the researchers to attain the maximum sulfur levels imposed for a near future by Governments (European Directive, 2003). Rhodococcus erythropolis IGTS8 is a natural and strictly aerobic microorganism able to remove the sulfur atom from dibenzothiophene (DBT) in a selective way (4S route (Oldfield et al., 1997)), obtaining 2hydroxibifenyl (HBP) and sulfate. Growth is carried out using the experimental procedure performed in previous works dealing with 
the inoculum built up, media composition and operational conditions (del Olmo et al., 2005a, 2005b). This work is focused to determine the oxygen uptake rate during the production of the biocatalyst. Four experiments were carried out at a Biostat B fermentor (Braun Biotech.) using as only variable the constant stirrer speed used: 150 , 250,400 and $550 \mathrm{rpm}$. Oxygen uptake rate have been determined by means of two methods: dynamic technique at different times during growth for few seconds to ovoid influences and from oxygen profile when the term dealing with oxygen transfer rate is known (predicted by the model proposed in a previous work (García-Ochoa and Gómez, 2004)). OUR values obtained from the techniques used present the same tendency in all the runs carried out: OUR values from dynamic technique is always lower than OUR values obtained from the oxygen profile. These values are modeled and the difference observed is explained due to the cellular economy principle: during the seconds employed in the dynamic technique determinations microorganism do not produce $4 \mathrm{~S}$ route enzymes.

\section{References}

del Olmo, C.H., Santos, V.E., Alcón A., García-Ochoa, F., 2005a. Biochem. Eng. J. 22, 229-237.

del Olmo, C.H., Alcón A., Santos, V.E., García-Ochoa, F., 2005b. Enzym. Microbiol. Technol. 37 (2), 157-166.

European Directive (2003/17/CE).

García-Ochoa, F., Gómez, E., 2004. Chem. Eng. Sci. 59, 24892501.

Oldfield, C., Pogrebinsky, O., Simmonds, J., Olson, E.S., Kulpa, C.H., 1997. (ATCC 53968). Microbiology UK. 143, 29612973.

\section{BE75}

Recuperation of antigenic protein produced by recombinant E. coli cultures Juan Gajardo ${ }^{1}$, Pablo Albistur ${ }^{1}$, Carolina Escobar ${ }^{1}$, Patricio Cataldo ${ }^{2}$, Claudia Altamirano ${ }^{1}$, Sergio Marshall ${ }^{2}$, Maria Elvira Zuniga-Hansen ${ }^{1}$ : ${ }^{1}$ School of Biochemical Engineering, Pontific Catholic University of Valparaiso, Chile; ${ }^{2}$ Laboratory of Immunology and Molecular Genetic, Pontific Catholic University of Valparaiso, Chile. E-mail: mzuniga@ucv.cl (M.E. Zuniga-Hansen)

It was studied different methods to recover a $P$. salmonis antigenic protein from recombinant $E$. coli cells. This protein has shown be highly effective in vivo vaccine. It has the ability to stimulate salmon immune system protecting them against of aggressive disease salmonid rickettsial syndrome resolving by this way a great problem of salmonid aquaculture. Biomass obtained from IPTG induced $E$. coli BL21 (DE3) Codon Plus culture was used for soluble and insoluble antigenic protein recovery. It was evaluated recuperation by glass bead mill, freezing and thawing, osmotic shock and lisozyme/EDTA treatments, all of them applied in single or combined way. Biomass was measured by dry weight of cells, soluble protein concentration was quantified according to Bradford method, and antigenic protein was identified by SDS-PAGE and Western-Blot analysis. Cells treated with lisozyme/osmotic shock and then glass bead mill the soluble protein was a $62.9 \%$ of the dry weight cell mass whereas using lisozyme/EDTA and glass bead mill as a single treatment only a 24.4 and $22.6 \%$ were obtained respectively. The freezing and thawing disruption treatment released less than $5 \%$ of soluble protein, as well as the osmotic shock procedure too. The SDS-PAGE and West- ernblot analysis revealed that the antigenic protein must be purified from the insoluble cell fraction when physical or mechanical disruption methods were employed and from the soluble cell fraction when chemical or enzymatic treatments were used. We propose investigate in further studies the inclusion bodies formation to design an efficient purification procedure for the target protein.

\section{BE76}

Use of immobilized peroxidase in oxidant compound analysis Marzouki-Mdelji Saida ${ }^{1}$, Limam Farid ${ }^{2}$, Ulber Roland ${ }^{3}$, Marzouki M. Nejib ${ }^{1}$ : $\quad{ }^{1}$ INSAT, Bioengineering laboratory, Tunis; ${ }^{2}$ ITC, Callinster 3, Hanover; ${ }^{3}$ INRST, Tunis. E-mails: mnmarzouki@yahoo.fr, mn.marzouki@insat.rnu.tn (M.M. Nejib)

The iso-peroxidase Pox 2 from garlic bulb Allium sativum that represented the major peroxidase activity was purified to homogeneity. The enzyme is monomeric and has a molecular mass of $36 \mathrm{kDa}$, and a $\mathrm{p} I$ around 9. The optimum temperature ranged between 25 and $40^{\circ} \mathrm{C}$, while optimum $\mathrm{pH}$ was around 5. Pox 2 appeared remarkably thermostable since it retained $50 \%$ of its activity at $50{ }^{\circ} \mathrm{C}$ for at least $6 \mathrm{~h}$. In addition, the enzyme was stable at a $\mathrm{pH}$ range from 3, 5 to 11 . Kinetic constants were calculated, the apparent $K_{\mathrm{m}}$ values were 500 and $150 \mu \mathrm{M}$ for gaïacol and $\mathrm{H}_{2} \mathrm{O}_{2}$, respectively. The high thermostability of Pox 2 may represent clear advantages in a number of processes including immobilizing peroxidase and use it as a biosensor to detect oxidant component as $\mathrm{H}_{2} \mathrm{O}_{2}$ and other peroxides. Immobilization of Pox 2 was achieved by binding covalently the enzyme to a sepharose matrix (bead and membrane VA epoxy). The immobilized peroxidase showed great stability at heat and storage than the soluble enzyme. The native enzyme retained $55 \%$ of its activity at $60^{\circ} \mathrm{c}$ for $10 \mathrm{mn}$ while the immobilized Pox 2 retained full activity for $35 \mathrm{mn}$ at the same temperature. In other side, the free enzyme retained full activity for at least one month and a half during storage at $25^{\circ} \mathrm{C}$, and lost $50 \mu \mathrm{m}$ of its activity after 2 months. The immobilized form of Pox 2 retained complete activity for 2 months at the same temperature. The immobilized enzyme was used to detect $\mathrm{H}_{2} \mathrm{O}_{2}$ in some food components such as milk and fruit juices. In a second study, same experiments were performed in order to detect the smaller quantity of added $\mathrm{H}_{2} \mathrm{O}_{2}$ to the farming milk.

\section{BE77}

The application of ABPs in target identification and drug discovery

Purpose: A new research field has been created to begin to address protein function at level of regulation of enzyme activity. This new area has been given the name chemical proteomics, or activity-based proteomics (ABPs), and makes use of small molecules that can covalently attach to catalytic residues in an enzyme active site. The selectivity of the chemically reactive group allows specific proteins or protein subset to be tagged, Purified and analyzed.

Methods: This molecule (ABPs) has three subsets: Tag, Linker, and Warhead. Warhead is a nucleophile and attach to active site. Linker is a polypeptide that makes a simple connection between warhead and tag. Tag is fluorcent or radioactive material that facilitates the detection of drugs.

Findings: Several diseases such as Cancer, rheumatoid arthritis and osteoporosis are associated with elevated levels of protease activity. Serine hydrolyses ABPs have been used to profile enzyme activity in a diverse range of cancer cell lines. In studies comparing metastatic 
and non-metastatic human breast cancer models, it was shown that the former exhibited a higher activity of a $\gamma$-glutathione- $S$-transferase, an enzyme that has not previously been associated with breast cancer. Discussion: Additionally, ABPs can be used to develop robust screens for small molecule inhibitors of a specific enzyme target within a large family of related enzymes. This method of inhibitor screening allows compounds to be assayed for both potency and selectivity against a set of related in complex biological samples. This technique is able to identify novel enzymatic proteins and drugs and has the potential to accelerate the discovery of new drug target.

\section{BE78}

Purification and characterization of a new CGTase from an alkaliphilic Bacillus clausii E16 Roberto Da Silva, H.F. Alves-Prado, E. Gomes, J.C. Rosa, L.J. Greene Biochemistry and Applied Microbiology Laboratory UNES-São José do Rio Preto, SP, Brazil. E-mail: dasilva@ibilce.unesp.br (R. Da silva)

A cyclodextrin glycosyltransferase (CGTase) from a new isolated strain from Bacillus clausii E16, was purified through Q-Sepharose, gel filtration chromatography and DEAE-Sephadex A-50. The mw of the pure enzyme was $75 \mathrm{kDa}$ with SDS-PAGE. The enzyme displayed optimum $\mathrm{pH}$ value and $\mathrm{pH}$ stability at $\mathrm{pH} 6.0$ and in range of 6.0-11.0, respectively. The optimum temperature and thermostability were at $55^{\circ} \mathrm{C}$ and up to $60^{\circ} \mathrm{C}$ by $1 \mathrm{~h}$, respectively. The $K_{\mathrm{m}}$ and $V_{\max }$ were $2.85 \mathrm{mg} / \mathrm{mL}$ and $80.0 \mu \mathrm{mol} / \mathrm{min} \mathrm{mg}$ and $0.83 \mathrm{mg} / \mathrm{ml}$ $13.45 \mu \mathrm{mol} / \mathrm{min} \mathrm{mg}$ Using maltodextrin and soluble starch, respectively. The isoeletric point was 4.8 and the $\mathrm{N}$-terminal region of the pure enzyme was sequencing by MALDI-TOF-MS. The ratio of $\alpha$-, $\beta$ - and $\gamma$-CD was 0.29:1.00:0.79 and 0:1:0 with maltodextrin and soluble starch at $2.5 \%$.

\section{BE79}

Application of magnetic separation technology for recovery of immobilised lipases Nadja Schultz ${ }^{1}$, Anke Neumann ${ }^{1}$, George Metreveli ${ }^{3}$, Matthias Franzreb $^{2}$, Christoph Syldatk ${ }^{1}$ ${ }^{1}$ Chair of Technical Biology, University of Karlsruhe, Engler Bunte Ring 1, D-76131 Karlsruhe; ${ }^{2}$ Forschungszentrum Karlsruhe, Institute of Technical Chemistry, Water- and Geotechnology; ${ }^{3}$ Inst. für Wasserchemie, Engler Bunte Ring 1,76131 KA. E-mail: nadja.schultz@ @iw.uni-karlsruhe.de (N. Schultz). url: www.fzk.de/itc-wgt (M. Franzreb)

First results on the development of the magnetic separation technology for the recovery of immobilised lipase from a 2-phase-system, which should be suitable for a large scale use in future, known as high gradient magnetic separation (HGMS) are presented. The application of immobilised lipases makes the reuse of the enzyme in a process possible and is therefore interesting for industrial applications. In this study immobilised lipase is used in a 2-phase-system. Here the new approach to recycle and reuse the lipase, immobilised on magnetic particles, from a 2-phase-system with the help of the new high gradient magnetic separator (HGMS) is examined in $30 \mathrm{~mL}$ scale. As model enzyme for the immobilisation on magnetic microparticles (polyvinyl alcohol (PVA), 1-2 $\mu \mathrm{m}$ ) the commercially available (NovoNordisc) Lipase A (CALA) from Candida antarctica was used. Necessary for screening of immobilisation methods and characterisation of the immobilised lipase (Candida antarctica) was the development of a robust, simple and rapid chromophoric activity assay. Therefore the pNPP-lipase assay was optimised for direct application on immobilised lipases (in preparation Schultz et al., 2005). Further more a pH-stat-assay for measuring the activity of free and immobilised CALA in a 2-phase-system of tributyrate and buffer was optimized for this system. Another important basis for the realisation of the recovery of immobilised lipases was to optimise the immobilization technique of the lipase. Furthermore approaches for the explication of generally empirical based immobilization techniques on insoluble support were made. Hereby we successfully applied the zeta potential measurement on the immobilization behaviour of the lipase CALA. For to determine the operating temperature for the biomagnetic separation procedure we studied stability analysis of free and immobilised lipase CALA at different temperatures $\left(37,25,4,-20^{\circ} \mathrm{C}\right)$ and at different $\mathrm{pH}$ values $(\mathrm{pH} 6,7$ and 8 ). The optimal temperature and $\mathrm{pH}$ value for the free and immobilised lipase was determined. Presently and constructively on the so far developed methods and techniques we intensively work on the demonstration and feasibility of the recovery of immobilized lipase from a 2-phase-system. Challenging approaches and first results on the recovery of immobilized lipase from a 2-phase-system in $30 \mathrm{~mL}$ scale were shown already.

\section{BE80}

Stationary phases for bioseparation of glycoproteins J. Aniulyte ${ }^{1}$, J. Liesiene ${ }^{1}$, B. Niemeyer ${ }^{2}$ : ${ }^{1}$ Department of Chemical Technology, Kaunas University of Technology (KTU), Radvilenu pl. 19, 50254 Kaunas, Lithuania; ${ }^{2}$ Institute of Thermodynamic, Helmut-SchmidtUniversity/University of the Federal Armed Forces Hamburg, Holstenhofweg 85, Hamburg D-22043, Germany

Nowadays glycomics raise new challenges for affinity chromatography related with an abundance of glycoconjugates in living organisms and with scaling-up of the preparative processes. Economics, efficiency and practicality dictate the search of novel chromatographic biospecific adsorbents that could contribute to enhancing the productivity of the affinity separation process. The purpose of the work was to prepare cellulose- and silica-based biospecific adsorbents with immobilized lectins and to evaluate them for the affinity chromatography of glycoproteins. Cellulose-based matrix Granocel and silica coated with hydrophilic polymers were used as a support. The effect of support characteristics, such as pore size, chemistry of active groups and their density on the support' surface on lectin immobilization and on the efficiency of adsorbents obtained were evaluated. Three different methods were used for the activation of the support: oxidation with sodium periodate, modification with pentaethylenhexamine (spacer arm of 18 atoms) and carbonyldiimidazole activation. ConA and WGA two lectins of different molecular weight and shape were selected to notice differences resulting from the size and diffusion behaviour. Chromatographic performances of the adsorbents were studied applying two different glycoproteins (GOD and fetuin) carrying specific terminal glycomoieties of mannose (GOD), and $N$-acetylglucosamine (fetuin) for specific interaction with ConA and WGA, respectively. The adsorbents demonstrated high affinity to glycoproteins with a sorption capacity in the column up to $7.4 \mathrm{mg}$ per $\mathrm{mL}$ support and a high recovery (up to 93\%). It was shown that spacer arm affected ligand coupling kinetic as well as the chromatographic behavior of the adsorbents obtained. The adsorption isotherms of GOD onto ConA adsorbents reveal an adsorption behavior with high and low affin- 
ity binding sites. The dissociation constant $K_{\mathrm{d}}$ of the ligand-sorbate complex is approximately $1 \times 10^{-6}$, and $0.4 \times 10^{-5} \mathrm{M}$, respectively. It was supposed that the second step is related to the sorption of solvated GOD onto already adsorbed GOD forming sorbate dimers.

\section{BE81}

Quantitative methods in high throughput screening of aqueous two phase systems Matthias Bensch, Björn Selbach, Jürgen Hubbuch Institute of Biotechnologie 2, Forschungszentrum Jülich, Germany

Purification of biopharmaceuticals is one of the most expensive and at the same time least understood steps in bioprocesses. During the process development for protein production, short time to market and the demand for cheap processes dominate today's process development. One way of reducing process costs is to implement integrative processes. Aqueous two phase systems (ATPS) combine the advantages of removing cell debris and simultaneously purifying and concentrating the target protein, however, to the cost of highly complex systems which are difficult to predict and optimize. Using high throughput screening techniques in the development of ATPS processes thus seems to be an ideal candidate for achieving both a reduced development time and an economical process without the need for preliminarily well characterized systems. In this study, we use the robotic system Tecan Freedom Evo ${ }^{\mathrm{TM}}$ as an automation platform for the evaluation of aqueous two phase systems. Central to this workstation are the integrated hardware as liquid handler, gripper, reader and centrifuge. We have created high throughput methods for rapid parameter estimations. As a first step, pipetting and mixing had to be calibrated for the use of highly viscous polymer solutions which are common in ATPS. The focus of the current work lies on the integration of the automatic preparation and analysis of two phase systems in microtiter plate scale. The robotic platform can now automatically create aqueous two phase systems and measure characteristic values such as binodal curves, protein concentrations and protein distributions between the two phases. The major bottleneck of HTS processes, namely the rapid analysis of impure systems, is tackled by using automated ELISA tools. Depending on the intended use, the high number of measured partition coefficients and yields can be used for modelling or rapid process design.

\section{BE82}

Optimization method for an ion exchange separation step Marcus Degerman, Niklas Jakobsson, Bernt Nilsson Department of Chemical Engineering, Lund University, P.O. Box 124, SE-221 00 Lund, Sweden

Today, most optimisations of chromatography separations are based on experimental work and rule of thumb. The PAT initiative has opened up for a model-based approach for downstream processing of pharmaceutical substances. This work uses a nonlinear chromatography model to optimize an ion exchange separation step. The general rate model with Langmuir MPM kinetics described the behaviour of the components in the column. The optimal operating points using both productivity and yield as objective functions were found. The optimizations were run with both IgG and BSA as target proteins respectively to compare their optimal operating points. The requirement on the optimal operating point was a purity of at least $99 \%$. This requirement was added to the optimization problem as a nonlinear inequality constraint. Flow rate, loading volume, start salt concentration in elution, elution gradient and cut points were used as decision variables in the optimization. The more retained component, BSA, was much easier to separate from $\mathrm{IgG}$ with a gradient elution than IgG from BSA while still retaining a high productivity and yield. The higher load volume at the optimal operating point, with BSA as target protein, causes a displacement of IgG and thereby improving the separation. A high productivity at the yield optimum was still possible with BSA as target protein. Both a lower productivity and yield was obtained with IgG as target protein.

\section{BE83}

Optimisation and robustness analysis of a hydrophobic interaction chromatography step Niklas Jakobsson, Marcus Degerman, Bernt Nilsson Department of Chemical Engineering, Lund University, P.O. Box 124, SE-221 00 Lund, Sweden

Process development, optimisation and robustness analysis for chromatography separations are often entirely based on experimental work and generic knowledge. The present study proposes a method of gaining process knowledge and assisting in the robustness analysis and optimisation of a hydrophobic interaction chromatography step using a model-based approach. Factorial experimental design is common practice in industry today for robustness analysis. The method presented in this study can be used to find the critical parameter variations and serve as a basis for reducing the experimental work. In addition, the calibrated model obtained with this approach is used to find the optimal operating conditions for the chromatography column. The methodology consists of threes consecutive steps. Firstly, screening experiments are performed using a factorial design. Secondly a kinetic-dispersive model is calibrated using gradient elution and column load experiments. Finally the model is used to find optimal operating conditions and a robustness analysis is conducted at the optimal point. The process studied in this work is the separation of polyclonal $\mathrm{IgG}$ from BSA using hydrophobic interaction chromatography.

\section{BE84}

Enhanced mercury biosorption by surface-engineered yeast displaying MerR inspected by new bacterial whole-cell biosensor Pavel Kotrba, Stanislav Vinopal, Pavel Vopalensky, Tomas Ruml Department of Biochemistry and Microbiology, ICT Prague, Technicka 3, Prague CZ-166 28, Czech Republic

The display of novel metal binding sites on the surface of the biosorbent represents potent tool to increase its binding capacity and improve selectivity. In this study, the $15.8 \mathrm{kDa}$ transcriptional regulator MerR of mercury-inducible mer operon of $T n 21$ exhibiting high affinity and selectivity towards $\mathrm{Hg}^{2+}$, was displayed on the surface of $S$. cerevisiae. To achieve this, merR was genetically fused with gene encoding $\mathrm{C}$-terminal domain of $\alpha$-agglutinin which resulted in covalent attachment of the of the fusion protein on the cell wall glucan via glycosylphosphatidylinositol anchor. To evaluate the performance of such modified whole-cell biosorbent with specific regard to $\mathrm{Hg}^{2+}$, we constructed a new biosensor $E$. coli strain, which utilizes kanamycine resistance gene as a reporter under the control of mer promoter. It allowed determination of $\mathrm{Hg}^{2+}$ in a range of $5-100 \mathrm{nM}$ by simply monitoring the growth in the $\mathrm{Hg}^{2+} / \mathrm{kanamycine-containing} \mathrm{media.}$ The effect of genetic engineering of $S$. cerevisiae surface by MerR became significantly pronounced in biosorption experiments with 
solutions containing $10 \mu \mathrm{M} \mathrm{Hg}^{2+}$ when modified cells accumulated 2.5 -fold more $\mathrm{Hg}^{2+}$ than the control strain expressing mere anchoring domain.

\section{BE85}

Sensitivity analysis of amino acids in simulated moving bed chromatography Ju Weon Lee, Chong Ho Lee, Yoon Mo Koo Center for Advanced Bioseparation Technology, Inha University, Inchon 402751, Korea. E-mail: ymkoo@inha.ac.kr (Y.M. Koo)

The difficulty of simulated moving bed (SMB) design is that the optimization of the operation conditions relies on the determination of accurate adsorption isotherms. Most SMB chromatograph is carried out under nonlinear conditions, and the nonlinear behavior should be considered properly in the equilibrium isotherms. The other difficulty is the SMB operation which has the characteristics of continuous process, all flow rates and switching time of valves should be maintained during the operation of SMB. If the disturbances of operating conditions and isotherm parameters are occurred, it affects the zone flow rates and the migration velocity of the solutes, and these effects change the internal profiles of the solutes. Therefore, it is the reason of decreasing the purity and the yield of products The objective of this work is to consider the sensitivity of isotherm parameters and operating parameters in SMB chromatography process. Two amino acids, phenylalanine and tryptophan, separation by SMB process is selected as control system.

\section{BE86}

Application of $\mathrm{pH}$ and $\mathrm{pO}_{2}$ probes during Bacillus caldolyticus fermentation: An additional approach in improving a feeding strategy Johannes Bader ${ }^{1}$, Boris Neumann ${ }^{2}$, Karima Schwab ${ }^{1}$, Milan Popovic ${ }^{1}$, Rakesh Bajpai ${ }^{3}{ }^{1}$ Studiengang Biotechnologie, Fachbereich V, TFH-Berlin, Seestr., 13347 Berlin, Germany ${ }^{2}$ Proteome Factory AG, Dorotheenstr. 94, 10117 Berlin, Germany; ${ }^{3}$ Department of Chemical Engineering, University of Missouri-Columbia, W2061 EBE, Columbia, MO, USA. E-mail: popovic@tfh-berlin.de (M. Popovic)

Bacillus caldolyticus, a thermophilic microorganism, is a good producer of thermostable liquefying $\alpha$-Amylase. During optimisation of initial and feeding media for fed-batch fermentation a two component feeding containing starch and casitone was found advantageous. To approach the optimal feeding rate the method published by Akesson et al., 2001 was extended to two component feeding. The key idea, discussed in this presentation, was using the $\mathrm{pO}_{2}$ and $\mathrm{pH}$ probing signals to determine if the feeding of one or the other component should be increased or decreased. Each of the probes offers information of different areas of feeding condition. To prevent excessive feeding of starch the $\mathrm{pH}$ probe is preferable. In case of excessive casitone feeding the $\mathrm{pO}_{2}$ probe responds in very authentic way enabling together with the $\mathrm{pH}$ signal reliable and reproducible evaluation of feeding strategy. However a congruent response of $\mathrm{pO}_{2}$ and $\mathrm{pH}$ probes means the approaching of the optimum feeding rate for both components.

\section{Reference}

Akesson, M., Hagander, P., Axelsson, J.P., 2001. Probing control of fed-batch cultivations: analysis and tuning. Contr. Eng. Pract. 9, 709-723.

\section{BE87}

Antibody immobilization by using the plasma polymerized acrylic acid R. Jafari, M.Tatoulian, F. Arefi-Khonsari Laboratoire de génie des procédés plasmas et traitement de surface, ENSCP, UPMC, 11 rue Pierre et Marie Curie, 75005 Paris, France

The objective of this work is therefore to produce a surface containing a high density of $\mathrm{COOH}$ functions on the polymer beads (PS) for the covalent immobilization of antibodies. We have investigated the plasma polymerization of Acrylic Acid in a fluidized bed reactor the polystyrene (PS) beads. For such application, there is a strong need to obtain stable plasma polymerized acrylic acid (PPAA) coating, resistant to washing with water. Different physico-chemical analyses have been used (water contact angle measurements (WCA), XPS and SEM analysis) to characterize the PPAA coating deposited on PS beads under different experimental conditions. The XPS results showed that the pretreatment of surface of the beads before deposition of acrylic acid plays an important role on the stability of PPAA layer. The instability of the coating is partially due the fact that under certain conditions the coatings are soluble in water and secondly due to the bad adhesion of the polymer beads which are hydrophobic to the growing PPAA coatings. XPS as well as ToF-SIMS gives evidence of the immobilization of the antibody. XPS results as well as static SIMS allows to detect nitrogen on the surface of the treated beads which proves the presence of the immobilized antibodies. Under optimum condition the PPAA coatings provides the possibility to show a nitrogen uptake which varies between 6.5 and $9 \%$ of the apparent stoicheiometry of the surface.

\section{Pharma Biotechnology}

\section{Discovery of Protein Therapeutics}

1

Activators of the GLP-1 receptor in diabetes treatment Jens Juul Holst Department of Medical Physiology, the Panum Institute, University of Copenhagen, DK-2200 Copenhagen, Denmark

GLP-1 (glucagon-like peptide-1), a peptide of 30 amino acids secreted by endocrine cells in the gut in response to meal ingestion, was discovered during a systematic search for gut factors capable of enhancing insulin secretion. It turned out to be the most efficacious insulin releaser known, and unlike other factors, was shown to retain its insulinotropic activity also in patients with type 2 diabetes. Subsequent research has documented that the peptide not only releases insulin from the beta cells, but also enhance all steps of insulin biosynthesis, up-regulates beta cell gene transcription, and has trophic effects on the beta cells. The latter includes both proliferation of existing cells, neogenesis from ductal precursor cells, and inhibition of apoptosis. The peptide also inhibits glucagon secretion, reduces gastric emptying and reduces appetite and food intake. Because of these actions, GLP-1 administered to patients with type 2 diabetes dramatically lowers blood glucose as well as glycated hemoglobin levels, and reduces body weight. However, natural GLP1 is extremely rapidly metabolized in the body, and the problem has been how to convert the unstable peptide into a clinically useful agent. The two main problems are its susceptibility to enzymatic degradation by ubiquitous dipeptidylpeptide peptidase IV (DPP-IV) and its rapid renal elimination. A related peptide, isolated from the 
saliva of a lizard, exendin-4, was found to be a full agonist of the GLP-1 receptor, to be resistant to DPP-IV and to be cleared more slowly by the kidneys. This peptide was highly effective in clinical studies and has now (30/4) been approved for diabetes treatment by the FDA. Other approaches include acylation of GLP-1 whereby it attaches to albumin in the body and acquires resistance to DPP-IV as well as a slow renal elimination. Also this analogue (Liraglutide) has favourable clinical effects. Fusion proteins of GLP-1 and larger, slowly eliminated proteins in the body are currently being evaluated. Small molecule, orally available inhibitors of DPP-IV have been demonstrated to protect endogenous GLP-1 from degradation and to be efficacious in both experimental and clinical diabetes, and numerous inhibitors are currently in clinical development. The incretin hormones are released from gut endocrine cells upon meal ingestion. They enhance glucose-induced insulin secretion and nay be responsible for up to $70 \%$ of postprandial insulin secretion. The incretin hormones are glucagon-like peptide-1 (GLP-1) and glucosedependent insulinotropic polypeptide (GIP). In patients with type 2 diabetes (2DM) the incretin effect is severely reduced or absent. In 2DM patients the secretion of GIP is normal, but its effect on insulin secretion is almost completely lost. GLP-1 secretion, on the other hand, may be impaired, but its insulinotropic actions are preserved and it may restore insulin secretion to near normal levels. Substitution therapy with GLP-1 might therefore be possible. GLP-1 is a product of the glucagon gene and its actions include: (1) potentiation of glucose-induced insulin secretion; (2) stimulation of the expression of $\beta$-cell genes essential for insulin secretion, including the insulin gene; (3) stimulation of $\beta$-cell proliferation and neogenesis (by enhancing endocrine differentiation of duct cells) and inhibition of $\beta$-cell apoptosis; (4) inhibition of glucagon secretion; (5) inhibition of gastrointestinal secretion and motility, notably gastric emptying; and (6) inhibition of appetite and food intake. These actions make GLP-1 particularly attractive as a therapeutic agent for 2DM. Thus, continuous subcutaneous administration of GLP-1 for 6 weeks resulted in a $5 \mathrm{mmol} / \mathrm{l}$ reduction in mean plasma glucose and a reduction in $\mathrm{HgbA1}$ of $1.3 \%$; a weight loss of $2 \mathrm{~kg}$; improved insulin sensitivity; improved $\beta$-cell function; and the treatment was associated with no significant side effects. Unfortunately, GLP-1 is rapidly destroyed in the body by the ubiquitous enzyme, dipeptidylpeptidase IV (DPP-IV). Clinical strategies therefore include: (1) the development of metabolically stable analogues of GLP-1 viz. activators of the GLP-1 receptor; and (2) inhibition of DPP-IV. Orally active DPP-IV inhibitors have proven successful in experimental diabetes and several companies are now trying to develop clinically suitable inhibitors. So far the clinical experience is limited, but recent clinical studies have provided proof of concept. Metabolically stable analogues/activators include the structurally related lizard peptide, exendin- 4 or analogues thereof, as well as GLP-1 derived molecules that bind to albumin and thereby assume the pharmacokinetics of albumin. These molecules are effective in animal experimental models of type 2 diabetes, and have been employed in clinical studies of up to 52 weeks' duration. On the basis of these studies it can be concluded that a therapy of type 2 diabetes mellitus based on stimulation of GLP-1 receptors is likely to be effective and to become a clinical reality within the not too distant future(1-4).

\section{References}

Holst, J.J., 2005. Glucagon-like peptide 1: physiology and therapeutic potential. Invited review. Curr. Opin. Endocrinol. Diab. 12, $56-62$.

Holst, J.J., Gromada, J., 2004. Role of incretin hormones in the regulation of insulin secretion in diabetic and nondiabetic humans. Am. J. Physiol. Endocrinol. Metab. 287, E199-E206.

Vilsboll, T., Holst, J.J., 2004. Incretins, insulin secretion and Type 2 diabetes mellitus. Diabetologia 47, 357-366.

Zander, M., Madsbad, S., Madsen, J.L., Holst, J.J., 2002. Effect of 6weeks course of glucagon-like peptide 1 on glycaemic control, insulin sensitivity, and beta-cell function in type 2 diabetes: a parallel-group study. Lancet 359, 824-830.

\section{2}

Mechanism of action and clinical experience of rFVIIa Ulla Hedner Research \& Development, Novo Nordisk A/S, 2880 Bagsvaerd, Denmark

Recombinant activated coagulation factor VII (rFVIIa) was developed to treat bleedings in hemophilia patients, who have developed inhibitors against FVIII or FIX, and has been demonstrated to have an efficacy rate of 80-90\% in major surgery as well as in serious bleedings in such patients. To use rFVIIa as a hemostatic agent in severe hemophilia is a new concept of treatment, not being a substitution therapy, but using a pharmacological dose of exogeneous rFVIIa to compensate for the lack of FVIII or FIX. The administration of extra rFVIIa has been found not only to bind to tissue factor (TF), but also to the negatively charged phospholipids surface of thrombin activated platelets. Hemostasis occurs on surfaces being initiated on the TF-expressing cells as a result of exposure of TF, not normally exposed to the circulating blood, following an injury to the vessel wall. TF is a true receptor protein with an intramembraneous part and an intracellular tail. Its ligand is FVII/FVIIa. As soon as TF is being exposed to the blood, it forms complexes with FVIIa already present in the circulation. These complexes activates FX and provide the initial limited amount of thrombin molecules activating the co-factors, FVIII and FV, as well as FXI and platelets. Following the thrombin activation of platelets, negatively charged phospholipids are being exposed on the outer surface of the platelets. On this surface most coagulation proteins bind tightly, facilitating the conversion of FX into FXa and the full thrombin burst, necessary for the formation of a tight fibrin hemostatic plug resistant against premature lysis. In hemophilia patients the initiation of hemostasis is essentially normal, but, since they lack FVIII or FIX, they do not form the FVIIIa-FIXa complex necessary for full thrombin generation on the activated platelet surface. As a consequence the fibrin plug formed in hemophilia is loose, fagile and easily dissolved resulting in continuous bleeding. Pharmacological doses of rFVIIa have been demonstrated to mediate direct binding of rFVIIa to the negatively charged thrombin activated platelet surface, thereby generating thrombin formation in the absence of FVIII/FIX. Through this mechanism hemostasis is generated in hemophilia patients independent of FVIII/FIX. Furthermore, by generating more thrombin at an increased rate the formation of stable, tight fibrin hemostatic plugs are facilitated. Such fibrin plugs are more resistant against premature lysis and help not only to initiate but also to maintain hemostasis. Based on its capacity of enhancing thrombin generation 
locally on the activated platelet surface, rFVIIa has been used to ensure hemostasis also in other situations than hemophilia, such as platelet defects including thrombocytopenia. Recently, rFVIIa was shown to be hemostatically effective in patients with profuse bleedings as a result of vast trauma and tissue damage. In these patients with a complex hemostasis pattern including a host of changes leading to an impaired hemostatic function, extra rFVIIa seems to help generate a burst of thrombin resulting in the formation of a stable hemostatic plug more resistant against the ongoing lysis. In patients with intracerebral haemorrhage, one single dose of $\mathrm{rFVIIa}$ recently was found to limit the expansion of the haemorrhage and thereby leading to improved functional outcome.

\section{3}

Use of genomics for prediction of T-cell epitopes Søren Buus Institute for Medical Microbiology and Immunology, Panum 18.3.12, Blegdamsvej 3, DK-2200 Copenhagen N, Denmark. Email: s.buus@immi.ku.dk

Complete genomes from several species including many pathogenic microorganisms are rapidly becoming available along with the corresponding "proteomes". Even at the peptide level, the diversity of proteome is enormous and easily represents a unique imprint of the originating organism. It is perhaps not surprising that the immune system considers peptides as key targets. Recent immunological advances have shown that MHC molecules act as peptide selectors for immune recognition. We have proposed to generate accurate predictions of peptide binding to MHC and used these to identify immunogenic epitopes directly from genomic data. We have developed an iterative data-driven immunobioinformatics approach where data is used to generate predictors, and predictors are used to select new and complementary data for the next iteration. We have demonstrated the superior performance of this approach compared to a random data selection approach. We have also developed an efficient approach to select the most informative MHC molecules to investigate. The resulting, immunobioinformatics resource represents an immediate and powerful application and interpretation of genomic data, and will enable a rational approach to immunotherapy in the future.

4

Recombinant allergens: from bench to bedside Oliver Cromwell Allergopharma Joachim Ganzer KG, 21465 Reinbek, Germany

Allergen specific immunotherapy is a causal treatment for IgEmediated allergic diseases such as hay-fever, and it has relied traditionally on preparations derived from aqueous extracts of various natural allergenic source materials. The cloning and production of an increasing number of allergens through the use of DNA technology has not only facilitated the characterisation and analysis of the allergenic proteins, but also provided the opportunity to use these recombinant proteins instead of natural allergen extracts for the diagnosis and therapy of allergic disease. Detailed physicochemical, biochemical and immunological characterisation are essential for the comparison of natural and recombinant proteins, and also provide a basis for developing derivatives. Chemically modified allergens with attenuated IgE-reactivity are currently used for immunotherapy in order to enable high doses to be achieved with a minimized risk of inducing allergic side reactions. DNA technology provides the opportunity to develop and produce hypoallergenic allergen variants using strategies including gene mutation. The design of such variants must ensure that $\mathrm{T}$ cell reactivity and immunogenic activity are retained in order to preserve therapeutic potential. The recombinant allergens and their derivatives have several advantages over natural allergen extracts. They are relatively easy to produce in consistent pharmaceutical quality; the problems of natural extract standardisation can be avoided completely; the relative concentrations of the individual allergens can be controlled to obtain optimal dosages; nonallergenic proteins are excluded; the possible risks of contamination are avoided. The first clinical trials with grass pollen allergens and birch pollen hypoallergenic variants have yielded very encouraging results.

5

Development of recombinant human polyclonal antibody therapeutics John Haurum Symphogen A/S, Denmark

The use of recombinant polyclonal antibodies (pAbs) may improve the treatment of disease caused by complex targets such as infectious agents, when compared to monoclonal antibody therapy. Symphogen has developed a method for reproducible production of target-specific fully human $\mathrm{pAb}$ compositions, so-called symphobodies. The antibody genes are first isolated from donors with an immune response against the target and antibodies are screened for specificity. Subsequently, the pAbs are expressed in mammalian cells using the Sympress technology, which is based on site-specific integration. This procedure ensures that each of the expression constructs encoding the antibody genes are stably integrated at the same site in each of the host cells, thereby eliminating genomic position effects and differential growth and production rates. Further, the Sympress technology comprises the generation of a polyclonal Working Cell Bank (pWCB) which is used as inoculation material for the manufacturing. These cells display sufficient genetic stability to enable a controlled GMP production of recombinant polyclonal antibodies. Symphogen's first product, Sym001, is a recombinant human polyclonal Rhesus D-specific symphobody preparation consisting of 25 different anti-Rhesus D antibodies. This product is intended to be used for treatment of Idiopathic Thrombocytopenia Purpura and prevention of Hemolytic Disease in newborns. Recombinant antiRhesus D symphobodies were produced and shown to be biologically active against Rhesus D. The expression technology provided a compositional reproducibility between batches which is sufficient for manufacturing of such a polyclonal product for clinical use. Scaledup production for clinical trials is currently ongoing.

\section{Tissue Engineering}

1

Human post-natal stem cells and tissue engineering for skin and cornea L. Germain, C. Paquet, D. Larouche, P. Carrier LOEX Laboratory, Saint-Sacrement Hospital (CHA) Quebec, Department of Surgery and Ophthalmology, Laval University, Quebec (Qc), Canada

Stem cells play an important role in renewing tissues such as skin and cornea. They are responsible for the continuous generation of the differentiated epithelium. We have characterized stem cells of the skin and cornea in situ and their fate in vitro in human skin reconstructed by tissue engineering using keratin (K)19. In the outer root sheath of the hair follicle, stem cells (label-retaining cells) present in the basal layer of the bulge area express K19 and present a loosely 
arranged keratin filament network and low levels of K14 protein in their cytoplasm. In addition, another stem cell population (also labelretaining) is present in the first suprabasal layers. These cells exhibit a very dense keratin network and express K17. Three-dimensional tissue constructs (dermis and epidermis) obtained by the self-assembly approach of tissue engineering allow the preservation of K19 positive stem cells in the basal layer of the epithelium. In the eye, the stem cells are located in the limbal part but not in central cornea and they express K19. The epithelium of reconstructed cornea is thinner compared to reconstructed skin and more transparent. The characterization of stem cells in reconstructed tissue is essential to evaluate the long-term survival of these tissues in vitro but also after grafting. These human reconstructed tissues are developed for fundamental (physiological, toxicological studies) and clinical applications such as transplantation for the permanent replacement of damaged organs. LG is holder of the Canadian Research Chair (CIHR) on stem cells and tissue engineering.

\section{2}

Nanocapsules: Coating for living cells and tissues Alessandra Gliozzi Physical Department, University of Genoa, 16146 Genoa, Italy

Hollow nanometer-sized capsules can be prepared by means of different techniques. First "nanocapsules" were liposomes, however they are too unstable for many medical or pharmaceutical applications. In contrast, recently developed polyelectrolyte capsules prepared by means of the layer-by-layer technique are much more stable and seem to be a very promising way for coating living cells or tissues in order to prevent or reduce their immune rejection after implantation. Several observations on single living cells encapsulated by the alternative adsorption of oppositely charged polyelectrolytes will be presented. The most relevant result is that cell preserve their metabolic activity, are still capable of dividing and performing specific functions. Moreover, a technique to immobilize in defined arrays coated cells expressing green fluorescent protein by using a microcontact printing of polyelectrolytes will be presented. Finally, tests performed to study the induction of fibrosis and vascularization by nanocapsules implanted in rat kidney and liver will be presented.

3

Cardiac tissue engineering T. Eschenhagen Institute of Pharmacology, University Medical Center Hamburg-Eppendorf, Germany

Over the past decade we have developed methods to generate spontaneously and synchronously beating tissue equivalents from neonatal rat heart cells in the culture dish. These tissue equivalents display the key morphological and functional features of intact myocardium and have been termed engineered heart tissue (EHT). To generate EHTs, heart cells are mixed with freshly neutralized, liquid collagen I, matrigel and growth supplements and grown in a circular casting mold around a central cylinder, which subjects the cells to a continuous mechanical load. This process is enforced by cyclic mechanical stretch. We use EHT mainly for two purposes, as a test bed for the effects of pharmacological or genetic manipulations and for cardiac repair. As a cell culture model, EHTs compare with standard 2D monolayer cultures of neonatal rat cardiac myocytes and freshly isolated adult cardiac myocytes. Advantages of EHTs are their functional similarities with intact heart muscles, the ability to easily measure force of contraction under mechanical load, the pos- sibility to transfect cardiac myocytes inside EHTs with adenovirus at high efficiency and the reproducibility in large series. A disadvantage is that contractile function as measured at the end of the culture period also integrates influences on tissue development, cell-cellconnections, extracellular matrix production and on non-myocytes. At present we are working on downscaling the EHT method to a 96well format for screening purposes. To use EHTs for cardiac repair we created multi-looped EHTs from five circular EHTs large enough to cover the infarct scar 14 days after coronary artery ligation in rats. EHTs survived and formed a layer of muscle tissue on top of the infarct scar. EHTs restored undelayed anterograde impulse propagation over the scar, prevented further ventricular dilatation, normalized enddiastolic pressure and relaxation, and partly restored contraction of the scar. Thus, the study provides evidence that implanting EHTs onto infarcted hearts can improve cardiac contractile function after myocardial infarction.

\section{4}

Invitro growth of osteoblast cells in Scaffolds Ghuncha Ambrin ${ }^{1}$, Aldo Crugnola ${ }^{2}$, Peter Hart ${ }^{3}$ : ${ }^{1}$ Department of Chemistry and Biochemistry, University of Massachusetts, Dartmouth, USA; ${ }^{2}$ Department of Plastic Engineering, University of Massachusetts, Lowell, USA; ${ }^{3}$ Department of Biology, University of Massachusetts, Dartmouth, USA. E-mails: g_gambrin@umassd.edu (Ghuncha Ambrin), aldo_crugnola@uml.edu (Aldo Crugnola), phart@umassd.edu (Peter Hart)

The goal of tissue engineering is the development of skin, bones and even organs to restore, maintain and improve tissue function within the body. The current paper focuses on the investigation of invitro growth of osteoblast cells in different types of scaffolds. Three of the scaffolds were made of PCL (polycaprolactone) $10 \%$ glycerol and $10 \% \mathrm{HCA}$ (hydroxylapetite). Two of the scaffolds were made by compression molding, and one was made by fused deposition modeling utilizing the Stratasys. The fourth Cerabio was a commercially available product totally ceramic. The pores in compression molding were obtained by putting in $75 \%$ volume of sugar either 150 and $595 \mu \mathrm{m}$ which was later removed by leaching. The fused deposition scaffold was made by placing the filaments in a predetermined arrangement. The Stratasys system was a computer designed model. The scaffolds were seeded with HFOB 1.19 human fetal osteoblast cell line with vigorous shaking overnight and incubating at $37^{\circ} \mathrm{C}$ and $5 \% \mathrm{CO}_{2}$. Observation of the seeded scaffolds was made after 3 days and 9 days of incubation. The seeded cells were stained with $\mathrm{BCIP} / \mathrm{NBP}$ at $37^{\circ} \mathrm{C}$ overnight. The cell proliferation in the 595 and $150 \mu \mathrm{m}$ scaffolds appeared approximately the same with a possible advantage of $595 \mu \mathrm{m}$. The Cerabio sample demonstrated the greatest proliferation among the four scaffolds studied and the Stratasys sample exhibited a different type of cell adhesion with the cells were clustered in the interstices of the structure.

\section{5}

Strategies to enhance reconstruction of in vitro engineered bone Denise Freimark, Ruth Freitag, Valérie Jérôme Chair of Process Biotechnology, University of Bayreuth, D-95448 Bayreuth, Germany. E-mail: denise.freimark@uni-bayreuth.de (D. Freimark)

Tissue engineering is emerging as an alternative to bone grafts for the regeneration of defects that do not heal spontaneously. Ultimately, the development of an optimum carrier and the identification 
of ideal inductive factors and cells may enable tissue engineering to provide an improvement over bone grafts in the future. Bone formation and repair require a complex cascade involving growth factors, cytokines and angiogenesis. At present the complexity of the molecular mechanisms that control gene expression in bone forming cells in embryo as well as in adult is not fully understood. Several factors like bone morphogenetic proteins (BMPs), transforming growth factor beta (TGF- $\beta$ ), vascular endothelial growth factor (VEGF) and insuline-like growth factor (IGF) have been identified and their ability to stimulate bone formation in vitro and in vivo has been investigated. While much is known about these factors per se, less is known about genetic regulation of artificially stimulated osteogenesis. Interestingly, some in vitro investigations showed that only optimal growth factors concentrations lead to effective bone formation whereas higher concentrations had deleterious effects which suggest some variation in the activated regulation pathways. Therefore, one of our goals is to analyze kinetics, dose-dependence and synergistic effects of growth factors and cytokines on regulation pathways of bone formation. Moreover, the optimal vascularization of the scaffold is a major hurdle in the development of engineered bone. It is well known that: (i) vascular invasion precedes bone growth and (ii) osteogenesis takes place in the vicinity of newly formed vessels. Thus, inadequate bone vascularization is associated with decreased bone formation. Further analysis of the intercommunication between endothelial cells and osteoprogenitors in co-culture systems could provide key information that could be thereafter used to solve this problem. We propose to add some new knowledge to this complicated puzzle. A first step in our investigation is the production of some of the growth factors mentioned above in recombinant form. These factors are expressed in a novel vector ( $\mathrm{pTriEx}^{\mathrm{TM}}$; Novagen) which allows recombinant protein production in prokaryotic or in eukaryotic systems with a single plasmid. Afterwards, we analyze potential synergistic effects of these factors as well as kinetic and dose-depend parameters on the genetic regulation of downstream pathways in osteoblasts culture. In parallel, we develop an in vitro system allowing us to investigate the intercommunication between endothelial cells and osteoprogenitors.

\section{Stem Cells}

\section{1}

Therapeutic potential of human embryonic stem cells Stephen Minger Kings College London, UK

There has been significant interest in the therapeutic and scientific potential of human embryonic stem (ES) cells since they were first isolated in 1998. If human ES cells could be differentiated into suitable cell types, stem cells might be used in cell replacement therapies for degenerative diseases such as Type I diabetes and Parkinson's disease, or to repopulate the heart following myocardial damage. However, there is a significant shortage of high quality human ES cell lines and few research groups have experience in the propagation and manipulation of these cells. We are addressing this important issue using the combined expertise of the Stem Cell Biology Laboratory and the Assisted Conception Unit at King's College, London. With local ethical approval and under licence from the UK Human Fertilisation and Embryology Authority, we have been establishing high quality human ES cell lines from a novel source of human embryos. To date, we have derived three human ES cell lines and are now focused on the generation of therapeutically important cell populations, including cells that may have clinical application in degenerative and traumatic injury to the brain and spinal cord, heart, retina and other target organs.

2

Stem cell therapy for Parkinson's disease Anders Björklund Wallenberg Neuroscience Center, Department of Physiological Sciences, Lund University, BMC A11, S-221 84 Lund, Sweden

Cell replacement therapy for Parkinson's disease is based on the idea that implanted dopamine neurons may be able to substitute for the lost nigrostriatal neurons. In rodent and primate models of Parkinson's disease it has been shown that transplanted dopamine neuroblasts can re-establish a functional innervation and restore dopaminergic neurotransmission in the area of the striatum reached by the outgrowing axons; that the grafted neurons are spontaneously active and release dopamine in an impulse-dependent manner, at both synaptic and non-synaptic sites; and that they can reverse or ameliorate some of the Parkinson-like motor impairments induced by damage to the nigrostriatal system. Clinical trials in patients with advanced Parkinson's disease have shown that dopamine neuroblasts obtained from fetal human mesencephalic tissue can survive and function also in the brains of PD patients, restore striatal dopamine release, and ameliorate impairments in motor behavior. The principal limitation of this approach is the problems associated with the use of tissue derived from aborted human fetuses, and the large numbers of donors needed to obtain good therapeutic effects. Until now, transplantation of dopamine neurons has focused primarily on differentiated neuroblasts and young postmitotic neurons, at the stage of neuronal development that is optimal for survival and growth of the grafted cells. However, progenitors taken at earlier stages of development might prove more effective. Efforts are now made to expand multipotent neural stem- or progenitor cells in vitro, and control their phenotypic differentiation into a dopaminergic neuronal fate. Initial results suggest that in vitro expanded cells can survive and function after transplantation to the striatum in the rat PD model, but the overall yield of surviving dopamine neurons has been very low. With further development, expanded progenitors or dopamine neuron precursors, possibly in combination with cell engineering techniques, may offer new sources of cells for replacement therapy in PD.

3

Future therapeutic products from human ES cells? A. Colman ES Cell International, Australia

Stem cell therapy has been very much in vogue for several years now. Like gene therapy before it, it has raised unrealistic hopes of cures being available imminently. Unlike gene therapy, it can cite proof of principle in the well established practice of bone marrow transplantation which is actually a good example of stem cell therapy. However, most of the uses that are now touted as targets for stem cell therapy, envisage the conversion of the stem cells into lineage restricted progenitor cells or more commonly, the final differentiated cell type. Such conversions are extremely difficult to initiate and control. The procedures involve the manipulation, differentiation, and expansion of cell cultures in the laboratory, with unknown long term effects on the genetics and physiology of the cells. These issues are compounded when one considers as source 
material, human embryonic stem cells, where not only the final cell product requires significant scrutiny, but also there are safety issues surrounding the persistence of undifferentiated cells. On top of all these challenges are commercial (for stem cell companies), clinical, and regulatory pressures which will impact heavily on the pace of progress. Nonetheless, despite all these hurdles, various academic groups and companies are making significant progress and examples of such developments in diabetes and cardiovascular repair will be given

4

Viscoelastic properties of human mesenchymal stem cells Wenxiao Pan ${ }^{1}$, Erik Petersen ${ }^{4}$, Ning Cai ${ }^{2}$, Gang Ma ${ }^{1}$, Jian Run Lee ${ }^{3}$, Zhiqin Feng ${ }^{1}, \underline{\text { Kin Liao }}^{2}$, Kam W. Leong ${ }^{5}{ }^{1}$ Biomedical Engineering Research Center, School of Electrical \& Electronic Engineering, Nanyang Technological University, Nanyang 637553, Singapore; ${ }^{2}$ Division of Bioengineering, School of Chemical \& Biomedical Engineering, Nanyang Technological University, Nanyang 639798, Singapore; ${ }^{3}$ School of Mechanical \& Aerospace Engineering, Nanyang Technological University, Nanyang 639798, Singapore; ${ }^{4}$ Division of Biomedical Sciences, Johns Hopkins in Singapore 138669, Singapore; ${ }^{5}$ Department of Biomedical Engineering, School of Medicine, Johns Hopkins University, Baltimore, MD 21205, USA

Stem cells have the unique ability to perpetuate themselves while continually replenishing tissues throughout the life of an organism. The era of cellular and tissue regeneration for the treatment of disease and the effects of aging has indeed begun. It has been known that mechanical factors play an important role in the regulation of cell physiology. It is therefore reasonable to believe that mechanical factors also play a significant role in the metabolic activity and differentiation of MSCs. In this study, we investigated the viscoelasticity of individual bone marrow-derived adult human Mesenchymal stem cells (hMSCs), and the role of specific cytoskeletal component - F-actin microfilaments on the mechanical properties of individual hMSCs. The mechanical properties of hMSCs were determined using the micropipette aspiration technique coupled with a viscoelastic solid model of the cell. For the hMSCs under control conditions the instantaneous Young"s modulus $E_{0}$ was found to be $886 \pm 289$ $(\mathrm{Pa})$, the equilibrium Young"s modulus $E_{\infty} 372 \pm 125(\mathrm{~Pa})$, and the apparent viscosity $2714 \pm 1626$ (Pas). After exposed to $2 \mu \mathrm{M}$ of chemical agent-cytochalasin D that disrupt the F-actin microfilaments, the Young's moduli of hMSCs decreased by up to $72 \%$ and the apparent viscosity increased by $167 \%$. These findings suggest that microfilaments are crucial in providing the viscoelastic properties of the hMSCs, and changes in the structure and properties of them may influence the mechanical properties of hMSCs significantly.

\section{Expression Systems and Novel Therapeutics}

1

Engineering of mammalian gene networks Martin Fussenegger ETH Institute for Chemical and Bio-Engineering, Switzerland

Pharmacologic transcription control of desired transgenes is essential for gene-function analysis, drug discovery, biopharmaceutical manufacturing, design of complex artificial regulatory networks, precise and timely reprogramming of key cell characteristics for gene therapy and engineering of preferred cell phenotypes for tissue engineering. Capitalizing on our recent advances in the design of small molecule-responsive transcription control modalities we have used conditional molecular interventions for (i) improvement of specific productivity in biopharmaceutical manufacturing, (ii) transdifferentiation of therapeutically relevant cell phenotypes and (iii) design of artificial microtissues. We will also report on a completely new dimension of transgene control as well as engineering of hysteretic and epigenetic gene networks in mammalian cells.

2

Use of virus-like particles for therapeutic vaccination against addiction and other chronic diseases Martin F. Bachmann Cytos Biotechnology AG, Zürich-Schlieren, Switzerland

Chronic diseases are a growing burden for the individual and society alike. One key factor driving this increase is an ageing population. Currently, there are 600 million persons aged 60 years or over and this number is predicted to triple by the middle of the 21 st century. Effective prevention of irreversible damage to major organ systems requires early diagnosis and treatment yielding significant quality of life to the individual and sparing valuable health care resources. Even when safe and effective medicines are available, a remaining problem to successful therapy are issues of patient compliance. Historically, vaccines have been one of the major advances towards the longevity we enjoy today, with compliance rates close to $100 \%$. Hence, vaccines for early treatment of chronic diseases are ideally positioned for long-term therapy, and will take away the burden of self-medication associated with orally active drugs. Here we will discuss a new generation of therapeutic vaccines based on virus-like particles (VLPs). By displaying target molecules in a highly repetitive manner on VLPs, it is possible to break B cell unresponsiveness in experimental animals as well as in humans. Using such vaccines in animals, chronic diseases such as hypertension, Alzheimer's disease, obesity and rheumatoid arthritis could be treated. Furthermore, vaccination against nicotine resulted in high nictotine-specific antibody titers in humans, greatly facilitating smoking cessation in immunized individuals.

3

Interdependence of the impact of methanol and oxygen supply on protein production with recombinant Pichia pastoris N.K. Khatri, F. Hoffmann Martin-Luther-University Halle-Wittenberg, Institute for Biotechnology, Halle D-06120, Germany. E-mail: f.hoffmann@biochemtech.uni-halle.de (F. Hoofmann)

The methylotrophic yeast Pichia pastoris is a potent expression system for secretion of recombinant proteins. Methanol as inductor of the foreign gene expression is also a substrate with high oxygen demand, which can lead to sudden oxygen depletion upon induction. Thus, supply rates of methanol and oxygen are major process parameter during protein production with recombinant Pichia pastoris. Limiting dosage of methanol allowed maintenance of oxygen sufficient conditions during production of a single chain antibody fragment, but the product was degraded from the C-terminal end. In contrast, full-length product accumulated with controlled methanol concentrations despite oxygen limitation. The volumetric methanol uptake rate are limited by the oxygen transfer capacity of the reactor. Higher methanol concentrations decreased the biomass yield and thereby increased the specific methanol uptake rate. This enabled prolonged production and yielded fivefold higher product concentrations. At the same time, the accumulation of small molecular 
weight contaminants was reduced. Dosage of pure oxygen accelerated methanol uptake, grow and production. Switching to DOstat mode upon oxygen depletion led to an arrest of product accumulation, in contrast to persistent methanol feeding. The productivity was tenfold higher than without oxygen. Combined with high methanol concentrations, however, fast methanol uptake led to toxication of the cells and early stop of production. Flow cytometry revealed that perturbation of oxygen metabolism was followed by partial lysis of the culture.

4

Recombinant production of soluble, active cystine bond contraining eukaryotic proteins in $\boldsymbol{E}$. coli Beatrix Fahnert ${ }^{1}$, Antje Neubauer $^{2}$, Anu Mursula ${ }^{1}$, Johanna Veijola ${ }^{1}$, Johanna Myllyharju ${ }^{2}$, Peter Neubauer ${ }^{1}{ }^{1}$ Bioprocess Engineering. Laboratory, Department of Medical Biochemistry and Molec. Biol., University of Oulu, FIN-90014 Oulu, Finland; ${ }^{2}$ Collagen Research Unit, Department of Medical Biochem. and Molec. Biol., University of Oulu, FIN-90014 Oulu, Finland. E-mail: peter.neubauer@oulu.fi (P. Neubauer)

Recombinant production of therapeutic proteins poses severe challenges due to their complexity (cystines, subunits, size). Formation of the correct disulphide bridges is a prerequisite for activity but difficult to achieve in a prokaryotic host. There proteins mainly fold post-translationally as opposed to eukaryotic co-translational folding. Thus the recombinant products are often not soluble and/or active. That is why different production parameters (e.g. host, induction conditions, temperature, compartment, proteinaceous fusion partners, co-expression of chaperones, foldases) are applied in order to gain functional recombinant proteins. The impact of all these strategies cannot be predicted and every problem of the production (expression, solubility, activity) might need to be solved for every target protein separately. Nevertheless, much effort has been put into this for almost 3 decades, because they are important targets for the pharmaceutical industry. Human growth factors influencing cellular proliferation and/or differentiation are one example. This case study gives an overview of strategies tested within the development of two processes leading to an optimised yield of active protein. Murine Wnt-1 (Wnt family) possesses 23 conserved cysteines (most likely all involved in disulphide bridge formation and one in posttranslational modification) and could only be successfully produced in E. coli (Fahnert, 2004) recently despite various attempts for many years. The other target protein is human collagen prolyl4-hydroxylase being a heterotetramer consisting of two $\alpha$-subunits and $\beta$-subunits each. The $\alpha$-subunit strongly aggregates if produced separately whereas the $\beta$-subunit is PDI and is suggested to have a chaperone function. Therefore a sequential induction strategy was proposed for this protein (Neubauer et al., 2005).

\section{References}

Fahnert. 2004. J. Biol. Chem. 279, 47520-47527.

Neubauer et al. 2005. Matrix Biol. 24, 59-68.

\section{5}

Moss cells in photobioreactors, a promising expression system for glycosylated proteins Alexander Lucumi, Clemens Posten Institute of Mechanical Process Engineering and Mechanics, Department of Bioprocess Engineering, University of Karlsruhe, Karlsruhe D-76131, Germany. E-mail: alexander.lucumi@mvm.uka.de (A. Lucumi)

The moss Physcomitrella patens has been recently recognized as an ideal producer of recombinant proteins with respect to glycosylation. Due to the elaborated post-translational capabilities of moss cells, the glycosylation patterns have been manipulated to obtain similar proteins to those found in animal cells. The protein expression using moss in suspension offers important advantages in comparison to other systems e.g. CHO cells. The recombinant proteins can be targeted into the mineral medium, simplifying the down stream processing. Moreover, there are neither known moss viruses nor plant viruses that are pathogenic for humans. The moss are cultivated axenically in a filamentous stage, the so called protonema. A pilot 301 tubular photoreactor is used to characterize the response of $P$. patens to variations on the culture conditions. The phototrophic culture in bioreactors is systematically investigated, where light quantity and quality, stress, concentration of phytohormones, and moss morphology influence the differentiation, growth, and protein expression. A tight control of the moss morphology in suspension, quantified by image analysis, has shown to be advantageous in order to delay the cell differentiation and maintain the carbon dioxide uptake in long bioreactor runs. The introduced perfused culture system by means of cross flow filtration allowed for a continuous product separation and concentration, and feed back of the productive cells. The characterization of this highly controlled culture system is presented and the potential of $P$. patens as an alternative tool for molecular farming is discussed.

\section{Small Interfering RNA}

1

Silencing the messenger: Harnessing RNA interference for therapy Judy Lieberman, Erwei Song, Pengcheng Zhu, Deborah Palliser CBR Institute for Biomedical Research, Harvard Medical School, Boston MA, 02115, USA

RNA interference (RNAi) is an evolutionarily conserved, endogenous mechanism for sequence-specific gene silencing that uses small double-stranded RNAs (called short interfering RNAs or siRNAs) to direct cleavage or prevent translation of homologous mRNAs. Harnessing RNAi for therapy presents an opportunity for potentially treating a wide variety of diseases. The main obstacle is delivering siRNAs into the cytosol of target cells in vivo. Although we were able to protect mice from autoimmune hepatitis by hydrodynamic tail vein injection of siRNAs targeting Fas, this delivery method is unlikely to be adaptable for human use. Alternate strategies to deliver siRNAs in vivo as small molecule drugs using currently available, clinically acceptable injection methods that have shown promise in mouse models will be discussed. These include local delivery to mucosal surfaces and delivery into specific cells via cell surface receptors using an antibody fragment fused to protamine. These siRNA complexes silence gene expression in vivo only in cells bearing the targeted receptor. Experiments showing efficient, 
effective and cell-specific delivery in a mouse tumor model will be discussed. In addition, encouraging preliminary data using RNAi for a microbicide to prevent sexually transmitted infection will be presented.

\section{2}

RNAi therapeutics Stefan Limmer Alnylam Europe AG, Germany

RNA interference (RNAi) holds significant progress as a therapeutic approach to siolence disease-causing genes, particularly those that encode "non-druggable" targets. The key hurdle for RNAi therapeutics is in vivo delivery. A critical requirement for achieving systemic RNAi in vivo is the introduction of "drug-like" properties, such as stability, cellular delivery and tissue biodistribution, into synthetic siRNAs. Our progress in achieving in vivo silencing of endogenous genes with chemically modified siRNAs will be discussed.

\section{3}

HIV-1 can evade RNAi-mediated inhibition by altering the secondary structure of its RNA genome Ellen M. Westerhout, Olivier ter Brake, Marcel Ooms, Monique Vink, Atze T. Das, Ben Berkhout Department of Human Retrovirology, Academic Medical Center, University of Amsterdam, Meibergdreef 15, 1105 AZ Amsterdam, The Netherlands

HIV-1 replication in human T cells can be inhibited by stable expression of a short hairpin RNA targeting the viral Nef gene (shRNA-Nef). However, HIV-1 escape variants emerge after prolonged culturing, and all but one escape mutant acquire a mutation in the shRNA-Nef target sequence. We observed single and multiple nucleotide substitutions, but also partial or complete deletion of the target sequence. These results demonstrate the sequencespecificity of this antiviral approach. We observed an inverse correlation between the level of resistance and the stability of the shRNA/target-RNA duplex for most of the escape mutants. However, two escape variants did not follow this pattern, including an escape mutant with a single point mutation at position -7 upstream of the target sequence. These mutants provide a much higher level of resistance than expected based on duplex stability, which is obviously not affected in the -7 mutant. We demonstrate that these mutants adopt an alternative RNA secondary structure that occludes the target sequence. This results in reduced shRNA-Nef binding and provides a novel mechanism for RNAi-resistance. To avoid viral escape, one should ideally target HIV-1 with multiple effective shRNAs against conserved genome sequences. We performed a large scale screening to identify such targets, and we have identified at least nine genome segments that can be targeted effectively with shRNAs. These potent antivirals are currently being assembled in a lentiviral vector for gene therapy applications in HIV-infected individuals. Furthermore, we will describe approaches to forecast viral escape routes and to effectively block such evolutionary paths with additional RNAi measures.

\section{Poster Abstracts Pharma Biotechnology (PB)}

\section{PB1}

Antibodies against electronegative ldl inhibit atherosclerosis in Idlr $^{-1-}$ mice D.M. Grosso ${ }^{1}$, L. Santos $^{2}$, A. Bonito ${ }^{2}$, M.H. Krieger ${ }^{2}$, D.S.P. Abdalla ${ }^{1}:{ }^{1}$ Faculty of Pharmaceutical Sciences, University of São Paulo, 05508-900 São Paulo, Brazil; ${ }^{2}$ Institute of Biology, University of Campinas, Campinas, Brazil. E-mail: dspa@usp.br (D.S.P. Abdalla)

In this study we analyzed the effect of antibodies against electronegative LDL on the development of atherosclerotic lesions in low-density receptor-deficient $\left(\mathrm{LDLR}^{-1-}\right)$ mice. Two groups of $\left(\mathrm{LDLR}^{-1-}\right.$ ) mice (eight females) fed $0.5 \%$ cholesterol-enriched chow were used. The first group received a monoclonal antibody against electronegative LDL $(100 \mu \mathrm{g})$ and the second one received PBS (controls). Additionally, other two groups (eight males) of $\left(\mathrm{LDLR}^{-/-}\right)$mice were treated with a polyclonal antibody against electronegative LDL $(100 \mu \mathrm{g})$ or PBS (controls). Antibodies were administered by intravenous route one week before starting the hypercholesterolemic diet and then every week over an experimental time of 21 days. Afterwards, quantification of atherosclerotic plaque area of heart and aortic arch was done by analysis of the slices stained with oil red/hematoxolin/light green with the Image Propus software. The passive immunization with either monoclonal or polyclonal antibodies against electronegative LDL significantly reduced the atherosclerotic plaque areas in atherosclerosis-prone LDLR $^{-1-}$ mice. In conclusion, antibodies against electronegative LDL administered by intravenous route may play a protective role in atherosclerosis. Supported by Fundação de Amparo à Pesquisa do Estado de São Paulo (FAPESP, scholarships to D.M.G., L.S. and A.B. and grants to M.H.K. and D.S.P.A.).

PB2

Asparaginase production by a recombinant Pichia pastoris strain harbouring Saccharomyces cerevisiae ASP3 gene Maria Antonieta Ferrara ${ }^{1}$, Neuza M.B. Severino ${ }^{2}$, Antonio C. Siani ${ }^{1}$, Nei Pereira Jr. ${ }^{3}$, Fernando A.G. Torres ${ }^{4}$, Elba P.S. Bon ${ }^{2}:{ }^{1}$ FarManguinhos/FIOCRUZ, Brazil; ${ }^{2}$ Instituto de Química/UFRJ, Rio de Janeiro, RJ, Brazil; ${ }^{3}$ Escola de Química/UFRJ, Rio de Janeiro, RJ, Brazil; ${ }^{4}$ Dep. Biologia Celular/UnB, Brasília, DF, Brazil. E-mail: ferrara@far.fiocruz.br (M.A. Ferrara)

The enzyme asparaginase from the procaryote Escherichia coli or Erwinia crysanthemi is used for the treatment of lymphoblastic leukaemia. The drug causes immunological reactions in despite of the treatment efficiency. Asparaginase may also be obtained from Saccharomyces cerevisiae and this enzyme could provide an alternative to its bacterial counterparts. In this study, the periplasmic nitrogen regulated asparaginase II from $S$. cerevisiae, that is coded by the $A S P 3$ gene, was cloned and expressed in the methylotrophic yeast Pichia pastoris under the control of the AOX1 gene promoter. The recombinant $P$. pastoris strain was cultured in shake flasks and in a 21 instrumented bioreactor. In both cases it was observed specific enzyme yields seven fold higher in comparison to that using a nitrogen derepressed strain of $S$. cerevisiae, reaching $800 \mathrm{U} / \mathrm{g}$ dry cell mass. High cell density cultures carried out in the 21 bioreactor, in which it was attained $107 \mathrm{~g}$ dry cell mass/l, resulted in a dramatic improvement in asparaginase fermentation. As such, it was measured enzyme yields of $85,600 \mathrm{U} / \mathrm{l}$ and productivities of $1083 \mathrm{U} / \mathrm{l} \mathrm{h}$. 


\section{PB3}

Drugs affecting the intracellular NADPH equilibrium influence light emission in whole cell - assays based upon the bacterial luciferase reporter Lorenzo Galluzzi, Matti Karp Department of Biochemistry and Food Chemistry, Biotechnology, University of Turku, Tykistokatu 6, Biocity 6th floor, 20540 Turku, Finland.E-mails: lorenzo.galluzzi@utu.fi; deadoc@libero.it; deadoc@aliceposta.it (L. Galluzzi)

The bacterial luciferase operon from the bacterium Photorhabdus luminescens has been used, since its first description, for exceptionally different applications. These ranged from the environmental monitoring to the cell tagging, from the analysis of cellular metabolism to the high-throughput screening of novel compounds. The wild-type luxCDABE operon has been engineered in countless ways (for instance by changing the order of the constituent genes, by optimizing the codon usage and by coupling it to many promoters) and has been expressed in prokaryotic and eukaryotic organisms in order to meet precise research and commercial needs. Upon the operon expression light is emitted as the side product of a chemical reaction catalyzed by the luciferase enzyme, an $\alpha \beta$ heterodimer encoded in luxA and luxB genes. The reaction involves the oxidation of a long-chain aliphatic aldehyde and reduced flavin mononucleotide $\left(\mathrm{FMNH}_{2}\right)$ with the liberation of excess free energy in the form of a blue-green light at $490 \mathrm{~nm}$. The luxCDE genes code for the polypeptides (transferase, synthetase, and reductase) forming the fatty acid reductase complex that catalyzes the conversion of fatty acids into the long-chain aldehyde required for the luminescent reaction. Noteworthy is that for the production of the substrates for the luciferase both ATP and NADPH are required, while neither is involved in the actual light emitting reaction (Wilson and Hastings, 1998). Recently, the coupling of the luciferase operon to regulated promoters lead to the construction of genetically modified bacteria able to sense the presence of chemicals and to respond, in a dosespecific manner, with light emission. This approach has been applied to the detection of antibiotics in samples from the food industry as well as to the detection of heavy metal ions in environmental samples (Kurittu et al., 2000; Bechor et al., 2002). In addition, it opened the possibility of screening wide libraries of new compounds looking for molecules with pre-determined features, able to induce the bioluminescent response by de-repressing the lux operon transcription when incubated with the appropriate bacterial sensor. The high throughput and low costs are the main advantages of this system, which shows as well a certain degree of specificity (Galluzzi and Karp, 2003; Galluzzi et al., 2004). Nevertheless, since the in vivo bioluminescence relies upon a complex network of biochemical reactions, under certain circumstances the light emission is not a direct consequence of the lux operon transcriptional induction but it more likely originates at a posttranslational stage. As a matter of fact, one can suppose that a change in the light emission will be observed whenever the concentration of one or more substrates for the lux $\alpha \beta$ reaction occurs. Consequently, all the molecules sharing the ability to impair the delicate chemical equilibrium regarding the compounds involved in bioluminescence will be sensed by the bacteria as inducing compounds. This, in turn, will result in a loss of specificity of the assay. We investigated this aspect of the whole-cell assays based upon the bacterial luciferase operon for drug discovery by means of a reporter plasmid in which the luxABCDE genes, rearranged and optimized for the

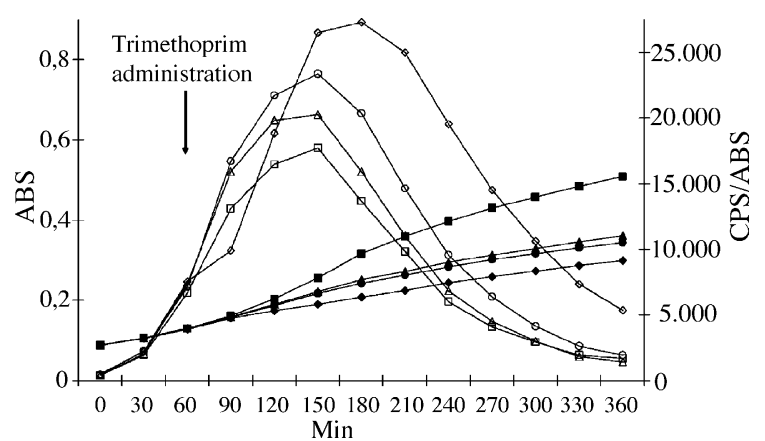

Fig. 1. Light emission from RN4220/pQacLux was induced with BC $1 \mu \mathrm{g} / \mathrm{ml}$ at time 0 . Uninduced cells (not shown) fail to emit light in a substantial extent (CPS/ABS values always under 1.200). After $60 \mathrm{~min}$ (black downward arrow) of incubation at $37^{\circ} \mathrm{C}$ under vigorous shaking the following concentrations of trimethoprim were added to the growing cells: $25 \mu \mathrm{g} / \mathrm{ml}$ (triangles), $50 \mu \mathrm{g} / \mathrm{ml}$ (circles) and $250 \mu \mathrm{g} / \mathrm{ml}$ (rumbles). Water was administered to control cultures (squares). Light emission was quantified every $30 \mathrm{~min}$ by means of the Wallac Victor 2 multilabel counter (Perkin-Elmer, Turku, Finland) and normalized to the absorbance, measured at $600 \mathrm{~nm}$ with the same device. Multi- 96 white-walled transparent-bottomed plates were used for the assays (Nalge Nunc, USA). Between the measurements the plates were kept at $+37^{\circ} \mathrm{C}$ under vigorous shaking. Filled symbols refers to the absorbance values (left side $Y$-axis); open symbols to the normalized count per seconds (right side $Y$-axis).

expression in Gram + organisms, are under the control of the QacR regulatory region from Staphylococcus aureus (Galluzzi et al., 2003). Non-pathogenic S. aureus RN4220 cells bearing the pQacLux plasmid (cultivated in LB broth supplemented with $0,5 \%$ D-glucose and $10 \mu \mathrm{g} / \mathrm{ml}$ chloramphenicol) emit light upon the specific transcriptional induction with quaternary ammonium compounds, widely used as surface disinfectants and in many over-the-counter drugs (Galluzzi et al., 2003). However, the incubation of the same cells with an inhibitor of the dihydrofolate reductase enzyme, trimethoprim (Sigma-Aldrich Chemie, Steinheim, Germany), enhanced in a dosedependant manner the light emission observed upon the induction with the optimal concentration $(1 \mu \mathrm{g} / \mathrm{ml})$ of benzalkonium chloride (BC). The extent of this increase in luminescence varied from 5-10\% to more than $200 \%$, according to the trimethoprim concentration and to the measurement time. Interestingly, when the same plasmid is carried by Escherichia coli XL1 cells, the lux operon is expressed constitutively (since the QacR regulatory region is not functional in XL1 cells) and at much higher levels than in induced RN4220 cells. Also in this experimental system the incubation with trimethoprim results in a dramatic increase of the luminescent signal from the cultures. The explanation for these observations can be found in the molecular mode of action of trimethoprim. The inhibition of dihydrofolate reductase, indeed, directly leads to the accumulation of its substrates, among which is NADPH, deeply entangled in the biochemical network of reactions centred on the light emission from the lux operon. NADPH provides the reducing power to restore the reduced flavin mononucleotide pool and it is as well involved in 


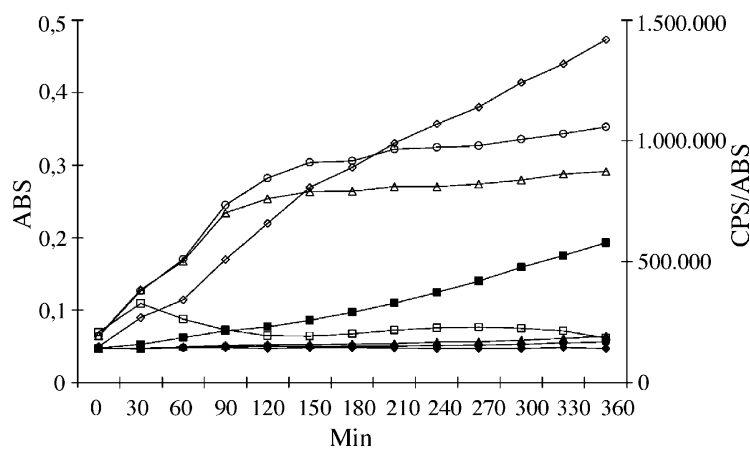

Fig. 2. XL1/pQacLux growing cells were incubated with the following concentration of trimethoprim: $25 \mu \mathrm{g} / \mathrm{ml}$ (triangles), $50 \mu \mathrm{g} / \mathrm{ml}$ (circles) and $250 \mu \mathrm{g} / \mathrm{ml}$ (rumbles). Water was administered to control cultures (squares). Light emission and absorbance measurements were performed as previously described for RN4220 cells. Filled symbols refers to the absorbance values (left side $Y$-axis); open symbols to the normalized count per seconds (right side $Y$-axis). The diverse level of growth observed for RN4220 and XL1 cells can be accounted by the different antimicrobial activity exerted by trimethoprim towards Gram - and Gram+ cells and by the lower activity of the promoter which in pQacLux plasmid drives the transcription of the selection marker (chloramphenicol acetyl transferase).

the production of the long-chain aldehyde, both substrates of the lux $\alpha \beta$ heterodimer (Wilson and Hastings, 1998). In conclusion, here we demonstrate that the use of light emission from the bacterial luciferase as a transcriptional reporter has to be very carefully controlled, since some molecules (here trimethoprim) could mimic to some extent a specific promoter activation by impairing the delicate intracellular biochemical equilibrium. On the reverse side of the coin,

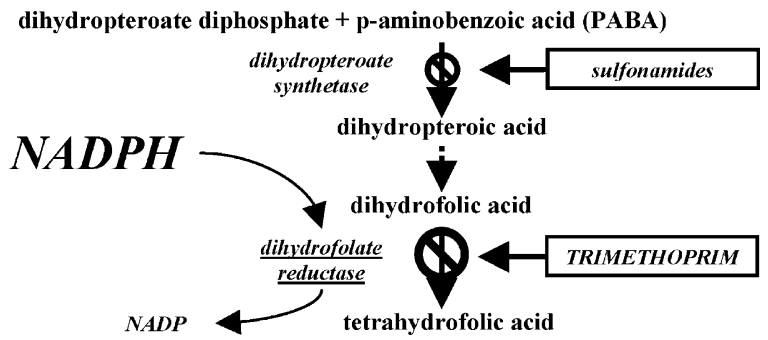

Fig. 3. Simplified scheme depicting the bacterial folate metabolic pathway. Only part of the reactions and compounds are reported. Trimethoprim inhibits the NADPH-dependant reduction of dihydrofolic acid into tetrahydrofolic acid catalyzed by dihydrofolate reductase. This results in the accumulation of both substrates, which become available for other reactions, and in the depletion of tetrahydrofolate, the major $\mathrm{C}_{1}$ carrier in the synthesis of purines, thymidine, glycine, methionine, and pantothenate in bacteria. For antimicrobial purposes trimethoprim is often associated with sulfonamides, with which it displays a synergistic activity (since they act on the same pathway but at an earlier reaction) (Scholar and Pratt, 2000). however, it has to be noted that the lux reporter system could be used in many different in vivo experimental setups, where the change in the intracellular concentration of NADPH, ATP or other metabolites would be sensibly detected.

\section{Acknowledgements}

Mrs. Lynnette Fernandez, Johanna Mäkeläinen, M.Sc. and Olli Rämö, M.Sc. are gratefully acknowledged for their help.

\section{References}

Bechor, O., Smulski, D.R., et al., 2002. J. Biotechnol. 94 (1), 125-132.

Galluzzi, L., Karp, M., 2003. J. Biomol. Screen 8 (3), 340-346.

Galluzzi, L., Virtanen, P., et al., 2004. Luminescence 19 (4), 225-227.

Galluzzi, L., Virtanen, P., et al., 2003. Biochem. Biophys. Res. Commun. 301 (1), 24-30.

Kurittu, J., Lonnberg, S., et al., 2000. J. Food Prot. 63 (7), 953-957. Scholar, M., Pratt, W.B., 2000. "The Antimicrobial Drugs" - Oxford University Press.

Wilson, T., Hastings, J.W., 1998. Annu. Rev. Cell Dev. Biol. 14, 197-230.

PB4

Comparative study of Pichia pastoris strains producers of five recombinant proteins $M$. Guerrero-Olazarán, E.L. Cab-Barrera, L. Rodríguez-Blanco, J.M. Viader-Salvadó Institute of Biotechnology, Biological Science School, Autonomous University of Nuevo León, San Nicolás de los Garza, N.L., 66450, México

We have carried out the construction of five Pichia pastoris strains harboring in their respective genome three different growth hormones cDNA's (22 and $20 \mathrm{kDa}$ human growth hormones and bovine growth hormone), a shrimp (Litopenaeus vannamei) trypsinogen cDNA and a bacterial (Bacillus subtilis) phytase gene. In all the cases, the same kind of expression vector and the same transformation technique were used. Each DNA was fused in frame to Saccharomyces cerevisiae alpha-factor secretion signal to lead the secretion of the foreign protein into the culture medium. The induction of each recombinant strain, all with $\mathrm{His}^{+}$and $\mathrm{Mut}^{+}$phenotype, was carried out in shake flaks using methanol buffered minimal medium (BMM) and growth rates on methanol determined. Production level of secreted proteins was evaluated by protein analysis by SDS-PAGE. Furthermore, the expression with three of the constructed strains was performed in a 5-L bioreactor and the level of secreted protein determined in each case. Both human growth hormones (HGHs) were secreted into the culture medium with a high degree of purity obtaining up to $64 \%$ of $\mathrm{HGHs}$ of total proteins in crude fermentation medium and $3-18 \mathrm{mg} / \mathrm{L}$ of HGHs. Neither bovine growth hormone, shrimp trypsinogen or bacterial phytase were detected in methanol induced cultures of the respective strains, in spite of the same culture conditions were used for all P. pastoris strains. The growth rates on methanol were different between some strains. In fermentor cultures the HGHs production were increased and shrimp trypsinogen was produced and secreted into the culture medium after improving the culture conditions. Bovine growth hormone was detected only when the culture conditions were modified. The protein production level for each strain was affected by the proprieties of each recombinant protein produced. 


\section{PB5}

Competitive advantages of the diagnostic method for invasive amoebiasis using preserved antigenic extracts without using enzymatic inhibitors M.S. Flores ${ }^{1,2 *}$, E. Tamez-Treviño ${ }^{2}$, R. Tijerina-Menchaca ${ }^{2}, F$. Bosques ${ }^{2}$, E. Rodríguez ${ }^{2}$, L.J. GalánWong $^{1}$, J.M. Viader-Salvadó ${ }^{1}$, M. Guerrero-Olazarán ${ }^{1}$, R. Rangel $^{2,3}$ : ${ }^{1}$ Instituto de Biotecnología, Facultad de Ciencias Biológicas. UANL. San Nicolás de los Garza, N.L., 66450, México; ${ }^{2}$ Facultad de Medicina, UANL. Monterrey, N.L., México; ${ }^{3}$ The University of Texas, USA; *U.S.A. Patents 5,459,042 and 5,861,263, Mexican Patents 209646 and 209648

We have patented a method to diagnose invasive amoebiasis using a novel assay that preserves antigenicity of extracts with high protease content without using enzymatic inhibitors (IC:MC). The available tests for serologic diagnosis of invasive amoebiasis like ELISA and indirect haemaglutination (IHA) do not have consistent results in endemic zones. Here we show the advantages of the assay and the validation of this diagnostic test for invasive amoebiasis. We demonstrated the reduction of proteolytic activity of IC:MC compared with the proteolytic activity of crude extract and crude extract with enzymatic inhibitors using assays. We displayed the IC:MC SDS-PAGE pattern and the Western Blot (WB) pattern useful for diagnosis. To search the clinical utility of this test we examined the WB obtained with sera from patients with different liver diseases; 90 patients had invasive amoebiasis and 45 patients had other liver diseases. The results were compared with those of IHA test. Also we have tested the accuracy of WB using sera from people with multiple intestinal parasites, like Giardia lamblia, Hymenolepis nana, Blastocystis hominis, Entamoeba coli, etc. The sensibility of the WB using the preserved amoebic antigens was $99 \%$, specificity was $100 \%$, positive predictive value was $100 \%$, negative predictive value was $98 \%$ and accuracy was $99 \%$. The WB did not exhibit cross reactions with sera from persons with intestinal parasites. Our test was better than the (IHA) test commonly used in endemic zones. These results show the improvement of using the preserved amoebic antigens in diagnostic tests. Also they prove the diagnostic accuracy of our new WB test.

\section{PB6}

Antibacterial and Antifungal Activity of Heracleum sphondylium subsp. artvinense Yasemin Kaçar ${ }^{1}$, Sema Tan ${ }^{2}$, Aysun Ergene ${ }^{2}$, Perihan Güler ${ }^{2}$, Semra Mirici ${ }^{3}$, Ergin Hamzaoğlu ${ }^{2}$, Ahmet Duran ${ }^{4}$, Sinem Yildirim ${ }^{2}:{ }^{1}$ Mersin University, Faculty of Science and Literature, Department of Biology, 33800 Mersin, Turkey; ${ }^{2}$ Kırıkkale University, Faculty of Science and Literature, Department of Biology, 71450 Yahsihan-Kırıkkale, Turkey; ${ }^{3}$ Akdeniz University, Faculty of Education, Department of Biology Education, 07400 Antalya, Turkey; ${ }^{4}$ Selcuk University, Faculty of Education, Department of Biology Education, 42300 Konya, Turkey

Turkey is covered yearly with a huge number of plant species. About 9222 species are condenced on the region that between Asia and Europe. Many plant species have been used in folkloric medicine to treat various ailments. Heracleum L (Apiaceae) is include over than 70 species on the world. This variety is represent with 17 species in Turkey that seven species are endemic. Heracleum sphondylium subsp. artvinense is endemic species for Turkey. Ethanolic and aquous extract of Heracleum sphondylium subsp. artvinense were investigated for their antimicrobial activities against six bacterial species (E. feacalis, E. coli, S. aureus, P. aeruginosa, L. monocytogenes, Shigella) and two yeast (C. albicans, C. krusei). Both ethanolic and aqueous extract of Heracleum sphondylium subsp. artvinense showed antimicrobial activity against the gram negative bacterium (Shigella) and gave the best activity against C.albicans.

\section{PB7}

Production of nanovesicles entrapping nucleic acids Tsutomu Ono; Mitsuhiro Morita, Shinji Sakai, Hiroyuki Ijima, Koei Kawakami, Department of Chemical Engineering, Kyushu University, Fukuoka 812-8581, Japan. E-mail: tono@chemeng.kyushu-u.ac.jp (T. Ono)

To develop artificial vectors allowing nucleic acid to transfect into mammalian cells are crucial for extending gene therapy. Synthetic vectors based on lipid molecules are particularly attractive because of their potential safety. However, the low encapsulation efficiency of nucleic acid is one of the problem to be solved. Recent advances in DNA-lipid complex have improved this drawback, and now some lipid molecules are used as transfection agents. In particular, cationic lipids interact with negatively-charged cell surfaces and nucleic acids. The former interaction results in delivery of the nucleic acids directly across the cell membrane. On the other hand, the latter interaction improves the efficiency of nucleic acids entrapment. In this study, we developed a preparation method of "nanovesicle" containing nucleic acids by using reverse micellar solubilization. Since DNA interacts spontaneously with cationic amphiphiles, complete extraction of the DNA molecules into an organic phase using dimethyl distearyl ammonium bromide (2C18AB), an oil-soluble cationic surfactant, is achieved. The re-encapsulation of the reverse micellar droplets solubilizing DNA by water-soluble surfactants facilitates the formation of nanovesicles. A high salt concentration at the re-encapsulation step promotes the production of nanovesicles containing DNA molecules. Eventually, more than $80 \%$ of DNA was encapsulated in this nanovesicles under the condition of $6 \mathrm{M} \mathrm{NaCl}$ and $5 \%(\mathrm{w} / \mathrm{v})$ cetyltridecyl ammonium bromide (CTAB). In addition, the nanovesicles prepared at $45^{\circ} \mathrm{C}$ was smaller than that prepared at room temperature. The resultant $2 \mathrm{C} 18 \mathrm{AB} / \mathrm{CTAB}$ nanovesicle was ca. $16 \mathrm{~nm}$. Asymmetric and chemically-modifiable vesicle surface are effective to design gene delivery system. Moreover, such a small DNA (or RNA) carrier has a potential for novel gene therapy application from skin.

\section{PB8}

siRNA-mediated down-regulation of cell-adhesion molecules therapeutic potential or not? Holger K. Riemann ${ }^{1}$, Ida Jørring ${ }^{1}$, Trine Moller ${ }^{1}$, Lars H. Pedersen ${ }^{1}$, Kim Holmstrom ${ }^{1}$, Thomas Brevig ${ }^{2}:{ }^{1}$ Bioneer A/S, Hørsholm, DK-2970, Denmark; ${ }^{2}$ Nunc A/S, Roskilde, DK-4000, Denmark. E-mail: hkr@bioneer.dk (H. Riemann)

The key players in clinically important inflammatory diseases, endothelial cells and leukocytes, communicate through membranebound cell adhesion molecules (CAM's). Obvious strategies for therapeutic intervention include specific means to affect the expression of CAM's on the cells involved. RNA-interference (RNAi) is a well known means to achieve specific gene-inhibition. For this study, two CAM's were chosen, namely vascular cell adhesion molecule1 (VCAM-1) as a target for inhibition, and intercellular adhesion molecule-1 (ICAM-1) as a non-target reference. We designed short 
interfering RNA (siRNA) oligos for VCAM-1 in order to selectively inhibit the expression of this CAM in cultured human vascular endothelial cells (HUVEC). Real-time RT-PCR showed an $80 \%$ down-regulation of VCAM-1 mRNA while the expression of ICAM1 remained unaffected. Neither CAM was affected by non-specific siRNA. In order to further substantiate the potential use of siRNA for therapeutic purposes, we have set out to investigate two things:

(1) Does VCAM-1-specific siRNA affect the amount of VCAM-1 on the surface of HUVEC?

(2) Is adhesion between leukocytes and endothelial cells affected by VCAM-1-specific siRNA?

\section{PB9}

Plasmid DNA production J.A.L. Santos, S.S. Freitas, D.M.F. Prazeres Centro de Engenharia Biológica e Química (CEBQ), Instituto Superior Técnico, 1049-001 Lisboa, Portugal. E-mail: josesantos@ist.utl.pt (J.A.L. Santos)

The manufacturing of plasmid DNA (pDNA) is crucial to obtain a consistent product for gene therapy applications. Although flowsheets for pDNA production are established on the basis of experience, simulation tools provide a valuable help for evaluating alternatives. A process designed to produce $23 \mathrm{~kg} \mathrm{pDNA} / \mathrm{year}$ is analysed with the SuperPro Designer tool. The target pDNA is amplified in Escherichia coli. After harvest, alkaline lysis is used to disrupt cells and release pDNA and impurities. Precipitations with isopropanol and ammonium sulphate are performed to concentrate/pre-purify pDNA prior to hydrophobic interaction chromatography. Experimental data is used as input for simulation. Inventory analysis identified water, yeast extract, tryptone, isopropanol and ammonium sulphate as the major raw materials. Major raw material costs (50\%) are related to fermentation components. Economic analysis indicates a unit production cost of $\$ 375 / \mathrm{g}$ pDNA. For a selling price of $\$ 1667 / \mathrm{g}$, the payback time was 1.2 years and the ROI was $88.6 \%$. An environmental analysis highlighted the replacement of the isopropanol precipitation for a microfiltration step as a benefit which would: (i) reduce the cost of raw materials (13.8\%), (ii) reduce the environmental impact associated with isopropanol (70\%) and (iii) reduce costs associated with the treatment/disposal of liquid waste (32.3\%).

\section{PB10}

Preparation of chitosan microspheres for controlled release of somatotropin $\underline{\text { S. Simsek }}{ }^{1}$, J. Akbuğa $a^{2}:{ }^{1}$ Department of Research and Development, Ilsan Hexal Pharmaceutical Company, 41400 Gebze, Kocaeli, Turkey; ${ }^{2}$ Department of Pharm. Biotechnology, Faculty of Pharmacy, Marmara University, 34668 Haydarpaşa, Istanbul, Turkey. E-mail: serhan_simsek@ilsan.com.tr (S. Simsek)

Introduction: Proteins and peptides have received extensive interest for their therapeutic applications in clinical applications. In order to achieve high administration efficacy of proteins, polymeric particulate carriers have been developed as an effective way to control the drug release profile and to protect the protein molecules from degradation. Somatotropin also known growth hormone is a protein hormone of about 190 amino acids. Growth hormone is of considerable interest as a drug used in both humans and animals. Chitosan a natural linear biopolyaminosaccharide is obtained by alkaline deacetylation of chitin. Properties such as biodegradability, low toxicity and good biocompatibility make it suitable for use in biomedical and pharmaceutical formulations. The aim of this study was to prepare chitosan microspheres containing somatotropin and to investigate these microsphere formulations in-vitro release properties.

Methods: Somatotropin-chitosan microspheres were prepared as follows: Chitosan was dissolved in acidic solution (2\%) containing polysorbate 80 . Sodium sulphate solution $(20 \% \mathrm{w} / \mathrm{v})$ containing somatotropin was added into the chitosan solution and mixed $500 \mathrm{rpm}$ for a hour. Resulting suspension was centrifuged 15,000 rpm $15 \mathrm{~min}$ at $4{ }^{\circ} \mathrm{C}$. The formed microspheres were freeze-dried and sieved. In-vitro release studies were performed in $\mathrm{pH} 7.4$ phosphate buffer and time interval samples were removed and analysed by Bradford protein assay method.

Results: Microspheres were obtained by using chitosan. Protein encapsulation efficiency was between 95 and $99 \%$. Average particle size of microspheres was between 48 and $57 \mu \mathrm{m}$. During to in-vitro release studies burst effect was observed with chitosan microspheres. For decreasing the burst effect gluteraldehit, betacyclodextrin and poly ethylene oxide were added to formulations.

Conclusion: According to our results modified chitosan microspheres are promising vehicles for controlled release somatotropine delivery.

\section{PB11}

Cancer immunotherapy using hyperthermia with magnetic

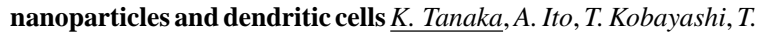
Kawamura, S. Shimada, K. Matsumoto, T. Saida, H. Honda Department of Biotechnology, School of Engineering, Nagoya University, Nagoya, Aichi 464-8603, Japan. E-mail: h041306d@ mbox.nagoyau.ac.jp (K. Tanaka)

Our hyperthermia system utilizes magnetic nanoparticle covered with lipid layer including cationic lipid (magnetite cationic liposomes, MCLs) as a heating mediator and necrotic cell death is induced by means of locally generating heat. In this process, heat shock proteins (HSPs) are strongly induced and released. Dendritic cells (DCs) are potent antigen-presenting cells (APCs) that play a pivotal role in regulating immune responses in cancer, which is in carrying antigens to APCs and in the maturation of DCs by acting as a danger signal. In the present study, we investigated the therapeutic effects of DC therapy combined with MCL-induced hyperthermia on B16 melanoma. In an in vitro study, when immature DCs were pulsed with B 16 cells heated at $43^{\circ} \mathrm{C}$ for $30 \mathrm{~min}$, MHC class I/II, costimulatory molecules CD80/CD86, and chemokine receptor CCR7 in the DCs were up-regulated, thus resulting in DC maturation. C57BL/6 mice bearing a B16 melanoma nodule were subjected to combination therapy using hyperthermia and DC immunotherapy. Mice were divided into four groups: group I (control), group II (hyperthermia), group III (DC therapy), group IV (hyperthermia + DC therapy). Complete regression of tumors was observed in $60 \%$ of mice in group IV, while no tumor regression was seen among mice in the other groups. Increased CTL and NK cell activity was observed on in vitro cytotoxicity assay using splenocytes in the cured mice treated with combination therapy, and the cured mice rejected a second challenge of B16 melanoma cells. This study has important implications for the application of MCL-induced hyperthermia plus DC therapy in patients with advanced malignancies as a novel cancer therapy. 


\section{PB12}

Fermentation of a marine bacterium for the production of cytotoxic compounds Vicky Webb ${ }^{1}$, Els Maas ${ }^{1}$, Eiichi Akaho ${ }^{2}$, Hiroto Kambara $^{2}$, Debbie Hulston ${ }^{1}$, Anna Kilimnik ${ }^{1}:{ }^{1}$ Marine Biotechnology, National Institute for Water and Atmospheric Research Ltd, Kilbirnie, Wellington New Zealand; ${ }^{2}$ Faculty of Pharmaceutical Sciences, Kobe Gakuin University, Kobe 651-2180, Japan

Marine bacteria are a potential source of novel compounds for the pharmaceutical industry. New Zealand marine bacteria were isolated from a variety of sources and initially bacterial supernatants were screened using a cell based MTT cytotoxic assay. Two bacteria were chosen for further fermentations in different media to optimise cytotoxic compound production. The media used were selected for their diverse ingredients. They were either carbon rich, nitrogen rich, starch rich or a basic seawater medium. The fermentations were extracted using ethyl acetate and methanol prior to assaying. The results showed that cytotoxic compound production was enhanced ten fold in the starch rich medium compared to the other media. The levels of cytoxicity appeared to be depended on the cell line used, with the epithelial lung carcinoma cell line A549 being more sensitive to the cytotoxic compounds than the human fibroblast cell line MRC-5.

\section{PB13}

Isolation and identification of marine bacteria from deep-sea sediments Els Maas ${ }^{1}$, Cara Brosnahan ${ }^{1}$, Vicky Webb ${ }^{1}$, Helen Neil $^{2}$, Phil Sutton ${ }^{2}{ }^{1}$ Marine Biotechnology, National Institute for Water and Atmospheric Research Ltd., Kilbirnie, Wellington New Zealand; ${ }^{2}$ Oceanography, National Institute for Water and Atmospheric Research Ltd., Kilbirnie, Wellington, New Zealand

Polystyrene micro plate activated by utilizing a common Co60 gamma source with a crotonic acid. The well surface have been studied in term of optical quality, protein (H-IgG) binding capacity and stability. A significantly enhanced total capacity and strength of binding to grafted surfaces was demonstrated as compared to passive adsorption of the proteins to untreated surfaces.The majority routine laboratory tests for the measurement of rheumatoid factor (RF) are semi quantitative and in order to achieve accurate, sensitive and specific RF assay, we are developed enzyme linked immuno sorbent assay (ELISA) for human IgM-RF kit. Stable and reproducible binding of antigen (Human- $\mathrm{IgG}$ ) to well of micro titer plate is a prerequisite for RF-ELISA kit. The principle of the assay was that the antibodies in serum of patients with rheumatoid arthritis (RA), commonly rheumatoid factor (RF) are directed to the Fc part of human IgG. In most cases RF belong to the IgM class, the well of micro titer plate are coated with antigen (H-IgM), and antibodies (H-IgG) binding to immobilized antigen is detected by adding enzyme conjugate (anti human-IgM-HRP-enzyme) to the wells, substrate was used for color reaction. The performance characteristics of the assay was, the coefficient of variation (C.V) of intra and inter assay was $4.4 \%$ and $2.2 \%$ respectively, the linearity is ranging from 86 to $112 \%$, and the recovery for three different sera is ranging from 89 to $106 \%$. The data presented in this paper indicated that the activation of polystyrene micro titer plate by the gamma rays could be use for preparation of IgM-RF kit and may be others immunoassay techniques.

\section{PB14}

Overcoming the nuclease barrier to gene expression during trafficking of plasmid DNA vectors A.R.Azzoni ${ }^{1}$, A. Tavares $^{2}$, G.A. Monteiro $^{1}$, D.M.F. Prazeres ${ }^{1}{ }^{1}$ Centro de Engenharia Biológica e Química (CEBQ), Instituto Superior Técnico, 1049-001 Lisboa, Portugal; ${ }^{2}$ Instituto Gulbenkian de Ciência, Rua da Quinta Grande 6, 2780-156 Oeiras, Portugal. E-mail: azzoni@ ist.utl.pt (A.R. Azzoni)

Inefficient nuclear delivery of plasmid DNA (pDNA) vectors is thought to be a bottleneck to gene transfer in gene therapy and DNA vaccination utilizing non-viral delivery systems. One of the main barriers found by pDNA vectors during trafficking to the nucleus is degradation by a population of endo/exo-nucleases. This barrier may be partially circumvented by shielding the pDNA from the nucleaserich cell environment with adjuvants or by using nuclease inhibitors. A different approach that has been studied at the CEBQ is the generation of pDNA vectors that are more resistant to nuclease action a priori. In this work, the nuclease barriers to gene expression are being studied aiming at the generation of pDNA with improved resistance to nucleases and thus higher transfection efficiency. By engineering the plasmid labile sequences, new plasmid vectors with an improved resistance to physical-chemical and biological degradation are being generated. This was indicated by an extended half-life of the supercoiled isoforms during storage and when the plasmids were exposed to nucleases present at eukaryotic cell lysates and mice plasma. The intracellular trafficking of the new plasmid vectors through the cytosol of mammalian cells was then assessed by fluorescence in situ hybridisation (FISH) and the expression of the reporter protein (eGFP) was detected by fluorescence microscopy.

\section{PB15}

Identification and evaluation of antibacterial phytochemicals of fishbone fern (Nephrolepis cordifolia) Rikhia Chakraborty ${ }^{1,2}$, Promod Kumar Verma ${ }^{2}$ : ${ }^{1}$ Department of Cancer Biology, Lerner Research Institute, 9500 Euclid Avenue Cleveland, OH 44195, USA, ${ }^{2}$ Department of Biotechnology, Guru Nanak Dev University, Amritsar, Punjab 143005, India. E-mail: riar5400@rediffmail.com (R. Chakraborty)

In this study, different aqueous and organic extracts from the fern, Nephrolepis cordifolia, were used for screening tests for antibacterial effects. Protein and lipid extracts were first tested for antibacterial activity. Subsequently, crude extracts of leaves, roots, and stems were prepared in methanol, ethanol, chloroform, hexane, petroleum ether, diethyl ether, and water using optimized standard protocols. Each fraction was tested for anti-microbial effect through Agar well-diffusion assay, and Paper disc method. The antibacterial spectrum against which the fern is active was thus determined. Dosagedetermination for optimum activity was also determined for each of the extracts. Bacillus and Staphylococcus were used as the indicator test-organisms. The results were very encouraging; being effective even at the 54th day, thus showing that the antibacterial properties were not due to any changes in external factors and physiological effects. Ethanol, methanol and chloroform extracts from the subaerial portions had strong anti-microbial properties. agar-well diffusion assay and paper-disc diffusion assay done for different solvent fractions were giving comparable results. $2.5 \mathrm{mg}$ was adequate for maximum effect against $B$. circulans, $S$. aureus, $S$. epididermis, and Streptococcus sp. $7.5 \mathrm{mg}$ was adequate as effective dosage for Kleb- 
siella pneumoniae. $10 \mathrm{mg}$ was required for Mycobacterium bovis, E. coli. Pseudomonas was not showing any susceptibility. Given the broad spectrum of activity, especially towards the gram-negative bacteria, Nephrolepis cordifolia is definitely a promising plant having pharmacological importance. For a full interpretation of the present results further investigations are necessary to elucidate the different physical and chemical parameters of the active principles and also to determine the mechanism of action. The present work highlights $N$. cordifolia as a plant having a broad spectrum of antimicrobial activity, a phenomenon very rarely observed in the plant kingdom.

\section{PB16}

Multiple antibiotic resistances in hospital soil-derived bacteria and effects of antimicrobial agents in garlic and honey Mousumi Chaudhury, S. Andhale, S.M. Dharmadhikari Departmet of Microbiology, Government Institute of Science, Dr. BA Marathwada University, Nipatniranjan, Aurangabad 431001, India. E-mail: mousumichaudhury@ rediffmail.com (M. Chaudhury)

Bacteria (Escherichia, Salmonella, Proteus, Staphylococci and Bacillus) were isolated from hospital soil using selective enrichment and growth on selective and differential medium, viz. MacConkey's agar, CLYED medium and Baird Parkers medium. Confirmation and species identification was carried out by biochemical and serological tests. From these isolates, three different pathogens were used to study multiple antibiotic resistances. E. coli BJ 83 showed resistance to ampicillin, streptomycin and cefurixime. S. typhi and $S$. aureus showed resistance to ampicillin and cefuroxime. Assay using Octadisc using E. coli and $S$. typhi showed a broader resistance pattern to antibiotics including amoxicillin, clavulanic acid, cephalexin, chloramphenicol, ciprofloxacin, and cotrimoxazole. The $\mathrm{R}$ plasmid profile was studied to understand the mechanism of drug resistance. To combat the problem of drug resistance, a strategy of combined antibiotic response of cultures were studied. Such a synergistic combination would possibly have the effect of overcoming multiple antibiotic resistances. For e.g. kanamycin resistance strains were inhibited in presence of kanamycin and cefotaxime. Further, the bactericidal activities of antimicrobials in honey and garlic were also tested. The MIC of honey was observed to be $4 \%$, while that of garlic was between 0.1 and $1 \%$. Honey and garlic were also found to inhibit the growth of organisms in the presence of antibiotics. Kanamycinresistant $E$. coli was unable to grow in presence of kanamycin and $0.08 \%$ garlic. These traditional agents, long used in Ayurvedic system of medicine in India, could be further explored for potent antimicrobial properties.

\section{PB17}

Comparison of two HBV DNA PCR commercial kits: PCR test interpretation must be done with great care Akbari Eidgahi, Mohammad Reza, Shabani Ali Akbar, Hadjighorbani Amir Hosein Center for Biotechnology Research, Semnan University of Medical Sciences and Semnan Management and Programing Org, Semnan, Iran. E-mails: akbari@ sem-ums.ac.ir (A. Eidgahi), mrakbari_2000@yahoo.com (M. Reza)

Hepatitis B virus (HBV) infection is s global health problem. Assays for HBV antigens and antibodies are widely available and standardized. Extremely sensitive qualitative PCR kits are also available for detection of HBV in serum. HBV PCR kit may be useful in assessment of occult hepatitis B in $\mathrm{HBcAb}$ positive alone subjects and carriers. A positive PCR results show presence of virus articles in serum without considering serologic results. But there are differences in efficiency of HBV DNA amplification kits. In this study we compare two commercially available HBV PCR kits for evaluation viremia of $\mathrm{HBsAg}$ positive carriers and $\mathrm{HBcAB}$ alone positive subjects.

Material and Methods: Of the 368 randomly selected subjects serologically examined for $\mathrm{HBV}, 49$ and 43 were positive for $\mathrm{HBsAg}$ and $\mathrm{HBcAb}$ alone respectively. Both later groups were tested for $\mathrm{HBV}$ DNA by two commercial kits, HBV PCR detection kit (Cinnagen, Iran) and HBV PCR test (Pazhohesh Azma, Iran). DNA extraction kits recommended by each manufacturer were used for HBV DNA extraction. Amplicons in both kits were a highly overlapped fragment in $5^{\prime}$ conserved sequence of viral genome.

Results: Of the $49 \mathrm{HBsAg}$ positive carriers, HBV DNA was detected in 37.4 and $65.3 \%$ using kit 1 and kit2 respectively. Only $28.6 \%$ were positive in both kits. In $\mathrm{HBcAb}$ positive subjects $(n=43), 23.3 \%$ were positive by kit 2 and all samples were negative when tested by kit 1 . There was a significant difference between two kits. Sensitivity of kit 1 and kit 2 were 48.6 and $91.4 \%$, respectively. Overall, kit 2 increased the detection rate of HBV DNA by $88.2 \%$.

Discussion: Our study show there is a significant variation between these two commercial kits especially in $\mathrm{HBcAb}$ positive subjects that the copy of virus is very low. From these results, it can be concluded that the unstandardized kits have not compatible results, and PCR test interpretation should be done with great care.

\section{PB18}

Isolation and characterization antibacterial bioactive compounds and peptides from Malaysian medicinal plants Benafri Asma $^{1}$, Abd-ElAziem Farouk ${ }^{1}$, Syed Z. Idid ${ }^{2}{ }^{1}$ Biomolecular Engineering Research Group, Department of Biotechnology Engineering, Kulliyyah of Engineering, International Islamic University Malaysia, Jalan Gombak, 53100, Kuala Lumpur, Malaysia; ${ }^{2}$ Biomedical Science Department, Kulliyyah of Science, International Islamic University Malaysia, Jalan Gombak, 53100, Kuala Lumpur, Malaysia

Ethanol, methanol and acetone extracts of various parts of three plant species (Annona muricata, Annona squamosa and labisia pumila) used in traditional Malaysian medicine were investigated for their antibacterial activity against fourteen species of pathogenic bacteria, i.e. eight Gram positive (Bacillus subtilis, Staphylococcus aureus,Staphylococcus saprophyticus, Staphylococcus epidermidis, MRSA, Streptococcus pyogens, Enterococcus feacalis and Micrococcus leteus) and six Gram negative (Escerichia coli, Klebsiella pneumonae, Salmonella typhi,, Pseudomonas aerugenosa, Proteus vulgaris, and Sarcina marcescens). The antibacterial activity of the extracts was evaluated based on the inhibition zone using plate diffusion method. Most of the extracts were active against both Gram positive and gram-negative bacteria, but Pseudomonas aerugenosa, Bacillus subtilis and sarcina marcescens, were more susceptible to almost all the extracts. Finally, further research will be done to elucidate the nature of the active compound and investigate for peptides by using protein gel immobilization bioassay. 


\section{PB19}

Short interfering RNA delivery and gene silencing using polymeric nanocarrier systems K.A. Howard ${ }^{1}$, X. Liu $^{1}$, D. Oupicky ${ }^{2}$, F. Besenbacher $^{1}$, J. Kjems ${ }^{1}$ : $^{1}$ iNANO, University of Aarhus, Denmark, ${ }^{2}$ Department of Pharmaceutical Sciences, Wayne State University, Detroit, USA

The effectiveness of a drug is determined by the ability to migrate through the body and reach target sites in therapeutically relevant levels. Nanocarriers for delivery of bioactive agents are being developed at iNANO to maximise drug payload at target sites. The inclusion of "biological triggers" into the nanocarrier design is used for modulation of cellular nucleic acid trafficking and increased target interaction. Chitosan and peptide-based polymers were used to formulate nanocarriers in the size range $30-250 \mathrm{~nm}$ containing small interfering RNAs (siRNAs) for gene silencing applications. PAGE analysis showed the structural integrity of the siRNA was maintained during particle formation. In systems composed of bioresponsive polymers, nanocarrier disassembly and siRNA release under cellular conditions were shown, using Atomic Force Microscopy. The time course for siRNA uptake into NIH cells was visualised using confocal microscopy. In addition, siRNA localisation within cells could be modulated by the composition of the polymer used. The ability of the nanocarrier system to mediate gene expression was investigated in a cell line stably expressing enhanced green fluorescent protein (eGFP). Furthermore, the various delivery systems were tested in a mouse model stably expressing the eGFP protein using both nasal and intravenous delivery routes.

\section{PB20}

Genetic modification of swine genome suitable for xenotransplantation Daniel Lipinski ${ }^{1}$, Wojciech Juzwa ${ }^{2}$, Joanna Zeyland ${ }^{2}$, Ewa Michalak ${ }^{2}$, Robert Kalak ${ }^{2}$, Andrzej Plawski ${ }^{1}$, Lucyna KatskaKsiazkiewicz $^{3}$, Bozenna Rynska ${ }^{3}$, Zdzisław Smorag ${ }^{3}$, Ryszard Slomski ${ }^{1,2}$ : ${ }^{1}$ Institute of Human Genetics, Polish Academy of Sciences, Poznan 60-479, Poland; ${ }^{2}$ Department of Biochemistry and Biotechnology, Agricultural University, Poznan, 60-637 Poland; ${ }^{3}$ Department of Animal Reproduction Biotechnology, Immuno- and Cytogenetics, National Research Institute of Animal Production, Balice 32-083, Poland. E-mail: lipin1 @ poczta.onet.pl (D. Lipinski)

The use of animals as a source of organs and tissues for xenotransplantation can overcome the growing shortage of human organ donors. However, the presence of xenoreactive antibodies in humans directed against swine Gal antigen present on the surface of xenograft donor cells leads to the complement activation and immediate xenograft rejection as a consequence of hyperacute immunological reaction. The graft of genetically modified organ of a swine depleted of enzyme $\alpha 1,3$-galactosyltransferase that is responsible for $\mathrm{Gal}$ antigen origin, would be tolerated with simultaneous administration of medicines decreasing other less severe immunological reactions. To prevent hyperacute rejection it is also possible to modify swine genome by human genes controlling enzymatic cascade of complement or modifying the set of donor's cell surface proteins. For this purpose genetic constructs containing inactivated $\alpha 1,3$-galactosyltransferase gene, human CD59, CD55 and CD46 genes controlling complement activation and human genes encoding $\alpha 1,2$-fucosyltransferase and $\alpha$-galactosidase enzymes modifying cell surface proteins were prepared. These genetic constructs were transfected into the pig foetal fibroblast using lipofection method. After selection, molecular and cytogenetic characteristic of cells with transgene integrated into the host genome were performed. Nuclear transfer of these cells can generate pigs with the appropriate genotype. Supported by SCSR grants 048/P05/2001/03 and 048/P05/2001/04.

\section{PB21}

Cloning, expression and biological activity of protease $2 \mathrm{~A}$ from coxsakievirus B3 Nader Maghsoudi $^{1}$, Mehdi Zeinoddini ${ }^{2}$, Seyyed Mohammad saide $\overline{\text { Hosseini Amini }}{ }^{2}$, M.A. Nasiri Khalili ${ }^{2}$, A.A. Deldar $^{2}:{ }^{1}$ Neuroscience Research center, Shahid Beheshti Medical Science Univ., Tehran, Iran; ${ }^{2}$ Biotechnology Research Center, M.A.U., Tehran, Iran. E-mail: maghsudi@yahoo.com (N. Maghsoudi)

Introduction: Protease 2A(2A-Pro) of coxsackievirus B3 (CVB3) plays major role in viral replication. In case of infection, viral proteins are being synthesized from viral mRNA using host biosynthesis machinery. 2A-Pro of virus, after being synthesized, exhibit two critical functions, cleavage of viral proteins and breaking eIF4G (eukaryotic initiation factor 4G-formerly called P220) which leads to host cell translational system shot-off. The enzyme plays essential role in viral replication and cellular damage. To understand pathogenicity of infection and also developing potent and selective inhibitors against picornavirus infection, it is necessary to prepare pure 2Apro enzyme. In this study an expression system with efficient and high yields was obtained.

Methods: cDNA of 2Apro was synthesized using in vitro infection of permissive host through reverse transcription process and was cloned in pET22b(+) and since 2A-Pro is a toxic product, naturally before induction its expression will act on the host and damage the cells. For this different hosts were checked and finally, $B L R(D E 3) p L y s S$ which carries an extra-plasmid for lysozyme expression that minimizes unwanted target protein production (leakage) was selected. On the other hand For biological activity assay, polyclonal antibodies against antigenic sites of $\mathrm{P} 220$ was prepared by synthesizing small peptides, corresponding to antigenic site of P220 coupling to KLH and injecting subcutanously to rabbit. Then, the enzyme and its substrate (HeLa cells lysate that contain P220) were incubated together for different times intervals.

Results: The recombinant product was confirmed by SDS polyacrylamide gel electrophoresis and immunoblot analysis. Also P220 cleavage by 2 Apro was assessed by SDS-PAGE and western blot analysis. Cleavage of $\mathrm{P} 220$ by r2A-pro was prominent after $24 \mathrm{~h}$. So recombinat 2A-Pro with good activity was prepared.

\section{PB22}

Application of Bombyx mori nuclear polyhedrosis virus (BmNPV) bacmid system on production of glycoprotein in larvae of silkworm Ayano Kageshima, Tatsuya Kato, Misun Kwon, Enoch Y. Park Department of Applied Biological Chemistry, Shizuoka University, Ohya 836, Suruga-ku, Shizuoka 422-8529, Japan

Bombyx mori nuclear polyhedrosis virus (BmNPV) bacmid system was applied on production of glycoprotein in larvae of silkworm. The bacmid system of Autographa californica nuclear polyhedrosis virus (AcNPV) has already been established and widely used. Since the AcNPV does not have a potential to infect silkworm we 
developed the first practical Bombyx mori nuclear polyhedrosis virus (BmNPV) bacmid system directly applicable for the protein expression of silkworm. By using this system, the green fluorescence protein and glycoprotein, human $\beta 1,3-N$-acetylglucosaminyltransferase 2 were successfully expressed in silkworm larvae not only by infection of its recombinant virus but also by direct injection of its bacmid DNA. Three different kinds of signal sequences were tested for the secretion of glycoprotein into hemolymph of silkworm. Signal peptides of prophenoloxidase-activating enzyme and bombyxin: insulin-like brain secretory peptide showed the highest secretion ratio, $99 \%$ of total expressed $\beta 1,3-N$-acetylglucosaminyltransferase 2 was secreted into hemolymph of silkworm. Using Bacmid system $50 \mathrm{mU} / \mathrm{ml}$ of $\beta 1,3-N$-acetylglucosaminyltransferase 2 was expressed in hemolymph of silkworm, which was 2-three times higher than that of insect cell. Silkworm is one of the most attractive hosts for large-scale productions of eukaryotic proteins as well as recombinant baculoviruses for gene transfer to mammalian cells. This method provides the rapid protein production in silkworm, is free from biohazard, thus will be a powerful tool for the future production factory of recombinant eukaryotic proteins and baculoviruses.

\section{PB23}

Development of transgenic pollen-derived edible vaccine Hee Sung Park ${ }^{1}$, Eun Hae Park ${ }^{1}$, Young Joo Huh ${ }^{1}$, Soo Sung Kim ${ }^{1}$, Yong Heo ${ }^{2}:{ }^{1}$ Department of Biotechnology, Catholic University of Daegu, Kyungbuk 712-702, Korea; ${ }^{2}$ Department of Occupational Health, Catholic University of Daegu, Kyungbuk 712-702, Korea. E-mail: hspark@cu.ac.kr (H.S. Park)

We have established an efficient system for foreign gene expression in lily (Lilium longiflorum) pollen in a transient mode. Pollen was transformed using Agrobacterium via vacuum filtration for $20 \mathrm{~min}$. The pollen germinated for $24 \mathrm{~h}$ was analyzed to confirm its foreign gene expression in molecular analysis. Mouse fed the transgenic pollen culture for 8 weeks showed immune reaction specific for pollen-derived recombinant protein. And the IgG level was highly elevated by one time boosting injection afterwards. The lily pollen system may be suggested as a novel type of biofactory for producing edible vaccine with rapidity.

\section{PB24}

Anti-apoptosis engineering with the $30 K c 6$ gene obtained from silkworm Shin Sik Choi, Won Jong Rhee, Tai Hyun Park School of Chemical and Biological Eng., Seoul National University, Seoul 151744 , Korea

The Chinese hamster ovary $(\mathrm{CHO})$ cell line producing recombinant human erythropoietin (EPO) was manipulated to express the $30 K c 6$ gene, which was originally obtained from a silkworm. The expression of $30 K c 6$ inhibited serum deprivation-induced apoptosis and increased the cell density and EPO expression level per unit cell by five- and two-folds, respectively. An increase in these two factors resulted in a 10-fold increase in the volumetric productivity of EPO. Compared with the controls, the oligosaccharide structures of the EPO synthesized by the cells expressing $30 K c 6$ showed greater homogeneity. The terminal sialylation of the glycans of EPO were promoted by the expression of $30 K c 6$. The positive effects of $30 K c 6$ expression on the cell viability and productivity were attributable to the stable maintenance of the mitochondrial activity. These results demonstrate that the $\mathrm{CHO}$ cell line genetically engineered with the
$30 K c 6$ gene has a great potential for use in the production of therapeutic proteins.

\section{PB25}

Evaluation of fucoidan-chitosan hydrogels on superficial dermal burn healing in rabbit: An in vivo Study A.D. Sezer ${ }^{1}, F$.

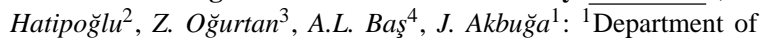
Pharm. Biotechnology, Faculty of Pharmacy, Marmara University, 34668 Haydarpaşa, Istanbul; ${ }^{2}$ Department of Pathology, Faculty of Veterinary, Selçuk University, 42075 Konya, Turkey; ${ }^{3}$ Surgery, Faculty of Veterinary, Selçuk University, 42075 Konya, Turkey; ${ }^{4}$ Pharmacology, Faculty of Veterinary, Selçuk University, 42075 Konya, Turkey. E-mail: adsezer@marmara.edu.tr (A.D. Sezer)

Introduction: Healing of dermal wounds with macromolecular agents such as natural polymers is one of the research areas of the pharmaceutical biotechnology. Fucoidan is a sulphated polysaccharide which is commonly obtained from seaweeds. Although a great number of studies on the different pharmacological properties of fucoidan are present, there is very limited information on the fucoidan-based system used in dermal burns. The aim of this study was to prepare chitosan hydrogel containing fucoidan and to investigate this hydrogel formulation for treatment of dermal burns on rabbits.

Methods: Fucoidan-chitosan hydrogels were prepared as follows: The polymers were dissolved in acidic solution and sonicated for removing the air-bubbles then the gels were stored at $+4{ }^{\circ} \mathrm{C}$ for in vivo studies. Seven adult male New Zealand white rabbits (mean weight, $3.8 \pm 0.6 \mathrm{~kg}$ ) were used for the evaluation of the gels on superficial dermal burns. The back of the rabbits were depilated and sedated. The wounds were made by circular stamp aluminium caps $\left(3.8 \mathrm{~cm}^{2}\right)$ at $80^{\circ} \mathrm{C}$. Four wounds were formed for each rabbit; (A) was treated with fucoidan-chitosan gel, (B) was treated with fucoidan solution, (C) was treated with chitosan gel (without fucoidan) and (D) as a negative control group. Biopsy samples were taken at 7,14 and 21 st days at the beginning of the study and each wound site was macroscopically observed and evaluated histopathologically.

Results: Oedema was not observed in all groups after 3 days treatment except controls. After 7 days treatment, fibroplasia and scar were observed on wounds treated with fucoidan-chitosan gel and fucoidan solution. The best regenerate dermal papillary formation and the fastest closure of wounds were observed in group A after 14 days treatment. The wound epithel elongation and thickness values were measured; A (5566 and $179 \mu \mathrm{m}), \mathrm{B}$ (3586 and, $146 \mu \mathrm{m}), \mathrm{C}$ (3666 and, $162 \mu \mathrm{m}), \mathrm{D}(3533$ and, $134 \mu \mathrm{m})$ at the end of the study.

Conclusion: Re-epithelization and contraction of the wound area which was treated with fucoidan-chitosan hydrogel were faster than the other groups. The fucoidan-chitosan hydrogel formulations can be suitable for the treatment of dermal burns.

\section{PB26}

Inhibition of HCV binding and entry to HepG2 cells by E1 antibody Ashraf A. Tabll, Khalid Atef, Mostafa K. EL-Awady Department of Biomedical Technology, National Research Center, Tahrir Street, Dokki, Cairo, Egypt. E-mail: ashraftabll@yahoo.com (A. Tabll)

Aim: To analyze the neutralizing -related activity of antibodies against $\mathrm{E} 1$ region of $\mathrm{HCV}$, specific polyclonal antibody was raised by immunized rabbits with synthetic peptide that had been derived 
from $\mathrm{E} 1$ region of $\mathrm{HCV}$ with the amino acid sequence $\mathrm{E} 1$ antibody [GHRMAWDMM).

Materials and methods: Hyper-immune HCV E1 antibodies were incubated over night at $4{ }^{\circ} \mathrm{C}$ with serum samples from patients positive for HCV RNA, with different viral load, ranged 7-11 million copes/ml, then incubated 90 min to HEPG2 cells. RT-PCR and flow cytometry were used to study the inhibition binding and entry effect of E1 antibody. Direct Immunostaining of E1 antibody conjugated with FITIC and Flow cytometry analysis showed reduced the mean fluorescence intensity in the samples pre-incubated with E1 antibody compared with samples without E1. Of 18 positive serum samples, $13(72 \%)$ samples showed completely inhibition of infectivity as detected by RT-PCR.

Conclusion: In house produced $\mathrm{E} 1$ antibody, blocks binding and entry of $\mathrm{HCV}$ virion infection to target cells suggesting the involvement of this epitope in virus binding and entry. Isolation of these antibodies that block virus binding and entry will be useful in providing potential therapeutic reagents and for vaccine development.

\section{PB27}

Folding and solubility of TNF $\beta$ are affected by structure and length of its N-terminus T. Preradov ${ }^{1}, M$. Drole ${ }^{1}, V$. Gaberc-Porekar ${ }^{1}$, V. Menart ${ }^{1,2}:{ }^{1}$ National Institute of Chemistry, Ljubljana, SI; ${ }^{2}$ Lek Pharmaceuticals d.d., Ljubljana, SI. E-mail: tanja.preradov.vasle@ki.si (T. Preradov)

Tumor necrosis factor beta (TNFß) is a pleiotropic cytokine mediating its activity through the same receptors as the structurally related TNF $\alpha$. Shortening of the N-terminal part has been reported to enhance its cytotoxic activity, with the explanation that removal of the flexible $\mathrm{N}$-terminus reduces steric interferences in the receptor binding process. For studies of relationship between the N-terminal protein structure and physicochemical properties, three different forms MetTNF $\beta$, His7TNF $\beta$ and $\triangle N 19 T N F \beta$ were expressed in E. coli. High solubility of $\triangle \mathrm{N} 19 \mathrm{TNF} \beta$ was expected, however, both analogs His7TNF $\beta$ and $\triangle \mathrm{N} 19 \mathrm{TNF} \beta$ were predominantly obtained in the form of inclusion bodies (IBs). On the other hand, MetTNF $\beta$ was equally distributed between the soluble and insoluble fraction. Most probably, the composition of the N-terminal part, such as the exposure of hydrophobic residues in the case of $\triangle N 19 T N F \beta$, or a mildly hydrophobic stretch of seven histidines appended to the natural hydrophobic N-terminus in the case of His7TNF $\beta$, lead to incorrect folding and force aggregate formation. Solubilization of IBs was performed under native and denaturing conditions using various concentrations of NLS, urea and $\mathrm{GndHCl}$. MetTNF $\beta$ IBs were easily dissolved with $0.2 \%$ NLS, while His7TNF $\beta$ and $\triangle N 19 T N F \beta$ demanded denaturing conditions. Structural differences in the $\mathrm{N}$ terminal part of various TNF $\beta$ proteins were also reflected in protein refolding characteristics and chromatographic behavior. Dilution, ultrafiltration and dialysis were used for refolding, and IMAC was chosen as the main chromatographic step. Our results suggest that not only the length of the N-terminal part of TNF $\beta$ but also the composition and exposure of certain amino acid residues affect its physicochemical properties.

\section{PB28}

Enzymatic modification of sphingomyelin Long Zhang, Lars Hellgren, Xuebing Xu BioCentrum-DTU.E-mail: 1z@biocentrum.dtu.dk (L. Zhang)
Due to its major role in maintaining the water-retaining properties of the epidermis, ceramide is of great commercial potential in cosmetic and pharmaceuticals such as hair and skin care products. Currently, chemical synthesis of ceramide is a costly process, and developments of alternative cost-efficient, high yield production methods are of great interest. In the present study, the potential of producing ceramide through enzymatic hydrolysis of sphingomyelin (SM) have been studied. SM is a ubiquitous membrane-lipid and rich in dairy products or by-products. In present study, we have optimized the production of ceramide from SM using phospholipase $\mathrm{C}$ from Clostridium perfringens. Water and enzyme amount had the biggest influence on SM hydrolysis in the system.

\section{PB30}

Isolation and cloning, expression and purification of Clostridium botulinum neurotoxin type $\mathbf{E}$ binding domain Seyed Latif Mousavi $^{1}$, Shideh Montaser Kouhsari ${ }^{2}$, Shahram Nazarian ${ }^{1}$, Iraj Rasooli ${ }^{3}$, J. Amani ${ }^{1}$ : ${ }^{1}$ Department of Biology, Faculty of science, Imam Hussein University, Tehran, Iran; ${ }^{2}$ Department of Biology, Faculty of science, Tehran University, Tehran, Iran; ${ }^{3}$ Department of Biology, College of Basic Science, Shahed University, Tehran, Iran. E-mail: kpnazari@ihu.ac.ir (S. Nazarian)

Botulinum neurotoxins constitute a family of bacterial toxins for botulism syndrome in human. The toxins bind with high affinity to nerve cells where they cause a complete inhibition and release of neurotransmitters and thereby produce flaccid paralysis. In this work we have reported isolation of the binding domain of type Eneurotoxin by PCR and expressed in a proper expression vector. The output of this investigation can be used as a tool to study the mechanism of binding of holotoxins and also can be useful to study the antibody production against botulism syndrome.

\section{PB31}

Isolation, cloning and expression gene encoding synaptobrevin protein (VAMP2) from Rat M.L.Mossavi ${ }^{1 *}$, J. Amani $^{1}$, Z. Safiry ${ }^{3}$, F. Ebrahimi ${ }^{1}$, Sh. Nazarian ${ }^{1}:{ }^{1}$ Department of Biology, Faculty of science, Imam Hussein University, Tehran, Iran; ${ }^{2}$ Department of Biology, Faculty of medicine, bageatallah University, Tehran, Iran. E-mail: mmousavi@ihu.ac.ir (M.L. Mossavi)

The synaptobrevin (VAMP2) a protein which play a key role in the fusion and exocytosis of the vesicle of mammalian nerves terminals this protein is substrate different serotypes of botulinum neurotoxins. Light chain of Clostridium Botulinum type B, D, F and $\mathrm{G}$ cleave end of neurons. Due to intraction of clostridium botulinum neurotoxin with VAMP2, this protein can be used as one of the toolsin the detection of poisings cause by this bacteria in the clinical laboratory using the enzyme with proof reading activity the above gene amplified by PCR technique for the production of the recombinant protein, the prokaryotic expression vectors (pET system) was used. A recombinant protein developed on poly acrylamide gel analyzed by western blotting and Eliza. 


\section{PB32}

Reduced maternal folic acid intake and MTHFR polymorphism as risk factors in Egyptian mothers of children with Down syndrome N.A. Meguid ${ }^{1}$, A.A. Dardir ${ }^{1}$, A.M. Rakha ${ }^{2}$, M.K. El Awady ${ }^{3}$ : ${ }^{1}$ Department of Research on Children with Special Needs, National Research Center, Cairo, Egypt; ${ }^{2}$ National Nutrition Institute, Cairo, Egypt; ${ }^{3}$ Biomedical Technology Department, National Research Center, Cairo, Egypt

Most children with Down syndrome (DS) are born to younger mothers ( $<35$ years). Recent reports linking Down syndrome (DS) to maternal polymorphisms at the methylenetetrahydrofolate reductase (MTHFR) gene locus have generated great interest among investigators in the field. In this study, forty mothers with their affected outcomes and 100 control mothers were included. All mothers were subjected to complete medical and nutritional history with special emphasis on folate intake through food or oral supplementation. Estimation of blood homocysteine level was done. Also we examined the two polymorphisms in genes encoding the folate metabolizing enzyme methylenetetrahydrofolate reductase (MTHFR), namely, $677 \mathrm{C}>\mathrm{T}$ and 1298A $>$ C. Folic acid intake from food and from vitamin supplements was significantly low (below the recommended daily allowance) in the group of mothers with DS children compared to control mothers $(P<0.01)$ using Student $T$-test. Blood homocysteine was normal in both control and DS mothers. The prevalence of the two polymorphisms, namely, 677C $>$ T and 1298A $>C$ in mothers of DS children (case mothers) $(n=40)$ was compared with controls $(n=100)$. Frequencies of MTHFR genotypes (CC, CT, and TT) at position 677 demonstrated no difference between the case and control groups. Genotype frequencies of MTHFR at position 1298A (AA, $\mathrm{AC}$, and $\mathrm{CC}$ ) were different among the case and control mothers. We here report the first study on a possible relation between DS with MTHFR 1298A > C genotypes in Egypt. Our results showed that MTHFR 1298A > C polymorphism is a remarkable genetic entity among Egyptian females with D.S. children. Sufficient folate intake and supplementation is an important preventive strategy in overcoming the risk of nondisjunction.

\section{PB33}

Analysis of separation of function rad52 mutants in Saccharomyces cerevisiae G. Lettier, H. Beck, C. Müller, U.H. Mortensen Center for Microbial Biotechnology, Technical University of Denmark, Denmark. E-mail: gl@biocentrum.dtu.dk (G. Lettier)

Homologous recombination (HR) is the mechanism that permits the creation of genetically engineered strains through gene targeting. In order to further develop gene targeting techniques, notably for higher eukaryotes such as filamentous fungi, it is of crucial importance to fully understand the molecular mechanisms behind mitotic HR. In Saccharomyces cerevisiae, a DNA double strand break (DSB) is an essential intermediate in meiotic HR. However, as HR occurs at a low rate in mitotic cells, it has been difficult to determine the nature of the event(s) that triggers it. Rad52 is a key protein involved in HR and is evolutionarily conserved from yeast to human. To shed light on the molecular events in HR, we have generated a large collection of defined rad52 mutant strains in the yeast S.cerevisiae. A screen of these mutants led to the identification of strains that fail to repair DNA DSBs, yet are proficient for homologous recombination. This result strongly suggests that DSBs may not be the major cause of spontaneous mitotic HR and gives new perspectives in respect to novel potential gene targeting substrates. We have analyzed these separation of function mutants in a variety of new assays to obtain a more detailed understanding of their controversial phenotype. Our latest results will be presented.

\section{PB34}

Antisense phosphorothioate oligonucleotide inhibition of hepatitis $\mathbf{C}$ Virus genotype 4 replication in HepG2 cells Mostafa $\mathrm{K}$. El Awady National Research Center Cairo Egypt

The outcome of interferone plus ribavirine treatment of hepatitis $\mathrm{C}$ virus (HCV) genotype 4 is unfortunately poor. Development of alternative therapy for this genotype is of a paramount importance. Inhibition of HCV gene expression in vitro by the use of antisense phosphorothioate oligodeoxynucleotides (S-ODN) against Internal Ribosomal Entry Site (IRES) elements were associated with favorable results. To assess S-ODN activity, previous studies utilized viral subgenomic or full cDNA fragments linked to reporter genes and transfected into adhered cells or in a cell free system. In the present study we utilized HepG2 cells infected with native HCV RNA of genotype 4 . The culture system presented herein was shown to support HCV replication on the following bases (1) consistent detection of both plus and minus RNA strands for 4 weeks in cells and in fresh culture supernatent, (2) ability of supernatent to infect naive HepG2 cells (3) consistent expression of core and E1 envelope proteins in infected cells throughout the 4 week culture. S-ODNs against AUG translation start site (S-ODN-1, nt 326-348) of the viral polyprotein precursor and stem loop IIId within the IRES region (S-ODN2, nt 264-282) were added to infected cells. Intracellular viral replication was monitored by nested RT-PCR of plus and minus strands. The results of these experiments demonstrated that intracellular replication of HCV genotype 4 was completely arrested after $48 \mathrm{~h}$ in culture using either S-ODN molecule (with more efficacy of S-ODN1 than S-ODN2) at concentrations as low as $1 \mu \mathrm{M}$. The inhibitory effect of $\mathrm{S}-\mathrm{ODN}$ appeared to be specific to HCV replication since equal levels of human glyceralehyde 3-phosphate dehydrogenase (GAPDH) gene expression were noted pre and post supplementation of S-ODNs. In conclusion, the present study provides evidence antisense phosphorothioate oligonucleotides have potent inhibitory effect on genomic replication of HCV genotype 4, the most common type in Egypt.

\section{PB35}

PCR-ELISA for detection shigatoxin of Shigella dysentery M.L. Mousavi, H. Ardestani, A. Karirmi, J. Salimian Department of Biology, Faculty of science, Imam Hussein University, Tehran, Iran. E-mail: g8102010@ihu.ac.ir (H. Ardestani)

A sensitive and specific PCR-ELISA was developed to detect shigella dysentery in food. The assay was based on the incorporation of degoxigenin-labeled dUTP and a biotin-labeled primer specific for shiga toxin genes during PCR amplification. The labeled PCR product were bound to streptoavidin-coated wells of a microtiter plat and detected by an ELISA. The ELISA detecting system was able to increase the sensitivity of the PCR assay by up to 100-fold, compared with a conventional gel electrophoresis. The detection limit of the PCR-ELISA was $0.1-10$ CFU dependent upon shigella dysentery serotypes and genotypes of shigatoxin. The entire procedure took about $4 \mathrm{~h}$. 


\section{PB36}

Isolation, cloning, expression and purification of SNAP-25 as a substrate of Botulinum Neurotoxin Type A and E M.L. Mossavi ${ }^{1}$,

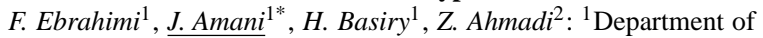
Biology, Faculty of science, Imam Hussein University, Tehran, Iran; ${ }^{2}$ Department of Biology, Faculty of medicine, bageatallah University, Tehran, Iran. E-mail: kpjamani@ihu.ac.ir (J. Amani)

Clostridial neurotoxin inhibit neurotransmitter relase by selective and specific intracellular proteolysis of synaptosomal associated protein of $25 \mathrm{KDa}$ (SNAP-25); synaptobrevin/VAMP-2 and syntaxin. SNAP-25 is one of the components that form docking complex in synaptic ends. This protein is substrate for botulinum neurotoxins type A and E. Each of these toxin serotypes specifically cleave SNAP25 in particular position and there by block docking and synaptic vesicle membrane fusion and finally prevent neurotransmitter exocytosis and transition of neurotic signals. In order to use the protein as a substrate for detection of different type of clostridium neurotoxin in vitro test, the protein was produced by recombinant technique. The DNA encoding SNAP-25 was isolated from rat brain by PCR using the two primer the amplified fragment colonel into expression vector pET32a.the expression protein was purified by affinity chromatography. confirm by different method the His tag fusion protein was digested with entrokinase.

\section{PB37}

Production of monospecific anti human alpha-1-antitrypsin and its efficiency in diagnosis of pulmonary emphysema Mamdouh M.Ali ${ }^{1}$, Ahmed M. Ibrahim ${ }^{1}$, Mohamed M. Abd ElFatah $^{2}$, Hala M. Ghanem ${ }^{2}$, M.M. Amriaa ${ }^{1}$, Gomaa Abuel-Nasr ${ }^{3}$ : ${ }^{1}$ National Research Center, Biochemistry Department, Division of Genetic Engineering and Biotechnology, Egypt; ${ }^{2}$ Biochemistry Department, Faculty of science, Ain Shams University, Egypt; ${ }^{3}$ Thoracic Surgical Department, Giza Chest Hospital, Egypt. E-mail: mmali1999@hotmail.com (M.M. Ali)

The present work deals with the preparation of pure alpha-1antitrypsin (AAT) protein from healthy subjects, which can be used in preparing its corresponding monospecific antibody in albino rabbits. This antibody was found very useful in the immuno-diagnosis of pulmonary emphysema. This study has been also concerned with the biochemical changes associated with AAT deficiency in pulmonary diseases. To fulfill this work, a group of healthy blood donors was selected for separation of pure AAT antigen from blood. The pure AAT was used for the preparation of anti-AAT, the purity and potency of antibody was checked by titration methods. The biochemical changes were studied in thirty subjects clinically divided into three groups including, control, heavy cigarette smokers with pulmonary emphysema, and non-smoking subjects with pulmonary emphysema. The activity of elastase and hydroxyproline (HP) level as a marker of elastin and collagen breakdown were assayed in bronchoalveolar lavage (BAL) fluid. The activity of AAT and to inhibit proteases as represented by tryptic inhibitory capacity (TIC) was evaluated. Serum ceruloplasmin, transferrin and IgA level as well as thiobarbituric acid reactive substances (TBARS) were estimated in all groups. Our data revealed that AAT and its TIC showed very highly significant decreased levels in all patients with emphysema as compared to control, while the elastase activity and HP level in BAL fluid were significantly increased in these patients. Serum TBARS was significantly increased in such patients associated with increasing level of both ceruloplasmin and transferrin. While, serum IgA was significantly increased. Furthermore, the biochemical changes were markedly changed in smokers with emphysema than non-smoking subjects. In conclusion, the preparation of anti-AAT on the local level is very important where less expensive and less time consuming and can be useful in immuno-diagnosis and prognosis of pulmonary diseases.

\section{PB38}

A new method combined with boosting and projective adaptive resonance theory for analysis of gene expression data from cancer patients Hiro Takahashi, Yasuyuki Murase, Hiroyuki Honda Department of Biotechnology, School of Engineering, Nagoya University, Nagoya 464-8603, Japan. E-mail: h041305d@ mbox.nagoyau.ac.jp (H. Takahashi)

An optimal and individualized treatment protocol based on accurate diagnosis is urgently required for the adequate treatment of patients. For this purpose, it is important to develop that a sophisticated algorithm that can manage large amount of data, such as gene expression data from DNA microarray, for optimal and individualized diagnosis. In our previous study, we developed the projective adaptive resonance theory (PART) as a gene filtering method and boosted fuzzy classifier with SWEEP operator (BFCS) as a modeling method. In the present study, we applied the combination of PART and BFCS (PART-BFCS method) to microarray data of brain tumor (central nervous system tumor) obtained from the website. The method enabled the selection of 14 important genes related to the prognosis of the tumor, i.e., sensitivity for combined therapy with surgery, radiotherapy, and chemotherapy mainly with vincristine, cisplatin, and cytoxan. The constructed model showed about $20 \%$ higher accuracy than that of the conventional method.

\section{PB39}

Genomic signal analysis of HIV variability based on RT gene P.D. Cristea, Rodica Tuduce D. Otelea Biomedical Engineering Center, University "Politehnica" of Bucharest, Romania. E-mail: pcristea@dsp.pub.ro (P.D. Cristea)

DNA sequences genotyped from 60 Clade F HIV-1 isolates from Romanian patients in the laboratory of the National Institute of Infectious Diseases "Matei Bals", Bucharest, Romania, have been studied. The symbolic sequences have been converted into digital genomic signals by using a complex quadrantal representation of the nucleotides described earlier. The cumulated phase and unwrapped phase of a complex genomic signal reflect the statistical distribution of bases and base-pairs, respectively. Independent Component Analysis of the genomic signals has been used to characterize the variability of the F subtype HIV strains isolated in Romania. The sequenced segment is of (about) 1302 base pairs, approximately aligning with the standard sequence of HIV-1 (accession NC001802 in GenBank) over the interval 1799-2430 bp. This segment, which is currently used for the standard identification and assessment of HIV-1 strains, comprises the protease (PR) gene and two thirds of the reverse transcriptase (RT) gene. Only results referring to the analysis of the RT gene region are presented here and used for extracting features of virion isolates and for establishing phylogenetic trees of the studied strains. The analyzed RT encoding segment has the length $1005 \mathrm{bp}$ and is located in the second interval (298-1302 bp) of the 
analyzed DNA segment, respectively along the 2096-3100 bp region of NC001802. Taking into account the mutations identified in these sequences, the samples were classified in three groups from the point of view of their resistance to current antiretroviral compounds: sensitive, resistant and multiresistant. The paper presents results for the isolates in which mutations leading to multiple drug resistance have been identified.

\section{PB40}

Over expression of SecB protein in $E$. coli enhances the periplasmic expression of human growth hormone $M$. Ghafari ${ }^{1,2}$, A. Zomorodipour ${ }^{2}{ }^{1}$ Islamic Azad University of Jahrom, Tehran, Iran; ${ }^{2}$ National Institute for Genet Eng and Biotechnol., Tehran, Iran. Email: maryamghafari2001@yahoo.com (M. Ghafari)

Among several proteins involved in the secretion pathway of proteins in E. coli, secB plays a key-important role in solubilization of preproteins before processing. In order to increase processing of a human growth hormone precursor (pelB::hGH) which appeared to have problem in processing efficiency, as a possible solution a regulated co-expression of a secB gene was considered. In this regard, we designed an arabinose-regulated secB expressing plasmid compatible with an IPTG/lactose-regulated pelB::hGH expressing plasmid. For the construction of the secB expressing plasmid the origin of replication and antibiotic resistant gene (Amp) of a pBAD vector was replaced by a p15A-Ori and a Kanamycin resistant gene, respectively. The expression and processing of pelB::hGH preprotein in the two-plasmid containing bacteria in a secB over-expression state was compared to that of the pelB::hGH expression in normal bacteria. Although a decline in total expression level of hGH during the overexpression of secB was observable, probably due to presence of two different expressing plasmids, but both the processing efficiency of pelB::hGH and the transport of mature protein into the periplasmic space was enhanced during prolonged arabinose induction.

\section{PB41}

Recombinant major cat allergen FeldI for diagnosis of cat allergy Marlena Szalata ${ }^{1,2}$, Daniel Lipinski ${ }^{2}$, Robert Kalak ${ }^{1}$, Paulina Tobola ${ }^{3}$, Joanna Lehmann ${ }^{1}$, Marta Pawlik ${ }^{1}$, Wojciech Juzwa $^{1}$, Karolina Wielgus ${ }^{1}$, Marek Pienkowski ${ }^{4}$, Ryszard Slomski ${ }^{2}$ : ${ }^{1}$ Department of Biochemistry and Biotechnology, Agricultural University, Poznan 60-637, Poland; ${ }^{2}$ Institute of Human Genetics, Polish Academy of Sciences, Poznan 60-479, Poland; ${ }^{3}$ Delta Pharma BV, Hengelo 7554 TR, The Netherlands; ${ }^{4}$ Allergic Diseases, Asthma and Immunology Clinic, P.C., Knoxville, TN 37917, USA. E-mail: marsz@au.poznan.pl (M. Szalata)

Current diagnostics and therapeutics for cat allergy are based on cat epithelial extracts. Natural allergen extracts contain a mixture of allergenic and non-allergenic components that are difficult to standardize. Recombinant allergens can improve diagnosis and de-sensitization against single component. Major cat allergen is a heterodimer composed of disulfide linked 7.8 and $10.1 \mathrm{kDa}$ polypeptide chains. Both chains of FeldI protein were obtained in E.coli system. Purification of recombinant proteins was performed in denaturing conditions using immobilized metal affinity chromatography specific for proteins with histidine tag. From $1000 \mathrm{ml}$ culture approximately $13 \mathrm{mg}$ protein for FeldI chain 1 and $43 \mathrm{mg}$ of FeldI chain 2 were obtained. After purification histidine tag was removed by hydrolysis with thrombin. Immunological activity of FeldI against serum of patients allergic to cat was narrowed to subgroup of patients allergic to FeldI protein by surface plasmon resonance. Immunological activity of each chain and renatured heterodimer was also tested using immunoprecipitation techniques against serum of population group.

\section{PB42}

Subdoligranulum variabile - a novel member of the human gut micro flora with a high prevalence Kim Holmstrøm, Trine Møller Bioneer A/S, Hørsholm, DK-2970, Denmark

In 2003 we isolated and cultured for the first time a bacterium from a human fecal sample representing a hitherto unknown member of the Clostridium leptum rRNA supra generic cluster. The C. leptum rRNA supra generic cluster represents one of the 3 major phylogenetic lineages within the human gut microbiota, and is characterized by having only a small proportion of its members actually identified by cultivation compared to the estimated numbers of bacteria contained in this group from culture-independent gut flora analyses. $S$. variabile is an obligate anaerobe Gram negative bacterium with a characteristic pleiomorphic coccoid-droplet-like cellular morphology. Its closest previously cultivated relative based on a $16 \mathrm{~S}$ rDNA phylogenetic analysis is Faecalibacterium prausnitzii, a rod-shaped and therefore easily distinguishable Gram negative bacterium present in high numbers in the human fecal micro flora. Based on 16S rDNA sequence we designed a $S$. variabile specific oligonucleotide probe for use in FISH analysis to estimate the prevalence of this "new" bacterium in fecal samples collected from healthy human beings. Interestingly, we observed a high proportion of $S$. variabile present in all tested samples, and in some instances we observed a higher prevalence than the more well-known group of bifidobacteria equally estimated by FISH analysis. Documentation of these results will be presented.

\section{PB43}

Isolation and characterization of antibiotic compounds obtained from Malaysian traditional medicines F. Abd. Hamid Ghouse ${ }^{1}$, Abd-ElAziem Farouk ${ }^{1}$, B.H. Ridzwan ${ }^{2}:{ }^{1}$ Biomolecular Engineering Research Group, Department of Biotechnology Engineering, Kulliyyah of Engineering, International Islamic University Malaysia, Jalan Gombak 53100, Malaysia; ${ }^{2}$ Biomedical Science Department, Kulliyyah of Science, International Islamic University Malaysia, Jalan Gombak 53100, Malaysia.

Several species of sea cucumbers, long an incumbent of traditional medicines were selected as the source of animal based antibiotic compounds. Swabs of the inner surface and coelomic fluid (inner fluid) samples from sea cucumber (Holothuria atra jaeger) were taken. Thirty strains of bacteria were isolated. These strains were grown in different antibiotic production media. Nine human pathogenic bacterial species were used as test agents and they are, $K$. pneumoniae, MRSA, M. luteus, S. thyphimurium, S. epidermitis, $S$. saprophyticus, B. subtillis, P. aeruginosa and S. pyogenes; Only four bacterial strains showed mild antibiotic activity against $S$. pyogenes and S. thyphimurium. Similar testing on two other species, H. scabra and $S$. variegatus will be carried out. Different media, especially antibiotic production enrichment media will also be used. Characterization will be done upon obtaining an antibiotic compound, which shows moderate to high activity against at least one of the nine human pathogens used. For plant based medicines, three rhizomes, "cekor," "jerangau" and "bonglai" were analyzed. Solvent extraction using 
ethanol, methanol and acetone was carried out, at a concentration of 20-50 mg per $\mathrm{ml}$ solvent. The filtrates were used for the antibiotic testing stage. All the three plant species showed moderate antibiotic activity against $M$. luteus, S. epidermitis, S. pyogenes and $S$. saprophyticus. Interestingly, the antibiotic activity increased when combinations of the herbal extracts were used.

\section{PB44}

Muscle engraftment of myogenic progenitor cells following intraarterial transplantation Estanislao Bachrach, Antonio L. Perez, Yeong-Hoon Choi, Ben MW Illigens, Susan J. Jun, Pedro del Nido, Francis X. McGowan, Sheng Li, Alan Flint, Jeffrey Chamberlain, Louis M. Kunkel Genomics Program, Enders 561, Howard Huges Medical Institute and Children's Hospital Boston, Harvard Medical School, 300 Longwood Avenue, Boston, Massachusetts 02115, USA

Cell-based therapy continues to be a promising avenue for the treatment of Duchenne muscular dystrophy, an X-linked skeletal muscle-wasting disease. Recently, we have demonstrated that freshly isolated myogenic progenitors contained within the adult skeletal muscle side population (SP) can engraft into dystrophic fibers of non-irradiated $m d x^{5 c v}$ mice after intravenous transplantation. Engraftment rates, however, have not been therapeutically significant, achieving at most $1 \%$ of skeletal muscle myofibers expressing protein from donor-derived nuclei. To improve the engraftment of transplanted myogenic progenitors, an intra-arterial delivery method was adapted from a previous procedure. Cultured, lentivirus transduced skeletal muscle SP cells were transplanted into the femoral artery of non-injured $m d x^{5 c v}$ mice. Based on the expression of microdystrophin and GFP transgenes in host muscle, sections of the recipient muscles exhibited $5 \%$ to $8 \%$ of skeletal muscle fibers expressing donor-derived transgenes. Further, donor muscle SP cells, which did not express any myogenic markers prior to transplant, express the satellite cell transcription factor Pax7 and the musclespecific intermediate filament desmin after extravasation into host muscle. The expression of these muscle-specific markers indicates that progenitors within the side population can differentiate along a myogenic lineage after intra-arterial transplantation and extravasation into host muscle. Given that femoral artery catheterization is a common, safe clinical procedure and that the transplantation of cultured adult muscle progenitor cells has proven to be safe in mice, our data may represent a step towards the improvement of cell-based therapies for DMD and other myogenic disorders.

\section{PB45}

Metabolic engineering design of an extracellular hGH synthesis system Birgül Şentürk ${ }^{1}$, Pınar Çalık ${ }^{2}$, Güzide Çalık ${ }^{1}$, Tunçer H. Özdamar ${ }^{1}$ : ${ }^{1}$ BRE Lab, Department of Chemical Engng, Ankara University, 06100 Ankara, Turkey; ${ }^{2}$ IBLab, Department of Chemical Engineering, METU, 06531 Ankara, Turkey. E-mail: ozdamar@eng.ankara.edu.tr (T.H. Özdamar)

Metabolic engineering design of an extracellular human growth hormone (hGH) synthesis system is based on cloning the DNA encoding human therapeutic protein together with the signal DNA sequence of an extracellular enzyme gene into a host-vector system. In this context, extracellular protease ( $s u b C$ ) signal DNA sequence, i.e. pre- signal DNA sequence, was fused into the frame in front of hGH mature DNA sequence, by the use of four primers designed using PCR-based gene splicing by overlap extension method. $B$. licheniformis chromosomal DNA and plasmid carrying hGH cDNA were used as templates in PCRs, respectively, for the amplification of the $s u b \mathrm{C}$ signal DNA sequence and hGH mature peptide sequence. For the fusion of two target genes, i.e. mature peptide sequence of hGH and, signal DNA sequences were amplified separately by PCRs. The primers used at the ends to be joined were designed as complementary to one another by including nucleotides at their $5^{\prime}$ ends that are complementary to $3^{\prime}$ portion of the other primer. These products were mixed in the next PCR reaction, where one strand from each fragment contains the overlap sequence at $3^{\prime}$ end. Extension of this overlap by DNA polymerase has yielded the recombinant hybrid-gene; and hybrid-gene serve as template for the continuation of reactions for the increase of the concentration in the microreactors. The hybrid gene fragment was first cloned into pUC19, and then sub-cloned to pMK4 E.coli-Bacillus shuttle plasmid. Thus, a new expression vector with high stability and high copy-number was obtained and transferred into host $B$. subtilis $168\left(\mathrm{spo}^{-}\right)$. The metabolic flux distributions calculated by the mass balance based stoichiometric model based on the proposed metabolic reaction network for r-B.subtilis were determined by using time profiles of the substrate, dry-cell, hGH, amino acids and organic acids concentrations as the constraints. On the basis of the intracellular bioreaction rates and the interactions with the bioreactor operation parameters, an in-depth insight will be provided for further metabolic engineering design for the extracellular hGH production in $\mathrm{r}-$ B.subtilis.

\section{PB46}

Medicines based on polypeptides consisting of 30 and more amino acid residues are widely spread in pharmaceutical market at the present time. Practically all polypeptide medicines known are prepared by general chemical synthesis that caused high cost of their production. That's why biotechnological way of polypeptide medicines preparation using recombinant gene expression in bacteria seems to be promising. During our work we design hybrid construction allowing solving a problem of specific cleavage of target polypeptide from the hybrid protein using system of protein splicing from New England BioLabs. In case of thymosin $\alpha_{1}$ production IMPAC system (Intein Mediated Purification with Affinity Chitin-binding Tag) has been used, where the modified Sce VMA from $S$. cerevisiae has been applied as intein. In contrast to other systems, IMPACT allows the preparation of target protein without using of serine protease and other factors that may cleave the hybrid protein. In the presence of thiol reagents, such as dithiothreitol, mercaptoethanol, or cystein the hybrid protein can be site-specifically cleaved to give intein, the target protein and small fragment of $\mathrm{N}$ extein. Using of this system does not allow to obtain the target protein with Ser, Cys or Thr on N-terminal of protein, because in those cases target protein and N-extein ligation product will be formed. Ser is $\mathrm{N}$-terminal amino-acid in thymosin $\alpha_{1}$. It was recently found that some metal ions essentially affect the splicing. We tried to use $\mathrm{ZnCl}_{2}$ and we have found that, in case of intein-thymosin $\alpha_{1}$, the maximal yield of target polypeptide and the minimal yield of splicing products are observed in the absence of dithithreitol and in the presence of $0.5-1 \mathrm{mM}$ zinc chloride in buffers on all stages of thymosin $\alpha_{1}$ isolation. The structure of recombinant thymosin $\alpha_{1}$ of human was confirmed by the determination of $\mathrm{N}$ - and $\mathrm{C}$ - terminal amino-acid sequences and by MALDI TOF mass-spectrometry. 


\section{PB47}

Mature embryos of five T. aestivum and five T. durum cultivars formed embryogenic callus on two different media. Embryos were removed from surface sterilised seeds and placed with the scutellum upwards on a solid agar medium containing the inorganic components of Murashige Skoog and $2 \mathrm{mg} / 1$ 2,4-dichlorophenoxyacetic acid (2,4-D) or $1 \mathrm{mg} / \mathrm{L}$ naphtalenacetic acid (NAA). The developed calli and regenerated plants were maintained on 2,4-D or NAA free MS medium. Wheat plants can be regenerated via two different systems. There were significant differences in percentage of callus induction and regeneration capacity on the different initiation medium. Among the T. aestivum cultivars, Yakar had the highest regeneration capacity in both induction medium. In $T$. durum cultivars, Kiziltan gave the highest regeneration capacity in MS + 2,4 D medium and Yilmaz gave the highest regeneration capacity in MS + NAA medium. A strong genotypic effect on the culture responses was found for both induction medium.

\section{PB48}

Folding and solubility of TNF $\beta$ are affected by structure and length of its $\mathbf{N}$-terminus T. Preradov ${ }^{1}, M$. Drole $^{1}, V$. Gaberc-Porekar ${ }^{1}$, V. Menart ${ }^{1,2}$ : ${ }^{1}$ National Institute of Chemistry, Ljubljana, SI; ${ }^{2}$ Lek Pharmaceuticals d.d., Ljubljana, SI. E-mail: tanja.preradov.vasle@ki.si (T. Preradov)

Tumor necrosis factor beta (TNF $\beta$ ) is a pleiotropic cytokine mediating its activity through the same receptors as the structurally related TNF $\alpha$. Shortening of the $\mathrm{N}$-terminal part has been reported to enhance its cytotoxic activity, with the explanation that removal of the flexible $\mathrm{N}$-terminus reduces steric interferences in the receptor binding process. For studies of relationship between the $\mathrm{N}$-terminal protein structure and physicochemical properties, three different forms MetTNF $\beta$, His7TNF $\beta$ and $\triangle \mathrm{N} 19 \mathrm{TNF} \beta$ were expressed in E. coli. High solubility of $\triangle \mathrm{N} 19 \mathrm{TNF} \beta$ was expected, however, both analogs His7TNF $\beta$ and $\triangle \mathrm{N} 19 \mathrm{TNF} \beta$ were predominantly obtained in the form of inclusion bodies (IBs). On the other hand, MetTNF $\beta$ was equally distributed between the soluble and insoluble fraction. Most probably, the composition of the $\mathrm{N}$-terminal part, such as the exposure of hydrophobic residues in the case of $\triangle \mathrm{N} 19 \mathrm{TNF} \beta$, or a mildly hydrophobic stretch of seven histidines appended to the natural hydrophobic N-terminus in the case of His7TNF $\beta$, lead to incorrect folding and force aggregate formation. Solubilization of IBs was performed under native and denaturing conditions using various concentrations of NLS, urea and GndHCl. MetTNF $\beta$ IBs were easily dissolved with $0.2 \%$ NLS, while His7TNF $\beta$ and $\triangle$ N19TNF $\beta$ demanded denaturing conditions. Structural differences in the Nterminal part of various TNF $\beta$ proteins were also reflected in protein refolding characteristics and chromatographic behavior. Dilution, ultrafiltration and dialysis were used for refolding, and IMAC was chosen as the main chromatographic step. Our results suggest that not only the length of the N-terminal part of TNF $\beta$ but also the composition and exposure of certain amino acid residues affect its physicochemical properties.

\section{PB49}

Influence of growth limiting factors on recombinant growth hormone expression in E.coli M. Morkunas, S. Alisauskaite, J. Sereikaite, V.-A. Bumelis Department of Chemistry and Bioengineering, Vilnius Gediminas Technical University, Vilnius, LT-10223, Lithuania. E-mail: mindaugas.morkunas@fm.vtu.lt (M. Morkunas)

High level expression of recombinant growth hormones in E.coli faces common problems such as protein aggregation and inclusion body formation. Discussions are raised whether it is more beneficial to obtain soluble protein but to loose expression rates. Here we describe an experiment based on the hypothesis that slower expression should result in at least partially soluble recombinant protein. Experiments were performed on bovine, chicken and mink growth hormones. Expression rate was controlled externally by adjusting cultivation temperature, media, inducer amount, and both induction and cultivation times. Another approach to the problem was performed through genetic manipulation. We changed strong T7 promoter to E. coli promoter consensus sequence thus reducing expression rate. Recombinant growth hormone was still found to form aggregates, even when expressed at extremely low levels several (2-8) percent of total intracellular protein. We developed optimization scheme of insoluble protein production and showed that expression rate minimization is not influencing recombinant growth hormone solubility in vivo thus suggesting an idea of sequence specific aggregation.

\section{PB50}

Quantitative mRNA analysis as a tool for optimization of recombinant protein production in cell cultures $M$. Bollok ${ }^{1}, A$.

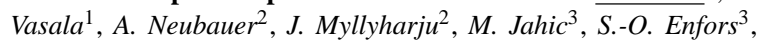
P. Neubauer ${ }^{1}$ : ${ }^{1}$ Bioprocess Engineering Laboratory, Department of Process and Environmental Engineering, University of Oulu, Oulu, Finland; ${ }^{2}$ Collagen Research Unit, Biocenter and Department of Medical Biochemistry, University of Oulu, Oulu, Finland; ${ }^{3}$ Department of Biotechnology, Royal Institute of Technology, Stockholm, Sweden. E-mail: monika.bollok@oulu.fi (M. Bollok)

To optimize the recombinant protein production in small-scale shake-flask system and high cell density fermentation, new tools have been developed at our laboratory which helps to get knowledge about the physiological state of the cell culture. These tools include (i) a quantitative monitoring system for cellular mRNAs based on a sandwich hybridization technique, and (ii) a wireless online monitoring tool (SENBIT), applicable for standard sensors such as $\mathrm{pH}, \mathrm{pO}_{2}$ and temperature for the continuous data collection from shake flasks. The SENBIT system is a new tool supplying valuable information for the optimisation of the expression of recombinant genes in shake flasks and allowing conclusions towards the reproducibility of shake flask cultures. The presentation will focus on use of the sensitive sandwich hybridization technology for the quantitative analysis of process relevant marker genes in different kind of microbial cell cultures with a focus on the production of recombinant proteins. Samples from shake flask cultures and high cell density fed-batch fermentations of the yeast Pichia patoris, have been analysed. Additionally the mRNA analysis was combined with the application of the SENBIT wireless system to study the production of a recombinant protein in shake-flask cultures of $P$. pastoris. Aside from P. pastoris, mRNA sandwich hybridization also was used to monitor product expression 
in fed-batch fermentation of E. coli for the production of a protein with two subunits by sequential induction.

\section{PB51}

Quantitative RNA analysis as a tool for optimization of tetrameric collagen prolyl 4-hydroxylase production in $E$. coli A. Neubauer ${ }^{1}$, M. Bollok ${ }^{2}$, J. Myllyharju $^{1}$, P. Neubauer ${ }^{2}:{ }^{1}$ Collagen Research Unit, Department of Medical Biochemistry and Molecular Biology, University of Oulu, FIN-90014 Oulu, Finland; ${ }^{2}$ Bioprocess Engineering Lab., Biocenter Oulu, University of Oulu, FIN-90014 Oulu, Finland. E-mail: antje.neubauer@oulu.fi (A. Neubauer)

Collagen prolyl 4-hydroxylase (P4H) involved in the biosynthesis of collagens is an $\alpha_{2} \beta_{2}$ tetramer. Recombinant expression of $\mathrm{P} 4 \mathrm{H}$ in $E$. coli was described recently (Neubauer et al., 2005). The construct for cytoplasmic expression contains the genes of both subunits in one plasmid under control of different promoters. The $\alpha$ subunit forms inactive aggregates, when expressed separately. In mammalian cells the $\beta$ subunit is available in large excess and keeps the $\alpha$ subunit in a soluble active form. To mimic this in the bacterial system, we induced both genes sequentially. After induction of the $\beta$ subunit with IPTG, expression of the $\alpha$ subunit was initiated with anhydrotetracycline. Here we use the analysis of the product mRNAs with a bead based sandwich hybridisation assay (SHA) (Rautio et al., 2003) for optimization of the fermentation procedure. A high $\mathrm{P} 4 \mathrm{H}$ activity was obtained if a high mRNA level of the $\alpha$ subunit could be maintained over a longer time. The obtained results illustrate the importance of the second induction for a high level expression of the $\mathrm{P} 4 \mathrm{H}$ tetramer. The cells need to be in a "healthy state" with low metabolic load to react efficiently to the second induction. The data illustrate the optimization of a fermentation process by monitoring mRNA levels which is of general interest for optimization of products which are difficult to detect.

\section{Acknowledgements}

This work was supported by the Health Science Council, the Academy of Finland and the TEKES-Neobio program.

\section{References}

Neubauer, A., et al. 2005. High-level production of human collagen prolyl 4-hydroxylase in E. coli. Matrix Biol. 24, 59-68.

Rautio et al., 2003. Sandwich hybridisation assay for quantitative detection of yeast RNAs in crude cell lysates. Microb. Cell Fact. 2 (4), 1-9.

\section{PB52}

Muscle engraftment of myogenic progenitor cells following intraarterial transplantation Estanislao Bachrach, Antonio L. Perez, Yeong-Hoon Choi, Ben MW Illigens, Susan J. Jun, Pedro del Nido, Francis X. McGowan, Sheng Li, Alan Flint, Jeffrey Chamberlain, Louis M Kunkel

Cell-based therapy continues to be a promising avenue for the treatment of Duchenne muscular dystrophy, an X-linked skeletal muscle-wasting disease. Recently, we have demonstrated that freshly isolated myogenic progenitors contained within the adult skeletal muscle side population (SP) can engraft into dystrophic fibers of non-irradiated $m d x^{5 c v}$ mice after intravenous transplantation. Engraftment rates, however, have not been therapeutically significant, achieving at most $1 \%$ of skeletal muscle myofibers expressing protein from donor-derived nuclei. To improve the engraftment of transplanted myogenic progenitors, an intra-arterial delivery method was adapted from a previous procedure. Cultured, lentivirus transduced skeletal muscle SP cells were transplanted into the femoral artery of non-injured $m d x^{5 c v}$ mice. Based on the expression of microdystrophin and GFP transgenes in host muscle, sections of the recipient muscles exhibited 5-8\% of skeletal muscle fibers expressing donor-derived transgenes. Further, donor muscle SP cells, which did not express any myogenic markers prior to transplant, express the satellite cell transcription factor Pax7 and the muscle-specific intermediate filament desmin after extravasation into host muscle. The expression of these muscle-specific markers indicates that progenitors within the side population can differentiate along a myogenic lineage after intra-arterial transplantation and extravasation into host muscle. Given that femoral artery catheterization is a common, safe clinical procedure and that the transplantation of cultured adult muscle progenitor cells has proven to be safe in mice, our data may represent a step towards the improvement of cell-based therapies for DMD and other myogenic disorders.

\section{PB53}

Production of non-hydroxylated recombinant collagen in barley Heidi Holkeri ${ }^{1}$, Eva Wahlström ${ }^{2}$, Anneli Ritala ${ }^{1}$, Julio Baez ${ }^{3}$, Kristiina Mäkinen ${ }^{2}$, Anna Maria Nuutila ${ }^{1}{ }^{1}$ VTT Biotechnology, P.O. Box 1500, 02044 VTT, Finland; ${ }^{2}$ Department of Applied Biology, P.O. Box 27, University of Helsinki, Helsinki 00014, Finland; ${ }^{3}$ FibroGen Inc. 225 Gateway, South San Francisco, CA 94080, USA

Collagen and its derived product gelatin are attractive mammalian proteins to be used as model for the production of complex heterologous proteins in plants. The availability of a recombinant product will provide a safer, more homogeneous product than the current animal-derived material. The aim of the project is to investigate the feasibility of a production system for the accumulation of recombinant collagen for conversion to gelatin using barley. The $5^{\prime}$-end of the Cocksfoot mottle virus (CfMV; genus Sobemovirus) genomic RNA sequence, called CfMV $\varepsilon$-element, has been shown to enhance recombinant protein synthesis in barley (WO 01/55298, Mäkinen et al., 1995). The $\varepsilon$-element will be used to study whether accumulation levels of complex mammalian proteins can be further increased, using collagen as a model that will serve as basis for exploring the expression of other complex proteins. This system can be study the production of barley-derived recombinant collagen for conversion to gelatin.

\section{PB54}

Effect of glycosylation inhibition on surface glycoproteins and survival of classical swine fever virus - a model for human hepatitis c virus B. Szewczyk ${ }^{1}$, J. Tyborowska ${ }^{1}$, E. Krol ${ }^{1}$, W. Szeja ${ }^{2}$, G. Grynkiewicz ${ }^{3}$ : $^{1}$ Department of Molecular Virology, University of Gdańsk, Gdańsk, Poland; ${ }^{2}$ Silesian Technical University, Gliwice, Poland; ${ }^{3}$ Pharmaceutical Institute, Warsaw, Poland

Classical swine fever virus (CSFV) is an animal pestivirus which can be used as a surrogate model to elucidate the role of envelope glycoproteins of closely related human hepatitis $\mathrm{C}$ virus (HCV). The necessity to use the surrogate models for $\mathrm{HCV}$ is due to the fact that this virus cannot be grown in vitro cultures. CSFV genome codes for three major antigenic glycoproteins which are located in the same cluster of genes; they are designated as E2 and E0 ( $\left.\mathrm{E}^{\text {rns }}\right)$ and E1. Glycoproteins form heterodimeric and homodimeric complexes on the 
external part of viral particles. It is generally accepted that envelope glycoproteins play a major role in the initial stages of viral infection both for CSFV and HCV. Formation of complexes is needed to effectively infect host cells. We have investigated the formation of glycoprotein dimers by immunoperoxidase monolayer assay and by immunoblotting (Western Blotting). Immunoblotting is a very useful technique in these studies because the complexes are formed via cysteine-cysteine disulphide bonds and they are retained during SDS-PAGE under non-reducing conditions. By modifying the glycoprotein genes and by arresting $\mathrm{N}$-glycosylation of E2 and E0 we have investigated which factors influence the formation of complexes. It has been found that some glycosylation inhibitors which act at the early stages of glycan chain processing influence, not only glycosylation, but also the stability of E2 protein, effectively inhibiting the formation of glycoprotein complexes and the yield of the virus. These inhibitors are potential agents for arresting the multiplication and spread of CSFV, and its relative - human HCV.

\section{PB55}

The red microalgal cells: a platform for recombinant protein production Kalpana Manandhar-Shrestha, Miri Lapidot, Yacob Weinstein, Shoshana (Malis) Arad Ben-Gurion University, P.O. Box 653, Beer-Sheva 84105, Israel. E-mail: kalpana@bgu.ac.il (K. Manandhar-Shrestha)

Recombinant proteins have been produced in a variety of heterologous protein expression systems. Eukaryotic unicellular algae have distinct advantages, e.g. it can synthesize complex protein that requires post-translational modification. Furthermore, microalgae can be grown in confined environment and thus prevents leakage of genes to the environment. Our group has developed a platform technology for the production of recombinant proteins in red microalga Porphyridium sp. We have constructed algal transformation plasmid vectors containing a CaMV $35 \mathrm{~S}$ promoter and polyA signal site. A Streptoalloteichus hindustanus bleomycin-resistant gene was used as the selective marker. We have expressed ovalbumin and hepatitisB surface antigen $(\mathrm{HBsAg})$ as model proteins. Transformation was carried out by agitating algal cells and vector DNA with glass bead. Transgenic lines were selected by growing algal cells on agar plate containing $6 \mu \mathrm{g} / \mathrm{ml}$ Zeocin. Positive transgenic lines were selected by screening the colonies by PCR and confirmed by DNA sequencing. Expression of ovalbumin and HBsAg protein was examined by Western blot analysis. Ovalbumin was found to be expressed inside the algal cells while small HBsAg was secreted into the medium due to presence of signal peptide. These findings indicate that red microalgae are capable of producing heterologous proteins.

\section{PB56}

Life-material exhibition as ethical interpretation Chang Shih-lung Biotechnology Industry Study Center, Taiwan Institute of Economic Research, Taipei 106, Taiwan. Email: schang@tier.org.tw

In this thesis we aim to explore the medical-related life-material exhibitions within NTU Hospital-the Humanity Building, and Taipei Mackay Hospital-the Historical Showroom as abecedarian clues to understand the burgeoning phenomenon-medical museums in Taiwan. Following these clues, we treat the whole context of medical museums, which transform values through situational construction, as the background to interpret the ethical implications of group val- ues that are transformed in the medical profession. In this thesis, we see medical museums, as the social prescription transforming medical profession, format the ritual context of situation ethics with the cultural construction of life-material. Multi-disciplinary interactions and visiting itinerary can be transferred to the exploring horizon of research approach through description and interpretation. Among them, we observe that humanistic elements have become essential equipment (or mat'eriel) of medical profession. Though humanistic equipment (or mat'eriel) has its bottleneck in the museum situation, it can also unblock new possibilities for museum exhibitions, ethical practice or life ethics.

\section{PB57}

Development of new anticancer drugs from secondary metabolites of marine origin José M. Padrón ${ }^{1,2,3}$, Fernando R.P. Crisóstomo $^{3}$, Tomás Martín ${ }^{3}$, Víctor S. Martín ${ }^{3}$, V.J. illar ${ }^{1}{ }^{1}$ Unidad de Investigación, Hospital Universitario NS de Candelaria, Ctra. del Rosario s/n, 38010 S/C de Tenerife, Spain; ${ }^{2}$ Instituto Canario de Investigación del Cáncer (ICIC), Red Temática de Investigación Cooperativa de Centros de Cáncer (RTICCC), Ctra. del Rosario s/n, 38010 S/C de Tenerife, Spain, ${ }^{3}$ Instituto Universitario de Bio-Orgánica "Antonio González", Universidad de La Laguna, C/ Astrofísico Francisco Sánchez 2, 38206 La Laguna, Spain. E-mail: jmpadron@ull.es (J.M. Padrón)

As part of a wide research program aimed at developing new antitumoral agents, we present herein a series of stereoisomeric derivatives of fused tetrahydrofuranes (fTHF) substituted with diverse protecting groups either at the primary or secondary hydroxyl groups. Unprotected derivatives were also synthesised to investigate the influence of substituents on the in vitro activity of fTHF.
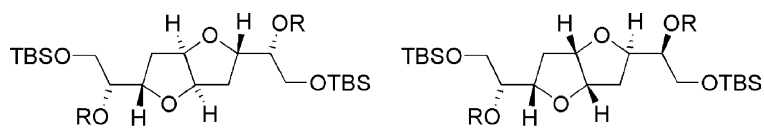

$\mathrm{R}=\mathrm{H}, \mathrm{Ac}$

Data on the synthesis, chemosensitivity and apoptosis induction by this series of fTHF will be correlated to substitution pattern, stereochemistry and protecting groups, as an aid to the rational design of novel antitumour drugs.

\section{Acknowledgements}

Supported by the MEC of Spain (PPQ2002-04361-C04-02) and the Canary Islands Government. JMP thanks ICIC for a postdoctoral fellowship. FRPC thanks CajaCanarias for a FPI fellowship. TM thanks the Spanish MCYT-FSE for a Ramón y Cajal contract.

\section{PB58}

Establishment of in vitro test systems for pulmonary edema resorption by peptide drug candidates Dominik Geiger, Aswin Mangerich, Rudolf Lucas, Klaus P. Schäfer, Inge Mühldorfer ${ }^{1}$ Department of Biotechnology, ALTANA Pharma AG, Konstanz, Germany; ${ }^{2}$ IMC University of Applied Sciences, Krems, Austria

TNF- $\alpha$ was found to up-regulate the rate of lung liquid clearance (LLC) in several animal models by the activity of its lectin-like tip domain. This activity can be mimicked by a circular peptide designated Tip. Tip was shown to induce LLC and consequently pulmonary edema resorption in different animal models. In order to 
study the mechanism of action of Tip, we established two in vitro test systems for edema resorption with the human lung epithelial cell line Calu-3:

(1) The ability of Calu-3 cells for spontaneous dome formation within confluent monolayers was utilized for quantitative examination of Tip's activity on active transepithelial fluid transport ("dome assay").

(2) The effects of Tip on bioelectrical properties of polarized cell monolayers were studied by using the Transepithelial Electrical Resistance (TEER) technology (“TEER assay”).

Dome assay experiments confirmed that dome formation is a sodium dependent process and that Tip is able to increase this process. TEER assay experiments proofed that Tip acts in a polarized and dose dependent manner.

In conclusion, there is strong evidence that the dome and the TEER assays are suitable systems for in vitro activity testing of Tip and other anti-edema peptide drug candidates and are useful for studies on their mechanism of action.

\section{PB59}

Large scale production of pDNA for gentherapy Jochen Urthaler, Hans Huber, Christoph Reinisch, Matthias Berkemayer Roman Necina, Boehringer Ingelheim Austria, Dr. Boehringerg. 5-11, 1121 Vienna, Austria. E-mail: jochen.urthaler@vie.boehringeringelheim.com (J. Urthaler)

Increasing safety concerns in gene therapy result in more stringent regulatory requirements. Those cover the complete process chain of cell banking, fermentation, and purification. A wide range of applications for pDNA requires gram to kilogram amounts for clinical trials and market supply. Economic, productive and robust processes are a prerequisite for low cost of goods (COGS). Therefore manufacturer of biopharmaceuticals need to address these considerations by developing new production processes meeting the new standards. Boehringer Ingelheim Austria developed a novel pDNA production process suitable for large-scale cGMP production. The process is based on $E$. coli fermentation. The process contains no components from animal origin. The optimized fermentation process yields up to $1 \mathrm{~g}$ pDNA/L fermentation volume. For isolation of pDNA from E. coli alkaline lysis in glass bottles or stirred tanks is commonly used. Cell wall structure is destroyed by a combination of alkaline $\mathrm{pH}$ and detergents. In our process alkaline lysis is operated in a closed, continuous system directly connected to clarification without using enzymes. We developed a scalable process for pDNA purification based on 3 different chromatographic principles. The capture step is carried out by hydrophobic interaction chromatography followed by anion exchange chromatography as intermediate step. Final polishing is carried out by conventional size exclusion chromatography in a group separation mode providing also buffer exchange and desalting for the final formulation. The process results in a pDNA drug substance of highest quality containing a low level of impurities (genomic DNA, RNA, proteins endotoxines) suitable for therapeutic applications. Depending on the conditions during fermentation and the used host homogeneities of greater than $95 \%$ or even $98 \%$ are possible, while a high over all yield can be achieved $(\sim 50 \%)$. During the development monolithic chromatography supports $\left(\mathrm{CIM}^{\circledR}\right)$ were compared with conventional resins and evaluated as potential alternatives. The complete process is monitored by a set of analysis covering cell banking to final purification. New sensitive methods based on HPCE, HPIEX and fluorometric measurement were developed.

\section{Industrial Biotechnology}

\section{Fine Chemicals and Biomaterials}

1

Engineering of $E$. coli for the production of L-Methionine Philippe Soucaille, Rainer Figge METabolic EXplorer SA, Biopôle Clermont-Limagne, F-63360 St Beauzire, France

Whereas many natural amino acids are currently produced by very cost efficient biological processes, the manufacture of Methionine is still performed by traditional chemical synthesis. This was mainly due to the poor performances of the currently available producing strains that inhibited the development and commercialization of a biological process to L-Methionine. Metabolic Explorer has recently reinvestigated and developed an efficient biological process that employs an engineered microorganism and utilizes a renewable starting material (corn sugar) as its feedstock and converts glucose into L-Methionine. We will describe (a) the general scheme for the engineering of the host organisms and (b) the general approach to maximize carbon and reducing equivalent throughput to L-Methionine. To highlight this effort, we will present the global approach developed to improve the process combining metabolic flux analysis, traditional protein biochemistry, molecular biology and fermentation optimisation.

\section{2}

Engineering Saccharomyces cerevisiae for xylose utilization Jack Pronk $^{1}$, Marko Kuyper ${ }^{1}$, Maurice Toirkens ${ }^{1}$, Ron Winkler ${ }^{2}$, Hans van Dijken ${ }^{1,2}$, Wim de Laat ${ }^{3}:{ }^{1}$ Kluyver Centre for Genomics of Industrial Fermentation and Department of Biotechnology, Delft University of Technology, Delft, The Netherlands; ${ }^{2}$ BIRD Engineering, Rotterdam, The Netherlands; ${ }^{3}$ Royal Nedalco, Bergen op Zoom, The Netherlands

Saccharomyces cerevisiae is an established 'work horse' of the fermentation industry and modern biotechnology has led to a spectacular expansion of the range of products that can be produced by this yeast. However, for the large-scale sustainable production of chemicals, it is equally important that the range of carbohydrate feedstocks be expanded. Especially relevant in this respect is the ability to consume the pentose sugars D-xylose and L-arabinose, which make up a substantial part of plant carbohydrates. Wild-type S. cerevisiae strains cannot metabolise D-xylose, but are capable of slowly metabolising its keto-isomer, D-xylulose. Therefore, efficient conversion of D-xylose into D-xylulose has long been a key issue in yeast metabolic engineering. Non-Saccharomyces yeasts that is capable of growing on D-xylose use two enzymes, xylose reductase and xylitol dehydrogenase for this purpose. While both enzymes have been successfully expressed in $S$. cerevisiae, this is not always compatible with efficient product formation. For example, in the case of ethanol production, the different cofactor specificities of these two oxidoreductases cause massive byproduct formation. Theoretically, introduction of a xylose isomerase, which catalyses the interconversion of xylose and xylulose, might circumvent these problems. 
However, it is notoriously difficult to express bacterial and Archaeal xylose isomerases in S. cerevisiae and, until recently, activities of heterologous xylose isomerases expressed in S. cerevisiae were vanishingly low, at least under physiological conditions. A breakthrough was reached when, in 2003, a xylose isomerase gene from the anaerobic fungus Piromyces was expressed in S. cerevisiae. While this led to high activities of xylose isomerase, these were not enough to enable fast growth or product (ethanol) formation. In this presentation, we will discuss how a combination of metabolic and evolutionary engineering led to fast and efficient xylose utilization by engineered Saccharomyces cerevisiae strains under aerobic as well as anaerobic conditions. Furthermore, we will illustrate how evolutionary approaches can be applied to facilitate the utilization of mixed substrates.

\section{References}

Kuyper, M., Harhangi, H.R., Stave, A.K., Winkler, A.A., Jetten, M.S., de Laat, W.T., den Ridder, J.J., Op den Camp, H.J., van Dijken, J.P., Pronk, J.T., 2003. High-level functional expression of a fungal xylose isomerase: the key to efficient ethanolic fermentation of xylose by Saccharomyces cerevisiae? FEMS Yeast Res. 4, 69-78.

Kuyper, M., Winkler, A.A., van Dijken, J.P., Pronk, J.T., 2004. Minimal metabolic engineering of Saccharomyces cerevisiae for efficient anaerobic xylose fermentation: a proof of principle. FEMS Yeast Res. 4, 655-664.

Kuyper, M., Hartog, M.M., Toirkens, M.J., Almering, M.J., Winkler, A.A., van Dijken, J.P., Pronk, J.T., 2005. Metabolic engineering of a xylose-isomerase-expressing Saccharomyces cerevisiae strain for rapid anaerobic xylose fermentation. FEMS Yeast Res. 5, 399-409.

Kuyper M., Toirkens M.J., Diderich, J.A., Winkler, A.A., Van Dijken, J.P., Pronk, J.T., in press. Evolutionary engineering of mixedsugar utilization by a xylose-fermenting Saccharomyces cerevisiae strain. FEMS Yeast Res. 5.

An up-scalable and efficient method to produce low molecular weight hyaluronic acid B.L. Fuhlendorff, B.M. Malle, K. SchwachAbdellaoui, K. Tommeraas, M. Weibye Novozymes Biopolymer A/S, Krogshoejvej 36, DK-2880 Bagsvaerd, Denmark. E-mail: bmm@novozymes.com (B.M. Malle)

Hyaluronic acid (HA) is a natural and linear polymer composed of $\beta$-1,3- $N$-acetyl glucosamine and $\beta$-1,4-glucuronic acid repeating disaccharide units with a molecular weight (MW) up to $6 \mathrm{MDa}$. It is a major constituent of the extracellular matrices and the synovial fluid. In the last decades, various fields of application including cosmetics, ophthalmology, rheumatology, tissue engineering and drug delivery have been explored, owing to the many important biological functions of HA and its unique physico-chemical properties. However, for some specific applications, the relatively high MW of HA is a limiting factor and the availability of low MW fractions would be highly desired. For food applications, low MW HA has been shown to penetrate the gastrointestinal barrier, thereby increasing the HA bioavailability. Moreover, low MW HA fractions are able to re-establish the HA content in the skin and can thus be used in cosmetics as anti-aging and anti-wrinkle agents. Finally, low MW HA has shown to prevent oxygen free radical damage in granu- lation tissue during wound healing. A range of methods has been described for the depolymerization of HA to low MW fractions. These techniques involve heat treatment, ultrasonication, UV/gamma irradiation, chemical and enzymatic degradation. We present results on a degradation process of HA originating from Bacillus subtilis fermentation into well-defined low MW fractions. The process developed in lab scale is safe, well-controlled and produce low MW HA fractions with narrow polydispersity. Moreover, the process is readily up-scalable. The low MW HA fractions are being evaluated in various cosmetic applications.

\section{4}

Industrial biotechnology and yeasts: Production of fine chemicals from D-glucose Danilo Porro ${ }^{1}$, Paola Branduardi ${ }^{1}$, Michael Sauer $^{2}$, Minoska Valli ${ }^{2}$, Diethard Mattanovich ${ }^{2}:{ }^{1}$ Department of Biotechnology and Bioscience, University of Milano Bicocca, Milan, Italy, 20126; ${ }^{2}$ Institute of Applied Microbiology, BOKU, University of Natural Resources and Applied Life Sciences, Vienna A-1190, Austria

Metabolic pathway manipulation for improving the properties and productivity of microorganisms is becoming an established concept. Metabolic engineering can be defined as the directed improvement of product formation or cellular properties through the modification of specific biochemical reactions or introduction of new ones with the use of recombinant DNA technology. A detailed analysis of the physiological means of the different pathways is needed to be able to introduce modifications aimed to the production of not only important metabolites, but also to understand the fundamentals of cell biology. Aimed to produce single compounds, metabolic engineering necessarily includes the modification of the cellular pathway(s) as well as the redirection of the energy toward the production itself. The existing metabolic engineering applications are the culmination of more than two decades of global experience developing processes for the production of fine chemicals, vitamins, nutraceuticals and animal nutritional aids such as amino acids. Based on the relative low complexity, the first biotechnological applications have been developed from microorganisms. Our laboratory has been engaged in this field since different years. Yeasts like Saccharomyces cerevisiae, Kluyveromyces lactis and Zygosaccharomyces bailii have been developed for the production of fine chemicals like lactic and ascorbic acids from D-glucose. In this contribution, we will present the last data obtained.

\section{5}

Methods to optimise threonine production with Escherichia coli Daniela Kruse, Nicole Dusch, Stephan Hans, Thomas Hermann, Ralf Kelle, Mechthild Rieping, Klaus Huthmacher Degussa AG, FA-FEBT, Kantstr. 2, 33790 Halle, Germany

Since 40 years amino acid production is in the focus of industrial microbiology. L-glutamate and L-lysine are produced with Corynebacterium glutamicum, while Escherichia coli is used for L-threonine production. The worldwide market of threonine drastically increases: In 1996 the amount of threonine produced worldwide was $15,000 \mathrm{t}$ and raised to $30,000 \mathrm{t}$ in 2000 and $45,000 \mathrm{t}$ in 2004. Concerning predictions of experts the demand of threonine will rise with a two-digit rate of economic growth within the next few years. Meanwhile the prices declined. These conditions enforce very efficient production processes. Beside the strain development 
and an optimised downstream procedure the fermentation process is an important target for productivity improvement. Strain development is dependent on detailed knowledge of the production strains. With innovative methods we are able to get a close look inside the cells under different culture conditions. These methods have been called 'omics' in recent literature. Knowledge about genome, transcriptome, proteome, phosphoproteome, metabolome and fluxome leads to new ideas for strain improvements. Data generated by these methods must be based on clearly defined culture conditions. Therefore, highly parallel fermentations have to be performed to generate biological parallel samples. Cutting-edge technical equipment is the basic requirement for experiments like this. Other requirements are optimised sampling for different analysis, technical parallels of analytical steps and a detailed statistical analysis of data. These procedures guarantee distinction between real data and data noise. Integration of all these data to a holistic model of the cell is the challenge for the future. By combination of a new strain, process and downstream improvements the plant productivity was increased drastically. We ended up in an optimized, fast and high yield process to scope challenges worldwide market comes up with.

\section{Reference}

Rieping, M., Hermann, T. (2002); Fermentation process for the preparation of L-Threonine; WO/0218543.

\section{Biocatalysis and Synthetic Application of Oxygenases}

\section{1}

Monooxygenases in biotechnology: A travelogue Rolf D. Schmid, Vlada Urlacher Institute for Technical Biochemistry, University of Stuttgart, D-70569 Stuttgart, Germany

P450 monooxygenases (cytochromes $\mathrm{P} 450$ ) are potentially quite useful biocatalysts, as they allow for the regioselective and stereoselective hydroxylation of activated as well as non-activated carbon atoms. In addition, the large number of members of the P450 superfamily exhibits a wide diversity of specificities from which a useful biocatalyst may be selected. From a technical point of view, however, they have significant drawbacks. Thus, they usually cannot be produced in large quantities nor recovered or stored without a severe loss in activity. Their catalytic activity is mostly quite low, and their operational stability leaves much to be desired. Most P450 enzymes require a complex protein/phospholipid machinery for activity, and the final electron donor in the reaction cascade, usually NADPH, does require extensive recycling to arrive at a commercially satisfactory process. Recently, the use of bacterial cytochromes as hydroxylation biocatalysts has received considerable attention. Some of them are natural fusion proteins, which contain the heme and the reductase domains on a single polypeptide chain. They are catalytically much more active compared to, e.g. cytochromes occurring in human tissue. We thus have set out to further improve the technical applicability of these enzymes, and have centered our activities around several bacterial cytochromes. It proved very useful to apply rational mutagenesis and directed evolution to these enzymes, leading to a surprising compatibility of mutant enzymes with a wide variety of substrates. Mechanisms for the limited stability of the enzymes were explored, leading to hybrid enzymes with enhanced stability, and the cofactor problem was alleviated using auxiliary enzymes or mediator-based technologies. As a result, a bioreactor based on microbial cytochromes was built and operated for several days.

\section{2}

Baeyer-Villiger monooxygenases: From genome analysis to structural details Marco W. Fraaije ${ }^{1}$, D.E. Torres Pazmiño ${ }^{1}$, E. Malito $^{2}$, A. Mattevi ${ }^{2}$, D.B. Janssen ${ }^{1}$ : ${ }^{1}$ Biochemical Laboratory, University of Groningen, Groningen Biomolecular Sciences and Biotechnology Institute, Nijenborgh 4, 9747 AG Groningen, The Netherlands; ${ }^{2}$ Department of Genetics and Microbiology, University of Pavia, via Abbiategrasso 207, 27100 Pavia, Italy. E-mail: m.w.fraaije@rug.nl (M.W. Fraaije)

Baeyer-Villiger monooxygenases represent useful biocatalytic tools as they can catalyze reactions, which are difficult to achieve using chemical means. However, so far only a limited number of these monooxygenases were available in recombinant form Kamerbeek et al. (2003). Using a recently described protein sequence motif Fraaije et al. (2002) and the available genome sequence information, we were able to identify and overexpress a number of novel bacterial BVMOs. One of the overexpressed BVMOs was found to be relatively stable as it originates from Thermobifida fusca, which grows at $\sim 60^{\circ} \mathrm{C}$. The enzyme was shown to be active on a broad range of substrates, preferring aromatic ketones Fraaije et al. (2005). The best substrate discovered so far is phenylacetone, hence its name: phenylacetone monooxygenase. We have solved the crystal structure of phenylacetone monooxygenase, which represents the first structure of a BVMO Malito et al. (2004). The crystal structure provides insight into the complex mechanism of catalysis mediated by FADcontaining BVMOs. By site-directed mutagenesis we have probed the role of several active-site residues. A crucial role is played by an arginine residue. As phenylacetone monooxygenase shares significant sequence identity ( $>40 \%)$ with all known NADPH-dependent BVMOs, many of the observed structural features seem to be conserved within this class of atypical monooxygenases. By homology modeling using the phenylacetone monooxygenase structure, catalytic properties of other Baeyer-Villiger monooxygenases can be explained or predicted.

\section{References}

Fraaije, M.W., Kamerbeek, N.M., van Berkel, W.J.H., Janssen, D.B., 2002. FEBS Lett. 518, 43-47.

Fraaije, M.W., Wu, J., Heuts, D.P., van Hellemond, E.W., Spelberg, J.H., Janssen, D.B., 2005. Appl. Microbiol. Biotechnol. 66, 393-400.

Kamerbeek, N.M., Janssen, D.B., van Berkel, W.J.H., Fraaije, M.W., 2003. Adv. Synth. Cat. 345, 667-678.

Malito, E., Alfieri, A., Fraaije, M.W., Mattevi, A., 2004. PNAS 101, 36, 13157-13162.

\section{3}

Screening for fungal Baeyer-Villiger monooxygenases $L$. Butinar $^{1}$, J. Friedrich ${ }^{1}$, V. Alphand ${ }^{2}{ }^{1}$ Laboratory of Biotechnology, National Institute of Chemistry, Ljubljana SI-1001, Slovenia; ${ }^{2}$ Groupe Biocatalyse et Chimie Fine CNRS FRE2712, Université de la Méditerranée, Marseille, France

The asymmetric form of the Baeyer-Villiger (BV) oxidation (transformation of ketones into lactones) is an important challenge 
for organic chemistry since the obtained lactones are valuable building blocks for synthesis of countless biologically active products. To date, enzymatic or microbial BV oxidations appears as more successful than their chemical counter-parts. (ten Brink et al.) Whereas most active BV monooxygenases are produced by bacteria (among which the well-studied enzyme of Acinetobacter calcoaceticus), only a few fungal strains expressing BVMO were described (Alphand et al., Carnell and Willetts). In order to increase the number of available biocatalysts which perform such an asymmetric biotransformations, a screening of fungi belonging to major groups of Zygo-, Ascoand Basidiomycetes was conducted using bicycloheptenone as testsubstrate. Surprisingly, a large number of the tested fungi were able to transform the substrate into one to four different lactone isomers. The yields, the enantio- and regio-selectivity of the reaction depended on the fungal strain.

\section{References}

Alphand, V., Furstoss, R., 2000. J. Mol. Catal. B 9, 209-17. Carnell, A., Willetts, A.,1992. Biotechnol. Lett. 14, 17-21. ten Brink, G.J., et al., 2004. Chem. Rev. 104, 4105-4123.

Pyranose oxidase from Trametes multicolour-application in biocatalysis $M$. Kujawa ${ }^{1}$, C. Leitner ${ }^{1}$, P. Halada ${ }^{2}$, J. Volc $^{2}$, B.M. Hallberg $^{3}$, C. $\overline{\text { Divne }^{3}, \text { C.K. Peterbauer }}{ }^{1}$, D. Haltrich ${ }^{1}:{ }^{1}$ Department of Food Sciences and Technology, BOKU-University of Natural Resources \& Applied Life Sciences, 1190 Wien, Austria; ${ }^{2}$ Institute of Microbiology, Academy of Sciences of the Czech Republic, 142 20 Praha 4, Czech Republic; ${ }^{3}$ Department of Biotechnology, KTH, Albanova University Center, 10691 Stockholm, Sweden. E-mail: magdalena.kujawa@boku.ac.at (M. Kujawa)

Pyranose oxidase (P2Ox) is a periplasmic enzyme that widely occurs in basidiomycetes. It catalyses the $C-2$ oxidation of several aldopyranoses to the respective 2-keto derivatives, transferring electrons to molecular oxygen to yield $\mathrm{H}_{2} \mathrm{O}_{2}$. $\mathrm{P} 2 \mathrm{Ox}$ is of interest for carbohydrate conversions, as its reaction products (2-keto sugars) can be attractive intermediates in the production of food ingredients. We cloned the gene encoding P2Ox, and subsequently amplified a cDNA clone by RT-PCR. The cDNA was inserted into a bacterial expression vector and successfully expressed in E. coli. Properties of the heterologous protein were compared to those of the native enzyme showing that they are essentially identical. Both the native as well as the recombinant enzyme were used in biotransformations of sugars. Recently, the 3D-structure of this tetrameric enzyme was elucidated. Based on structural information, several enzyme variants containing point mutations were constructed and further characterised. Two of these variants (E542K and E542R) displayed improvements in stability and certain kinetic properties thus making them attractive for biocatalytic applications.

\section{5}

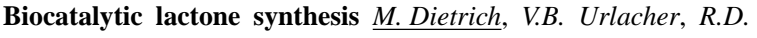
Schmid Institute of Technical Biochemistry, University of Stuttgart, Stuttgart, Germany, 70569. E-mail: itbmdi@ @itb.uni-stuttgart.de (M. Dietrich)

Lactones are important compounds for the fragrance and flavour industry. Right now the production of lactones is dependant on the import of crude materials from tropical countries. In this project, we want to tackle the manufacture of lactones via a biocatalytic route using P450 Monooxygenases. Cytochrome P450 Monooxygenases catalyse the oxyfunctionalization of non-activated $\mathrm{C}$-atoms. CYP102A1 from Bacillus megaterium, CYP102A2 and CYP102A3 from Bacillus subtilis are soluble fusion proteins comprising $\mathrm{P} 450$ monooxygenase and FAD/FMN reductase domains in one polypeptide chain. All three enzymes are highly homologous fatty acid hydroxylases. Especially, CYP102A1 also known as P450 BM-3 is well characterized and shows high activity compared to other P450 monooxygenases. The aim of the work is to change selectivity but conserve the high activity that is typical for those enzymes. Using methods of structure modelling, rational protein design and directed evolution new mutants of these enzymes with changed regioselectivity are obtained. Products of conversion with monooxygenases are intermediates in the production of lactones.

\section{Applied Biocatalysis and Carbohydrate Engineering}

\section{1}

Biocatalytically-enabled assembly of functional and responsive carbohydrate-based nanostructures Jonathan S. Dordick, Guangyu Zhu, George John, Jun Li Department of Chemical and Biological Engineering, Rensselaer Polytechnic Institute, Troy, NY 12180, USA

The interface of biology and materials science has led to new materials with unique structural and functional properties, and new process technologies with the ability to produce, from "bottoms up", a wide range of biomimetic structures. These materials and their designs have broad application as catalysts, sensors, and devices for use in synthesis, cell and tissue engineering, bioanalysis and screening, and nanoelectronics. We have focused on the generation of sugar-based nanostructures, complete with tailored selectivities and biocatalytic activities at the molecular and nanoscales. These include biocatalytically-generated carbohydrate derivatives that selfassemble with high precision to give novel architectures with functional and responsive properties.

2

Izumoring: A strategy for total production of rare sugars Ken Izumori Rare Sugar Research Center, Kagawa University, Kagawa 761-0795, Japan. E-mail: izumori@ag.kagawa-u.ac.jp

We found a new enzyme, D-tagatose 3-epimerase (DTE), that epimerize all ketohexoses at C-3 position. This epimerase catalyze not only between $\mathrm{D}$-tagatose and $\mathrm{D}$-sorbose, but also $\mathrm{D}$-fructose $=\mathrm{D}$ psicose, $\mathrm{L}$-sorbose $=\mathrm{L}$-tagatose, and $\mathrm{L}$-psicose $=\mathrm{L}$-fructose. This new enzyme offered us a useful key tool to connect all ketohexoses using hexitols as intermediates. The figure shows that all eight ketohexoses can be connected with DTE and polyol dehydrogenases (PDH) in a ring. Using this ring, we can easily find the pathway to transfer D-fructose to D-tagatose via D-psicose using DTE and PDH. Various aldose isomerases transform ketohexoses to the corresponding aldohexoses. So, we can connect all 16 aldohexoses with 8 ketohexoses using the enzyme. Finally, all hexoses, 8 ketohexoses, 10 hexitols and 16 aldohexoses are connected using enzyme reactions in a ring structure (not shown). This kind of strategy is effective also on transformation of tetroses and pentoses. Now, we can produce all monosaccharides; tetroses, pentoses and hexoses by enzyme reac- 


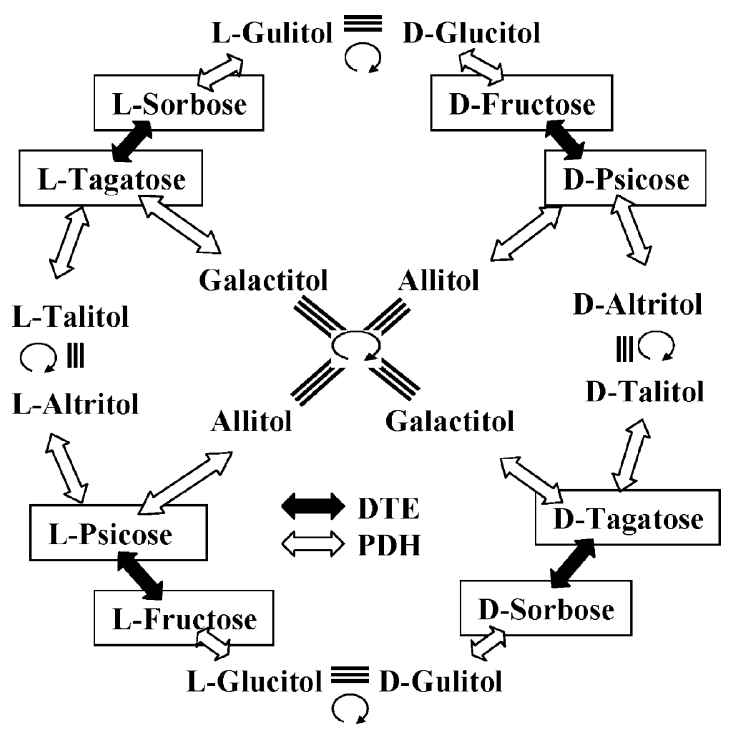

tions. The bioproduction strategy of all rare sugars (Monosaccharides that are rare in nature) is illustrated using ring form structures named as Izumoring. We have already succeeded to produce D-psicose in large scale and are now in the progress of mass production of various rare sugars from natural and cheap sugars using Izumoring.

3

Bioprocess development for chiral intermediates Christian Wandrey Institute of Biotechnology, Forschungszentrum Jülich $\mathrm{GmbH}$, Jülich D-52425, Germany

Chiral alcohols, diols, amino alcohols and chiral acids (e.g. hydroxy acids and amino acids) play an important role in pharma and agro synthesis. In the past such chiral intermediates were obtained by racemic resolution via chiral reductions using prochiral precursors. Here the problem of cofactor regeneration arises. This problem could be solved by enzyme-coupled or substrate-coupled cofactor regeneration using formate or isopropanol as reducing agent. Alternatively, whole cell bioreductions were developed where glucose is used as the reducing agent. In recent years "designer microorganisms" were developed in which oxidoreductases (e.g. alcohol dehydrogenases) were over-expressed in Escherichia coli. In such cases, cofactor regeneration was achieved intracellularly with isopropanol as the reducing agent or by coexpression of a formate dehydrogenase, so that once again format could be used for reduction. Another route to obtain chiral intermediates is a fermentative approach using classical pathways (like the aromatic amino acid pathway). Here, the pathway is interrupted after the intracellular production of chorismate. New chiral intermediates can be obtained by over expression of additional genes, which catalyzed the production of chorismate derivatives leading to cyclohexadiene-transdiols and the corresponding amino cyclitols. The last example can be regarded as an example of industrial biotechnology where glucose is used as starting material (white biotechnology). Here bioprocess development is carried out in an integrated approach, in which molecular biochemical engi- neering cares for the optimal intracellular metabolic network and the classical biochemical engineering cares for the optimal environment of the cell in a fermenter. Examples will be given which reach (in cooperation with industrial partners) up to kilogram scale.

\section{4}

A new approach for the production of DHAP: The system of biotransformations Michael Schümperli, Matthias Heinemann, Stephan Gomolka, Anne Kümmel, Sven Panke Institute of Process Engineering, ETH Zürich, 8092 Zürich, Switzerland. E-mail: schuemperli@ipe.mavt.ethz.ch (M. Schümperli)

Biotransformations are usually involved in just one or very few separate reactions in organic syntheses. The development of a cell-free "System of Biotransformations" (SBT), in which a set of enzymes acts in a coordinated fashion in a one-pot synthesis, lead to increased catalytic complexity, selectivity and yield, as well as facilitated operation at reduced costs. The example chosen to prove the usefulness of the SBT-approach is the production of dihydroxyacetone phosphate (DHAP). DHAP, a C3-compound from glycolysis, is an important precursor for asymmetric $\mathrm{C}-\mathrm{C}$-bond formation. So far, the production of DHAP is difficult and expensive. For the construction of the DHAP-producing SBT, E. coli's glycolysis is isolated from the metabolism to an as large as possible extent by the construction of a multi-KO-mutant. A culture is grown in an appropriate medium, homogenized in the production buffer, and used as the catalytic system. High production yields can be achieved since the production pathway is almost completely isolated from the metabolic network. The employed DHAP-producing SBT provides not only a path from glucose to the product, but also an integrated ATP-regeneration and NAD-recycling system. In first experiments with a tpi-KO-mutant, a DHAP-production yield on glucose of $32 \%$ could be achieved, without optimizing the system. The system remained active for more than $24 \mathrm{~h}$. Up to now, ATP cannot be applied in catalytic concentrations, but has to be present in equimolar amounts to glucose. The production yield could be increased by $10 \%$ through the addition of phosphate ions as substrate to the reaction, enabling the system to utilize ATP more efficiently. These experiments indicate that the SBT-approach is viable and a large potential remains to improve the DHAP-producing SBT.

\section{5}

Development of pectate lyases for industrial applications M. Bjornvad, K.S. Johansen, C. Andersen Novozymes A/S, Research \& Development, DK-2880 Bagsvaerd, Denmark. E-mail: cara@novozymes.com (C. Andersen)

For some years Novozymes have manufactured a pectate lyase for scouring of textile as an ecological alternative to the traditional harsh chemical treatment, and recently, we at Novozymes discovered additional applications for pectate lyases. However, to be commercially attractive more robust pectate lyses had to be developed. In this paper, we will demonstrate how we for two different pectate lyases have improved their stability significantly. As the two enzymes are quite similar in sequence and structure, it was a new discovery for us to find that different concepts of protein engineering had to be used in our attempt to stabilize each individual pectate lyase. The stability of one enzyme was improved by substitutions in the internal of the structure whereas the stability of the other pectate lyase was primarily improved by changing surface residues. Starting with 
knowledge from structural analysis, we have applied rational based protein engineering resulting in few selected variants. Also random based protein engineering combined with screening of hundreds of thousands variants was used. In conclusion: the project team showed that by synergistic use of the two approaches, we were able to move faster towards a solution and eventually we succeeded finding new stabilized pectate lyase variants, applicable for new business areas.

\section{Biofuels}

2

The potential role of alternative (renewable) fuels in the U.S. Lonnie O. Ingram Director of the Florida Center for Renewable Chemicals and Fuels, University of Florida, IFAS, Gainesville, FL, USA

The importance energy independence as a national goal equals or exceeds that of the moon landing in 1990. The development of a new industry to produce fuel ethanol from woody biomass would increase national security, improve employment and the environment, and provide substantial relief from the debt of imported petroleum. Costs associated with the rapid development of this new industry ( $\sim \$ 1.4$ billion per year) could be paid by re-assigning 1 cent per gallon from existing Federal Gasoline taxes, a small price to pay for future energy independence. The corn-to-ethanol industry continues to make a remarkable contribution to our liquid fuel needs through expansion. Today, one row of every six rows of corn is converted into fuel ethanol in the U.S. However, this expansion will be limited to $3-4 \%$ of total automotive fuel by the economics of corn costs and production. Corn can do more! Corn stover is the single most abundant agricultural residue in the US and can be used as a feedstock to produce $60-80$ gallons of ethanol per dry ton. Further expansion with other biomass feedstocks such as agricultural and municipal residues (lignocellulose, woody biomass) could produce over 100 billion gallons of fuel ethanol annually according at a recent joint report by the USDA and DOE (April, 2005). Current technology has been demonstrated at pilot scale for the production of fermentable sugars from hemicellulose by dilute acid hydrolysis and for the hydrolysis cellulose using fungal cellulose enzymes. Biocatalysts such as recombinant Escherichia coli have been developed and demonstrated for the efficient conversion of all sugar constituents of biomass to ethanol. A national goal for the full-scale deployment of current technology to produce biomass-based fuel ethanol will allow the US to reduce imported petroleum by $50 \%$. Together with increased efficiencies of hybrid vehicles, energy independence could be achieved within 10-20 years. Similar gains could be realized by many nations around the world to provide new manufacturing and employment, redistributing wealth and ensuring a cleaner, healthier environment.

\section{3}

Bioethanol production using thermophilic bacteria Marie Just Mikkelsen, Birgitte K. Ahring EMAB, Biocentrum-DTU, 2800 Lyngby, Denmark

The industry of bioethanol production is facing the challenge of redirecting the process from fermentation of relatively easily convertible but expensive starchy materials, to complex but inexpensive lignocellulosic biomass. On lignocellulosic hydrolysates, Gram-positive thermophilic bacteria have unique advantages over the conventional ethanol production strains. The primary advantages are their natural broad substrate specificities, and in some strains, a high tolerance to lignocellulosic hydrolysates. Moreover, ethanol fermentation at high temperatures also has the advantages of high productivities and substrate conversions, low risk of contamination and facilitated product recovery. Some thermophilic bacteria naturally produce primarily ethanol from most sugar monomers present in lignocellulosics, but modifications are still necessary to increase ethanol yields.

4

Physiological responses to furfural and HMF and the link to other stress pathways $\underline{S}$.W. Gorsich, P.J. Slininger, Z.L. Liu Crop Bioprotection, National Center for Agricultural Utilization Research ARS/USDA, 1815 N. University St., Peoria, IL 61604, USA. E-mail: gorsichs@ncaur.usda.gov (S.W. Gorsich)

The release of useable sugars from lignocellulose biomass for industrial fuel-ethanol fermentation is often facilitated by a weak acid hydrolysis step. As a consequence, inhibitors such as furfural and 5-hydroxymethylfurfural (HMF) are formed as degradation products of xylose and glucose, respectively. Moreover, the fermentative end-product of ethanol is also inhibitory. These, and other inhibitors present an environment, which elicits the expression of stress-related genes in Saccharomyces cerevisiae. Recently, 65 S. cerevisiae genes have been identified as important in furfural stress tolerance. When furfural is present, yeast with these genes disrupted grows poorly compared to wild-type yeast. A sub-class of these genes suggests that yeast grown under furfural-induced stress may rely upon similar pathways as cells grown under various other stresses, including oxidative, heat, and sorbate. To investigate this link further, we analyzed stress-induced phenotypes such as ROS activity, DNA damage, and membrane damage in wild-type and mutant yeast exposed to furfural or HMF stress. Moreover, we investigated whether overexpression of this sub-class of genes would provide protection from furfural-induced stress and oxidative damage.

5

Furan reduction capacity of Saccharomyces cerevisiae strains in fermentation of dilute-acid hydrolyzates Anneli Nilsson ${ }^{1}$, Tobias Modig $^{1}$, Marie F. Gorwa-Grauslund ${ }^{2}$, Bärbel Hahn-Hägerdal ${ }^{2}$, Gunnar Lidén ${ }^{1}$ : ${ }^{1}$ Department of Chemical Engineering, Lund University, P.O. Box 124, S-221 00 Lund, Sweden; ${ }^{2}$ Department of Applied Microbiology, Lund University, P.O. Box 124, S-221 00 Lund, Sweden. E-mail: Anneli.Nilsson@chemeng.lth.se (A. Nilsson)

Micro-organism to be used in fermentation of lignocellulose hydrolyzates should preferably have three characters: (a) high ethanol tolerance, (b) resistance to inhibitors found in the hydrolyzate, and (c) a broad substrate utilization range, since the hydrolyzate contains several sugars. In addition to the possibility of controlling the level of potential inhibitors, fed-batch fermentations also permit the parallel uptake of several different monomeric sugars. Two strains of Saccharomyces cerevisiae, CBS 8066 (a commonly used laboratory strain) and TMB 3000 (a strain isolated from a spent sulfite liquor fermentation plant), were characterized in batch and fed-batch fermentation of a dilute-acid hydrolyzate from spruce. The strains had different abilities to ferment spruce hydrolyzate. The study suggests that the furan reduction capacity of a yeast strain is 
a key factor for its performance in fermentation of lignocellulosic hydrolyzate.

\section{Microbial Metabolomics and Secondary Metabolites}

\section{1}

Prospects for the biosynthesis of antitumoral polyketides in bacteria and fungi Christian Hertweck Leibniz Institute for Natural Products Research and Infection Biology, HKI, Jena, Germany. Email: Christian.Hertweck@hki-jena.de

Polyketides constitute a structurally highly diverse group of natural products that possess broad ranges of pharmacological properties and represent a major source for novel cancer therapeutics. However, these compounds may be sub-optimal in regard of activity, selectivity, availability and unwanted side effects. In addition, the sustainable production of these valuable metabolites can be a challenge. Studying the molecular basis of the biosynthetic pathways may set the basis for improving the production and for rationally engineering derivatives with altered bioactivity profiles, e.g. through targeted knockouts, mutasynthesis Ziehl et al. (2005), and swapping of pathway genes. Our results in elucidating and manipulating the biosynthesis of selected antitumoral polyketide metabolites from bacteria (aureothin, chartreusin) and fungi (cytochalasines, rhizoxin) are presented. Analyses at the genetic and biochemical levels provided new insights into several unusual biosynthetic features, e.g. non-linear polyketide assembly for the nitroaryl-substituted polyketide aureothin He and Hertweck $(2003,2005)$, an oxidative rearrangement cascade in the chartreusin pathway Xu et al. (2005), and a fungal iterative PKSNRPS hybrid synthase Schuemann and Hertweck (2005) involved in cytochalasin biosynthesis. The most surprising result was obtained from elaborating the biogenesis of the antimitotic agent rhizoxin from Rhizopus sp., which allowed for a significant improvement in large-scale production Partida-Martinez and Hertweck (2005).

\section{References}

He, J., Hertweck, C., Chem. Biol. 2003, 10, 1225-1232; Chem. Bio. Chem. 2005, 6, 908-912.

Partida-Martinez, L., Hertweck, C., 2005.

Schuemann, J., Hertweck, C., 2005.

Xu, Z., Jakobi, K., Welzel, K., Hertweck, C., in press. Chem. Biol. 12.

Ziehl, M., He, J., Dahse, H.-M., Hertweck, C., 2005. Angew. Chem. Int. Ed. 44, 1202-1205.

\section{2}

Analysis and Engineering of glycopeptide biosynthesis

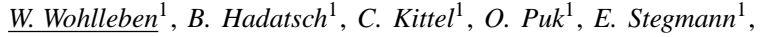
R. Süßmuth ${ }^{2}$, S. Weist ${ }^{2}$ : ${ }^{1}$ Institute of Microbiology, Microbiology/Biotechnology, Eberhard-Karls-University Tuebingen Auf der Morgenstelle 28, 72076 Tuebingen, Germany; ${ }^{2}$ Institute of Chemistry, Department of Organic Chemistry, Eberhard-Karls-University Tuebingen, Auf der Morgenstelle 18, 72076 Tuebingen, Germany

Glycopeptides such as vancomycin and teicoplanin are the drugs of last resort for the treatment of severe infections caused by antibiotic resistant Gram-positive bacteria. Glycopeptides inhibit the peptidoglycan biosynthesis by binding as dimers to the D-Ala-D-Ala termini of the cell wall precursors. Amycolatopsis balhimycina synthesizes the vancomycin-type glycopeptide balhimycin, whose structure and biological properties greatly resemble vancomycin and which only differs by its glycosylation pattern. Using a "Reverse Genetics" approach we have identified the $66-\mathrm{kb}$ gene cluster encoding the biosynthesis of balhimycin. By a combination of genetics, biochemistry and analytical organic chemistry, we were able to elucidate the biosynthetic pathway and to assign functions to almost all genes of the cluster. The biosynthesis starts with the pathway-specific provision of the non-proteinogenic amino acids $\beta$-hydroxytyrosine $(\beta-\mathrm{Ht})$, hydroxyphenylglycine $(\mathrm{Hpg})$ and dihydroxyphenylglycine (Dpg) which form together with ( $N$-methyl)-leucine and asparagine the heptapeptide backbone of balhimycin. Dpg is synthesized via a polyketide synthase mechanism (PKSIII) similar to that known from plant chalcon/stilben synthases (Pfeifer et al., 2001). For the $\beta-\mathrm{Ht}$ synthesis three genes are essential which form an operon (Puk et al., 2004): BpsD, an NRPS binds a tyrosine molecule, which is then hydroxylated by the P450 monooxygenase OxyD. The perhydrolase Bhp is required for the release of $\beta-\mathrm{Ht}$. Subsequently BhaA, a NADH/FAD-dependent halogenase catalyzes the chlorination of $\beta$-Ht to form chloro- $\beta$-hydroxytyrosine (Puk et al., 2002), which is needed to stabilize the dimerization. The amino acids are linked by non-ribosomal peptide synthetases (Recktenwald et al., 2002), and the aromatic side chains are interconnected by $\mathrm{P} 450$ monooxygenases; a series of reactions which lead to the first antibiotically active intermediate. Inactivation of the oxygenase genes revealed the order of the cyclization steps (Bischoff et al., 2001): The oxygenases act in a stepwise fashion in the sequence OxyB, OxyA and OxyC. The resulting cross-linked heptapeptide is then finally modified by methylation and glycosylation. The biosynthesis is regulated by the StrR-type regulator Bbr, which was shown to bind in front of different operons of the balhimycin gene cluster. This ensures the coordinated expression of the biosynthetic genes. The non-producing mutants, defective in the supply of the non-proteinogenic amino acids, were used as recipients in cloning experiments as well as in approaches of precursor-directed biosynthesis by feeding chemically synthesized alternative precursors. Thus, novel balhimycin derivatives were generated (Weist et al., 2002, 2004).

\section{References}

Bischoff et al., 2001. Angew. Chem. Int. Ed. 40, 4688-4691.

Pfeifer et al., 2001. J. Biol. Chem. 276, 38370-38377.

Puk et al., 2004. J. Bacteriol. 186, 6093-6100.

Puk et al., 2002. Chem. Biol. 9, 225-235.

Recktenwald et al., 2002. Microbiology 148, 1105-1118.

Weist et al., 2002. Angew. Chem. Int. Ed. 41, 3383-3385.

Weist et al., 2004. J. Am. Chem. Soc. 126, 5942-5943.

\section{3}

Spectroscopy guided discovery of novel bioactive microbial natural products Thomas Ostenfeld Larsen, Michael Edberg Hansen CMB Biocentrum-DTU, Technical University of Denmark, 2800 Lyngby, Denmark

The task of finding novel bioactive natural products is usually bioassay driven. Often a certain type of compound (e.g. polyketide, alkaloid) turns out to be active in an assay. When having generated a promising hit in a bioassay the normal procedure in the drug discovery process usually is to produce a large number of structurally analogous compounds either by traditional chemical synthesis or by combinatorial chemistry in order to study structure activity relation- 
ships and to find even more active lead compounds. Alternatively to chemical synthesis of analogues Nature can be explored for structurally similar compounds by UV-spectroscopy guided screening. This work will present a new method for the systematic and automated computer assisted search of full UV spectra in large number of datafiles for both dereplication of known and discovery of new natural products based on the use of the new mathematical algorithm $X$-hitting.

\section{4}

Exploring the substrate spectrum of the antibiotic producing bacteria Saccharopolyspora erythraea P. Krabben, P. Oliveira, F. Baganz, J. Ward Department of Biochemical Engineering, University College London, London WC1E 7JE, UK

Knowledge of substrate utilisation capabilities play an important role in the development of genome scale metabolic models (Borodina et al., 2005) and refinement of first generation annotations. Furthermore, knowledge of the product formation during catabolism of different substrates provides valuable information about the distribution of metabolic fluxes and thereby forms a basis for rational strain improvement. We present here, data on the substrate utilisation capabilities and the corresponding product formation of S. erythraea. This analysis will help in improving the production of erythromycin and provide clues to the activation of the cryptic secondary metabolic pathways present in the $S$. erythraea genome.

\section{Reference}

Borodina, I., et al., (2005). Genome-scale analysis of Streptomyces coelicolor A3(2) metabolim. Genome Res. in press.

\section{5}

A structured model to represent rifamycin B fermentation in complex media Pramod Wangikar, P.M. Bapat, S. Bhartiya, K.V. Venkatesh Department of Chemical Engineering, Indian Institute of Technology, Bombay, Powai, Mumbai 400 076, India. E-mail: pramodw@iitb.ac.in (P. Wangikar)

Modeling of growth and product formation on complex media containing multiple substitutable substrates is a challenge. Complex media offers the organism multiple choices of carbon and nitrogen substrates including free amino acids, peptides, soluble and insoluble proteins in addition to the defined sources such as glucose and ammonium sulfate. We present a structured model that accounts for growth and product formation kinetics of rifamycin B fermentation in a multi-substrate complex medium. The model considers the organism to be an optimal strategist with a mechanism to regulate the uptake of the substrate combinations. Further, we assume that the uptake of a substrate depends on the level of a key enzyme, which may be inducible. The model also considers control parameters as fraction of flux through a given metabolic branch. The control parameters are obtained using a simple multi-variable constrained optimization. The model parameters were rigorously estimated via a specifically designed experimental plan. The model correctly predicts the experimentally observed growth and product formation kinetics and the regulated simultaneous uptake of the substitutable substrates under different fermentation conditions. The model and the model parameters provide useful insights into the growth and product formation strategy of this industrially important process. This presentation will describe the experimental results, the model development and the relevant model parameters for A. mediterranei 5699 .

\section{Cell Factories}

1

Cell factory for succinic acid production: Genome-scale analysis and metabolic engineering of Mannheimia succiniciproducens Sang Yup Lee Department of Chemical and Biomolecular Engineering and Department of BioSystems, BioProcess Engineering Research Center and Bioinformatics Center, Korea Advanced Institute of Science and Technology, 373-1 Guseong-dong, Yuseong-gu, Daejeon 305-701, Republic of Korea. E-mail: leesy@ kaist.ac.kr

The recent surge in oil price and the increasing concern on our environment have generated much interest in the production of chemicals from renewable resources. Succinic acid, also called as amber acid, is a four-carbon dicarboxylic acid, which can be used as a precursor of numerous products including biodegradable polymers, green solvents, pharmaceuticals, and bulk and fine chemicals. A new capnophilic bacterium named Mannheimia succiniciproducens MBEL55E was isolated from the rumen of Korean cow. This bacterium can produce large amounts of succinic acid along with some other metabolites such as lactic, formic and acetic acids. We have completely sequenced the genome of $M$. succiniciproducens and charaterized its genome content in the context of metabolic pathways. We then constructed the genome scale in silico metabolic network for metabolic flux analyses, and carried out metabolic flux analysis under varying environmental conditions. Based on the in silico analyses results, we selected several target genes to be manipulated for enhanced succinic acid production. Detailed results of metabolic engineering based on genome-scale information will be reported.

\section{Acknowledgements}

This work was supported by the Korean Systems Biology Research Grant from the Ministry of Science and Technology, LG Chem Chair Professorship, IBM SUR program and BK21 program.

2

Inverse Metabolic Engineering Gregory Stephanopoulos, Yong-Su Jin, Hal Alper MIT, Department of Chemical Engineering, Room 56-469, Cambridge, MA 02139. E-mail: gregstep@mit.edu (G. Stephanopoulos)

We have been developing tools for Inverse Metabolic Engineering in order to identify gene targets that improve the phenotype of industrial strains and cells for medical applications. To this end, we create genomic fragment libraries from a source organism and use it to transform the host organism. Cells are properly selected in environments that favor the phenotype of interest and genes enriched in these cells are sequenced and used in follow up transformations of cells with specific genetic backgrounds. This overall strategy is complemented with additional tools for modulating gene over-expression, gene deletion, and high throughput clone isolation. We will demonstrate applications of this strategy to the identification of gene targets for improved xylose assimilation in recombinant Saccharomyces cerevisiae and improved lycopene production in Escherichia coli. 
3

Enzymic analysis of NADPH metabolism in Penicillium chrysogenum: Presence of a mitochondrial NADPH dehydrogenase in 及-lactam-producing cultures Diana Harris, Jasper Diderich, Zita van der Krogt, Marijke Luttik, Walter van Gulik, Johannes van Dijken, Jack Pronk Department of Biotechnology, Delft University of Technology, Julianalaan 67, 2628 BC Delft, The Netherlands. Email: d.m.harris@tnw.tudelft.nl (D. Harris)

Based on assumed reaction network structures, NADPH availability has been proposed to be a key constraint in $\beta$-lactam production by Penicillium chrysogenum. In this study, NADPH metabolism was investigated in glucose-limited chemostat cultures of an industrial P. chrysogenum strain. Enzyme assays confirmed the NADPspecificity of the dehydrogenases of the pentose-phosphate pathway and the presence of NADP-dependent isocitrate dehydrogenase. Pyruvate decarboxylase/ NADP-linked acetaldehyde dehydrogenase and NADP-linked glyceraldehyde-3-phosphate dehydrogenase were not detected. Although the NADPH requirement of penicillin-Gproducing chemostat cultures was calculated to be 1.5-1.7-fold higher than that of non-producing cultures, activities of the major $\mathrm{NADPH}$-providing enzymes were the same. Isolated mitochondria showed high rates of antimycin A-sensitive respiration of NADPH, thus indicating the presence of a mitochondrial NADPH dehydrogenase that oxidizes cytosolic NADPH. The presence of this enzyme in $P$. chrysogenum has important implications for stoichiometric modelling of central carbon metabolism and $\beta$-lactam production and may provide an interesting target for metabolic engineering.

4

The use of multi-parameter flow cytometry for characterisation and monitoring of insect cell-baculovirus fermentations in a mechanically-agitated bioreactor Bojan Isailovic ${ }^{1}$, Alvin $W$. Nienow $^{1}$, Ian W. Taylor ${ }^{2}$, Ryan Hicks ${ }^{2}$, Christopher J. Hewitt ${ }^{1}$ : ${ }^{1}$ Biochemical Engineering, Centre for Formulation Engineering, School of Engineering (Chemical Engineering), The University of Birmingham, Edgbaston, B15 2TT, UK; ${ }^{2}$ AstraZeneca, Mereside, Alderley Park, Macclesfield, Cheshire SK10 4TG, UK. E-mail: BXI897@bham.ac.uk (B. Isailovic)

Bacteria and mammalian cells have been traditionally used as hosts for commercial recombinant protein production. However, in recent years, the insect cell-baculovirus system has emerged as a potentially attractive recombinant protein expression vehicle. Although flow cytometry has been used widely for analysis of mammalian and microbial cells, there is very little information on applications of this powerful technique in insect cell culture. Here we compared cell ratiometric counts and viability (propidium iodide and calcein AM) of Sf-21 cell cultures using flow cytometry to those determined by more traditional methods using a haemocytometer and the trypan-blue exclusion dye. Flow cytometry has also been used to monitor various parameters during cultures of Sf21 infected with the recombinant Autographa californica Nuclear Polyhedrosis Virus (AcNPV) containing the inserted nucleic acid sequence amFP486 coding for AM-Cyan coral protein, which emits natural green fluorescence.
5

Promoter engineering for tuning gene expression in yeast Elke Nevoigt $t^{1,2}$, Hal Alper ${ }^{1}$, Curt Fischer ${ }^{1}$, Gregory Stephanopoulos ${ }^{1}:{ }^{1}$ Department of Chemical Engineering, Massachusetts Institute of Technology, Cambridge, MA 02139, USA; ${ }^{2}$ Department of Microbiology and Genetics, Berlin University of Technology, D-13353 Berlin, Germany. E-mail: E.Nevoigt@LB.TU-Berlin.de (E. Nevoigt)

Complete elucidation of the genetic control of a metabolic flux requires the availability of fine-grained expression levels of the gene(s) of interest. We developed a collection of promoters of varying strength for tuning gene expression in the yeast $S$. cerevisiae. Engineered promoters were obtained through random mutagenesis of the constitutive TEF1 promoter. Eleven mutated promoters were selected by fluorescence-activated cell sorting (FACS) spanning gradually increasing activities between about 8 and $120 \%$ compared to the native $T E F 1$ promoter. Data were also confirmed at the level of mRNA via RT-PCR. By introducing selectable markers in front of the different $T E F 1$ promoter mutations, we provided plasmid collections, which can be directly used to amplify promoter replacement cassettes for genomic integration of the fine-grained promoter collection in front of any yeast gene.

\section{Poster Abstracts Industrial Biotechnology (IB)}

IB1

Cloning and selection of L-arabinase gene from Compost microorganism Mi-Sun Ahn, Hyoung-Joo Lee, Yeon-Woo Ryu* Department of Molecular Science and Technology, Ajou University, Wonchon-dong, Youngtong-gu, Suwon 442-749, Korea. E-mail: anmint@ajou.ac.kr (M.-S. Ahn)

L-Arabinose, widely distributed in plant kingdom, is a component of plant cell wall. L-Arabinose does not abundantly exist in free state in plants, but usually in corn hull, sugar beet pulp, gum arabic, mesquite gum, as the polysaccharide such as arabinoxylan and arabinogalactan. To produce arabinose from agricultural wastes, we screened arabinogalactan degradable strain from compost. Thereafter, putative arabinase gene from this strain was cloned (B1029 TS2-8). As a result of spectrometric assay using $\rho$-nitrophenyl $\alpha$ L arabinofuranoside, recombinant showed 3-4-fold higher activity than wild type E. coli strain. After enzymatic reaction with corn fiber, B1029 TS2-8 produced $2.15 \mathrm{~g} / \mathrm{L}$ of L-arabinose, which was detected on HPLC. However, the enzyme activity was very low. So, we are transferring the gene into expression vector system. Further characterization study and enzyme engineering to enhance the activity toward corn fiber will be presented in poster.

IB2

Production of alkaline protease by a halotolerant bacillus pumilus isolated from Van Lake in Turkey Ozlem Ates ${ }^{1}$, Dilek Kazan ${ }^{1,2}$, Ebru Toksoy Oner ${ }^{1}:{ }^{1}$ Department of Chemical Engineering, Marmara University,Goztepe 34722 Istanbul, Turkey; ${ }^{2}$ The Scientific and Technical Research Council of Turkey (TUBITAK), Research Institute for Genetic Engineering and Biotechnology (RIGEB), Marmara Research Center Campus (MRC), Gebze 41470 Kocaeli, Turkey. E-mail: atesozlem@ @otmail.com (O. Ates)

There are only a limited number of hypersaline areas all over the world, which include several locations in Turkey such as Van Lake 
located in Eastern region of Turkey. Isolation and identification of halophilic and hyperhalophilic microorganisms from such locations is essential for the determination of biodiversity in Turkey. High-level production of extremozymes from these microorganisms has also many economical advantages due to their stability at extreme reaction conditions. Proteolytic enzymes are the most important group of enzymes produced commercially. Of these, proteases produced by alkalophilic microorganisms are investigated not only in scientific areas such as protein chemistry and protein engineering but also find wide application in food, pharmaceutical, leather and detergent industries. In this study, 24 microorganisms isolated from Van Lake were screened for the presence of extracellular alkaline protease activity. The optimum screening temperature and $\mathrm{pH}$ were determined as $37^{\circ} \mathrm{C}$ and $\mathrm{pH} 9.5$, respectively. One of the isolates that could grow at $0-20 \%$ salinity reached highest levels of extracellular alkaline protease activity. This best producer, which was identified as the halotolerant Bacillus pumilus, was found to produce alkaline protease both in the presence and absence of $\mathrm{NaCl}$. To improve enzyme production yields, culture conditions such as medium composition, growth $\mathrm{pH}$ and temperature were optimized. The effect of different carbon sources, organic and inorganic nitrogen sources on the production of alkaline protease was studied. Whereas a mixture of inorganic and organic nitrogen sources induced high protease production, use of only an organic nitrogen source supported poor enzyme production. Halotolerant Bacillus pumilus produced maximum alkaline protease activity when maltose, yeast extract and sodium nitrate were used as carbon source, organic and inorganic nitrogen sources, respectively.

\section{Acknowledgement \\ This project was supported by TUBITAK through project TBAG 2321-103T069.}

\section{IB3}

Heterologous proteins production in Zygosaccharomyces bailii: Expression system optimisation Paola Branduardi ${ }^{1}$, Laura Dato $^{1}$, Davide Cattaneo ${ }^{1}$, Michael Sauer ${ }^{2}$, Diethard Mattanovich ${ }^{2}$, Danilo Porro ${ }^{1}{ }^{1}$ Dipartimento di Biotecnologie e Bioscienze, Università degli Studi di Milano-Bicocca, Piazza della Scienza, 2-20126 Milano, Italy; ${ }^{2}$ Institute of Applied Microbiology, University of Natural Resources and Applied Life Sciences, Muthgasse 18, A-1190 Wien. E-mail: paola.branduardi@unimib.it (P. Branduardi)

In the market of biochemical products a very important role is played by heterologous proteins production, and despite recent advances in mammalian cells exploitation, yeasts can still present advantages as host systems. Among them, the spoilage yeasts belonging to the Zygosaccharomyces genus have become, due to some peculiar properties, significantly attractive. In particular, Z. bailii is characterized by acid resistance, osmotolerance to high sugar and ethanol concentration combined with high biomass yield. Despite still little is known about its genetics and cellular biology, our group is working on its development and exploitation for recombinant productions with an integrated approach coupling physiological study with the creation of molecular tools for heterologous proteins production. We previously described and did a patent application regarding the first techniques necessary to transform this yeast and to express and secrete different proteins derived from different sources. Here we present and discuss the last advances in optimization of heterol- ogous protein expression In particular, on one side we present a reproducible strategy for target gene deletion, leading to the first $Z$. bailii auxotrophic mutant, and on the other we show the improvement of gene dosage and plasmid stability by building a set of multicopy expression vectors based on the sequences of the Z. bailii $2 \mu$-like endogenous plasmid pSB2 and an integrative plasmid.

\section{IB4}

In vivo functional analysis of $\boldsymbol{\gamma}$-butyrolactone autoregulator receptor in Streptomyces clavuligerus ATCC27064, a clavulanic acid producer S.U. Choi, S.J. Kang, M.K. Kim, H.R. Park, Y.I. Hwang Division of Food Science and Biotechnology, Kyungnam University, Masan 631-701, Republic of Korea. E-mail: suchoi@kyungnam.ac.kr (S.-U. Choi)

All the known $\gamma$-butyrolactone autoregulator receptors are highly conserved in the DNA binding motif present in their N-terminal portions and have been proposed to play roles as transcriptional regulators in antibiotic production and/or morphological differentiation. Previously, Kim et al. reported that the cloned scaR in Streptomyces clavuligerus has several characteristics of the autoregulator receptors in the genus Streptomyces. In this study, to clarify the in vivo function of $s c a R$, a scaR-disrupted strain was constructed by means of homologous recombination after introducing a scaR-disruption construct via transconjugation from $E$. coli. No difference in morphology was found between the wild-type strain and the scaR disruptant. However, the scaR disruptant showed a 1.8-fold higher production of clavulanic acid than the wild-type strain. The phenotype was restored to the original wild-type phenotype by complementation with intact $s c a R$. Therefore, the autoregulator receptor, ScaR, acts as a negative regulator of biosynthesis of clavulanic acid but plays no role in cytodifferentiation of $S$. clavuligerus.

\section{IB5}

Expression of lactate dehydrogenase genes of Rhizopus oryzae in Aspergillus niger Şeyda Açar ${ }^{1}$, Luisa Trindade ${ }^{2}$, Meral Yücel ${ }^{3}$, Haluk Hamamcl ${ }^{4}$, Leo H. De Graaff ${ }^{2}{ }^{1}$ Department of Biotechnology, Middle East Technical University, Turkey; ${ }^{2}$ Department of Microbiology, Fungal Genomics Section, Wageningen University, The Netherlands; ${ }^{3}$ Department of Biological Sciences, Middle East Technical University, Turkey; ${ }^{4}$ Department of Food Engineering, Middle East Technical University, Turkey. E-mail: seyda@metu.edu.tr (Ş. Açar)

Lactate dehydrogenase catalyses the production of lactate from pyruvate. It is the first target for many researches on lactic acid producer microorganisms like Rhizopus oryzae. In the present study based on the known sequences of $R$. oryzae $l d h \mathrm{~A}$ and $l d h \mathrm{~B}$ genes Skory (2000), they were cloned and expressed in a citric acid producer fungus Aspergillus niger. The Aspergillus niger strains expressing $l d h \mathrm{~A}$ or $l d h \mathrm{~B}$ gene resulted in increased production of lactate in Aspergillus niger. Among 50 transformants tested 4 ldh $\mathrm{A}$ and $5 l d h \mathrm{~B}$ expressing strains were found to have higher lactate dehydrogenase activity compared to wild type in the conditions tested. The highest specific activity obtained with $l d h \mathrm{~A}$ transformants was only 2.5 times of the wild type while this was 10 times for one of the transformants expressing $l d h \mathrm{~B}$. In addition to increased lactate production citric acid production was also increased. However, gluconic acid production ceased in the $l d h \mathrm{~A}$ or $l d h \mathrm{~B}$ expressing $A$. niger strains. The production of lactic acid in A. niger transformants and 
lactate dehydrogenase A and lactate dehydrogenase B enzymes are being investigated in the chosen strains.

\section{Reference}

Skory, C. D. (2000) Isolation and expression of lactate dehydrogenase genes from Rhizopus oryzae, Appl. Environ. Microbiol. 66, 2343-2348.

\section{IB6}

Microbial $9 \alpha$-hydroxylation of pregnane steroids $B$. Angelova, $S$. Mutafov, T. Avramova, L. Stefanova The Stephan Angeloff Institute of Microbiology, Bulgarian Academy of Sciences, Sofia, Bulgaria. E-mail: blaga@microbio.bas.bg (B. Angelova)

Selection of N source suitable for production of Rhodococcus $\mathrm{sp}$. biomass for the purposes of microbial transformation of $5 \alpha \mathrm{H}$-epoxypregnanolone $(5 \alpha \mathrm{H})$ and $\Delta^{5}$-epoxy-pregnenolone $\left(\Delta^{5}\right)$ into their $9 \alpha$-hydroxy-derivatives was carried out. Three dehydrated and three non-dehydrated $\mathrm{N}$ sources were tested. The transformation reaction was carried out in phosphate buffered medium containing $1 \mathrm{~g} \mathrm{~L}^{-1}$ of the steroid substrate and $0.1 \mathrm{~g} \mathrm{~L}^{-1}$ cells. The steroids were determined by HPLC. The transformation resulted in formation of three derivatives appearing in the reaction medium in the sequence: $\Delta^{4}$ 3-keto-; $9 \alpha$-hydroxy- and $9 \alpha, 20 \beta$-hydroxy-epoxy-pregnenolone. A strong influence of the $\mathrm{N}$ source on the hydroxylating activity of the biomass was observed. Triptose (Difco) gave a cell depot actively hydroxylating $5 \alpha \mathrm{H}$ without any significant accumulation of the $\Delta^{4}$ 3-keto-derivative. The most effective accumulation of hydroxylated derivatives of $\Delta^{5}$ was observed with biomass grown on freshly prepared meat extract while the commercial products Triptose (Difco), Meat Extract (Difco) and Lactalbumin (Flika) gave valuable information about the dynamics of the transformation process.

\section{IB7}

Biobleaching of kraft cellulose pulp by Poliporus versicolor Aysun Ergene ${ }^{1}$, Nazif Kolankaya ${ }^{2}:{ }^{1}$ Kirıkkale University, Faculty of Science and Literature, Department of Biology, Yahsihan, Kırıkkale, Turkey; ${ }^{2}$ Hacettepe University, Faculty of Science, Department of Biology, Beytepe, Ankara, Turkey

The suitability of culture supernatant from Poliporus versicolor for use in the biobleaching of kraft cellulose pulp was investigated. $P$. versicolor was found to grow on mycological broth (1\% soytone, $4 \%$ D-glucose and $0.5 \%$ cellulose pulp). Maximal extracellular ligninase production was detected after7 day ( $7 \mathrm{nkat})$. The optimum biobleaching conditions are $30^{\circ} \mathrm{C}$ and $\mathrm{pH}: 4.8$, with 10 days. In this condition $P$. versicolor decreased the Kapa number from 38.55 to 19.42 and increased brigthness from 28 to 32.7 in 10 day treatment.

\section{IB8}

Xylanase production, purification and characterization from a soil isolate, Bacillus M-13 Ayşsegül Ersayin ${ }^{1}$, Aytaç Kocabaş ${ }^{1}$, B. Zümrüt Ogel $^{2}$, Ufuk Bakir ${ }^{3}:{ }^{1}$ Biotechnology Department, Middle East Technical University, Ankara, Turkey; ${ }^{2}$ Food Engineering Department, Middle East Technical University, Ankara, Turkey; ${ }^{3}$ Chemical Engineering Department, Middle East Technical University, Ankara, Turkey, 06531. E-mail: ubakir@metu.edu.tr (U. Bakir)

Xylan is a major component of the plant cell wall, representing up to $35 \%$ of the dry weight. Xylan molecule is a complex polymer consisting of a $\beta$-D-1,4-linked xylanopyranoside backbone substituted with acetyl, arabinosyl and glucuronosyl side chains. Hydrolysis of the xylan backbone is mainly catalysed by endo- $\beta$ 1,4-xylanases (EC 3.2.1.8). Many bacterial and fungal species are able to utilize xylan as a carbon source. Interest in the enzymology of xylan hyrdolysis has increased because use of xylanases in bioconversion of agricultural wastes to valuable products like single cell protein, xylo-oligosaccharides and fuel, in bio-bleaching processes, food and animal feed industries. In this study, xylanolytic nature of a soil isolate Bacillus spp., Bacillus M-13, has been shown. Bacillus M-13 produced multiple xylanases when grown on a liquid medium containing agricultural wastes as the sole carbon source. Various agricultural wastes including corn-cobs and cotton waste, with and without pretreatments were used to maximize enzyme production. The major xylanase having molecular weight of $20 \mathrm{kDa}$ upon SDSPAGE and a pI of 9.1 upon IEF was partially purified by liquid chromatographic techniques 150 -fold with $40 \%$ recovery, including gel filtration, ion exchange and hydrophobic interaction chromatography.

\section{IB9}

Determination of inactivation mechanism of enzymes in laun-

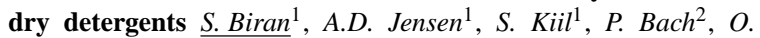
Simonsen ${ }^{2}{ }^{1}$ CHEC Research Center, Department of Chemical Engineering, Technical University of Denmark, DK-2800 Kgs. Lyngby, Denmark; ${ }^{2}$ Novozymes A/S, DK-2880 Bagsværd, Denmark. E-mail: sb@kt.dtu.dk (S. Biran)

Enzymes are important constituents in the laundry detergents due to their contribution to shortening washing times, reduction of energy and water consumption by lowering washing temperatures, provision of environmentally friendlier wash-water effluents and fabric care. However, they can loose a significant part of their activity in the chemically-hostile detergent matrix over a time period of several weeks. Therefore, improving the storage stability of enzyme granulates is the main challenge in the development of a new product. The complexity of the detergent matrix implies the presence of a complicated mechanism involved in the inactivation of the enzymes. A combination of factors, such as oxidation by $\mathrm{H}_{2} \mathrm{O}_{2}$ released by the bleaching agents, humidity, high temperature, autolysis of enzymes, high local $\mathrm{pH}$ in a granule, oxygen, and other detergent components, plays a role in the activity loss. An experimental investigation on the inactivation of the solid-state enzyme during storage has been initiated. The release rate of $\mathrm{H}_{2} \mathrm{O}_{2}$ from the bleaching agent, sodium percarbonate, was determined using a simple and accurate method for measuring the gas phase $\mathrm{H}_{2} \mathrm{O}_{2}$ concentration. The deactivation kinetics of pure enzyme was determined as a function of gas phase $\mathrm{H}_{2} \mathrm{O}_{2}$ concentration and humidity. The preliminary results indicate that humidity plays a significant role in the inactivation mechanism of the detergent enzyme due to a possible increase in the mobility of the enzyme molecule and the surface area exposed to destructive agents. The effect of main granulate ingredients on the stability of the enzyme was investigated and the extent of the protection of each component was estimated. The study is important for the revealing of the phenomena occurring in the detergent matrix during storage. Understanding the inactivation mechanism provides a valuable tool for the development of more effective protective coatings and stabilizers. 


\section{IB10}

Enzymatic synthesis of sugar vinyl esters catalyzed by protease from Bacillus subtilis Rosângela Balaban Garcia, Marta Costa, Mauricio Rodrigues Borges, Fábio Fernandes Fagundes Chemistry Department, Federal University of Rio Grande do Norte, Natal 1662, Brazil

The use of biosurfactants in cosmetic industry has attracted great attention of biotechnological researchers because they consist of two inexpensive, renewable and easily accessible starting agricultural materials: sugar and oil/fat. Carbohydrate based products are non-toxic, biocompatible and biodegradable. In addition, the enzymatic processes present many advantages in comparison with the chemical methods, which employ high temperatures in the presense of alkalin catalysts, high-energy consuption and low selectivity of products. Sugar esters present vast application, such as for antibiotics, biomaterials, surfactants, cosmetics and so on. We investigated the synthesis of sugar vinyl esters, using protease-catalysed transesterefication method applying protease from Bacillus subtilis. Sucrose $0.125 \mathrm{M}$ and vinyl ester $0.5 \mathrm{M}$ has been mixed in dimethylformamide at $160 \mathrm{rpm}$ of agitation. At first, we have studied the effects of protease from Bacillus subtilis concentrations $(5,10,20$ and $40 \mathrm{mg} / \mathrm{ml}$ ) as catalyst. Afterwards, the influence of the temperature (30 and $\left.50{ }^{\circ} \mathrm{C}\right)$. After that, the influence of the molar ratios $(1: 1 ; 1: 2 ; 1: 4 \mathrm{~m} / \mathrm{m})$ between vinyl laurate $(\mathrm{CH} 3(\mathrm{CH} 2) 10 \mathrm{COOCH}-\mathrm{CH} 2)$ and sucrose. Subsequently, we investigated the effects of water amount, using 0 , 5,10 and $20 \%$ of water in DMF. The conversion ratios of sucroseto-sucrose esters were determined decreasing sucrose measurement with HPLC. The results showed that the best conditions to produce high activity on the enzymatic reaction was by using $40 \mathrm{mg} / \mathrm{mL}$ of protease from Bacillus subtilis at $30^{\circ} \mathrm{C}$, molar ratio of $1: 4$ (vinyl laurate:sucrose) and adding $10 \%$ of water in DMF. Finally, we succeeded in the characterization of vinyl sugar ester, which was produced after 25 hours of reaction by ${ }^{13} \mathrm{C}$ NMR. The results confirmed the $\mathrm{C1}^{\prime}-$ substituted sugar mono-ester (1'-O-vinyl lauroyl sucrose).

\section{IB11}

Effects of oxygen transfer on $\alpha$-amylase production by $B$. amyloliquefaciens Nurhan Güngör, Güzide Calık BRE Lab, Department of Chemical Engng, Ankara University, 06100 Ankara, Turkey

$\alpha$-Amylase (E.C. 3.2.1.1) a commercially important enzyme used in food, textile, detergent, brewing, paper, and animal feed industries; hydrolyses $\alpha-1,4$ glucosydic bonds in amylose, amylopectin, and related polysaccharides. Optimum medium composition and influence of bioreactor operation parameters on $\alpha$-amylase production with high yield and selectivity were determined together with the metabolic flux distributions using B. amyloliquefaciens (NRRL B-14396), which is found to be a good producer of the enzyme. Systematic investigation of oxygen transfer in relation with the metabolic fluxes for $\alpha$-amylase is not available in the literature. Shake-flask experiments were conducted in $0.5 \mathrm{dm}^{3}$ air-filtered bioreactors in orbital shakers with agitation and heating controls (B. Braun, Certomat-BS1). Laboratory-scale bioreactors were composed of agitation, heating, foam, dissolved-oxygen and $\mathrm{pH}$-controlled 1.0 and $3.5 \mathrm{dm}^{3}$ systems (B. Braun, Biostat Q; and Chemap). After separation of the cells with a Sorval RC28S ultracentrifuge, $\alpha$-amylase activity was measured by the DNS method Bernfeld (1955). Amino acid concentrations were determined with a HPLC (Waters), pro- tein and organic acid concentrations were measured with a HPCE (Waters, Quanta 4000E) Çalik et al. (1998). Oxygen transfer characteristics in the bioreactor systems were calculated using the Dynamic method Rainer (1990). In the mass flux balance-based analyses, a pseudo-steady state approximation for the intracellular metabolites and the accumulation rates of the extracellular metabolites measured throughout the fermentations in considerations of the biochemical feature of the system were used to acquire the flux distributions. In laboratory scale, the effects of different $\mathrm{C}$ sources, i.e. glucose, fructose, maltose, lactose and soluble starch; $\mathrm{N}$ sources, i.e. $\left(\mathrm{NH}_{4}\right)_{2} \mathrm{HPO}_{4},\left(\mathrm{NH}_{4}\right)_{2} \mathrm{SO}_{4}$, and $\mathrm{NH}_{4} \mathrm{Cl}$ and/or their concentrations; and the operation parameters, $\mathrm{pH}$ and temperature on cell growth, substrate utilization, $\alpha$-amylase and by-product concentrations, and $\alpha$-amylase activity were investigated. In pilot scale, the fermentation and oxygen transfer characteristics of the bioreaction system together with the metabolic fluxes were determined.

\section{References}

Bernfeld, P., 1955. Methods in Enzymol. 1:149-159.

Çalık, P., Çalık, G., Özdamar, T.H., 1998. Enzyme Microb. Technol. 23, 451-461.

Rainer, B.W., 1990. Chem. Biochem. Eng. 4:185-196.

\section{IB12}

Recovery of Geldanamycin from fermentation broth using adsorbent resins John T. Casey, Padraig K. Walsh, Daniel G. $O$ 'Shea School of Biotechnology and National Institute of Cellular Biotechnology, Dublin City University, Dublin 9, Ireland. E-mail: john.casey3@mail.dcu.ie (J. Casey)

Geldanamycin is a Benzoquinone Ansamycin produced as a secondary metabolite by the Actinomycete, Streptomyces hygroscopicus var. geldanus, in submerged culture. It is a broad-spectrum antibiotic and exhibits an anti-tumour activity through its interaction with the Heat Shock Protein 90 family of chaperone proteins. The optimal recovery of geldanamycin from fermentation broths is the focus of the presented work. The application of adsorbent resins was assessed and the viability of developing a Solid Phase Extraction process for geldanamycin was determined. It was found that recovery of geldanamycin from fermentation broth was possible using adsorbent resins and the use of resins facilitated the recovery of a product stream of high purity. The composition of the fermentation broth had an impact on the performance of the resins and it was found that assessing performance on the basis of experimentally derived data was more apt than studying the kinetics of adsorption alone. Adsorptive processes are, by their nature, difficult to optimise and this was found to be the case when optimising the recovery of geldanamycin from partially clarified fermentation broth. Considerable effort was required to optimise geldanamycin adsorption, via examining the effect of environmental conditions and process system configuration, and geldanamycin desorption, via examining the effect of environmental conditions and investigating selective elution patterns.

\section{IB13}

Production and characterization of compatible solutes from Marinococcus ssp. exploiting a microfiltration bioreactor C. Maresca ${ }^{1}$, A. Catapano $^{1}$, L. Buommino ${ }^{1}$, M.A. Tufano $^{1}$, E. Galinski ${ }^{2}$, M. De Rosa ${ }^{1}$, C. Schiraldi ${ }^{1}:{ }^{1}$ Department of Experimental Medicine, Second University of Naples, Naples 80138, Italy; 
${ }^{2}$ Institute of Microbiology \& Biotechnology, Meckenheimer Allee 168, 53115 Bonn, German. E-mail: angela.catapano@unina2.it (A. Catapano)

It is well known that halophilic eubacteria synthesize compatible solutes in order to face the high ionic strength environment in which they proliferate. These biomolecules are gaining more and more importance as biotechnological tools in a wide array of applications, and the recently developed novel bioprocesses enabled large-scale production of these compounds and therefore commercial distribution. However, there is still interest in the optimization of the production process of sole hydroxyectoine that was demonstrated to have a superior stabilization capacity. In this research project, we optimized growth conditions of Marinococcus M52 to obtain high yield of hydroxyectoine. Their production proved faster in the batch experiments at a higher oxygen supply, even if the stationary phase was comparable in all cases. The MF experiments showed a final biomass which was 5-fold that obtained in the corresponding batch process. In addition the monitoring of compatible solutes production showed that in the last $24 \mathrm{~h}$ experiment hydroxyectoine accounted for $80-90 \%$ of the total content, accumulating up to $13-15 \%$ of the cell dry weight. Studies for improving downstream process for ectoine and hydroxyectoine recovery showed that short permeabilization cycles in water are effective in a temperature range between $45^{\circ} \mathrm{C}$ and $55^{\circ} \mathrm{C}$ using a ratio 1:4/biomass:water. Moreover, we evaluated the ability of ectoine to stabilize lactic acid bacteria during freeze-drying and to protect human cells from heat stress. In particular, the compatible solutes were added to the medium of confluent keratinocytes before subjecting the cells to heat stress, or LPS insult. RT-PCR and Western blot analysis demonstrated the hsp70B' gene over-expression in heat stressed human keratinocytes treated with ectoine. Finally, we demonstrated that even at low concentration $(50 \mathrm{mM})$ these compatible solutes are able to diminish cell death in lactic acid bacteria due to lyophilization procedure.

\section{IB14}

Application of metabolic control analysis (MCA) on the enzymatic hydrolysis of model cellulose substrates Chia-Wen Chang ${ }^{1}$, Natalija Andersen ${ }^{1,2}$, Katja Johansen ${ }^{2}$, Jens Nielsen $^{1}$, Lisbeth Olsson ${ }^{1}{ }^{1}$ Center for Microbial Biotechnology, BioCentrum, Technical University of Denmark, DK-2800 Kgs. Lyngby, Denmark; ${ }^{2}$ Novozymes A/S, Krogshoejvej 36, DK-2800 Bagsvaerd, Denmark. E-mail: cwc@biocentrum.dtu.dk (C.-W.C. Chang)

Among all existing alternative energy sources, biomass-derived bioethanol is especially advantageous since it is clean, sustainable and potentially inexpensive. The actual production of bioethanol is divided into a pre-treatment step, an enzymatic hydrolysis step and a fermentation step. While fermentation has been practiced by humans for centuries, our knowledge of enzymatic hydrolysis is still limited. Nevertheless, it is well accepted that hydrolysis is a synergism among three classes of enzymes, $\beta$-glucosidase, endoglucanase and cellobiohydrolase. Furthermore, a complete and efficient hydrolysis is only achieved when the enzymes are in the correct proportion. The common enzyme proportions have so far been based on the natural enzyme abundance as produced by the microorganism or mainly been determined by a trial-and-error approach. In this study, however, we used metabolic control analysis (MCA) as a modelling tool to gain fundamental knowledge about enzymatic hydrolysis and to design an optimal enzyme mixture. Using Gepasi, a free software, the degree of control of each reaction step or each enzyme towards the overall hydrolysis can be calculated. Our hypothesis is that the amount of each enzyme used for hydrolysis should be proportional to the degree of control of the enzyme. With MCA, a significant amount of time, labour and reagents can be saved on developing hydrolysis enzyme mixture. Furthermore, this study should demonstrate the usefulness of MCA on understanding enzyme-catalyzed reactions outside the cell.

\section{IB15}

Process Optimization for Fed-Batch Fermentation of Bacillus thuringiensis subsp. israelensis Arindam Chaudhury, Gopinathan $C$ Department of Biotechnology, University of Calicut, Calicut, Kerala 673635 India. E-mail: g_achaudhury@umassd.edu (A. Chaudhury)

Bacillus thuringiensis $(\mathrm{Bt})$ is a desirable biopesticide because of its low cost and lack of toxicity. The use of Bt in developing countries is limited due to process complications and economic non-feasibility of the fermentation process. In the present study, we have shown how regional production, using inexpensive alternatives for carbon and protein sources, can effectively reduce the cost of mass production of Bt. While using alternative media supplements, the biomass production, nor the larvicidal activity was hampered. In addition, the positive effects of sparged aeration and the indispensable role of yeast extract were also proved. This work provides the first experimental proof of delineating the sporulation process and delta-endotoxin production. The role of various buffering agents and additives in increasing biomass production and early sporulation were also investigated.

\section{IB16}

Enhanced Coenzyme Q10 contents by DO control and restricted respiration in Agrobacterium tumefaciens Gi-SubChoi ${ }^{1}$, YongSung Kim ${ }^{1}$, Jin-Ho Seo ${ }^{2}$, Yeon-Woo Ryu ${ }^{1 *}$ : ${ }^{1}$ Department of Molecular Science and Technology, Ajou University, Suwon, 443-749, Korea; ${ }^{2}$ School of Agricultural Biotechnology, Seoul National University, Seoul 151-742, Korea. E-mail: plasmid@ajou.ac.kr (G.-S. Choi)

For the production of Coenzyme $\mathrm{Q}_{10}\left(\mathrm{CoQ}_{10}\right)$, an electron carrier in the respiration chain with antioxidant activity. With decrease of dissolved oxygen level from 20 to $5 \%$, the intracellular $\mathrm{CoQ}_{10}$ content increased about 4 -fold, yielding $2 \mathrm{mg}$ per g-dry cell weight at $5 \%$ dissolved oxygen level. Azide significantly increased the intracellular $\mathrm{CoQ}_{10}$ content, with the highest value of $5.3 \mathrm{mg}$ per $\mathrm{g}$ dry cell weight in the presence of $0.45 \mathrm{mM}$ of sodium azide. However, DNP (up to $200 \mu \mathrm{M}$ ) and $\mathrm{H}_{2} \mathrm{O}_{2}$ (up to $10 \mu \mathrm{M}$ ) did not affect the intracellular $\mathrm{CoQ}_{10}$ content, indicating proton gradient release and oxidative stress do not affect the synthesis of $\mathrm{CoQ}_{10}$. These results show that restricted electron flux by limited oxygen supply and the addition of azide increases the intracellular $\mathrm{CoQ}_{10}$ content.

\section{IB17}

Monitoring a Pichia pastoris fermentation using FT-IR/ATR $\underline{\text { John Dahlbacka }}^{1}$, Jan Weegar ${ }^{1}$, Niklas von Weymarn ${ }^{2}$, Kaj Fagervik ${ }^{1},{ }^{1}$ Åbo Akademi University, Faculty of Chemical Engineering, Finland; ${ }^{2}$ Helsinki University of Technology, Laboratory of Bioprocess Engineering, Finland. E-mail: John.Dahlbacka@abo.fi (J. Dahlbacka) 
Fourier transform infrared spectroscopy (FT-IR), combined with in situ heat sterilizable attenuated total reflection (ATR) probes, constitutes a promising and versatile technique for on-line monitoring of bioprocesses. The FT-IR enables rapid determinations of the medium composition without the requirement of sample withdrawal and preparation. In this work the concentration levels of the substrates glycerol and methanol were monitored on-line in Pichia pastoris cultivation. Partial least squares (PLS) models were used for obtaining the concentration readings. The glycerol concentration measurement proved to be very reliable and reproducible in the glycerol batch phase. However, the on-line information regarding the glycerol concentration was not utilized for any process control purposes. On the other hand, the availability of on-line information about the methanol concentration proved to be crucial for the successful implementation of the cultivations. The temperature strategy in the methanol fed-batch phase utilized temperatures as low as $10^{\circ} \mathrm{C}$. In order to keep the metabolic activity at a reasonable level the culture was therefore pushed towards the maximal substrate consumption rate, rather than being a conventional substrate limited fed-batch. As a consequence methanol accumulation occurred on occasions. Without on-line information about the concentration this accumulation, if sustained, would have resulted in a poisoning of the culture, either from methanol itself, or perhaps more importantly from formaldehyde. Therefore, it can be concluded that the FT-IR/ATR instrument was very useful in this application.

\section{IB18}

Grey-box modeling of continuous cultures of Aspergillus oryzae under oxygen limitation Florin Paul Davidescu, Sten Bay Jørgensen Department of Chemical Engineering, Denmark Technical University, Building 229, DK-2800 Lyngby, Denamrk. E-mail: fpd@kt.dtu.dk (F.P. Davidescu)

Modeling biochemical reaction network in microorganisms still represents a challenge due to the very large number of enzyme catalyzed biochemical reactions, to the very complex system and to the many feed-forward and feedback regulation mechanisms. The presented approach to model such a system is based on the stochastic grey-box modeling framework proposed by Kristensen et al. (2003). This methodology consists of parameters estimation based on a prediction error method followed by different statistical tests for parameter significance and for model (in-) validity. The methodology furthermore allows estimation of unknown functional relations, e.g. kinetic rates. A set of experimental data Zangirolami (1998) obtained during continuous cultures of a high enzyme producing Aspergillus oryzae strain. The oxygen concentration was decreased stepwise and the substrate concentration was modified from one experiment to other. A model proposed by Agger et al. (1998) is investigated on these data. The primary interest is to develop a physiologically feasible model, also at the low oxygen concentrations often found in industrial practice.

\section{References}

Agger et al., 1998. Growth and product formation of Aspergillus oryzae during submerged cultivations: Verification of a morphologically structured model using fluorescent probes, Biotechnol. Bioeng. 57, 321-329.
Kritensen, et al., 2004. A method for systematic improvement of stochastic grey-box models, Computers and Chemical Engineering, 28, pp 1431-1449.

Paul davidescu, F., fpd@kt.dtu.dk, Biochem. Eng.

Zangirolami, T.C., 1998. Modeling of growth and product formation in submerged cultures of filamentous fungi, Ph.D thesis, Lyngby, Department of Chemical Engineering, Technical University of Denmark.

\section{IB19}

The development of an optimised fermentation media for Geldanamycin production Lynne F. Dobson, Daniel G. O'Shea School of Biotechnology and National Institute of Cellular Biotechnology, Dublin City University, Dublin 9, Ireland. E-mail: lynne.dobson2@mail.dcu.ie (L. Dobson)

Microbially produced secondary metabolites such as antibiotics have tremendous economic importance. Streptomyces spp. have long been identified as sources of antibiotics and chemotherapeutic compounds, synthesising over 4000 bioactive compounds. Geldanamycin is a novel chemotherapeutic agent produced by Streptomyces hygroscopicus var. geldanus in submerged fermentation. Initial studies have focused on optimisation of media design through understanding and controlling metabolic routes of biosynthesis within the cell. Geldanamycin is a by-product of the Shikimate or aromatic amino acid biosynthesis pathway. Stimulation of this pathway and concomitant production of Geldanamycin is achievable through amino acid control. Increasing concentrations of primary carbon source greatly influence biomass generation and product formation, as does the inclusion of cations such as magnesium and calcium to the fermentation media. Optimisation of production media through balancing minerals, nitrogen, and carbon sources has significantly improved antibiotic yields in shake flask cultures and the development process will be extended into pilot scale through the use of bioreactors.

\section{IB20}

Modulation of PEP carboxylase activity in silico and its effect on glutamate production in Corynebacterium glutamicum Naomi Fawcett, Ida Hakansson, Charlie Bryce, Mansi El-Mansi Applied Microbiology and Biotechnology Research Group, School of Life Sciences, Napier University, Edinburgh, EH10 5DT, Scotland. Email: m.el-mansi@napier.ac.uk (M. El-Mansi)

Synopsis: During growth of Corynebacterium glutamicum on glucose or other glycolytic intermediates, PEP carboxylase fulfils an anaplerotic function as it replenishes intermediary metabolism with biosynthetic precursors that are essential for growth and glutamate production. Under these conditions, PEP carboxylase plays a central role and this in turn is characterised by a high flux control coefficient thus rendering this enzyme an ideal target for metabolic interventions. Further analysis in silico revealed that any increases in the concentration of the enzyme was accompanied by increases in flux through the enzyme itself as well as glutamate formation, presumably as a consequence of sustaining a high intracellular level of $\alpha$-ketoglutarate; the immediate precursor for glutamate biosynthesis. 


\section{IB21}

A combined approach to enhance periplasmic expression of human growth hormone in Escherichia coli, using a modified signal peptide from Alpha Amylase gene of Bacillus licheniformis S.K. Falsafi ${ }^{1,2}$, A. Zomorodipour ${ }^{2}:{ }^{1}$ Islamic Azad University of Jahrom, Iran; ${ }^{2}$ Department of Mol Genet. National Inst for Genet Eng \& Biotechnol., Tehran, Iran. E-mail: soheil_falsafi@yahoo.com (S.K. Falsafi)

The alpha amylase gene signal peptide, originated from a strain of Bacillus licheniformis, was shown to be able to transport its native protein, when expressed in $E$. coli the competence of the fusion protein being processed and translocated through the inner membrane is highly dependent on the amino acid sequences in the signal peptide. Therefore, in order to increase the expression efficiency of BLA signal peptide, we reconstructed the BLA signal peptide coding fragment with the following modifications. Two rare codons of $\mathrm{Arg}_{6}$ (CGG) and $\mathrm{Arg}_{10}$ (CGA) and codons for $\mathrm{Leu}_{15}$ (TTA) and $\mathrm{Pro}_{23}(\mathrm{CCT})$, in the signal peptide were substituted with their corresponding E. coli major codons. Two other changes, including $\mathrm{Phe}_{20}$ (TTC) $\rightarrow \mathrm{Leu}_{20}$ (CTG) and $\mathrm{Ala}_{28}(\mathrm{GCG}) \rightarrow \mathrm{Met}_{28}(\mathrm{ATG})$, were also introduced to increase the processing efficiency. The hGH-expressing plasmid equipped with the modified BLA (BLAF2) was subjected for further expression analysis in a T7-based expression system. The results obtained from the protein patterns of the induced bacteria indicates in high expression level of hGH preprotein (hGH::BLAF2) followed by efficient transfer of the mature hGH to E. coli periplasm.

\section{IB22}

Different dephosphorylation pathways of bacterial phytatedegrading enzymes and their applications Malaysian rice bran utilization Abd-ElAziem Farouk ${ }^{1}$, Ralf Greiner ${ }^{2}$, Hamzah Mohd Salleh ${ }^{1}:{ }^{1}$ Biomolecular Engineering Research Group, Department of Biotechnology Engineering, Kulliyyah of Engineering, International Islamic University Malaysia, Jalan Gombak, 53100 Kuala Lumpur, Malaysia; ${ }^{2}$ Centre for Molecular Biology, Federal Research Centre for Nutrition and Foods, Haid-und-Neu-Straße 9, D-76131 Karlsruhe, Germany

myo-Inositol hexakisphosphate (IP6) has been demonstrated to have a wide range of health benefits such as prevention and therapy of various cancers, amelioration of heart disease, and prevention of renal stone formation as well as complications from diabetes. On the hand, lower phosphorylated forms of inositol, especially inositol trisphosphate (IP3) and inositol tetrakisphosphate (IP4) are important signal transduction molecules within the cells both in plants and the animal kingdom. It has been hypothesized that at least the anticancer function of IP6 is mediated via these lower inositol phosphates. The diversity and practical unavailability of the individual myo-inositol phosphates preclude their investigation. Phytases, which catalyze the sequential hydrolysis of phytate, render production of defined myoinositol phosphates in pure form and sufficient quantities. Different phytases may result in different positional isomers of myo-inositol phosphates and therefore different biochemical properties. Phytases differing in pH optima, substrate specificity, and specificity of hydrolysis have been identified in plants and microorganisms. In this paper the dephosphorylation pathway of the novel PhyFAUIA1 was compared to other bacterial phytate degrading enzymes. Preliminary results have shown that PhyFAUIA1 converted IP6 into IP5 (myoinositol 1,2,3,5,6 pentakisphosphates) and another isomer, which is yet to be elucidated.

\section{IB23}

Characterization of the novel $\beta$-peptidyl aminopeptidase (BapA) from Sphingomonas sp. 3-2W4 that cleaves synthetic $\beta$ peptides Birgit Geueke, Hans-Peter E. Kohler Environmental Microbiology, EAWAG, 8600 Duebendorf, Switzerland. E-mail: birgit.geueke@eawag.ch (B. Geueke)

Non-natural peptides, which are capable of evoking a specific biological response, are currently receiving much attention. Oligomers of $\beta$-amino acids ( $\beta$-peptides) are representing a group of pharmaceutically interesting peptides because of their very high stability towards enzymatic degradation and their ability to mimic the structure of naturally occurring biologically active peptides. The pharmaceutical potential on the one hand and the high stability on the other hand aroused interest for studies on the environmental fate and the degradation behaviour of this class of compounds. A novel bacterial strain (Sphingomonas sp. 3-2W4) that was capable of degrading short $\beta$-peptides was isolated from an enrichment culture. The $\beta$ peptide degrading enzyme was purified and its gene sequence was determined (bapA). The gene encodes a $\beta$-peptidyl aminopeptidase (BapA) of 402 amino acids that is synthesized as preprotein with a signal sequence of 29 amino acids. It belongs to the $\mathrm{N}$-terminal nucleophile (Ntn) hydrolase superfamily and is the first peptidase that is capable of cleaving amide bonds in $\beta$-peptides composed of synthetic $\beta$-amino acids. The biochemical properties of recombinant BapA were investigated regarding its substrate specificity and possible application in the synthesis of $\beta$-peptides.

\section{IB24}

To produce efficient strains of Agaricus bitorquis (Quel.) Saccardo, which are resistant to high temperatures $\underline{P \text { Guler, A. }}$ Ergene, S. Tan Kirikkale University, Faculty of Science and Literature, Department of Biology, Yahsihan-Kirikkale

In this study, the culture mushroom Agaricus bitorquis (Quel.) Sacc. the growth of the mycelium and the fructifications under high temperature is examined. The spores taken from the mushrooms that were collected from nature were grouped as A, B, C, D, E. The spores were inoculated into malt extract agar and incubated at $30^{\circ} \mathrm{C}$ and primer mycelium was produced. The mycelium discus taken from primer mycelium in $8 \mathrm{~mm}$ diameter were inoculated into the center of malt extract agar and incubated at $30,32,34,36$ and $38^{\circ} \mathrm{C}$ separately. During the incubation period the growth of the mycelium were measured. During the growing period the radial growth speed of the mycelium were taken as criteria. The best mycelium growth for all groups was seen at $30^{\circ} \mathrm{C}$. At $36^{\circ} \mathrm{C}$ the E group mycelium and at $38^{\circ} \mathrm{C}$ other group's mycelium did not grow. These temperatures were determined as thermal lethal point for the groups. From all the mycelium produced from all temperatures spawn was prepared and with the results taken from these, spawn calendar is prepared. In this research, the spawn was inoculated to compost with mixing system and separately put in culture rooms, temperatures as 30 and $32^{\circ} \mathrm{C}$. At this level the culture mushroom production techniques were used. The harvested mushrooms were inspected morphologically. At this morphological inspections the cap width, cap tissue thickness, stalk thickness and stalk long ness was taken as criteria. In the study 
the best growth was seen at D group mushrooms and this group mushrooms tyrosinase's activities were measured and graphics were made.

\section{IB25}

Effects of pulsed UV on bovine viral diarrhea virus inactivation and biological properties of FBS Masoud Hosseini ${ }^{1,2}$, Mahmood Karimie-Azar ${ }^{1}$, Morteza Raie ${ }^{2}$, Parvin Jamshidi ${ }^{2}$, Ali-Reza Zareh $^{3}:{ }^{1}$ Department of Biology, University of Shahid Beheshti, Tehran, Iran; ${ }^{2}$ Central Veterinary Diagnostic and Quality Control of Biologics and Pharmaceutical Laboratory, Tehran, Iran; ${ }^{3}$ LaserPolymer Laboratory, Atomic Energy Organization, Tehran, Iran. E-mail: Masoud_Hosseini@Yahoo.com (M. Hosseini)

Introduction: Viral contamination of biological products; cause many problems in viral diagnostic laboratories, blood transfusion organizations, and biological producers. Bovine viral diarrhea virus (BVDV), from the Pestivirus genus, is the most common viral contamination in (fetal) bovine serums (FBS). Also, BVDV used as a module, for study hepatitis $\mathrm{C}$ virus inactivation due to its similarity in structure and genome. Pulsed UV lights (PUVLs) have this potential to inactivate known and unknown or reemerging viruses as well as prions.

Materials and methods: Two PUVL with the wavelengths of 355 and $266 \mathrm{~nm}$, were produced by Q-switched $\mathrm{Nd}^{3+}$ :YAG laser in its third and forth harmonic, respectively. The energy of each pulse for $355 \mathrm{~nm}$ was $12.7 \mathrm{~mJ} / \mathrm{cm}^{2}$ and for $266 \mathrm{~nm}$ was $35.2 \mathrm{~mJ} / \mathrm{cm}^{2}$. BVDV were produced and titrated in MDBK cell line. MDBK and FBS were already checked for non-cytopathic or cytopathic pestiviruses, using related Ag-ELISA kit. BVDV suspended in solution with the dilution of 1:2 before exposure. The quartz tube with the minimum $\mathrm{UV}$-absorption in compare with air, used as a container for exposed solutions. Calculation of the virus titer, $10^{4.3} \mathrm{TCID}_{50} / \mathrm{ml}$, was done based on the Reed and Muench method. BVDV suspended in PBS was exposed into the $3.52-352 \mathrm{~J} / \mathrm{cm}^{2}$ of PUVLs with the wavelength of $355 \mathrm{~nm}$ and also, was exposed into the $1.27-92.25 \mathrm{~J} / \mathrm{cm}^{2}$ of PUVLs with the wavelength of $266 \mathrm{~nm}$. Furthermore, BVDV suspended in FBS was exposed into the $88,176,352$ and $704 \mathrm{~J} / \mathrm{cm}^{2}$ of PUVLs with the wavelength of $355 \mathrm{~nm}$ and also, was exposed into the 6.35 , $25.4,63.5,127$ and $190.5 \mathrm{~J} / \mathrm{cm}^{2}$ of PUVLs with the wavelength of $266 \mathrm{~nm}$.

Results: The minimum dose for inactivation of BVDV suspended in PBS with the 355 and $266 \mathrm{~nm}$ wavelengths of PUVLs, were 352 and $92.25 \mathrm{~J} / \mathrm{cm}^{2}$, respectively. Also, the minimum dose for inactivation of BVDV suspended in FBS with the 355 and $266 \mathrm{~nm}$ wavelengths of PUVLs, were 704 and $127 \mathrm{~J} / \mathrm{cm}^{2}$, respectively. To evaluate the FBS quality to support cell culture, treated FBS with the dose of $190.5 \mathrm{~J} / \mathrm{cm}^{2}$ of $266 \mathrm{~nm}$ PUVLs was used to grow Vero cell line in 12 successive passages. The viability of cells in two study groups was identical. The statistical evaluation of two treated groups showed no significant difference, in 12 passages.

Conclusion: Because inexpensive equipment can be used to produce PUVLs capable of handling different volumes of biologics with operational ease, this viral inactivation technique is cost effective for relevant industries. The procedure has the potential to be combined synergically with other inactivation method. PUVLs offer a new, nonadditive and chemically safe alternative for the treatment of FBS to inactivate adventitious viruses and to preserve the biological activity necessary for the propagation of cell culture.

\section{IB26}

Characterization and gene cloning of the g-resorcylic acid decarboxylase for application to selective production of g-resorcylic acid $Y$. Iwasaki ${ }^{1}, Y$. Ishii ${ }^{2}, K$. Kino ${ }^{1}, K$. Kirimura ${ }^{1}$ : ${ }^{1}$ Dept. Appl. Chem., Sch. Sci. Eng., Waseda Univ., Tokyo, Japan; ${ }^{2}$ BME, ASMeW, Waseda Univ., Tokyo, Japan. E-mail: iwasaki@ moegi.waseda.jp (Y. Iwasaki)

For selective production of $\gamma$-resorcylic acid $(\gamma$-RA, 2,6-dihydroxy-benzoic acid) from resorcinol (RE, 1,3dihydroxybenzene) under mild conditions, we screened various microorganisms and found the reversible $\gamma$-RA decarboxylase (Rdc) as a novel enzyme applicable to carboxylation of RE to form $\gamma$-RA, in a bacterial strain Rhizobium radiobacter WU-0108 ${ }^{1)}$. Rdc catalyzed the decarboxylation of $\gamma$-RA, and regio-selective carboxylation of RE to form $\gamma$-RA, without formation of $\alpha$-RA and $\beta$-RA. The molecular weight of Rdc was estimated to be $130 \mathrm{kDa}$ by gel-filtration, and that of the subunit was determined to be $34 \mathrm{kDa}$ by SDS-PAGE, suggesting that Rdc is a homotetrameric structure. The gene encoding Rdc was sequenced, and a site-directed mutagenesis study revealed that the two histidine residues at positions of 164 and 218 in Rdc are essential for the catalytic activity of Rdc. Through the reactions using E. coli cells highly expressing $r d c, 6.7 \mathrm{mM}$ $\gamma$-RA was produced from $15 \mathrm{mM} \mathrm{RE}$ at $30^{\circ} \mathrm{C}$ for $16 \mathrm{~h}$, with a yield of $45 \%$.

\section{Reference}

Ishii, Y. et al., 2004. Biochem. Biophys. Res. Commun., 324, 611-620.

\section{IB27}

Laccase biosynthesis in stirred fermenters Teresa Jamroz, Stanislaw Ledakowicz, Barbara Sencio Department of Bioprocess Engineering, Technical University, Lodz PL 90-924, Poland

Industrial applicability of enzymes is closely related to development of efficient methods of their production. Currently, significant interest in lignolytic enzymes, including laccase, has been observed. Laccase is an enzyme applied in various industrial branches and environmental processes. Broad laccase applicability induces researchers to develop urgently efficient methods for its commercial production. Laccase (EC.1.10.3.2. p-diphenol oxidase) is produced by cap mushrooms from the class Basidomycetes. This is the so-called white rot fungi which in natural conditions appears on both living and dead wood. As shown in the practice of biotechnological processes, high-efficient strains have low resistance to destructive factors in bioreactors. Hence, to preserve a proper morphology and physiological state of an organism, strictly determined culture conditions must be obeyed. This is very important in the case of Basidomycetes for which submerged culture in the liquid phase is not a natural habitat. Results of studies on laccase production from Cerrena unicolor family are discussed. Cultivation of active biomass was carried out in stirred tank and rotating disc bioreactors of different volume (B. Braun of working volume $12 \mathrm{dm}^{3}$; FAS-01 of working volume $3.5 \mathrm{dm}^{3}$ ). Experiments in both fermenters were made at impeller revolutions 200 and $300 \mathrm{~min}^{-1}$, on a modified Lindberg substrate. Significant differences in the rate and yield of laccase production were reported. An almost three times higher values of laccase activity were obtained in the B. Braun fermenter, at rotational speed $300 \mathrm{~min}^{-1}$. 


\section{IB28}

Filtration system for suspended biomass in Aspergillus niger single stage continuous culture Malgorzata Janczar, Jerzy J. Pietkiewicz Food Biotechnology Department, University of Economics, Wroclaw 53-345, Poland. E-mail: malgorzata.janczar@ae.wroc.pl (M. Janczar)

To retain suspended cells in bioreactor a filtration process can be used. The biomass is concentrated by withdrawing cell-free culture broth. If the desired product is dissolved in the broth (extracellular production), the procedure enables the continuous harvest in the cellfree permeate. An application test of filtration system for suspended biomass of Aspergillus niger in submerged single stage continuous culture was presented in this report. The system is easy to construct and there is a possibility of its sterile exchange during cultivation. The culture medium contained the following substances $\left(\mathrm{g} / \mathrm{dm}^{3}\right)$ : white sugar, $150.0 ; \mathrm{NH}_{4} \mathrm{NO}_{3}, 1.5 ; \mathrm{MgSO}_{4} \cdot 7 \mathrm{H}_{2} \mathrm{O}, 0.2 ; \mathrm{KH}_{2} \mathrm{PO}_{4}$, $0.2 ; \mathrm{FeSO}_{4} \cdot 7 \mathrm{H}_{2} \mathrm{O}, 0.05$. Fermentations were carried out in the lab bioreactor BIOMER 10. The bioreactor was a standard CSTR (continuous stirred tank bioreactor) with working volume of $5 \mathrm{dm}^{3}$. High citric acid concentration in culture medium $\left(P=108.9 \mathrm{~g} / \mathrm{dm}^{3}\right)$, high yield of citric acid $\left(Y_{\mathrm{P} / \mathrm{S}}=72.6 \%\right)$ and high efficiency coefficient $\left(K_{\text {ef }}=71.1\right)$ were observed in single stage continuous culture with biomass retention.

\section{IB29}

Oxygen transfer regulates benzaldehyde lyase production in E. coli Pınar Çalık, IBLab, Department of Chemical Engineering, METU,06531 Ankara, Turkey.E-mail: pcalik@metu.edu.tr

The effects of oxygen transfer rate on benzaldehyde lyase (BAL) production by pUC18::bal carrying recombinant Escherichia coli on a defined medium with $8.0 \mathrm{~kg} / \mathrm{m}^{3}$ glucose were investigated in order to fine-tune the bioreactor performance, in $V=3 \mathrm{dm}^{3}$ batch bioreactors at five different conditions with the parameters at, i.e. $Q_{\mathrm{O}} / V_{\mathrm{R}}=0.5 \mathrm{vvm}$ and $N=250,375,500,750 \mathrm{~min}^{-1}$ and; $Q_{\mathrm{O}} / V_{\mathrm{R}}=0.7 \mathrm{vvm}$ and $N=750 \mathrm{~min}^{-1}$. The concentrations of the product and by-products amino acids and organic acids were determined in addition to BAL activities. Medium oxygen transfer rate conditions and uncontrolled $\mathrm{pH}$ operation at $\mathrm{pH}_{\mathrm{O}}=7.25$ are optimum for maximum BAL activity, i.e. $860 \mathrm{U} / \mathrm{cm}^{3}$ at $12 \mathrm{~h}$, and productivity and selectivity. On the bases of the data, response of the intracellular bioreaction network of r-E. coli to oxygen transfer conditions were analysed using a mass-flux balance based stoichiometric model that contains 102 metabolites and 133 reaction fluxes. The results reveal that metabolic reactions are intimately coupled with the oxygen transfer conditions. Oxygen transfer rate showed diverse effects on the product formation by influencing metabolic pathways and changing metabolic fluxes. Metabolic flux analysis was helpful to describe the interactions between the cell and the bioreactor by predicting the changes in the fluxes and the rate controlling step(s) in the metabolic pathways. Therefore, knowing the distribution of the metabolic fluxes during the growth, and BAL and by-product formations provide new information for understanding physiological characteristics of the $\mathrm{r}-E$. coli, and reveals important features of the regulation of the bioprocess and opens new avenues to successful application of metabolic engineering.

\section{IB30}

Isoniazid increase the permeability of mycobacterial cell wall to hydrophobic compounds Malgorzata Korycka-Machala, Anna Rumijowska-Galewicz, Jaroslaw Dziadek Laboratory for Genetics and Physiology of Mycobacterium, Centre for Medical Biology, Polish Academy of Sciences, Lodz, Poland. E-mail: mkorycka@cbm.pan.pl (M.Korycka-Machala)

Saprophytic Mycobacterium strains belong to the best known microorganisms which have been applied to the pharmaceutical industry for the production of steroid drugs. The mycobacterial cell wall is the permeation barrier to chemical compounds, including lipophiles. Using isoniazid (INH), the inhibitor of the mycolic acids biosynthesis, we were able to demonstrate increased AD production and susceptibility to antimicrobial agents. The process of sterol transformation and products accumulation was monitored using gas chromatography. Isoniazid was shown to intensify $\beta$-sitosterol side-chain degradation by Mycobacterium sp., and accumulation of 4-androstene-3,17-dione (AD) and 1,4-androstadien-3,17-dione (ADD), which are the starting materials in the biotechnology of medically important steroids. To confirm these results, the sensitivity of the bacteria to antimycobacterial drugs was performed. The minimum inhibitory concentration $\mathrm{MIC}_{50}$ of rifampicin and erythromycin decreased markedly in the presence of INH.

\section{Acknowledgement}

This work was supported by grant nr 3P04C 06923 of the Committee for Scientific Research.

\section{IB31}

CMOS chip technology for electrophysiological and metabolic monitoring of cell cultures G. Krause ${ }^{1}$, M. Lehmann ${ }^{2}$, I. Freund ${ }^{2}$, R. Ehret ${ }^{3}$, E. Schreiber ${ }^{1}$, A. Podssun ${ }^{1}$, W. Baumann ${ }^{1}$ : ${ }^{1}$ Institute of Cell Biology and Biosystems Technology, University of Rostock, Germany; ${ }^{2}$ Micronas GmbH; Freiburg, Germany; ${ }^{3}$ Bionas GmbH; Rostock, Germany

For the purpose of high-throughput screening and to reduce experiments with animals in pharma biotechnology biosensor systems gain importance. The principle of a biosensor is the combination of cultured cells and a sensorchip device, which allows the monitoring of cellular activity. In contrast to traditional analytics with a biosensor you can measure on-line cellular activity change caused by an effector as well as the restored activity after privation of the effector (re-native activity). CMOS technology can be used for the realisation of various biological sensorchips such as adhesion sensorchips, metabolical sensorchips and electrophysiological sensorchips. The standard CMOS technology allows a high reproducibility of the chips, the integration of electronic components on the chip, which reduces the amount of external devices and the combination of different sensors on one chip. In cooperation with the semiconductor company Micronas and the biotech company Bionas we have realised different types of CMOS sensorchips to measure adhesion of a cellular monolayer with interdigitated electrodes (IDES), metabolical activity via acidification with ion-sensitive field effect transistors (ISFET) and sponteneous neuronal network activity with passive palladium electrodes. 


\section{IB32}

Effect of the laccase-mediator system on the yellowing of kraft pulps Manuela Lageiro ${ }^{1}$, Cristina Maximo ${ }^{1}$, M. Costa-Ferreira ${ }^{1}$, Jorge Gominho $^{2}$, Ana Lourenço ${ }^{2}$, Helena Pereira ${ }^{2}:{ }^{1}$ Department of Biotechnology; National Institute for Engineering, Technology and Innovation-INETI, 1649-038 Lisboa, Portugal; ${ }^{2}$ Centro de Estudos Florestais, Departamento de Engenharia Florestal, Instituto Superior de Agronomia, 1349-017 Lisboa, Portugal

Microbial agents have been applied to the different stages of pulp and paper processing. The work presented describes a study on the effect of applying ligninolytic enzymes, such as a laccase plus mediator system, on a variety of different types of pine and eucalyptus pulps and subsequently subjecting these to different ageing processes. Industrial pulps were obtained from different Portuguese pulp and paper companies. The pulps used were (1) unbleached pine pulp from Portucel Tejo; (2) unbleached eucalyptus pulp from Portucel Setúbal; (3) bleached eucalyptus pulp from Portucel Setúbal; and (4) pulp made from recycled paper from Renova S.A. Several types of handsheets were produced with 2 different grammage namely, 60 and $180 \mathrm{~g} / \mathrm{m}^{2}$. The prepared handsheets were subject to an aging sequence in three different chambers: ultraviolet radiation (wavelength of $280 \mathrm{~nm})$, temperature $\left(19^{\circ} \mathrm{C}\right)$ and moisture $(70 \%)$; and thick saline fog at a concentration of $1 \%$ and temperature of $35^{\circ} \mathrm{C}$. In order to evaluate the effect of moisture cycles and temperature, two aging sequences were used for each type of handsheet. In the first, the moisture varied $(60,80$ and $100 \%)$, while the temperature was held constant $\left(25^{\circ} \mathrm{C}\right)$; in the second the temperature varied (60, 70 and $80{ }^{\circ} \mathrm{C}$ ) and the moisture was held constant $(50 \%)$. Following the aging phase, the handsheets were subject to several chemical (viscosity and index Kappa) and physico-mechanical (colour, tensile breaking strength, stretch and the bursting strength) tests in order to characterize the effect of the aging conditions. Results will be presented describing the effect of application of the laccase-mediator system on the optical and mechanical properties of the prepared handsheets.

\section{Acknowledgement \\ Fundação para a Ciência e a Tecnologia, project POCTI/ AGR/47309/02.}

\section{IB33}

Oxygen stress in Aspergillus niger A. Diano, T. Hagemann, L. Olsson, J. Nielsen Center for Microbial Biotechnology, Technical University of Denmark, Kgs-2800 Lyngby, Denmark. E-mail: aud@biocentrum.dtu.dk (A. Diano)

Aspergillus niger is a filamentous fungi widely used in industry. Its growth as freely dispersed hyphae leads to an increase in the medium viscosity and to problem of mass transfer, especially oxygen transfer. Oxygen acts both as final electron acceptor in the mitochondrial chain and as nutrient for the biosynthesis of unsaturated fatty acids and sterols. Thereby, a lack of oxygen affects the $\mathrm{NADH} / \mathrm{NAD}$ ratio, the ATP production, the growth and has a strong influence on the physiology of the microrganism. In the present study, the metabolic changes of $A$. niger in response to a lack of oxygen was investigated using oxygen limited chemostats combined with nitrogen pulse. Under these conditions, the main consequence of a sudden decrease of oxygen availability is an increase in the mannitol production. This work showed that the mannitol biosynthesis, involving the enzyme mannitol-1-P dehydrogenase, helps the reoxidation of NADH when the final electron transport acceptor, oxygen, is limiting.

\section{IB34}

Investigation of the lipase activity of the bacteria isolated from olive mill wastewater Sevgi Ertuğrul ${ }^{1}$, Nur Koçberber ${ }^{1}$, Gönül Dönmez ${ }^{1}$, Serpil Takaç ${ }^{2}$ Department of Biology Faculty of Science Ankara University 06100 Beşevler Ankara Turkey; ${ }^{2}$ Department of Chemical Engineering Faculty of Engineering Ankara University 06100 Tandoğan, Ankara, Turkey

The bacteria that could grow on media containing olive mill wastewater (OMW) were isolated and their lipase production capacities were investigated. The strain possesing the highest lipase activity among 17 strains grown on tributryin agar medium was identified as Bacillus sp. The effect of $\mathrm{pH}$ on the lipase activity of the strain was investigated in tributryin medium and $\mathrm{pH} 6$ was found to be the optimal. The liquid medium composition was improved by adding different carbon sources and fatty acids into tributryin medium -omitted tributryin - to increase the enzyme activity. The cultivations were performed at $30^{\circ} \mathrm{C}$ and $\mathrm{pH}$ 6. Lipase activity of the Bacillus sp. was measured spectrophotometrically through the hydrolysis of $p$-nitrophenol palmitate. Among the media containing different compositions of tricapryn, trimyristin, tributyrin, triacetin, Tween 80 , glycerol-trioleate, glycerol-trioctanoate, glycerol-tridodecanoate, OMW, glucose, and whey; the medium consisted of $20 \%$ whey $+1 \%$ glycerol-trioleate was found to give the highest lipase activity. Cultivation of Bacillus sp. in the optimum medium at $\mathrm{pH}=6$ and $30^{\circ} \mathrm{C}$ for $64 \mathrm{~h}$ was resulted in the extracellular and intracelluar lipase activities of 15 and $168 \mathrm{U} / \mathrm{ml}$, respectively.

\section{Acknowledgement}

This study was supported by Ankara University Biotechnology Institute (project no: 2004-151 and 2005-164).

\section{IB35}

Effect of osmotic pressure on kinetics of cell growth and cellular metabolism in stem cell research and biotechnological industries H.El-Enshasy ${ }^{1}$, T. Esmail ${ }^{2}$, M. El-Demellawy ${ }^{2}$, S.T. Yang ${ }^{3}$ : ${ }^{1}$ Mubarak City for Scientific Research and Technology Applications, New Burg Al-Arab, Alexandria, Egypt; ${ }^{2}$ Faculty of Chemical Engineering, Ohio State University, USA. E-mail: enshasy@yahoo.com (H.A. El Enshasy)

Under different abiotic stresses, cell growth and metabolic activity are highly influenced in all types of living organisms Medium osmolality is usually one of those factors affecting different types of biological systems in different ways. However, even in the same organ of higher eukaryotes the degree of osmoregulation mechanism is highly variable in different types of cells. Therefore, studying the effect of osmotic stress on mammalian cell is very important subject for particular cell line. The effect of hyperosmotic pressure on the kinetics of cell growth of and metabolic activity of mesenchymal stem cells (MSCs) and two industrially important cell lines, hybridoma cells and human embryonic kidney cell (HEK) were investigated in batch cultures at different osmotic pressures in the range from 325 to $500 \mathrm{mOsm} \mathrm{kg}^{-1}$. In case of MSCs cells, the maximal specific growth rate $[\mu]$ of $0.029\left[\mathrm{~h}^{-1}\right]$ associated with the highest specific glucose consumption rate $\left[-q_{\mathrm{gluc}}\right]$ of $0.1129 \times 10^{-6}$ 
[ $\mu \mathrm{mol}$ cells ${ }^{-1} \mathrm{~h}^{-1}$ ] was obtained in medium of $375 \mathrm{mOsm} \mathrm{kg}^{-1}$. In case of hybridoma cells, osmotic pressure showed not only influence on the kinetics of cell growth and metabolism but also on the monoclonal antibody production. The maximal MAb production was obtained in case of cells cultivated under osmotic pressure of $375 \mathrm{mOsm} \mathrm{kg}^{-1}$. Further increase in osmotic pressure resulted in significant reduction in growth rate as well as MAb production. On the other hand, HEK cells were more sensitive to osmotic pressure in industrially used serum free medium and the addition of serum decreased the inhibitory effect of high osmotic pressure on the cells.

\section{IB36}

Growth and metabolite formation during batch and chemostat cultures of Kluyveromyces marxianus CBS $\mathbf{6 5 5 6}$ Gustavo G. Fonseca $^{1,2}$, Andreas K. Gombert ${ }^{2}$, Elmar Heinzle ${ }^{1}$, Christoph Wittmann ${ }^{1}$ : ${ }^{1}$ Biochemical Engineering, Saarland University, Saarbrücken, Germany; ${ }^{2}$ Chemical Engineering, São Paulo University, Brazil

Kluyveromyces marxianus CBS 6556 is a potentially interesting yeast strain characterized by a high capacity of conversion of substrate into biomass. However, this yeast has been only marginally studied so far. Therefore, we performed a metabolic characterization in batch and chemostat cultures at dilution rates of $0.10,0.25$ and $0.5 \mathrm{~h}^{-1}$. The specific rate of $\mathrm{O}_{2}$ consumption $\left(\mathrm{qO}_{2}\right)$ increased with dilution rate from 2.87 to $11.09 \mathrm{mmol}(\mathrm{g} \mathrm{DW})^{-1} \mathrm{~h}^{-1}$. The respiratory coefficient remained almost stable around 1.0 for all metabolic states investigated. Even at the dilution rate of $0.5 \mathrm{~h}^{-1}$, which is close to the maximum growth rate of the strain of $0.56 \mathrm{~h}^{-1}$, no significant overflow metabolism was observed. The concentration of extracellular metabolites increased with the dilution rate, but remained below $6 \%$ of the carbon consumed as glucose. All carbon balances closed near $100 \%$ underlining the consistency of the data. In contrast to $S$. cerevisiae the respiratory capacity of K. marxianus CBS 6556 is not strongly influenced by the dilution rate in aerobic chemostat or batch cultures, indicating its high potential for biomass-directed applications.

\section{IB37}

A Thermostable L-arabinose Isomerase for enzymatic production of D-tagatose $O$. Hansen, F. Jørgensen, P. Stougaard Department of Enzyme Technology, Bioneer A/S, Kogle Allé 2, DK-2970 Hørsholm, Denmark. E-mail: och@ bioneer.dk (O. Hansen)

$\mathrm{D}$-tagatose, an isomer of D-fructose, is a low-calorie bulk sweetener with a sweetness equivalent to sucrose. D-tagatose has obtained GRAS approval for use as a food ingredient, and is currently produced by chemical isomerization of D-galactose, which may readily be obtained by hydrolysis of lactose. Structurally, D-galactose is closely related to L-arabinose, and it has previously been shown that some variants of L-arabinose isomerase ( $\mathrm{raA}$ ) may catalyze the conversion of D-galactose to D-tagatose, in addition to the metabolic conversion of L-arabinose to L-ribulose. We have screened a number of bacterial araA enzymes for their ability to catalyze the isomerization of D-galactose to D-tagatose. The best enzyme was found in the thermophilic bacterium Thermoanaerobacter mathranii (DSM11426). The araA gene of T. mathranii was cloned, sequenced and expressed in E. coli. Amino acid sequence comparisons of the T. mathranii sequence and other known araA sequences showed a relatively low sequence identity of about $30 \%$, indicating a distant phylogenetic relationship to the other members of the L-arabinose isomerase group. The T. mathranii enzyme was thermostable with optimal activity at $65^{\circ} \mathrm{C}$ and it required manganese ions. Unlike other araA variants, the T. mathranii enzyme showed $K_{\mathrm{m}}$ values in the same order of magnitude for L-arabinose and D-galactose, suggesting that this enzyme is a versatile isomerase capable of isomerizing structurally related aldoses. The enzyme was immobilized by chemical cross-linking of a crude E. coli cell homogenate, and the immobilized enzyme efficiently converted D-galactose into D-tagatose. Currently, we are developing an enzymatic method for industrial production of D-tagatose using the immobilized enzyme.

\section{IB38}

Evolution of hydrodynamic conditions during the submerged monoxenic culture of the entomopathogenic nematode Steinernema carpocapsae in an internal-loop air-lift bioreactor: culture medium containing whey or agave-juice (Agave spp.) R. Sanjuan-Galindo ${ }^{1}$, M.A. Islas-López ${ }^{1}$, L. Medina-Torres ${ }^{2}$, A.I. Rodríguez-Hernández ${ }^{1}$, N. Chavarría-Hernández ${ }^{1}$ : ${ }^{1}$ Instituto de Ciencias Agropecuarias, Universidad Autónoma del Estado de Hidalgo. Av. Universidad km 1, Rancho Universitario, Tulancingo, Hidalgo CP 43600, México; ${ }^{2}$ Facultad de Química, UNAM, Ciudad Universitaria, CP 04510, México

The agricultural production can be negatively affected by different pest insects (PI) and the use of chemical insecticides (ChI) has been the traditional method for controlling PI during decades. Nevertheless, there are various ecological implications due to the extensive application of ChI. A viable alternative for the use of ChI in certain agro-systems, is the use of entomopathogenic nematodes (EPN) of the genera Steinernema and Heterorhabditis that are natural pathogens for different PI; besides, the presence of a symbiotic bacterium is necessary for an effective entomopathogenic activity can take place. The nematode/bacterium complex does not represent a risk for the environment. Different authors propose that the best alternative for the massive production of EPN is the submerged culture within bioreactors; nevertheless, more research is required to have really robust processes. Particularly, information regarding the actual hydrodynamics during EPN production and its relation with the EPN productivities are scarce, among other aspects. The present study deals with the hydrodynamic characterisation during the production of the EPN Steinernema carpocapsae and its symbiont bacterium Xenorhabdus nematophilus, in submerged monoxenic culture in an internal-loop air-lift bioreactor $\left(V_{\mathrm{L}}=4.5 \mathrm{~L}\right)$ using two culture media: one of them containing whey and the other one, agave juice, aguamiel, (Agave spp.). Process viscosity of the culture broth was determined along the time, exhibiting a maximum value of $20 \mathrm{mPa}$ s. Moreover, it was determined that the hydrodynamic conditions were always located within the laminar region $(R e<500)$. At the experimental conditions tested, it can be inferred that the EPN productivity are more sensitive to changes in the culture medium composition than on the prevailing hydrodynamic conditions during the fermentations.

\section{IB39}

Characterization of a bacteriocin and a fungicide of Bacillus thuringiensis S. Jaoua, F. Kamoun, F.Driss, H. Mejdoub, M. Kallassy-Awad, N. Zouari Laboratoire des Biopesticides, Centre de Biotechnologie de Sfax, PO Box 'K', 3038, Sfax, Tunisia 
Bacillus thuringiensis is a Gram-positive bacterium used as a biological pest control agent. Moreover, it is able to produce, several biologically active molecules such as bacteriocins and hydrolytic enzymes among which chitinases that play double roles, fungicide and improving the insecticidal effect of $B$. thuringiensis deltaendotoxins. A newly isolated B. thuringiensis subsp. kurstaki strain BUPM4, was shown to produce a novel bacteriocin named Bacthuricin F4. The highest bacteriocin activity was found in the growth medium and evidenced in the late exponential growth phase. Upon purification of Bacthuricin F4, the specific activity was increased 100 -fold. This bacteriocin was heat-stable up to $70^{\circ} \mathrm{C}$ and resisted up to $\mathrm{pH}$ 3.0. Its molecular mass, determined by mass spectrometry was $3160.05 \mathrm{Da}$. Direct $\mathrm{N}$-terminal sequencing of Bacthuricin F4 revealed the following sequence: DWTXWSXL. The latter was unique in the databases. Bacthuricin F4 was active against Bacillus species while it had little or no effect on Gram-negative bacteria. The bacteriocin produced by the $B$. thuringiensis strain BUPM4 respond to both criteria of thermostability and stability to low pHs. Thus, it could be used as a source of bacteriocin active against related species of Bacillus harmful for agricultural products and as food preservative. The other example of antimicrobial compound produced by $B$. thuringiensis is a chitinase. We describe the selection of B. thuringiensis high chitinase-producing strain BUPM255, and the characterization and the heterologous expression of a novel chitinase encoding gene. The cloning and sequencing of the corresponding gene named chi 255 showed an open reading frame of $2031 \mathrm{bp}$, encoding a 676 amino acid residue protein. Both nucleotide and amino acid sequences similarity analyses revealed that the chi255 is a new chitinase gene, presenting several differences from the published chi genes of $B$. thuringiensis. The identification of chitin hydrolysis products resulting from the activity, exhibited by Chi255 through heterologous expression in E. coli revealed that this enzyme is a chitobiosidase. The addition of the sequence of chi255 to the few sequenced $B$. thuringiensis chi genes might contribute to a better investigation of the chitinase "structure-function" relation.

\section{IB41}

Cloning and characterization of S-Adenosyl-L-methionine synthetase from Pichia ciferrii DSCC 7-25 Kwon-Hye Ko ${ }^{1}$, Gee-Sun Yoon $^{1}$, Gi-Sub Choi ${ }^{1}$, Joo-Won Suh ${ }^{2}$, Yeon-Woo Ryu $u^{1 *}:{ }^{1}$ Department of Molecular Science and Technology, Ajou University, Suwon, South Korea, 443-749; ${ }^{2}$ Department of Biological Science, Institute of Bioscience and Biotechnology, Myongji University, Yongin 449-728, Korea. E-mail: 7424550@ @anmail.net (Kwon-Hye Ko)

S-adenosyl-L-Methionine(SAM) has an important role for DNA methylation and cell signaling. SAM was synthesized from methionine and ATP by SAM synthetase and play an pivotal function in the primary and secondary metabolism of cells. Recent studies have revealed in the effect of SAM in case of morphological differentiation in both eukaryotes and prokaryotes. The $P$. ciferrii produces large quantities of sphingoid base. Tetraacetylphytosphingosine(TAPS), which is a precursor of sphingolipid, could be used for the production of pharmaceuticals and cosmetics. We isolated SAM gene from $P$. ciferrii and cloned it into expression vector for $E$. coli and $P$. pastoris, respectively. An $1.2 \mathrm{~kb}$ SAM-s gene fragment was isolated by Low-strigency PCR using degenerated primer. By the analysed primary sequence deduced from DNA sequence, this gene included conserved domains similar with other well-known SAM synthetase. First of all, SAM synthetase gene cloned pGEM-T vector and subcloned into histidine tagging system to purify the expressed protein using metal chelating resin. Typical characteristic analysis of this enzyme is underway.

\section{IB42}

Design of a system of biotransformations by means of stoichiometric network analysis Anne Kümmel, Michael Schümperli, Matthias Heinemann Sven Panke Institute of Process Engineering, ETH Zürich, 8092 Zürich, Switzerland. E-mail: kuemmel@ipe.mavt.ethz.ch (A. Kümmel)

Metabolic networks offer a large variety of different synthesis pathways starting from cheap substrates and leading to interesting high-value compounds, i.e. metabolites. In case an interesting pathway can be disconnected from the remaining metabolic network, the perforated cell or the crude extract could be used for a one-pot multi-step synthesis of the desired compound. Pathway isolation, achieved by deletion of genes encoding gene products enabling side reactions, interferes with the viability of the organism, which is a requisite for the production of the system of biotransformation (SBT). In this work, a rational systems biology-derived approach is presented for the design of a SBT. It is illustrated for a SBT allowing for production of dihydroxyacetone phosphate. The design procedure comprises three steps: (i) A production pathway is identified in the metabolic network of E. coli. The E. coli pathway is complemented by two additional enzymes in order to obtain a fully energy and redox balanced production pathway. (ii) An optimal combination of gene knockouts is designed and a suitable growth medium composition is identified, both by a model-based approach: Flux Balance Analysis of a genome-scale metabolic network is used to predict enzyme expression within the wild-type organism on different media, while a mixed-integer optimisation is employed to identify viable mutants As this approach strongly depends on the quality of the FBA prediction, available regulatory information on the usage of metabolic pathways and thermodynamic constraints were taken into account. (iii) Thermodynamic analysis of the obtained, partially branched SBT reaction cascade revealed the extent of loss in yield by the remaining side reactions. In summary, this systems biology-driven approach potentially enables the substitution of a elaborative multi-step synthesis process by a one-pot enzyme reaction cascade.

\section{IB43}

Possible solutions to mutual shading problem: A key to highdensity microlagal cultures Tae Ryeon Heo, Choul-Gyun Lee Institute of Industrial Biotechnology, Inha University, Incheon 402-751, Korea. E-mail: leecg@inha.ac.kr (C.-G. Lee)

Algal biotechnology is drawing increasing interest due to its potential as a source of valuable pharmaceuticals, pigments, carbohydrates, and other fine chemicals. Currently, its application is being extended to the areas of wastewater treatment and agriculture. However, lack of suitable photobioreactors (PBRs) makes the cost of algally-derived compounds higher than those derived by chemical synthesis and thus has prevented widespread use of algal cultures. The culture of algae prior to the late 1940s was apparently restricted to laboratory scale operations. Experiments on outdoor algal mass production began in the late 1940s with nearly concurrent development of experimental culture facilities in Germany and the United 
States. For the next two decades, outdoor mass culture of algae was undoubtedly the hottest topic in the algal biotechnology area. Recent developments of high-density PBRs enable the production of valuable biologically active compounds by algal mass cultures. However, light is almost always the limiting factor in high-density photobioreactors. Key factors for successful photobioreactors will be discussed and various photobioreactors will be analyzed and compared for their advantages and disadvantages. The new techniques, such as pigment redcution and application of flashing light and lumostatic operation, will be discussed for possible solutions to overcome the light limitation in high-density microalgal cultures.

\section{IB44}

Comparison of rapid extraction methods for high quality fungal DNA for PCR-applications S.L. De Maeseneire, M. De Mey, M. De Groeve, T. Dauvrin, W. Soetaert, E.J. Vandamme Department of Biochemical and Microbial Technology, University of Ghent, Ghent B-9000, Belgium. E-mail: Sofie.DeMaeseneire@UGent.be (S. De Maeseneire)

A quick and reliable method for screening fungal transformants for specific genetic modifications is essential for many molecular applications, for example when one tries to develop a transformation system for a new fungal host. Southern analysis is laborious and time consuming. Several colony hybridisation methods have been developed for the analysis of a large number of transformants. Unfortunately these methods suffer from different disadvantages such as non-specific binding, a limited usability to screen for specific integration and the fact that these procedures always take a few days (Van Zeijl et al., 1998). Recently, methods for PCR-based analysis of fungal transformants have been described. Most of these methods require high quality DNA. Many methods for DNA extraction from fungi have been described in the past few years. These methods often are tedious, time consuming, costly or limited to a small number of samples each time. Most of the available protocols include the growth of mycelium in a liquid culture, followed by freeze-drying or maceration in liquid nitrogen and grinding of the frozen material to break the cell walls (Cassago et al., 2002). Lately a few methods have been described to isolate DNA from fungi suitable exclusively for PCR and appropriate for the simultaneous treatment of a large number of samples. Some methods also describe the direct use of mycelium (Cooke et al., 1997) in the PCR-reaction mixture. We compared the applicability of a few rapid DNA extraction methods for Myrothecium gramineum and tested the resulting DNA samples on there suitability for PCR-applications. Myrothecium gramineum is a filamentous ascomycete used in ongoing research as a new cloning and expression host. Five methods were tested. In four of these methods DNA was extracted from mycelium (Goodwin et al., 1993 and Aljanabi et al., 1997) or spores (Ferreira et al., 1997 and Xu et al., 1995) prior to PCR. A fifth assay used mycelium straight in the PCR-reaction mixture. Only this last method seemed useful for Myrothecium to isolate DNA suitable for PCR. Fragments up to 2000bp were amplified.

\section{IB45}

Optimization of lactic acid production from cheese whey Marcelo T. Leite, Raquel M. Santos, Eloízio J. Ribeiro, Mar$\cos$ A.S. Barrozo Chemical Engineering College, Federal University of Uberlândia, Uberlândia-MG 38408-100, Brazil. E-mail: leitemarcelo@terra.com.br (M.T. Leite)
Cheese whey is a liquid effluent from cheese-making processes. There is an increased interest in the economic utilization of whey produced by the dairy industries, because the whey is a pollutant, due mainly to its lactose content. The goal of this work was to find the most suitable values of some fermentation parameters for lactic acid production from whey by a lactic acid bacterium, Lactobacillus helveticus (ATCC 15009). The effects of lactose content, temperature, $\mathrm{pH}$ and the supplementation with yeast extract were investigated using surface response methodology. A composite central design was used with three center points, making a total of 27 operational conditions. The region of maximum production is outlined by the following intervals: temperature around $40^{\circ} \mathrm{C}$; lactose concentration between 70 and $85 \mathrm{~g} / \mathrm{L}$; concentration of yeast extract between 20 and $25 \mathrm{~g} / \mathrm{L}$; $\mathrm{pH}$ between 7 and 7.5.

\section{IB46}

Experimental development, instrumentation and control of a novel vacuum extractive fermentation process for ethanol production Daniel I.P. Atala, Francisco Maugeri Department of Food Eng./Unicamp, 13081-970, Campinas, SP, Brazil. E-mail: maugeri@fea.unicamp.br (F.M. Filho)

Fermentation studies on a continuous fermentative process coupled to a vacuum flash evaporator were carried out in lab scale equipment. The phases of this work consisted in an assembly and instrumentation of the prototype and elaboration of a supervisory system coded in LabVIEW 6.1, which allows the data acquisition and control through personal computers. The experiments in continuous fermentation used Saccharomyces cerevisiae and sugar cane molasses as substrate. The analytical follow up was done through analysis of total reducing sugars, ethanol, glycerol, dry mass and viable cells. The system worked for months uninterruptedly, producing an alcoholic solution at the condenser with $50^{\circ} \mathrm{GL}$. The fermentation operated with concentrations of ethanol at $5^{\circ} \mathrm{GL}$, which is a weakly inhibitory value for the yeast of the process, even when fed with concentrated cane molasses, containing up to $330 \mathrm{~g} / \mathrm{L}$ of sugar. The result meets the initial goal, which was to operate the system with low level of ethanol and to guarantee high productivity, even in high concentrations of sugar in the feeding. The results showed that system productivity was superior to that of the conventional continuous process.

\section{IB47}

Batch and fed batch production of $\mathrm{L}(+)$ lactic acid $\underline{S}$. Mirdamadi, A. Rajabi, A. Akbarzadeh, J. Vaez, B. Farahmand, B. Momen Pilot Biotechnology Dep., Pasteur Institute of IRAN, Tehran-13164, Iran. E-mail: Mirdamadi@pasteur.ac.ir (S. Mirdamadi)

Lactic acid (LA) is a versatile chemical, used as an acidulant, flavor and preservative in the food, pharmaceutical, leather and textile industries, and for production of biodegradable poly Lactic acid (PLA). L(+)Lactic acid is the only optical isomer for use in pharmaceutical and food industries because human body is only adapted to assimilate this form. In this research, lactic acid production was improved on 201 fermentor. In our experience, among six strains of Lactobacillus were examined for the production of $\mathrm{L}(+)$ lactic acid, Lactobacillus casei ssp. casei ATCC 39392 was selected as a highest $\mathrm{L}(+)$ lactic acid producer. Optimized medium used for lactic acid production contained (per 1) $80 \mathrm{~g}$ glucose, $50 \mathrm{~g}$ whey powder and $20 \mathrm{~g}$ corn steep powder. For a homofermentative process, $\mathrm{pH}$ 
6.0 was found to be optimal. In order to avoid product inhibition, the produced lactic acid was neutralized using calcium hydroxide. Maximum production and productivity of Lactic acid in batch system, were $81 \mathrm{~g}$ and $1.35 \mathrm{~g} / \mathrm{lh}$, but in fed batch system, after 3 feeds of glucose, production and productivity increased up to $360 \mathrm{~g}$ and $4 \mathrm{~g} / \mathrm{lh}$.

\section{IB48}

Saleh A. Mohamed Molecular Biology Dept., National Research Centre, Cairo, Egypt

An extracellular polygalacturonase (PGII) from Trichoderma harzianum was purified to homogeneity by two chromatography steps using DEAE-Sepharose and Sephacryl S-200. The molecular weight of T. harzianum PGII was $31,000 \mathrm{Da}$ by gel filtration and SDS-PAGE. PGII had isoelectric point of 4.5 and optimum $\mathrm{pH}$ of 5.0. PGII was very stable at the $\mathrm{pH}$ 5.0. The extent of hydrolysis of different pectins by enzyme was decreased with increasing of degree of esterification (DE). PGII had very low activity toward nonpectic polysaccharides. The apparent $\mathrm{Km}$ value and $\mathrm{Kcat}$ value for hydrolyzing polygalacturonic acid (PGA) were $3.4 \mathrm{mg} / \mathrm{ml}$ and $592 \mathrm{~s}^{-1}$, respectively. PGII was found to have temperature optimum at $40^{\circ} \mathrm{C}$ and was approximately stable up to $30^{\circ} \mathrm{C}$ for $60 \mathrm{~min}$ of incubation. All the examined metal cations showed inhibitory effects on the enzyme activity. 1, 10 phenanthroline, Tween 20, Tween 80 , Triton X-100 and SDS had no effect on the enzyme activity. The rate of enzyme catalyzed reduction of viscosity of solutions of PGA or pectin was higher three times than the rate of release of reducing sugars indicating that the enzyme had an endo-action. The storage stability of the enzyme in liquid and powder forms was studied, where the activity of the powder form was stable up to one year. These properties of T. harzianum PGII with appreciable activity would be potentially novel source of enzyme for food processing.

\section{IB49}

Preparation of horseradish peroxidase and its application in diagnostic kits Tarek M. Mohamed, Biochemistry Division, Chemistry department, Tanta University, Tanta, Egypt

Preparation of peroxidase from horseradish, which could be used for commercial applications such as diagnostic kits, was occurred through a simple reproducible method consisting of extraction, ammonium sulphate precipitation, filtration through Non-Binding Protein Filter and lyophilization. The purification method was developed allow the preparation of $33 \mathrm{mg}$ of enzyme from $1 \mathrm{Kg}$ of horseradish roots. The one mg of enzyme contains 900 units of peroxidase. This value is similar to that produced by Sigma (50-1000 unit $\mathrm{mg}^{-1}$ powder). The final preparation is salt free reddish brown powder with free ammonia content less than $0.01 \mu \mathrm{g}^{-1}$ units. The RZ value (A400/A280) of the enzyme, which is a good criterion of purity and heme content, was 2.6. The lyophilized enzyme was stable at $-20^{\circ} \mathrm{C}$ for at least one year. The liquid form of the enzyme in presence of $0.1 \%$ sodium azide was stable up to 25 days at $4{ }^{\circ} \mathrm{C}$, while it lost most of activity at room temperature in the same period. The properties of horseradish peroxidase including $\mathrm{Km}$, optimal $\mathrm{pH}$ and temperature, activation energy, thermal stability and effect of different compounds were studied. The applicability of this enzyme in determination of serum glucose was performed. The analysis of glucose in human sera gave results using the kit containing the prepared peroxidase similar to those obtained with a commercial glucose kit.

\section{IB51}

Lactobionic acid production using lactose oxidase: From laboratory to 600 L scale Mikkel Nordkvist ${ }^{1}$, Per Munk Nielsen ${ }^{2}$, Peter Budtz ${ }^{3}$, John Villadsen ${ }^{1}$ : ${ }^{1}$ Center for Microbial Biotechnology, Technical University of Denmark, DK-2800 Lyngby, Denmark; ${ }^{2}$ Novozymes A/S, DK-2880 Bagsværd, Denmark; ${ }^{3} \mathrm{Chr}$. Hansen A/S, DK-2970 Hørsholm, Denmark. E-mail: mnq@biocentrum.dtu.dk (M. Nordkvist)

Currently, lactobionic acid is mainly a high-price specialty product used, e.g. in solutions for organ stabilization. However, lactobionic acid can also be used as a biodegradable cobuilder in detergents, and it has several applications in food technology. With lower production costs it has the potential to become a bulk chemical. The kinetics for the oxidation of lactose to lactobionic acid by a new carbohydrate oxidase was studied in a $1 \mathrm{~L}$ bio-reactor with control of $\mathrm{pH}$, temperature, and dissolved oxygen. The byproduct hydrogen peroxide has a negative influence on the lactose oxidase enzyme, and hence additional experiments were made with addition of catalase to remove hydrogen peroxide, thereby also providing extra oxygen. On the basis of the experiments in $1 \mathrm{~L}$ scale, experiments were performed in a $600 \mathrm{~L}$ reactor equipped with a new system for dispersion of air to supply the necessary oxygen for the oxidation. The aeration system in the large scale reactor was able to supply oxygen sufficiently fast to give the same production rate, at low values of the air flow rate and the energy input, as was obtained in the high-performance laboratory reactor.

\section{IB52}

Characterization of recombinant D-Psicose 3-epimerase from Agrobacterium tumefaciens producing D-Psicose from D-Fructose Yong-Joo Lee, Hye-Jung Kim, Yeung-Soo Kim, Eun-Kyung Hyun, Deok-Kun Oh Department of Bioscience and Biotechnology, Sejong University, Seoul 143-747, Korea. E-mail: deokkun@sejong.ac.kr (D.-K. Oh)

The non-characterized gene previously proposed as D-tagatose 3-epimerase from Agrobacterium tumefaciens was cloned and expressed in Escherichia coli. The expressed enzyme was purified by affinity chromatography on HisTrap HP, desalting chromatography on HiPrep 16/60, and gel filtration chromatography on Sephacryl S-300 HR with a final specific activity of $8.89 \mathrm{U} / \mathrm{mg}$. Using MALDITOF-MS, the native protein was estimated to have a molecular mass of 32,600 Da and a monomeric structure. The purified enzyme exhibited maximal activity at $50^{\circ} \mathrm{C}$ and $\mathrm{pH} 7.5$ without the addition of metal ions and at $60^{\circ} \mathrm{C}$ and $\mathrm{pH} 7.0$ with $1.0 \mathrm{mM} \mathrm{Mn}^{2+}$. Among various metal ions, $\mathrm{Mn}^{2+}$ was the most effective divalent cation for $\mathrm{D}$-fructose epimerization activity. The addition of $\mathrm{Mn}^{2+}$ significantly increased the thermal stability and the epimerization activity with other ketoses such as D-psicose, D-tagatose, D-ribulose, D-sorbose, and D-xylulose. The activity, substrate affinity, maximum velocity, and catalytic efficiency $\left(k_{\mathrm{cat}} / K_{m}\right)$ of the enzyme for D-psicose were higher than those for D-tagatose, which suggests that the enzyme is not D-tagatose 3-epimerase but D-psicose 3-epimerase. The equilibrium ratio between D-psicose and D-fructose was $37: 63$ at $60^{\circ} \mathrm{C}$ with $1.0 \mathrm{mM} \mathrm{Mn}^{2+}$. When the enzyme was used at $14 \mathrm{U} / \mathrm{ml}$, Dpsicose was produced at $211 \mathrm{~g} / \mathrm{l}$ from $700 \mathrm{~g} / \mathrm{l} \mathrm{D}$-fructose containing 
$1 \mathrm{mM} \mathrm{Mn}{ }^{2+}$ after $120 \mathrm{~min}$, corresponding to a conversion yield of $30.2 \%$.

\section{IB53}

The role of ammonium ions in glucosamine formation during the citric acid fermentation process by Aspergillus niger M. Papagianni ${ }^{1}$,F. Wayman ${ }^{2}$, M. Mattey ${ }^{2}:{ }^{1}$ Department of Hygiene and Technology of Food of Animal Origin, School of Veterinary Medicine, University of Thessaloniki, Thessaloniki 54006, Greece; ${ }^{2}$ Department of Bioscience, University of Strathclyde, Glasgow, G1 1XW, UK. E-mail: mp2000@ vet.auth.gr (M. Papagianni)

Stoichiometric modeling of the citric acid fermentation process by Aspergillus niger, in 12-L stirred tank reactor, indicates that $\mathrm{NH}_{4}{ }^{+}$ ions combine with a $\mathrm{C}$-containing metabolite inside the cell to form a nitrogen compound which is then excreted by the mycelium. The close correlation between calculated and experimental profiles indicates that this metabolic process is rapid and takes place before the $\mathrm{C}$-structure of the glucose has been greatly altered by glycolysis or the pentose phosphate pathway. HPLC analysis identified glucosamine as the product of this relationship. A clear effect of medium concentration of $\mathrm{NH}_{4}{ }^{+}$on glucosamine formation was observed when fermentations carried out with optimal and sub-optimal ammonium concentrations. $\left(\mathrm{NH}_{4}\right)_{2} \mathrm{SO}_{4}$ addition when medium nitrogen was depleted, enhanced the formation of new cells from the tips of fragmented hyphae and led to glucosamine accumulation in amounts depending to pulse concentration. The fungus reacts in excess ammonium by converting it to glucosamine, to be utilized later when a regeneration process takes place with fragmentation of vacuolated hyphae and subsequent regrowth, depending always on the culturing conditions. However, depending on carbon and ammonium concentration in medium, glucosamine can be secreted in concentrations as high as $50 \mathrm{~g} / \mathrm{L}$.

\section{IB54}

Isolation, identification and characterization of a highly efficient palatinose producer, Enterobacter sp. FMB1 Sang-Eun Park, Mi-Hyun Cho, Cheon-Seok Park Department of Food Science and Biotechnology, KyungHee University, Yongin 449-701, South Korea. E-mail: cspark@khu.ac.kr (C.-S. Park)

About 1000 microorganisms originated from traditional Korean food origin were screened for efficient palatinose production. An isolate designated FMB1 was exceptionally efficient in sucrose-palatinose conversion activity. Conversion of sucrose into palatinose by FMB1 was much faster than a reference strain of Erwinia rhapontici. FMB1 is a Gram negative, facultatively anaerobic, motile, noncapsulate, and straight rod-shaped bacterium producing acid from glucose. Based on API and 16S rDNA analyses, FMB1 was determined to be Enterobacter $\mathrm{sp}$. The maximum conversion of $10 \%$ sucrose to palatinose and trehalulose by Enterobacter $\mathrm{sp}$. FMB 1 was achieved within $6 \mathrm{~h}$. The preliminary DNA sequencing result of the gene corresponding to sucrose isomerase of Enterobacter sp. FMB1 revealed that it showed $87 \%$ similarity to that of Klebsiella sp. (??).

\section{IB55}

Bacillus subtillis BMRII production of alkaline proteases M. Lageiro, J. Santos, J. Moura, A. Reis, J. Costa-Ferreira Department of Biotechnology, INETI, Lisbon, Portugal
Within the scope of an R\&D project developed in collaboration with leather tanning Portuguese industrial partners a screening of new proteases to be used in the industrial process was performed. A Bacillus subtilis strain isolated from alkaline spent purge liquor was shown to be a promising protease producer. Microorganism growth was studied for optimisation of temperature, agitation, $\mathrm{pH}$ and medium composition either for biomass or proteases production. Optimal growth temperature is different for maximum biomass growth $\left(40^{\circ} \mathrm{C}\right)$ and optimal proteolytic activity $\left(43^{\circ} \mathrm{C}\right)$ yielding biomass specific growth rate of 1.6 and $1.4 \mathrm{~h}^{-1}$, respectively. The achieved proteolytic activities were 5.7 and $7.2 \mathrm{U} / \mathrm{mL}$ of protease, respectively. Optimised medium composition $(7 \mathrm{~g} / \mathrm{L}$ beef extract, $4 \mathrm{~g} / \mathrm{L}$ yeast extract, $5 \mathrm{~g} / \mathrm{L}$ peptone and $0.4 \mathrm{~g} / \mathrm{L} \mathrm{CaCl}_{2}$ ) yielded a specific growth rate of $1.5 \mathrm{~h}^{-1}$ and $13.9 \mathrm{kU} / \mathrm{L}$ of protease, in shake flask experiments. Bioreactor experiments (from 1 to $16 \mathrm{~L}$ ) with the selected medium were performed at $43{ }^{\circ} \mathrm{C}$ in order to test aeration rate (1 and $2 \mathrm{vvm}$ ), stirring (300-700 rpm) and $\mathrm{pH}$ (uncontrolled, controlled at 7 and 8). Best protease activity was $64 \mathrm{U} / \mathrm{mL}$ in $2 \mathrm{~L}$ bioreactor without $\mathrm{pH}$ control at $500 \mathrm{rpm}$ and $2 \mathrm{vvm}$. The proteolytic extract was characterized and compared to commercial bates. Results indicate that these proteases can be employed in the purge phase of the leather tanning process in industry.

\section{IB56}

Bacillus megaterium - a versatile tool for production and secretion of heterologous proteins Rebekka Biedendieck, Martin Gamer, Dieter Jahn, Marco Malten Institute of Microbiology, Technical University Braunschweig, Spielmann-strasse 7, D-38106 Braunschweig, Germany. E-mail: m.malten@tu-bs.de (M. Malten)

The Gram positive bacterium Bacillus megaterium is known for its capacity to produce exoenzymes including amylases, proteinases, and penicillin amidase at industrial scale. Here, we describe the development of various vectors for the production and export of recombinant heterologous proteins employing $B$. megaterium signal peptides. The target gene can be cloned directly adjacent to the signal peptide coding sequence (Bart et al., 2005). This arrangement allows for a correct $\mathrm{N}$-terminal sequence of the mature protein after processing by the signal peptidase SipM. Using this newly developed protein production and export system Lactobacillus reuteri 121 levansucrase (van Hijum et al., 2001) was secreted in significant amounts $(\sim 4 \mathrm{mg} / \mathrm{l})$ into the growth medium. Fusion of the recombinant levansucrase to affinity tags allowed one-step purification of the recombinant protein from the growth medium. However, fused peptide tags resulted in a decreased secretion of the fusion protein. $1.4 \mathrm{mg} \mathrm{His}_{6}$-tagged levan-sucrase were purified per litre of culture. The system was further enhanced via coexpression of a gene for the signal peptidase SipM (Malten et al., 2005a) and deletion of the gene for the extracellular protease NprM. Developed new tools allow for various strategies of integrated high level production, export and purification of heterologous proteins in B. megaterium. Methods for high-throughput screening of secreted enzymes are under development. The determined sequence of the B. megaterium genome, studies using high-cell density cultivations (Malten et al., 2005b) and proteome data from batch fermentations implicate new targets for directed genetic optimization of B. megaterium production and secretion strains. Novel strong and inducible promoters are currently under investigation. 


\section{References}

Barg et al., 2005. Microbial Processes and Products. Humana Press Inc., Totowa, pp. 165-184.

Malten et al., 2005. Biotechnol. Bioeng. 89, 206

Malten et al., in press. Biotech. Bioeng. 218.

van Hijum et al., 2001. FEMS Microbiol. Lett. 205, 323-328.

\section{IB57}

$\boldsymbol{R}$-Styrene oxide production by ethene utilizing bacteria $\underline{\text { Toru Matsui }}^{1}$, Naoya Shinzato ${ }^{1}$, Hisashi Saeki ${ }^{2}$, Hitoshi Matsuda ${ }^{2}$ : ${ }^{1}$ Center of Molecular Biosciences, University of the Ryukyus, Okinawa 903-0213, Japan; ${ }^{2}$ Japan Energy Co., Japan. E-mail: tmatsui@comb.u-ryukyu.ac.jp (T. Matsui)

Optically active epoxides are considered as the potential intermediate for chiral drugs synthesis. Although $S$-styrene oxide (SO) have been extensively investigated using styrene monooxygenase from Pseudomonas sp., microbial production of $R$-SO with high enantiomeric excess was hardly examined. In this study, $R$-SO producing bacteria from styrene was screened using various alkene assimilating bacteria. $R$-SO with the highest ee (ca. 100\%ee) was obtained using ethene utilizing bacteria, identified as Mycobacteirum sp., while produced relatively lower at around $70 \%$ ee when using propene utilizing bacteria. The alkene monooxygenase gene homologue sequence amplified from the genomic DNA revealed a significant similarity to that of etnABC. These bacteria also showed stereoselective degradation of racemic SO, suggesting that the produced SO might be further stereoselectively degraded to increase the ee. The ethene utilizing bacteira produced not only $R$-SO but also $S$-epichrolhydrin at high ee.when using arylchloride as the substrate.

\section{Acknowledgement}

This research was supported by Nagase Science and Technology Foundation.

\section{IB59}

The secretion efficiency of the Escherichia coli Sec pathway is dependent on the growth phase but not on protein size F.J.M. Mergulhão, G.A. Monteiro Centro de Engenharia Biológica e Química, Instituto Superior Técnico, Av. Rovisco Pais, 1049-001 Lisbon, Portugal. E-mail: filipem@alfa.ist.utl.pt (F. Mergulhão)

The secretion efficiency of the Escherichia coli Sec pathway was evaluated through the expression of Green Fluorescent Protein and Human Proinsulin fusion proteins. Translocation to the periplasm is dependent on the growth phase of the bacterial culture and the highest secretion efficiency is attained in mid-exponential phase. Secretion performance is independent of protein size $(17-42 \mathrm{kDa})$ and even when the amino acid composition of the secreted proteins is very similar, the amino acid distribution within the protein can affect translocation. In silico prediction analysis suggests that proteins that are prone to form $\alpha$-helix structures are more efficiently translocated. Culture medium composition plays an important role on secretion performance with the highest secretion results being obtained in minimal medium.

\section{IB60}

Mohammad Reza Nejadmoghaddam Avesina Research Centre, Medical Diagnostic Reagents \& Biological Products, Chamran Highway, 19835-177 Tehran, Iran
Streptokinase is a common fibrinolytic drug. That is used in thrombolytic therapy for long time. To compare with another thermbolytic drugs like tPA, it has lot of advantages. In this present research DNA was extracted from S. equisimilis H46A for the first time in Iran. Streptokinase gene was amplified by using two forward primers and one reverse primer. A common restriction enzyme, BamH-I, was used for cloning. Both ends of the PCR products (full length: $1323 \mathrm{bp}$ and mature section: $1245 \mathrm{bp}$ ) and the restriction site on MCS of PQE-30 vector were digested. In this study, PQE-30 expression vector was used with high level expression ability for production of recombinant fusion streptokinase with simplifying the purification by employing affinity-metal chromatography method. In addition, the cloning results were controlled by double digestion and sequencing.

\section{IB61}

Oxidation of iso-alkanes by a gaseous hydrocarbon assimilating mold, Scedosporium sp. A-4 Masayuki Onodera, Satoshi Takesono, Kiyoshi Toda Department of Applied Chemistry and Biotechnology, Niigata Institute of Technology, Kashiwazaki, Niigata 945-1195, Japan. E-mail: onodera@acb.niit.ac.jp (M. Onodera)

Oxidation of short-chain iso-alkanes was studied with propanegrown resting mycelia of Scedsporium sp. A-4. Isobutane was oxidized to tert-butanol, but not to isobutanol. Isobutanol was used for growth, but both isobutene and tert-butanol were not used for growth. Isopentane was oxidized to 3-methyl-1-butanol, 2-methyl2-butanol, and 3-methyl-2-butanol but not to 2-methyl-1-butanol. 2-Methylpentane was oxidized to 4-methyl-1-pentanol, 2-methyl-2pentanol, and 4-methyl-2-pentanol but not to 2-methyl-1-pentanol or 2-methyl-3-pentanol. 3-Methylpentane was not oxidized. Oxidation of branched alcohols was also studied.

\section{IB62}

Application of NADPH-dependent 2.5-diketo-gluconic acid reductase for production of $\mathrm{L}$-ascorbic acid Claudia Pacher ${ }^{1,2}$ : ${ }^{1}$ Division of Food Biotechnology, Department of Food Sciences and Technology, BOKU, University of Natural Resources and Applied Life Sciences, Muthgasse 18, A-1190 Vienna, Austria; ${ }^{2}$ Research Centre Applied Biocatalysis, Petersgasse 14, A-8010 Graz, Austria. E-mail: Claudia.pacher@boku.ac.at

Ascorbic acid is an organic acid with various applications in the food and pharmaceutical industries. At present, the majority of commercially manufactured Vitamin $\mathrm{C}$ is synthesized via the Reichstein process, which is highly energy-consuming, involves considerable quantities of organic solvents and gives an overall yield of about $50 \%$. Therefore, during the past decades a lot of research was done to develop biotechnological alternatives for the synthesis of Reichstein intermediates by enzymatic or fermentative means, which show some advantages regarding costs and environmental friendliness. One of the fermentation routes runs via 2.5-diketo-D-gluconic acid (2.5-KDG), produced by Pectobacter (Erwinia) cypripedii. This compound has been reduced by a NADPH-dependent 2.5-diketogluconic acid reductase (DKR) from Corynebacterium glutamicum to the key intermediate 2-keto-L-gulonic acid (2-KLG) before chemical rearrangement leads to the final product. For economical reasons we wanted to express DKR heterologously. Based on our long term experience with coenzyme regeneration we wanted also to perform the reaction in homogeneous solution. The spent coenzyme of 
NADPH-dependent DKR has been regenerated by a second isolated NADP-dependent enzyme like glucose dehydrogenase. We describe here the recombinant production, purification and characterization of DKR and the results of enzymatic 2-KLG formation by using the recombinant enzyme in a homogeneous system with conjugated coenzyme regeneration.

\section{IB63}

Regulation of GRP78 Transcription by substances of microbial origin H.R. Park, J.H. Hwang, S.U. Choi, Y.I. Hwang Division of Food Science and Biotechnology, Kyungnam University, Masan 631-701, Republic of Korea. E-mail: parkhy@kyungnam.ac.kr (H.R. Park)

GRP78 residing in Endoplasmic reticulum (ER) functions as a molecular chaperon by associating transiently with incipient proteins as they traverse the ER and aiding in their folding and transport. Furthermore, the protein can also be induced under various stress condition such as glucose starvation, inhibition of protein glycosylation by tunicamycin, blockage of vesicular trafficking by brefeldin A and ER-calcium-ATPase pump inhibition by thapsigargin. Thus, substances that directly down and up- regulate grp78 transcription are expected to be useful for treatment of cancer and Alzheimer's disease, respectively. In the course of our screening program to obtain substances, which regulate grp78 expression, we first constructed an assay system monitored by the expression of a reporter gene. HeLa cells, which are transformed with luciferase gene under the control of grp78 promoter designated as HeLa $78 \mathrm{C} 6$ cells, respond sensitively to luciferase grp78 induction by ER stress such as treatment of tunicamycin. By using this screening system, we isolated pyrisulfoxin as an up-regulator of grp78, and valinomycin, citreoviridin and alternariol as down-regulators. Detailed studies on other biological activities were now under way.

\section{IB64}

Pyranose dehydrogenase from Agaricus bisporus Christoph Sygmund $^{1}$, Roman Kittl ${ }^{1}$, Dorothea Fragner ${ }^{1}$, Jindrich Volc ${ }^{2}$, Dietmar Haltrich ${ }^{1}$, Clemens Peterbauer ${ }^{1}:{ }^{1}$ Division of Food Biotechnology, Department of Food Sciences and Technology, BOKU, University of Natural Resources and Applied Life Sciences, Muthgasse 18, A-1190 Vienna, Austria; ${ }^{2}$ Institute of Microbiology, Academy of Sciences of the Czech Republic, Videnska 1083, CZ-142 20 Praha 4, Czech Republic. E-mail: Christoph.Sygmund@boku.ac.at (C. Sygmund)

$\mathrm{PDH}$ is an enzyme that was described only several years ago in a number of ecologically related litter-decomposing fungi (Agaricales, Gasteromycetales). It catalyzes the C-3 and/or C-2 oxidation of several aldopyranoses to the respective keto sugar derivates. PDH shows a very broad substrate range, oxidizing almost all major sugar components of wood polysaccharides, and is implicated to play a role in lignocellulose degradation. Agaricus bisporus, the white button mushroom, is an economically significant agricultural crop. The cultivation, which is done by solid-substrate fermentation on straw- and hay-based composted substrate, is sometimes seen as one of only few economically feasible methods for bioconversion of lignocellulosic agricultural waste material. Deeper insight in the physiological role of PDH may provide help for mushroom growers to increase yield, improve quality or make new sources of raw materials utilizable. We amplified a fragment of the $p d h$ gene with degenerated primers derived from internal peptide sequences. The screening of a genomic library led to the isolation of the PDH gene. Subsequently we amplified a cDNA clone by RT-PCR and investigated the transcriptional regulation by different carbon sources on a defined minimal medium.

\section{IB65}

Optimization of monoclonal antibody production processes with simulation and scheduling tools Demetri Petrides, Charles Siletti INTELLIGEN Inc., Scotch Plains, NJ 07076, USA. E-mail: dpetrides@intelligen.com (D. Petrides)

This presentation will review the state of the art in batch process simulation and scheduling tools and their applications in the design and debottlenecking of integrated biopharmaceutical processes. A systematic methodology will be presented for identifying and eliminating size, time, and throughput bottlenecks that limit production in single and multi-product facilities. The methodology will be illustrated with an industrial case study dealing with the optimization of a multi-product facility that produces therapeutic monoclonal antibodies (MABs). MAB processes are characterized by a long bio-reaction time (e.g., 1.5-2 weeks for fed-batch operation and 1-2 months for perfusion operation). The cycle time for processing a lot in the recovery and purification train typically takes $3-4$ days. Consequently, one way of increasing throughput is by installing extra bioreactors that operate in staggered mode and utilize the same recovery train. The result is that multiple batches may be at different stages of completion at any given time. Since cleaning equipment (e.g., CIP skids) and buffer preparation and holding tanks are shared by multiple steps across many batches, this type of operation leads to time/scheduling bottlenecks that constrain the cycle time and the throughput of a process. The problem becomes more challenging in the context of multi-product facilities and when constraints imposed by the limited availability of resources are considered. Our methodology and its computer implementation will illustrate how to systematically identify and eliminate such bottlenecks. The industrial case study will provide a real world example of the methodology.

\section{IB66}

Application of two stage continuous cultures of Aspergillus niger for citric acid biosynthesis Jerzy J. Pietkiewicz, Malgorzata Janczar, Wladyslaw Lesniak Food Biotechnology Department, University of Economics, Wroclaw 53-345, Poland. E-mail: jerzy.pietkiewicz@ae.wroc.pl (J.J. Pietkiewicz)

The aim of the work was application test of submerged two stage single stream continuous cultures of Aspergillus niger for citric acid production from sucrose. Studies were carried out in lab fermenters with working volume of $5 \mathrm{dm}^{3}$. The bioreactors were standard CSTRs. In two stage continuous cultures (TSCC) high mycelium growth and high citric acid production were observed in the first bioreactor. There was almost four times lower growth of biomass rate and about three times lower citric acid production rate in the second bioreactor. Studies on influence of dilution rate in race from 0.0098 to $0.0230 \mathrm{dm}^{3} /\left(\mathrm{dm}^{3} \mathrm{~h}\right)$ on course and efficiency of TSCC showed, that the highest citric acid yield $\left(Y_{\mathrm{P} / \mathrm{S}}=86.3 \%\right)$, high volumetric rate of its production $\left(R_{\mathrm{Pc}}=0.958 \mathrm{~g} /\left(\mathrm{dm}^{3} \mathrm{~h}\right)\right)$ and the highest biosynthesis efficiency coefficient $\left(K_{\mathrm{ef}}=82.7\right)$ were obtained with dilution rate $D=0.0148 \mathrm{dm}^{3} /\left(\mathrm{dm}^{3} \mathrm{~h}\right)$. There was also high citric acid concentration $\left(P=129.5 \mathrm{~g} / \mathrm{dm}^{3}\right)$ and low residual sugar concentration $\left(S_{\mathrm{k}}=6.9 \mathrm{~g} / \mathrm{dm}^{3}\right)$ in 
the medium flowing out the second bioreactor in those cultures.

\section{IB67}

Synthesis by beta-galactosidase in aerobic fermentation by Kluyveromyces marxianus Eloízio Júlio Ribeiro, Líbia Diniz Santos Marquez, Gisele Duque B. Sousa Faculdade de Engenharia Química, Universidade Federal de Uberlândia, Uberlândia 38400-902, Brazil. E-mail: ejribeiro@ufu.br (E.J. Ribeiro)

The beta-galactosidases in commercial use are of different origins and yeast and fungal lactases present the greatest interest. The yeast lactases present neutral optima $\mathrm{pH}$ and are suitable for the hydrolysis of lactose in milk. In this work, the aim was to study the influence of aeration in the production of beta-galactosidase in batch fermentations with Kluyveromyces marxianus ATCC 46537. The medium composition for culture was as follows (in $\mathrm{g} / \mathrm{L}$ ): lactose PA 50, yeast extract 5, $\left(\mathrm{NH}_{4}\right)_{2} \mathrm{SO}_{4} 4$ and $\mathrm{KH}_{2} \mathrm{PO}_{4}$ 2. The fermentations was carried out at $30^{\circ} \mathrm{C}, \mathrm{pH} 5.5$, at $200 \mathrm{rpm}$ starting with an initial cellular concentration of $1 \times 10^{7}$ cels $/ \mathrm{mL}$, with different aeration rates. The cells were disruped with chloroform $2 \%$ (v/v) as solvent. The enzymatic activity was determined as initial rate of lactose hydrolysis at defined conditions. The studies have revealed the importance of aeration on Kluyveromyces marxianus in the growth and beta-galactosidase synthesis. The enzymatic activity of fermented medium with $0.5 \mathrm{vvm}$ was $50 \%$ higher than one without aeration. Furthermore, the cellular growth was faster in the aerobic fermentation than in the anaerobic one. The aeration has taken an important place in the enzymatic synthesis and in the cellular growth, however the results have shown that the aeration rate increase of $0.5-1.5 \mathrm{vvm}$ has not implied a increase in the cellular growth neither in the enzymatic reached activity.

\section{IB68}

Immobilization of beta-galactosidase of Aspergillus oryzae in sodium alginate Eloízio Júlio Ribeiro, Gustavo Paiva Ribeiro, Fernanda Ferreira Freitas Faculdade de Engenharia Química, Universidade Federal de Uberlândia, Uberlândia 38400-902, Brazil. E-mail: ejribeiro@ufu.br (E.J. Ribeiro)

The lactose presents in the milk has a solubility of only $20 \%$ at $30^{\circ} \mathrm{C}$, and a high percentage of the world population presents intolerance to this sugar, due to the low or absence of the activity of the lactase enzyme in the organism. To minimize such problems, the most viable alternative for nourishing dairy products is the enzymatic hydrolysis of milk, although it is an expensive process due to the high cost of the beta-galactosidase enzyme. An alternative that has been greatly studied is the immobilization of this enzyme, originated from many different sources. There are several immobilization procedures for this enzyme, however, a procedure considered ideal was not obtained yet. The objective of this work was to study the immobilization process of beta-galactosidase from Aspegillus Oryzae in sodium alginate with commercial gelatin. In the immobilization process was studied the glutaraldehyde influence for 1,3 and $5 \%$, in the presence of commercial gelatin at the concentration of $2 \%$ at the immobilization medium. The activities of the immobilized enzymes were obtained in a stirred micro-reactor, at the temperature $30^{\circ} \mathrm{C}, \mathrm{pH}$ 4.5 with a $50 \mathrm{gL}^{-1}$ lactose solution in acetate buffer. The experimental results showed that the immobilized biocatalyst that presented the larger initial activity was the one obtained at the immobiliza- tion medium that contained 5\% of glutaraldehyde. After 20 daily determinations of enzymatic activities, a fall of 30, 40 and $24 \%$ was verified in the enzymatic activities for the immobilized biocatalysts using glutaraldehyde at 1, 3 and 5\%, respectively, however, in all cases, the enzymatic activity reached the half of their initial activity after 10 determinations.

\section{IB69}

Hydrolysis of sucrose by immobilized beta-fructofuranosidase in silica Eloízio Júlio Ribeiro, Ubirajara Coutinho Filho Faculdade de Engenharia Química, Universidade Federal de Uberlândia, Uberlândia 38400-902, Brazil. E-mail: ejribeiro@ufu.br (E.J. Ribeiro)

Invertase, known as beta-fructofuranosidase (EC 3.2.1.26), plays a catalytic role in the conversion of sucrose into glucose and fructose. It is largely used in the food industry to prevent the crystallization of sucrose in sugar mixtures and can be used in enzyme reactors for hydrolysis of sucrose. The objective of this work was to study the kinetic of sucrose hydrolysis by immobilized betafructofuranosidase in a continuous recirculation reactor, evaluated the enzyme stability and determine the effective half-life of immobilized enzyme. Invertase was covalently immobilized on sillanized controlled pore silica. Nonlinear fitting were used to determine the kinetic parameters for substrate and product inhibition observed in the enzymatic hydrolysis of sucrose. The kinetics studies of immobilized invertase were carried out in a continuous recirculating reactor. The half-time of enzyme inactivation $\left(t_{1 / 2}\right)$ was calculated from the initial rates of the remaining enzyme activity. The model of inhibition by substrate and product adequately represented the enzymatic hydrolysis. The fructose effect was competitive inhibition $\left(K_{\mathrm{F}}=3.1022 .10^{-4} \mathrm{~mol} / \mathrm{mL}\right)$ and the glucose effect was noncompetitive inhibition $\left(K_{\mathrm{G}}=2.2521 .10^{-4} \mathrm{~mol} / \mathrm{mL}\right)$. The effective diffusivity of sucrose into the support was shown to be the same as for sucrose in dilute solution $\left(0.75 \times 10^{-5} \mathrm{~cm}^{2} / \mathrm{s}\right.$ at $\left.40^{\circ} \mathrm{C}\right)$. The half-time of enzyme inactivation $\left(t_{1 / 2}\right)$ was $1656 \mathrm{~h}$. Controlled pore silica showed to be an excellent immobilizing support. The immobilized invertase was very stable at temperatures lower than $50^{\circ} \mathrm{C}$. The intrinsic parameters $\left(K_{\mathrm{I}}, K_{\mathrm{F}}, K_{\mathrm{G}}\right.$ and $\left.V_{\mathrm{M}}\right)$ were shown to be similar to the apparent values.

\section{IB70}

Changes in the mycobacterial cell wall skeleton in the presence of isoniazid Anna Rumijowska-Galewicz ${ }^{1}$, Malgorzata KoryckaMachala ${ }^{1}$, Katarzyna Lisowska ${ }^{2}$, Jaroslaw Dziadek ${ }^{1}$ : ${ }^{1}$ Laboratory for Genetics and Physiology of Mycobacterium, Centre for Medical Biology, Polish Academy of Sciences, Lodz, Poland; ${ }^{2}$ Department of Industrial Microbiology and Biotechnology, University of Lodz, Poland. E-mail: arumijow@ @bm.pan.pl (A. Rumijowska-Galewicz)

The low permeability of mycobacterial cell wall envelopes is a result of the unique composition and organization of the cell wall lipids. The permeability of mycobacterial cell wall can be changed by means of partial disintegration of its compounds. The aim of present work was to characterize the changes in the cell wall skeleton (CWS) and non covalently bound free lipids under the influence of isoniazid, the inhibitor of mycolic acids biosynthesis. Fatty acid (FAMEs) and mycolic acid methyl esters (MAMEs) obtained from all tested preparations were analyzed by GC/MS analysis. The analysis of free lipids and CWS revealed distinct changes in the composition of the frac- 
tions obtained from the cells exposed to action of the isoniazid. The changes in the quantity of fatty acids in the INH-treated cells indicates that INH interferes with the synthesis of lipidic compounds of the mycobacterial cell wall also. The decreased amount of covalently bound mycolic acids in the CWS is responsible for the enhanced penetration of hydrophobic compounds through the cell wall.

\section{Acknowledgement}

This work was supported by grant nr 3P04C 06923 of the Committee for Scientific Research.

\section{IB71}

Optimisation of inoculum for laccase production Barbara Sencio, Teresa Jamroz, Stanislaw Ledakowicz Department of Bioprocess Engineering, Technical University, Lodz, PL 90-924, Poland

The enzyme laccase (EC.1.10.3.2. p-diphenol oxidase) is a subject of research in many centres dealing with improvement of bioprocesses with the use of different white rot fungi species. Most strains that produce this enzyme in vitro require inductors initiating its biosynthesis. When Cerrena unicolor was applied, it was found that the strain produced laccase very efficiently without additional toxic compounds. To specify optimum conditions for laccase production in a submerged culture, research was undertaken to obtain the most efficient inoculum C. unicolor. The goal of this research was to determine the effect of form and incubation time of inoculum on enzymatic activity of the laccase producing strain. The experimental inoculum was the mycelium prepared on a solid and liquid substrate. Basing on results obtained, it was found that the laccase yield was the highest in the cultures where the mycelium was grown on a solid substrate. Maximum activity of the $C$. unicolor strain was achieved on the 16th day of culture, and the amount of laccase produced was higher by, ca. $30 \%$ as compared to the mycelium obtained from the liquid substrate. Results of these experiments were used to continue studies on the impact of inoculum age. Experiments were carried out using an inoculum incubated for 1-3 weeks at the temperature $30^{\circ} \mathrm{C}$ in a Certomat BS1 shaker at $110 \mathrm{rpm}$. The best results in the $C$. unicolor strain culture were achieved using a 7-day-old inoculum.

\section{IB72}

Effect of alcohol treatment on hydrolytic activity of Candida rugosa lipase Serpil Takaç, A. Ezgi Ünlü Department of Chemical Engineering, Institute of Biotechnology, Ankara University, 06100 Tandoğan, Ankara, Turkey

Candida rugosa lipase (CRL) was treated with 20, 40, and 60\% concentrations of methanol (M), ethanol (E), 2-propanol (2P) and 1-butanol (1B) to investigate the changes in its hydrolytic activity toward $p$-nitrophenylacetate. The treatment included the following steps at $+4{ }^{\circ} \mathrm{C}$ : (i) stirring CRL with phosphate buffer for $24 \mathrm{~h}$; (ii) treating the solutions with alcohols; (iii) stirring treated-CRL for $24 \mathrm{~h}$; (iv) centrifugation at $10,000 \mathrm{rpm}$ for $30 \mathrm{~min}$; (iv) dialysis the supernatant against bidistilled water for $39 \mathrm{~h}$. The activity of CRLs was followed for $120 \mathrm{~h}$ at $37^{\circ} \mathrm{C}$ in the presence and absence of isooctane. The enzyme activity was measured spectrophotometrically and the protein concentration was measured by Lowry's method. It was found that the recovered protein did not change considerably with the type of alcohol; however, decreased with alcohol concentration. In the presence of isooctane, specific activities of the untreated and treated-CRLs increased compared with those obtained in the absence of isooctane. 1B-CRLs and E-CRLs showed higher activities than MCRLs and 2P-CRLs whereas untreated-CRL exhibited higher activity than M-CRLs, E-CRLs and 2P-CRLs. The highest and the lowest activities were obtained with 20\% 1B-CRL and 60\% 2P-CRL, respectively. The changes occur in the structure of CRL after treatments were investigated by electrophoretic analysis.

\section{Acknowledgement}

This study was supported by Ankara University Biotechnology Institute (Project No: 89).

IB73

Deep cold anabiosis as a state of different genera of industrial microorganisms, providing unlimited storage terms A. Tsutsayeva, A. Ananyina, L. Balyberdina, L. Stepanyuk Cryoimmunology Department, Institute for Problems of Cryobiology, Cryomedicine of the National Academy of Sciences of Ukraine, Kharkov 61015, Ukraine. E-mail: cryo@online.kharkov.ua (A. Tsutsayeva)

Different genera, species and strains of microorganisms were found to posses different cryoresistance. Optimal ways for cryopreservation of microorganisms-producers of antibiotics, microorganisms, used in food industry, agriculture and veterinary have been developed. It was demonstrated, that non-lethal damages could occur in cryopreserved microorganisms after their returning to physiological culture conditions, which were manifested in Streptomyces' hypha fragmentation, that of cyanobacteria's, streptococci's chains as a result there was an increase in a number of colony-forming units, a reversible inhibition of microorganisms' proliferative activity in Bacillus thuringiensis and lactic streptococci, stimulation of the enzyme processes and antibiotic production. Non-lethal damages are repaired during microorganism culturing in the first passage. The cause of non-lethal damages is a reversible inhibition of biosyntheses of protein and nucleic acids respiratory activity. The repairing of non-lethal damages is accompanied by the production of stressproteins, different from heat shock proteins.

\section{IB74}

Effect of pH in the 2-propanol treatment of Candida rugosa lipase on its enantioselectivity in the hydrolysis of racemic Naproxen methyl ester Serpil Takaç, A. Ezgi Ünlü Department of Chemical Engineering, Ankara University, 06100 Tandoğan, Ankara, Turkey

Candida rugosa lipase (CRL) was treated with 2-propanol (2P) at the $\mathrm{pH}$ values of $1.5,4,6,7.5,9$ and 12 to investigate the changes in its enantioselectivity in the hydrolysis of racemic Naproxen methyl ester. The treatment included the following steps at $+4{ }^{\circ} \mathrm{C}$ : (i) stirring CRL with different buffer solutions to maintain the desired $\mathrm{pH}$ values for $24 \mathrm{~h}$; (ii) treating the solutions with $40 \% 2 \mathrm{P}$; (iii) stirring treated-CRLs for $24 \mathrm{~h}$; (iv) centrifugation at $10,000 \mathrm{rpm}$ for $30 \mathrm{~min}$; (iv) dialysis the supernatant against bidistilled water for $39 \mathrm{~h}$. Hydrolyses of racemic Naproxen methyl ester to form $S$-Naproxen were performed in shaking flasks at $200 \mathrm{rpm}$ and $37^{\circ} \mathrm{C}$ for $192 \mathrm{~h}$ in isooctane-phosphate buffer solution biphasic system using treatedCRLs with the activity of $7.75 \mathrm{U}$. The concentrations of the enantiomers of Naproxen methyl ester and Naproxen were determined with HPLC. It was found that the treatment $\mathrm{pH}$ has an important role on the enantioselectivity and conversion. The highest enantiomeric excess for the substrate, for the product, enantiomeric ratio, and con- 
version were obtained with CRL treated at $\mathrm{pH} 1.5$ as $39,98,181$ and $29 \%$, respectively. These values were followed with $2 \mathrm{P}$ treated CRL at pH 12 as 35, 98, 121 and 27\%. However, lower enantiomeric excesses, conversions and enantiomeric ratios were obtained at the treatment $\mathrm{pH}$ values between 1.5 and 12 .

\section{Acknowledgement}

This study was supported by Ankara University Biotechnology Institute (Project No: 89).

\section{IB75}

Biosynthesis of oxalic acid from fatty acids by Aspergillus niger D. Witkowska, W. Rymowicz, I. Musial, D. Lenart Departament of Biotechnology and Food Microbiology, Agricultural University of Wrocław, Wrocław 50-375, Poland. E-mail: wit@ozi.ar.wroc.pl (D. Witkowska)

The effects of fatty acids, nitrogen (as $\mathrm{NH}_{4} \mathrm{NO}_{3}$ ), phosphorus (as $\left.\mathrm{KH}_{2} \mathrm{PO}_{4}\right), \mathrm{pH}$ value, manganese $\left(\mathrm{Mn}^{2+}\right)$, iron $\left(\mathrm{Fe}^{2+}\right)$ and methanol concentration on growth and production of oxalic acid from post refining fatty acids by a mutant of Aspergillus niger XP in submerged fermentation experiments was studied. Of the A. niger strains screened, A. niger XP was identified as the best oxalate producer on lipids. The influence of the $\mathrm{pH}$ on oxalic acid formation shows that the maximum production rate and higher concentration of product are observed at the $\mathrm{pH}$ ranging from 4 to 5 . With a medium containing $50 \mathrm{~g}$ fatty acids/l, the production reached a maximum of $68 \mathrm{~g}$ oxalic acid/1 after 7 days. The addition of $1.5 \%$ (w/v) methanol to seed culture increased the product yield and concentration of oxalic acid but decreased the amount of an undesired by-product (citric acid). Under this condition, the maximum oxalate productivity (14-18 g/l days) was maintained for 2-4 days of fermentation. Other results of the experiments show that supplementation of the production medium with manganese and iron enhances oxalate production. Fatty acids proved to be a very good substrate for oxalic acid production by $A$. niger XP giving excellent yields and productivity at low $\mathrm{pH}$. The results are very promising as they may lead to cheap alternative processes for oxalic acid production from renewable lipid resources.

\section{IB76}

Cloning and heterologous expression of a laccase orthologue from Aspergillus fumigatus and functional analysis of the associated gene cluster Banu Yalcindag, Zumrut Begum Ogel, Ufuk Bakir Department of Food Engineering, Middle East Technical University, Ankara 06531, Turkey. E-mail: banuy @ metu.edu.tr (B. Yalcindag)

Laccase (E.C. 1.10.3.2, p-benzenediol:oxygen oxidoreductase), which is an enzyme belonging to the multi-copper oxidase family, catalyzes the oxidation of a broad variety of polyphenols with a preference for $p$-isomers, which are converted to $p$-quinones. Fungi generally contain several laccases which have been found to be involved in delignification, melanin synthesis and pathogenesis. Laccase has also important potential application areas especially in food and chemical industries. After Aspergillus fumigatus genome data were released, research on functional analysis of laccases has been initiated in our laboratory. Laccase genes of Aspergillus nidulans, yA and tilA, and laccase and multi-copper oxidase genes of Aspergillus fumigatus, abr 2 and $a b r 1$, were used to analyze A. fumigatus genome for laccases. This sequence analysis resulted in 4 probable laccase genes, one of which was the previously cloned $a b r 2$ gene. In this study, one of these genes (Aflacl) was further characterized. After sequence alignment and characterization studies, Aflacl was predicted to have $2128 \mathrm{bp}$ having six introns, which makes the protein 606 amino acid long, and the predicted protein sequence showed 63\% homology with the dihydrogeodin oxidase of Aspergillus terreus and 38\% homology to the laccase 2 of Botryotinia fuckeliana. Aflacl gene is found within an uncharacterized gene cluster containing genes with homology to glutathione- $S$-transferase, polyketide synthase, $o$-methyl transferase, and others. The information obtained from sequence analysis was employed in designing PCR-primers to amplify the Aflacl gene, followed by cloning onto pAN52-1 and pAN52-4 vectors for heterelogous expression in Aspergillus sojae. In addition, by the use of RT-PCR, Aflacl cDNA will be cloned and expressed in Escherichia coli. Furthermore, gene silencing studies will be performed to enlighten the function of $A f l a c l$ and associated gene cluster.

\section{IB77}

Growth stability of photosynthetic bacteria: Effects of supplemental illumination and ferrous ion concentration Reza Yegani, Satoshi Yoshimura, Tomohisa Katsuda, Shigeo Katoh Graduate School of Science and Technology, Kobe University, Kobe, Japan

Stability of growth rate of photosynthetic cells is an important factor in designing of effective photobioreactors especially in long term operations. In our experiments, in order to keep operational parameters almost constant, a semi-continuous culture method was developed. In this method, a part of culture broth containing grown cells was repeatedly replaced by fresh medium at a predetermined time interval. The replacement of broth with fresh media could keep the cell concentration, volume of broth and distribution of light intensity constant at initial values throughout the cultivation. It was shown that in one side illumination with a halogen lamp, if the ratio of the light intensity at the front side of a flat plate photobioreactor to that at the rear side was kept lower than 4, the growth rates was sustained in constant levels. However, at higher ratios the growth was followed by rapid decrease after 5-6h. Supplemental illumination with a fluorescent lamp from the rear side of the flat plate photobioreactor could sustain almost stable growth rate. Beside of the illumination conditions, increased ferrous ion concentrations in medium could keep the stability of growth rate even in unstable illumination conditions, while consumed ferrous ion was slight.

\section{IB78}

Production of metabolites involved in detoxification mechanisms of yeast cells Manuela Rollini, Matilde Manzoni DISTAM, Section of Industrial Microbiology, University of Milan, Italy

Glutathione (GSH) plays a pivotal role in protecting cells from by-products generated by oxidative metabolism. These characteristics make this active tripeptide an important drug for the treatment of liver diseases and is of interest in the food additive industry, therapeutics and sport nutrition. In the first part of the research, a screening was carried out among 48 yeast strains, to find out those able to accumulate higher GSH intracellular levels. Two Saccharomyces cerevisiae strains proved to be the best GSH producers $(1.3 \% \mathrm{dw})$, in every samples the presence of $S$-adenosyl-methionine in traces $(0.2 \% \mathrm{dw})$ was also evidenced. $S$-Adenosyl-methionine (SAM) plays a role in the immune system, maintains cell membranes, participates in detoxification reactions and in the manufacture of brain chemicals and 
cartilage. The second part of the research was aimed at increasing, in a post fermentative procedure, GSH levels present inside the cells at the end of the growth phase. Moreover, time course of SAM intracellular levels, to be related with accumulated GSH, was also monitored. Cells were then suspended in an appropriate solution containing mineral salts, glucose and the aminoacids, precursors of the two studied molecules. According to this procedure, GSH intracellular levels reached $4.6 \% \mathrm{dw}$ after $48 \mathrm{~h}$ incubation. Moreover, GSH levels can be related to SAM production (up to $1.3 \% \mathrm{dw}$ ). The presence, in several samples, of intermediate metabolites, such as cystathionine and omocysteine, proved the establishement of an intracellular equilibrium between GSH and SAM; this behaviour represents a promising starting point for the set-up of a microbial process for the simultaneous production of the two studied molecules.

\section{IB79}

Continuous citric acid production by Yarrowia lipolytica yeast in a membrane reactor W. Rymowicz, M. Robak, A. Rywinska, I. Musial, B. Zarowska Departament of Biotechnology and Food Microbiology, Agricultural University of Wroclaw, Wroclaw 50-375, Poland. Email: rymowicz@ozi.ar.wroc.pl (W. Rymowicz)

Three acetate mutants of $Y$. lipolytica yeasts, which varied in colony morphology (rough and smooth), were employed for continuous citric acid production from glucose and fructose syrup in a membrane reactor with cell recycle. The strains were compared for their product yields, specific acid production rates and ratios of citric acid to isocitric acid. Experiments shoved that glucose syrup was a better substrate for citric acid production by $Y$. lipolytica. Citric acid concentration in the effluent ranged from 80 to $120 \mathrm{~g} / \mathrm{l}$, depending on the yeast strain used. All $Y$. lipolytica strains produced very low amounts of isocitric acid. Its concentration did not exceed $3 \mathrm{~g} / \mathrm{l}$. Based on the results of these experiments, smooth strain AWG-7 was found to be the most suitable for citrate production both from glucose and fructose syrup during long time continuous processes $(500 \mathrm{~h})$. In the steady state, the highest citrate productivity $(1.3 \mathrm{~g} / \mathrm{lh})$ was obtained with this strain, when the feed medium contained $200 \mathrm{~g} / \mathrm{l}$ of glucose and dilution rate (D) was $D=0.0131 / \mathrm{h}$. Supplementation of the feed medium with bacto-pepton improved the productivity, citric acid yield and stability of the continuous process in the cell recycle fermentation system.

\section{IB80}

Dyeing properties of natural indigo derived from Polygonum tinctorium J.-S. Hwang ${ }^{1}$, J.-N. Kim ${ }^{1}$, Y.-J. Wee ${ }^{2}$, H.-W. Ryu ${ }^{2}$, J.-S. Yun ${ }^{3}$, H.-K. Jang ${ }^{4}$, S.-H. Kim ${ }^{4}$ : $^{1}$ Department of Material Chemical and Biochemical Engineering, Gwangju, South Korea; ${ }^{2}$ School of Biological Sciences and Technology, Chonnam National University, Gwangju, South Korea; ${ }^{3}$ BioHelix, South Korea; ${ }^{4}$ Senoco Co., Naju, Jeonnam, South Korea

For textile dyeing with natural dyes, indigo has an almost unique position as the most blue natural dye. Due to the importance of indigo, considerable research has been conducted to replace the chemical synthesis of the dye by an application of biotechnological methods. Therefore, we investigated several characteristics of natural indigo derived from Polygonum tinctorium and its dyeing properties using silk fabrics, such as washing, perspiration, and light fastnesses.

\section{Acknowledgement}

This work was financially supported by Program for Cultivating Graduate Students in Regional Strategic Industry from Korea Industrial Technology Foundation.

\section{IB81}

Optimization of the expression of his-tagged Bacillus subtilis glycine oxidase in Escherichia coli and its use to obtain $\alpha$ keto acids I. Martínez-Martínez, J.D. Lozada-Ramírez, F. GarcíaCarmona, Á. Sánchez-Ferrer Department of Biochemistry and Molecular Biology (A), Faculty of Biology, Universidad de Murcia, E-30071 Murcia, Spain. E-mail: alvaro@um.es (Á.Sánchez-Ferrer)

Glycine oxidase is the product of the yjbR gene of Bacillus subtilis that was predicted by sequence homology to be a flavoprotein similar to sarcosine oxidase. Glycine oxidase catalyzes the oxidative deamination of various primary and secondary amino acids (e.g. sarcosine, $N$-ethylglycine, and glycine) and D-amino acids to form the corresponding $\alpha$-keto acids and hydrogen peroxide. Previous investigations reported on the cloning and production of the glycine oxidase gene in Escherichia coli was up to $1 \mathrm{U} / \mathrm{g}$ cell. The present works has improved the expression of the recombinant His-tagged glycine oxidase by 15 -fold by using pET28a and Rosetta cells under the optimal IPTG, temperature and time of induction. The protein obtained represented $30 \%$ of total soluble proteins in crude extract. The enzyme was purified to near homogeneity using IMAC with a 95\% recovery and with and specific activity of $1.21 \mathrm{U} / \mathrm{mg}$ protein. The enzyme was active towards glycine, sarcosine and different D-amino acids, having in general, a basic $\mathrm{pH}$ optimum. The kinetic parameters were also studied, showing a Km range from 0.3 to $300 \mathrm{mM}$. The enzyme was immobilized, and used to obtain pyruvic acid ( $\alpha$-keto acid) from D-alanine with a good yield.

\section{Acknowledgements}

This work was partially supported by MEC and FEDER (BIO2004-00439), and Fundación Séneca CARM (00842/PI/01).

\section{IB82}

Ionic liquids as media for the biocatalytic preparation of lipophilic derivatives of natural compounds with enhanced antioxidant activity A.C. Polydera ${ }^{1}$, L.D. Tsironis ${ }^{2}$, M.H. Katsoura ${ }^{1}$, F.N. Kolisis ${ }^{3}$ A.D. Tselepis ${ }^{2}$, H. Stamatis $^{1}$ : $^{1}$ Laboratory of Biotechnology, Department of Biological Applications and Technologies, University of Ioannina, 45110 Ioannina, Greece; ${ }^{2}$ Laboratory of Biochemistry, Department of Chemistry, University of Ioannina, 45110 Ioannina, Greece; ${ }^{3}$ Laboratory of Biotechnology, School of Chemical Engineering, Technical University of Athens, 15780 Athens, Greece

The enzymatic synthesis of lipophilic derivatives of various natural antioxidants including flavonoid glycosides, as well as derivatives of cinnamic acid, was performed using various immobilized lipases in ionic liquids such as 1-butyl-3-methylimidazolium tetrafluoroborate (bmim- $\mathrm{BF}_{4}$ ) and 1-butyl-3-methylimidazolium hexafluorophosphate (bmim- $\mathrm{PF}_{6}$ ). The influence of various reaction parameters on the catalytic behavior and the selectivity of lipases was pointed out. A response surface methodology was applied for the optimization of the yield and the productivity of the biocatalytic process. The antioxidant activity of the biocatalytically prepared lipophilic derivatives of natural antioxidants, as expressed on $\mathrm{Cu}^{2+}$-induced oxidation of low-density lipoprotein (LDL) and total serum, was investigated. 


\section{IB83}

Process strategy for reduction of proteolysis in Pichia pastoris fermentations Jan Weegar ${ }^{1}$, John Dahlbacka ${ }^{1}$, Noora Sirén ${ }^{2}$, Niklas von Weymarn ${ }^{2}$, Kaj Fagervik ${ }^{1}$ : ${ }^{1}$ Faculty of Chemical Engineering, Åbo Akademi University, Finland; ${ }^{2}$ Laboratory of Bioprocess Engineering, Helsinki University of Technology, Finland. E-mail: Jan.Weegar@abo.fi (J. Weegar)

The yeast Pichia pastoris is a popular host organism for production of recombinant proteins. It is, however, common that the products are degraded by proteases towards the end of the fermentation, resulting in productivity and purity decreases. Proteolysis of recombinant proteins in P. pastoris fermentations is affected by the temperature and $\mathrm{pH}$ of the growth medium. In this study, it was shown that decreasing the temperature from 30 to $10^{\circ} \mathrm{C}$ during the induction phase effectively prohibited proteolysis. On the other hand, the temperature decrease resulted in a reduced maximal methanol consumption rate, which subsequently resulted in a culture highly sensitive to residual methanol. The temperature was slowly decreased according to a predetermined trajectory. As the temperature reached values below $12{ }^{\circ} \mathrm{C}$, the methanol concentration had to be closely monitored and the substrate feed rate adjusted in order to prohibit methanol poisoning as well as to maintain the culture as a substrate limited fed-batch. Measurement of protease concentrations revealed that proteases were present at $10^{\circ} \mathrm{C}$, but at this temperature the proteolysis rate was evidently effectively reduced. The recombinant protein produced could almost totally be recovered (i.e. high purity) with this process strategy compared to a constant high temperature culture $\left(30^{\circ} \mathrm{C}\right)$ where severe breakdown of the product was observed.

\section{IB84}

Investigations on two enzymes decreasing the yield of 2,5-diketoD-gluconate in Erwinia cypripedii Franz Wuehrer ${ }^{1,2}:{ }^{1}$ Division of Food Biotechnology, Department of Food Sciences and Technology, BOKU, University of Natural Resources and Applied Life Sciences, Vienna A-1190, Muthgasse 18 , Austria; ${ }^{2}$ Research Centre Applied Biocatalysis, Petersgasse 14, A-8010 Graz, Austria. E-mail: franz.wuehrer@boku.ac.at

With the growing of an ecological conscience in the public opinion, more and more industrial processes are analyzed for a possible ecologically beneficial alternative. For the production of ascorbic acid, which is nowadays done by the Reichstein process, a lot of research was done to develop biotechnological alternatives for the synthesis of Reichstein intermediates by enzymatic means, which show some advantages regarding costs and environmentalfriendliness. Besides of two-stage fermentation, our approach is to design a tailor-made organism that produces 2-keto-L-gulonic acid, which is the direct precursor of ascorbic acid from glucose or gluconic acid and which can easily be converted to the final product by conventional methods. Erwinia (Pectobacter) cypripedii, which is a natural producer of 2,5-diketo-D-gluconic acid, was selected as a suitable host for a 2,5-diketo-D-gluconate reductase from Corynebacterium glutamicum. To increase the yield of the desired compound we investigate two 2-keto-reductases in the host organism that diminish the yield of 2-keto-L-gulonate by reducing the compound to L-idonic acid, or by metabolisation of intermediates. These two enzymes were investigated with vari- ous biochemical and molecular biological methods, which will be presented.

\section{IB85}

Overproduction of bioinsecticides by heat and salt stress and control of dissolved oxygen in cheap media of Bacillus Thuringiensis Nabil Zouari, Dhouha Ghribi, Samir Jaoua Laboratoire des Biopesticides, Centre of Biotechnology of Sfax, Tunisia, BP:K, 3038 Sfax, Tunisia

Bioinsecticides based on preparations of spores and insecticidal crystal-proteins (ICPs) produced by the bacterium Bacillus thuringiensis $(\mathrm{Bt})$ proved to be a high tool for fighting some agricultural pests and vectors of diseases. However, the use of Bt preparations as commercial insecticides would be prohibitively expensive because it is not easy to reach cheap overproduction of ICPs during large-scale fermentation. Here, we report possibilities to improve delta-endotoxins production as a consequence of responses of $\mathrm{Bt}$ strains to low levels of heat and salt stress. Each stressor results differently in the improvement of delta-endotoxins production, but both were shown to be most efficient at the beginning or the midexponential phase of the cultures which become resistant at the stationary or the sporulation steps. Heat stress caused increase of $84 \%$ of synthesis yields of the sporulating cells, In contrast, salt caused increase of $25 \%$ of spores counts, corresponding to $28 \%$ toxins production improvement. Combined effects of both stressors lead to toxins production improvement of $66 \%$, yield improvement of $40 \%$. We focused on the overcome of carbon repression catabolite, closely related to oxidative metabolism, by an adequate control of dissolved oxygen in the cheap media we formulated for Bt insecticides production. We showed that an equilibrium between the high density of vegetative cells and their ability to synthesize toxins during their sporulation was necessary to take into account. $40 \%$ increase of ICPs production was reached into 31 fermenter Combination of mutagenesis, heat and salt stress and oxidative metabolism control allowed more than $100 \%$ improvement of delta-endotoxins. These results are of great importance in practical point of view, since high bioinsecticides concentrations could be produced without decrease of the yields of their production.

\section{IB86}

Mechanism and function of the intramembrane-cleaving protease rhomboid Marius Lemberg ${ }^{1}$, Javier Menendez $z^{2}$, Christopher Koth ${ }^{2}$, Matthew Freeman ${ }^{1}{ }^{1} \mathrm{MRC}$ Laboratory of Molecular Biology, Cambridge, UK; ${ }^{2}$ Ontario Center for Structural Proteomics, Toronto, Canada

Rhomboids are a family of intramembrane serine proteases that are widely conserved throughout evolution. Among diverse functions discovered so far, rhomboids participate in intercellular signalling, parasite invasion, membrane dynamics and bacterial quorum sensing, making them potentially valuable therapeutic targets. The identification of physiological substrates and of selective inhibitors will be key towards their evaluation as drug targets. We have developed an in vitro cleavage assay to monitor rhomboid activity in the detergent solubilised state, enabling the first isolation of a highly pure rhomboid with catalytic activity. Analysis of purified mutant proteins suggests that rhomboids use a serine protease catalytic dyad instead of the previously proposed triad, and gives insights into subsidiary functions like ligand binding and water supply. 


\section{Acknowledgement}

This work was supported principally by EMBO and the MRC.

\section{IB87}

Fed-batch cultivation of Haematococcus pluvialis under illumination with LEDs for production of astaxanthin Abdolmajid Lababpour, Tomohisa Katsuda, Shigeo Katoh Department of Molecular Science and Material Engineering, Kobe University, Kobe, Hyogo 657-8501, Japan

Photosynthetic microalga Haematococcus pluvialis is a most promising microorganism for production of astaxanthin, which has powerful antioxidant activity and is used both for human being as a food supplement and cosmetic; as well as animal farming such as salmon and poultry. The deficiency of nutrients in batch culture of $H$. pluvialis decreases the growth of cells and increases the induction of astaxanthin as an induction factor. Therefore, it is impossible to reach to high cell concentrations in batch cultures. In previous experiments, the medium replacement increased the cell concentration, while accumulation of astaxanthin was not induced without other factors. In this work, the effects of fed-batch addition of culture medium on the cell growth and astaxanthin production in $H$. Pluvialis cultures were studied. H. pluvialis was cultivated in $50 \mathrm{~cm}^{3}$ culture medium containing sodium acetate, yeast extract, $\mathrm{L}$-asparagines, $\mathrm{MgCl}_{2} \cdot 6 \mathrm{H}_{2} \mathrm{O}, \mathrm{FeSO}_{4} \cdot 7 \mathrm{H}_{2} \mathrm{O}$ and $\mathrm{CaCl}_{2} \cdot 2 \mathrm{H}_{2} \mathrm{O}$ (pH 6.8). Light was supplied by panels of blue or red LED lamps. The temperature was kept at $20^{\circ} \mathrm{C}$ and the culture was mixed with a magnetic stirrer. In fed-batch cultures of $H$. pluvialis, the cell concentration and production of astaxanthin increased in comparison with those in batch culture. In addition, the operation in feb-batch manner is easier than medium replacement from industrial viewpoints for production of astaxanthin.

\section{IB88}

Studies on isolation and purification lovastatin hydrolase Waldemar Kurek, Dominik Koszelewski, Ryszard Ostaszewski Institute of Organic Chemistry PAS, 01-224 Warsaw, Kasprzaka 44/52, Poland

Simvastatin and similar compounds are wide used as antihypercholesterolemic agents. Simvastatin is obtained by C-methylation of side chain of Lovastatin. This process is not perfect and some unreacted lovastatin is present in the reaction mixture. Simvastatin is separated from lovastatin using fungi Clonostachys compactiuscula. Using of living microbials has some disadvantages such as high cost, difficult product separation from the reaction mixture. We work on overcome these difficulties by separation and immobilization of enzyme that hydrolyses lovastatin ammonium salt in presence of simvastatin ammonium salt. Our results of purification and immobilization Lovastatin hydrolase will be presented.<smiles>CC[C@H](C)C(=O)O[C@H]1C[C@@H](C)C=C2C=C[C@H](C)[C@H](CC[C@H]3CC(O)CC(=O)O3)[C@H]2[C@H]1CC[C@H](C)C(C)C</smiles>

\section{IB89}

Investigation of peptide antibiotics produced by Trichoderma strains isolated from winter wheat rhizosphere A. Szekeres ${ }^{1}$,

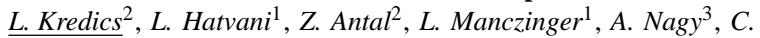
Vágvölgyi $^{1}$ : ${ }^{1}$ Department of Microbiology, University of Szeged, P.O. Box 533, H-6701 Szeged, Hungry; ${ }^{2}$ Hungarian Academy of Sciences, University of Szeged, Microbiological Research Group, Hungry; ${ }^{3}$ Pilze-Nagy Ltd. ,Kecskemét, P.O. Box 407, Hungary. Email: kredics@bio.u-szeged.hu (Laszlo Kredics)

Species of the imperfect filamentous fungal genus Trichoderma with teleomorphs belonging to the Hypocreales order of the Ascomycota division are of great economic importance as sources of enzymes, antibiotics, as plant growth promoters, decomposers of xenobiotics, and as commercial biofungicides. Peptaibols and related Peptaibiotics (PrPs) are secondary metabolites constituting a family of fungal peptide antibiotics which is constantly growing since alamethicin was isolated from cultures of Trichoderma viride. These compounds are linear, amphipathic polypeptides composed of 5-20 amino acids and usually containing several non-proteinogenic amino acid residues, which are representing characteristic building blocks of the structure. One hundred and twenty Trichoderma strains were isolated from roots of winter wheat grown in agricultural fields of southern Hungary. The identity of species was examined based on morphological and molecular characters. The presence of PrPs-producing strains among the isolated Trichoderma strains was detected by biological tests and the antibiotics were partially purified using a multistep chromatography procedure involving exclusion chromatography, adsorption chromatography and thin-layer chromatography. About $20 \%$ of the isolates proved to be able to produce PrPs. The antibacterial activity of the compounds was tested against Staphylococcus aureus, Bacillus subtilis, Micrococcus luteus and Escherichia coli, while the antifungal effect was recorded against Fusarium oxysporum, F. culmorum, Rhizoctonia solani and Pythium debaryanum.

\section{Acknowledgements}

This work was supported by grants F037663 of the Hungarian Scientific Research Fund and grant OMFB-00219/2002 of the Hungarian Ministry of Education.

\section{IB90}

Pyranose dehydrogenase from Agaricus meleagris Roman Kittl $^{1}$, Christoph Sygmund ${ }^{1}$, Magdalena Kujawa ${ }^{1}$, Jindrich Volc ${ }^{2}$, Dietmar Haltrich ${ }^{1}$, Clemens Peterbauer ${ }^{1}{ }^{1}$ Division of Food Biotechnology, Department of Food Sciences and Technology, BOKU, University of Natural Resources and Applied Life Sciences, Muthgasse 18, A-1190 Vienna, Austria; ${ }^{2}$ Institute of Microbiology, Academy of Sciences of the Czech Republic, Videnska 1083, CZ-142 20 Praha 4, Czech Republic

Keto sugars have long been implicated as attractive intermediates or substrates for further chemical or enzymatic reactions, to generate a number of synthetic sugar derivatives and fine chemicals. The quinone-dependent pyranose dehydrogenase (PDH) purified of the basidiomycete fungus Agaricus meleagris catalyzes with high specificity the oxidation of the C-3 of glycosidically bound D-glucose, whereas in contrast it oxidizes simultaneously the C-2 and C-3 of free D-glucose. Considering the broad substrate tolerance, $\mathrm{PDH}$ provides a new convenient tool for high yield production of 3-keto- 
oligosaccharides and 3-keto-glycosides. Availability of the enzyme is, however, hampered by the very slow growth and low production rates of the fungus. Cloning of the encoding gene and production of the protein by heterologous expression are therefore a prerequisite not only for any biotechnological application, but further scientific investigations as well. On the basis of peptide sequences degenerated primers were designed, and the resulting PCR fragment was used as a probe to isolate the gene from a genomic library. Two very similar genes encoding previously uncharacterized proteins were also found, and flanking regions were amplified using RAGE-PCR. Furthermore cDNA clones of all genes were isolated by RT-PCR.

\section{IB91}

Dyeing properties of natural indigo derived from Polygonum tinctorium J.-S. Hwang ${ }^{1}$, J.-N. Kim ${ }^{1}$, Y.-J. Wee ${ }^{2}$, H.-W. Ryu ${ }^{2}$, J.-S. Yun ${ }^{3}$, H.-K. Jang ${ }^{4}$, S.-H. Kim ${ }^{4}:{ }^{1}$ Department of Material Chemical and Biochemical Engineering, Gwangju, South Korea; ${ }^{2}$ School of Biological Sciences and Technology, Chonnam National University, Gwangju, South Korea; ${ }^{3}$ BioHelix, Naju, Jeonnam, South Korea; ${ }^{4}$ Senoco Co., Naju, Jeonnam, South Korea

For textile dyeing with natural dyes, indigo has an almost unique position as the most blue natural dye. Due to the importance of indigo, considerable research has been conducted to replace the chemical synthesis of the dye by an application of biotechnological methods. Therefore, we investigated several characteristics of natural indigo derived from Polygonum tinctorium and its dyeing properties using silk fabrics, such as washing, perspiration, and light fastnesses.

\section{Acknowledgement}

This work was financially supported by Program for Cultivating Graduate Students in Regional Strategic Industry from Korea Industrial Technology Foundation.

\section{IB92}

The behavior of phytate degrading enzymes isolated from Malaysian Zea mays root in rice bran media Anis Shobirin Meor Hussin ${ }^{1}$, Abd-ElAziem Farouk ${ }^{1}$, Hamzah Mohd Salleh ${ }^{1}$, Ralf Greiner ${ }^{2}$ : ${ }^{1}$ Biomolecular Engineering Research Group, Department of Biotechnology Engineering, Kulliyyah of Engineering, International Islamic University Malaysia, Jalan Gombak, 53100 Kuala Lumpur, Malaysia; ${ }^{2}$ Centre for Molecular Biology Federal Research Centre for Nutrition and Foods, Haid-und-Neu-Straße 9, D-76131 Karlsruhe, Germany

Phytate degrading enzymes catalyze the step-wise release of phosphate from phytate, the principle storage form of phosphorus in plant seeds and pollen. They are widespread in nature, occurring in plants and microorganisms, as well as in some animal tissues. Phytate-degrading enzymes have been studied intensively in recent years because of the great interest in such enzymes for phytate degradation and their application for animal feed and human health. From over isolate 140 screened isolates of phytate degrading enzymes, three isolates from the root Malaysian Maize plantation have shown phytate degrading activity. The production of phytate degrading enzyme was studied using different concentrations $[\%, w / v]$ of rice bran media during different stages of cultivation. The dephosphorylation of phosphate from rice bran phytate has shown regulatory effect on the secretion of bacterial phytases. In this conference, we will present data for the characterization of the enzymes.

\section{IB93}

A novel bacterial highly specific phytate-degrading enzyme (PhyFAUIA1) with promising properties for industrial applications Abd-ElAziem Farouk ${ }^{1}$, Ralf Greiner ${ }^{2}$, Hamzah Mohd Salleh $^{1},{ }^{1}$ Biomolecular Engineering Research Group, Department of Biotechnology Engineering, Kulliyyah of Engineering, International Islamic University Malaysia, Jalan Gombak, 53100 Kuala Lumpur, Malaysia; ${ }^{2}$ Federal Research Centre for Nutrition and Foods, Centre for Molecular Biology, Haid-und-Neu-Straße 9, D-76131 Karlsruhe, Germany

In this paper we present the properties of a phytase purified from a bacterium isolated from Malaysian wastewater, which might find application as an animal feed supplement. The phytase described herein is a periplasmic enzyme. The phytase was purified about 180fold to apparent homogeneity using ion-exchange chromatography and gel-filtration with a recovery of $10 \%$ referred to the phytatedegrading activity in the crude extract. The enzyme exhibits an activity of about $1106 \mathrm{U} \mathrm{mg}^{-1}$. Gel filtration of the native enzyme on a calibrated Sephacryl S-200 column gave a molecular mass of the phytase of $42,000 \pm 1500 \mathrm{Da}$ with elution position being measured by determination of enzyme activity. Lower molecular mass species or higher molecular mass aggregates were not observed. The phytase appeared homogeneous by polyacrylamide gel electrophoresis under non-denaturing conditions at $\mathrm{pH} 8.3$ and 4.8 and gave a single protein band upon SDS gel electrophoresis after Coomassie staining of the gels. These results indicate that the phytase could be regarded as homogeneous. The estimated molecular mass after SDS-PAGE indicated that the phytase having a molecular mass of $41,500 \pm 2500 \mathrm{Da}$. Consequently, this enzyme is a monomeric protein. The purified enzyme had a single $\mathrm{pH}$ optimum at $\mathrm{pH} 4.5$ and was virtually inactive above $\mathrm{pH}$ 7.0. At $\mathrm{pH} 3.0,40 \%$ and at $\mathrm{pH} 2.5,20 \%$ of the activity at optimal $\mathrm{pH}$ was observed. The effect on enzyme stability was studied in the $\mathrm{pH}$ range $1.0-9.0$ at $4{ }^{\circ} \mathrm{C}$. Within 14 days the phytase did not lose any activity in the $\mathrm{pH}$ range from 3.0 to 8.0 , but at $\mathrm{pH}$ values below 2.0 a rapid decline in activity was observed. At $\mathrm{pH} 1.5,72 \%$ and at $\mathrm{pH} 9.0,65 \%$ of the initial activity was lost during $24 \mathrm{~h}$. In the range of temperatures studied, $10-80^{\circ} \mathrm{C}$, the optimum temperature for the enzyme was found to be $65^{\circ} \mathrm{C}$. The apparent activation energy was estimated at $\mathrm{pH} 4.5$ from the slope of $\log V_{\max }$ versus $1 / T$. The data showed excellent linearity from 15 to $65^{\circ} \mathrm{C}$. The Arrhenius activation energy for the hydrolysis of phytate was calculated to be $37.5 \mathrm{~kJ} / \mathrm{mol}$. In order to check thermal stability, the purified enzyme was incubated at different temperatures, cooled to $4{ }^{\circ} \mathrm{C}$ and assayed using the standard phytase assay. The enzyme lost no activity in $10 \mathrm{~min}$ at temperatures up to $65^{\circ} \mathrm{C}$. When exposed for $10 \mathrm{~min}$ at $70^{\circ} \mathrm{C}$, it retained $50 \%$ and at $80^{\circ} \mathrm{C} 12 \%$ of the initial activity. In order to determine the substrate selectivity of the purified phytase, several phosphorylated compounds in addition to phytate, were used for $K_{\mathrm{M}}$ and $V_{\max }$ estimation by detecting the release of the phosphate ion during hydrolysis using formation of a soluble phospho-molybdate complex in an acidic water-acetone mixture. Only phytate was identified as a substrate. The kinetic parameters for the hydrolysis of phytate were determined to be $K_{\mathrm{M}}=0.15 \mathrm{mmoll}^{-1}$ and $k_{\text {cat }}=1164 \mathrm{~s}^{-1}$ at pH 4.5 and $37^{\circ} \mathrm{C}$. Like other bacterial phytatedegrading enzymes, the purified enzyme showed substrate inhibition. The activity of the purified enzyme was inhibited at substrate concentrations $>7 \mathrm{mM}$. The study of the effect of metal ions on enzyme 
activity showed that none of them had an activating effect when used at a concentration between $10^{-4}$ and $10^{-3} \mathrm{M} . \mathrm{Mg}^{2+}, \mathrm{Ca}^{2+}, \mathrm{Mn}^{2+}$, $\mathrm{Co}^{2+}, \mathrm{Ag}^{+}, \mathrm{Hg}^{2+}$, and $\mathrm{Cu}^{2+}$ had little or no effect on enzyme activity, while $\mathrm{Zn}^{2+}, \mathrm{Fe}^{2+}$, and $\mathrm{Fe}^{3+}$ showed strong inhibitory effects. The reduced phytate-degrading activity in the presence of $\mathrm{Fe}^{2+}$ and $\mathrm{Fe}^{3+}$ is attributed to a lower phytate concentration in the enzyme assay because of the appearance of a Fe-phytate precipitate. When compounds which tend to chelate metal ions, such as $o$-phenanthroline, EDTA, oxalate, citrate or tartrate, were tested for their effect on enzyme activity, it was noted that none of them was inhibitory at a concentration from $10^{-4}$ to $10^{-3} \mathrm{M}$. Fluoride, a known inhibitor of different phytate-degrading enzymes from bacteria and the hydrolysis product phosphate as well as its structural analogs molybdate, wolframate and vanadate were found to be strong inhibitors of the purified enzyme. Flouride inhibited the hydrolysis of phytate with a $K_{i}$ value of $112 \mu \mathrm{moll}^{-1}$

\section{IB94}

Facilitation of expression and purification of antimicrobial peptide by fusion with baculoviral polyhedrin in Escherichia coli Wei Quande, Young Soo Kim, Jeong Hyun Seo, Hyung Joon Cha Department of Chemical Engineering, Pohang University of Science and Technology, Pohang 790-784, South Korea. E-mail: hjcha@postech.ac.kr (H.J. Cha)

Several fusion strategies have been developed for the expression and purification of small antimicrobial peptides (AMPs) in recombinant bacterial expression systems. In the present work, we investigated the use of the baculoviral polyhedrin (Polh) protein as a novel fusion partner for production of a model AMP (halocidin 18 subunit; Hal18) in Escherichia coli. The recombinant Hal18 AMP could then be hydroxylamine cleaved from the fusion protein and easily recovered by simple dialysis and centrifugation. This was facilitated by the fact that Polh was soluble in the alkaline cleavage reaction but became insoluble during dialysis at a neutral $\mathrm{pH}$. Importantly, recombinant and synthetic Hal18 peptides showed nearly identical antimicrobial activities against E. coli and Staphylococcus aureus, which were used as representative Gram-negative and -positive bacteria, respectively. These results demonstrated that baculoviral Polh can provide an efficient and facile platform for production or functional study of target AMPs.

\section{IB95}

Metabolic engineering of succinic acid production in Saccharomyces cerevisiae D. Cimini, K.R. Patil, G. Lettier, C. Schiraldi, J. Nielsen CMB, BioCentrum-DTU, Technical University of Denmark, DK-2800 Kgs. Lyngby, Denmark. E-mail: dc@biocentrum.dtu.dk (D. Cimini)

Extensive industrial and food additive applications of succinate have attracted much effort towards finding an environment-friendly alternative to the petrochemical production processes. It is very attractive to engineer $S$. cerevisiae for succinate production because of its Generally Regarded As Safe (GRAS) status, ease of genetic manipulation and fermentation. We approached this metabolic engineering problem with a two-step methodology combining modern computational as well as molecular biology tools. In the first step we identified potential metabolic engineering targets leading to high Succinate yield and productivity, with the aid of genome scale metabolic model and a bi-level optimization framework using flux balance analysis and quadratic programming. In the next step, various deletion mutants are being constructed and characterized for physiology and succinate production. Results from these experiments then will be used to improve the predictions in computational models. So far, we have constructed a Saccharomyces cerevisiae mutant deleted in Sdh3, which encodes a major subunit of SDH-complex converting succinate to fumarate in mitochondria. The physiology of $\operatorname{Sdh} 3 \Delta$ mutant has been characterized in aerobic and anaerobic batch cultivations and in glucose limited chemostat at dilution rate as low as $0.027 \mathrm{~h}^{-1}$. In aerobic batch fermentations, the mutant showed reduced maximum specific growth rate as compared to the wild-type, and it was incapable of growing on ethanol as sole carbon source, as predicted from the model. Interestingly, the mutant showed much higher specific growth rate in anaerobic conditions, close to the wildtype strain. Moreover, the chemostat cultivations indicate that the critical dilution rate of the mutant is below $0.027 \mathrm{~h}^{-1}$. This opens further opportunities to investigate interesting behavior of the mutant and the underlying regulatory processes to improve our understanding of yeast mitochondrial metabolism.

\section{IB96}

A modelling study on enzymatic hydrolysis of milk lactose by a sonifier Neslihan Şener, Dilek Kiliç Apar, Belma Kin Özbek Department of Chemical Engineering, Yıldız Technical University, Davutpaşa Campus, 34210 Esenler/Istanbul, Turkey. E-mail: dkilic@yildiz.edu.tr (D.K. Apar)

Lactose is the dominant carbohydrate in milks which are, in turn, the only significant natural sources of lactose. A large number of people do not digest lactose properly due to a lack or inactivity of the intestinal beta-galactosidase and they suffer from intestinal dysfunctions - gas, abdominal pain and diarrhea - if their diet contains lactose. Moreover, lactose is a sugar with a high BOD, low sweetness, low solubility, when compared to the products of its hydrolysis (glucose and galactose) and being a hygroscopic sugar has a strong tendency to adsorb flavours and odours. The hydrolysis of this sugar is very attractive towards the improvement of processes for the production of ice cream and other refrigerated dairy products and it could be very interesting for the development of additives for animal and human alimentation. The enzymatic hydrolysis of lactose is carried out by beta-galactosidases, enzymes that are widely distributed in nature, appearing in micro-organisms, plants and animal tissues. The present investigation describes the effects of the sonication process parameters on enzymatic hydrolysis of milk lactose and enzyme stability. Bandelin Sonopuls Sonicator was used for the lactose hydrolysis experiments. $\beta$-Galactosidase enzyme used is produced from Kluyveromyces marxianus. The reactions were carried out in $250 \mathrm{ml}$ of milk. The process variables for the sonicator are duty cycle, acoustic power and enzyme concentration. The amount of residual lactose concentration $(\mathrm{g} / \mathrm{l})$ and residual enzyme activity (\%) against time were investigated versus process variables. Beside of this; the mathematical models depending on the operating conditions were also derived by using the experimental data of lactose concentration and enzyme activity. 


\section{IB97}

Effect of temperature, ph and viscosity on enzymatic hydrolysis of corn gluten Dilek Kiliç Apar, Belma Kin Özbek Department of Chemical Engineering, Yıldız Technical University, Davutpaşa Campus, 34210 Esenler/Istanbul, Turkey. E-mail: dkilic@yildiz.edu.tr (D.K. Apar)

Over the last decade, the use of plant protein hydrolysates alternative to animal protein hydrolysates in human nutrition has broadly expanded. Protein hydrolysates are often used in different nutritional formulations, such as supplementation of drinks to enhance their nutritional and functional properties, or special medical diets. There are many processes which employ protein hydrolysis and hydrolytic products. Among these processes, the use of enzymes allows selective hydrolysis of protein and produces a potentially safer and more defined material. In the present study, the effect of the temperature, $\mathrm{pH}$ and viscosity on the hydrolysis of corn gluten was investigated using a stirred batch reactor system. The corn gluten was hydrolysed by using Neutrase enzyme, a bacterial protease produced by a selected strain of Bacillus amyloliquefacien. The reactions were carried out in 0.21 of aqueous solutions containing $1 \%(\mathrm{w} / \mathrm{v})$ corn gluten and $0.4 \%(\mathrm{v} / \mathrm{v})$ enzyme. The degree of hydrolysis (\%) and soluble protein concentration depending on the time were investigated at the temperatures $40,45,50,55$, and $60^{\circ} \mathrm{C}$; and at the $\mathrm{pH}$ values $6.5,7$, 7.5 , and 8 . To investigate the effect of viscosity, the various amounts of glycerol was added to the reaction solutions to produce viscosities in the range of $1.415-13.43 \mathrm{cp}$. The degree of hydrolysis (DH) was computed by using $\mathrm{pH}$-stat method. For the soluble protein determination in the hydrolysates samples, the Folin-Lowry method (1951) was used.

\section{IB98}

Production of biopolymers by mixed microbial cultures from propionate and acetate P.C.Lemos, L.S. Serafim, M.A.M. Reis REQUIMTE, Departamento de Química, CQFB, FCT/UNL, 2829-

516 Caparica, Portugal. E-mail: pac@fct.unl.pt (P.C. Lemos)

Polyhydroxyalkanoates (PHA), one of the most promising bioplastics for the partial replacement of synthetic polymers like polypropylene, are polyesters produced by bacteria as intracellular storage reserves of carbon and energy. The industrial production of PHA is achieved by pure cultures in its natural state or using genetically engineered organisms. The main obstacle to the replacement of synthetic plastics by biopolymers is their great cost difference. Research on the field of biopolymers synthesis using mixed cultures and waste organic carbon sources as substrates prove to decrease substantially the production costs of PHA. The optimization of PHA production under aerobic feeding conditions (ADF) was achieved recently in our group, obtaining the highest value of PHA content stored by mixed cultures ( $79.2 \%$ of cell dry weight). In this work only a homopolymer of polyhydroxybutyrate (PHB) was obtained and since it is a highly crystalline and brittle material its application field is limited. The mechanical and thermal properties of PHA can be varied to a great extend by adjusting the monomer composition. The incorporation of different monomeric units, other than $\mathrm{HB}$, in the polymer chain, originates copolymers with improved mechanical properties. Optimization of PHA production from propionate by a mixed culture was studied varying the carbon and ammonia concentrations. Propionate only, acetate alone or a mixture of acetate and propionate were tested. Copolymers of hydroxybutyrate and hydroxyvalerate, $\mathrm{P}(\mathrm{HB} / \mathrm{HV})$, with different compositions were obtained. Consequently polymer properties could be manipulated by feeding the selected volatile fatty acid composition.

\section{IB99}

The pharmaceutically important plant species of Glycyrrhiza sp. (called licorice) is an important commercial product used as a natural sweetener, anti-inflammatory, anti-cancer and anti-diabetic agents. Agrobacterium rhizogenes transformation system was used for the hairy root cultures of licorice G. uralensis. After inoculation of aseptic stem segments the ability of hairy root formation was scored for a period of 6 weeks. Mean transformation frequency ranged from $27 \%$ (for 8196 up to $31 \%$ (for 15834 ). Some transformed genotypes showed significant differences in roots weight, flavonoids and glycyrrhizin (Gl) production. The cotransformation rate of licorice intact explants cultivars with LBA 9402 TL-DNA and the 35S GUS gene showed an average of more than $35 \%$. These obtained root cultures were additionly elicited with extracts of biotic elicitor Acremonium sp. (endomycorhizal fungus), and were used as an in vitro system to metabolites production. The transformed and elicited hairy roots of G.uralensis were obtained by infection of A. rhizogenes 8196 have produced Gl at an yield of $4.5 \%$ dry weight on the period of culture as a 30 days. According to tentative analyses the hairy roots cultures of Glycyrrhiza species produced flavonoids (liquiritigenin and liquiritigen). More high levels ( $3.42 \mathrm{~g} / \mathrm{l})$ of the total flavonoids production have been identificated on the strains which transformed by LBA 9402. This study involved any difference among elicitor treatments and incubation periods for the optimal meabolites production. Clearly, the selection of an effective Agrobacterium strain for the production of transformed root cultures is highly dependent on the plant species, and must be determined empirically.

\section{IB100}

Production, purification and characterization of Scytalidium thermophilum phenol oxidases Didem Sutay ${ }^{1}$, Ufuk Bakir ${ }^{1}$, Zumrut B. Ogel ${ }^{2}:{ }^{1}$ Chemical Engineering Department, Middle East Technical University, Inonu Bulvari, 06531 Ankara, Turkey; ${ }^{2}$ Food Engineering Department, Middle East Technical University, Inonu Bulvari, 06531 Ankara, Turkey. E-mail: ubakir@metu.edu.tr (U. Bakir)

Phenol oxidases (POs) are a group of enzymes which are responsible for oxidation of various phenolic compounds in the presence of molecular oxygen. There are different types of POs present in nature and three major groups of these enzymes are laccases (E.C. 1.10.3.2, p-benzenediol: oxygen oxidoreductase), catechol oxidases (E.C. 1.10.3.1, o-diphenol oxidoreductase) and tyrosinases (E.C. 1.14.18.1, monophenol monooxygenase). Another group of enzymes, peroxidases (E.C. 1.11.1.7), can also be considered as a member of PO family. POs have very wide substrate range and final oxidation products of these substrates are quinones, which are highly reactive molecules and polymerize into brown, red or black waterinsoluble compounds. POs are very common in nature, they can be found in almost all plants, animals and microorganisms. Pigmentation and protection from the environment are main functions of these enzymes. POs have different applications in food, pharmaceutical, textile industries and waste-water treatment systems. The objective of this study was PO production by the thermophilic fungus, Scytalidium thermophilum, purification and characterization of 
the enzyme. For this purpose, enzyme production was performed either in a shaker-incubator or a temperature, $\mathrm{pH}$ and dissolved oxygen controlled $2 \mathrm{~L}$ bioreactor (Probiotem) to optimize enzyme production medium composition and bioreactor parameters. As the carbon and nitrogen sources, $4 \%$ glucose and $0.4 \%$ yeast extract were determined as the optimal concentrations, respectively. Copper, gallic acid and tannic acid were determined to increase enzyme production. Purification was performed by using membrane and chromatographic techniques. Hydrophobic, ion exchange and gel filtration columns were used by using a FPLC system; Äkta Prime (Amersham Biosciences). Especially the Phenyl Sepharose ${ }^{\mathrm{TM}}$ High Performance column appeared to be very efficient for PO purification from Scytalidium thermophilum. Purified PO have been characterized by electrophoretic techniques and kinetic studies.

\section{IB101}

Isolation of lipolytic microorganisms from subtropical soils. cloning, purification and characterization of a novel esterase from strain Pseudomonas sp. CR-611 Núria Prim, Cristian Ruiz, Cristina Bofill, F.I. Javier Pastor, Pilar Diaz Department Microbiology, University of Barcelona. Av. Diagonal 645, 08028-Barcelona, Spain. E-mail: nprim@ub.edu (N. Prim)

Microorganisms or their enzymes are used in a wide range of biotechnological activities such as polymer hydrolysis, synthesis of added-value compounds, sample decontamination, etc. Thus, there is an increasing interest for isolating new enzymes and new enzymeproducing organisms for their use in industrial conversions (Cherry and Fidantsef, 2003). Among these enzymes, lipases, esterases, cellulases, xylanases and pectinases play an important role in many biotechnological processes like those related to pulp and paper processing. Three samples of subtropical forest soil from Puerto Iguazú (Argentina) were used for the isolation of autoctonous microorganisms growing in an organic matter-rich environment. A total of 724 pure cultures of bacteria and fungi were obtained and their hydrolytic activities on polysaccharide and lipidic substrates were assayed using olive oil, tributyrin, cholesterol esters, xylan, cellulose and pectin as substrates. Among the isolates analysed, 449 were active on one or more of the substrates evaluated, and 43 of them degraded all substrates. Nearly half of the strains displayed lipolytic activity, whereas the number of strains active on xylan, cellulose, pectin and cholesterol esters, was much lower. The 76 strains bearing the highest hydrolytic activities were selected and stored for further characterization (Ruiz et al., 2005). Among them, strain CR-611, one of the most active isolates on tributyrin, was selected for identification and characterization of its lipolytic system. Lipolytic strain CR-611 was identified by morphological, physiological and phylogenetic tests, as a Pseudomonas sp., closely related to $P$. fluorescens. SDS-PAGE and zymogram analysis (Diaz et al., 1999) of cell extracts and supernatants from the strain revealed a complex lipolytic system consisting of at least two lipolytic enzymes. Sequence alignment and clustering of previously described Pseudomonas lipases and esterases allowed the design of different sets of primers for the isolation of the lipase/esterase coding genes. A gene coding for an esterase with homology to family VI bacterial lipases (Arpigny and Jaeger, 1999) was isolated and cloned in Escherichia coli. The cloned enzyme was further purified and characterized, showing preference for short fatty acid esters and displaying a typical Michaelis-Menten kinetics, with no interfacial activation. The substrate profile, together with the kinetic behaviour and sequence similarity of the cloned enzyme to family VI bacterial esterases, allowed to identify this enzyme as an esterase and was named EstA6. Maximum activity was achieved on MUF-butyrate at $55^{\circ} \mathrm{C}$ and $\mathrm{pH} 8.5$, suggesting that it could be of interest for biotechnological purposes.

\section{Acknowledgments}

This work was partially financed by the Scientific and Technological Research Council (CICYT, Spain), grant REN2001-3224, by the III Pla de Recerca de Catalunya (Generalitat de Catalunya), grant 2001SGR-00143, and by the Generalitat de Catalunya to the "Centre de Referència en Biotecnologia" (CeRBa).

\section{References}

Arpigny, J.L., Jaeger, K.E., 1999. Biochem. J. 343, 177-183.

Cherry, J.R., Fidantsef, A.L., 2003. Curr. Opi. Biotechnol. 14, 438-443.

Diaz, P., Prim, N., Pastor, F.I.J., 1999. BioTechniques 27, 696-700.

Ruiz, C., Pastor, F.I.J., Diaz, P., 2005. Lett. Appl. Microbiol. 40, 218-227.

\section{IB102}

Optimization of oxygen supply in microbial xylitol production

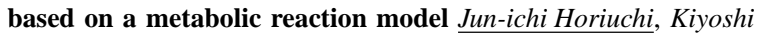
Tada Bioprocess Engineering Laboratory, Department of Chemical System Engineering, Kitami Institute of Technology, Hokkaido 090-8507, Japan. E-mail: horiuchi@betta.chem.kitami-it.ac.jp (J.-i. Horiuchi)

Microbial xylitol production from agricultural wastes has recently attracted much attention from industries because it has potentials to realize the cheaper production of xylitol with low environmental impact (Tada et al., 2004). In order to realize the effective xylitol production by a xylose utilizing yeast, the oxygen supply is a key for maximizing xylitol yield over consumed xylose $\left(\mathrm{Y}_{\mathrm{xl}}\right)$ because the intracellular xylitol metabolism is strongly influenced by the amount of available oxygen. In the present work, we tried to apply a metabolic reaction model in order to determine the optimal oxygen transfer rate (OTR) in a fermentor for maximizing xylitol yield. Corn cob hydrolysates containing $25 \mathrm{~g}$-xylose/L was employed as medium for xylitol production by computer-controlled batch cultures using Candida magnoliae (FERM P-16522, AIST). A metabolic reaction model considering main xylitol metabolisms including glycolysis, pentose-phosphate pathway, TCA cycle and cell synthesis was developed. The model allows to estimate various intracellular metabolic flux distributions including a xylitol production rate. The oxygen uptake rate to maximize the ratio of xylitol production rate over xylose consumption rate corresponds to the OTR condition to maximize a xylitol yield over xylose consumed. Based on the metabolic reaction model, the OTR was optimized by a linear programming, the optimal OTR and the maximum xylitol yield were estimated as $0.5 \mathrm{mmol} \mathrm{O}_{2} / \mathrm{Lh}$ and $0.81 \mathrm{~g}$-xylitol/g-xylose, respectively. The experimental verification using the optimal OTR demonstrated that the xylitol yield was greatly improved to $0.75 \mathrm{~g}$-xylitol/g-xylose from $0.6 \mathrm{~g}$-xylitol/g-xylose in our previous study. 


\section{Reference}

Tada, K., Horiuchi, J.-i., Kanno, T., Kobayashi, M., 2004. Microbial xylitol production from corn cobs using Candida magnoliae. J. Biosci. Bioeng. 98 (3), 228-230.

\section{IB103}

Expression of a bacterial sugar phosphate transporter in $S$. cerevisiae to release L-glycerol 3-phosphate accumulated by metabolic engineering Almut Popp, Huyen Thi Thanh Nguyen, Ulf Stahl, Elke Nevoigt Department of Microbiology and Genetics, University of Technology, 13353 Berlin, Germany. E-mail: a.popp@lb.tu-berlin.de (A. Popp)

In contrast to glycerol, its phosphorylated precursor L-glycerol-3phosphate (L-G3P) is retained by the plasma membrane. Therefore, engineered yeast strains accumulate L-G3P in the cytosol resulting in low overall yield of the desired product and laborious downstream processing. A suitable sugar phosphate transporter in the yeast plasma membrane would overcome these limitations. The glycerol3-phosphate transporter (GlpT) of E.coli is an antiporter and naturally mediates the uptake of L-G3P in exchange with inorganic phosphate. We assume that this transporter would also mediate the excretion of accumulated L-G3P into a phosphate-rich medium if it was present in the plasma membrane of engineered yeast. Despite many inconsistencies in codon usage, we were able to express the bacterial GlpT gene in yeast. Expression was monitored by a c-Myc tag added to the $\mathrm{C}$ - or $\mathrm{N}$-terminal hydrophilic tail, respectively. The quantity of the construct with N-terminal tag clearly exceeds the quantity of the construct with C-terminal tag. Both gene products are located in the endoplasmic reticulum, as shown by immunofluorescence microscopy. Obviously, yeast's transmembrane protein sorting machinery does not recognise it as a substrate for the secretory pathway.

\section{IB105}

Metabolic flux analysis of $\mathbf{C}$ - and $\mathbf{P}$-limited shikimic acid producing E. coli Gaspard Lequeux ${ }^{1}$, Louise Johansson ${ }^{2}$, Jo Maertens ${ }^{1}$, Peter Vanrolleghem ${ }^{1}$, Gunnar Lidén ${ }^{2}$ : ${ }^{1}$ BIOMATH, Ghent University, Coupure Links 653, 9000 Gent, Belgium; ${ }^{2}$ Department of Chemical Engineering, Lund University, P.O. Box 124, 22100 Lund, Sweden. E-mail: Gaspard.Lequeux @ biomath.ugent.be (G. Lequeux)

Metabolic flux analysis (MFA) was applied to decipher why Plimited E. coli fermentations are more optimal for shikimic acid production in comparison with glucose-limited fermentations. As MFA allows obtaining insight in the intracellular flux distribution over different pathways by only measuring net production and consumption rates of metabolites, under the condition that parallell pathways are removed. To this end a detailed metabolic network model was created and checked for consistency, dead-ends, and parallell pathways. Several fermentations were performed at different dilution rates (ranging from 0.05 to $0.3 \mathrm{~h}^{-1}$ ) and different limitations (phosphate and glucose). The E. coli strain used was W3110 with genetic modifications in the aromatic amino acid pathway to enhance shikimic acid production. For each dilution rate, a metabolic model was solved. This way, the evolution of each flux can be followed with respect to the dilution rate. The P-limited cultures showed a better yield which can be explained by the diminished excretion of dehydro-shikimic acid (as is known from literature) and a reduction of the hydrolysis of ATP.

\section{IB106}

Transcript levels of alternative oxidase gene (aoxl) under the conditions of citric acid production in Aspergillus niger Takasumi Hattori, Kuniki Kino, Kohtaro Kirimura Department of Applied Chemistry, School of Science and Engineering, Waseda University, Tokyo, Japan. E-mail: takasumi@suou.waseda.jp (T. Hattori)

Alternative oxidase is a terminal oxidase in respiration chain, which is a branched chain of cytochrome pathway, and inhibited by salicylhydroxamic acid (SHAM), but not by cyanide. The citric acid-producing fungus Aspergillus niger WU-2223L has a cyanide-insensitive and SHAM-sensitive respiration catalyzed by the alternative oxidase (Kirimure et al., 1999) and did not produce citric acid when cultivated with SHAM (Kirimure et al., 2000). In this study, the transcript levels of alternative oxidase gene (aoxl) (Kirimure et al., 1999) and activities of alternative oxidase under the conditions of citric acid production were examined during 9 days-cultivation. The amount of aoxl mRNA was determined by Northern blot analysis, and the specific activity of alternative oxidase as that of duroquinol oxidase. The transcript level and the activity were highest at 2 days at log-phase, decreased during 2 and 4 days, and thereafter maintained at low levels. However, the transcript and alternative oxidase activity was constitutively detected during whole the cultivation periods under the conditions of citric acid production. The sequence analysis of aoxl chromosomal DNA revealed the presence of potential binding site of cyclic AMP responsive element (CRE), stress responsive element (STRE) and heat shock factor (HSF) in its upstream region. These results indicated that the expression of alternative oxidase was regulated in the transcription level and alternative oxidase contributes as the main respiration chain during citric acid production. Transcription level?669 Transcript level?754 Transcription? Gene transcription? Expression?683,000 expression analysis?3300 transcriptional analysis?791 transcription analysis?321 Transcription level levelは・・・段階というのがおおうく使用例が少ない。China, Ukrinaとか・・?

\section{References}

Kirimura, K., et al., 1999. Curr. Genet. 34, 472-477.

Kirimura, K., et al., 2000. Biosci. Biotechnol. Biochem. 64, 2034-2039.

\section{IB107}

RNA-mediated gene silencing in Trichoderma harzianum Sonia Sousa $^{1}$, Jesús Delgado-Jarana ${ }^{1}$, Antonio Llobell ${ }^{1}$, Manuel Rey ${ }^{2}:{ }^{1}$ Instituto de Bioquímica Vegetal y Fotosíntesis, Av. Américo Vespucio, 49, 41092 Seville, Spain; ${ }^{2}$ Newbiotechnic S.A., Parque Industrial A-49 (PIBO), 41110 Bollullos de la Mitación, Seville, Spain. E-mail: sousa@ibvf.csic.es (S.S. González)

Trichoderma strains are considered to be among the most useful fungi in industrial enzyme production, agriculture and bioremediation. Metabolic versatility displayed by these fungi makes it a very amenable source of new gene products. Functional analysis of candidate genes goes by two complementary ways: gene overexpression and loss-of-fuction mutants generation. A few expression systems are available mainly to direct constitutive gene expression in catabolite repression conditions. Following a genomic approach, we have recently cloned some gene promoters with high expression in glucose in Trichoderma harzianum CECT2413. A main goal in a gene 
functional analysis is the generation of knock-out mutants. Up to date, there is no reference about successful gene disruptions in $T$. harzianum mainly due to a very low homolog recombination frequency. RNA-mediated gene silencing has been shown as an efficient tool to diminish or totally abolish specific gene expression. Especially those strategies based on the use of hairpin constructs allow rapid and easy generation of strains with reduced levels of mRNA from genes of interest. We have used the T. harzianum CECT2413 $t s s 1$ promoter to direct the expression of a hairpin DNA construct that induced the appearance of small-interfering RNAs (siRNAs) and the silencing of the uidA reporter gene in a previous uidA overexpressing strain. Reduced levels of mRNA and GUS activity correlated with the presence of siRNAs. This is the first report on RNA-mediated gene silencing in Trichoderma and constitutes a useful and promising tool for functional genomic studies in fungal systems.

\section{IB108}

Analysis of the metabolic response of Escherichia coli to quantitative modulations of the glucose-6-phosphate dehydrogenase based on ${ }^{13} \mathrm{C}$-labelling experiments Cécile Nicolas, Fabien Létisse, Stéphane Massou, Philippe Soucaille, Jean-Charles Portais. E-mail: fabien.letisse@insa-toulouse.fr (F. Létisse)

Microorganisms have an efficient capacity for adapting their metabolism in response to genetic or environmental changes, and understanding metabolic robustness has become an emergent issue. Part of the robustness originates from the network organization of metabolic systems, where the interplay between all available biochemical reactions provides alternative mechanisms for compensating the perturbations. Recently, ${ }^{13} \mathrm{C}$-metabolic flux analysis $\left({ }^{13} \mathrm{C}\right.$-MFA) has been applied to Escherichia coli knock-out mutants lacking key enzymes to determine the phenotypic effects of structural changes in the metabolic network, providing further evidences for compensatory phenomena. The aim of our on-going work is to understand how the central metabolism in E. coli responds to quantitative alterations at a specific key-point of the metabolic network. The glucose-6-phosphate dehydrogenase (G6PdH), a key enzyme in the central metabolism for which the effects of deleting the gene (zwf) has been already described (Zhao et al., 2004), was chosen as the target. To this aim we have generated a set of expression mutants, i.e. mutants having each a fixed level of expression of the $z w f$ gene. Four different levels of expression, leading respectively to G6PdH activity 2; 2.9; 5.7 and 14 times higher than in the WT strain, have been obtained. For each mutant, transcriptomics analysis will be carried out and compared to both the zwf- and WT strains to detect changes in the network structure, and the distribution of fluxes will be measured using ${ }^{13} \mathrm{C}$-MFA. The flux maps obtained for the various strains will be compared to evaluate the quantitative response of the central metabolic network to imposed and increased G6PdH activity. Metabolic control analysis will be applied to provide insights onto the sensitivity of the measurable metabolic fluxes to G6PdH activity. Combination of transcriptomics and fluxomics approaches will provide information on the nature and extent of the compensatory mechanisms. Because the activity of a single enzyme is tuned at different levels in knock-out and expression mutants, this investigation provides a situation that mimics gene-level regulation of metabolism.

\section{IB109}

Fuel ethanol production with entrapped biosystems RebrošMartin, Rosenberg Michal Department of Biochemical Technology, Slovak University of Technology, Bratislava 812 37, Slovakia. E-mail: martin.rebros@stuba.sk (M. Rebroš)

With the depletion of the world's petroleum supply, there has been an increasing worldwide interest in ethanol as an alternative, nonpetroleum source of energy. This fact caused increased interest in the new ethanol technology fermentation process research. As reported before, bacteria Zymomonas mobilis possesses more advantages than Saccharomyces cerevisiae, microorganism used for ethanol production in industrial scale. For that reason, we have focused on the fermentation studies of this facultative bacterium in free and immobilized form. The immobilization of the cells into polyvinylalcohol (PVA) hydrogel lens-shaped capsules LentiKats, improved the batch fermentation process efficiency nine times. Starch, the substrate considered as one of the best of renewable energy source is considered as fuel ethanol feedstock. Due to Z. mobilis disability of maltose, maltotriose and dextrin utilization, the starch has to be converted into glucose monomers. This pre-fermentation step can overcharged whole ethanol production process. This ineffective part of the process was resolved with immobilization of glucoamylase into LentiKats. The system with immobilized enzyme and cell was stabile in continuous mode for 60 days without any significant change in the system efficiency. The combination of cell and enzyme immobilization can significantly improve the efficiency and the cost of ethanol production in industrial scale.

\section{Acknowledgements}

This work was supported by MEGA a.s. (Czech Republic) (www.mega.cz) and following VEGA Grants: 1/2391/05 and $1 / 2390 / 05$.

\section{IB110}

Fermentation of an inhibitory dilute acid-hydrolysate from spruce using a fed-batch procedure combined with cell-reuse Andreas Rudolf, Gunnar Lidén Department of Chemical Engineering, Box 124, Lund University, SE-221 00 Lund, Sweden. E-mail: Andreas.rudolf@chemeng.lth.se (A. Rudolf)

A well-controlled addition of hydrolysate to the fermentation has proved very efficient in reducing yeast inhibition due to compounds formed during lignocellulose hydrolysis. Furthermore, using high cell mass concentrations has been another way of avoiding the negative impact of the inhibitory compounds. If possible, the yeast should therefore be re-used in the process. In the present work a dilute-acid hydrolysate from spruce was fermented using a fed-batch procedure with reutilization of yeast. The fermentation procedure worked satisfactorily, with more than $98 \%$ of fermentable sugars consumed in each of the four consecutive fed-batch fermentations performed. The ethanol yields on fermentable sugars reached $0.45 \mathrm{~g} / \mathrm{g}$. There was continued cell growth in the repeated fed-batch experiments, with an average cell yield on fermentable sugars of $0.06 \mathrm{~g} / \mathrm{g}$. In contrast, only about $20 \%$ of the fermentable sugars were consumed within $24 \mathrm{~h}$, when the fermentation of the hydrolysate was run in a batch process. The work shows the potential to re-use the yeast in a suitably designed process.

\section{Reference}

Zhao, J., et al., 2004, Metab. Eng., 6, 164. 


\section{IB111}

Metabolic engineering as a business process reengineering endeavour H. Aleem, F. Mavituna School of MACE, School of Chemical Engineering and Analytical Science, University of Manchester, Manchester M60 1QD, UK. E-mail: h.aleem@manchester.ac.uk (H. Aleem)

Metabolic engineering is defined by Bailey in his seminal 1991 paper as "the improvement of cellular activities by manipulation of enzymatic, transport, and regulatory functions of the cell with the use of recombinant DNA technology". The manipulation of these functions ultimately results in the manipulation of metabolism, which is the purpose of many biotechnological processes. Metabolic engineering has sought its methods and tools in mathematics and the physical sciences, and later in Information Technology (IT) leading to the proliferation of Bioinformatics. In this work we propose a novel approach to metabolic engineering that regards it as a Business Process Reengineering (BPR) endeavour. Hammer and Champy in their celebrated 1993 book define BPR as "the fundamental rethinking and radical redesign of business processes to achieve dramatic improvements in critical contemporary measures of performance, such as cost, quality, service and speed". Our thesis is that metabolic engineering with its goal of reengineering the metabolism of the microorganism, is equivalent to business process reengineering (BPR) in business and management. Indeed this is essentially what metabolic engineering does to the cell through the use of recombinant DNA technology, which can be viewed as a radical redesign of the metabolic process. After all, it causes changes that cannot be attained otherwise, and whose purpose is to achieve dramatic improvements in cellular activities such as the increase in production of some metabolites by orders of magnitude. The cost incurred by the process, which is metabolism in this case, can be, for example, energy requirements or change to a cheaper substrate. In this study we elucidate this parallelism between the two with emphasis on modelling of metabolism and how the concepts of business process modelling can be applied to it. The purpose is not to produce a model, but rather to introduce the modelling methodology and demonstrate its utilisation and benefits and outline its limitations and challenges. We believe that the novelty of our work lies in applying a new paradigm in approaching metabolic engineering that has not been considered previously.

\section{IB112}

Thermophilic ethanol production from wheat straw hydrolysate in continuous culture Tania I. Georgieva, Birgitte K. Ahring BioCentrum-DTU, Technical University of Denmark, Building 227, DK-2800 Lyngby, Denmark. E-mail: tig@biocentrum.dtu.dk (T. Georgieva)

Ethanol production from lignocellulosic biomass has attracted widespread attention as an unlimited low cost renewable source of energy to transportation fuels due to increasing petroleum use and air pollution towards greenhouse gases. Wheat straw available as agricultural residue has been considered as a potential lignocellulosic substrate for industrial bioethanol production. A major technical obstacle to commercialize bioethanol production form lignocellulose (such as wheat straw) is associated with a lack of microorganism able to rapidly and efficiently ferment both hexose and pentose sugars into ethanol and to tolerate the inhibitors present in undetoxified hydrolysates. Currently used industrial mesophilic microorganisms
(Saccharomyces cerevisiae and Zymomonas mobilis) are excellent ethanol producer from glucose, however, they are not able to ferment other sugars such as xylose, which is the second most abundant sugar in lignocellulose. Thermophilic anaerobic bacteria have been considered for ethanol production from lignocellulosic biomass as an alternative to mesophilic ethanol producing strains, predominantly because of their abilities naturally to ferment the whole diversity of sugars found in lignocellulosic biomass. An increase attention to thermophilies for ethanol production have also arise from broad spectrum of advantages regarding industrial scale ethanol fermentation such as high growth and metabolic rates, low oxygen solubility, reduced risk of reactor contamination, and cost savings via mixing, cooling and facilitated product recovery. In addition, simultaneous co-fermentation of glucose and xylose in a single operation unit could substantially reduced the ethanol production cost. Research has been attempted to study the potential of using a thermophilic anaerobic bacterium for continuous ethanol fermentation of lignocellulosic biomass, with particular emphasis on effectiveness of our strain to ferment undetoxified wet oxidized wheat straw hydrolysate with respect to sugar (glucose and xylose) conversion and ethanol yield. The experiment was carried out in a lab-scale reactor operated at $70^{\circ} \mathrm{C}$ with wheat straw hydrolysate as a substrate in concentrations from 20 to $80 \mathrm{wt} . \%$ equivalent to total sugar mixture of $11-38 \mathrm{~g} / \mathrm{l}$. Wheat straw hydrolysate $(\mathrm{WOH})[200 \mathrm{~g} / 1$ wheat straw, $92.4 \%$ dry matter (DM)] was prepared using wet oxidation pretreatment process followed by enzymatic saccharification with commercial enzymes mixture of Celluclast1.5, and Novozym188 (Novozymes, Denmark). Both xylose and glucose sugars were simultaneously converted to ethanol. The sugar utilization was higher than $90 \%$, and high ethanol yields were achieved. Reactor shows good long-term performance (124 days) in terms of operation stability and reactor contamination.

\section{IB113}

Maltotriose utilization in lager strains: MTT1 encodes a maltotriose transporter J. Dietvorst ${ }^{1}$, J. Londesborough ${ }^{2}$, H.Y. Steensma ${ }^{1,3}:{ }^{1}$ Institute of Biology Leiden, Leiden University, Wassenaarseweg 64, 2333 AL Leiden, The Netherlands; ${ }^{2}$ VTT Biotechnology, P.O. Box 1500, FIN-02044, Finland; ${ }^{3}$ Department of Biotechnology, Delft University of Technology, Julianalaan 67, 2628 BC Delft, The Netherlands. E-mail: dietvorst@rulbim.leidenuniv.nl (J. Dietvorst)

Maltotriose is the second most abundant fermentable sugar in wort and due to incomplete fermentation, residual maltotriose in beer may cause problems in the brewing industry. To study genes that might improve utilization of maltotriose we used a library with DNA from brewer's strains and a laboratory strain and identified a new transporter encoded by MTT1. MTT1 gave lager strain A15 the ability to grow on $\mathrm{YP} / 2 \%$ maltotriose in the presence of $3 \mathrm{mg} / \mathrm{l}$ of the respiratory inhibitor antimycin A. This transporter gene shares $74 \%$ similarity with $M P H 2$ and $M P H 3,62 \%$ similarity with $A G T 1$ and $91 \%$ similarity with $M A L 61$ and MAL31. Moreover, MTT1 shares even higher similarity (98\%) with the S. pastorianus mtyl gene (M. Salema-Oom, unpublished, NCBI Accession number AJ491328). Purified radiolabeled maltotriose and radiolabeled maltose were used to study sugar uptake of lager strains A15 and WS34/70, and of A15 containing MTT1 or MTT1alt, a more efficient, altered version of this gene lacking the 66 basepairs from the $3^{\prime}$ end and containing 57 base- 
pairs of vector sequences. These transport studies show that MTT1 and, especially, MTT1alt encode maltose transporters with relatively high activity towards maltotriose compared to maltose. This study is part of a multi-disciplinary project, funded by the European Union (contract no. QLK1-CT-2001-01066) focusing on the development of high-gravity resistant brewer's yeast strains.

\section{IB114}

Metabolic engineering of L-phenylalanine pathway in Bacillus subtilis Yasemin Demirci ${ }^{1}$, Pınar Çalık ${ }^{2}$, Güzide Çalık ${ }^{1}$, Tunçer H. Özdamar ${ }^{1}$ : ${ }^{1}$ BRE Laboratory, Department of Chemical Engineering, Ankara University, 06100 Ankara, Turkey; ${ }^{2}$ IBLab, Department of Chemical Engineering, METU, 06531 Ankara, Turkey. E-mail: ozdamar@eng.ankara.edu.tr (T.H. Özdamar)

Metabolic engineering design of a recombinant L-phenylalanine (Phe) production system is based on coordinated overexpression of the flux-controlling genes in the aromatic-amino acid pathway. Based on the insights gained by the work carried out in our laboratories (Özçelik et al., 2004), aroH for the reaction R96 at the branch-point chorismate that connects the preceding reactions of the aromatic group amino acid pathway to the proceeding reactions towards Phe, was predicted as the first-, and aroA for DAHP synthase (R89) predicted as second-metabolic engineering sites. aroA gene was cloned next to $\operatorname{aroH}$, by the use of four primers designed using PCR-based gene splicing by overlap extension method. The genes were amplified separately by PCRs. The primers used at the ends to be joined were designed as complementary to one another by including nucleotides at their $5^{\prime}$ ends that are complementary to the $3^{\prime}$ portion of the other primer. These products were mixed in the next PCR reaction, where one strand from each fragment contains the overlap sequence at $3^{\prime}$ end. Extension of this overlap by DNA polymerase has yielded the recombinant two-gene product; and the two-gene product serve as template for the continuation of reactions for the increase of the concentration in the micro-reactors. The two-gene fragment was first cloned into pUC19, and then sub-cloned to pMK4 E. coli-Bacillus shuttle plasmid. The new expression vector with high stability and high copy-number was obtained and transferred into host $B$. subtilis. The metabolic flux distributions calculated by the mass balance based stoichiometric model based on the metabolic reaction network for $\mathrm{r}$ B.subtilis were determined by using time profiles of the substrate, dry-cell, Phe and other amino acids, and organic acids concentrations as the constraints. On the bases of calculated intracellular fluxes of recombinant $B$. subtilis carrying pMK4::aroA::aroH, an in-depth analyses of the metabolic engineering design will be presented.

\section{Reference}

Özçelik, İ., Çalık, P., Çalık, G., Özdamar,T.H., 2004. Metabolic engineering of aromatic group amino acid pathway in Bacillus subtilis for L-phenylalanine production. Chem. Eng. Sci. 59 (22-23), 5019-5026.

\section{IB115}

Optimization of ethanol production from starch using respiratory - deficient recombinant Saccharomyces cerevisiae Ebru Toksoy Öner ${ }^{1}$, Stephen G. Oliver ${ }^{2}$, Betül Kırdar ${ }^{3}:{ }^{1}$ Department of Chemical Engineering, Marmara University, Goztepe 34722 Istanbul, Turkey; ${ }^{2}$ School of Biological Sciences, University of Manchester, 2.205 Stopford Building, Oxford Road, Manchester M13 9PT, UK; ${ }^{3}$ Department of Chemical Engineering, Boğaziçi University, Bebek 80815 Istanbul, Turkey. E-mail: etoksoy@eng.marmara.edu.tr (E.T. Öner)

The $100 \%$ respiratory-deficient nuclear petite amylolytic Saccharomyces cerevisiae NPB-G strain capable of excreting a hybrid protein possessing both $\alpha$-amylase and glucoamylase enzyme activities was generated and its employment for direct fermentation of starch into ethanol was investigated under both shake flask and controlled bioreactor cultivation conditions. When compared with a standard host strain, higher ethanol concentrations and yields were achieved with the nuclear petite strain under both cultivation conditions. Further improvement in ethanol production was achieved by the use of an initial glucose supplement. Comparison of the ethanol fermentation performances of the respiratory-deficient NPB$\mathrm{G}$ and the parental respiratory-sufficient WTPB-G strain showed an increase of, ca. $48 \%$ in both ethanol production yields and ethanol productivities with the respiratory-deficient strain. Response surface methodology (RSM) was used as a statistical tool to optimize the initial yeast extract and starch contents of the medium, which resulted in a substantial increase in the stability of the expression plasmid in both strains with concomitant improvement in their amylolytic potentials. High ethanol yields on substrate values of the bioreactor cultures, that were very close to the theoretical yield, indicated that the amylolytic respiratory-deficient strain developed in this study was very effective in the direct fermentation of starch into ethanol.

\section{IB116}

Establishing a biotechnology educational framework to support a knowledge-based economy in Puerto Rico Rosa Buxeda, Lorenzo Saliceti-Piazza Industrial Biotechnology Program, University of Puerto Rico, Mayagüez Campus, Mayaguez 00680, Puerto Rico. E-mail: rbuxeda@uprm.edu (R. Buxeda)

Industrial Biotechnology has been identified as a major thrust area of economic development within the past five years for the island of Puerto Rico. The portfolio of biotechnology manufacturing investments in the island has passed the two billion dollar mark. World known companies like Amgen, Abbott and Eli-Lilly lead these investments, which have catalyzed a strong technology transfer to the island. A strong collaboration between industry and academia was needed to provide a well-trained workforce for these company startups. As a result, the University of Puerto Rico, Mayagüez Campus (UPR-M) developed four initiatives which are part of the educational pipeline in biotechnology. These are: (i) an Industrial Biotechnology Program, a 5-year BS degree which contains a novel curriculum with courses from science and engineering with undergraduate research and industrial internships as part of the degree requirements; (ii) an Industrial Biotechnology Learning Center, which provides customized biotechnology and bioprocessing training modules, including lectures and hands-on experiences to train and develop the workforce needed in the biotechnology manufacturing plants; (iii) 
a Biotechnology Summer Camp, which addresses the high school student population and its main purpose is to educate and advise on the different career paths that can be followed in the field of biotechnology; and (iv) a Biotechnology Center for Research and Training in Bioprocessing, that will address the development of corporatesponsored research projects to strengthen links between industry and academia in order to build up a knowledge-based economy. Our paper will describe each initiative in detail as well as its outcomes and impact on Puerto Rico's knowledge-based economy goals.

\section{IB117}

Isolation of acid phosphatase from sweet potato and immobilization using different adsorbent D. Omay, Y. Güvenilir, N. Deveci Istanbul Technical University, Department of Chemical Engineering, Maslak 34769, Istanbul, Turkey. E-mail: omayd@itu.edu.tr (D. Omay)

Phosphatase enzymes occur in a wide range of plant and animal tissues. They catalyze the hydrolysis of phosphate bonds in organic phosphates, between the phosphate group and rest of the molecule. Immobilization of enzymes and biological compounds is currently gaining importance due to its wide variety of applications in the food and pharmaceutical industries and also its biomedical applications. It was reported that enzymes can be activated by complexation with polysaccharides such as chitin or chitosan. The aim of this experimental study was determined as partial purification and isolation of acid phosphatase enzyme and its immobilization. The purification was realized by applying centrifugation, ammonium sulfate precipitation and dialysis respectively. The specific activity of the supernatant was $0.1 \mathrm{U} / \mathrm{mg}$ and after $80 \%$ saturation this value increased $0.64 \mathrm{U} / \mathrm{mg}$. Furthermore, acid phosphatase was investigated using different adsorbent (chitin, chitosan, synthetic zeolite and raw zeolite) and evaluated the storage stability and re-usability of the immobilized acid phosphatase. It was estimated that, acid phosphatase activity was shielded the ratio of $94,96,99$, and $92 \%$ by using raw zeolite, synthetic zeolite, chitin and chitosan respectively under $12 \mathrm{~h}$ operation condition.

\section{IB118}

Plasmid-dependent methylotrophy and its significance for the Biology of thermotolerant bacterium Bacillus methanolicus Øyvind M. Jakobsen ${ }^{1,2}$, Michael C. Flickinger $^{3}$, Svein Valla ${ }^{1}$, Trond E. Ellingsen ${ }^{2}$, Trygve Brautaset ${ }^{1}$ : ${ }^{1}$ Department of Biotechnology, Norwegian University of Science and Technology, Norway; ${ }^{2}$ SINTEF Applied Chemistry, SINTEF, Norway; ${ }^{3}$ BioTechnol. Institute, Department of Biochemistry, Molecular Biology and Biophysics, University of Minnesota, USA

Aerobic methylotrophs can utilize one-carbon $\left(\mathrm{C}_{1}\right)$ compounds such as methane and methanol as a sole $\mathrm{C}$-source for growth and energy. The majority of research on these organisms has focused on their biochemical novelity and commercial viability. For the industrial production of bulk products such as the amino acids lysine and glutamate raw material costs and abundance are important, and $\mathrm{C}_{1}$ sources are thus attractive compared to sugars. Bacillus methanolicus can secrete up to $55 \mathrm{~g} / 1$ of glutamate upon methanol growth at $50{ }^{\circ} \mathrm{C}$ (thermotolerant and methylotroph) and mutants producing $35-40 \mathrm{~g} / \mathrm{l}$ of L-lysine have been selected. We study the genetics for conversion of methanol into biosynthesis of glutamate and lysine, and in the present report we unravel the regulation of genes impor- tant for the consumption and tolerance level for $\mathrm{C}_{1}$ compounds by $B$. methanolicus. B. methanolicus has a methanol dehydrogenase gene $(m d h)$ for oxidation of methanol into formaldehyde and a ribulose monophosphate (RuMP) pathway for assimilation of formaldehyde. We recently discovered that $m d h$ and five RuMP genes are carried by natural plasmid pBM19 in this bacterium and this represented the first documentation of plasmid-dependent methylotrophy in any microorganism. We here use real-time PCR to analyse the regulation of plasmid- and chromosomally located RuMP genes, in cells upon methylotrophic and non-methylotrophic growth. High methanol concentrations in the growth medium is cell toxic and the mechanisms for this sensitivity of B. methanolicus is poorly understood. Our results indicate that plasmid pBM19 plays a fundamental role for this trait as well and the impact of our results on the biotechnological applications of this bacterium is discusssed.

\section{References}

Brautaset, T., Williams, M.D., Dillingham, R.D., Kaufmann, C., Bennars, A., Crabbe, E., Ellingsen, T.E., Flickinger, M.C., 2003. The role of Bacillus methanolicus citrate synthase gene, citY, in regulatiing the secretion of glutamate in lysine-secreting mutants. Appl. Environ. Microbiol. 69, 3986-3995.

Brautaset, T., Jakobsen, Ø.M., Flickinger, M.C., Valla, S., Ellingsen, T.E., 2004. Plasmid-dependent methylotrophy in thermotolerant Bacillus methanolicus. J. Bacteriol. 186, 1229-1238.

\section{IB119}

Production of plant proteinase from jack fruit seeds (Artocarpus Integrifolis) and its influence on rheological and sensory characteristics of low fat yogurt El-Sayed El-Tanboly Dairy Sciences Department, National Research Centre, Dokki, Cairo, Egypt

Adding a proteolytic enzyme extraction from Jack Fruit (Artocarpus integrifolis) in combination of fermentation process in low fat yogurts manufacture was tried to improve yogurt flavour and rheological properties. Experimental yogurts milk contained control, 3.9 (T1), 7.8 (T2) and 11.7 (T3) units/ml milk from crude extracts of plant proteinase. The $\mathrm{pH}$ of the product treated with crude proteinase was lower than the control. However, The rate of acidity development during storage slightly increased with increasing the addition of crude proteinase level and progress of storage period of yogurt. The proteolytic activity of all yogurts gradually increased until the end of storage period (15 days). Yogurts made from milk treated with crude proteinase preparations were less firm compared with control at all storage periods, where T3 showed more less firm after 15 days of storage being $20.17 \mathrm{~g} / 100 \mathrm{~g}$. Generally, increasing units of plant proteinase preparations decreased the firmness. On the other hand, yogurt made from milk pretreated with plant proteinase had higher syneresis, and apparent viscosity than the untreated product. The greatest viscosity was found in T2 and T3 of 433 and $479 \mathrm{mPa} s$ respectively, compared with control of $299 \mathrm{mPa}$ s at 15 days storage. The results indicated that there is an inverse relationship between the amount of units of crude proteinase preparations and susceptibility of yogurt to syneresis. The $\mathrm{T} 2$ gained the highest scores ( 85 points) followed by the control ( 81.5 points) after 15 days of storage, while yogurt of T3 showed a low scoring being 75. From the foregoing results, it is recommend to use Jack Fruit (Artocarpus integrifolis) as a source of plant proteinases and utilize it to develop a high quality yogurt at a level of 7.8 units of plant proteinases $/ \mathrm{ml} \mathrm{milk.}$ 


\section{IB120}

Characterization of the oat 1 gene encoding a novel bi-functional $\omega$-aminotransferase involved in the catabolism of arginine and lysine in Penicillium chrysogenum L. Naranjo ${ }^{1,3}$, M. Lamas-

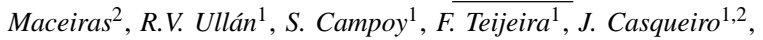
J.F. Martín ${ }^{1,2}$ : ${ }^{1}$ Instituto de Biotecnología de León (INBIOTEC), Parque Científico de León, Av. Real, 1, 24006 León, Spain; ${ }^{2}$ Área de Microbiología, Facultad de Cs. Biológicas y Ambientales, Universidad de León, Campus de Vegazana, s/n, 24071 León, Spain; ${ }^{3}$ Centro de Biotecnología, Fundación Instituto de Estudios Avanzados (IDEA), Caracas 1015-A, Venezuela. E-mail: lnaranjo@idea.org.ve (L. Naranjo)

The Penicillium chrysogenum oat1 gene encoding a class III omega-aminotransferase has been cloned and characterized. This enzyme that converts lysine into 2-aminoadipic semialdehyde is important in providing 2-aminoadipic acid, a precursor of penicillin and other $\beta$-lactam antibiotics. The enzyme is related to ornithine5 -aminotransferases and to lysine-6-aminotransferases encoded by the lat gene located in the bacterial cephamycin gene clusters. Expression of oat1 is induced by lysine, ornithine and arginine and repressed by ammonium ions. AreA-binding consensus sequences and an 8-bp direct repeat associated with arginine induction in Emericella (Aspergillus nidulans) have been found in the oat 1 promoter region. Deletion of the oat1 gene resulted in the loss of omegaaminotransferase activity. The deletion mutants were unable to grow on ornithine or arginine as sole nitrogen sources and showed a reduced growth on lysine. Complementation of the deleted mutant with the oat 1 gene restored growth on ornithine, arginine and lysine to the levels of the parental strain and omega-aminotransferase activity. The strong expression of oat 1 gene after induction by the basic amino acids may provide additional 2-aminoadipic acid for the formation of the 2-aminoadipyl-cysteinyl-valine tripeptide for $\beta$-lactam biosynthesis.

\section{IB121}

Morphological characterisation of two high producing strains of Penicillium chrysogenum carrying a disruption in the NADPH-dependent glutamate dehydrogenase $\underline{K}$. Rueksomtawin, J. Thykaer, H. Noorman, J. Nielsen Center for Microbial Biotechnology, BioCentrum-DTU, Technical University of Denmark, DK2800 Lyngby, Denmark. E-mail: kr@ biocentrum.dtu.dk (K. Rueksomtawin)

Metabolic engineering has proven to be useful in optimisation of $\beta$-lactam production, e.g. constructing superior strains with multiple copies of the $\beta$-lactam gene cluster. It is however, of equal importance to gain insight into other aspects of the metabolism to establish a general overview in order to apply metabolic engineering for further improvement of the production strains. In that context, the redox metabolism is essential, as it functions as a tightly controlled connection between the different parts of the metabolism. In order to investigate this role of the redox metabolism in more detail, the $g d h A$-gene, encoding the NADPH-dependent glutamate dehydrogenase, was disrupted in two industrial strains of Penicillium chrysogenum. During physiological characterisation of the two strains it became apparent that considerable changes in the morphology had occurred due to the genetic alteration. Since the morphology is an important parameter in process optimisation, an examination of the morphology of the two strains was undertaken. In this work, the morphological differences between the $g d h A$-disrupted strains and the reference strains were comprehensively investigated both during growth on solid media and submerged growth in a flow-through growth chamber. With the advance development of computerized image analysis techniques, the key morphological properties of the individual hyphal elements were quantified. In comparison to the reference strains, the disruption of the $g d h A$ gene resulted in a morphological change from short hyperbranched hyphal elements to long elongated hyphal elements with less branches. In the parallel studies with Aspergillus nidulans and its corresponding $g d h A$-deleted strain, no difference in the morphology was observed.

\section{IB122}

Hetrologous expression of 6-methylsalicylic acid synthase in Saccharomyces cerevisiae and Aspergillus sp. Songsak Wattanachaisaereekul, Torsten Bak Regueira, Anna Eliasson Lantz, Michael Lynge Nielsen, Jens Nielsen Center for Microbial Biotechnology, BioCentrum-DTU, Technical University of Denmark, 2800 Kgs. Lyngby, Denmark. E-mail: swa@biocentrum.dtu.dk (S. Wattanachaisaereekul)

Polyketides (PK) represent one of the largest groups of natural products and are found in fungi, bacteria and plants. Since many useful polyketides either originate from sources that are difficult or even impossible to cultivate or are produced in inadequate amounts, we are interested in expressing polyketide synthases (PKSs) in heterologous hosts. Saccharomyces cerevisiae, Aspergillus niger and Aspergillus nidulans were chosen as initial hosts, because the techniques necessary to cultivate and manipulate these strains genetically are well established. 6-Methylsalicylic acid synthase (6-MSAS) was chosen as a model PKS. Replicative plasmids carrying the genes encoding 6-MSAS from Penicillium patulum and phosphopantetheinyl transferase (PPTase), respectively, were transformed into $S$. cerevisiae. In addition, an integrative vector was designed and the gene encoding 6-MSAS was integrated in the yeast chromosome. Batch cultivations on galactose minimal media were performed. The results are presented and in particular the effect of expression mode and type of PPTase (bacterial versus fungal) is discussed. Furthermore, the progress of the work on expressing 6-MSAS in A. niger and A. nidulans using an integrative vector system is presented and discussed.

\section{IB123}

\section{Biobased Chemicals: Today, Not Tomorrow}

The valorisation of functionalized chemicals from biomass resources compared to the conventional fossil production route Ben Brehmer, Wageningen UR Agrotechnology \& Food Sciences, Workgroup: Valorisation of Plant Production Chains, P.O. Box 17, 6700 AA Wageningen, The Netherlands. E-mail: ben.brehmer@wur.nl

\section{Dissertation Abstract}

\section{The Problem and the Solution}

At some undisclosed point in the foreseeable future, cheap fossil fuels resources will become depleted and the industry will be forced to pursue more difficult reserves with increasingly high extraction costs. Most alternatives available and under research do not consider price as the main motivation for replacement, but focus solely on 
sustainability and environmental benefits. Sustainability is an interesting word as there are enough fossil resources scattered around the world to be sustainable in quantity but not sustainable in price. Seeing that fossil fuels are derived from prehistoric biomass it is not at all presumptuous to assume that every application and product can be replaced by the biomass of today. In fact, many highly specialised pharmaceutical chemicals already have a biomass origin. Yet, not all of the uses of fossil fuels need to be replaced by a comparable carbon based source, such as biomass. Energy and transportation in particular do not necessary need to rely on carbon cleavage, whereas practically all of the petrochemicals contain a carbon backbone. The main stipulation in substituting fossil-based chemicals with bio-based chemicals is availability and cost. It is proposed that already today, by utilising existing, recently developed and developing technology, it is economically advantageous for many chemicals to derive from biomass, in particular the functionalized chemicals. The only way to validate this conjecture is to develop a complete comparative life cycle analysis. As opposed to a traditional LCA, the "multicriterion" developed here will revolve around energy flows and process efficiency in terms of exergy. The aim is to assess the optimum route with the best production options along the whole production chain while determining any possible limiting factors. Using this tool, a systematic production matrix relating several logical source crops and a few key chemicals of varying derivative levels can be created and compared to the conventional fossil routes. Combined with economic considerations and some unambiguous environmental factors, the investigation will provide all the information relevant to the industry. The goal is to create an objective and reliable simulation system ratifying the economic and environmental feasibility of exploiting biobased chemicals today and indicate the steps necessary for further improvement.

\section{IB124}

Biosynthesis of multi-enzymatic preparation from Aspergillus niger IBT-90 useful in textile fabric treatment Rita Pyc ${ }^{1}$, Jadwiga Sojka-Ledakowicz ${ }^{2}, \quad$ Tadeusz Antczak ${ }^{1}, \quad$ Joanna Lichawska ${ }^{2}{ }^{1}$ Institute of Technical Biochemistry, The Technical University of Lodz, Lodz 90-924, Poland; ${ }^{2}$ Textile Research Institute, Lodz 92-103, Poland

Among many methods of producing enzymatic preparations, i.e. by liquid surface fermentation - LSF, submerged fermentation - SmF or solid state fermentation - SSF, this last is most advantageous. Cultivation in solid state means fermentation on a matrix formed by industrial and agricultural wastes. Most often filamentous fungi-due to the lack of available water in the foundation - are the efficient, competitive microorganisms applied in solid state bio-conversion. The aim of research works carried out at the Institute of Technical Biochemistry of the Technical University of Lodz and at Textile Research Institute, Lodz was defining optimal conditions for biosynthesis and testing the possibility of applying multi-enzymatic preparation from Aspergillus niger IBT-90 in the treatment of woven fabrics made of natural cellulose fibres. As the result of biosynthesis optimization process, malt sprouts, wheat barn and beet pulp were selected as the best media to obtain enzymes of high pectinolytic activity maintaining at the same time high activity of cellulolytic enzymes and xylanase. The highest obtained activities reached: $2000^{\circ} \mathrm{PM}$ for total pectinolytic activity, $7 \mathrm{~J} / \mathrm{ml}$ for endoglucanase and $527 \mathrm{~J} / \mathrm{ml}$ for endoxylanase and they were 2.4-3.0 times higher than those achieved before optimizing process. Performed research works demonstrated that the optimum activity of applied enzymatic system is obtained in the range of $\mathrm{pH} 4.6-4.8$. Woven fabrics made of flax and cotton fibre blends, subjected to bio-pre-treatment, were evaluated with reference to their sorption properties. Comparative evaluation of liquid sorption by woven fabrics allowed to notice efficient enhancement of fibres' sorption capabilities after pre-treatment using enzymes system from Aspergillus niger IBT-90. This offers the possibility of substitution of alkali scouring of linen-cotton woven fabrics before their bleaching by bio-treatment.

\section{IB125}

Pathways for central carbon metabolism in Actinobacter Anna Eliasson Lantz, Jens Nielsen, J. Stefan Rokem ${ }^{*}$ Center for Microbial Biotechnology, BioCentrum-DTU, Kgs. Lyngby DK-2800, Denmark; *Permanent address: Department of Molecular Genetics \& Biotechnology, The Hebrew University of Jerusalem, Jerusalem IL-91120, Israel. E-mail: ael@biocentrum.dtu.dk (A.E. Lantz), sr@biocentrum.dtu.dk (J.S. Rokem)

The phylum Actinobacter includes many bacteria of industrial importance both for accumulation of primary and secondary metabolites. Both primary and secondary metabolites are dependent on precursors and cofactors that are provided by the central carbon metabolism of microorganisms. There are alternative pathways for catabolism of carbon either via the Embden Meyerhof Parnas (EMP) and pentose phosphate (PP) pathway or through the Entner Doudoroff (ED) pathway. The EMP pathway is energetically more favorable and has therefore been presumed to be the dominating route for carbon metabolism in bacteria producing secondary metabolites. However, primary metabolism is poorly studied for most Actinobacter species as focus traditionally has been on secondary metabolism. With the aim to gain more knowledge about the diversity of central carbon metabolism within the phylum Actinobacter, 17 different strains were collected from various sources and strain collections. The strains were grown in minimal medium with supplement of standard vitamin solution and $\left[1-{ }^{13} \mathrm{C}\right]$ glucose as carbon source. The ${ }^{13} \mathrm{C}$-labeling patterns of proteinogenic amino acids were determined by GC-MS analysis. Through this method, the fluxes in the central carbon metabolism during balanced growth were estimated and pathways for carbon metabolism were determined. In particular the labeling patterns of alanine and valine were of interest as they are derived from pyruvate and therefore can be used to distinguish between whether glucose is metabolized through the ED pathway or the EMP pathway.

\section{IB126}

Physiology of bahlimycin formation by Amycolatopsis balhimycina Anna Eliasson Lantz, Jetter Thykar, J. Stefan Rokem*, Jens Nielsen Center for Microbial Biotechnology, BioCentrumDTU, Kgs. Lyngby DK-2800, Denmark; *Permanent address: Department of Molecular Genetics \& Biotechnology, The Hebrew University of Jerusalem, Jerusalem IL-91120, Israel. E-mail: ael@biocentrum.dtu.dk (A.E. Lantz), sr@biocentrum.dtu.dk (S. Rokem)

Glycopeptides are an important natural class of antibiotics, exhibiting activity against Gram positive bacteria, including methicillin-resistant Staphylococcus aureus strains. A novel acti- 
nobacter, Amycolatopsis balhimycina produces the glycopeptide balhimycin. The balhimycin aglycone is identical to the aglycon of vancomycin, which is a commercial glycopeptide. As A. balhimycina appears to be accessible to genetic modifications and the biosynthetic cluster responsible for balhimycin production is published, this genetically well-characterised academic strain can serve as a platform strain for production of vancomycin analogues derived through combinatorial biosynthesis. The understanding of the physiology of this microorganism is essential for the efficient accumulation of potentially commercial secondary metabolites. The bacterium is capable of growth in a fully defined minimal medium, with the production of balhimycin. The strain was grown at either nitrogen or phosphate limitation and balhimycin accumulation was followed at these conditions. Flux analysis of balhimycin production by Amycolatopsis balhimycina based on ${ }^{13} \mathrm{C}$ labelling experiments was performed.

\section{IB127}

Regulation of terpenoid biosynthesis in zygomycetes Vera Kouzina, Enrique Cerdá-Olmedo Departamento de Genética, Universidad de Sevilla, Sevilla E-41080, Spain. E-mail: kouzina_v@yahoo.es (V. Kouzina)

The Zygomycetes Blakeslea trispora and Phycomyces blakesleeanus accumulate beta-carotene, ubiquinone (coenzyme Q), various different sterols, and other terpenoids, all of them produced via the mevalonate pathway. These fungi are used or could be used for the industrial production of these terpenoids, edible oil, chitosan, and various organic acids. By measuring the specific radioactivity of terpenoids made from radioactive mevalonate, leucine or acetate in the presence of excess glucose in wild types and mutant strains we have concluded that these fungi have separate subcellular compartments for the production of carotene, sterols and triacylglycerols. The terpenoid moiety of ubiquinone is synthesized in the same compartment as ergosterol. These compartments contain separate pools of all their common metabolites, beginning from acetyl-CoA. Mevalonate carbon atoms do not find their way back to general metabolism, i.e., these fungi lack the "shunt" pathway. The compartments are regulated independently. The very large variations in carotene content caused by many environmental and genetic changes are not accompanied by variations in the ubiquinone content. The ubiquinone content increases when the cultures grow on leucine or acetate as carbon sources and is not affected by illumination. Phycomyces, but not Blakeslea, increases the production of ubiquinone in presence of oligomycin.

\section{IB128}

Shared features of lincomycin and anthramycin antibiotic gene clusters Markéta Jelínková, Markéta Koběrská, Jan Kopecký, Jiří Janata Institute of Microbiology, Academy of Science of the Czech Republic, Vídeňská 1083, 14220 Prague 4, Czech Republic. E-mail: marketaj@biomed.cas.cz (M. Jelínková)

Lincomycin, produced by Streptomyces lincolnensis, is important, clinically used antibiotic. Its gene cluster consists of 27 putative open reading frames with biosynthetic or regulatory functions ( $\operatorname{lmb}$ genes) and three resistance genes $(\operatorname{lm} r A, \operatorname{lm} r B, \operatorname{lm} r C)$. The organization of transcription units was determined. The analysis of the lincomycin biosynthetic gene transcripts in various cultivation stages revealed the genes with putative regulatory functions which are transcribed in early stages of cultivation. Previous analysis of biosynthetic pathways of lincomycin and functionally different anthramycin antibiotics (anthramycin, sibiromycin, tomaymycin, mazetharmycin and porothramycin) indicates that the genetic information on the lincomycin and anthramycin biosynthesis should share common elements (genes), both biosynthetic and regulatory. Hybridization experiments demonstrated presence of several analogues of $\operatorname{lmb}$ genes involved in the biosynthesis of anthramycin produced by Streptomyces refuineus and porothramycin produced by Streptomyces albus.

\section{IB29}

Effect of various calcium salts on the erythromycin production by Saccharopolyspora erythraea M. Rostamza ${ }^{1}, A$. Noohi ${ }^{1}$, J. Hamedi $i^{2 *}$ : ${ }^{1}$ Department of Biology, Faculty of Science, Science and research branch, Islamic Azad University, Tehran, Iran; ${ }^{2}$ Microbial Biotechnology Lab., Department of Biology, Faculty of Science, University of Tehran, Tehran, Iran. E-mail: jhamedi@khayam.ut.ac.ir (J. Hamedi)

Calcium carbonate has the positive effect on the erythromycin, however, because of its low water solubility, caused to clogging the spargers of fermenters and fouling the microfilters. In this research, various soluble calcium salts was added to the fermentation medium and their effect the growth of Saccharopolyspora erythraea and erythromycin production was studied in the complex medium consisted soy bean meal, dextrin and starch as major ingredients. The fermentation conditions were $220 \mathrm{rpm}, 8$ days at $30^{\circ} \mathrm{C}$. The results obtained showed that there is no significant difference between erythromycin concentrations in the medium containing calcium lactate and calcium carbonate. However, erythromycin concentrations in the other calcium salts containing media were less than to calcium carbonate containing medium. Optimum concentration of calcium lactate for erythromycin production was $10 \mathrm{~g} / 1$.

\section{IB130}

Comparison of biosynthetic gene clusters of lincomycin and celesticetin Markéta Koběrská, Jan Kopecký, Markéta Jelínková, Ladislav Čermák, Jiř́ Janata Institute of Microbiology, Academy of Science of the Czech Republic, Vídeňská 1083, 14220 Prague 4, Czech Republic. E-mail: malb@biomed.cas.cz (M. Koběrská)

Lincosamides and its derivatives are clinically important antibiotics. Comparison of gene cluster coding for lincomycin biosynthesis and newly identified cluster of genes for celesticetin biosynthesis revealed new information on functions of several genes common for both biosynthetic pathways. The celesticetin gene cluster was identified by screening the cosmid library of Streptomyces caelestis with heterologous probes based on lincomycin biosynthetic genes involved in a part of biosynthesis shared by both antibiotics. Sequence analysis of the cluster revealed 24 putative ORFs, out of which 18 are lincomycin biosynthetic genes analogues, four are specific for celesticetin biosynthesis and one codes for resistance. The gene cluster is bounded with transposase genes on both sides. In order to clarify function of three putative regulatory genes of lincomycin biosynthesis, the insertional inactivation with the PCR targeting system in Streptomyces lincolnensis was done and resulted in differently reduced production of lincomycin. 


\section{IB131}

A new algorithm for novelty detection and de-replication of spectroscopic analysis of complex mixtures of natural products Michael Edberg Hansen, Jørn Smedsgaard, Thomas Ostenfeld Larsen CMB BioCentrum-DTU, Technical University of Denmark, 2800 Kgs. Lyngby, Denmark

We present a new algorithm called "X-hitting" for automatic identification of novel bioactive compounds based on full spectroscopic characters of highly complex mixtures of natural product. One of the most dramatic advances in recent drug discovery has been the increase in screening capacity throughput and data handling. Therefore, analysis has become the bottleneck in the drug discovery process. The algorithm presented here is investigated and demonstrated on identifying potentially new bioactive compounds. In addition method is shown to have a high performance for automatic identification of known structures. These tasks are referred to as new-hitting and cross-hitting, respectively. Finally, the receiver operating characteristics (ROC) is introduced to the research field as an important tool for evaluating the performance of the "compound predictor". Through examples it is shown, that known cross-hits are identified with high proficiency, and that the new-hitting works on finding new targets represented by analogues and structurally new compounds.

\section{IB132}

Functional analysis of a gene encoding the $\gamma$-butyrolactone autoregulator receptor in Streptomyces natalensis ATCC27448 producing natamycin Y.I. Hwang, K.M. Lee, J.Y. Kim, S.U. Choi, H.R. Park Division of Food Science and Biotechnology, Kyungnam University, Masan 631-701, Republic of Korea. E-mail: yihwang@kyungnam.ac.kr (Y.-I. Hwang)

A gene encoding a $\gamma$-butyrolactone autoregulator receptor that have a common activity as DNA-binding transcriptional repressors, controlling secondary metabolism and/or morphological differentiation in Streptomyces was cloned from a natamycin producer, Streptomyces natalensis, and its function was evaluated by in vivo. PCR using the primers designed from two highly conserved regions of Streptomyces autoregulator receptors gave a 102-bp band, the sequence of which revealed high similarity to the expected region of a receptor gene. By genomic Southern hybridization with 102-bp insert as a probe, a 687-bp intact receptor gene $(s n g R)$ was obtained from $S$. natalensis. In vivo to clarify the function of $s n g R$, a $s n g R$ disrupted strain was constructed, and a phenotype was compared with the wild-type strain. The $s n g R$ disruptant started natamycin production $6 \mathrm{~h}$ earlier and showed a 4.6-fold-higher production of natamycin than the wild-type strain. In addition, sporulation was earlier and 10 -fold abundance. The phenotype indicates that the autoregulator receptor protein of $S$. natalensis acts as a primary negative regulator of the biosynthesis of natamycin and is related to regulation of sporulation.

\section{IB133}

Exploring the biocatalytic potential of the novel thermostable Baeyer-Villiger monooxygenase: Phenylacetone monooxygenase Daniel E. Torres Pazmiño ${ }^{1}$, Gonzalo de Gonzalo ${ }^{2}$, Gianluca Ottolina ${ }^{2}$, Giacomo Carrea ${ }^{2}$, Dick B. Janssen ${ }^{2}$, Marco W. Fraaije ${ }^{1},{ }^{1}$ Biochemical Laboratory, Groningen Biomolecular Sciences and Biotechnology Institute, University of Groningen, Nijen-

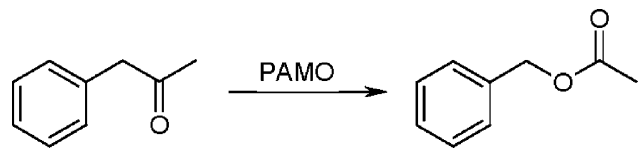

Fig. 1. Conversion of phenylacetone by phenylacetone monooxygenase (PAMO).

borgh 4, 9747 AG, Groningen, The Netherlands; ${ }^{2}$ Instituto di Chimica del Riconoscimento Molecolare, CNR, via Mario Bianco 9, 20131 Milano, Italy. E-mail: d.e.torres.pazmino@ rug.nl (D.E. Torres Pazmiño)

Baeyer-Villiger monooxygenases (BVMOs) represent useful biocatalytic tools as they can catalyze reactions which are difficult to achieve using chemical means. However, only a limited number of these atypical monooxygenases are available in recombinant form. Using a recently described protein sequence motif, a putative BVMO was identified in the genome of the thermophilic actinomycete Thermobifida fusca. The NADPH-dependent and FAD-containing monooxygenase is active with a wide range of aromatic and aliphatic ketones and sulfides. Genetic and kinetic data suggest that phenylacetone is the physiological substrate of the enzyme. Previously, it was reported that this BVMO exhibits only a moderate enantioselectivity with $(R, S)-\alpha$-methylphenylacetone. This poster we will show an overview of the biocatalytic potential of the enzyme as explored so far. Interestingly the enzyme has been found to perform highly enantioselective oxidations with a range of ketones and sulfides. This again indicates that this novel thermostable oxidative biocatalyst can be a useful tool for the synthesis of chiral building blocks.

\section{IB134}

Enzymatic synthesis of $S$-adenosyl-homocysteine using recombinant $\boldsymbol{S}$-adenosyl-homocysteine hydrolase from $\boldsymbol{C}$. glutamicum I. Martínez-Martínez; J.D. Lozada-Ramírez; A. Sánchez-Ferrer, F. García-Carmona Department of Biochemistry and Molecular Biology (A), Faculty of Biology, Universidad de Murcia, E-30100 Murcia, Spain. E-mail: gcarmona@um.es (F. García-Carmona)

The enzyme $S$-adenosylhomocysteine hydrolase (AdoHcyase) catalyzes hydrolysis of $S$-adenosylhomocysteine (AdoHcy), an inhibitor of transmethylation reactions, into adenosine (Ado) and homocysteine (Hcy). The catalysed reaction is reversible, and the equilibrium is strongly displaced in direction of the synthesis of AdoHcy when reaction occurs in vitro. Nevertheless, its biotechnological application resides in the synthesis of antivirals. For it, we selected between different producing microorganisms of the enzyme, the gram positive bacterium Corynebacterium glutamicum. After designing the specific oligonucleotides, the gene was expressed in Escherichia coli using the expression system pET28a+ with IPTG. The electrophoretic analysis under denaturing conditions, shows a clear induction and over-expression of a protein with a MW of $49 \mathrm{kDa}$. On the other hand, the immobilization of this recombinant enzyme in a solid support allows to use it as a catalyst for the synthesis of AdoHcy. The enzyme was purified by IMAC thanks to the presence of N-terminal $6 \times$ HIS tag end, and immobilized in Eupergit $\mathrm{C}$ for the optimization of the production of AdoHcy, a product of high value. 


\author{
Acknowledgements \\ This work was partially supported by MEC and FEDER \\ (BIO2004-00439), and Fundación Séneca CARM (00842/PI/01).
}

\section{IB135}

Sequencing, cloning, expression, purification and characterization of a novel Cytochrome $\mathbf{P 4 5 0}$ monooxygenase from Rhodococcus rubber Luo Liu, Rolf D. Schmid, Vlada B. Urlacher Institute of Technical Biochemistry, Stuttgart University, Allmandring 31, 70569 Stuttgart, Germany. E-mail: itbvur@itb.unistuttgart.de (L. Liu)

The Cytochrome P450 monooxygenases are heme-containing proteins, which catalyze a wide range of oxidative reactions (WerckReichhart and Feyereisen, 2000). A monooxygenation activity was observed for the strain Rhodococcus ruber DSM 44319. A P450-like gene fragment was amplified by PCR using degenerated primers. For identification of regions that flank this P450-like DNA fragment, the method "directional genome walking using PCR" was applied (Mishra et al., 2002). The full size gene encoding a cytochrome P450 enzyme was amplified by PCR from genomic DNA and cloned into the vector $\mathrm{pET} 28 \mathrm{a}(+)$ for heterologous expression in Escherichia coli BL21(DE3) cells. The enzyme was purified using metal affinity chromatography. The primary protein structure suggests, that this enzyme is a natural self-sufficient fusion protein consisting of a ferredoxin, a reductase and a P450 monooxygenase. The reductase activity was determined using an exogenous electron acceptor cytochrome $c$. The reductase domain of this $\mathrm{P} 450$ monooxygenase showed a strong preference for NADPH over NADH. The substrate spetrum was investigated. In the presence of NADPH the P450 enzyme shows hydroxylation activity towards 7-ethoxycoumarin, naphthalene, indene, acenaphthene, toluene and fluorene.

\section{References}

Mishra, R.N., Singla-Pareek, S.L., Nair, S., Sopory, S.K., Reddy, M.K., 2002. Directional genome walking using PCR. BioTechniques 33, 830-834.

Werck-Reichhart, D., Feyereisen, R., 2000. Cytochromes P450: a success story. Genome Biol. 1 (6), 3003.1-3003.9.

\section{IB136}

Selective production of monoglyceride consisted of conjugated linoleic acid by Penicillium lipase Yomi Watanabe ${ }^{1,2}$, Yoshie Yamauchi-Sato $^{3}$, Toshihiro Nagao ${ }^{1}$, Satoshi Negishi ${ }^{3}$, Tadamasa Terai $^{4}$, Takashi Kobayashi ${ }^{1}$, RolfD. Schmid ${ }^{2}$, Yuji Shimada ${ }^{1}:{ }^{1}$ Osaka Municipal Technical Research Institute, Osaka, Japan; ${ }^{2}$ Stuttgart University, Stuttgart, Germany; ${ }^{3}$ The Nisshin OilliO Group, Ltd, Yokosuka, Japan; ${ }^{4}$ Osaka Institute of Technology, Osaka, Japan

Conjugated linoleic acid (CLA) is a group of $\mathrm{C} 18$ fatty acid (FA) containing two conjugated double bonds. It is expected to prevent cancer, adipositas, atherosclerosis etc, and is commertially available in the primary form of free FA, containing almost equal amounts of 9cis,11trans- and 10trans,12cis-CLA. It is therefore desired to be converted to a palatable form. For this purpose, we have previously proposed two enzymatic ways to convert CLA to monoglyceride, an emulsifier, with Penicillium camembertii lipase; (1) sequencial esterification-glycerolysis, (2) esterification at low tem- perature. These methods, however, are time- and energy-consuming. Esterification of CLA with glycerol under ambient pressure by the lipase produces equal amounts of mono- and diglycerides. In contrast, it was newly found that the reaction under reduced pressure supressed the formation of diglycerides and achieved to produce $90 \%$ monoglyceride at $95 \%$ esterification.

\section{IB137}

Improving the thermal stability of cellobiohydrolases I (Cel7A) from $T$. reesei by site directed evolution Frits Goedegebuur ${ }^{1}$, Lydia Dankmeyer $^{1}$, Peter Gualfetti ${ }^{2}$, Brad Kelemen ${ }^{2}$, Edmundo Larenas ${ }^{2}$, Paulien Neefe ${ }^{1}$, Pauline Teunissen ${ }^{1}$, Colin Mitchinson ${ }^{2}{ }^{1}$ Genencor International BV, Archimedesweg 30, 2333CN Leiden, The Netherlands; ${ }^{2}$ Genencor International Inc., 925 Page Mill Road, Palo Alto, CA 94304, USA

Genencor International has been working to produce improved enzyme products for economic conversion of ligno-cellulosic biomass to fermentable sugars. Most of this work was performed under a subcontract with the U.S. Department of Energy for cellulose cost reduction for biomass conversion. Cellulolytic biomass conversion is performed in nature by a complex mixture of enzymes. Cellobiohydrolases play a key role and all effective cellulase mixtures contain a large excess of cellobiohydrolases over endoglucanases, suggesting that it is the exoglucanase activity that is limiting. The fundamental dependence of reaction rate on temperature predicts that large increases in performance, and decreased enzyme cost, would be achieved if the enzymatic conversion could be operated at elevated temperatures. Industrial strains of Trichoderma reesei produce cellulases at very high levels and low cost. However, T. reesei CBH1 (Hypocrea jecorina Cel7A) does not have sufficient stability to survive and perform at high temperatures. This poster shows the thermal stability improvement in $T$. reesei $\mathrm{CBH} 1$ by site directed evolution. Sites with increased thermal stability properties were combined and evolved in high temperature stable CBHI variants.

\section{IB138}

Studies on lipases immobilization for peptidomimetic functionalization Dominik Koszelewski, Ryszard Ostaszewski Institute of Organic Chemistry PAS, Warsaw 02-224, Kasprzaka 44/52, Poland

The evaluation of lipases as biocatalysts for organic chemistry can be carried out, at laboratory scale, by using soluble enzymes for biotransformations in aqueous media. However, the industrial exploitation of such an enormous potential should require a suitable protocol for immobilization of lipases. The binding of lipases on suitable pre-existing supports should greatly improve the performance of industrial reactors allowing us a continuous use or re-use of such interesting biocatalysts. In addition, lipases, like most enzymes, are not perfect chemical catalysts. Lipases may be unstable and they may not have the optimal activity nor the optimal enantio or regioselectivities. In this way, immobilization of lipases, together with its relevance for the performance of each different industrial reactor, could be also used as a tool to improve and optimize some of these parameters. That is, immobilization of lipases, far from an already solved problem, constitutes an exciting field of research in the promising area of industrial bio-organic chemistry. 


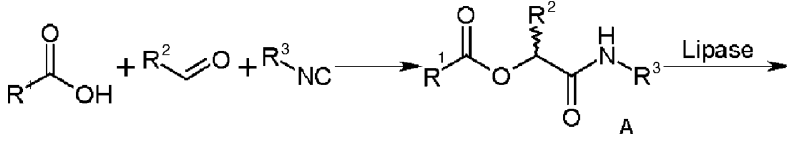

In this work we would like to present useful immobilization methods of several lipases. The lipase immobilised were used for enzymatic hydrolysis of peptidomimetics of structure A. Type of immobilzation used can changed the enantioselectivity of biocatalyst prepared. The absolute configuration of products $\mathrm{B}$ and $\mathrm{C}$ obtained in enzymatic reactions were assigned by chemical correlation.

\section{IB140}

Discovery of an epoxide forming monooxygenase from the metagenome Erik W. van Hellemond, Marco W. Fraaije, Dick B. Janssen Biochemical Laboratory, Groningen Biomolecular Sciences and Biotechnology Institute, University of Groningen, Nijenborgh 4, 9747 AG Groningen, The Netherlands. E-mail: E.W.van.Hellemond@ rug.nl (E.van Hellemond)

Two-component flavin-dependent monooxygenases form an interesting class of flavoenzymes. They consist of two separate proteins; a monooxygenase component, which catalyses an oxygenation reaction in the presence of reduced flavin, and a flavin reducing component, which reduces flavin (FAD or FMN) using NAD(P)H as an electron donor. A well-known example of this class of monooxygenases is styrene monooxygenase (Otto et al., 2004). Due to the ability to form enantiopure epoxides, which are relevant building blocks for the pharmaceutical industry, styrene monooxygenases form a valuable class of enzymes for biocatalysis. While screening a metagenomic library for oxidative enzymes, an indigo-producing clone was found. Sequencing the particular clone revealed an inserted fragment of environmental DNA encoding a two-component monooxygenase $(\mathrm{SmoAB})$, consisting of a monooxygenase (SmoA) and a flavin reductase $(\mathrm{SmoB})$ component. The monooxygenase component shows homology with known styrene monooxygenases. While sequence homology among the styrene monooxygenases is high (>95\% seq. id.), SmoA only displays a moderate sequence homology ( $\sim 50 \%$ seq. id.). The substrate specificity for SmoAB is currently being investigated.

\section{Reference}

Otto, K., Hofstetter, K., Rothlisberger, M., Witholt, B., Schmid, A.J., 2004. Bacteriol. 186 (16), 5292-5302.

\section{IB141}

Biotransformation of citronellol in rose-oxide using agroindustrial waste Mario Maróstica Jr., Gabriela Macedo, Gláucia Pastore Department of Food Science, UNICAMP, P.O. Box 6121, Campinas, CEP 13083-862, São Paulo, Brazil

Many investigations over the recent years have been directed to the production of natural aroma compounds. Through biotransformation and bioconversion, aroma compounds considered as "natural" can be produced starting from monoterpenes, generating high value products as rose oxide. Rose oxide is found in small amounts in some essential oils such as Bulgarian rose oil and geranium oil. (-)-Rose oxide is an impacting flavor compound and has a small threshold: $0.5 \mathrm{ppb}$. Application of agro-industrial residues in bioprocess on one hand provides alternative substrates, and on the other hand helps

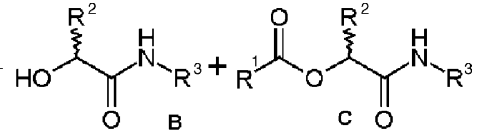

solving pollution problems, that might be caused by the disposal of this residue in nature. The liquid cassava waste, is originated by the pressing of cassava roots. It is considered as a "harmful" pollutant waste due to its high organic charge and presence of cyanide. On the other hand, can be considered rich in nutrients that can be used in other applications. It was found that sporulated surface cultures of Penicillium sp. were able to convert citronellol into cis- and trans-rose oxides. Other bioproducts were 3,7-dimethyl-5-octen-1,7diol and 3,7-dimethyl-6,7-epoxy-1-octanol. No chemical oxidation or auto-oxidation products were detected in liquid control broths. The experiments were conducted at $30^{\circ} \mathrm{C}$ and $160 \mathrm{rpm}$. When the medium was cassava, the production of rose oxide, 3,7-dimethyl-5octen-1,7-diol and 3,7-dimethyl-6,7-epoxy-1-octanol were insignificant reaching trace amounts. But when the mycelium developed in cassava medium and than transferred to mineral medium (citronellol as $\mathrm{C}$-source) the concentrations of rose oxide increased dramatically, reaching $70 \mathrm{mg} / \mathrm{L}$ for the cis-isomer and $30 \mathrm{mg} / \mathrm{L}$ for trans-isomer.

\section{IB142}

Mathematical modeling of enzymatic hydrolysis of cellulosic model substrates Natalija Andersen ${ }^{1,3}$, Katja S. Johansen ${ }^{2}$, Michael Michelsen ${ }^{3}$, Erling H. Stenby ${ }^{3}$, Lisbeth Olsson ${ }^{1},{ }^{1}$ Center for Microbial Biotechnology (CMB), BioCentrum-DTU, Denmark; ${ }^{2}$ Novozymes A/S, Krogshoejvej 36, DK-2880, Bagsværd, Denmark; ${ }^{3}$ IVC-SEP, Department of Chemical Engineering-DTU, Denmark. E-mail: na@biocentrum.dtu.dk (N. Andersen)

A mechanistic mathematical model of enzymatic degradation of Avicel and Phosphoric Acid Swollen Cellulose (PASC) has been proposed. The model is based on the degree of polymerization (DP) of starting substrate, and follows its decline with time, to the final end product - glucose. Three enzyme classes, namely, endoglucanase (EG), cellobiohydrolase ( $\mathrm{CBH}$ ) and $\beta$-glucosidase (BG) are all individually incorporated in the model. The model is, additionally, taking into account cooperative action of the involved enzymes, as well as effects of enzyme inhibition by end-products, cellobiose and glucose. To be able to describe the complex process of enzymatic hydrolysis with a set of differential equations certain assumptions needed to be introduced. Those assumptions represent the simplification of an up-to-date knowledge of both substrate and enzyme structure, but also enzyme mode of action. For example, one of the often asked questions is: "What is happening with shorter cellooligosacharides (DP 7 and up) laying on the surface of cellulose chain? Are they being adsorbed to the core cellulose chain or partly solubilized to a hydrolysis broth?" To give answers to these questions and confirm mathematical modeling real enzyme hydrolysis data are needed. In this work, four well characterized, highly purified mono-component enzymes from Humicola insolens (two EG and two $\mathrm{CBH}$ ) and one BG from Aspergillus niger were used to hydrolyze Avicel and PASC. By careful choice of catalyst, some enzyme specific characteristics like presence or absence of cellulose binding domain will also be incorporated into the model. Hydrolysis experiments were initially performed by distinct mono-component enzymes, to confirm the 
basic characteristics of each of the enzyme classes. Soluble hydrolysis products (DP 1-6) were analyzed by HPLC and detection of non-soluble, higher-DP polysaccharides was performed by technique of polysaccharide analysis using carbohydrate gel electrophoresis.

\section{IB143}

Optimisation of halogenase enzyme activity $K$. Muffler $^{1}, M$. Retzlaff $^{2}, K .-H$. van Pée ${ }^{3}, \quad R$. Ulber $^{1}{ }^{1}{ }^{1}$ Technische Universität Kaiserslautern, Germany; ${ }^{2}$ Technische Universität München, Germany; ${ }^{3}$ Technische Universität Dresden, Germany. E-mail: muffler@rhrk.uni-kl.de (K. Muffler)

Halogenases provide the opportunity of a regioselective and stereospecific halogenation of organic subtrates in contrast to the class of haloperoxidases. These enzymes allow a gentle synthesis of halogenated organic molecules and are capable to halogenate in specific positions (Hammer et al., 1997; Keller et al., 2000), whereas traditional organic synthesis often failures or mainly leads to byproducts (Hasegawa et al., 1999), e.g. halogenation of tryptophan in other positions than position 3. Our current research is focussed on tryptophan-5-halogenases, because the 5-Br/Cl-tryptophan could be applied as a pharmacologically attractive precursor of serotonin. In our work we describe the optimization of an enzyme assay respectively the enzyme activity. For this purpose we use a genetic algorithm. The responsible gene of the $\mathrm{FADH}_{2}$ dependent enzyme was cloned from Streptomyces sp. origin into a Pseudomonas fluorescens strain. However, the optimization procedure was done with the purified his-tagged tryptophan-5-halogenase, which was easily obtained from the crude extract of the lysed cells by application of immobilized metal affinity chromatography. The application of algorithms allows an optimization of the multidimensional search problem leading to a global optimum in the search space in contrast to the traditional used one-factor-at-a-time method. Latter often failures, because this method does not reflect possible influences the parameters can have on each other.

\section{References}

Hammer, P.E., Hill, D.S., Lam, S.T., van Pée, K.-H., Ligon, J.M., 1997. Appl. Environ. Microbiol. 63, 2147-2154.

Hasegawa, M., Yamada, K., Hagahama, Y., Somei, M., 1999. Heterocycles 51, 815-2821.

Keller, S., Wage, T., Hohaus, K., Hölzer, M., Eichhorn, E., van Pée, K.-H., 2000. Angew. Chem. Int. Ed. 39, 2300-2302.

\section{IB144}

Effect of organic solvent type on the enantioselectivity of Candida rugosa lipase in the hydrolysis of racemic Naproxen methyl ester in biphasic reaction system Serpil Takaç, Deniz Mutlu Department of Chemical Engineering, Institute of Biotechnology, Ankara University, 06100 Tandoğan, Ankara, Turkey

Hydrolysis of racemic Naproxen methyl ester to produce $S$ Naproxen was carried out in the biphasic system using isooctane, cyclohexane, hexane and toluene with Candida rugosa lipase after stirring in the phosphate buffer $(\mathrm{pH} 7.5,0.02 \mathrm{M})$ for $2 \mathrm{~h}$ at $+4{ }^{\circ} \mathrm{C}$ and centrifuging. The hydrolyses were carried out in shaking flasks for $120 \mathrm{~h}$ at $200 \mathrm{rpm}$ and $37^{\circ} \mathrm{C}$ with the initial substrate concentration of $0.034 \mathrm{M}$. The concentrations of the enantiomers of racemic Naproxen methyl ester in organic solvents and those of Naproxen in buffer solution were determined with HPLC. It was found that the enantiomeric excess for substrate $\left(e_{s}\right)$, enantiomeric ratio $(E)$ and conversion $(x)$ decreased in the following order: isooctane $>$ cyclohexane $>$ hexane $>$ toluene. The enantiomeric excess for product $\left(e_{\mathrm{p}}\right)$ was found to be the same for isooctane, cyclohexane and hexane where the lowest ee $e_{p}$ was obtained in toluene. The highest $\mathrm{ee}_{\mathrm{s}}, \mathrm{ee}_{\mathrm{p}}, E$ and $x$ values achieved in isooctane at the residence time of $120 \mathrm{~h}$ were $91,95,130$, and $49 \%$, respectively.

\section{Acknowledgement}

This study was supported by Ankara University Biotechnology Institute (Project No: 89).

\section{IB145}

Carbon regulation of gibberellin and bikaverin biosynthesis in Fusarium fujikuroi S. Distel, B. Mehta, J. Avalos, M.C. Limón Departamento de Genética, Universidad de Sevilla, Avenida de Reina Mercedes, 6, 41012 Sevilla, Spain. E-mail: carmenlimon@us.es (M.C. Limón)

Fusarium fujikuroi (Gibberella fujikuroi mating group C) produces multiple secondary metabolites such as gibberellins and bikaverin. Gibberellins are terpenoid hormones that induce growth and regulate various stages of development in plants. They have numerous applications in agriculture industry. Bikaverins are polyketides that have toxicity against different organisms because they inhibit respiration. Regulation of polyketide and gibberellin synthesis by nitrogen has been intensively studied in Fusarium but little is known about their regulation by carbon source. Our main interest is to understand the regulation of biosynthesis of these compounds in F. fujikuroi IMI 58289. To investigate this regulation the organism was grown in high nitrogen medium under submerged conditions and then transferred to nitrogen-free media having various concentrations of different carbon sources. The gibberellin production was not affected significantly. On the other hand bikaverin was synthesized enormously when sucrose was used as the only carbon source. High production in sucrose required a minimal amount of the sugar, but did not change appreciably above this threshold along a wide range of concentration. The bikaverin synthesis was repressed when glucose coexisted with sucrose in the medium. The effect of the $\mathrm{C}$ source on the expression of key genes, cps/ks (copalyl diphosphate synthase/kaurene synthase) for gibberellin and $p k s 4$ (polyketide synthase) for bikaverin biosynthesis is currently under investigation.

\section{Acknowledgements}

Financed by a Marie Curie Re-integration Grant MERG-CT2004-006378 and Consejería de Educación y Ciencia de la Junta de Andalucía.

\section{IB146}

Efficient plant regeneration from seeds of water lily (Nymphaea alba) under in vitro conditions Hasan Huseyin Atar ${ }^{1}$, Sule Sumlu ${ }^{1}$, Khalid Mahmood Khawar ${ }^{2}$, Sebahattin Ozcan ${ }^{3}$ : ${ }^{1}$ Aquatic Plant Biotechnology Section, Department of Fisheries, Faculty of Agriculture, University of Ankara, Turkey; ${ }^{2}$ Department of Field Crops, Institute of Biotechnology, Faculty of Agriculture, University of Ankara, Turkey; ${ }^{3}$ Department of Field Crops, Faculty of Agriculture, University of Ankara, Turkey

Water lily (Nymphaea alba) is an important and popular aquatic perenniel plant. It has been used for ornamental and pharmaceutical purposes in Turkey and various countries. Because of the develop- 
ment of dormancy and loss of viability in seeds with the passage of time, it lacks any systematic propagation from seeds and is typically propagated through rhizomes. This restricts large scale cultivation of this plant. In vitro propagation of plants is an effective means for rapid multiplication of species, in which conventional methods have limitations. In the pesent study we have analysed the role of various growth promoters and the effects of dark and light incubation on germination of $N$. alba seeds. The results indicate that in vitro propagation of $N$. alba from seeds can be applied as an efficient method of multiplication.

\section{Acknowledgements}

The study was funded by the State planning commisson (DPT) Turkey and The University of Ankara vide Projects No. 120640.

\section{IB147}

Study of operation condition of biofilter using fibril-form matrix for odor gas removal Don-Hee Park, Gwi-Taek Jeong, Kyoung-Min Lee, Hyun-June Park School of Biological Science and Technology, Chonnam National University, Gwangju 500-757, South Korea. Email:dhpark@chonnam.ac.kr (D.-H. Park)

This research was performed for developing of biological treatment process of odor gas such as MEK, $\mathrm{H}_{2} \mathrm{~S}$, and toluene, which is generated from the food waste recycling process. To establish the operational conditions of odor gas removal by small-scale biofiltration equipment, it was continuously operated by using toluene as a treating odor object. When the odor treating microorganisms were adhered to fibril form biofilter, high removal efficiency over $93 \%$ was obtained by biofilm formation. At $400 \mathrm{ppm}$ of inlet odor gas concentration and $10 \mathrm{~s}$ of retention time, the removal efficiency was 76 and $93 \%$ in first stage reactor and second stage reactor, respectively. However, the removal efficiency remained over $97 \%$ at the operational conditions above $15 \mathrm{~s}$ of retention time.

\section{IB148}

Regulation of treating with GMO's in transition countries (Eastern Europe counties) Dzvenyslava Kachur National University, Kyiv-Mohyla Academy, Ukraine

Post Soviet Countries are going through the transition stage and are extremely sensitive to new technology, economics or social changes and globalization processes. That is why decision-making and system of regulation of the use of GMO's are very sensitive to number of factors. Three levels of factors, the most influential to decision-making: global, regional and local; are identified at the research paper. Global level depends on external policy of leaders of GMO regulations. USA and EU have the biggest influence on transition countries, though their positions completely differ. As USA was leader in inventing GMOs, it is lobbing newly created biotechnological industry. In EU and other European countries lobbing of biotechnological industry was not as strong as in USA. Thus, their National law is stricter. World Trade Organization and International Agreements are also part of Global level. Regional level. Geographical position of country is also very important because every regulation system depends a lot on regulation that is implemented in neighboring countries. Countries do not exist in vacuum; they are linked territorially, politically, economically and socially with neighboring states. Regulation systems of the transition countries in the Eastern Europe can be divided into three types: those who have no system of regulation of GMO (Belarus, Romania, Hungary and Ukraine); who approved some variety that are treated as safe to the market (Poland, Moldavia and Georgia) and who approved all of the GMOs (Bulgaria, Croatia and Russia [before 2004]). But even if a country declares not to use GMO it is rather difficult to control import of such products, because of the lack of the testing laboratories, corruption of the state employees, agreements on intellectual property, and institutional country problems. In spite of global and regional tendency of GMOs related regulation the most important part is the local level, namely the national regulation system. Depending on national level we are choosing priorities at higher levels. As an example of Post Soviet Countries Ukraine is taken, as Ukraine is one of the largest countries and one of the biggest exporters of agricultural products in Europe. Research includes the analysis of attitude to GM product, their potential risks and benefits of three categories that influence decision-making the most: farmers, GM experts and Non-Governmental Organizations.

\section{IB149}

Recombinant microorganism development for extracellular benzaldehyde lyase production Hande Kaya ${ }^{1}$, Plnar Çalık ${ }^{1}$, Tunçer H. $\ddot{O}_{z \text { damar }}{ }^{2}$ : ${ }^{1} \mathrm{IBLab}$, Department of Chemical Engineering, METU, 06531 Ankara, Turkey; ${ }^{2}$ BRE Laboratory, Department of Chemical Engng, Ankara University, 06100 Ankara, Turkey. E-mail: e119497@metu.edu.tr (H. Kaya)

In this study, the extracellular production of the benzaldehyde lyase (BAL, EC 4.1.2.38) that catalyses the synthesis the enantiopure 2-hydroxy ketones for drug syntheses, by Bacillus sp., was aimed. For this purpose, the signal DNA sequence of an extracellular Bacillus enzyme, i.e., serine alkaline protease, was fused in front of the $b a l$ gene (accession number AX349268) from Pseudomonas fluorescens Biovar I, using PCR-based gene splicing by overlap extension (SOE) method. B. licheniformis (DSM 1969) chromosomal DNA was used as SAP gene (accession number X03341) template for the synthesis of SAP signal sequence. bal gene was amplified by using the plasmid carrying bal gene, pUC18::bal. Thereafter, the signal peptide of SAP with its own promoter was fused in front of the bal gene by SOE method. The hybrid gene first cloned into pUC19 plasmid, thereafter sub-cloned into pBR373, pMK4 and pHV1431 shuttle vectors. The Escherichia coli-Bacillus plasmids carrying the hybrid gene pre(subC)-bal was transferred into Bacillus subtilis npr-apr-, and Bacillus licheniformis. The influence of the host Bacillus species on BAL production on a defined medium with glucose was investigated in bioreactor systems. For each of the recombinant (r-) Bacillus species, effects of initial glucose concentration on cell growth and BAL production were investigated; and, physiological differences and similarities between the wild-type and r-Bacillus species are discussed. Thereafter, the benzaldehyde lyase production capacities of recombinant $E$. coli and $B$. subtilis are compared in terms of cell concentration and BAL volumetric and specific activities. For the comparison bal gene was cloned into pRSETA vector which is under the control of strong T7 promoter and expressed in E. coli BL21 (DE3) pLysS strain. The variations in by-product distributions with each recombinant organism and yields are also discussed. 


\section{IB150}

Characterization of the D-xylulose 5-phosphate/D-fructose 6phosphate phosphoketolase gene, xpkL, from Cellulomonas flavigena L. Amaya-Delgado, M.E. Hidalgo-Lara, M.C. MontesHorcasitas Biotechnology and Bioengineering Department, CINVESTAV IPN, D.F., Mexico City, C.P. 07360, Mexico. E-mail: lamaya@cinvestav.mx (L. Amaya-Delgado)

Phosphoketolases (EC 4.1.2.9) are thiamine diphosphate (ThDP)-dependent enzymes, that play a crucial role in the pentose phosphate pathway (PPP) of heterofermentative and facultative homofermentative lactic acid bacteria, and of the D-fructose 6phosphate shunt of bifidobacteria. Reports affirm that Cellulomonas flavigena can use the PPP when is cultured under anaerobic conditions. A genomic library of $C$. flavigena constructed in $\lambda$-ZAP Express vector was screened. Four positive clones were isolated, in vivo excised and the resulting $\mathrm{pBK}-\mathrm{CMV}$ phagemids, each containing a $4.0 \mathrm{~kb}$ insert, were characterized by restriction analysis and DNA sequencing. The open reading frame (ORF) of the Dxylulose 5-phosphate/D-fructose 6-phosphate phosphoketolase gene, $x p k L$, was located from nucleotide 54 to 2466. The $x p k L$ ORF encoded a 804 amino acids-residue polypeptide (XPKL) with a calculated molecular mass of $89,000 \mathrm{Da}$, a value coincident with that estimated by comparative SDS-PAGE (about 90,000 Da). A putative ribosome binding site (GGGAGC) is present 11-5 nucleotide upstream of the translational start of the XPKL polypeptide. The C. flavigena XPKL polypeptide sequence was $66 \%$ identical to Dxylulose 5-phosphate/D-fructose 6-phosphate phosphoketolase from Bifidobacterium sp., Bifidobacterium gallinarum, Gloeobacter violaceus and Bifidobacterium adolescentis. This analysis also revealed highly conserved regions.

\section{IB151}

Characterization and functional analysis of Lactococcus lactis RNase III Sandra Cristina Viegas, Mónica Amblar, Cecilia Maria Arraiano Instituto de Tecnologia Química e Biológica, EAN, 2784505 Oeiras, Portugal. E-mail: sviegas @itqb.unl.pt (S. Viegas)

Lactococcus lactis is a main diacetyl-producing bacteria by citrate metabolism in dairy products. The transport of citrate in these bacteria is dependent on citrate permease that is encoded by citP gene. Previous studies of the citQRP operon in Escherichia coli mutants showed that citP message is considerably stabilized in RNase III mutant. So, in the context of the citrate metabolism research, the characterization of the lactococcal RNase III enzyme is very important for the dairy industry. RNase III is an endoribonuclease which has an important role in rRNA processing and control of gene expression. With the aim of studying lactococcal RNase III we have cloned the $r n c$ gene from L. lactis ssp lactis IL1403 in the broad host range pLS1RGFP vector. This plasmid includes the $g f p$ gene, encoding the green fluorescent protein (GFP), cloned under the control of the PM promoter, that is inducible by maltose. Maltose induction of the lactococcal $r n c$ expression showed a 5-fold increase of $r n c$ transcription from this plasmid. Activity assays for lactococcal RNase III were standardized using crude extracts and a substrate specific for B. subtillis RNase III. The results showed that this substrate was specifically cleaved by lactococcal RNase III and its activity induced by maltose. Lac-rnc was cloned in pET15 vector and the corresponding six-histidine-tagged RNase III protein was overproduced in E. coli BL21 (DE3) strain by IPTG induction. The protein was purified by affinity chromatography using HPLC system and was shown to be active by in vitro activity assays using the LacRNase III specific substrate mentioned above. We have also cloned lactococcal $r n c$ gene and studied its expression in an E. coli rnc deletion mutant $(\Delta r n c)$. Complementation assays performed in E. coli demonstrate that the lactococcal RNase III (Lac-RNase III) is able to process rRNAs and to regulate the levels of Polynucleotide Phosphorylase (PNPase). These results demonstrate that the lactococcal enzyme is able to substitute the Ec-RNase III not only in the rRNA processing, but also in the processing of mRNAs. The amount of lactococcal $r n c$ transcript in an E. coli $\Delta r n c$ strain was 3.3-fold higher than in the wild type strain, suggesting that the E. coli RNase III triggers the degradation of the heterologous $r n c$ mRNAs. The results obtained have shown that Lac-RNase III is an interesting enzyme for biotechnological purposes.

\section{IB152}

Co-immobilization of dextranase and dextransucrase into Caalginate for the facilitated enzymatic synthesis of isomaltose Frank A. Erhardt, Hans-Joachim Jördening, Klaus Buchholz Technical University of Braunschweig, Chair for Carbohydrate Technology, Braunschweig, Germany. E-mail: f.erhardt@tu-bs.de (F.A. Erhardt)

Objectives: The pharmaceutical and food industry has an increasing demand for selectively glycolized active agents. In our application isomaltose can be synthesized by immobilized dextransucrase, which transfers a glycosyl residue from sucrose (substrate) to glucose (acceptor). As the reaction proceeds, isomaltose can act as an acceptor and is converted into undesired follow-up products called isomalto-oligosaccharides, IMOs. We investigate on two approaches to avoid $I M O$ formation, selective adsorption of isomaltose (Ergezinger et al., 2005) and the co-entrapment of dextranase adsorbate, which breaks IMOs down to isomaltose.

Results and conclusions: The first part of our research concerns the adsorption of dextranase on bentonite, which complies with Langmuir model. At complete saturation $\left(0.8 \mathrm{~g} \mathrm{~g}^{-1}\right)$ our immobilisate exhibits an activity of $16,000 \mathrm{U} \mathrm{g}^{-1}$. A kinetic analysis does not reveal significant differences between the adsorbed and free form of enzyme $\left(K_{\text {m,bentonit }}: 14.1 \pm 0.7 \mu \mathrm{M}\right.$ versus $\left.K_{\mathrm{m} \text {,free }}: 13.0 \pm 0.7 \mu \mathrm{M}\right)$. Thus, bentonite displays a high binding capacity paired with favorable kinetic properties. Beyond that we investigate the activity of dextransucrase in co-immobilisates, which is reduced during coimmobilization due to interactions with the adsorbate. Among various co-immobilisates, the one containing dextranase bound to preblotted bentonite imparts the highest activity ( $40 \%$ as compared to control: immob. dextransucrase). The molar yield coefficient of coimmobilisates $Y_{\text {isomaltose/sucrose }}$ surpasses coefficient of control by $13 \%$. Further on we will characterize mass transfer of dextranase substrate into alginate matrix as well as bentonite-dextransucrase interactions.

\section{Acknowledgements}

This project is part of the collaborative research centre SFB 578 "Development of biotechnological processes by integrating genetic and engineering methods", which is supported by the German Research Foundation DFG. 


\author{
Reference \\ Ergezinger, M., Bohnet, M., Berensmeier, S., Buchholz, K., 2005. \\ Integrierte enzymatische synthese und adsorption von isomaltose \\ in einem mehrphasenbioreaktionsreaktor. CIT 77, 167-171.
}

\section{Food Biotechnology}

\section{Enzymes and Functional Ingredients}

1

Glucansucrases: Versatile catalysts for the synthesis of functional food G. Richard, E. Fabre, C. Moulis, G. Joucla, C. Albenne, G. Potocki-Veronese, P. Monsan, M. Remaud-Simeon BiotechnologyBioprocess Laboratory, UMR CNRS 5504, UMR INRA 792, INSA, 135 Rangueil Avenue, 31077 Toulouse Cedex 4, France

Glucansucrases from family 70 of glycoside-hydrolases are transglucosidases that produce $\alpha$-glucans from sucrose, a very cheap substrate, without any use of nucleotide activated sugars. Based on sequence analyses, these enzymes have been classified in two families, the family 70 and the family 13 of glycoside hydrolases. Among the natural diversity existing in family 70 in which are found the glucansucrases produced by lactic acid bacteria, three enzymes have been selected for their distinctive specificities: dextransucrase from L. mesenteroides NRRL B-512F (DSR-S), which catalyses almost essentially the synthesis of $\alpha-1,6$-linkages, alternansucrase from L. mesenteroides NRRL B-1355 (ASR), which produces alternan polymer formed of $\alpha-1,6-$ and $\alpha-1,3$-alternated linkages and finally dextransucrase from L. mesenteroides NRRL B-1299 (DSRE), which is responsible for the synthesis of a branched dextran composed of about $70 \%$ of $\alpha-1,6$-linkages in the main chain and $30 \%$ of $\alpha-1,2$-branched linkages. For all these enzymes, the natural polymerase activity can be shifted towards oligosaccharide production or gluco-conjugate syntheses by introducing acceptors in the reaction medium. A number of sugar acceptors have been successfully glucosylated with the view of developing new functional food products. Acceptor glucosylation yield as well as acceptor reaction product structures were shown to be highly dependant on the enzyme specificity. Consequently, using glucansucrases of distinctive specificities and varying the acceptors give access to a large variety of applications. Amylosucrase, the sole glucansucrase found in Family 13 of glycoside-hydrolases is also of great interest for functional food applications. This enzyme is able to synthesize highly resistant amylose from sucrose. Again the reaction conditions can be used to modulate the yield and the size of amylose. The aim of our work is to further develop the applications of these enzymes via rational and combinatorial engineering. The most recent results obtained in this field will be discussed.

2

Novel food structure engineering concepts with enzymes Johanna Buchert VTT Biotechnology, Espoo, Finland

Food structure is a very important quality attribute in food choice, since it affects not only the sensory perception of texture, but also release of flavour. Enzymes offer specific means to engineer food structure by creating cross-links to food biopolymers, i.e. to proteins and/or carbohydrates. Enzymatic cross-linking of food biopolymers can be exploited to create novel types of structures to foods without any need of added food ingredients. Laccases and peroxidases can be used to crosslink ferulic acid containing carbohydrates, such as sugar beet pectin or arabinoxylan. Proteins can be crosslinked by different oxidative or transferase type of enzymes. Transglutaminases can crosslink protein via formation of isopeptide bond between glutamine and lysine residues. Laccase and peroxidase can oxidize tyrosine residues to corresponding radicals, which in turn can further react with different groups in proteins. Tyrosinases, on the other hand, oxidize tyrosine to a quinone, which can further react with aromatic ring, amine and thiol groups present in proteins. The biopolymer networks formed can be further engineered by combining adequate processing to the enzyme treatment. In this work the potential of enzymatic food structure engineering is reviewed.

3

Asparaginase-mediated reduction of acrylamide formation in baked, fried, and roasted products Hanne Vang Hendriksen, Beate Kornbrust, Steffen Ernst, Mary Stringer, Hans Peter Heldt-Hansen, Peter Østergaard Novozymes A/S, DK-2880 Bagsvaerd, Denmark. E-mail: hvhe@novozymes.com (H.V. Hendriksen)

In 2002, it was discovered that acrylamide is formed in several potato and grain-based foods (e.g. chips, French fries, toasted bread, biscuits, cereals) and in coffee, all of which have been prepared at high temperatures. The level of this potential carcinogen in the final food appears to range from 50 to $4000 \mathrm{ppb}$. Later that year, the mechanism of acrylamide formation was unraveled, demonstrating that asparagine and reducing sugars are the precursors for acrylamide. This pointed to several potential enzymatic approaches to remove the root cause of the problem by degrading the precursors in situ. Here, we demonstrate that asparaginase treatment leads to a more efficient reduction in acrylamide than alternative enzymatic treatments. Asparaginase from Aspergillus oryzae is used to reduce acrylamide formation significantly in laboratory models of a range of common food products. Examples are French fries, biscuits, crisp bread, and fabricated chips. The sensory qualities appear to be constant. The implications for scaling up the processes for industrial food production are discussed.

\section{4}

Effect of cultivation conditions on folate content in yeast: Exploring the potential of yeast as a bio-enrichment vehicle for folate in foods Sofia Hjortmo ${ }^{1}$, Johan Patring ${ }^{2}$, Jelena Jastrebova ${ }^{2}$, Thomas Andlid ${ }^{1}$ : ${ }^{1}$ Department of Chemical and Biological Engineering, Chalmers University of Technology, PO Box 5401, 40229 Gothenburg, Sweden; ${ }^{2}$ Department of Food Science, Swedish University of Agricultural Sciences, PO Box 7051, 75007 Uppsala, Sweden. E-mail: sh@fsc.chalmers.se (S. Hjortmo)

Over the past 10 years, the interest in health benefits of the B vitamin folate has increased considerably. A good folate status may hinder progression of several diseases such as neural tube defects and downs syndrome in foetus, as well as cancer, dementia, Alzheimer's disease and cardiovascular disease in adults. It is however not easy to reach the recommended intake and new strategies have to be developed to increase the folate status. In this project we explore the use folate producing microorganisms for this purpose. Many yeasts have the ability to synthesise folate de novo and can thus serve as a source for humans. Folate enrichment in fermented foods could be much improved by using starter cultures better at producing folate compared to traditional strains. This is, e.g. applicable to bread making 
and kefir. A second approach is to use yeast as a production organism to produce natural folates for fortification. Here we investigate and discuss the folate content in SKQ2n, a diploid strain of Saccharomyces cerevisiae, when cultured in different media and at different stages of growth. The aim is to gain a basal knowledge of the folate production profile, forms of folate produced and degree of leakage to the surrounding medium, in relation to the culturing medium and physiological state of the cells.

\section{5}

Enzymatic conversion of starch to antioxidants, antimicrobials and fine chemicals, the Anhydrofructose technology Shukun $Y u$ Danisco Innovation, Danisco A/S, Langebrogade 1, PO box 17, DK 1001 Copenhagen K, Denmark

We at Danisco A/S (Copenhagen, Denmark) have revealed a new starch degrading pathway by the discovering several new enzymes and metabolites in fungi and algae. These new enzymes include glucan lyases, dehydratases and tautomerases, which proved to be useful in biocatalysis. These new metabolites proved to be useful as both antioxidants and antimicrobials for food and non-food applications. This pathway is named as Anhydrofructose pathway of starch and glycogen degradation. This technology is referred to as the Anhydrofructose technology.

\section{References}

Andersen, S.M., Lundt, I., Marcussen J., Yu, S., 2002. 1,5-AnhydroD-fructose; a versatile chiral building block: biochemistry and chemistry. Carbohydr. Res. 337, 873-890.

Lee, S.S., Yu, S., Withers, S.G., 2003. Detailed dissection of a new mechanism for glycoside cleavage: the $\alpha-1,4$-glucan lyase. Biochemistry 42 (44), 13081-1390.

Yu, S., 2000. $\alpha-1,4-G l u c a n$ lyase, a new class of starch and glycogen degrading enzyme. Trends Glycosci. Glycotechnol. 12, 381-389.

Yu, S., 2004. $\alpha-1,4-G l u c a n$ lyase, a new starch processing enzyme for production of 1,5-anhydro-D-fructose. Sugar Industries/Zuckerindustrie 129 (1), 26-30.

Yu, S., Christensen, T.M.I.E., Kragh, K.M., Bojsen, K., Marcussen, J., 1997. Efficient purification, characterization and partial amino acid sequencing of two $\alpha-1,4$-glucan lyases from fungi. Biochim. Biophys. Acta 1339, 311-320.

Yu, S., Bojsen, K., Svensson, B., Marcussen, J., 1999. $\alpha-1,4-G l u c a n$ lyases producing 1,5-anhydro-D-fructose from starch and glycogen have sequence similarity to alpha-glucosidases. Biochim. Biophys. Acta 1433 (1-2), 1-15.

Yu, S., Refdahl, C., Lundt, I., 2004. Enzymatic description of the anhydrofructose pathway of glycogen degradation I. Identification and purification of anhydrofructose dehydratase, ascopyrone tautomerase and $\alpha-1,4$-glucan lyase in the fungus Anthracobia melaloma. Biochim. Biophys. Acta 1672, 120-129.

\section{Omics in Food Biotechnology}

\section{1}

-Omics for food: Protein and gene expression profiling towards a better understanding of nutritional health M. Affolter, G.E. Bergonzelli, L.B. Fay, C. Garcia-Rodenas, L.V. Lopes, L. MarvinGuy, D.M. Mutch, A. Panchaud, F. Raymond, A. Schumann, G. Williamson, M. Kussmann Nestlé Research Centre (NRC), Nestec Ltd., Vers-chez-les-Blanc, CH-1000 Lausanne 26, Switzerland
Diet is evolving from nourishing populations via providing essential nutrients to improving health of individuals through nutrition. Modern nutritional research focuses on health promotion and disease prevention, on protection against toxicity and stress, and on performance improvement. As a consequence of these ambitious objectives, the disciplines "nutrigenetics" and "nutrigenomics" have evolved. Nutrigenetics asks the question how individual genetic disposition, manifesting as single-nucleotide polymorphisms (SNPs), copy-number polymorphisms (CNPs) and epigenetic phenomena, affects susceptibility to diet. Nutrigenomics addresses the inverse relationship, i.e. how diet influences gene transcription, protein expression and metabolism. The mid-term objective of nutrigenomics is integrating genomics (gene analysis), transcriptomics (gene expression analysis), proteomics (global protein analysis) and metabolomics (metabolite profiling) to define a "healthy" phenotype. The long-term deliverable of nutrigenomics is personalised nutrition for maintenance of individual health and prevention of disease. The major challenges for-omics in nutrition and health still lie ahead of us, some of which apply to -omic disciplines in general while others are specific for-omic discovery in the food context: (i) the integration of gene- and protein expression profiles with metabolic fingerprints is still in its infancy as we need to understand how to (a) select relevant sub-sets of information to be merged, and (b) resolve the issue of the different time-scales, at which transcripts, proteins and metabolites appear and act; (ii) the definition of health and comfort is less of a clear-cut case than the one of disease; (iii) -omics in nutrition must be particularly sensitive: it has to reveal rather many subtle than a few abundant signals to detect early deviations from normality; (iv) in the food context, health cannot be uncoupled from pleasure, that is, food preference and nutritional status are interconnected. Transcriptomics serves to put proteomic and metabolomic markers into a larger biological perspective and is suitable for a first "round of discovery" in regulatory networks. Metabolomics, the comprehensive analysis of metabolites, is an excellent diagnostic tool for consumer classification. The great asset of this platform is the quantitative, non-invasive analysis of easily accessible human body fluids like urine, blood and saliva. This feature also holds true to some extent for proteomics, with the constraint that proteomics is more complex in terms of absolute number, chemical properties and dynamic range of compounds present. Proteomics in the context of nutrition and health has the potential to (a) deliver biomarkers for health and comfort, (b) reveal early indicators for disease disposition, (c) assist in differentiating dietary responders from non-responders, and, last but not least, (d) discover bioactive, beneficial food components. Independent of the context of application, proteomics represents the only platform that delivers not only markers for disposition or condition but also targets of intervention: the only way to intervene in a biological condition and to modulate its outcome is interfering with the proteins involved. It is evident that not only comprehensive analyses with one discovery platform (lateral integration of information) are required but also vertical integration between different -omic levels are indispensable for a deeper understanding of disposition, health, environment and diet (Desiere, 2004). A major "vertical integration issue", to date unresolved, is given by different timescales of transcript production, protein expression and metabolite generation (Nicholson et al., 2004). The transcript machinery usually responds fast to an external stimulus (seconds to minutes), the 
proteins may be expressed within minutes to hours (and have a halflife from minutes to even months) and metabolites vary significantly during the day and depend on latest dietary input. This means that data, which seem to correlate qualitatively (e.g. reflecting the same pathway), may not necessarily be related time-wise. Rather, they may represent different responses at different time points and, possibly, to different stimuli. Comprehensive -omic analyses is an essential building block of "systems biology", which can be defined as follows (Clish et al., 2004): Systems biology is the comprehensive analysis of the dynamic functioning of a biological system (cell, organ, organism or even ecosystem) at gene, protein and metabolite (or higher organizational) level, achieved by comparison of two defined biological states of this system, typically before and after perturbation. While a comprehensive list of components (genes, proteins, metabolites) of a given biological system is a pre-requisite for this kind of research, the main reasoning for the "system view" is that only information on the interactions between the components gives clues to function of the entire network. A systems biology approach has recently demonstrated the power of proteomics to dissect immunity and inflammation. Toll-like receptor recognition and signalling was elucidated and showed, how bacterial "barcodes" are read and interpreted in order to trigger an adapted immune response (Aderem and Smith, 2004). In order to address some of the challenging objectives of-omics-driven nutritional research, we have addressed (a) the effect of early antibiotic administration on the maturation of intestinal tissues, (b) protein discovery in human milk, (c) the effects of polyunsaturated fatty acids on gene expression and lipid profile in the liver, and (d) biomarkers for intestinal stress.

(a) Antibiotics and gut maturation: The effects of early administration of antibiotics on intestinal maturation were assessed at the gene expression level in a rat model.

(b) Human milk: Rapid enrichment and iterative, consolidated identification of immunologically relevant milk proteins was achieved through the employment of restricted-access media and a tailored proteomic strategy (Labéta et al., 2000; LeBouder et al., 2003; Panchaud et al., 2005).

(c) Fatty acids and liver transcriptome/lipidome: Epidemiological studies have correlated higher intakes of poly-unsaturated fatty acids (PUFAs) with lower incidence of chronic metabolic disease. The molecular mechanisms regulated by PUFA consumption were examined assaying the liver transcriptome and lipid metabolome of mice fed a control and a PUFA-enriched diet (Mutch et al., 2005).

(d) Gut stress markers: As a first step, we catalogued protein expression along the jejunum, ileum and colon of the rat intestine and found gut segment-specific proteins (Marvin-Guy et al., 2005). The innovative combination of a neonatal separation model with proteomic analysis allowed us to study, whether early life psychological stress may impact the adult gut neuromuscular protein expression and the approach revealed specific protein biomarkers.

\section{References}

Aderem, A., Smith, K.D., 2004. A systems approach to dissecting immunity and inflammation. Semin. Immunol. 16, 55-67.
Clish, C., C.B., et al., 2004. Integrative Biological Analysis of the APOE*3-Leiden Transgenic Mouse. OMICS. 8, 3-13.

Desiere, F., 2004. Biotechnol. Annu. Rev. 10, 51-84.

Labéta, M.O., Vidal, K., Rey-Nores, J.E., Arias, M., Vita, N., Morgan, B.P., Guillemot, J.C., Loyaux, D., Ferrara, P., Schmid, D., Affolter, M., Borysiewicz, L.K., Donnet-Hughes, A., Schiffrin, E.J., 2000. Innate recognition of bacteria in human milk is mediated by a milk-derived highly expressed pattern recognition receptor, soluble CD14. J. Exp. Med. 191, 1807-1812.

LeBouder, E., Rey-Nores, J.E., Rushmere, N.K., Grigorov, M., Lawn, S.D., Affolter, M., Griffin, G.E., Ferrara, P., Schiffrin, E.J., Morgan, B.P., Labéta, M.O., 2003. Soluble forms of Toll-like receptor (TLR)2 capable of modulating TLR2 signaling are present in human plasma and breast milk. J. Immunol. 171, 6680-6689.

Marvin-Guy, L., Lopes, L.V., Affolter, M., Courtet-Compondu, M.C., Wagnière, S., Bergonzelli, G.E., Fay, L., Kussmann, M., in press. Proteomics of the rat gut: analysis of the myenteric plexuslongitudinal muscle preparation. Proteomics.

Mutch, D., Grigorov, M., Berger, A., Fay, L.B., Roberts, M.A., Watkins, S.M., Williamson, G., German, J.B., 2005. An integrative metabolism approach identified stearoyl-CoA desaturase as a target for an arachidonate-enriched diet. FASEB J.

Nicholson, J.K., Holmes, E., Lindon, J.C., Wilson, I.D., 2004. The challenges of modeling mammalian biocomplexity. Nat. Biotechnol. 22, 1268-1274.

Panchaud, A., Kussmann, M., Affolter, M., in press. Rapid enrichment of bioactive milk proteins and iterative, consolidated protein identification by MudPIT technology. Proteomics.

\section{2}

Omics for engineering lactic acid bacteria Willem $M$. de Vos Wageningen Center for Food Sciences, Wageningen University, The Netherlands. E-mail: willem.devos@wur.nl. Url: http://www.wcfs.nl/

Lactic acid bacteria (LAB) are high AT-rich gram-positive bacteria that have a well-established record in industrial food fermentations where they contribute to conservation, flavour and texture. In addition, several LAB are used as food-grade hosts for the production of enzymes, peptides or metabolites. Finally, LAB are exploited in functional foods that contribute to the health and well-being of the consumer. A variety of metabolic engineering approaches have allowed for the improvement of many attributes of LAB. These approaches have been facilitated by the possibility of uncoupling of growth and metabolite production in LAB, the wealth of genetic tools that allow modulation of gene expression in a dynamic range, and the determination of several complete LAB genomes (de Vos et al., 2005). We have developed Lactobacillus plantarum as a paradigm for LAB engineering by experimental and modelling approaches, the application of functional and comparative genomics, and the implementation of other post-genomics avenues (Kleerebezem et al., 2003; Smid et al., 2005; de Vos et al., 2004). Examples of optimizing the production of vitamins and other cofactors, the impact of these engineering approaches on the global transcription and metabolite profiles, and determining the $L$. plantarum activity in the human host will be discussed. 


\section{References}

de Vos, W.M., Bron, P.A., Kleerebezem, M., 2004. Post-genomics of lactic acid and other food-grade bacteria to discover gut functionality. Curr. Opin. Biotechnol. 15, 86-93.

de Vos, W.M., Kleerebezem, M., Kuipers, O.P., in press. Genetics, metabolism and application of lactic acid bacteria. FEMS Microbiol. Rev.

Kleerebezem, M., et al., 2003. Complete genome sequence of Lactobacillus plantarum WCFS1. Proc. Natl. Acad. Sci. U.S.A. 100, 1990-1995.

Smid, E., Molenaar, D., Hugenholtz, J., de Vos, W.M., Teusink, B., 2005. Functional ingredient production: application of global and metabolic models. Curr. Opin. Biotechnol. 16, 190-197.

3

Global transcript profiling of potato tuber using serial analysis of gene expression Kåre L. Nielsen, Karen Grønkjar, Karen G. Welinder, Jeppe Emmersen Department of Life Sciences, Aalborg University, Sohngaardsholmsvej 49, DK-9000 Aalborg, Denmark

Solanum tuberosum (potato) is the fourth major crop worldwide and used for food, feed and biotechnological applications. To fully realize the biosynthetic potential for production of starch, protein and metabolites, we conducted an extensive quantitative profiling of the expressed genes of mature potato tuber. A total of 58,322 SAGE (serial analysis of gene expression) tags of $19 \mathrm{nt}$ representing 22,235 different tags were analyzed. The 695 tags seen 10 or more times were assigned a tentative function by comparison to homologous genes. Contrary to the transcript profile of rice seedlings (Gibbings et al., 2003) the storage organ of potato is not dominated by transcripts encoding storage proteins. Transcripts for four types of protease inhibitors, a metallothionein and a lipoxygenase were more prominent than patatin isoforms.

\section{Reference}

Gibbings, et al., 2003. Plant Biotechnol. J. 1, 271-285.

\section{4}

Proteome and transcriptome analysis of Lactococcus lactis stress responses Morten Ejby ${ }^{1}$, Ljiljana Nesic ${ }^{2}$, Natascha H. Beyer ${ }^{3}$, Allan K. Nielsen ${ }^{4}$,Marzanna Pulka-Amin ${ }^{1}$, Michael D. Rasmussen ${ }^{4}$, Peter Roepstorff ${ }^{3}$,Karin Hammer ${ }^{1}$, Mogens Kilstrup ${ }^{1}{ }^{1}$ Microbial Physiology and Genetics, BioCentrum-DTU, Denmark; ${ }^{2}$ Biochemistry and Nutrition, BioCentrum-DTU, Denmark; ${ }^{3}$ Protein Group, University of Southern Denmark, Odense, Denmark; ${ }^{4}$ Novozymes, Bagsværd, Denmark

The lactic acid bacterium Lactococcus lactis is used extensively in the production of fermented milk products. During cheese production the bacterium experiences many changes in its immediate environment, as a result of its own reactions. The most severe change is the accumulation of lactic acid, which changes the $\mathrm{pH}$ of the medium until growth is totally inhibited. We have focused upon a survey of these dairy related stress responses, as a means of constructing more robust strains. When L. lactis starter cultures are produced in rich media, they will experience an initial period with purine limitation after being added to the milk substrate, a stress condition that in several studies have been found to induce cross resistance towards a number of other stresses. We have analyzed both general purine nucleotide (ATP and GTP) and specific GTP limitation in chemi- cally defined medium, using both proteomics and transcriptomics. The differential expression analyses were performed with a custom designed DNA microarray of PCR amplified probes. The two stress conditions resulted in very different stress responses, both at the transcriptomic and proteomics level. From a new study on the temporal expression pattern of L. lactis during growth in milk, we present preliminary data showing differential expression of genes and proteins of the purine stress stimulon as well as other stress stimulons.

\section{5}

Physiology and global transcriptional profile of Saccharomyces cerevisiae glucose repression mutants $\underline{R}$. Usaite, J. Nielsen, L. Olsson Center for Microbial Biotechnology, BioCentrum-DTU, Technical University of Denmark, DK-2800 Lyngby, Denmark. E-mail: ru@biocentrum.dtu.dk (R. Usaite)

Cell physiology of the yeast Saccharomyces cerevisiae glucose repression mutants $\Delta s n f 1, \Delta s n f 4$ and $\Delta s n f 1 \Delta s n f 4$ was studied in batch and glucose limited chemostat cultivations. Detailed physiological studies were performed on cells grown in batch using glucose, galactose, or glucose-galactose mixture as a carbon source. During growth on glucose-galactose mixtures it was shown that after glucose was consumed, galactose consumption remained repressed for about $15 \mathrm{~h}$ in $\Delta s n f 1$ or $\Delta s n f 4$ mutants, and for more then $40 \mathrm{~h}$ in $\Delta s n f 1 \Delta s n f 4$ mutant, whereas it only lasted $6 \mathrm{~h}$ in wild-type cells. The global transcriptional response in the glucose repression mutants was studied using chemostat cultures. S. cerevisiae wild type and the mutants were grown in glucose limited aerobic chemostats at a dilution rate of $0.1 \mathrm{~h}^{-1}$. Biological triplicates were performed for each strain. To identify transcriptional responses of the glucose repression mutants, statistical tests, clustering method and a model-driven analysis method were used. The global transcription data analysis experiments showed that genes involved in hexose transport, carbohydrates metabolism, respiration, and signal transduction were differently expressed in $\Delta s n f l$ and $\Delta s n f 4$ mutants comparing to wild type cells. Combination of gene expression data and gene-scale metabolic model indicated changes in the metabolic sub-networks among studied glucose repression mutants.

\section{Genomics in Food Science}

Assessing molecular functions of food constituents by genomics technologies Hannelore Daniel Molecular Nutrition Unit, Technical University of Munich, Am Forum 5, D-85350 FreisingWeihenstephan, Germany

Genomics technologies have recently been introduced into food and nutrition science for identifying targets of molecular actions of nutrients as well as non-nutrient components of foods. Changes in the transcriptome, proteome and metabolome have been determined for assessing the molecular actions of zinc as an essential micronutrient and of flavonoids in processes such as colon carcinogenesis and atherosclerosis. Zinc is essential for the structural and functional integrity of cells and plays a pivotal role in the control of gene expression. Zinc deficiency effects in human cells and an animal model (rats) were analyzed by microarrays and showed that a low intracellular zinc concentration caused major alterations in the steady-state mRNA levels of several hundred target genes-dependent on the tissues studied including liver, brain, muscle, intestine and kidney. 
Proteome analysis from the same samples by 2D-PAGE followed by peptide mass fingerprinting via MALDI-TOF-MS identified similarly a large set of proteins with altered expression level but allowed a common theme of action to be identified. Although pleiotropic in first view, the obtained pattern of zinc-affected genes/proteins may represent a reference for defining the zinc regulon in mammalian cells. Flavonoids occurring in large number in plant species are considered protective agents in a variety of processes including inflammation and cancer development. We have studied the effects of around 80 selected flavonoids in a screening program and identified for compounds such as flavon, genistein or quercetin by genomics technologies their putative mode of action in colon cancer models and endothelial cells. As part of a collaborative effort employing human endothelial cells and blood mononuclear cells from a human intervention trial with soy isoflavones (genistein/daidzein) the effects of the flavonoids on the stress-response to oxidized LDL and homocystein was analyzed. A set of markers of anti-inflammatory and anti-apoptotic activity was identified for genistein and daidzein and cell biological studies confirmed that both compounds prevented programmed cells death in stressed endothelial cells.

\section{3}

Genomics to detect extreme thermal resistant bacterial spore formes and steer (real-time) adjustments of food processing $\underline{\text { S. Brul }}^{1,2}$, B.J.F. Keijser ${ }^{1}$, H. van der Spek ${ }^{1}$, S.J.C.M. Oomes ${ }^{2}$, R. Montijn ${ }^{3}:{ }^{1}$ Department of Molecular Biology and Microbial Food Safety, Swammerdam Institute for Life Sciences, University of Amsterdam, 1018 WV Amsterdam, UK; ${ }^{2}$ Department of Microbiology, Unilever Food Research Center, Vlaardingen, The Netherlands; ${ }^{3}$ Department of Microbiology, TNO Quality of Life, Zeist, The Netherlands. E-mail: brul@ science.uva.nl (S. Brul)

In the food processing industry, unwanted presence of extremely heat-resistant bacterial endospores creates major problems due to their capability to survive classical thermal treatments and their ability to subsequently germinate and form actively growing vegetative cells. Screening of spoilage isolates using genomic typing techniques to visualise putative genome-based biomarkers allowed us to classify strains according to the degree of thermal resistance of their spores. In addition, we showed that sporulation in the presence of ingredients rich in calcium ions promotes thermal resistance of developing spores and correlates with the expression of specific (marker) genes (see Oomes and Brul, 2004). Finally, the molecular program that forms the basis of spore germination has been assessed using genome-wide expression analysis. Noticeably genes involved in DNA-repair were transiently expressed in germinating wild-type spores. Also, surprisingly, it was found that spores contain significant levels of ribosomal and messenger RNAs. Degradation of these RNA molecules upon spore thermal injury was found to be characteristic for their thermal resistance and predictive for their subsequent outgrowth behaviour. This finding is currently being patented. The information on spore presence, predictions of their thermal resistance and process survival chances, is used to structure a process management system to facilitate optimal food quality assurance and allow for real time analysis in case of the need for quality control. The information on spore presence, predictions of their thermal resistance and process survival chances is now being integrated. This is used to formulate the conditions for a process management system with state of the art food production quality assurance, which allows for real time analysis in case of the need for quality control.

4

The interplay of the pectinase spectrum of Aspergillus niger as revealed by DNA microarray studies Elena Martens, Jac Benen, Johan van den Berg, Peter Schaap Fungal Genomics Group, Laboratory of Microbiology, Wageningen University, Dreijenlaan 2, 6703 HA Wageningen, The Netherlands. E-mail: elena.martens@wur.nl (E. Martens)

The saprobic fungus Aspergillus niger is an efficient producer of extracellular enzymes many of which show carbohydrate modifying activities. These enzymes have GRAS status and therefore are widely used in the food and feed industry. After determination of the genomic sequence of $A$. niger by DSM, it was estimated that only a fraction of the potential of secreted enzymes is currently characterised. Database mining using the proprietary genome sequence has resulted in the identification of more then 80 genes encoding enzymes involved in the depolymerisation of the back bone and the site chains of the complex polysaccharide pectin. Additional enzymatic activities required to remove methyl and acetyl esters, present in pectin were also observed. By using DNA microarrays we have sought to gain insight into the complex regulation of all the genes involved in pectin degradation. A. niger was cultivated on sugar beet pectin and on the monomeric constituents of pectin, viz. galacturonic acid, rhamnose and xylose. Subsequently the corresponding transcriptomes were analysed. We will report on our findings concerning the regulation of the expression of the genes involved in the degradation of pectin and its main constituent-galacturonic acid and the consequences for the interplay of the encoded (novel) enzymes.

5

Proteomic detection of protein oxidation in fish during frozen storage and tainting Inger V.H. Kjarsgård Danish Institute for Fisheries Research, DTU Building 221, Kgs. Lyngby DK-2800, Denmark.E-mail: ivk@dfu.min.dk

Since reactive oxygen species (ROS) are formed in all living organisms a wide range of antioxidative enzyme systems are present to keep the system in balance. When an animal is slaughtered the cellular anti-oxidative capability is reduced, resulting in an accumulation of ROS followed by an increased oxidation of DNA, lipids and proteins. Generally lipid oxidation is a well-known problem, causing increased rancidity during prolonged storage, of especially fatty fish. The implications of protein oxidation are, however, more unclear also in respect to quality decay of fish. Protein oxidation causes a wide variety of amino acids modifications, where of the most studied is carbonylation of proline, argenine, lysine or threonine. These carbonyl groups can be labelled with 2,4-dinitro-phenylhydrazine. Combining two-dimensional gel electrophoresis with immunoblotting enables the detection of carbonyl groups for each single protein. The results presented here, reveal that both during frozen storage and tainting of rainbow trout protein oxidation/carbonylation increases, furthermore there is an increase in oxidation/carbonylation for distinct proteins. 


\section{Farm Animal Genomics}

\section{1}

Ethical and societal aspects of farm animal genomics Anne-Marie Neeteson European Forum of Farm Animal Breeders, Benedendorpsweg 98, 6862 WL Oosterbeek, The Netherlands

Society is concerned about food, animal welfare, food safety, new technologies, scientists and industry. These elements are all present in genomics for farm animals. Therefore, it is important to build awareness in scientists and industry, start a dialogue with stakeholders and society, and to be transparent in a pro-active way. This paper will address the issues at stake for scientists and industry, when it comes to genomics and animal health. It will combine the results of imperical, ethical and sociological efforts in three EU funded projects. (a) The definition of sustainable breeding and sustainable breeding scenarios were worked out in Sustainable European Farm Animal Breeding And Reproduction (SEFABAR, http://www.sefabar.org/). (b) In 2005, a Code of Good Practice for Farm Animal Breeding Organisations (http://www.sefabar.org/code-efabar) was developed. (c) The proper use of genomics in relation to infectious diseases in production animals, and the role of the scientist in the development in new technologies in this field, are being addressed in European Animal Disease Genomics Network of Excellence (EADGENE, http://www.eadgene.org/). Some observations are that: (1) genomics does not concern changing the gene. However, acceptability of any discovery dealing with living beings and edible products, is not obvious just like that! Animals have a symbolic and emotional load. (2) Genes are still related to eugenics, in the mind of people. Genes as such cause reluctance, but it is seen as positive if the use of medication can be reduced, and if animals will be better resistant to disease. (3) Consumers are in favour of consumer education, compulsory labelling and the imposition of minimum standards. The inclination to pay more for foods produced according to desired standards relates closely to income level. (4) Animal welfare is the major issue citizens mention as a concern. The focus of breeding organisations on productivity should be counterbalanced by serious attention to the animal's needs in order to avoid unnecessary negative impact on the welfare of the animals. (5) When technical specialists and lay people communicate, they tend to use different languages: they use the same words, but with rather different interpretations. So transparency of breeding practices and clear definitions of terminology will be essential for effective communication among all stakeholders. (6) Food safety and human health are the major concern for most people, when it comes to making a choice.

2

Structural and functional genome analysis in the pig Merete Fredholm The Royal Veterinary and Agricultural University, Institute of Animal and Veterinary Basic Sciences, Division of Genetics

During the latest decades research within the field of animal genomics has in general been following the same strategies as those used within the field of human genomics, although with much less resources. The porcine genome has been characterized intensively through the development of linkage maps, comparative maps and physical maps. Until a few years ago it had not been anticipated that it would be possible to embark on whole genome sequencing of animals genomes. However, because of technological developments and much lower costs for sequencing, several animal genomes have now been assembled/are on the way to being assembled. The initial step towards sequencing the porcine genome was taken by the Sino-Danish Pig Genome Project. The efforts within this project have now generated approximately 3.84 million genomic shotgun sequences and 700.000 expressed sequence tags (ESTs). The shotgun sequences have been included in a three-species alignment to make an initial evolutionary analysis. The results show that pig is much closer to human than mouse is. The ESTs represent $5^{\prime}$-end sequences from a total of 98 non-normalized cDNA libraries. Based on assembly and annotation of the ESTs the structure of the porcine transcriptome has been analysed. The relevance of assembling the porcine genomic sequence is justified both from the perspective of sustainable animal breeding and from the fact that the porcine model is an important research platform because of the anatomical, physiological, biochemical and metabolically similarities with man. Examples of functional genomic studies both aimed at sustainable animal breeding and aimed at exploiting the pig as a model for medical studies will be discussed.

\section{3}

Functional genomics and reproduction in farm animals François Hatey ${ }^{1}$, Philippe Monget ${ }^{2}:{ }^{1}$ Laboratoire de Génétique Cellulaire, Centre INRA de Toulouse, 31326, Castanet, Tolosan cedex, France; ${ }^{2}$ Physiologie de la Reproduction et des Comportements, Centre INRA de Tours, 37380 Nouzilly, France

Genomics refers to global, systematic and high throughput approaches that allow collecting large amount of data and thus offer new possibilities for analysis and understanding biological processes. We will present some new knowledge related to reproduction in farm animals resulting from three different strategies.

(1) Functional analysis of gene and protein expression: The transcriptome and the proteome analysis allowed to identify new genes and proteins whose expression is associated with processes of ovarian follicular growth and atresia as well as oocyte maturation in bovine and porcine species, maturation of spermatozoa in the different compartments of epididymis.

(2) Utilization of genetic and physical maps: These maps, as well as the sequence of human genome and the sequence of the genome of domestic animals, allowed the identification of new genes playing key roles in reproduction. For example, the identification of Booroola, Inverdale, Cambridge and Belclare mutations recently highlighted the role of Bone-Morphogenetic Protein system in the regulation of ovulation rate in females. Similarly, several QTLs involved in the decrease of fertility of high productive dairy cattle are tracked.

(3) Utilization of bioinformatics tools to identify new genes involved in reproduction: Recently, 100 of new oocyte-specific genes have been identified by using in silico strategies of subtraction of several cDNA libraries in the mouse. The identification of orthologous genes is now currently under development in cattle, pig, poultry and trout. This powerful strategy can be developed in other tissues involved in reproduction. 
The use of genomics for enhanced quality of life Jan W.M. Merks, Barbara Harlizius IPG, Institute for Pig Genetics B.V., P.O. Box 43, 6640 AA Beuningen, The Netherlands

Farm animals produce food as cost effectively as possible, however this may have negative side effects for their health and welfare. Trade off processes between production on one hand and reproduction and health on the other hand play a crucial role. The principles of selective breeding for the best of naturally occurring variation has proven to be able to balance an increased level of production and quality of life for the animal. Every year, the economic value of the genetic gain achieved by the breeders and carried over to the producers is $1.5 \%$ of the economic value of EU farm animal production. Consequently, a conservative estimate of the gain from animal breeding is, every year $€ 1.2$ billion in Europe. Recent developments, such as the sequencing of the genomes of the human, chicken and cow, together with high throughput laboratory techniques, means that there are new opportunities to enhance quality of life. The goal of this paper is to give an overview of the options offered by genomics for enhanced quality of life with focus on identifying relevant gene variants and technologies for large scale tracking and tracing. Selective breeding for the best of naturally occurring variation remains the same as in traditional systems, but by pinpointing the relevant gene variants along genomics it is possible to identify directly the animals best selected for high production without comprising health and welfare. The combination of full genome sequences, software tools, study of functional physiological processes cost-effective high-throughput SNP genotyping and comparative mapping have the (proven) potential to identify relevant gene variants, e.g. pork color, boar taint, general disease resistance. Functional mutations have direct option of application in breeding programs. Unfortunately this is not the case for genetic markers due to cost of genotyping and inconsistent phenotypic effects. New technologies for SNP genotyping are cost effective and enable large scale genotyping (1.000 of animals/day). A selection of the best technology and strategic use of these opportunities enable tracking and tracing. The application of this technology offers new opportunities for quality of life, both for animal and humans.

\section{Functional Foods and Probiotics}

The influence of synbiotic consumption on cancer risk biomarkers in previously resected colon cancer subjects John Kevin Collins $^{1,2,5}$, Yvonne Clune ${ }^{1}$, Karen Meaney ${ }^{1}$, Maurice O'Donoghue ${ }^{1}$, Annett Klinder ${ }^{7}$, Monika Roller ${ }^{8}$, Pernilla Karlsson ${ }^{6}$, Michael Bennett ${ }^{4}$, Michael O'Riordan ${ }^{4}$, Colum Dunne ${ }^{3}$, Gerald O'Sullivan ${ }^{3,4}$, Joseph Rafter ${ }^{6}$, Bernhard Watzl ${ }^{8}$, Gerhard Rechkemmer ${ }^{9}$, Beatrice L. Pool-Zobel ${ }^{7}:{ }^{1}$ Department of Medicine, University College Cork, Cork, Ireland; ${ }^{2}$ Department of Microbiology, University College Cork, Cork, Ireland; ${ }^{3}$ Cork Cancer Research Centre, University College Cork, Cork, Ireland; ${ }^{4}$ The Mercy University Hospital, University College Cork, Cork, Ireland; ${ }^{5}$ Alimentary Pharmabiotic Centre, University College Cork, Cork, Ireland; ${ }^{6}$ Department of Medical Nutrition, Karolinska Institutet, Sweden; ${ }^{7}$ Institute of Nutrition, Friedrich Schiller University of Jena, Germany; ${ }^{8}$ Federal Research Centre for Nutrition and Food, Karlsruhe, Germany; ${ }^{9}$ Technical University Munich, Germany
Background: Studies have shown that prebiotic and probiotic consumption alters the gastrointestinal flora, modulates the immune system, inhibits genotoxicity and has a protective effect on colon carcinogenesis. However, the effect of synbiotic consumption on these parameters in subjects at risk of colon cancer has not until now been investigated.

Aim: To determine if a synbiotic (prebiotic and probiotics together) modulates cancer risk biomarkers in human subjects at risk of colon cancer.

Methods: A 12-week randomised, double blind, placebo controlled, ethically approved trial of a food supplement containing Lactobacillus GG, Bifidobacterium Bb-12 and Raftilose Synergy 1 (prebiotic) was performed in 37 colon cancer subjects who had undergone 'curative resection'. Faecal and blood samples were obtained before (T1, week 0 ) midway through (T2, 6 weeks) and following intervention (T3, 12 weeks). Rectal biopsies were obtained at T1 and T3. The effect of synbiotic consumption on the faecal flora was assessed using standard plate count techniques. Genotoxic damage was measured in single cells derived from biopsies using the comet assay. FW was prepared by diluting faeces 1:1 in DMEM, ultracentrifugation and sterile filtration. The genotoxic (comet assay) and cytotoxic potential (almar blue assay) of FW was determined. Peripheral blood mononuclear cells were isolated from blood and cytokine production in vitro assayed by ELISA. Natural killer cell cytotoxic activity and the phagocytic and respiratory burst activity of monocytes and granulocytes in whole blood were determined by flow cytometry.

Results: In the synbiotic group faecal numbers of bifidobacteria significantly increased $(P<0.001)$ and lactobacilli increased although not significantly $(P=0.0674)$ while coliforms decreased $(P<0.05)$. Enterococci, Clostridium perfringens and bacteroides were unaffected. In the placebo group bifidobacteria decreased $(P<0.001)$, the other bacterial groups were unaffected. In biopsies genotoxic damage was increased in the placebo group at T3 versus T1 $(P=0.0301)$ but was unchanged in the synbiotic group. The genotoxic and cytotoxic potential of FW was unaltered. Synbiotic consumption significantly increased $(P<0.05)$, IFN- $\gamma$ production by PBMCs but IL-2, IL-10, IL-12, and TNF- $\alpha$ production was unaffected. Natural killer cell, phagocytic and respiratory burst activities were unaltered.

Conclusion: Synbiotic consumption did not have a strong immunomodulatory effect on the systemic immune system in this study, nor did it influence the genotoxic and cytotoxic potential of FW. However, synbiotic consumption altered the composition of the gut flora to a more beneficial composition as well as protecting against genotoxic damage in vivo, suggesting a protective effect of synbiotics against colon carcinogenesis.

2

Influence of environmental conditions on exopolysaccharide formation in Lactobacillus reuteri Emma Arsköld, Malin Svensson, Halfdan Grage, Peter Rådström, Ed W.J. van Niel Applied Microbiology, Lund Institute of Technology, Lund University, P.O. Box 124, SE-221 00 Lund, Sweden

Lactobacillus reuteri is used today in a variety of dairy products as a probiotic bacterium. Several lactic acid bacteria have the ability to produce different kinds of exopolysaccharides (EPS), which have the potential to be used as an alternative biothickener. However, the yield of EPS is too low to be profitable in the food industry. To 
optimise the environmental conditions for EPS formation a $L$. reuteri strain was chosen for a factorial design study. The factors used in this experiment were temperature $\left(30-43^{\circ} \mathrm{C}\right), \mathrm{pH}(4.5-6.5)$ and sucrose concentration $(50-150 \mathrm{~g} / \mathrm{l})$; for each factor three different values were chosen. The strain was grown in batch mode using a semi-defined medium at constant $\mathrm{pH}$ and sucrose as the carbon source. The results obtained with 27 fermentations revealed that the highest EPS formation was found at $37^{\circ} \mathrm{C}, \mathrm{pH} 4.5$ and $100 \mathrm{~g} / \mathrm{l}$ of sucrose. Sucrose did not further affect the EPS formation above a concentration of $100 \mathrm{~g} / \mathrm{l}$. Temperature and $\mathrm{pH}$ were significant for the EPS formation, but only temperature was significant for growth. A central composite design study was chosen for further optimization of the $\mathrm{pH}$ and sucrose concentration for maximum EPS formation. Also the gene expression of the sucrase enzyme responsible for EPS formation was investigated using qRT-PCR. The data were used to develop a model for growth and EPS formation.

3

Characterization of a large panel of lactic acid bacteria derived from the human gut for their capacity to polarize dendritic cells H.R. Chistensen ${ }^{1,2}, H$. Frøkiar ${ }^{1}$ : ${ }^{1}$ BioCentrum-DTU, Biochemistry and Nutrition Group, Kgs. Lyngby, Denmark; ${ }^{2}$ Bioneer A/S, Hørsholm, Denmark

Dendritic cells (DC) play a pivotal immune regulatory role in the Th1, Th2 and Treg cell balance. DC are present in the gut mucosa and may thus be target for modulation by gut microbes. Here, we screened a large panel of human gut-derived Lactobacillus and Bifidobacterium spp. for DC polarizing capacity: bone marrow-derived murine DC were exposed to lethally irradiated bacteria and cytokine and DC surface markers were analyzed. Substantial differences were found among strains in their capacity to induce proinflammatory cytokines, while the differences for anti-inflammatory cytokines were less pronounced. Bifidobacteria were weak IL-12, IL-23 and TNF- $\alpha-$ inducers, while both strong and weak cytokine-inducers were found among the strains of Lactobacillus. Remarkably, strains weak in IL12 induction inhibited IL-12, IL-23 and TNF- $\alpha$ production induced by otherwise strong cytokine-inducing strains, while IL-10 production remained unaffected. Those lactobacilli with greatest capacity to induce IL-12 were also most effective in up-regulating surface markers. Surface marker up-regulation was however reduced in the presence of weak IL-12-inducing strains. In conclusion, human Lactobacillus and Bifidobacterium spp. polarize differentially DC maturation. Thus, the potential exists for Th1/Th2/Treg-driving capacities of the gut DC to be modulated according to composition of gut flora including ingested probiotics.

4

Cell surface-associated glycolytic enzymes from Lactobacillus plantarum 299v mediate adhesion to human epithelial cells and extracellular matrix proteins $\underline{S . M . M a d s e n}$, J. Glenting, A. Vrang, P. Ravn, H.K. Riemann, H. Israelsen, M.R. Nфrrelykke, A.M. Hansen, M. Antonsson, S. Ahrné, H.C. Beck Bioneer A/S, Hørsholm DK2970, Denmark

Among the main selection criteria of lactic acid bacteria for probiotic use, the ability to adhere to intestinal epithelial cells, mucus, or extracellular matrix proteins is considered important. Using a proteom based approach we identified a group of novel surface proteins that are non-covalently bound to the cell wall of the probi- otic bacterium Lactobacillus plantarum 299v. Surface proteins were extracted and analysed by gel electrophoreses followed by mass spectrometry analysis. The surface proteins included glycolytic enzymes like, e.g glyceraldehyde 3-phosphate dehydrogenase, which usually is a typical intracellular enzyme. A collection of Lactobacillus species was screened and the phenomenon of surface-associated glycolytic enzymes was found in many of the analyzed species. This is to our knowledge the first example of surface-associated glycolytic enzymes in probiotic bacteria. However, in pathogenic bacteria these enzymes are well known and their surface localization is involved in adhesion to human epithelial cells and invasion. We suspect that the presence of these enzymes on the surface of probiotic bacteria could prevent adhesion of pathogenic bacteria and possibly also involve other probiotic activities such as immune modulation. Binding studies showed that the surface-associated glycolytic enzymes of Lb. plantarum 229v were able to bind to Caco- 2 intestinal cells and extracellular matrix proteins like fibronectin.

5

Exopolysaccharides producing strains with probiotic potentialities V. Valli, O.F. Restaino, I. Marzaioli, C. Schiraldi, M. Cartenì, M. De Rosa Department of Experimental Medicine, Biotechnology and Molecular Biology Section, Medical School, Second University of Naples, Naples 80138, Italy. E-mail: francesca..restaino@unina2.it (O.F. Restaino)

Scientific and industrial interest on exopolysaccharides (EPS) synthesized by microorganisms and on their chemico-physical properties has been quickly growing in the last years. Many strains of Lactobacilli produce EPS allowing them to adhere to human mucosae and therefore to have probiotic effects such as the stimulation of immune response and even antitumoral activity of these molecules has been claimed. Futhermore prebiotic actions of EPS beneficially affect the human host health improving the properties of indigenous microflora. In this research we have studied two novel interesting Lactobacilli strains: Lactobacillus plantarum DSMZ 12028 and a particular human isolated strain of Lactobacillus crispatus that have probiotic potentialities and are good EPS and L(+)-lactic acid producers. The aim of this work has been to characterize these strains and their metabolites (EPS, organic acid and bacteriocins), to state them as probiotics and to study their adhering ability on human cells. We have studied the physiology of L. plantarum and L. crispatus in shaking flasks as well as their optimal fermentation conditions to obtain high cell density cultures suitable for use as starters in the food industry and eventually for probiotic preparations. Fermentation experiments have been performed in a bioreactor equipped with microfiltration (MF) modules using a semidefined medium and various carbohydrates in different culture conditions (aeration, temperature). The kinetic of EPS production has been followed and according to results both strains have shown a growth-related production ranging from 200 to $400 \mathrm{mg} / \mathrm{l}$. In vitro studies concerning the ability of these strains to adhere to human mucosae are in progress as well as the structural characterization of the exopolisaccharides. 
Targeted delivery of compression probiotic cells with multiparameter flow cytometry Ben Huckle, Christopher J. Hewitt, Zhibing Zhang, Biochemical Engineering, Centre for Formulation Engineering, School of Engineering (Chemical Engineering), The University of Birmingham, Edgbaston B15 2TT, UK. E-mail: bdh850@bham.ac.uk (B. Huckle)

Previous studies have shown that compression coating improves the storage stability of freeze-dried Lactobacillus acidophilus, although this stability is related to the degree of cell injury, which in turn is related to the compression pressure used. Compression coating has also been found to improve the survival of freeze dried $L$. acidophilus during exposure to simulated gastric fluid (SGF). The aim of the present work is to create a compression coated $L$. acidophilus formulation, with targeted release at the terminal ileum and beginning of the colon in the human gastrointestinal tract. Dissolution studies were performed using a phosphate buffer with a $\mathrm{pH}$ of 2 and 6.8 , to simulate gastric fluid and intestinal fluid (SIF), respectively. Cell viability was monitored using multi-parameter flow cytometry (MPFC), together with traditional CFU/ml counts. MPFC was used to identify live, dead and stressed cell populations, using the fluorescent stains propidium iodide (PI), 3,3'-dihexylocarbocyanine iodide $\left(\mathrm{DiOC}_{6}(3)\right)$ and To-Pro-3. Results show that an enteric coating material, Eudragit L100-55, is both suitable for compression coating, and enhancing the survival of cells when exposed to SIF. Pectin USP 100 has also been shown to promote targeted release of the cells.

\section{Plant Genomics and Natural Products}

A target for medicinal plant metabolic engineering-the opium poppy Papaver somniferum Toni $M$. Kutchan Leibniz-Institut für Pflanzenbiochemie, Weinberg 3, 06120 Halle/Saale, Germany

The opium poppy Papaver somniferum contains more than 80 tetrahydrobenzylisoquinoline-derived alkaloids. It is the source of the narcotic analgesics codeine and morphine, which accumulate in specialized internal secretory cells called laticifers. In the aerial parts of the plant, the laticifer cells are anastomosed, forming an articulated network. Laticifers are found associated with the vascular bundle in all plant parts. The morphinan alkaloids morphine, codeine and thebaine are found both in roots and in aerial plant parts and specifically accumulate in vesicles within laticifers. The benzo[c]phenanthridine alkaloids sanguinarine and 10-hydroxysanguinarine are found in root tissue. The syntheses of sanguinarine and of the tetrahydrobenzylisoquinoline latex alkaloid laudanine are completely understood at the enzyme level. Nearly all enzymes of morphine biosynthesis have also been described. In more recent years, cDNAs encoding enzymes of alkaloid biosynthesis in $P$. somniferum have been isolated and characterized. The cell-specific localization of several of the enzymes of morphine, sanguinarine and laudanine biosynthesis has also been described. With knowledge of many of the gene of alkaloid formation and their sites of expression, the metabolic engineering of $P$. somniferum for tailored alkaloid profiles is now being undertaken. An Agrobacterium tumefaciens-based transformation and a regeneration protocol have recently been developed specifically for narcotic Tasmanian cultivars. The various cDNAs encoding genes of alkaloid biosynthesis in P. somniferum are being systematically reintroduced into the plant to achieve engineered plants with altered alkaloid profiles. The first results have now been obtained with sense and antisense genes stably expressed in a regenerated Tasmanian cultivar. The ultimate goal of exploiting the genes of alkaloid biosynthesis is to produce transgenic medicinal plants of specific alkaloid content that would facilitate commercial production and improve our understanding of the factors that regulate biosynthesis as well as provide experimental systems with which to investigate the ecological role of alkaloids in planta.

2

Integrated transcript and metabolite profiling for gene discovery in plant natural product pathways Richard A. Dixon, Lahoucine Achnine, Bettina Deavours, Mohammed Farag, Marina Naoumkina, Lloyd W. Sumner Plant Biology Division, Samuel Roberts Noble Foundation, Ardmore, OK 73401, USA

The rich diversity of chemical structures found in the plant kingdom arises in large part from a limited number of basic chemical scaffolds (e.g. terpene, polyketide) that are modified by a limited number of chemical substitution types (hydroxylation, glycosylation, acylation, prenylation, $O$-methylation, etc.). Much of the diversity is brought about by the substrate- and/or regio-specificities of the substitution enzymes. In contrast to the large collections of gene sequence and transcript level data available on-line, little detailed information exists on the plant (secondary) metabolome. Promiscuity of substrate specificity in vitro may complicate attempts to assign functions to genes of secondary metabolism accessible to researchers through various cDNA library collections. Using the isoflavonoid and triterpene pathways in Medicago species as examples, we describe how integrated metabolite and transcript profiling approaches can aid functional genomics, help explain metabolic regulation, and provide tools for assessing the impacts of genetic modifications in plant secondary metabolism.

\section{3}

Metabolic engineering of dhurrin in transgenic Arabidopsis plants with marginal inadvertent effects on the metabolome and

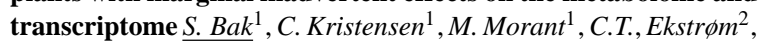
C.E. Olsen ${ }^{2}$, B.L.M $\overline{\text { L }}$ ller ${ }^{1}$ : ${ }^{1}$ Plant Biochemistry Laboratory, Department of Plant Biology, Center for Molecular Plant Physiology (PlaCe), Denmark; ${ }^{2}$ Department of Natural Sciences, Royal Veterinary and Agricultural University, 40 Thorvaldsensvej, DK-1871 Frederiksberg C, Copenhagen, Denmark

Focused and non-targeted approaches were used to assess the impact associated with introduction of new high flux pathways in Arabidopsis thaliana by genetic engineering. Transgenic A. thaliana plants expressing the entire biosynthetic pathway for the tyrosine derived cyanogenic glucoside dhurrin as accomplished by insertion of CYP79A1, CYP71E1, and UGT85B1 from Sorghum bicolor accumulated $4 \%$ dry-weight dhurrin with marginal inadvertent effects on plant morphology, free amino acid pools, transcriptome and metabolome. In a similar manner, plants expressing only CYP79A1 accumulated $3 \%$ dry-weight of the novel tyrosine derived glucosinolate, $p$-hydroxybenzylglucosinolate with no morphological pleitropic effects. In contrast, insertion of $C Y P 79 A 1$ plus CYP71E1 resulted in stunted plants, transcriptome alterations, accumulation of numerous new glucosides derived from detoxification of intermediates in the dhurrin pathway, and in loss of the brassicaceae specific UV protec- 
tants sinapoyl glucose and sinapoyl malate as well as kaempferol glucosides. The accumulation of new glucosides in the plants expressing CYP79A1 and CYP71E1, was not accompanied by induction of glycosyltransferases, demonstrating that plants are constantly prepared to detoxify novel xenobiotics. The pleiotrophic effects observed in plants expressing sorghum CYP79A1 and CYP71E1 were complemented by retransformation with S. bicolor UGT85B1. Accordingly, insertion of high flux pathways directing synthesis and intracellular storage of high amounts of natural products is achievable in transgenic plants with marginal inadvertent effects.

\section{4}

Genome-wide analysis of $5^{\prime}$ untranslated region introns in Arabidopsis thaliana-Distinct function in gene transcription dynamics? Jeppe Madura Larsen, Brian Stougaard Vad, Søren Molgaard, Kell Andersen, Mads N. Davidsen, Klaus D. Grasser Department of Life Sciences, Aalborg University, 9000 Aalborg, Denmark

In recent years it has been shown that introns to some extent can regulate expression in the eukaryotic cell. Insertion of introns in the $5^{\prime}$ UTR in Arabidopsis genes has shown to increase gene expression at both RNA and protein level. In order to investigate if $5^{\prime}$ UTR introns have distinct characteristics, we analyse these in the well annotated Arabidopsis thaliana genome published by the Arabidopsis Genome Initiative. 12,898 loci annotated with full-length cDNAs were analysed and 1989 loci $(15.4 \%)$ containing 5'UTR introns were isolated. We studied if the genes containing these introns showed different patterns in alternative splicing and gene function (gene ontology classification) compared to the remaining genes not containing this intron type. 1802 of the isolated loci $(90.6 \%)$ contained only one $5^{\prime}$ UTR intron and these were used for further analysis, where it was investigated if the $5^{\prime} \mathrm{UTR}$ introns had a characteristic size distribution. Genes containing transcripts with $5^{\prime}$ UTR introns were more subjected to alternative splicing $(9.63 \%$ versus $2.39 \%)$ and had a tendency to be more involved in cell regulatory functions compared to genes without this intron type. It was also found, that $5^{\prime}$ UTR introns was characteristically size-distributed. We identified thee predominant sizes of approximate 110, 270 and $390 \mathrm{bp}$ compared to only one for ORF introns. This suggests widespread multiple splicing events in $5^{\prime}$ UTR introns. The results presented here suggest that $5^{\prime}$ UTR introns have distinct characteristics and function in gene transcription dynamics.

\section{5}

Transgenic tobacco plants carrying CYP11A1 gene of bovine cytochrome P450 $_{\text {SCC }}$ N.A. Kartel ${ }^{1}$, S.G. Spivak ${ }^{2}$, I.N. Berdichevets ${ }^{1}$, D.G. Yarmolinsky $^{1}$, T.V. Maneshina ${ }^{1}$, G.V. Shpakovski ${ }^{3}$ : ${ }^{1}$ Institute of Genetics and Cytology, NASB, Minsk 220072, Belarus; ${ }^{2}$ Institute of Bioorganic Chemistry, NASB, 220141, Belarus; ${ }^{3}$ Shemyakin-Ovchinnikov Institute of Bioorganic Chemistry, RAS, Moscow 117871, Russia. E-mail: n.kartel@igc.bas-net.by (N.A. Kartel)

Cytochrome P450 monooxygenases appear to be involved in the biosynthetic pathways of a large variety of primary and secondary metabolites in microbial, animal and plant cells. In particular, cytochrome $\mathrm{P}^{4} 50_{\mathrm{SCC}}$ catalyzes the conversion of cholesterol into pregnenolone-the precursor of all steroid hormones in mammalian steroidogenic tissues. Cytochromes P450 are also involved in the biosynthesis of different plant steroid derivates that play important role in regulation of plant growth and development. Therein, investigation of possible influence of cytochrome $\mathrm{P}^{4} 50_{\mathrm{SCC}}$ expression on plant regulatory system is of a great interest. This report devoted to the investigation of transgenic tobacco plants, which have been generated by the transformation with recombinant plasmid $p G B P 450 f$ constitutively expressing CYP11A1 cDNA of the bovine cytochrome $\mathrm{P} 450_{\text {SCC }}$. The transgenic state of the plants was confirmed by Southern blot analysis. Transgenic plants are phenotypically different from the control ones. In particular, they obtained a substantially higher growth rate and are larger than wild type plants. We have demonstrated that incubation of fragments of the transgenic plants leaves in $\left[{ }^{14} \mathrm{C}\right]$-labeled cholesterol containing medium results in formation of the radioactively labeled product with chromatographic mobility corresponding to pregnenolone. The presence of this metabolite in the steroid fraction of lipid extracts obtained from the transgenic plants leaves was confirmed by gas chromatography mass spectrometry (GC-MS) method. The data obtained indicate that cytochrome $\mathrm{P}^{4} 50_{\text {SCC }}$ synthesized in transgenic plants displays its specific catalytic activity.

\section{Poster Abstracts Food Biotechnology (FB)}

\section{FB1}

Biotechnological production of glucose isomerase enzyme with Streptomyces olivochromogenes for production of fructose syrup from hydrol M. Hashemiravan, A. Sadat Barikani Department of Food Science and Technology, Azad University (Pishva, Varamin Unit), Institute of Food Science and Agriculture, Tehran, Iran

The use of glucose isomerase for isomerization and production of fructose syrup was performed by selected industrial strain of Streptomyces olivochromogenes PTCC 1457. Growth of microorganism and production of enzyme in different culture media was studied, and effects of different parameters such as phosphate and aeration was evaluated. The growth of microorganism at $28^{\circ} \mathrm{C}$, caused a production of high amount of enzyme. The production of enzyme was considered in two culture media (A and B). Medium (A) was selected for the higher production amount of enzyme. The highest amount of enzyme production was seen in medium A, which was $36.4 \mathrm{GIU} / \mathrm{ml}$, after $80 \mathrm{~h}$. The use of baffles in culture flasks, increased the amount of enzyme production, four times more. The production of enzyme was increased, 1.25 times more, in phosphate deficient medium. The cells containing enzyme (intra cellular glucose isomerase) was separated by centrifuge, and extraction and release of enzyme was performed by ultra sonication, that is a physical-mechanical method. This method released about $89.9 \%$ of total intracellular enzyme. The best length of time for sonication was found to be $4 \mathrm{~min}$. Experiments showed that optimum $\mathrm{pH}$ and temperature of the enzyme were $7.5-8$ and $80^{\circ} \mathrm{C}$, respectively. The highest activity of the enzyme was observed at $\mathrm{pH} 5.8$ for up $120 \mathrm{~min}$. At the time of isomerization reaction the existence of magnesium ions showed to be necessary and omission of this ion cause a decrease of enzyme activity in isomerization process, but this effect was not necessary for the enzyme activity results showed that treatment of glucose syrup at temperatures of 40,60 and $80^{\circ} \mathrm{C}$, by the enzyme, caused $48 \%, 50 \%$ and $52 \%$ of isomerization, respectively. 


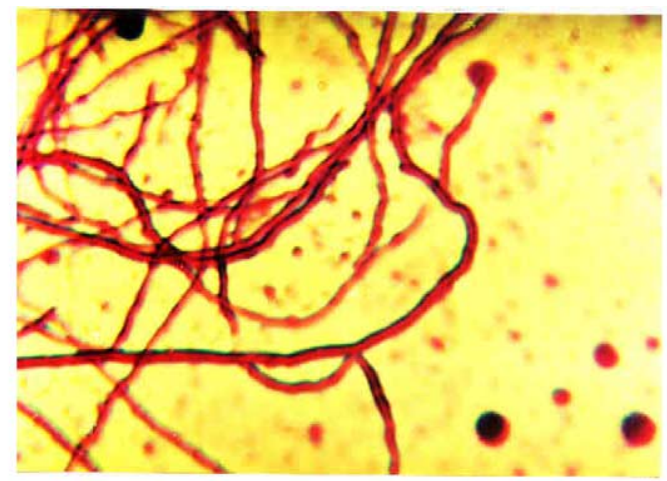

\section{streptomyces olivochromogenes before sonication}

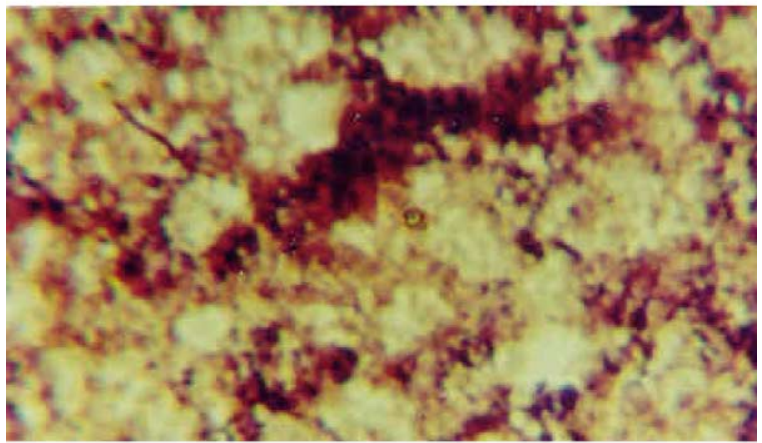

streptomyces olivochromogenes after sonication

FB2

Efficiency increase of high acetic acid production with the use of Acetobactereace Iranian native strains mutation $M$. Hashemiravan ${ }^{1}$, A. Alirezasadat Barikani ${ }^{2}$ : ${ }^{1}$ Department of Food Science and Technology, Azad University (Pishva, Varamin Unit) Institute of Food Science and Agriculture, Iran; ${ }^{2}$ Young Iranian Researchrer's Club, Tehran, Iran

The first step in the present research is the isolation of Acetobactereace native strains from fruits (such as grape or apple) and fresh vinegar. This separation has been done with the use of effective isolation methods and optimized mediums. Ten strains were isolated effectively, the bio chemicals test were performed, each of them were detected, classified and nominated with a special code. Two methods have been used for mutation:

(a) mutation with the ultraviolet radiation;

(b) mutation with nitrous acid.

In the first method the microbial cells were treated with us radiation for different periods of $15,30,45$ and $60 \mathrm{~s}$, and the effect of the UV mutation was assessed. As a result, the period of $45 \mathrm{~s}$ was determined as the optimum mutation periods. In the second method, the microbial cells were first washed with the acetate buffer $0.2 \mathrm{M}$ with the $\mathrm{pH}$ of 4.5 , then $10 \mathrm{ml}$ nitrous acid $0.07 \mathrm{M}$ was added and was mixed for $2-10 \mathrm{~min}$. Finally the sampling was done in the periods of 2, 4, 6, 8 and $10 \mathrm{~min}$ and was transferred to a plate containing the medium of ethanol-phenol red-Agar. The two methods have been compared with each other. Each method has its own advantages and disadvantages. The mutation pathway in method (b) is more stable and conducted, while mutation with the UV radiation method, change the position of thiamine-cytosine bases absolutely randomly. Finally, the best mutant site was inoculumed with the medium containing alcohol $16 \%$, acetozyme GZ $0.6 \mathrm{~g} / \mathrm{l}$, acetozyme D $1 \mathrm{~g} / \mathrm{l}$, acetic acid $1.5 \%$ and 11 double distilled water. The acetic acid was produced in the rate of $16 \mathrm{~g} / 100 \mathrm{ml}$. Also the mutant strains were detected with scanning electron microscope (SEM) and interesting photographs were taken from mutant cell. The performed experiments were planned with the use of Taguchi statistical method (Qualitek 4). This research has been performed in the IROST as the thesis of Ph.D. in food biotechnology industry.

\section{FB3}

Microfluidic isotachophoretic analysis of milk proteins in epoxy(poly)dimethylacrylamide coated glass chips $C$.-H. Brogren ${ }^{1,2}, J$.

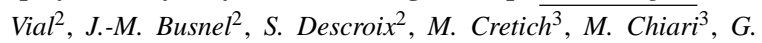
Peltre ${ }^{2}{ }^{1}$ Department of Medical Biochemistry and Genetics, University of Copenhagen, DK-2200, Denmark; ${ }^{2}$ Laboratoire Environnement et Chimie Analytique, Ecole Superieure de Physique et de Chimie Industrielles, Paris F-75005, France; ${ }^{3}$ Institute of Biocatalysis and Molecular Recognition, Consiglio Nazionale delle Ricerche, Milano I-20131, Italy

Isotachophoresis has almost exclusively been applied for contracting and stacking samples ions before zone electrophoretic separation of proteins. This study attempts to apply microfluidic isotachophoresis (ITP) as a high resolution analytical method for proteins. Beta-lactoglobulin and other milk proteins with slightly different $\mathrm{p} I$ were labelled with Fluorescent Red 646 and analysed by the Micralyne $\mu \mathrm{TK}$ system using microfluidic glass chips, either with simple cross (SC) or double cross (TT) injection or designed 2D-ITP-CZE chips with double TT injection and SC for transfer to the second dimension CZE channel, efficiently non-covalently coated with $0.6 \%$ (w/v) epoxy-polydimethylacrylamide to lower electroendoosmosis. Capillary zone electrophoresis (CZE) in borate or phosphate buffer was reproducibly perform for more than 75 consecutive runs using Upchurch $^{\mathrm{TM}}$ reservoirs glued to the wells to enable larger buffer volumes and greater run-to-run stability. Finally, isotachophoretic anionic separation of the proteins were done using phosphate $(\mathrm{pH}$ $8.1)$ or chloride $(\mathrm{pH} 8.5)$ as leading ion and $\varepsilon$-amino-caproic acid (pH 8.9) as terminating ion. The effect of narrow cut ampholytes as spacers needs further investigations. The perspective aim is to combine the migrating ITP separated zones with second dimension capillary zone electrophoresis as a new microfluidic proteomic 2Danalysis.

\section{FB4}

Genotypical differences affecting the response of Pisum sativum to differing boron/iron applications E.E. Hakki, U. Zeynep, M. Hamurcu, A. Tamkoc, M.B. Babaoglu, S. Gezgin Department of Field Crops, Faculty of Agriculture, Selcuk University, Kampus, Konya 42079, Turkey. E-mail: eehakki@ selcuk.edu.tr (E.E. Hakki)

Boron and iron are among the microelements required for the proper development of the vegetative and generative tissues of plants. Though iron is present in high amounts in almost all soil types, its 
bioavailability to crops is extremely reduced, hence most of the plants face an iron defficiency problem and while on one side crop productions effected, on the other hand the nutrition problems come up to human through contagious nutritional chains. Boron is also among the most problematic micronutrients of the major crop plantation areas of Turkey. Both defficiency and toxicity problems exist in a total of about $50 \%$ of the Central Anatolian soil where pea is among the legumes cultivated. Application of varying levels of boron and iron combinations in greenhouse and the analysis of plant aquisition via ICP-AES as well as determination of the effects of the element combinations both in morphological and molecular levels are the aim of our studies. The genetic bases of the response differences of plant genotypes to B and/or Fe, were investigated through the applications of molecular marker techniques. Considerable growth rate and stem size differences were detected within the parents (wild-type versus cultivar) and the F9 plants. The presence of efficient genotypes to high micronutrient levels are expected to help us increase the cultivation of the crop in problematic areas as well as in exploring the molecular bases of the microelement uptake mechanisms.

\section{FB5}

Increase in sulfite production by accelerating sulfate uptake in brewing yeast T. Fujimura, Y. Kodama, Y. Nakao, N. Nakamura, W. Miki Institute for advanced technology, Suntory Ltd., Mishima-gun, Osaka 618-8503, Japan

Sulfite plays a role as an antioxidant, which stabilizes beer flavor. Therefore, it is important to control the sulfite concentration during fermentation. Sulfite is produced as an intermediate in the sulfate assimilation pathway in yeast. We have already reported that over-expression of a lager yeast-specific SSU1 gene, encoding a sulfite efflux pump, leads to increase of sulfite production (Fujimura et al., 2003). In the present work, we have clarified that there are two types of SUL2 genes (ScSUL2 and non-ScSUL2) each encoding a high affinity sulfate permease in lager brewing yeast. Eighty percent and $86 \%$ identity are found by comparing the DNA sequences and the deduced amino acid sequences, respectively. A comparative functional analysis of the two genes has been performed aimed at achieving further increases in sulfite production by accelerating sulfate uptake. Over-expression of SCSUL2 and non-ScSUL2 has been achieved by transformation of lager brewing yeast, Saccharomyces pastorianus. Experiments have been done with and without expression of non-ScSSU1. The resultant transformants have been evaluated by fermentation tests in wort. Over-expression of either ScSUL2 or non-ScSUL2 failed to show significant effect on sulfite formation. A combination of over-expression of non-ScSUL2 and non-ScSSU1 resulted in two-fold higher sulfite production compared with overexpression of only non-ScSSU1 and four-fold higher compared with the parental strain. These results suggest that the non-Sc-gene types significantly contribute to sulfite production in lager brewing yeast.

\section{Reference}

Fujimura, T., et al., 2003. Functional analysis of SSU1 genes in lager brewing yeasts. 23rd International Specialized Symposium on Yeast, Hungary.

\section{FB6}

Screening of soil bacteria phytate-degrading enzymes from the roots of Malaysian Zea mays Anis Shobirin Meor Hussin ${ }^{1}$, Abd-ElAziem Farouk ${ }^{1}$, Hamzah Mohd. Salleh ${ }^{1}$, Ralf Greiner ${ }^{2}$ : ${ }^{1}$ Biomolecular Engineering Research Group, Department of Biotechnology Engineering, Kulliyyah of Engineering, International Islamic University Malaysia, Jalan Gombak, 53100 Kuala Lumpur, Malaysia; ${ }^{2}$ Centre for Molecular Biology, Federal Research Centre for Nutrition and Foods, Haid-und-Neu-Straße 9, D-76131 Karlsruhe, Germany

Phytases (myo-inositol hexakisphosphate phosphohydrolase) catalyse the release of phosphate from phytate (myo-inositol hexakisphosphate), the predominant form of phosphorus in cereal grains, oilseeds and legumes. Possible applications of phytases have been suggested in animal nutrition to increase mineral bioavaliability and to decrease phosphate pollution in area of intensive life stock management and in human health. Zea mays is one of the cereals that contain high amount of phytate as the major phosphate storage compound. Over 200 bacteria were isolated and screened for phytases from the halosphere, rhizosphere and endophyte of Malaysian maize plantation. The phytase activity of the isolates was screened by a modification of the ammonium molybdate method. The highest extracellular phytase activity was detected from bacteria that isolated from the endophyte of the maize root. In this paper, results for 24 isolates chosen for media, temperature and $\mathrm{pH}$ optimization will be presented.

\section{FB7}

Production of plant proteinase from Jack Fruit seeds (Artocarpus integrifolis) and its influence on rheological and sensory characteristics of low fat yogurt El-Sayed El-Tanboly Dairy Sciences Department, National Research Centre, Dokki, Cairo, Egypt

Adding a proteolytic enzyme extraction from Jack Fruit (Artocarpus integrifolis) in combination of fermentation process in low fat yogurts manufacture was tried to improve yogurt flavour and rheological properties. Experimental yogurts milk contained control, 3.9 (T1), 7.8 (T2) and 11.7 (T3) units/ml milk from crude extracts of plant proteinase. The $\mathrm{pH}$ of the product treated with crude proteinase was lower than the control. However, The rate of acidity development during storage slightly increased with increasing the addition of crude proteinase level and progress of storage period of yogurt. The proteolytic activity of all yogurts gradually increased until the end of storage period (15 days). Yogurts made from milk treated with crude proteinase preparations were less firm compared with control at all storage periods, where T3 showed more less firm after 15 days of storage being $20.17 \mathrm{~g} / 100 \mathrm{~g}$. Generally, increasing units of plant proteinase preparations decreased the firmness. On the other hand, yogurt made from milk pretreated with plant proteinase had higher syneresis, and apparent viscosity than the untreated product. The greatest viscosity was found in T2 and T3 of 433 and $479 \mathrm{mPas}$, respectively, compared with control of $299 \mathrm{mPa}$ s at 15 days storage. The results indicated that there is an inverse relationship between the amount of units of crude proteinase preparations and susceptibility of yogurt to syneresis. The T2 gained the highest scores ( 85 points) followed by the control ( 81.5 points) after 15 days of storage, while yogurt of T3 showed a low scoring being 75 . From the foregoing results, it is recommend to use Jack Fruit (Artocarpus integrifolis) as 
a source of plant proteinases and utilize it to develop a high quality yogurt at a level of 7.8 units of plant proteinases $/ \mathrm{ml} \mathrm{milk.}$

\section{FB8}

Evaluation of distilled white lees for tartaric acid recovery and economic nutrient utilization J.M. Domínguez ${ }^{1}$, B. Rivas ${ }^{1}, N$. de la Torre ${ }^{1}$, A.B. Moldes ${ }^{1}$, M. Castro ${ }^{2}$, J. González ${ }^{2}$ : ${ }^{1}$ Departmento de Ingeniería Química, Universidad de Vigo (Campus Ourense), Edificio Politécnico, As Lagoas, 32004 Ourense, Spain; ${ }^{2}$ Vitivinicola do Ribeiro S.C.G., Valdepereira s/n. Ribadavia 32415 Ourense, Spain. E-mail: jmanuel@uvigo.es (J.M. Domínguez)

An effective process for the chemical-biotechnological utilization of distilled white lees was studied. A first treatment with hydrochloric acid allowed the solubilisation of tartaric acid. The influence of temperature, amount of $\mathrm{HCl}$ and reaction time were considered through an experimental design. Under the optima conditions $77 \mathrm{~g} / \mathrm{L}$ from white distilled lees and $45.6 \mathrm{~g} / \mathrm{L}$ from red distilled lees were recovered. The tartaric acid was precipitated as calcium tartrate so that it can be isolated from the rest of the raw material compounds. The solid residue was used as an economic nutrient for lactic acid production by Lactobacillus pentosus using trimming wastes as substrate. The lactic acid concentrations and volumetric productivities achieved were similar to those obtained using distilled lees without tartaric acid recovery as nutrient.

\section{FB9}

Preparation and characterization of Toasted Wine: A sweet table wine J.M. Domínguez ${ }^{1}$, B. Rivas ${ }^{1}$, N. de la Torre ${ }^{1}$, A.B. Moldes ${ }^{1}$, S. Cortés ${ }^{2}:{ }^{1}$ Departmento de Ingeniería Química, Universidad de Vigo (Campus Ourense), Edificio Politécnico, As Lagoas, 32004 Ourense, Spain; ${ }^{2}$ Estación de Viticultura e Enoloxía de Galicia (EVEGA), Ponte San Clodio s/n, 32427, Leiro (Ourense), Spain. E-mail: jmanuel@uvigo.es (J.M. Domínguez)

Toasted Wine was traditionally produced in Galicia, northwest of Spain. Nowadays this technique is being recovered. Grapes after harvesting are air dried in order to concentrate sugars, acids and flavor compounds. Raisings are pressed to obtain a must with high sugars concentrations. Two different grape wines were prepared concentrating the sugars up to 37 and 57 Brix, respectively. In order to get a better knowledge of the problems involved, synthetic media simulating the grape musts were prepared. Theses musts were used to optimize the initial sugar concentration, the amount of nutrients required, the optimum temperature to carry out the fermentation and the influence of the type and amount of yeast. Under the best conditions some fermentations with grape must were carried out to produce wines with intense aroma and flavor notes and high residual sugar concentrations.

\section{FB10}

Isolation of bacteria producing biological response modifier found in Korean style fermented soybean paste B.K. Lee $e^{2}$, J.Y. Kim ${ }^{2}$, S.W. Hong ${ }^{1}$, K.S. Chung ${ }^{1}$ : ${ }^{1}$ Department of Biological Resources and Technology, Yonsei University, Wonju 220710, Republic of Korea; ${ }^{2}$ Department of Microbiology, College of Medicine, Yonsei University, Seoul 120-749, Republic of Korea. Email: kschung@dragon.yonsei.ac.kr (K.S. Chung)

In this studies, Bacillus sp. E1 strain was isolated from Koreanstyle fermented soybean paste and it was producing the biological response modifier (BRM). The BRM activated the B cell selectively. It was identified the Bacillus licheniformis E1. The BRM was purified by ion-exchange chromatography and gel filtration. Chemical properties of BRM: Molecular weight of BRM was estimated to be about 1,594,000 Da. Sugar content of BRM was $33.0 \%$ (w/w) and glucosamine (35.1 mol\%) was the high level. Protein content of BRM was $4.3 \%(\mathrm{w} / \mathrm{w})$ and serine $(17.2 \mathrm{~mol} \%)$ was the high level. Infra-red absorption spectrum was showed the characterization of glycoprotein. Biological properties of BRM: The BRM which isolated from fermented soybean paste was similar to that of Bacillus licheniformis E1 by immuno-fluorescence assay. We confirmed that the BRM was capsular substance of B. licheniformis E1.

\section{FB11}

Production of $\alpha$-amylase by Aspergillus oryzae with potato nitrogen concentrate as the sole nitrogen source Marie-Astrid Dolnik ${ }^{1}$, Eric Thaller ${ }^{1}$, Ferdinand Karner $^{1}$, Bernhard F. Adamitsch ${ }^{1}$, Werner A. Hampel ${ }^{1}$, Ulrich Stifter ${ }^{2}$, Eduard Taufratzhofer ${ }^{2}$, Marnik M. Wastyn ${ }^{2}$ : ${ }^{1}$ Institute of Chemical Engineering, Vienna University of Technology, A-1060 Vienna, Austria; ${ }^{2}$ Zuckerforschung Tulln GmbH, A-3430 Tulln, Austria. E-mail: bernhard.adamitsch@tuwien.ac.at (B.F. Adamitsch)

Potato nitrogen concentrate (PNC) is a highly viscous liquid with high complex nitrogen content produced from the protein-fraction in potato starch extraction. The concentrated extract is rich in minerals and $\alpha$-amino nitrogen. Although $\alpha$-amylase nowadays is mainly produced exploiting Bacillus production systems there is still considerable demand for fungal $\alpha$-amylase from Aspergillus oryzae origin. The aim of the experiments to be reported here was to investigate, if PNC can replace commonly used complex nitrogen sources in the production of fungal $\alpha$-amylase. The following data have been measured in PNC pretreated by diluting to $1 / 2$ and clarifying by centrifugation. Total-N: $8.4 \mathrm{~g} \mathrm{~N} / \mathrm{L} ; \alpha$-amino-N: $2.8 \%$ (w/v) (as glycine); soluble protein (Bradford): $51.2 \mathrm{mg} / \mathrm{L}$ (as BSA); total carbohydrates: $110.0 \mathrm{~g} / \mathrm{L}$; reducing sugars: $5.6 \mathrm{~g} / \mathrm{L}$; dry weight: $57.3 \%(\mathrm{w} / \mathrm{w})$. In the following experiments nitrogen sources were replaced on the basis of their $\alpha$-amino nitrogen content. The carbon source for all experiments was maize starch. The formation of $\alpha$-amylase by A. oryzae ATCC 1011 in shake flasks - using PNC (centrifuged or not), yeast extract, malt extract, casein hydrolysate or meat extract - was compared to "standard" cultivation with Corn Steep Liquor. The experiments showed only small differences in $\alpha$-amylase titers using complex nitrogen sources. No remarkable differences were observed in the resulting biomass. In general no differences in enzyme productivity and biomass formation could be seen after $50 \mathrm{~h}$ of incubation. Especially the bench top bioreactor experiments indicated an optimal fermentation time of about $100 \mathrm{~h}$. Cultivations of A. oryzae ATCC 1011 were carried out in bench top bioreactors. Comparing cultivations in a medium with PNC as the sole complex nitrogen source to one containing CSL as such no significant differences both in the formation and amount of $\alpha$-amylase and the fungal growth were observed. Thus PNC might be able to replace complex nitrogen sources such as CSL or even the more expensive yeast extract and casein hydrolysate in fungal amylase production systems. 


\section{FB12}

Glucuronate reductase from the ballistosporous yeast Sporobolomyces oryzicola Michael Trost, Alexander Vogl, Ferdinand Karner, Bernhard F. Adamitsch Institute of Chemical Engineering, Vienna University of Technology, Getreidemarkt 9/166-41, A-1060 Vienna, Austria. E-mail: bernhard.adamitsch@tuwien.ac.at (B.F. Adamitsch)

Glucuronate reductase (E.C. 1.1.1.19) is involved in the metabolism of inositol and catalyzes the conversion of D-glucuronic acid to L-gulonic acid with NADPH as a cosubstrate posterior to the oxidation of inositol to glucuronic acid by the enzyme inositol oxygenase. Although the yeast Sporobolomyces oryzicola (Nakase and Suzuki, 1986) is not able to grow on inositol as the sole carbon source, intracellular glucuronate reductase can be found in cells grown in a medium containing D-glucuronic acid. The enzyme could be a useful tool in the design of a specific quantitative assay for glucuronic acid, e.g. in so called Energy Drinks. The organism was grown in media containing either glucose and glucuronic acid or only glucuronic acid and Difco yeast nitrogen base. Whereas growth on both media was similar in shake flask culture, hardly any growth in either medium was observed in bench top bioreactors. The influence of dissolved oxygen tension was investigated and the relevant data will be shown. The formation of intracellular glucuronate reductase activity by $S p$. oryzicola is inducible by media containing glucuronic acid. No activity is found in cells grown in a medium containing only glucose as the carbon source. Besides the activity against D-glucuronic acid, activities against 5-ketogluconate and - at very low levels against galacturonic acid and the lactone of glucuronic acid were detected. The enzyme activity is stable up to $35^{\circ} \mathrm{C}$. The $\mathrm{pH}$ has relatively low influence on the activity against glucuronate, whereas the reduction rate of 5-ketogluconic acid is optimal at $\mathrm{pH} 7.0-7.5$ with significantly lower values at $\mathrm{pH} 6.0$ and 8.0, respectively. Data on the kinetics of the conversion of both glucuronate and 5-ketogluconate will be shown.

\section{Reference}

Nakase, T., Suzuki, M., 1986. J. Gen. Appl. Microbiol. 32, 149-155.

\section{FB13}

Iron bioavailability from carrot juice fermented by homo- and hetero-fermentative lactic acid bacteria by combined in vitro digestion and Caco-2 cells S.W. Bergkvist, T. Andlid, A.-S. Sandberg Chalmers University of Technology, Department of Chemical and Biological Engineering/Food Science, Box 5401, SE-402 29 Göteborg, Sweden. E-mail: sbt@fsc.chalmers.se (S.W. Bergkvist)

The multiple nutritional and functional impacts of food fermentation on human health have been widely accepted (Reddy and Pierson, 1994; Hugenholtz et al., 2002). However, the related role of the involved microorganisms to the nutritional effect from the fermented food is still not well defined and the mechanisms involved are still largely unknown. The present study was to investigate iron bioavailability in carrot juice fermented by two selected LAB strains, $L$. pentosus FSC1 and Ln. mesenteroides FSC2. After digestion by GI enzymes, the juice was supplied to fully differentiated caco- 2 cells to study iron uptake and transepithelial transport by caco- 2 cells from the digested juice. Our data revealed strain specified changes in iron bioavailability in carrot juice fermented by these two strains. After in vitro digestion with pepsin and pancreatic-bile enzymes, the best yield of soluble iron was from Ln. mesenteroides FSC2 fermented juice. Surprisingly, the $L$. pentosus FSC1 fermented juice yielded about five times higher uptake iron as compared to fresh juice, while Ln. mesenteroides FSC2 fermented juice was not significantly different from the fresh juice. Interestingly, the transepithelial transferred iron across the cell line was however better from Ln. mesenteroides FSC2 fermented juice than from $L$. pentosus FSC1 fermented juice. To summarise, our study showed that level of soluble iron after in vitro digestion does not necessary indicate iron absorption, especially in the case of LAB fermented food. Data on improved iron uptake from $L$. pentosus FSC1 fermented juice indicated exiting of promoter(s) for iron absorption in such juice that is not related to the production of organic acids and lowering $\mathrm{pH}$ effect.

\section{References}

Hugenholtz, J., Sybesma, W., Groot, M.N., Wisselink, W., Ladero, V., Burgess, K., van Sinderen, D., Piard, J.C., Eggink, G., Smid, E.J., Savoy, G., Sesma, F., Jansen, T., Hols, P., Kleerebezem, M., 2002. Metabolic engineering of lactic acid bacteria for the production of nutraceuticals. Antonie Van Leeuwenhoek 82 (1-4), 217-35.

Reddy, N.R., Pierson, M.D., 1994. Reduction in antinutritional and toxic components in plant foods by fermentation. Food Res. Int. 27 (3), 281-290.

\section{FB14}

Contribution of cassava biotechnology on sustainable development in tropical agriculture Peng Zhang, Herve Vanderschuren, Martin Stupak, Wilhelm Gruissem Institute of Plant Sciences, ETH-Zürich LFW E17, 8092 Zurich, Switzerland. E-mail: zhang.peng@ipw.biol.ethz.ch (P. Zhang)

The tropical root crop cassava (Manihot esculenta Crantz) is a major source of food for approximately 1 billion people worldwide. In sub-Saharan Africa, more than 200 million people rely on cassava as their major source of dietary energy. In many parts of Africa and Latin America, cassava leaves are a vegetable source for daily uptake. Cassava is grown mostly by poor farmers under marginal environmental conditions and in areas where few other crops can sustain competitive yields. The crop is therefore fundamental for subsistence farming and food security, but it is also very susceptible to stresses common in the areas and conditions where it grows. In many parts of Africa, reliable cassava production is strongly impacted by infections with the African cassava mosaic geminiviruses (CMGs), a rapidly spreading disease that causes large yield losses. In the coastal areas of east Africa, cassava production now is threatened by another devastating disease, cassava brown streak disease (CBSD). Cassava plants are also frequently attacked by many pests, such as cassava hornworm and stemborers. Several reports also indicate that greater leaf longevity, especially under drought conditions, could be important for increasing yields and/or the stability of production in cassava, as well as improve the access to an important nutrient source. Conventional breeding efforts have attempted to address the constraint to cassava production, but with limited success. The new tools of biotechnology can change this situation by offering new approaches to the challenges of cassava. These new technologies have the potential to make cassava much more productive, a better source of nutrients, and profitable to grow, hence, greatly contributing on the sustainable development of tropical agriculture. Recently 
we have developed biotech cassava with value-add traits, including resistance to cassava mosaic virus, prolonged leaf life and insect resistance. New strategies are also explored to increase protein content of cassava storage roots. We are currently undertaking pilot studies with two teams of leading scientists and experts for projects to test ACMV-resistant transgenic cassava lines in Africa and lines with extended leaf retention at CIAT, Colombia under field conditions. This development of substantially equivalent improved transgenic cassava lines is part of a larger study to analyze the need, effectiveness and biosafety of biotech cassava for agricultural production. The goal of the pilot studies will be the development and coordination of a broader project that produces important and novel scientific results, valuable information on the need and impact of biotechnology at the subsistence farming level, and a sound scientific basis for the development of guidelines for biosafety assessments and release of transgenic organisms into the environment and agricultural production in Africa and Latin American countries.

\section{FB15}

Effects of different pre-treatments on obtaining haploid plants via anther culture in Capsicum A. Naci Onus, Kamile Ulukapl, A. Gül Ince Nurgül Ercan Department of Horticulture, Faculty of Agriculture, Akdeniz University, Antalya 07070, Turkey

This study was conducted to reveal the effects of different pretreatments on obtaining haploid plants by using the anther culture in pepper Capsicum annum L. cultivars Demre Sivrisi and Sirena. Buds were collected at uninucleate microspore stage. Anthers collected from buds were cultured in MS medium containing different hormones and hormone concentrations. Experiment results revealed that when Sirena anthers were pre-treated cold at $+4^{\circ} \mathrm{C}$ for $24 \mathrm{~h}$ and kept in darkness at $25^{\circ} \mathrm{C}$ for a period of 1 week gave good results. In the case of Demre Sivrisi anthers were pre-treated cold at $+4{ }^{\circ} \mathrm{C}$ for $48 \mathrm{~h}$ and kept in darkness at $25^{\circ} \mathrm{C}$ for a period of 1 week gave good results. On the other hand no cold pretreatment to anthers resulted with low embryo formation. Similar results were also observed on the anthers kept at $35^{\circ} \mathrm{C}$ for 1 week as callus was produced in some petri dishes but no regeneration was observed. As a conclusion, since no cold pretreatment to anthers resulted with low embryo formation it is possible to say that cold pretreatment should be applied to anthers in pepper another culture studies.

\section{FB16}

Modelling of Debaryomyces hansenii UFV-170 metabolism in a xylose-to xylitol bioconversion $F$. Sampaio ${ }^{3}, \underline{\text { P. Torre }^{1}}{ }^{1}$, D. De Faveri $^{1}$, B. Rivas ${ }^{2}$, P. Perego ${ }^{1}$, F.M Lopes Passos ${ }^{3}$, J.M. Domínguez ${ }^{2}$, A. Converti ${ }^{1}$ : ${ }^{1}$ Department of Chemical and Process Engineering, Genoa University, Via Opera Pia 15, 16145 Genoa, Italy; ${ }^{2}$ Department of Chemical Engineering Technology, Vigo University, Campus of Ourense, 32004 As Lagoas, Spain; ${ }^{3}$ Department of Microbiology and Food Technology, Federal University of Vicüosa, Av. P.H. Rolfs s/n, 36571-000 Vicüosa, Minas Gerais, Brazil. E-mail: paolo.torre@dichep.unige.it (P. Torre)

The yeast $D$. hansenii UFV-170 was tested in this work in batch experiments in synthetic media at constant initial substrate concentration $\left(100 \mathrm{~g} \mathrm{~L}^{-1}\right)$ under variable oxygenation conditions. To get additional information on its fermentative metabolism, a stoichiometric network was proposed on the basis of the general knowledge available in the literature on xylose metabolism in pentose- fermenting yeasts and the specificities of XR and XDH activities in $D$. hansenii and checked through a bioenergetic study performed using the experimental data of product and substrate concentrations. It can be stressed that under strongly oxygen-limited conditions xylitol production was negligible, whereas under semi-aerobic conditions maximum xylitol production $\left(P_{\max }=76.6 \mathrm{~g} \mathrm{~L}^{-1}\right)$ and yield $\left(Y_{\mathrm{P} / \mathrm{S}}=0.73 \mathrm{~g} \mathrm{~g}^{-1}\right)$ were obtained. A progressive decrease in these parameters was observed under fully aerobic conditions, suggesting that xylitol-producing yeasts require limited oxygen conditions, which is species-dependent. The proposed model, which utilizes the experimental specific rates of substrate consumption and product formations, allows estimating the main bioenergetic parameters. Besides, it proved to be an effective tool to investigate different metabolic situations and showed how they can influence the flux distribution of the carbon source and the bioenergetics of this biosystem.

\section{FB17}

The effect of disinfectants on fungi Anne Svendsen, Pernille Skouboe Bioneer A/S, Hørsholm DK-2970, Denmark

Prevention of mould spoilage of foods can only be carried out successfully, if the species, which are actually spoiling the food product, are known. A very limited number of fungal species has been associated with the spoilage of each food category. Proper disinfection of production facilities is very important to avoid mould spoilage. Resistance of moulds to disinfectant treatments are known and different species have shown different response to the same disinfectant. To obtain proper disinfection it is important to know the resistance of the spoilage fungi against different disinfectants. In this study the effect of disinfectants on the spoilage fungi of cheese, rye bread, liver paté and fruit juice was investigated. In collaboration with five food companies the dominating species responsible for spoilage of each food product were isolated and used for testing. Commercial disinfectants and disinfectants "under development" were tested. Tests were performed in suspension and on surfaces, the methods used were modified after EN 1650 and EN 13697. Considerable variability in fungicidal effect among the species was observed. Some disinfectants were ineffective at low temperature. Some disinfectants showed different effect in suspension and on surfaces, resulting in an effective kill in suspension and almost no effect on surface. The identification of effective disinfectants in the food industry includes: (1) testing against the specific spoiling species of the food product, (2) testing on surface, not only in suspension, (3) test parameters adapted to the food manufacturing plant.

\section{FB18}

Synthesis of prebiotic galacto-oligosaccharides by novel betagalactosidases of Lactobacillus spp. Barbara Splechtna ${ }^{1,2}$, Thu-Ha Nguyen ${ }^{1,2}$, Klaus D. Kulbe ${ }^{2}$, Dietmar Haltrich ${ }^{2}:{ }^{1}$ Applied Biocatalysis Research Centre, Petersgasse 14, A-8010 Graz, Austria; ${ }^{2}$ Division of Food-Biotechnology, Department of Food Sciences and Technology, Vienna University of Natural Resources and Applied Life Sciences (BOKU), Muthgasse 18, A-1190 Vienna, Austria. E-mail: barbara.splechtna@a-b.at (B. Splechtna)

Intensified research efforts in recent years confirm the major importance of the microbial flora in the gastro-intestinal tract for human health. Ingestion of prebiotic oligosaccharides increases the number of the desirable bacteria like Bifidobacteria and Lactobacilli in the colon. We are looking at beta-galactosidases from Lacto- 
bacillus spp. for the production of galacto-oligosaccharides (GOS) because we speculate that the enzymes of probiotics will form GOS with high prebiotic potential. In this present study, purified betagalactosidases of selected Lactobacillus strains were used for the production of GOS from lactose. Different enzyme reactor set-ups, both discontinuous and continuous, were tested and compared. Temperatures up to $37^{\circ} \mathrm{C}$ and $\mathrm{pH}$ values between 6 and 6.5 were required for satisfactory enzyme stability during the process. Enzyme source, substrate concentration and the level of substrate conversion were found to be critical process parameters for GOS yields and composition. Yields of up to $40 \%$ (w/w) of total sugars were achieved when the initial lactose concentration was $200 \mathrm{~g} / \mathrm{l}$. Capillary electrophoresis (CE) and HPLC with pulsed amperometric detection were the analytical tools for investigating the influence of reactor type, enzyme source and conversion level on GOS composition. The prebiotics market is increasing rapidly and is expected to more than double until 2010 to about 180 million $€$ world-wide. Therefore, the development of enzymatic processes on an industrial scale is a high priority goal of our research.

\section{FB19}

Microbial formulation of fermented foods: A new method to select microbial ecosystems efficient to generate a diversity of sensory properties H.E. Spinnler, C. Bonaïti, F. Irlinger LGMPA,

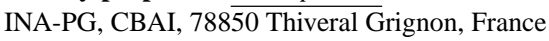

Starter addition does not always succeed in improving standardisation and quality of the complex sensory properties of traditional fermented foods. In many cases the added strains do not grow as well as the environmental strains present in the production plant. Here, a method of geometric simplification (by dichotomy) of a complex ecosystem found on a raw milk Livarot (82 strains) was tested on cheese curd. By a limited number of cultures, successively, 40 out of 82,20 out of 40 and 10 out of 20 strains were selected on the basis of two criteria (i) respect of the taxonomic proportion, (ii) generation by the daughter ecosystems of an odour close to the one of the mother ecosystem. Finally a sub-ecosystem of 10 strains gave an odour similar to the one of the more complex mixture. The use of molecular methods (PCR-SSCP) permitted to follow the main species growing. Mother and daughter ecosystems were characterized by sensory analysis and GC-MS. Probably because of an important redundancy of the strain functions, the method was very efficient. This method may permit to improve a lot the set up of mixture of strains and species used in fermented food industry.

\section{FB20}

Effect of the dilution rate on the exopolysaccharide production by Bifidobacterium longum ATCC 15707 C. Shene, M. Rubilar, S. Bravo Universidad de la Frontera, Chemical Engineering, Av. Francisco Salazar 01145, Casilla 54-D Temuco, Chile

Exopolysaccharides (EPS) producing lactic acid bacteria are used in dairy industry (cheese and yogurt) due to the rheological properties that these compounds confer to the products. Preliminary results also suggest the use of EPS as health-promoting (anti-tumor and immunostimulatory actions) ingredients. Bifidobacteria are grampositive bacteria natural inhabitants of the gut of warm-blooded animals and man. A number of investigations have shown that bifidobacteria promote host health mainly because of the reduction proliferation of some pathogenic bacteria through acid synthesis.
In this work results obtained in the experiments carried out to test the capability of $B$. longum ATCC 15707 to synthesize EPS are presented. Continuous culture fermentations were carried out at dilution rates between 0.04 and $0.44 \mathrm{~h}^{-1}$. Composition of the culture media was that of the MRS broth. Biomass concentration presents higher values $\left(2.9-3.2 \mathrm{~g} \mathrm{~L}^{-1}\right)$ at dilution rates between 0.1 and $0.2 \mathrm{~h}^{-1}$. Biomass growing at these rates is difficult to pellet and adheres to the fermentor walls behavior that was not observed at other growth conditions. EPS from cultures grown at these rates were preparated and fractionated.

\section{Acknowledgment}

Authors wish to thank the Chilean CONICYT for the economical assistance given through the project Fondecyt 1050602.

\section{FB21}

Influence of HPLT treatment on the inactivation of $B$. subtilis cells $\underline{T . S h e n}^{1}$, G. Urrutia Benet ${ }^{2}$, S. Brul ${ }^{1}$, D. Knorr ${ }^{2}$ : ${ }^{1}$ URDV, Post box 114, 3130 AC Vlaardingen, The Netherlands; ${ }^{2}$ TUBER, Königin-Luise-Str. 22, D-14195 Berlin, Germany. E-mail: tong.shen@unilever.com (T. Shen)

High pressure-low temperature (HPLT) inactivation processes were performed on Bacillus subtilis vegetative cells at various conditions. At atmospheric pressure, lowering the temperature to as low as $-45^{\circ} \mathrm{C}$ was found to have minor anti-microbial effects. Upon application of high pressure various phase transitions occurred in the microbial suspensions under study. After pressure treatment at 150-450 MPa, cells were plated under optimal conditions to assess cell viability. Treatments at $250-450 \mathrm{MPa}$ and $-25^{\circ} \mathrm{C}$ were the most effective in inactivation. In these cases, ICE I-III solid-solid phase transition was observed. In addition, we hypothesised that intracellular thawing (solid-liquid phase transition) had already occurred while the extracellular surrounding was undergoing solid-solid phase transition. This double effect is suggested to be key in mediating the observed large drop in viability. We speculate that more cells survived after treatment at $-45^{\circ} \mathrm{C}$ compared to the same treatment at $-25^{\circ} \mathrm{C}$ because both the extra- and intracellular surrounding remained fully frozen. At $-45^{\circ} \mathrm{C}$ a solid-solid phase transition was observed when pressure was higher than $350 \mathrm{MPa}$. A metastable state of ICE I was observed at $250 \mathrm{MPa}$ treatment. Results from the current study will be presented (see also Shen et al., IFSET in press). The data call for a mechanistic evaluation of the effects of HPLT as an anti-microbial treatment. Such data are currently being gathered and will be used in defining optimal HPLT process conditions for the food industry.

\section{FB22}

The influence of Saccharomyces cerevisiae, Kloeckera apiculata and Candida pulcherrima mixed cultures on the selected alcohols formation during model fermentation Pawel Satora, Tadeusz Tuszynski Department of Fermentation Technology and Technical Microbiology, Food Technology Faculty, Agricultural University, Cracow, Poland. E-mail: psatora@ar.krakow.pl (P. Satora)

For the study five yeast species were chosen, isolated from successive stages of plum fruits spontaneous fermentation: from the Beginning (Candida pulcherrima, Kloeckera apiculata, Saccharomyces cerevisiae W4), Middle (S. cerevisiae W54) and Final Fermentation (S. cerevisiae $\mathrm{K} 1$ ). To characterize the potential influence of yeast mixed cultures on the selected alcohols formation, Wick- 
erham synthetic medium (10\% glucose) was fermented by mixed cultures of two and three yeast species. After distillation, ethanol, propanol, isobutanol, isoamyl alcohols, hexanol and 2-phenylethanol were determined using gas chromatography. Findings were compared with the results obtained after monoculture fermentations. The use of mixed cultures resulted in increasing of glucose utilization rate, ethanol and fusel alcohols formation (except propanol) and decreasing of methanol synthesis. The samples fermented using two yeast species characterized higher (about 10\%) amount of volatile compounds in relation to monocultures. It takes note of especially high level of ethanol (av. $\left.44.3 \mathrm{~g} / \mathrm{dm}^{3}\right)$, methanol $\left(16.7 \mathrm{mg} / \mathrm{dm}^{3}\right)$ and isoamyl alcohols $\left(47.6 \mathrm{mg} / \mathrm{dm}^{3}\right)$. The positive feature of triple cultures using was limitation of methanol and fusel alcohols synthesis that was accompanied by relatively high ethyl alcohol production (av. $41.5 \mathrm{~g} / \mathrm{dm}^{3}$ ).

\section{FB23}

Production of a fructooligosaccharide enriched sugar syrup from sucrose Andrelina M.P. Santos ${ }^{1}$, Francisco Maugeri ${ }^{2}{ }^{1}$ Laboratory Device and Nanostructure/UPFE, 50711-970, Recife, PE, Brazil; ${ }^{2}$ Department of Food Eng./Unicamp, 13081-970, Campinas, SP, Brazil. E-mail: lia_pinheiro@yahoo.com.br (A.M.P. Santos)

The consumption of sugar syrup becomes increasingly significant in industrial processes due to economic advantages and the easy of use. The production of sucrose syrup using enzymatic hydrolysis represents the safest alternative, once the reaction does not produce any toxic or undesirable substance. This work consists on the production of sugar syrup by immobilized inulinase from Kluyveromyces marxianus, with two alternatives process: (a) syrup enriched with fructooligosaccharides or solely with glucose and fructose. The process is comprised by the following stages: production and purification of the enzyme in optimized conditions, immobilization of the enzyme in solid support and the conversion of sucrose in a fixed bed bioreactor with the immobilized enzyme. The final composition of the product can be a mixture of glucose, fructose, sucrose and fructooligosaccharides or a mixture of fructose and glucose, according to the operational conditions. The bioreactor can be operated continually for approximately 5 months with the same biocatalyst. The product from this process is ideal for applications in the food products such as sweet, candies, chocolates, yogurts, etc. Besides, the prebiotics properties of the fructooligosaccharides, is a beneficial stimulant of the intestinal flora, which gives to the product a functional property.

\section{FB24}

Studies on plant microbial interactions using Azotobacter sp. as bio-inoculants towards soil fertility Baljeet Singh Saharan Faculty of Biotechnology, JCDM College of Engineering, Sirsa 125055, India. E-mail: baljeet.saharan@gmx.de, baljeet_br@yahoo.co.uk (B.S. Saharan)

High nitrogen fixing, phytohormone producing isolates of Azotobacter, Azospirillum, acetobacter and Pseudomonas were used as inoculants on wheat and cotton with varying doses of nitrogen under field conditions. Bio-inoculants were selected on the basis of yield, dry weight and survival rate of bacteria under field conditions. Seeds of wheat variety WH 711 were treated with different biofertilizers using nitrogen level of 90,120 and $150 \mathrm{~kg} \mathrm{ha}^{-1}$ and one level of P, i.e. $60 \mathrm{~kg} \mathrm{ha}^{-1}$ in field along with control.
Under field conditions, maximum yield was obtained with Azotobacter chroococcum E 12 at $90\left(2506 \pm 0.04 \mathrm{~kg} \mathrm{ha}^{-1}\right)$ as well as $120 \mathrm{~kg} \mathrm{ha}^{-1}\left(2817 \pm 0.07 \mathrm{~kg} \mathrm{ha}^{-1}\right)$ followed by $A$. chroococcum HT $57\left(2482 \pm 0.16 \mathrm{~kg} \mathrm{ha}^{-1}\right)$ and AVK $51\left(2474 \pm 0.37 \mathrm{~kg} \mathrm{ha}^{-1}\right)$. Whereas, with $120 \mathrm{~kg} \mathrm{ha}^{-1}$ highest yield was observed with Mac 27 $\left(2833 \pm 2.59 \mathrm{~kg} \mathrm{ha}^{-1}\right)$ followed by E12 $\left(2817 \pm 0.91 \mathrm{~kg} \mathrm{ha}^{-1}\right)$ and AVK $51\left(2804 \pm 0.16 \mathrm{~kg} \mathrm{ha}^{-1}\right)$. Maximum height at $90 \mathrm{~kg} \mathrm{ha}^{-1}$ was observed with Mac 27 inoculation $(71.1 \pm 5.24 \mathrm{~cm})$ followed by AVK $51(70.8 \pm 4.70 \mathrm{~cm})$ and HT $57(70.3 \pm 1.76 \mathrm{~cm})$. Various chosen strains were tested with Desi (HD 123) and American cotton (H 1098) under similar pot and field conditions as for wheat in the following season. Plant height and yield were determined at the time of harvesting whereas survival rate was monitored at various intervals of time. Survival rate of inoculated bacteria was determined after 30, 80, and 135 days. Highest survival rate was observed in Mac $68\left((3.34 \pm 2.56) \times 10^{6}\right)$, which decreased after 80 and 135 days, respectively. $(3.38 \pm 1.48) \times 10^{5},(1.53 \pm 0.92) \times 10^{5}$ with Mac 68 and $(2.97 \pm 2.01) \times 10^{5}$ and $(1.26 \pm 3.01) \times 10^{5}$ with HT 54 , respectively. Maximum boll weight was with AVK 51 (76.2 $\pm 2.34 \mathrm{~g}$ boll wt. plant $\left.{ }^{-1}\right)$ followed by Pseudomonas $(71.3 \pm 1.77 \mathrm{~g})$, AC 18 $(61.5 \pm 1.73 \mathrm{~g})$ and Ala27 $(61.4 \pm 2.79 \mathrm{~g})$ boll no. plant ${ }^{-1}$ was maximum with Ala 27 and AVK $51\left(46 \pm 2.59\right.$ plant $\left.^{-1}\right)$ followed by Pseudomonas $\left(34 \pm 0.07\right.$ plant $\left.^{-1}\right)$. Maximum height and dry matter was obtained with Pseudomonas $(179.7 \pm 1.97 \mathrm{~cm})$ and AVK521 $(146.7 \pm 3.49 \mathrm{~cm})$ with variety HD 123 under field conditions. Net saving of $20 \%$ nitrogen was observed using A. chroococcum (E 12 and AVK 51) bioinoculants for wheat and cotton, respectively.

\section{FB25}

Antioxidative properties and characteristic of tempeh albumins A. Rozwandowicz ${ }^{1,2}$, M. Koziolkiewicz ${ }^{1}$, T. Nurmi ${ }^{2}$ : ${ }^{1}$ Faculty of Biotechnology and Food Sciences, Technical University of Lodz, Lodz 90924, Poland; ${ }^{2}$ Life Sciences and Business, Turku Polytechnic, Turku 20520, Finland. E-mail: anita.rozwandowicz@turkuamk.fi (A. Rozwandowicz)

To characterize the antioxidative properties of tempeh-fermented food prepared from Vicia faba (L. Kontu) with the use of Rhizopus oligosporus, the sulfhydryl groups content and surface aromatic hydrophobicity of albumins were investigated. The results obtained for tempeh albumins were compared with raw Vicia faba and bovine serum albumin (BSA). These results indicate that tempeh fermentation increased antioxidative activity of albumins. The measurements of antioxidative activity were carried out with the use of 1,1-diphenyl-2-picrylhydrazyl (DPPH) and 2,2'-azinobis-(3ethylbenzothiazoline-6-sulfonic acid (ABTS). The albumins of Faba bean-tempeh have possessed much higher activity for scavenging free radicals as measured with the DPPH and ABTS $(49.4 \%$ and $41.1 \%)$ than raw seeds $(27.9 \%$ and $23.2 \%)$ and BSA $(5.8 \%$ and $13.4 \%$ ), respectively. It has been also found that tempeh fermentation process increased 2.5 times sulfhydryl groups content ( $43.2 \mu \mathrm{M} / \mathrm{mg}$ of albumins) as compared to raw seeds $(17.5 \mu \mathrm{M} / \mathrm{mg}$ of albumins). The tempeh albumins have possessed lower surface aromatic hydrophobicity than raw seeds (352.3 FI and 692.1 FI, respectively). 


\section{FB26}

Orange peel characterization and generation of fermentable sugars solutions for the biotechnological production of food additives $\underline{\text { B. Rivas }}^{1}$, J.M. Domínguez ${ }^{1}$, P. Torre ${ }^{2}$, J.C. Parajo ${ }^{1}:{ }^{1}$ Department of Chemical Engineering, Vigo University (Campus of Ourense), Polytechnic Building, As Lagoas, 32004 Ourense, Spain; ${ }^{2}$ Department of Chemical and Process Engineering, Genoa University, Via Opera Pia 15, 16145 Genoa, Italy. E-mail: brivas@ uvigo.es (B. Rivas)

The citrus processing industry generates in Mediterranean area around 3 millions tonnes of orange peel as byproduct from the extraction of citrus juices in industrial plants. In order to avoid ecological problems and provide an extra profit, this residue was studied in order to generate a suitable substrate for the fermentation process oriented to the production of food additives. Orange peels were characterized and the data collected allowed quantifying a $97 \%$ of this waste. Soluble sugars $(21.2 \%)$, cellulose $(17.0 \%)$ and pectin $(42.5 \%)$ were identified as more important fractions. This material was submitted to two hydrolysis techniques, prehydrolysis (with diluted sulfuric acid) and autohydrolysis (with water) under different experimental conditions. Autohydrolysis was selected as the most appropriate technique for the production of suitable fermentation media. Finally, the liquors obtained at $130^{\circ} \mathrm{C}$ and liquid:solid ratio of $8 \mathrm{~g} / \mathrm{g}$, containing $38.2 \mathrm{~g} / \mathrm{L}$ of sugars, without additional nutrients, were employed to citric acid production by Aspergilus niger CECT 2090 (ATCC 9142, NRRL 599). The influence of the addition of calcium carbonate and methanol were studied. Under the best conditions an effective conversion of sugars into citric acid was attained, showing the viability of the production of fermentable solutions from this industrial waste.

\section{FB27}

High gravity fermentations for low calories beer production Maya Piddocke ${ }^{1}$, Thomas Sørensen ${ }^{2}$, Sven Pedersen ${ }^{2}$, Lisbeth Olsson ${ }^{1}$ : ${ }^{1}$ Center for Microbial Biotechnology, BioCentrum-DTU, Technical University of Denmark, Denmark; ${ }^{2}$ Starch, Research \& Development, Novozymes A/S, Laurentsvej 51-53, DK-2880 Bagsvaerd, Denmark. E-mail: mpd@biocentrum.dtu.dk (M. Piddocke)

Today there is an increasing interest in using high gravity fermentation in brewing. High-gravity fermentation involves production of beer wort of up to $18^{\circ} \mathrm{P}$ or even higher and results in beer that has more consistent product quality. The main aim of this study is an increased understanding of how Brewer's yeast respond to the various stress factors imposed during high gravity beer fermentation and the consequences these stress factors have on the gene regulation and its consequences on the metabolite levels (both intra- and extracellular). Higher attenuation of the wort will be achieved by two different techniques: by the addition of highly fermentable adjuncts such as sucrose or glucose syrups and by mashing with addition of microbial enzymes such as pullulanases and glucoamylases. In the first part of the study model fermentation conditions are established, where the sugar uptake and product formation can be studied in details. Characterization of the carbohydrate profile is analyzed by HPLC. As flavour changes may occur at higher gravities, it is important to study changes in formation of secondary metabolites, especially esters. Transcriptome and metabolome analysis will be used to establish how the stressful conditions prevailing under high gravity fermentations may influence the secondary metabolism in
Saccharomyces cerevisiae. Furthermore, analytical aroma characterization of final beer will be studied by SPME and GC-MS. Detailed analysis of the effect of different stress factors on the cellular response using DNA arrays and metabolite profiling will be carried out. DNA arrays will be employed to evaluate if specific metabolic pathways are up-regulated or down-regulated as a consequences of the stress factors.

\section{FB28}

Operational stability of $A$. niger CECT2088 naringinase immobilized in PVA beads M.D. Busto, V. Meza, N. Ortega, M. Perez-Mateos Department of Biotechnology and Food Science, University of Burgos. Plaza Misael Bañuelos, s/n. 09001 Burgos, Spain. E-mail: mapema@ubu.es (M. Perez-Mateos)

Naringin, a bitter compound that occurs in citrus fruit juices, may be converted to a nonbitter form by enzyme hydrolysis. The enzymatic complex naringinase was produced in Aspergillus niger CECT2088 cultures with naringin as inducer (Pérez-Mateos et al., 2004). Crude extracts from A. niger and purified naringinase from Penicillium decumbens were immobilized into a polymeric matrix of polyvinyl alcohol (PVA) hydrogel cryostructured in liquid nitrogen. The operating stability of the PVA-naringinase beads was tested using synthetic citric juice (Gray and Olson, 1981). Immobilized enzymes reduced $40 \%$ the naringin content at $20^{\circ} \mathrm{C}$ and $\mathrm{pH} 3.2$. Furthermore, immobilized preparations from Aspergillus and Penicillium could be re-used through six cycles $(144 \mathrm{~h})$ remaining $70 \%$ and $38 \%$ catalytic efficiency, respectively.

\section{Acknowledgment \\ Financial support from "Ministerio de Ciencia y Tecnología" and FEDER (no. AGL2003-08006/ALI).}

\section{Reference}

Gray and Olson, 1981. J Agric. Food Chem. 29, 1298-1301.

Pérez-Mateos, et al., 2004. Eur. J. Biochem. 271 (1), 230.

\section{FB29}

Otimizing the fermentation broth for tanase production by a new isolated strain Paecilomyces variotii Vania Battestin, Gláucia Pastore, Gabriela Macedo Department of Food Science, UNICAMP, P.O. Box 6121, Campinas, CEP 13083-862 São Paulo, Brazil

Tannase is an inducible enzyme that catalyses the breakdown of ester linkages in hydrolysable tannins, resulting in gallic acid and glucose. The fermentation broth can use by-products as wheat bran, rice or oats, adding tannic acid. The use of by products or residues rich in carbon source for fermentation purposes an alternative to solve pollution problems that can be caused by an incorrect environmental disposal. In the present study we have optimized the production of an extracellular tannase by a new isolated Paecilomyces variotii using response surface methodology. The first step was to identify the variables having a significant effect on enzyme production. The variables evaluated were temperature, residues ratio (coffe: wheat bran), concentration of tannic acid, salt solution during 3, 5 and 7 days of fermentation time. Results showed that temperature, residues ratio (coffe: wheat bran) and tannic acid had significant effects on tannase production. Commercial wheat bran (CWB) and coffe rusk residues $(\mathrm{CR})$ were used as solid substrate. For fermentation the medium was composed by, CWB:CR were mixed with distilled water and transferred into $250 \mathrm{~mL}$ capacity Erlenmeyers flasks and auto- 
claved at $120^{\circ} \mathrm{C}$ for $20 \mathrm{~min}$. The medium was then inoculated with spores $\left(5.0 \times 10^{7}\right)$ and the flaks were incubated at $32^{\circ} \mathrm{C}$. Tannase was assayed according to the methodology of Mondal et al. (2001). According to the statist analyses, the optimum conditions to produce tannase was the range of temperature $\left(29-34^{\circ} \mathrm{C}\right)$; tannic acid (8.5-14\%); residues percent (coffe: wheat bran) (50:50) and 5 days fermentation time. The enzyme production increased 8.6 times more enzyme production than that was obtained before this optimization.

\section{FB30}

Controlling mixed fermentation of lactic acid bacteria and yeast using chitosan Y.F. Hong, Y.H. Park Department of Molecular Science and Technology, Laboratory of Applied Microbiology Graduate School, Ajou University, Suwon 443-749, South Korea. E-mail: yhpark@ajou.ac.kr (Y.H. Park)

Yeast and lactic acid bacteria are two major microbial groups of the most fermented products. A large variety of fermented foods and beverage are made by the activities of both yeast and lactic acid bacteria, simultaneously or successively. During the spontaneous mixed fermentation of lactic acid bacteria and yeast population, it is extremely difficult to control microbial species due to the complexity of the microorganism involved. Therefore, we have compared the antimicrobial activity of chitosan against two lactic strains, Lactobacillus plantarum and Lb. brevis, and yeast strains, Saccharomyces cerevisiae to investigate the possible use of non toxic biopolymer chitosan for selective control in mixed culture. The lactobacilli were more sensitive to the inhibitory activity of chitosan than S. cerevisiae. The results suggest the possible use of low molecularweight-chitosan for the control of food fermentation in which both groups of organisms frequently occur together.

\section{FB31}

The effect of vegetable oils on astaxanthin production of Phaffia rhodozyma and Xanthophyllomyces dendrorhous Csaba Vágvölgyi, Gyöngyi Lukács, Miklós Takó, Árpád Csernetics, Tamás Papp Department of Microbiology, Faculty of Sciences, University of Szeged, P.O. Box 533, Szeged H-6701, Hungary. E-mail: pappt@bio.u-szeged.hu (T. Papp)

Astaxanthin ( $3,3^{\prime}$-dihydroxy- $\beta, \beta$-carotene- $4,4^{\prime}$-dione $)$ is one of the most important carotenoid product. It is used primarily as food colorant and animal feed additive. Their effective antioxidant properties linked to a preventive action on various types of cancer and an enhancement of the immune response could lead to expanded commercial applications. Among the natural microbial source available, the closely related red pigmented yeasts Phaffia rhodozyma and Xanthophyllomyces dendrorhous are of great biotechnological interest. These yeasts have desirable properties as biological sources of pigment, including rapid metabolism and producing high cell densities in fermentor, but the commercial production of astaxanthin is limited by the relatively low content in wild-type strains. The purpose of this study was to determine whether the different vegetable oils had an effect on the carotenoid production in $P$. rhodozyma. Effects of media supplemented with corn germ oil, wheat germ oil, sesame-seed oil, palm oil, pumpkin-seed oil, coconut grease, olive oil (extra virgin), olive oil (sanza), sunflower-seed oil and cottonseed oil were tested. Studies were performed on both a Phaffia and a Xanthophyllomyces strain. Yeast was grown in yeast-pepton-glucose liquid medium complemented with the appropriate vegetable oil in different concentrations $(0.5-2, \mathrm{v} / \mathrm{v}, \%)$. After four days the total carotenoid production was determined spectrophotometrically, and it was referred to dry cell mass. Palm oil increased significantly the carotenoid production of the Phaffia strain, while a similar effect on the Xanthophyllomyces strain could be observed with coconut grease. Composition of carotenoid compounds in the strains was determined by thin layer chromatography.

\section{Acknowledgments}

This research was supported in part by grants from the Hungarian Scientific Research Fund (OTKA T37471, F46658 and D48537) and the Hungarian-Spanish Intergovernmental S \& T Cooperation Programme (OMFB00103/2005).

\section{FB32}

Over-expression of isoprene biosynthetic enzymes in the $\beta$-carotene producer zygomycete Mucor circinelloides Tamás Papp $^{1}$, Árpád Csernetics ${ }^{1}$, Antonio Velayos ${ }^{2}$, Enrique A. Iturriaga $^{2}$, Arturo P. Eslava ${ }^{2,3}$, Csaba Vágvölgyi ${ }^{1}:{ }^{1}$ Department of Microbiology, Faculty of Sciences, University of Szeged, P.O. Box 533, H-6701 Szeged, Hungary; ${ }^{2}$ Área de Genética, Departamento de Microbiología y Genética, University of Salamanca, Salamanca, Avda. Campo Charro s/n 37007, Spain; ${ }^{3}$ Centro Hispano-Luso de Investigaciones Agrarias, University of Salamanca, Salamanca, Avda. Campo Charro s/n 37007, Spain. E-mail: pappt@bio.u-szeged.hu (T. Papp)

Mucor circinelloides has been involved to study the carotene biosynthesis genesis of fungi. This fungus is more amenable to molecular techniques than the others traditionally used in carotenogenic studies (e.g. Blakeslea trispora and Phycomyces blakesleeanus). Moreover, Mucor has a great advantage: it is a dimorphic organism. This type of morphology is preferred by the fermentation industry because yeast-like growth allows the submerged culture, when usually higher biomass production can be achieved and cells can be more easily separated from the media. $\beta$-Carotene is a terpenoid-type chemical compound likewise to sterols, quinones or chlorophylls. The production can be increased by improving the non-carotene specific terpenoid biosynthesis. This can be carried out by the overexpression of the genes responsible for the ratelimiting steps of these pathways. In this study, polyethylene glycol mediated transformations of $M$. circinelloides protoplasts were performed with autoreplicative expression vectors containing the known terpenoid genes of $M$. circinelloides (e.g. isoA encoding farnesyl pyrophosphate synthase and $\operatorname{car} G$ encoding geranylgeranyl pyrophosphate synthase). Carotene production of the transformants and the wild-type strains were analysed by high-performance liquid chromatography (HPLC). Transformants harbouring plasmids with isoA or car $G$ produce about 1.5 times more carotene than the recipient strain, while carotene production increased about two times in the co-transformants containing both type of plasmids.

\section{Acknowledgments}

This research was supported in part by grants from the Hungarian Scientific Research Fund (OTKA T37471, F46658 and D48537) and the Hungarian-Spanish Intergovernmental S \& T Cooperation Programme (OMFB00103/2005). 


\section{FB33}

Identification of biotechnologically important Rhizopus strains on the basis of high affinity iron permease (FTRI) sequences Ildikó Nyilasi ${ }^{1}$, Tamás Papp ${ }^{2}$, Miklós Takó ${ }^{2}$, Erzsébet Nagy ${ }^{1}$, Csaba Vágvölgyi ${ }^{2}:{ }^{1}$ HAS-USZ Microbiology Research Group, Department of Microbiology, University of Szeged, P.O. Box 533, H-6701 Szeged, Hungary; ${ }^{2}$ Department of Microbiology, Faculty of Sciences, University of Szeged, P.O. Box 533, H-6701 Szeged, Hungary. E-mail: pappt@bio.u-szeged.hu (T. Papp)

Members of the genus Rhizopus are important from biotechnological aspects in consequence of their effective extracellular enzyme, alcohol and organic acid production. Moreover, Rhizopus strains are used for fermentation of various foods, because they are capable of transforming soybeans into edible products. The high affinity iron permease (FTRl) contains both highly conservative and variable regions applicable for phylogenetic comparisons. The aim of this study was the comparative analysis of this gene of different Rhizopus species in order to elaborate a simple and fast method to identify these fungi at a species and subspecies level. Conserved regions of Candida albicans and Rhizopus oryzae FTR1 genes (Fu et al., 2004) have been analysed to design degenerate primers for polymerase chain reaction. They were used to amplify the homologous regions from different strains of $R$. oryzae, $R$. microsporus, $R$. stolonifer and $R$. niveus. Isolates of the similarly thermophilic Rhizomucor miehei and $R$. pusillus, as well as a strain of $M$. rouxii were involved in the study as outgroups. Deduced protein sequences were aligned and phylogenetic analysis was performed. Surprisingly the $R$. oryzae isolates formed a group completely different with a significant distance from the $R$. microsporus isolate. $R$. niveus is currently not distinguished from $R$. stolonifer var. stolonifer because of morphological considerations. However, phylogeny of FTR1 gene sequences, in agreement with earlier results based on RAPD data (Vágvölgyi et al., 2004), raise the need to handle $R$. niveus as a separate species. Sequences and PCR primers useful for identification of all tested Rhizopus strains were elaborated.

\section{Acknowledgments}

This research was supported in part by grants from the Hungarian Scientific Research Fund (OTKA T37471, F46658, D48537) and GVOP-3.1.1.-2004-05-0471.

\section{References}

Fu, Y., Lee, H., Collins, M., et al., 2004. FEMS Microbiol. Lett. 235, 169-176.

Vágvölgyi, Cs., Heinrich, H., Ács, K., et al., 2004. Ant. Leeuwenhoek $86,181-188$.

\section{FB34}

Role of water on the lipase mediated hydrolysis of lutein diesters in non-conventional media Juan Mauricio Mora Pale, Eduardo Bárzana Facultad de Química, Lab E-314, Universidad Nacional Autónoma de México, México D.F. 04510, México. Email: mmpfacquim@yahoo.com.mx (J.M.M. Pale)

Lutein is considered a nutraceutic compound that has developed an increasing interest since it is one of the two carotenoids that are located in the macula of the human eye. Its consumption is associated with the prevention of age related macular disease (AMD). Industrially, lutein may be produced using a saponification step of a mixture of lutein diesters that are previously extracted with hexane from natural sources. Our proposal is to improve the process by catalyzing the same reaction using microbial lipases during the extraction step with hexane. Additionally, the use of supercritical fluids represents an extension of enzymology in non-conventional media with process and environmental advantages. This work was developed using extracts from Marigold flower (Tagetes erecta) in hexane and supercritical carbon dioxide $\left(\mathrm{SC}-\mathrm{CO}_{2}\right)$ where the lutein esters were hydrolyzed by two commercial lipases: lipase B from Candida antarctica (Novozym 435) and lipase from Mucor miehei (Lipozyme RM 1M). In particular, we focused our interest in the role of water in the system. Interestingly our results show an inverse dependence of the initial reaction rate with respect to the initial water activity (awi) for both lipases, a phenomena that seems to be related to the partition of substrates and products in the solid support)and the hexane phase as a function of water. When $\mathrm{SC}-\mathrm{CO}_{2}$ was used as solvent an increase in the consumption rate of lutein diesters occurred, reaching conversions of $70 \%$ in $24 \mathrm{~h}$. For hexane, the same conversion was reached after $160 \mathrm{~h}$. This result suggests a significant effect of the media on the reaction that can be related to shifts in the partition of compounds that bring the substrates in closer contact with the enzyme. This work also demonstrates that lutein hydrolysis seems to be another potential application of commercial immobilized lipases in the food/nutraceutical market.

\section{FB35}

Immobilization of $\boldsymbol{\beta}$-galactosidase from Lactobacillus sp. on chitosan and Eupergit C Thu-Ha Nguyen ${ }^{1,2}$, Barbara Splechtna ${ }^{1,2}$, Klaus D. Kulbe ${ }^{2}$, Dietmar Haltrich ${ }^{2}:{ }^{1}$ Applied Biocatalysis Research Centre, Petersgasse 14, A-8010 Graz, Austria; ${ }^{2}$ Division of FoodBiotechnology, Department of Food Sciences and Technology, Vienna University of Natural Resources and Applied Life Sciences (BOKU), Muthgasse 18, A-1190 Vienna, Austria. E-mail: thu-ha.nguyen@a-b.at (T.-H. Nguyen)

The enzyme of interest in this work is $\beta$-galactosidase from $\mathrm{Lac}$ tobacillus sp. (EC 3.2.1.23). $\beta$-Galactosidases catalyze the hydrolysis and transgalactosylation of $\beta$-D-galactopyranosides (such as lactose). An attractive biocatalytic application is found in the transgalactosylaction potential of these enzymes which is based on the catalytic mechanism of $\beta$-galactosidases. The products of transgalactosylation, galacto-oligosaccharides, are non-digestible carbohydrates which meet the criteria of 'prebiotics' and therefore have attracted increasing attention. To produce these 'prebiotic' galactooligosaccharides, an inexpensive and efficient process is desired. Immobilization of the enzyme $\beta$-galactosidase on an insoluble support is an attractive tool to make the process of lactose conversion more economical because the enzyme can be recovered and reused during continuous operation. In this present study, we aimed at immobilizing $\beta$-galactosidase from Lactobacillus $\mathrm{sp}$. by covalent linkages on two solid supports which are commonly used for protein immobilization: chitosan and Eupergit $\mathrm{C}$. The protein-binding capacity, the immobilization yield, $\mathrm{pH}$ and temperature dependency of activity and stability, and the kinetic parameters of immobilized enzymes were studied. Higher activity retention of the immobilized enzymes over a broader $\mathrm{pH}$ range and at higher temperatures compared to those of the free enzyme was observed. The immobilized enzymes were evaluated in terms of transgalactosylation activity and stability for a 
potential application in a lactose conversion process. The prebiotics market is at high demand therefore the development of the process to produce 'prebiotic' galacto-oligosacharides efficiently and inexpensively is our particular interest.

\section{FB36}

Evaluation of genetic diversity among mandarins germplasm in INRA Morocco by molecular markers $N$. Handaji ${ }^{1}$, J. Carlier $^{2}$, L. Cabrita ${ }^{2}$, H. Ben Yahia ${ }^{1}$, J.M. Leitao ${ }^{2}{ }^{1}$ BP257 INRA, Kénitra, Maroc, Portugal; ${ }^{2}$ FERN, Universidade do Algarve, Campus de Gambelas, 8005-Faro, Portugal. E-mail: nhandaji2002@yahoo.fr (N. Handaji)

Citrus, particularly mandarins and clementines, are among the most economically important fruit crops in Morocco. Besides morphological traits multiple molecular markers have been used for the caracterisation of Citrus germplasm. The main aim of this study was to evaluate the Moroccan mandarin germplasm and to identify specific polymorphisms among accessions sharing identical name. Eighty mandarin and two sweet orange varieties were analyzed by DNA markers. ISSR markers were amplified using $3^{\prime}$ anchored primers and analysed by agarose gel electrophoresis. AFLP markers analyses were performed using three primer combinations. The Dice coefficient was used to estimate genetic similarities and the UPGMA algorithm was utilised to generate a phenogram depicting the genetic relationships among the acessions. The selection of 9 primers out of the 31 ISSR primers primarily assayed, allowed us to maximize the average number of amplified fragments analyzed per reaction (7.3), and the percentage of informative polymorphisms (49\%). The three combinations of EcoRI/MseI primers revealed 73 reliable AFLP markers, 35 (47\%) of witch were polymorphic. The range of fragment sizes varied from 100 to $650 \mathrm{bp}$. Contrasting with the phenotypic diversity for agronomic and fruit quality traits, very low variability at the DNA level has found among mandarins, which always showed a high $(S>0.81)$ coefficient of genetic similarity. The molecular marker analyses allowed the clarification of ambiguous denominations and the establishment of phenological relationships. The mandarin cultivars have been clustered into several different sub groups. This study allowed the identification of one ISSR marker, distinct and specific for the Clementine Sidi Aissa and some AFLP markers specific to Maroc late and W. Navel. Many hybrids used in this study presented high coefficient of the similarity with one of their parents, such as Siamelo and King of Siam $(S=0.92)$, Fortuna and Clementine $(S=0.93)$, Kara and King of Siam $(S=0.92)$.

\section{Acknowledgments}

This work was partially funded by the program for Scientific Cooperation CNRST (Morocco) and ICCTI (Portugal), the International Foundation for Science (IFS) support in Stockholm (Sweden).

\section{FB37}

Identification of Morocco hybrid mandarin "Nadorcott" using isozymes, ISSR, RAPD and AFLP N. Handaji ${ }^{1}, J_{\text {. } \text { Carlier }^{2}, L}$ L. Cabrita $^{2}$, H. Ben Yahia ${ }^{1}$ J.M. Leitao $\bar{o}^{2}{ }^{1} \mathrm{BP} 257$, INRA, Kénitra Maroc, Portugal; ${ }^{2}$ FERN, Universidade do Algarve, Campus de Gambelas, 8005-Faro, Portugal. E-mail: nhandaji2002@yahoo.fr (N. Handaji)

The new Morocco mandarin variety Nadorcott became very important in the international market because of high quality, good size, easy peeling and absence of seeds. Also known as Afourer or W. Murcott, this variety was selected in 1981-1982 at the Afourer Experimental Station, INRA, located near to Beni Mellal city, as an original tree among several 18-year-old Murcott honney (C. reticulata $\times C$. sinensis) seedling trees. In order to shed additional light on the genetic origin of this variety, we have carried out isozyme and DNA fingerprinting analyses. For better interpretation of the Nadorcott molecular profiles, others mandarin cultivars, among which Murcott honney, were also analyzed by molecular markers. Three enzymatic systems (IDH, PGM and PGI) permitted the discrimination between Nadorcott and his female parent Murcott honney. The molecular patterns displayed by these cultivars point out the sexual origin of Nadorcott and discard the previously assumed hypothesis for its origin as a mutation of a nucellar zygote. The ISSR and RAPD markers analyses allowed the identification of Kinnow; Du japon, Vietnam; and Swett lime as the genetically most closely related mandarins $(S \sim 0.95)$ to Nadorcott. Strong genetic similarity was also found with the Clementine group, a possible male parent of Nadorcott. The analysis by AFLP markers confirmed the hybrid origin of Nadorcott and the high genetic relatedness $(S=0.93)$ of this mandarin to its putative female parent Murcott honey, and other cultivars as Kinnow $(S=0.94)$ and Clementine $(S=0.93)$. The possibility of an accurate molecular identification of Nadorcott by specific molecular markers is of paramount importance for the protection and management of this original Moroccan citrus variety.

\section{Acknowledgments}

The authors thank Dr. Hamid Narjiss (Director of Morocco INRA) for the instruction to identify Nadorcott mandarin by molecular markers and helpful discussions regarding this paper. This work was partially funded by the program for Scientific Cooperation CNRST (Morocco) and ICCTI (Portugal), the International Foundation for Science (IFS) support in Stockholm (Sweden).

\section{FB38}

Evaluation of distilled white lees for tartaric acid recovery and economic nutrient utilization J.M. Domínguez ${ }^{1}, B$. Rivas ${ }^{1}, N$. de la Torre ${ }^{1}$, A.B. Moldes ${ }^{1}$, M. Castro ${ }^{2}$, J. González ${ }^{2}$ : ${ }^{1}$ Departmento de Ingeniería Química, Universidad de Vigo (Campus Ourense), Edificio Politécnico, As Lagoas, 32004 Ourense, Spain; ${ }^{2}$ Vitivinicola do Ribeiro S.C.G., Valdepereira s/n, Ribadavia 32415 Ourense, Spain. E-mail: jmanuel@uvigo.es (J.M. Domínguez)

An effective process for the chemical-biotechnological utilization of distilled white lees was studied. A first treatment with hydrochloric acid allowed the solubilisation of tartaric acid. The influence of temperature, amount of $\mathrm{HCl}$ and reaction time were considered through an experimental design. Under the optima conditions $77 \mathrm{~g} / \mathrm{L}$ from white distilled lees and $45.6 \mathrm{~g} / \mathrm{L}$ from red distilled lees were recovered. The tartaric acid was precipitated as calcium tartrate so that it can be isolated from the rest of the raw material compounds. The solid residue was used as an economic nutrient for lactic acid production by Lactobacillus pentosus using trimming wastes as substrate. The lactic acid concentrations and volumetric productivities achieved were similar to those obtained using distilled lees without tartaric acid recovery as nutrient. 


\section{FB39}

Optimization of callus induction and regeneration media for Iranian cultivated rice (Oryza sativa L.) A. Mohammadi Department of Agronomy and Plant Breeding, Karaj Azad University, Iran. E-mail: a-mohamadi@kiau.ac.ir, mobin_ab@yahoo.com (A. Mohammadi)

To introduce of foreign genes for the important crop plants such as rice, we need a reproducible efficient procedure for regeneration of the calli through somatic embryogenesis. For this intention, we established the best callus induction medium for Tarom mahalli and Deilamani cultivars and created the method that the regeneration frequency was reached to $48 \%$. Calli were induced from scutellar tissues of mature seeds on MS medium supplemented with three level of 2,4-D (2, 2.5 and $\left.3 \mathrm{mg}^{-1}\right)$ and N6 medium supplemented with five level of 2,4-D $\left(1.5,2,2.5,3\right.$ and $\left.3.5 \mathrm{mg}^{-1}\right)$. For Deilamani cultivar the best medium was N6 with $1.5 \mathrm{mg} \mathrm{l}^{-1} 2,4-\mathrm{D}$ and for Tarom mahalli the same medium with $2 \mathrm{mg}^{-1}$ were the best. In subculture media, sucrose was used instead of maltose. For regeneration analysis of plantlets, We used two-factorial experiment in base of CRD; one factor was regeneration media with six levels (MS medium supplemented with five amount of kinetin and: NAA $\left(\mathrm{mg}^{-1}\right)[(6: 2)$, (4:2), (2:0.5), (3:1), (2:1), respectively] and $0.2 \mathrm{mg}^{-1}$ 2,4-D and $2 \mathrm{mg} \mathrm{l}^{-1}$ BAP). Other factor was dehydration process with three levels (without dehydration, dehydration with two layers of filter paper for $30 \mathrm{~min}$ [prior to transfer to the regeneration medium], and third factor was factor of 2 with substitution of sucrose with maltose [after 2 weeks; 2 sucrose: 1 maltose]). We conclude that maltose due to changing in osmolarity proceeding can elevate the regeneration frequency to $48 \%$. Therefore, type of carbon source is critical in callus induction and regeneration.

\section{FB40}

Production of carotenoids by bacterial and yeasts strains transformed by crt genes from Pectobacterium carotovorum Márová Ivana, Hrdličková Jana, Kubešová Jitka, Kočí Radka, Vidláková Tereza Faculty of Chemistry, Brno University of Technology, Purkyňova 118, 61200 Brno, Czech Republic. E-mail: marova@fch.vutbr.cz (M. Ivana)

Carotenoids are the most widespread natural pigments with important biological activities and applications mainly in food and feed industry. At present many ways including genetic engineering are developed to reach higher production of naturally formed carotenoids using microbial producers. In this work cloning and expression of crt gene cluster from Pectobacterium carotovorum in recipient bacterial strain $E$. coli $\mathrm{DH} 5 \alpha$ as well as in yeast strain $S$. cerevisiae was tested. Plasmid vector pHSG298 with inserted crt genes was used for transformation of chemically competent $E$. coli DH5 $\alpha$ cells, while in $S$. cerevisiae shuttle vector pAUR135 was used. Transformants were selected based on resistance to antibiotics, formation of orange-coloured transformant colonies, analysis of recombinant plasmid size and LC/MS analysis of carotenoids produced by recombinant cells. The yield of individual carotenoids (lutein, beta-carotene, lycopene) obtained from various bacterial transformants was several fold higher than in natural producer (lutein: $0.2-1.2 \mu \mathrm{g} / \mathrm{g}$ of d.w., beta-carotene: $0.1-1.4 \mathrm{mg} / \mathrm{g}$ of d.w.). The highest yield obtained in transformed strain was $63.5 \mu \mathrm{g} / \mathrm{g}$ of lutein and $15.4 \mu \mathrm{g} / \mathrm{g}$ of beta-carotene. The yield of biomass and carotenoids in. transgenic S. cerevisiae was comparable to some industrial red yeast strains ( $4.5 \mathrm{mg}$ of total carotenoids $+32 \mathrm{mg}$ ergosterol/1; $36 \mathrm{~g} / \mathrm{l}$ of biomass). So, transgenic yeasts could be suitable for large scale production of carotenoids and/or enriched biomass, while transgenic bacterial producers are perspective above al for high production of rare carotenoids as lutein or lycopene using transformation by specific genes of crt gene cluster.

\section{Acknowledgment}

This work was supported by the project MSM 0021630501 of the Czech Ministry of Education, Youth and Sports.

\section{FB41}

Effect of heating conditions of grape seeds on the antioxidant activity of grape seed extracts S.C. Lee, J.H. Park, S.J. Kim, A.R. Rhim Division of Food Science and Biotechnology, Kyungnam University, Masan 631-701, Republic of Korea. E-mail: sclee@kyungnam.ac.kr (S.C. Lee)

Two forms of grape seeds, whole and powdered forms, were heated at four different temperatures- $50,100,150$ and $200^{\circ} \mathrm{C}$. After heating, grape seeds were extracted with $70 \%$ ethanol $(0.1 \mathrm{~g}$ grape seed $10 \mathrm{~mL}$ of $70 \%$ ethanol), and total phenol contents (TPC), radical scavenging activity (RSA) and reducing power of the extracts were determined. Thermal treatment of grape seed increased the antioxidant activity of extracts. The maximum TPC and RSA of whole grape seed extract (WGSE) were achieved when the seeds were heat-treated at $150{ }^{\circ} \mathrm{C}$ for $40 \mathrm{~min}$, while that of powdered grape seed extract (PGSE) were at $100^{\circ} \mathrm{C}$ for $10 \mathrm{~min}$, and were greater than that of the non-treated control. According to the GC-MS analysis, several low-molecular-weight phenolic compounds were newly formed in the WGSE heated at $150^{\circ} \mathrm{C}$ for $40 \mathrm{~min}$. These results indicated that antioxidant activity of GSE was affected by heating conditions (temperature and time) and physical conditions of grape seeds at the time of heat treatments.

\section{FB42}

Analysis of the unexpected phenotypic consequences associated with plant transformation Jonathan Latham, Allison Wilson, Ricarda Steinbrecher EcoNexus, 6, Canon Frome Court, Ledbury HR8 2TD, UK. E-mail: jrlatham@gn.apc.org (J. Latham)

Transgenic plants often exhibit unexpected phenotypes. Such phenotypes could arise from pleiotropic effects associated with the transgene, or they could arise from other sources. A recent EcoNexus report underlined the potential for the process of plant transformation to result in genetic damage to the transformed plant (Genome Scrambling-Myth or Reality? Transformation-induced Mutations in Transgenic Crop Plants: http://www.econexus.info/). The report showed that mutations arising at the site of transgene insertion are often substantial, frequently resulting in loss or rearrangement of chromosomal DNA and insertion of multiple superfluous DNA fragments. Unintended mutations were also documented at other locations in the plant genome. Such transformation-induced mutations could provide an explanation for unexpected phenotypes in transgenic plants. We decided to survey regulatory documents and the scientific literature for instances of unexpected phenotypic consequences arising in transgenic plants. This poster documents the preliminary results of our survey. It is intended to assess the range and frequency of unexpected consequences and to examine whether there is sufficient data available to determine their origin. It is our 
belief that investigating the origin of these unexpected phenotypes should be a principal aim of biosafety research.

\section{FB43}

Biotechnological use of exogenous stress factors in production of carotenoids by red yeasts $\underline{R}$. Koci, I. Marova, M. Drabkova, T. Hulinova Faculty of Chemistry, Brno University of Technology, Purkyňova 118, 61200 Brno, Czech Republic. E-mail: kocir@fch.vutbr.cz (R. Koci)

Biotechnological production of metabolites such as carotenoids could be of high interest because of their antioxidative and antimutagenic activities in human body. Production of these metabolites by microbial cells is dependent on cultivation conditions. So, presence of exogenous stress in cultivation environment could stimulate biosynthetic pathways of desired metabolites. Two non-conventional yeast strains, Rhodotorula glutinis and Sporidiobolus salmonicolor, were chosen for study of carotenoid production useful in feed industry. Hydrogen peroxide, sodium chloride and/or their combinations were used as exogenous stimulators of carotenoid pathway. Presence of exogenous stress led to important overproduction of pigments as well as of supplementary studied substances (ergosterol, glycerol). Higher adaptability of yeast cells was observed not only in cultivations with one type of stress. Combination of stress factors in cultivation media induced significant increase of pigment formation. Moreover, under controlled conditions in laboratory fermentor $S$. salmonicolor produced about eight-fold amount of $\beta$-carotene $(230 \mu \mathrm{g} / \mathrm{g})$ in medium with $2 \% \mathrm{NaCl}$ and $5 \mathrm{mM} \mathrm{H}_{2} \mathrm{O}_{2}$ than in control sample. Similar result was observed in R. glutinis cultivated in presence of $2 \% \mathrm{NaCl}$ in inoculation medium only $(200 \mu \mathrm{g} / \mathrm{g}$ of $\beta$-carotene). The use of stressed biomass of red yeasts in feed industry could have positive effect not only in animal and fish feeds because of high content of physiologically active substances, but it could influence nutritional value and organoleptic properties of final products for human nutrition.

\section{Acknowledgment}

This work was supported by the project MSM 0021630501 of Czech Ministry of Education.

\section{FB44}

Critical effects of culture pH on the production of exopolysaccharides in submerged mycelial culture of Ganoderma lucidum Hyun Mi Kim, Jong Won Yun Department of Biotechnology, Daegu University, Kyungsan, Kyungbuk 712-714, South Korea. E-mail: jwyun@daegu.ac.kr (J.W. Yun)

The culture $\mathrm{pH}$ significantly affects mycelial growth and morphology, exopolysaccharide (EPS) formation, and their molecular properties during submerged cultures of a medicinal mushroom Ganoderma lucidum. When the culture $\mathrm{pH}$ shifts from 3 to 6 , mycelial growth $(12.5 \mathrm{~g} / \mathrm{l})$ and EPS production $(4.7 \mathrm{~g} / \mathrm{l})$ were favorable compared with other $\mathrm{pH}$-control strategies. The mycelial morphology was also significantly varied upon culture $\mathrm{pH}$ : a feather-like pellets were found when the $\mathrm{pH}$ was controlled shifting from 6 to 3 at day 4 , which was regarded as undesirable morphological form for EPS production. Compositional analyses revealed that the ratios and chemical compositions of the EPS formed in bottom or top fractions of ethanolic precipitates were significantly different upon culture $\mathrm{pH}$. The molecular characteristics of the EPS were further investigated using a size exclusion chromatography/multi-angle laser light scattering (SEC/MALLS) system.

\section{FB45}

Purification and characterization of a $\beta-N$-acetylhexosaminidase from Ginseng (Panax gingseng C. Meyer) roots Byeong Sik Yang, Jong Kook Moon, Do Hyun Jo Department of Molecular Science and Technology, Ajou Universisty, SuWon 442-749, South Korea. E-mail: dhj832@ajou.ac.kr (D.H. Jo)

Plant $\beta$ - $N$-acetyl-hexosaminidase (Hex) (EC 3.2.1.52) is reported to have diverse physiological roles like fruit ripening, degradation of reserved glycoproteins in germinating seed and chitin-elicited lignification. In this paper we report the purification and characterization of Hex from Korean Ginseng roots. After extraction with citrate-phosphate buffer, Hex was purified to homogeneity using ion exchanger chromatography, hydrophobic interaction chromatography and gel filtration. Its molecular weight was determined using gel filtration and mass spectrometer. Enzymatic parameters were studied with 4-methyl-umbelliferyl- $N$-acetylglucosaminide as substrate.

\section{FB46}

The effect of heat stress and weak organic acids on Escherichia coli and a comparison of its recovery by the plate count method and flow cytometry Monica S. Talsania, Christopher J. Hewitt, Peter Fryer Biochemical Engineering, Centre for Formulation Engineering, School of Engineering (Chemical Engineering), The University of Birmingham, Edgbaston B15 2TT, UK. E-mail: mxt128@bham.ac.uk (M.S. Talsania)

Due to the importance of microbiology for human health, methods have been developed to enumerate viable bacteria. Dilution plating is seen to be the 'gold standard' for proof of a cells viability. However, the success of this method relies on post sampling growth, which is limited by our ability to grow cells in the laboratory. Additionally, stressed or sub-lethally damaged cells, remain undetected. Single cell measurements can provide rapid detailed physiological information, and the assessment of population heterogeneity. This work compared the recovery of stressed $E$. coli as measured by the number of $\mathrm{CFU} / \mathrm{ml}$ and by multi-parameter flow cytometric analysis. Weak organic acids and high temperature-short time processing (HTST) were used to stress the cells both methods commonly used during food preservation. It was shown here that flow cytometry is a powerful tool for the enumeration and detailed analysis of any non-culturable microbial population, which is important because cytotoxic compounds and heat stresses used in food preservation often have a growth inhibiting effect but not necessarily a lethal one.

\section{FB47}

Paul G. Kovalenko Molecular Biology \& Genetics NASU, Zabolotnogo Str. 150, 031473 Kyiv, Ukraine

The pharmaceutically important plant species of Glycyrrhiza sp. (called licorice) is an important commercial product used as a natural sweetener, anti-inflammatory, anti-cancer and anti-diabetic agents. Agrobacterium rhizogenes transformation system was used for the hairy root cultures of licorice G. uralensis. After inoculation of aseptic stem segments the ability of hairy root formation was scored for a period of 6 weeks. Mean transformation frequency ranged from $27 \%$ (for 8196 up to $31 \%$ (for 15,834 ). Some transformed genotypes showed significant differences in roots weight, flavonoids and glycyrrhizin (Gl) production. The cotransformation rate of licorice intact explants cultivars with LBA 9402 TL-DNA and the 35S GUS gene showed an average of more than $35 \%$. These obtained root cultures 
were additionally elicited with extracts of biotic elicitor Acremonium sp. (endomycorhizal fungus), and were used as an in vitro system to metabolites production. The transformed and elicited hairy roots of G. uralensis were obtained by infection of A. rhizogenes 8196 have produced $\mathrm{Gl}$ at an yield of $4.5 \%$ dry weight on the period of culture as a 30 days. According to tentative analyses the hairy roots cultures of Glycyrrhiza species produced flavonoids (liquiritigenin and liquiritigen). More high levels ( $3.42 \mathrm{~g} / \mathrm{l})$ of the total flavonoids production have been identificated on the strains which transformed by LBA 9402. This study involved any difference among elicitor treatments and incubation periods for the optimal meabolites production. Clearly, the selection of an effective Agrobacterium strain for the production of transformed root cultures is highly dependent on the plant species, and must be determined empirically.

\section{FB48}

Ayse Gul Nasircillar Akdeniz University, Biology, Akdeniz Univ. Faculty of Art-Science, Biological Department, 07058 Antalya, Turkey

Mature embryos of five T. aestivum and five T. durum cultivars formed embryogenic callus on two different media. Embryos were removed from surface sterilised seeds and placed with the scutellum upwards on a solid agar medium containing the inorganic components of Murashige Skoog and $2 \mathrm{mg} / \mathrm{L}$ 2,4-dichlorophenoxyacetic acid (2,4-D) or $1 \mathrm{mg} / \mathrm{L}$ naphthalenacetic acid (NAA). The developed calli and regenerated plants were maintained on 2,4-D or NAA free MS medium. Wheat plants can be regenerated via two different systems. There were significant differences in percentage of callus induction and regeneration capacity on the different initiation medium. Among the T. aestivum cultivars, Yakar had the highest regeneration capacity in both induction medium. In $T$. durum cultivars, Kiziltan gave the highest regeneration capacity in MS + 2,4-D medium and Yilmaz gave the highest regeneration capacity in MS + NAA medium. A strong genotypic effect on the culture responses was found for both induction medium.

\section{FB49}

Triosephosphate isomerase has no control on the glycolytic flux and metabolic shift in Lactococcus lactis IL1403 Christian Solem, Brian Koebmann, Peter Ruhdal Jensen Microbial Physiology and Genetics, BioCentrum-DTU, Technical University of Denmark, Building 301, DK-2800 Kgs. Lyngby, Denmark. E-mail: brk@biocentrum.dtu.dk (B. Koebmann)

The glycolytic enzyme triosephosphate isomerase (TPI), which catalyses the interconversion of the triosephosphates dihydroxyacetone phosphate (DHAP) and glyceraldehyde-3-phosphate (GAP), was studied for its control on glycolysis and mixed acid production in Lactococcus lactis IL1403. We constructed a number of $L$. lactis strains in which the TPI activity was modulated from $3 \%$ to $217 \%$ of the wild-type level. The enzyme was found to be present in high excess with $3 \%$ TPI activity supporting $30 \%$ of the wildtype glycolytic flux, and with $24 \%$ of the wildtype TPI activity the glycolytic flux was essentially unchanged. Measurements of the upstream metabolites glucose-6-phosphate (G6P), fructose-1,6bisphosphate (FBP) and DHAP were essentially unchanged for TPI activities from $24 \%$ to $217 \%$, and only in the strain with $3 \%$ TPI activity we observed a significant increase in the intracellular DHAP concentration. Homolactic product formation was preserved throughout the interval of TPI activity studied, though a small increase in the amount of acetate and formate production was observed in the strain expressing TPI at the lowest level (3\% TPI activity). The finding of an increased mixed acid pattern under intracellular conditions with a high DHAP concentration is in contrast to earlier data from literature, which indicated that the triosephosphates play an important role in regulation of pyruvate metabolism in L. lactis with a negative effect on the mixed acid flux.

\section{FB50}

Alcohol-induced surface attachment of Listeria monocytogenes: Which mechanisms lie behind? Bettina Knudsen ${ }^{1}$, Hanne Jarmer ${ }^{2}$, Susanne Knфchel ${ }^{1}$, Anne Gravesen ${ }^{1}$ : ${ }^{1}$ Department of Food Science, KVL; ${ }^{2}$ Center for Biological Sequence analysis, DTU. E-mail: bekn@kvl.dk (B. Knudsen)

We have recently shown that alcohols induce the adhesion of L. monocytogenes at low temperatures, presumably accompanied by enhanced exopolysaccharide (EPS) production. However, little is known about the mechanisms involved in the formation of biofilm and EPS by L. monocytogenes. In the present project, we show that deletion of selected regulatory and up-regulated genes did not abolish attachment, though the degree of alcohol-induction in some cases was affected. We are applying bioinformatics to search for homologues in L. monocytogenes of known EPS genes from various gram positive bacteria. This has revealed candidate genes involved in the synthesis of EPS, such as genes encoding glycosyltransferases. Moreover, we are at present performing DNA microarray analysis for the EGDe strain grown at $10{ }^{\circ} \mathrm{C}$ in the presence of $2.5 \%$ isopropanol. This data should, combined with the bioinformatic results, give us a good indication of the genes involved in alcohol-induced surface attachment.

\section{FB51}

Application of repetitive-PCR in research on the cheese nonstarter microflora C.-H. Brogren, P. Christiansen, S. Sergianitis, E.W. Nielsen, Y. Ardö, F.K. Vogensen Department of Food Science, The Royal Veterinary and Agricultural University, Rolighedsvej 30, DK-1958 Frederiksberg C, Denmark

Repetitive-PCR (rep-PCR) was applied in research on non-starter lactic acid bacteria (NSLAB) in cheese. We first showed that strains previously differentiated by pulsed field gel electrophoresis (PFGE) also could be differentiated by rep-PCR. This was partially due to slight changes in the PCR conditions that allowed reproducible amplification of 7-9 kb bands. More than 20 bands were obtained for most strains. A clear differentiation was also obtained between Lactobacillus paracasei, Lactobacillus plantarum, Lactobacillus curvatus and Lactobacillus danicus (a new species found in Danish and Estonian cheeses and traditional starter cultures). We found that this technique is highly reproducible, e.g. identical profiles in three different PCR-machines, two different DNA isolation procedures, and different trained personnel. We applied the developed rep-PCR technique to confirm that survivors after heat treatment, were the actual strains introduced and not due to post-pasteurization contamination. We also showed that when we added a cocktail combination of five strains as protecting cultures to cheese, two to three members of this cocktail was dominating the cheese NSLAB microflora. In control cheeses without the cocktail in most cases other strains dominated, but in a few cases we were able to show cross-contamination between cheese vats. These data indicate that the rep-PCR will be useful to 
follow development of adjunct cultures as well as provide a reproducible subspecies (e.g. strain) differentiation. Rep-PCR is a much quicker and less labour requiring procedure than PFGE, and is apparently a much more reproducible technique than what has been seen for RAPD.

\section{FB52}

Dynamic modeling of Lactococcus lactis metabolism and its dynamic behavior for lactate secretion and regulatory characteristics Jinwon Lee, Ui Sub Jung, Hye Won Lee Department of Chemical and Biomolecular Engineering, Sogang University, Seoul, South Korea, 121-741. E-mail: jinwonlee@ sogang.ac.kr (J. Lee)

Dynamic metabolic model for Lactococcus lactis has been developed in order to analyze a time-dependent behavior of lactate secretion mechanism and probe its regulatory roles. The model was used to compare and analyze the lactate metabolism through in silico simulation and in vitro experimental measurements Most of all pyruvate branch point seems to play a major role in producing lactate, and the results of metabolic control coefficient analysis recommend to increase lactate dehydrogenase activity and to decrease NADH oxidase activity. For obtaining more realistic data, we have added some measured flux data including some intermediate metabolites. By combining the simulation results and experimental measurements, we could establish more reliable and robust systematic lactate secretion model. In addition, an efficient parameter estimation method was used to test the exactness of the reported kinetic parameters.

\section{FB53}

LC-MS contra direct infusion-MS for profiling of secondary metabolites in fungi Jørn Smedsgaard CMB, BioCentrum-DTU Technical University of Denmark 2800 Lyngby, Denmark. E-mail: js@biocentrum.dtu.dk

What to choose - the fast or the detailed - strategy to get informative profiles of secondary metabolite produced by fungi in culture. Chemo-diversity and lead discovery calls for high throughput techniques, but do we need columns will direct infusion ESI-MS (DiMS) do the job. The latter may give matrix effects and lacks resolution resulting in loss of information, while LC-MS analysis takes time and challenge the data processing. Results from nano-ESI DiMS and LC-MS analyses of the same extracts important Penicillium species are compared. These results illustrate advantages and problems using these techniques for rapid profiling of fungal secondary metabolites, reviling that matrix effects in DiMS do not seriously hampers detection of important metabolites while the specificity and certainty, for e.g. de-replication is much higher in LC-MS.

\section{FB54}

Phenotypic classification of fungi is essential in food biotechnology Ulf Thrane Center for Microbial Biotechnology, BioCentrumDTU, Søltofts Plads 221, Technical University of Denmark, DK2800 Kgs. Lyngby, Denmark. E-mail: ut@ biocentrum.dtu.dk

Fungi are of great importance in food and food production. The intended use of fungi as cell factories for production of food ingredients is an upcoming issue in food biotechnology; however, this brings up a possible contamination with mycotoxins as a major issue. A reliable identification of the producer strains is crucial as a correct identification at species level following an updated taxonomy is the key to information on functional characters, e.g. useful metabo- lites and potential mycotoxins, growth conditions, resistance, etc. Unfortunately, many mycological reports do not specify the taxonomy used or do not pay sufficient attention to taxonomical systems based on classification by functional characters - in contrast they are using a nucleotide sequence based phylogeny, which conveys little - if anything - about function of the organism. This situation is a major challenge for biotechnologists and mycologists in the years to come and will be highlighted by illustrative examples.

\section{FB55}

Viability and stability enhancement of Lactobacillus gasseri 5714 A. Garach Domech, U. Cuenca, M.J. Guardia Alba Departamento de Fermentación, Puleva Biotech, S.A. 18004 Granada, Spain. E-mail: albertogarach@pulevabiotech.es (A.G. Domech), ucuenca@pulevabiotech.es (U. Cuenca), mjguardia@pulevabiotech.es (M.J. Guardia)

The commercial interest in functional foods containing sufficient amounts of living probiotics is paralleled by the increasing scientific attention to the beneficial effect in the digestive tract. A daily intake of viable cells is proposed to ensure probiotic effect on consumer's health. One of the approaches which seems to be feasible to enhance probiotic viability and stability is to improve the fermentation conditions. During batch fermentation the viability of Lactobacillus gasseri 5714 decreases after reaching a maximal value apparently indicating cell death. In this work, the apparent loss of viability can be avoided during fed-batch fermentation. A three-fold increase in viability is obtained when nutrient concentration was controlled compared with the viability reached in batch cultures. As a consequence, higher biomass concentration and lower specific lactic acid production were obtained. A mathematical model was developed to simulate and describe the effect of nutrient limitation on growth, viability, glucose consumption and lactic acid production.

\section{FB56}

Contribution to the metabolic adaptation to food restriction in rabbits (preliminary results) $S$. van $\mathrm{Harten}^{1}$, S. Borges $^{2}$, P. Cravo $^{2}$, L.A. Cardoso ${ }^{1}:{ }^{1}$ Instituto de Investigação Científica Tropical, CVZ, Lisboa, Portugal; ${ }^{2}$ Instituto de Higiene e Medicina Tropical, Lisboa, Portugal. E-mail: svharten@gmail.com (S. van Harten)

In order to understand metabolic differences between two breeds of rabbits (Halop $\mathrm{AB}$ and oryctolagus cuniculus algirus) during food restriction, the activities and expression of key enzymes and hormones of the rabbit were studied. Animals from each breed were divided in two groups (ad libitum and restricted), revealing the results a similar difference in glycemic levels between fed and underfed rabbits, with a restriction of $50 \%$ of ad libitum feeding in the wild animals (decrease of $23 \% \mathrm{LW}$ ) and $16 \%$ of that ingestion in the Halop breed (decrease of $32 \% \mathrm{LW}$ ). The activities of glutamine synthetase and glutaminase show a higher reduction of these enzymes in the wild animals superior to that of the Halop breed, compromising, in this way, the ammonium detoxification and the entry of residual carbonated groups of the protein catabolism into the Krebs cycle. In the latter animals, a rapid mobilization capacity of triacylglycerols (TGA) appears to exist, with a rapid catabolism of fatty acids leading to their oxidation. The wild breeds' results reveal a rise of circulating TGA, reflecting difficulties in the lipolysis and mobilization of NEFA for oxidation. In these underfed animals, phosphoenolpyruvate and pyruvate suffered a large increase and oxaloacetate a decrease. The 
Halop breed revealed results that indicate a diminution of glycolisis, being glucoses' energy substituted by carbonated chains of lipolysis and protein catabolism. Hormone results showed a higher decrease in insulin, T3 and IGF-1 in the underfed Halop animals. In order to confirm the biochemical results, relative quantification of enzyme expression was studied by real time-PCR.

\section{FB57}

Knowledge, attitudes and perceptions of Kenyans on GM foods and their detection in food samples

Since the introduction of genetically modified (GM) crops in 1996, the area under their cultivation has globally increased from 1.7 million hectares in 1996 to 67.7 in 2003. The number of countries adopting GM crops also rose from one country, the USA, in 1996 to 20 in 2003. Despite numerous successes public opinion still questions the ecological, moral, ethical considerations and issues concerning altering the natural state of the organisms. In this study, a survey of food shoppers' knowledge, attitudes and perceptions of GM foods was carried out in food outlets in Nairobi. The food outlets were determined by simple random sampling. Using systematic sampling, shoppers were interviewed at targeted imported food products. Focus group discussions were also conducted with farmers at city markets. The survey reflected views of a systematic sample of 387 shoppers in seven food outlets between November and December of 2003. It revealed knowledge at $20 \%$, with positive attitudes and good perceptions towards GM foods $\left(\chi^{2}=42.873\right.$, d.f. $\left.9, P<0.001\right)$. Seventy nine percent of shoppers were willing to buy and consume GM foods $\left(\chi^{2}=61.321\right.$, d.f. $\left.2, P<0.001\right)$. Cross-tabulation of shopper's position on various issues raised in the survey showed a strong correlation between the respondents' respective knowledge, attitudes, and perceptions $(r=0.84)$. Nineteen percent of food sampled tested positive for GMs. Poisson statistics were used to calculate the number of sample sequences. The statistical tools were obtained from SPSS version 11.5. The results of this study will be of great interest in determining the use and adoption of GM crops in Kenya. It will also guide the development of national foreign food policy on GM foods. The technology should be embraced as soon as it is acceptable to alleviate, drought, famine and hunger estimated to be affecting 3.3 million Kenyans today, mostly children.

\section{FB58}

Consumers and GM foods: The case of Turkey Özlen $\ddot{O}_{z g e n}{ }^{1}$, Mustafa Yildiz ${ }^{2}:{ }^{1}$ Department of Family and Consumer Sciences, School of Home Economics, University of Ankara, Ankara 06130, Turkey; ${ }^{2}$ Department of Field Crops, Faculty of Agriculture, University of Ankara, 06110 Ankara, Turkey

The future development of food biotechnology depends on consumer acceptance. Scientists are aware that consumer attitudes will have a crucial impact on the process of the food biotechnology. Because, food is one of the central features in human life. Consumers' attitudes and trusts in the institutions will determine how gene technology will be used in food sector, in the future. Recently, research concerned with consumer aspects of GM foods accelerated. But in Turkey, the literature that deals with this subject is very limited and sparse. Therefore, this research was carried out on the Turkish consumers with the purpose of analyzing the consumers' awareness, assessments about benefits-risks, market place and labelling, and trusts in institutions, towards GM foods. This study was based on interviews with consumers who have recently purchased from major malls, during shopping hours. Of the four major malls, voluntary male and female consumers were included in the research if they had main or secondary responsibility for household shopping. The questionnaire form was applied to subjects through face-to-face individual interview. The data were analyzed by using statistical methods according to explanatory variables, including age, gender and educational level. Findings indicated that consumers' awareness and views about GM foods were connected to selected demographic characteristics. The results of this study can be important for consumer educators, marketing managers and policy makers.

\section{FB59}

Benefit-risk perceptions and moral beliefs of Turkish consumers towards transgenic products Özlen Özgen ${ }^{1}$, Haluk Emiroğlu ${ }^{2}$, Mustafa Yildiz ${ }^{3}$, Ayşe Sezen Taş ${ }^{1}:{ }^{1}$ Department of Family and Consumer Sciences, School of Home Economics, University of Ankara, 06130 Ankara, Turkey; ${ }^{2}$ Faculty of Law, University of Bilkent, 06590 Ankara, Turkey; ${ }^{3}$ Department of Field Crops, Faculty of Agriculture, University of Ankara, 06110 Ankara, Turkey

The use of biotechnology in production has generated considerable debate involving the benefits-risks and moral beliefs associated with its use. Consumer acceptance of genetically modified product is a critical factor will affect the future of this technology. This study was planned and conducted to determine the relationship between product/process related benefit perceptions, product/process related risk percentions and moral beliefs of consumers towards transgenic products. A total of 400 university educated consumers, consisting of 200 males and 200 females, employed at the ministries selected by random sampling method in Ankara, were included into study. Interview techniques were used in the gathering the research materials. The interview instrument had been prepared considering previous research and literature. Answers given to sentences typed Likert were scored, used "Varimax analysis technique" for validity. In order to test the reliability of questionnaire were calculated "Cronbach Alpha" as inner consistency coefficient. The $t$-test were performed for determining the differences dependent on gender and age variables between product related benefit perceptions, process related benefit perceptions, product related risk perceptions, process related risk perceptions and moral beliefs of consumers. The examination of relationships between product/process related benefit perceptions, product/process related risk perceptions and moral beliefs of consumers was made by correlation analysis technique. It is thought that the results of this study are important both for scientists and social scientists.

\section{FB60}

Knowledge, opinion and interest on agrobiotechnology by university students $I$. Monsalve, $\underline{\text { M Cámara }}$ Dpto. Nutrición y Bromatología II, Bromatología, Facultad de Farmacia, Universidad Complutense de Madrid, Plaza Ramón y Cajal s/n 28040 Madrid, Spain. E-mail: mcamara@farm.ucm.es (M. Cámara)

The application of the DNA recombinant technology for food production is generating a great debate in our society with the participation of scientists trying to explain the way of obtaining these new foods and which are their implications; environmentalist groups and anti-biotechnology associations that systematically are against to the application of this technology; legislatives bodies and the public in 
general, represented by consumers' organizations that expresses their right to be informed. Considering that university students will be the future professionals and consumers their opinion on this topic will be decisive in its success or failure, this research is aimed to performed a global and comparative study of the agrobiotechnology perception by students from different areas of knowledge and studies. This study was carried out during the academic years 2000-2004, being analyzed a total of 1516 valid surveys. The designed questionnaire included 30 questions relatives to: evaluation of the own knowledge and interest on the topic; evaluation of the information sources mainly used by university students to obtain nutritional information; the opinion about GM food labelling; risks/benefits perception; purchasing intention and support of biotechnology. Results obtained showed that $37 \%$ of the university students interviewed have a clear positive perception of biotechnology, mainly the students of the Health Sciences area. These students understand the scientific terminology and they use the university as the main source of information. They support the development of the biotechnology and they consider that in a future it will report them benefits. Other group (11\%) has a clear negative perception, they are mainly students from Law and Art History. They do not understand the scientific terminology, they consider that biotechnology will cause them risks, and as a consequence they don't have intention of buying these foods.

\section{FB61}

Review of Agrobiotechnology application for edible vaccines development $E$. Cebadera, $M$. Cámara Dpto. Nutrición y Bromatología II, Bromatología, Facultad de Farmacia. Universidad Complutense de Madrid, Plaza Ramón y Cajal s/n, 28040 Madrid, Spain. E-mail: mcamara@farm.ucm.es (M. Cámara)

The technology of the DNA recombinant can be also used to introduce in the plants genome the gene that codes a protein of interest for their use as antigen. The application of Agrobiotechnology has allowed the development of a new generation of vaccines that try to reduce or to eliminate the inconveniences of the classic ones. For the new vaccines design, the detailed knowledge of the biology of the pathogen is considered. With this knowledge the genes implied in virulence can be inactivated or modified selectively. The term "edible vaccines" it is usually applies to the use of edible parts of the plants (tubers, fruits, leaves, etc.) genetically modified with the purpose to produce specific components (antigens) of a pathogen (virus, bacteria, etc.) against which is wanted to protect a person or animal. However, oral is not the best vaccination route since the quantity of antigen for an efficient immunization it is usually high, being also needed, the co administration of an adjuvant that stimulates the immune answer. On the other hand, it is also important to highlight that the levels of antigen accumulation in transgenic plants is usually lower than the necessary ones. Another problem is the irregular accumulation of the antigen in the different parts of the plants, thus difficult the appropriate control of the doses. The edible vaccines that at the moment are in clinical phases of research (Phase I) are the following ones: vaccine against the Enteritis caused by Escherichia coli. expressed in potato plants; vaccine against infections caused by the virus Norwalk, expressed in potato plants and tobacco; vaccine against the virus of the Hepatitis B expressed in tobacco. Other fruits under experiment are banana and tomato.

\section{FB62}

Differentiation of Bifidobacterium longum strains using realtime PCR with SYBR Green I dye and melting curve analysis M. Shuhaimi ${ }^{1}$, Z. Suhaili ${ }^{1}$, A.M. Ali ${ }^{2}$, A.M. Yazid ${ }^{3}$, G. Farouk ${ }^{4}$, A.B. Sipat ${ }^{5}:{ }^{1}$ Department of Microbiology, Universiti Putra Malaysia, 43400 UPM Serdang, Selangor, Malaysia; ${ }^{2}$ Department of Molecular and Cell Biology, Universiti Putra Malaysia, 43400 UPM Serdang, Selangor, Malaysia; ${ }^{3}$ Faculty of Food Sciences and Technology, Universiti Putra Malaysia, 43400 UPM Serdang, Selangor, Malaysia; ${ }^{4}$ Department of Biotechnology and Engineering, Kulliyah of Engineering, International Islamic University, Gombak, Kuala Lumpur, Malaysia; ${ }^{5}$ Department of Biochemistry, Faculty of Biotechnology and Biomolecular Sciences, Universiti Putra Malaysia, 43400 UPM Serdang, Selangor, Malaysia. E-mail: shuhaimi@putra.upm.edu.my (M. Shuhaimi)

A pair of PCR primers for the rapid detection of Bifidobacterium longum BB 536, B. longum JCM 1210 and B. longum JCM 1217 were successfully designed based of $b s h$ gene previously described in $B$. longum BB 536 and B. longum SBT 2928. The primers have revealed the $b s h$ gene fragment with the size of approximately $151 \mathrm{bp}$ in all the $B$. longum tested. G/C content of the amplified fragments was analysed and two strains (B. longum BB536 and B. longum JCM 1210) showed the same percentage of $G / C$ content than the other strain (B. longum JCM 1217). In general, real-time PCR melting curve analysis correlated well with $\mathrm{G} / \mathrm{C}$ content analysis since the melting temperature of B. longum BB536 and B. longum JCM 1210 was determined to be $87.3^{\circ} \mathrm{C}$ and melting temperature of $B$. longum JCM 1217 was $87.7^{\circ} \mathrm{C}$. We conclude that real-time PCR amplification plot and melting curve analysis could be used as a powerful tool to rapidly detect as well as to differentiate strains of B. longum.

\section{FB63}

Removal of tannin from pomegranate juice by enzymatic method M. Ardjmand ${ }^{1}$, A. Seif Cordi ${ }^{2}$, F. Karbassi ${ }^{3}:{ }^{1}$ Postgraduate Faculty of Islamic Azad University, Tehran South Branch, Tehran, Iran; ${ }^{2}$ Chemical Engineering Department, Sharif University, Tehran, Iran; ${ }^{3}$ Chemical Engineering Department, Science and Research Campus, Islamic Azad University, Tehran, Iran

Tannin is polyphenolic component having some antioxidant properties and exists in many plants and fruits. In pomegranate juice this component causes turbidity and haze. During fruit juice clarification by conventional gelatin method, all poly phenolic substances which are responsible for antioxidant activity are removed and as a result the quality of the product is reduced. In the present study tannase enzyme (tannin acyl hydrolase; EC 3.1.1.20) was used to decompose tannin to gallic acid and glucose and as the result the amount of turbidity of the juice is decreased, however, the antioxidant properties remain unchanged since tannin is not decomposed and not separated in the juice as it occurs in the gelatin method. The amount of gallic acid in pomegranate juice samples before and after addition of tannase was measured using HPLC tests and the optimum temperature, the enzyme and juice contact time, $\mathrm{pH}$, and solvent concentration for clarification of pomegranate juice were obtained as $45^{\circ} \mathrm{C}, \mathrm{pH}=5.5,2 \mathrm{~h}$ and $50 \mathrm{mM}$ citrate buffer, respectively. The potential benefits of enzymatic clarification of pomegranate juice, that is preservation of antioxidant activity and hence increasing the quality of the fruit product, in comparison to that of conventional 
clarification method by gelatin introduce a new technique in turbidity and haze removed in tannin containing fruit juices.

\section{FB64}

Effect of food additives on the inhibitory activity of chitosan against foodborne pathogens Y.F. Hong, Y.H. Park Department of Molecular Science and Technology, Laboratory of Applied Microbiology Graduate School, Ajou University, Suwon 443-749, South Korea. E-mail: yhpark@ajou.ac.kr (Y.H. Park)

The objectives of this study are to investigate the inhibitory effect of low molecular weight chitosan for its use as biopreservatives of foods. The inhibitory activity of chitosan against Escherichia coli and Staphylococcus aureus was investigated by determining the effect of chitosan treatment on bacterial growth during culture and its effect on the viability of non-growing cells. The activity of chitosan was decreased considerably for both strains when sucrose was added to TS broth containing chitosan. The addition of ethanol affected little on the inhibition of chitosan against $S$. aureus. The influence of $\mathrm{NaCl}$ on the activity of chitosan was similar to the influence of sucrose and ethanol. However, the experiments with non-growing cells showed that the ethanol enhance drastically the antibacterial activity of chitosan on $S$. aureus. The results presented in this paper demonstrate that its antibacterial activity may be affected considerably by common food additives such as sucrose, ethanol and $\mathrm{NaCl}$.

\section{FB65}

Control analysis as a tool to understand the formation of the las operon in Lactococcus lactis Brian Koebmann, Christian Solem, Peter Ruhdal Jensen Microbial Physiology and Genetics, BioCentrum-DTU, Technical University of Denmark, Building 301, DK-2800 Kgs. Lyngby, Denmark. E-mail: brk@biocentrum.dtu.dk (B. Koebmann)

In Lactococcus lactis the enzymes phosphofructokinase (PFK), pyruvate kinase $(\mathrm{PK})$ and lactate dehydrogenase (LDH) are uniquely encoded in the las operon. We have applied Metabolic Control Analysis to study the role of this organisation. Earlier work showed that $\mathrm{LDH}$ at wildtype level has zero control on glycolysis and growth rate but high negative control on formate production $\left(C_{\mathrm{LDH}}^{J_{\text {formate }}}=-1.3\right)$. We find that PFK and PK have zero control on glycolysis and growth rate at the wildtype enzyme level but both enzymes exert strong positive control on the glycolytic flux at reduced activities. PK has high positive control on formate $\left(C_{\mathrm{PK}}^{J_{\text {frrmate }}}=0.9-1.1\right)$ and acetate production $\left(C_{\mathrm{PK}}^{J_{\text {acetate }}}=0.8-1.0\right)$, whereas PFK has no control on these fluxes. Decreased expression of the entire las operon resulted in a strong decrease in growth rate and the glycolytic flux. Increased las expression resulted in a slight decrease in the glycolytic flux. At the wildtype level the control was close to zero on both glycolysis and the pyruvate branches. The sum of control coefficients for the three enzymes individually was comparable to the control coefficient found for the entire operon at the wildtype level; the strong positive control by PK almost cancels out the negative control by LDH on formate production. The analysis suggests that co-regulation of PFK and PK provides a very efficient way to regulate glycolysis, and co-regulating PK and LDH allows the cells to maintain homolactic fermentation during regulation of glycolysis around wildtype level.

\section{FB66}

Cloning and expression of caprine prochymosin in Saccharomyces cerevisiae and Kluyveromyces lactis María C. Vega-
Hernández, Jesús Villar, Félix Claverie-Martín Unidad de Investigación, Asociada al Centro de Investigaciones Biológicas-CSIC, Hospital Universitario N.S. de Candelaria, 38010 Santa Cruz de Tenerife, Spain. E-mail: fclamar@gobiernodecanarias.org (F. Claverie-Martín)

Bovine chymosin is used extensively in cheese production because of its specificity and low proteolytic activity. We are interested in the caprine chymosin as an alternative because in the Canary Islands cheese has traditionally been made using goats milk with extract from the abomasum of newborn goats as coagulating factor. We isolated and characterized the prochymosin cDNA from the abomasum of milk-fed kid goats. This cDNA predicts a polypeptide of 381 amino acid residues, with a signal peptide and a proenzyme region of 16 and 42 amino acids, respectively. The caprine preprochymosin has $99 \%$ and $94 \%$ identity with the corresponding lamb and calf sequences. The cDNA fragment encoding prochymosin was fused in frame to the killer toxin signal sequence in a constitutive vector, and to the $\alpha$-factor signal sequence-FLAG in an inducible expression vector. Kluyveromyces lactis PM3-5C, K. lactis SEL1, characterized by a "supersecreting" phenotype, and Saccharomyces cerevisiae $\mathrm{BJ} 3505$ were transformed with the recombinant plasmids. Activated culture supernatants of yeast transformants showed milk-clotting activity. The FLAG-prochymosin fusion was purified from $\mathrm{BJ} 3505$ culture supernatants by affinity chromatography. After activation at acid $\mathrm{pH}$, proteolytic activity assayed toward casein fractions showed that the recombinant caprine chymosin specifically hydrolyzed $\kappa$-casein. The recombinant caprine enzyme could be an alternative milk coagulant in cheese making.

\section{FB68}

Carbon and nitrogen limitation in lipid accumulation of Schizochytrium G13/2S grown in continuous culture E. Ganuza, R. Colin, A. Anderson, S. Hopkins, M. Izquierdo Grupo de Investigación en Acuicultura, Instituto Canario de Ciencias Marinas, Aptdo de correos 56, Telde Las Palmas de Gran Canaria 35200, Spain. Email: enekoganuza@hotmail.com (E. Ganuza)

Lipid accumulation in Schizochytrium G13/2S was studied under batch and continuous culture. Different glucose and glutamate source concentrations were supplemented in a defined medium. During batch cultivation, lipid accumulation occurred towards the end of the growth phase but ceased when cell proliferation stopped. Under continuous culture, as dilution rate decreased from 0.08 to $0.02 \mathrm{~h}^{-1}$, both cell dry weight and total fatty acid content (TFA) of the cell increased. With a constant dilution rate of $0.04 \mathrm{~h}^{-1}$, nitrogen limitation induced lipid synthesis (28\% TFA) as described for other lipid-accumulating organisms. However, with carbon-limited conditions, some lipid accumulation was still possible, the TFA being $22 \%$. Finally, the batch and continuous culture methods are compared for docosahexaenoic acid $(22: 6, n-6)$ production.

\section{FB69}

Effect of food additives on the inhibitory activity of chitosan against foodborne pathogens Y.F. Hong, Y.H. Park Department of Molecular Science and Technology, Laboratory of Applied Microbiology Graduate School, Ajou University, 443-749 Suwon, South Korea. E-mail: yhpark@ajou.ac.kr (Y.H. Park)

The objectives of this study is to investigate the inhibitory effect of low molecular weight chitosan for its use as biopreservatives of 
foods. The inhibitory activity of chitosan against E. coli and Staphylococcus aureus was investigated by determining the effect of chitosan treatment on bacterial growth during culture and its effect on the viability of non-growing cells. The activity of chitosan was decreased considerably for both strains when sucrose was added to TS broth containing chitosan. The addition of ethanol affected little on the inhibition of chitosan against $S$. aureus. The influence of $\mathrm{NaCl}$ on the activity of chitosan was similar to the influence of sucrose and ethanol. However, the experiments with non-growing cells showed that the ethanol enhance drastically the antibacterial activity of chitosan on $S$. aureus. The results presented in this paper demonstrate that its antibacterial activity may be affected considerably by common food additives such as sucrose, ethanol and $\mathrm{NaCl}$.

\section{Environmental Biotechnology}

\section{Physiology of Microbial Communites in Wastewater Treat- ment}

1

Ecophysiology and genomics of nitrite-oxidizing bacteria important for wastewater treatment Holger Daims ${ }^{1}$, Denis LePaslier ${ }^{2}$, Eva Spieck ${ }^{3}$, Michael Wagner ${ }^{1}{ }^{1}$ Department of Microbial Ecology, University of Vienna, Austria; ${ }^{2}$ Genoscope, Evry, France; ${ }^{3}$ Biozentrum Klein Flottbek, Abteilung Mikrobiologie, Universität Hamburg, Germany

Nitrite-oxidizing bacteria catalyze an essential step of nitrogen elimination in biological wastewater treatment. Recently, novel and yet uncultured nitrite-oxidizing Nitrospira-like bacteria were found to be abundant in municipal and industrial wastewater treatment systems where they outcompete Nitrobacter, which has long been considered as the organism responsible for nitrite oxidation in bioreactors. Despite the importance of Nitrospira-like bacteria for wastewater treatment and for nitrogen fluxes in natural ecosystems, little is known about their ecophysiology and interactions with other organisms. Cultivation-independent molecular techniques were applied to investigate the diversity, distribution, and physiological and genetic features of Nitrospira-like bacteria in nitrifying activated sludge and biofilm. A surprisingly high diversity of these organisms was found to exist in these engineered and in natural habitats. Moreover, significant physiological differences could be identified among various phylogenetic sublineages in the genus Nitrospira. Quantitative co-localization analyses performed by novel image analysis software revealed that these metabolic features are reflected by the spatial organization of nitrifiers living in biofilm and activated sludge flocs. Based on an environmental genomics approach the genome of a Nitrospira-like bacterium found in activated sludge is being analyzed. Results obtained so far point at unexpected physiological capabilities of this organism, and allow us to propose that Nitrospira-like bacteria may also play roles in the bioremediation of (per)chlorate and chlorite.

2

Ecophysiology of bacteria involved in N- and P-removal in full-

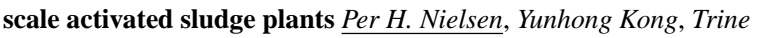
$R$. Thomsen, Jeppe L. Nielsen Deptartment of Life Sciences, Section of Environmental Engineering, Aalborg University, Sohngaardsholmsvej 57, DK-9000 Aalborg, Denmark
The activated sludge process is the most common way to remove organic matter, nitrogen and phosphorus from wastewater by microbiologically means. Knowledge about the microorganisms involved is fundamental for optimisation of existing plants and development of new plants and process designs. Many of the bacteria believed to be involved in nitrification, denitrification, biological phosphorusremoval, and removal of organic matter in full scale plants are now identified by use of molecular methods. Recent developments in experimental approaches have allowed the study of the ecophysiology of these uncultured and potentially important bacteria, thus providing a better understanding of their function in full-scale activated sludge ecosystems. Relatively few dominant species in each functional group (e.g. denitrifiers and polyphosphate accumulating organisms) seems to be present. Some species appear to be very specialized regarding nutrient requirements while others are more versatile.

\section{3}

Modification of the microbiological method for mercury remediation from industrial wastewater $P$. Gluszcz ${ }^{1}, S$. Ledakowicz ${ }^{1}, K$. Zakrzewska ${ }^{1}$,W.-D. Deckwer ${ }^{2}{ }^{1}$ Faculty of Process and Environmental Engineering, Technical University of Lodz, 90-924 Lodz, Poland; ${ }^{2} \mathrm{GBF}$, German Research Centre for Biotechnology, D-38124 Braunschweig, Germany. E-mail: pgluszcz@p.lodz.pl (P. Gluszcz)

A new method for mercury remediation from industrial wastewater based on the enzymatic reduction of mercury by live mercury resistant bacteria immobilized on the pumice particles has been developed in GBF, Germany, and implemented in the industrial scale (Unknown). The experience gained during operation of this instalation led to the idea, that the process of bioremediation may be integrated in one bioreactor with the sorption of mercury from wastewater, by immobilization of the bacteria directly on the activated carbon. For this it was necessary to define several significant parameters of the activated carbon used and the sorption process itself. The paper presents results of the equilibrium and kinetics investigations of the process of mercury sorption from aqueous solutions onto seven different types of activated carbon. The effective diffusion coefficients in the particles were obtained from the transient-state experiments and the sorption isotherms, saturation capacity of the sorbents and its dependence on the temperature and $\mathrm{pH}$ were identified. Then the hydrodynamic and sorption characteristics of the activated carbon bed in a laboratory-scale fixed-bed bioreactor were investigated in different process conditions (mercury concentration, volumetric flow rate, temperature, $\mathrm{pH}$ ). The results (effective capacity of the bed, dispersion and diffusion coefficients, mass transfer coefficient) enable implementation of this bioreactor for modified, integrated process of mercury bioremediation from industrial wastewaters.

\section{Acknowledgement \\ Research supported by the grant KBN 4 T09C 01325.}

\section{Reference}

http://www.gbf.de/mercury_remediation1/pilotplant1.html.

4

Comparative study of $\mathrm{Cr}(\mathrm{VI})$ reduction ability of Ochrobactrum tritici strain 5 bvl1 and Microbacterium sp. strain 3a R. Francisco $^{1}$, A.L. Kanouté ${ }^{2}$, M.C. Alpoim ${ }^{2}$, P.V. Morais ${ }^{1,2}$ : $^{1}$ Instituto do Ambiente e Vida, Departamento de Zoologia, 3004-517 Coimbra, Portugal; ${ }^{2}$ Departamento de Bioquímica, Faculdade de Ciências e Tecnologia 
da Universidade de Coimbra, Apartado 3126, 3001-401 Coimbra, Portugal. E-mail: rmfrancisco@ netcabo.pt (R. Francisco)

Bacterial $\mathrm{Cr}(\mathrm{VI})$ reductases convert the very mobile toxic $\mathrm{Cr}(\mathrm{VI})$ to the less toxic and less mobile $\mathrm{Cr}(\mathrm{III})$. The ability to reduce $\mathrm{Cr}(\mathrm{VI})$ was studied on cell extracts of Ochrobactrum tritici strain 5 bvl1 and Microbacterium sp. strain 3a. Both microorganisms were isolated from the same sample of chromium-contaminated sludge, taken from a wastewater treatment plant. While in the first case activity was found to be associated with the intracellular soluble extract, in the second case it was a process occurring extracellularly. $\mathrm{Cr}(\mathrm{VI})$ reduction by the intracellular soluble extracts of strain 5 bvl1 required the presence of NADH or NADPH as electron-donor, while the extracellular fraction of strain 3a only used NADPH. Several studies were made on strain 5 bvl1 intracellular soluble extracts. A $K_{\mathrm{m}}$ of $26.11 \mu \mathrm{M} \mathrm{Cr}(\mathrm{VI})$ and a $V_{\max }$ of $5.75 \pm 0.13 \mathrm{nmol} \mathrm{Cr}(\mathrm{VI}) \mathrm{min}^{-1} \mathrm{mg}^{-1}$ protein were estimated from the Lineaweaver-Burk plot and Michaelis-Menten non-linear regression. The temperature and the $\mathrm{pH}$ optima for $\mathrm{Cr}(\mathrm{VI})$ reduction were $37.5^{\circ} \mathrm{C}$ and 5.0, respectively.

\section{Extremeophiles}

\section{1}

Genome sequence of the Crenarchaeote Hyperthermus butylicus and thermostable enzymes of biotechnological and industrial interest Lanming Chen ${ }^{1}$, Kim Brügger ${ }^{1}$, Peter Redder ${ }^{1}$, Roger A. Garrett ${ }^{1}$, Hans-Peter Klenk ${ }^{2}$, Markus Stark ${ }^{2}:{ }^{1}$ Danish Archaea Centre, Institute for Molecular Biology and Physiology, Copenhagen University, Sølvgade 83H, 1307 Copenhagen K, Denmark; ${ }^{2}$ e.gene Biotechnologie GmbH, Poeckinger Fussweg 7a, 82340 Feldafing, Germany

Hyperthermus butylicus is an anaerobic hyperthermophilic crenarchaeon, isolated from the solfataric sea floor off Sáo Migel island, Azores (Zillig et al., 1990). H. butylicus grows at up to $108^{\circ} \mathrm{C}$ (optimally between 95 and $106^{\circ} \mathrm{C}$ ) at $\mathrm{pH}$ 7. It can utilize peptides, polysaccharides, and other substrates, as carbon sources to produce acetate, butyrate, and $n$-butanol. The capability to produce enzymes (e.g. hydrolases, DNA and RNA polymerases, etc.) that can tolerate and function at temperatures $20^{\circ} \mathrm{C}$ higher than most other thermophilic archaea, renders $H$. butylicus of particular interest to the biotechnology industry. The complete genome sequence of $H$. butylicus was determined and it contains $1,667,186 \mathrm{bp}$ on a single circular chromosome. 1695 protein encoding genes were identified which use a high level of UUG and GUG start codons. Many of these were assigned functions on the basis of sequence comparisons. Our analyses revealed some unusual metabolic properties in H. butylicus. Several sugar transporters were identified, although the set of genes required for glycolysis is incomplete. Moreover, genes encoding enzymes converting glucose to trioses are absent and no genes encoding enzymes of the pentose phosphate cycle or the KDPG pathway were detected. The $H$. butylicus genome encodes many proteases and peptidases although the Lon proteases, encoded in all other archaeal genomes, are absent. Although it was reported that $H$. butylicus does not utilize free amino acids in the media, genes for amino acid transporters were identified, and several proteins involved in di- or oligo-peptide transport are encoded. Genes encoding signal peptidases are absent. We will summarize gene products of special biotechnological interest.

\section{Reference}

Zillig et al., 1990. J. Bact. 172, 3959-3965.

2

Hot genomics: Insights in the thermophilic lifestyle of Thermus thermophilus from its complete genome Holger Brüggemann ${ }^{1,2}$, Anke Henne ${ }^{1}$, Gerhard Gottschalk ${ }^{1}{ }^{1}$ Göttingen Genomics Laboratory, Institute of Microbiology and Genetics, University of Göttingen, Germany; ${ }^{2}$ Institut Pasteur, Unité de Génomique des microorganismes pathogènes, Paris, France

Thermus thermophilus is an extremely thermophilic, halotolerant bacterium, which was originally isolated from a natural thermal environment. Recently completed genome sequences of two strains, HB27 and HB8, provide a solid foundation for investigating many aspects of thermophilic lifestyle; these range from molecular stability determinants to key elements of organismic physiology. In addition, the species has considerable biotechnological potential; many thermostable proteins isolated from members of the genus Thermus are indispensable in research and in industrial applications. The closely related genera Thermus and Deinoccoocus belong to a distinct branch of bacteria called the Deinococcus-Thermus group. Genome comparison of $T$. thermophilus and D. radiodurans, a mesophilic organism, which exhibits high resistant to radiation, oxidative stress, and desiccation, is of particular interest for the identification and exploration of thermophilic determinants. A large number of orthologs with a high degree of sequence identity are shared between the two species. This opens the opportunity for comparative studies of conformational and chemical thermostability of proteins, as well as for the identification of specific traits for each organism, explaining their unique physiological properties and their intriguing differences in stress tolerance. Although strains HB27 and HB8 share a highly conserved chromosome, striking differences can be found between their megaplasmids, which encode a huge proportion of genes not found in the genome of D. radiodurans. Possible contributions made by the megaplasmids to a thermophilic lifestyle will be discussed.

3 Extremophiles from Çamalti Saltern Area in Turkey Bahar Yavuztürk $^{1}$, Ebru Toksoy Öner ${ }^{2}$, Aziz Akın Denizci ${ }^{3}$, Dilek Kazan $^{2,3}$ : ${ }^{1}$ Department of Biology, Marmara University, Goztepe 34722 Istanbul, Turkey; ${ }^{2}$ Department of Chemical Engineering, Marmara University, Goztepe 34722 Istanbul, Turkey; ${ }^{3}$ The Scientific and Technical Research Council of Turkey (TUBITAK), Research Institute for Genetic Engineering and Biotechnology (RIGEB), Marmara Research Center Campus (MRC), Gebze 41470 Kocaeli, Turkey. E-mail: baharyavuzturk@yahoo.com (B. Yavuztürk)

Microorganisms that can live in high temperatures, extreme $\mathrm{pH}$ and high salt concentration are called extromophiles. Extromophilic microorganisms have extended our knowledge and understanding of fundamental questions such as the origin of life. The ability to grow in extreme conditions and to produce stable proteins makes extremopliles very attractive for the researchers and also for the industry. Extremozymes from extremophiles have a great economic potential in many industrial processes, including agricultural, chemical and pharmaceutical applications. Concurrent development of protein engineering will increase the application of enzymes from extremophiles in industry. Turkey has vast and various ecologi- 
cal areas, and so it has a broad microbial diversity. Based on the extremophilles which defined in the scope of this project, halophilic microorganisms produced industrially important proteins were isolated from Çamaltı Saltern Area in Izmir, Turkey. In this work, growth of isolates at different temperature, salinity and $\mathrm{pH}$ values were investigated to determine the effects of various growth conditions. Eight isolates grow at $\mathrm{pH}$ between 6.50 and 8.50 and two isolates at 6.50-7.50. They grow at temperature between 37 and $55^{\circ} \mathrm{C}$ and salt concentration between $3 \%$ and $25 \%$. The results of some phenotypic characters showed that they are gram $(-)$ and oxidase, ürease, DNAse and nitrate reduction are (-), and catalase $(+)$. They used $\mathrm{D}(+)$ glucose, maltose, lactose, sucrose, $\mathrm{L}(+)$ arabinose, $\mathrm{D}(+)$ mannose, glycerol and four of isolates used $\mathrm{D}(+)$ xylose as a carbon source. The isolates resistant to erythromycine, ampicilin sulbactam, cefoxitin, penicillin, bacitracin, novabiocin, amikacin and sensitive to ceftazidine, ciprofloxacin, amoxycillin/clavulanic acid, imipenem, chloromphenicol, ceftazidime/clavulanic acid, aztreonam, cefepime, cefotaxime, cefoperazone amoxicillin.

\section{Acknowledgement}

This project was supported by TUBITAK through project TBAG 2321-103T069.

\section{From Waste to Value}

\section{1}

The development of an integrated biorefinery concept for production of fuels and chemicals derived from lignocellulosic biomass Birgitte K. Ahring, Marie J. Mikkelsen, Slawek Dabrowski, Tania I. Georgieva, Frank D. Haagensen BioCentrum-DTU, Technical University of Denmark, DK-2800 Lyngby, Denmark

The technology of producing renewable energy sources such as ethanol, methane and hydrogen from biomass holds the potential of creating in-house energy resources while lowering the emission of greenhouse gasses as demanded by the Kyoto protocol. Recently, goals were defined for the European Union determining that 5.75\% of the transportation fuel has to come from biofuels in year 2010. A large-scale implementation of biofuels into the transportation sector will demand that lignocellulosic biomass, which is found in a surplus throughout the World is used as the raw material for the production process. The presentation will include a comprehensive description of the special bio-refinery concept developed in Denmark for production of biofuels and other valuable products from straw. The concept includes several innovative steps such as a pre-treatment method using wet oxidation, on-site production of enzymes and a continuous fermentation process using a genetic modified thermophilic bacterium. By co-producing several biofuels in the plant optimal use of the biomass has been assured and the price of for instance of bioethanol is getting close to conventional oil-based fuels. Optimizing each step in the bio-refinery, while having the full integration in mind, will be the way to make an economical viable biofuel production. In the presentation we will present our road map for achieving this goal in the nearest future.

\section{2}

Fuel ethanol from forest residues-technical and economic challenges Guido Zacchi, Mats Galbe, Christian Roslander, Anders Wingren Chemical Engineering, Lund University, P.O. Box 124, S22100 Lund, Sweden
Replacement of gasoline by liquid fuels produced from renewable sources is a high-priority goal in many countries worldwide. One such fuel, which has been found well suited, is ethanol. It may be produced from various lignocellulosic materials, such as forest and agricultural residues, which are fairly inexpensive. To compete with gasoline the production cost must be substantially lowered. Ethanol production from lignocellulose comprises the following main steps: hydrolysis of hemicellulose, hydrolysis of cellulose, fermentation, separation of lignin, recovery and concentration of ethanol and wastewater handling. The enzymatic hydrolysis and fermentation can either be run separately (SHF) or combined into a simultaneous saccharification and fermentation (SSF). The latter has been shown to result in higher ethanol yields than SHF. Some of the most important factors to reduce the cost are: efficient utilisation of the raw material by high ethanol yields, high productivity, high ethanol concentration in the feed to distillation and process integration in order to reduce capital cost and energy demand. In the last years we have performed several studies on the hydrolysis and fermentation of various forest and agricultural residues in a mini-pilot to improve the overall yield of ethanol and to reduce the energy demand and production cost. Steam pretreatment, with small addition of acid catalyst, has resulted in sugar yields close to $90 \%$ of the theoretical for various types of raw materials, e.g. spruce, salix and corn stover. The SSF has been developed and optimized to give high yield of ethanol. For spruce an ethanol yield of about $80 \%$ of theoretical based on the composition of the raw material has so far been obtained using a two-stage steam-pretreatment of $\mathrm{SO}_{2}$ impregnated raw material followed by SSF. Improvements of the SSF step, in the form of high dry matter content, recirculation of process streams and adapted yeast have resulted in ethanol concentrations around $45 \mathrm{~g} / \mathrm{l}$ leading to substantial reduction in energy demand and production cost. These improvements have been assessed by techno-economic evaluation to determine the effect on the ethanol production cost. The process has been further optimised by process integration to further reduce the energy demand. The ethanol production cost was estimated to be around $0.38-0.46$ Euro/l ethanol assuming a yearly capacity of 200000 tonnes raw material (dry matter).

3

Production of bioethanol from spent grain, a by-product of beer production Sho Shindo, Tadanori Tachibana, Akita Research Institute of Food and Brewing, Akita-city, Akita 010-1623, Japan. E-mail: shindo@arif.pref.akita.jp (S. Shindo)

The breweries generate one million tons of spent grain every year, and about $20 \%$ of the spent grain is recycled in Japan. Therefore, it is environmentally and economically significant to consider the production of ethyl alcohol as biomass energy using the spent grain from the breweries industry. Ethyl alcohol production from spent grain with immobilized yeast cells was investigated. Spent grains were liquefied by a steam explosion treatment to obtain liquefied sugar. When $1 \mathrm{~kg}$ of wet spent grain was treated under the $30 \mathrm{~kg} / \mathrm{cm}^{2}$ pressure for $1 \mathrm{~min}$ using a $5 \mathrm{~L}$ steam explosion reactor, $60 \mathrm{~g}$ of total sugar was obtained from the liquefied spent grain. Furthermore, 1.3\% (w/v) of glucose, $0.4 \%(w / v)$ of xylose, and $0.1 \%(w / v)$ of arabinose were produced when the liquefied spent grain was treated with glucoamylase, cellulase, and hemicellulase enzymes. Ethyl alcohol production was carried out by immobilized Sacchromyces cereviseae 
and immobilized Yamadazyma stipitis simultaneously from liquefied spent grain. Both yeast cells were immobilized on the glass beads carrier. Xylose and arabinose were consumed after glucose was consumed completely during ethyl alcohol production. 5.8\% (v/v) ethyl alcohol was produced from liquefied spent grain that was adjusted $17 \%$ of initial sugar concentration after 2 days.

4

Lipase catalyzed synthesis of biodiesel from castor oil in organic solvent Fábio Fernandes Fagundes, Rosângela Balaban Garcia, Marta Costa, Mauricio Rodrigues Borges, Chemistry Department, Federal University of Rio Grande do Norte, Postal Code 1662, Natal, Brazil

The vegetable oils constitute a resource of renewable potential for the production of fuels, becoming a viable alternative when compared to the diesel from petroleum. Among the vegetable oils, the extracted oil of the castor plant seeds is a promising alternative source because it is constituted mainly of the ricinoleic acid (12-hydroxy-9octadecenoic) that represents $90 \%$ of the total constitution of the oil approximately. The biodiesel obtained from castor oil can be defined chemically as being a mixture of methyl esters or ethyl esters of carboxylic acids synthesized by transesterification reaction of the existent triglyceride and an alcohol of little chain through the use of alkaline or enzymatic catalysts. In this work, we described the results found in castor oil with different degrees of purity. Initially, it was made a rheological characterization followed by structural characterization (RMN 13C, RMN 1H and infrared) and thermal characterization (DTG, DTA and DSC) of the crude and refined castor oil. It has been also measured the hydroxyl tenor, acidity index, saponification index and iodine index in different oils. Later, these results were used to evaluate possible differences in the quality of the biodiesel (ethyl esters) produced in the enzymatic alcoholysis of the castor oil catalyzed by lipases (Novozym 435, Liposyme RM IM and Lipozyme TL IM). The degree of substitution of castor oil derivative was performed by titration with $0.1 \mathrm{~N} \mathrm{HCl}$ and confirmed by TLC analysis and the results showed conversion rates about $90 \%$.

\section{5}

Different dephosphorylation pathways of bacterial phytatedegrading enzymes and their applications Malaysian rice bran utilization Abd-ElAziem Farouk ${ }^{1}$, Ralf Greiner ${ }^{2}$, Hamzah Mohd Salleh ${ }^{1}$ : ${ }^{1}$ Biomolecular Engineering Research Group, Department of Biotechnology Engineering, Kulliyyah of Engineering, International Islamic University Malaysia, Jalan Gombak, 53100 Kuala Lumpur, Malaysia; ${ }^{2}$ Centre for Molecular Biology, Federal Research Centre for Nutrition and Foods, Haid-und-Neu-Straße 9, D-76131 Karlsruhe, Germany

myo-Inositol hexakisphosphate (IP6) has been demonstrated to have a wide range of health benefits such as prevention and therapy of various cancers, amelioration of heart disease, and prevention of renal stone formation as well as complications from diabetes. On the hand, lower phosphorylated forms of inositol, especially inositol trisphosphate (IP3) and inositol tetrakisphosphate (IP4) are important signal transduction molecules within the cells both in plants and the animal kingdom. It has been hypothesized that at least the anticancer function of IP6 is mediated via these lower inositol phosphates. The diversity and practical unavailability of the individual myo-inositol phosphates preclude their investigation. Phytases, which catalyze the sequential hydrolysis of phytate, render production of defined myoinositol phosphates in pure form and sufficient quantities. Different phytases may result in different positional isomers of myo-inositol phosphates and therefore different biochemical properties. Phytases differing in $\mathrm{pH}$ optima, substrate specificity, and specificity of hydrolysis have been identified in plants and microorganisms. In this paper the dephosphorylation pathway of the novel PhyFAUIA1 was compared to other bacterial phytate degrading enzymes. Preliminary results have shown that PhyFAUIA1 converted IP6 into IP5 (myoinositol 1,2,3,5,6-pentakisphosphates) and another isomer, which is yet to be elucidated.

\section{New Trends in Bioremediation and Wastewater Treatment}

\section{1}

New visions on life style and application of anammox bacteria Boran Kartal ${ }^{1}$, Wouter van der Star ${ }^{2}$, Mike Jetten ${ }^{1,2}$, Cristian Picioreanu ${ }^{2}$, Mark van Loosdrecht ${ }^{2}$, Marc Strous ${ }^{1}$ : ${ }^{1}$ Institute of Water and Wetland research, Department of Microbiology, Radboud University Nijmegen, Toernooiveld 1,6525 ED Nijmegen, the Netherlands; ${ }^{2}$ Department of Biotechnology, TU Delft, Julianalaan 67, 2628 BC Delft, the Netherlands. E-mail: M.Jetten@science.ru.nl; URL: www.anammox.com, www.iwwr.science.ru.nl; www.microbiology.science.ru.nl (M. Jetten)

In a denitrifying pilot plant reactor, a new obligately anaerobic ammonium oxidation (anammox) process with great potential for nitrogen removal for high strength wastewater was discovered. After transfer of the complex microbial community to a laboratory SBR system, a highly enriched population, dominated by a single anaerobic chemolithoautotrophic bacterium related to the Planctomycetes was obtained. The bacterium was purified via percoll centrifugation and characterized as 'Candidatus Brocadia anammoxidans'. Survey of different wastewater treatment plants using anammox specific 16S rRNA gene primers and anammox specific oligonucleotide probes revealed the presence of at least four other anammox bacteria, tentatively named 'Candidatus Kuenenia stuttgartiensis', 'Candidatus Brocadia fulgida', 'Candidatus Scalindua wagneri' and 'Candidatus Scalindua brodae'. A close relative of the two Scalindua species, 'Candidatus Scalindua sorokinii' was found to be responsible for about $50 \%$ of the nitrogen conversion in the anoxic zone of the Black Sea and in the Benguela upwelling system along the Namibian coast, making anammox an important player in the global nitrogen cycle. Electron microscopic studies of all five anammox bacteria showed that several prokaryotic membrane-bounded compartments are present inside the cytoplasm, which are surrounded by unique ladderane lipids. Hydroxylamine oxidoreductase, a key anammox enzyme, was present exclusively inside one of these compartments, named the 'anammoxosome'. Unique peptides fragments of the purified $\mathrm{HAO}$ were used to locate the hao gene in genome assembly of 'Candidatus Kuenenia stuttgartiensis'. The implementation of the anammox process in the treatment of wastewater with high ammonium concentrations was started at the treatment plant in Rotterdam, the Netherlands, where it is combined with the partial nitrification process SHARON. The estimated price for nitrogen removal with partial nitrification and anammox is about 0.75 euro $/ \mathrm{kg} \mathrm{N}$. 
Gas lift reactors could sustain the highest anammox capacity at $8.9 \mathrm{~kg} \mathrm{~N}$ removed $/ \mathrm{m}^{3}$ reactor per day. An alternative configuration of anammox is the oxygen-limited CANON process in which aerobic ammonium-oxidizing bacteria protect anammox bacteria from oxygen and produce the necessary nitrite. Maximum nitrogen removal with CANON in gas lift reactors was $1.5 \mathrm{~kg} \mathrm{~N} / \mathrm{m}^{3}$ reactor per day. Using several different conditions and parameters, the competition and co-existence of aerobic and anaerobic ammonium-oxidizing bacteria were modeled. In addition to ammonia, urea was also converted after a 2-week adaptation in the CANON system. Recently it was shown that anammox bacteria can use organic acids as additional energy source.

3

Bioreactor design strategies for bioremediation applications Murray Moo-Young, WA Anderson Department of Chemical Engineering, University of Waterloo, Waterloo, Ont., Canada N2L 3G1

Bioreactors are central to the bioremediation of contaminated environments of water, air or soil. In all three areas of application, bioreactor design is critical to the development of new or improved processes. This overview focuses on the physical limitations of bioreactors caused by biological requirements. The information is based on our own research findings. The need for more applicationsoriented bioremediation research becomes apparent. For technoeconomic reasons, the airlift type has often been the bioreactor of choice for most bioremediations. However, lack of adequate understanding of the quantitative effects of operating conditions on its performance has been an ongoing concern. These effects have been characterized for engineering implementation. To enhance productivity, innovative pretreatment techniques of the polluted sources have also been developed using photocatalytic and chemical oxidation methods. Case studies on petrochemical-contaminated water and soil reveal significant enhancement potentials. Other studies on microbial biofilters for air bioremediation indicate that the active mass of the biological consortia is not sufficiently understood for rational design.

\section{4}

Analysis and retrofit design of wastewater treatment facilities using process simulation tools Demetri Petrides, Alexandros Koulouris, INTELLIGEN, Inc., Scotch Plains, NJ 07076, USA. Email: dpetrides@intelligen.com (D. Petrides)

Process simulators have been used in the petroleum and chemical industries for over four decades to facilitate the design of new processes and optimize the performance of existing ones. Similar benefits can be derived from the use of such tools in the environmental arena, particularly in the field of physical and biological treatment of municipal and industrial wastewater. Specifically, process simulators can be used to evaluate and improve options for: (1) more efficient removal of nutrients (e.g., organic nitrogen and phosphorous) that cause eutrofication, (2) estimation and control of volatile organic compound (VOC) emissions from open tanks, and (3) more efficient removal and control of hazardous compounds. The potential benefits will be illustrated with cases studies involving both municipal and industrial wastewater facilities.

\section{Poster Abstracts Environmental Biotechnology (EB)}

\section{EB1}

Precipitation of metal by Desulfovibrio sp. in presence of petroleum R. Pérez, J.M. Gómez, A. Ábalos, D. Cantero Study Centre of Industrial Biotechnology, Faculty of Natural Sciences, University of East, Santiago of Cuba, CP 90500, Cuba. E-mail: rmaria@cebi.uo.edu.cu (R. Perez)

The microbial reduction of metals has showed recent interest as these transformations can play crucial roles in the cycling of both inorganic and organic species in a range of environments and, if harnessed, may offer the basis for a wide range of innovative biotechnological processes. Under certain conditions, however, microbial metal reduction can also mobilise toxic metals with potentially calamitous effects on human health. Some effluents present heavy metals as soluble compounds, several microorganisms have the capacity to precipitate these metals as insoluble compounds, and this fact allows the collection and separation of these metallic precipitates from contaminated medium. Sulfate-reducing bacteria (SRB), under anaerobic conditions, oxidize simple organic compounds (such as acetic acid and lactic acid) by utilizing sulfate as an electron acceptor and generate hydrogen sulfide. Hydrogen sulfide reacts with heavy metal ions to form insoluble metal sulfides that can be easily separated from a solution. The purpose of this work was study the capacity of Desulfovibrio sp. cultures to reduce mixtures of the heavy metals in presence or not of petroleum. For it the experimental design $2^{k}(k=5)$ was carried out. The five studied factors were $\mathrm{Cr}, \mathrm{Cu}, \mathrm{Mn}, \mathrm{Zn}$ and petroleum. The study was carry out with Desulfovibrio sp. Batch studies were performed in $50 \mathrm{ml}$ sealed bottles with different concentrations (Cr(III)-10 ppm, $\mathrm{Cu}$ (II)$5 \mathrm{ppm}, \mathrm{Mn}(\mathrm{II})-10 \mathrm{ppm}, \mathrm{Zn}(\mathrm{II})-15 \mathrm{ppm}$ ) of metal sulfate and $2 \mathrm{~g}^{-1}$ of petroleum. During batch incubation the dissolved concentration of metal studied in supernatant were decreased to undetectable levels for $\mathrm{Zn}(70-100 \%)$, however with $\mathrm{Cu}(40-60 \%), \mathrm{Mn}(40-70 \%)$ and $\mathrm{Cr}(50-80 \%)$. The development of continuous process with sulfatereducing bacteria seems to be a suitable alternative to reduce metals in solution from contaminated media such as industrial or mine effluents. After these preliminary results, some experiments in course are focused to study that purpose.

\section{EB2}

Reduction of odour emissions from livestock buildings using a bioscrubbing system Morten Øgendahl, Nawaf Abu-Khalaf, Jens J $\phi$ rgen Lonsmann Iversen Department of Biochemistry and Molecular Biology, University of Southern Denmark, DK-5230 Odense M, Denmark. E-mail: tvede@bmb.sdu.dk (M. Øgendahl)

A bioscrubbing system for reducing odour emissions from livestock buildings is presented. The bioscrubbing system consists of two separate units; an absorption column and a water purification module. The absorption column is mounted in the ventilations stacks in the livestock buildings absorbing odorants in the effluent air flow. The odorants are absorbed in a spray of droplets formed by a grid of high pressure nozzles in the inlet of the absorption column. The spray of droplets is extracted from the air flow and pumped to a centrally located water purification module, an inverse three phase fluidised bed bioreactor, where the bio-degradation of the absorbed odorants occurs. The bioreactor features a split sparging system for maximum mixing and aeration. The cleaned water is recirculated 
to the absorption column. An electronic tongue will quantify key odorants in the bioreactor. The absorption column is designed to be retrofitted into existing livestock building ventilation systems. The water purification module is constructed in standard size units simplifying scaling to match the requirements of individual applications. The total bioreactor volume is increased by increasing the number of standard bioreactors.

\section{EB3}

This work describes a "light off" toxicity bioassay sensor based on whole cell genetically modified bioluminescent bacteria. The biosensor was constructed by mating between the environmentally isolated phenol-degrading Acinetobacter sp. strain DF4 and the plasmid PUTK2 that is an Inc $\mathrm{P} \beta$ plasmid with the bioluminescence genes luxCDABE inserted into a genetic region involved in plasmid replication and transfer. Subsequently, the bioreporter designated DF4/PUTK2 and used to investigate phenolics toxicity. Among examined phenolics, pentachlorophenol, catechol and nitrophenol recorded the fastest effect on the bioluminescence of bioreporter DF4/PUTK2 over incubation period of $350 \mathrm{~min}$. The effect of various concentrations of phenol and its derivatives either in an individual, duple or triple mixture forms on the bioluminescence response of the constructed bioreporter DF4/PUTK2 were also examined. Significant reduction of the bioluminescence was observed whenever a mixture contained pentachlorophenol, catechol and nitrophenol, respectively. To develop a system appropriate to commercialize, the constructed bioreporter DF4/PUTK2 was subjected for immobilization in microtiter plates using several entrapment gels. After a selection of materials was tried, LB/Agar was chosen as the most suitable candidate material.

\section{EB4}

Quantification of odorants in an air wet scrubber using direct aqueous injection-gas chromatography (DAI-GC) and solid phase extraction-gas chromatography (SPE-GC) methods Nawaf Abu-Khalaf $^{1}$, Kim F. Haselmann ${ }^{2}$, Jens Jørgen Lønsmann Iversen ${ }^{1}:{ }^{1}$ Department of Biochemistry and Molecular Biology, University of Southern Denmark, Campusvej 55, DK-5230 Odense M, Denmark; ${ }^{2}$ Department of Chemistry, University of Southern Denmark, Campusvej 55, DK-5230 Odense M, Denmark. E-mail: nawaf@bmb.sdu.dk (N. Abu-Khalaf)

Characterization of key odour compounds in an air wet scrubber is presented. The key odour compounds represent five chemical groups, i.e. sulphide, alcohol, volatile fatty acids (VFAs), phenol and indole. Direct aqueous injection (DAI) and solid phase extraction (SPE) methods were used before injection of key odorants into the gas chromatography-flame ionisation detection (GC-FID). The DAI and SPE methods were efficient in the identification of odour compounds in the wet scrubber. The SPE method had a high recovery and can be more effective in the identification of compounds at low initial concentration. However, DAI showed a better linearity and a lower limit of detection (LOD) than the SPE method. The DAI method was the method of choice for characterization, as it is cheaper, easier to handle and highly applicable. At least two odorants, phenol and 1-butanol, were quantified successfully using the DAI method. Their LOD was less than their odour detection limit in the wet scrubber. DAI method can be used as a reference measurement method for any further analytical application, e.g. electronic tongue.

\section{EB5}

Camalti saltern area as a source of industrially important enzymes Nilar Acaroğlu ${ }^{1}$, Ebru Toksoy Öner ${ }^{2}$, Aziz Akın Denizci ${ }^{3}$, Dilek Kazan ${ }^{2,3}:{ }^{1}$ Department of Biology, Marmara University, Goztepe 34722 Istanbul, Turkey; ${ }^{2}$ Department of Chemical Engineering, Marmara University, Goztepe 34722 Istanbul, Turkey; ${ }^{3}$ The Scientific and Technical Research Council of Turkey (TUBITAK), Research Institute for Genetic Engineering and Biotechnology (RIGEB), Marmara Research Center Campus (MRC), Gebze 41470 Kocaeli, Turkey. E-mail: nilaracaroglu@yahoo.com (N. Acaroğlu)

Recent developments in biotechnology enabled the widespread use of microbial enzymes in textile, detergent, food and dairy industries and also in various environmental applications. Microorganisms which live at extremes of temperature, $\mathrm{pH}$ and salinity, produce extremozymes that offer many exciting opportunities for their use in clean production. In this study, microorganisms were isolated from Camaltı saltern area in İzmir, Turkey. Effect of medium salinity on the growth of these microorganisms was determined. Seven out of 10 isolates required salt for growth. The salinity ranges at which growth was detected were: $5-25 \%$ for two isolates, $6-25 \%$ for one isolate, $7-25 \%$ for two isolates and $8-25 \%$ for one isolate. The isolates were also screened for their capability of producing industrially important enzymes such as amylase, protease, lipase, xylanase and cellulose which are widely used not only in textile, detergent, food and dairy industries but also in various environmental applications. All of the isolates were found to be producers of both amylase and xylanase enzymes at varying salinity array within 5-30\% salt concentration range at $\mathrm{pH}$ 7.0. Extracellular protease activity was detected in the medium of all isolates grown at 5, 10, 15, 20 and 25\% salinity at both $\mathrm{pH} 7.0$ (optimum growth $\mathrm{pH}$ ) and $\mathrm{pH}$ 9.0. Out of 10 isolates, 9, 10 and 9 were found to produce cellulase enzyme when the salt concentrations were 5, 10 and $15 \%$, respectively. At $20 \%$ salt concentration, only one isolate was found to be cellulase enzyme producer. None of the isolates were found to produce lipase enzyme at 5-30\% salt concentration range.

\section{Acknowledgement}

This project was supported by TUBITAK through project TBAG 2321-103T069.

\section{EB6}

Investigation of thin semiconductor coatings and their antibacterial properties Arcan Erkan, Ufuk Bakir, Gurkan Karakas Chemical Engineering Department, Middle East Technical University, Ankara 06531, Turkey. E-mail: ubakir@metu.edu.tr (U. Bakir)

Glass and ceramic tiles are very widely used industrial materials. In most cases, periodical cleaning is required to maintain their optical properties such as transparency and visual aspects. Because of the ever-growing demand for healthy living, there is a keen interest in materials capable of killing harmful microorganisms. The application of these tiles in care facilities to reduce the spread of infections, in public and residental places to improve hygienic conditions are of general interest. In this study the aim is developing methods to apply thin film coatings on glass tiles to make them anti-bacterial by utilizing photocatalysis and investigating their anti-bacterial properties. Semiconductors because of their reasonable band gap energies find great attraction through this purpose. The photocatalytic property of semiconductors are used in this process. Oxidising radicals are 
formed on the coated surfaces and these radicals attacks the organic pollutants and bacteria on contact with the surface. Titanium dioxide $\left(\mathrm{TiO}_{2}\right)$ coated surfaces are considered to be very effective against organic and inorganic materials, as well as against bacteria. In the experimental procedure coating solution is prepared by sol-gel technique. After pretreatment of surfaces, the coating solution is applied on the surfaces by dip-coating method. After appropriate thermal treatments, to achieve thin, dense and strong coatings, indicator microorganism is directly applied on the coated surfaces and illuminated under solar simulater light source. Finally, the number of surviving microorganisms are determined. In this study, the effects of titanium dioxide $\left(\mathrm{TiO}_{2}\right)$, tin oxide $\left(\mathrm{SnO}_{2}\right)$ solutions and metal doping to these coating solutions on anti-bacterial function were investigated. As a result of this study, the number of Escherichia coli that is used as indicator microorganism, on $\mathrm{TiO}_{2}$ and $\mathrm{SnO}_{2}$ coated glasses with respect to the control glass reduced by $80-85 \%$ and $40-45 \%$, respectively. Doping with metals increased the activity of the coatings, hence the number of surviving microorganisms decreased.

\section{EB7}

Activity of a methanogenic ecosystem during the primary contact with a solid support S. Michaud, N. Bernet, P. Buffière, J.P. Delgenès INRA-LBE, Avenue des Etangs, F-11100 Narbonne, France

In this paper, the biological activity during the first initial contact between a methanogenic sludge and a solid support was investigated in batch experiments, at different solid concentrations, using two different granular solid materials and with glucose as the main organic substrate. In all cases, the introduction of a solid material in a methanogenic suspended biomass induced a response of the anaerobic microorganisms, after a lag phase during which biological activity was not detected. This lag phase could be the consequence of a physical stress induced by the first contact between microbial cells and the solid surface. This lag phase was not observed when the biomass used originated from a biofilm reactor, i.e. using a biomass previously exposed to a solid material. A change in the metabolism of organic matter from catabolism and methane production toward production of other compounds could be observed, characterised by a sharp decrease of the methane yield in the anaerobic system. Analyses of the gas and liquid phases did not show the production of any new gaseous or soluble compound as the biological end product of this activity. This suggests the production of non-soluble compounds by an anabolic pathway, which could indicate the initiation of biofilm formation. This metabolic activity was shown to be directly correlated to the ratio between the solid surface introduced and the microorganism concentration in the anaerobic culture $\left(\mathrm{m}^{2} \mathrm{~g}_{\mathrm{Vs}}{ }^{-1}\right)$. From kinetic observations, it could be observed that acetogenic methanogenesis recovered more rapidly than syntrophic propionate and butyrate degradation.

\section{EB8}

Evaluating microbial diversity of hydrocarbon degrading bacteria Cleantis Braithwaite, Howard Rosser, Tawfiq Al-Ibrahim, Hussain, AL-BANDI Research and Development Center, Saudi Aramco, Dhahran, Saudi Arabia

The analysis of microbial diversity with molecular methods is central to isolating and identifying new and potential biocatalysts resources for research and industry. The ability to degrade hydrocarbon components of petroleum is widespread among bacteria, and is an effective method for remediation of a variety of ecosystems. Due to the high carbon content of oil and the low levels of other nutrients essential for microbial growth, treatment of oil with phosphorus and nitrogen is generally required to enhance the growth of hydrocarbon-degrading bacteria and to stimulate oil sludge degradation. In this research study, three types of oily sludges from a gas plant, refinery, and terminal facilities were treated with nutrients. To assess the microbial diversity, both Biolog culture method and culture independent polymerase chain reaction (PCR,) denaturing gradient gel electrophoresis (DGGE) methods were used. Nutrient addition significantly improved oil sludge degradation. We identified and characterized several hydrocarbon degrading bacterial strains that have the ability to convert petroleum. These bacteria included representatives both gram positive and gram-negative genera. There were slight difference in the quantity and type of hydrocarbon degrading bacteria found in the three sites. This is the first molecular analysis of hydrocarbon degrading microbial population in Saudi Arabian Operations.

\section{EB9}

Expression of functional recombinant mussel adhesive protein Type 3A in Escherichia coli Youngsoo Gim, Dong Soo Hwang, Hyung Joon Cha Department of Chemical Engineering, Pohang University of Science and Technology, Pohang 790-784, South Korea. E-mail: hjcha@postech.ac.kr (H.J. Cha)

Mussel adhesive proteins, including the 20-plus variants of foot protein type 3 (fp-3), have been suggested as potential environmentally friendly adhesives for use in aqueous conditions and in medicine. Here we report the novel production of a recombinant Mytilus galloprovincialis foot protein type 3 variant A (Mgfp-3A) fused with a hexahistidine affinity ligand in Escherichia coli, and its $\sim 99 \%$ purification with affinity chromatography. Recombinant Mgfp-3A showed a superior purification yield and better apparent solubility compared to those of the previously reported recombinant M. galloprovincialis foot protein type 5 (Mgfp-5). The adsorption abilities and adhesion forces of purified recombinant Mgfp-3A were compared with those of Cell-Tak (a commercial mussel extract adhesive) and Mgfp-5 using QCM analysis and modified AFM, respectively. These assays showed that the adhesive ability of recombinant Mgfp-3A was comparable to that of Cell-Tak but lower than that of recombinant Mgfp-5. Collectively, these results indicate that recombinant Mgfp-3A may be useful as a commercial bioadhesive or an adhesive ingredient in medical or underwater environments.

\section{EB10}

Dearomatisation of $p$-cresol using Pseudomonas sp. Mousumi Chaudhury ${ }^{1}$, Sami Sarfaraz ${ }^{2}$, Swapna Thomas ${ }^{2}$, Leela Iyengar ${ }^{2}:{ }^{1}$ Department of Microbiology, Government Institute of Science, Dr. BA Marathwada University, Nipatniranjan, Aurangabad 431001, India; ${ }^{2}$ Department of Chemistry, Indian Institute of Technology (IIT), Kanpur 208016, India. E-mail: mousumichaudhury@ rediffmail.com (M. Chaudhury)

Cresol, a monomethylated phenol, is an aromatic compound. The Environmental Protection Agency (EPA) has determined the carcinogenic potential of cresol. Various options are being examined for the degradation of cresol because of their unavoidable large scale production and toxicity. Many aromatic hydrocarbons can be used as 
electron donors aerobically by species of Pseudomonas, thus leading to the ring cleavage of these compounds. In the present study, Pseudomonas strains were isolated from activated sludge collected from sewage treatment plant. It was repeatedly transferred onto nutrient agar plate to check the purity of the culture. The organism was grown aerobically in an inorganic medium with $p$-cresol as the solitary carbon source. Pseudomonas was confirmed by the expression of green pigment, Gram staining and biochemical tests including $\mathrm{KOH}$, catalase, nitrate reduction and carbohydrate fermentation reaction. Inoculation status was used to determine the rate of degradation of $p$-cresol. The effect of temperature on $p$-cresol degradation was studied. Moreover, the effect of different concentrations of the aromatic compounds on Pseudomonas as well as substrate variability was also documented. Phenolic intermediates were estimated colorimetrically using 4-aminoantipyrene, Folin-Lowry method, UV spectrophotometry and HPLC. The results indicated that Pseudomonas could degrade up to $300 \mathrm{mg} / \mathrm{L}$ of $p$-cresol within $10 \mathrm{~h}$. Pseudomonas sp. exhibited good metabolic versatility and degraded other aromatic compounds including m-cresol and $p$-hydroxybenzoic acid. We conclude that this strain of Pseudomonas has excellent potential for bioaugmenting the degradation of $p$-cresol-containing waste water treatment units.

\section{EB11}

Recycling of waste cooking oil for lipase production by Yarrowia Lipolytica Alberto Domínguez, María A. Longo, M. Angeles Sanromán Department Chemical Engineering, University of Vigo, Campus Lagoas-Marcosende, 36310 Vigo, Spain. E-mail: mlongo@uvigo.es (A. Dominguez)

A considerable amount of waste cooking oil is produced by the restaurant industry worldwide. This poses a significant environmental and economic problem, since high oil and grease concentrations in the sewage system could lead to pipes occlusion and decreased efficiency in water treatment operation plants. Therefore, sending these wastes to recycling companies or hazardous waste processors is usually required. Yarrowia lipolytica, a well-known lipase producer, requires the presence of lipidic compounds (i.e. vegetable oils) to boost enzyme biosynthesis. In this work, the suitability of waste cooking oil as lipase inducer in submerged cultures of this yeast has been assessed. If successful, this procedure could allow both the degradation of an abundant waste and its valorisation as a raw material for the production of a high added value product. The microorganism was grown in a liquid medium to which various amounts of waste cooking oil were added. Biodegradation degrees up to $80 \%$ (measured as decrease in COD) were obtained after 3 days of treatment. Also, initial glucose concentration in the basal medium seemed to influence the efficiency of the process. On the other hand, addition of waste oil led to a significant increase in lipase production (more than two-fold), compared to oil-free cultures. Moreover, chain-length specificity of the produced enzymes was significantly different: high activity towards medium chain length esters was found, which hinted to the occurrence of both lipases and esterases.

\section{Acknowledgements \\ This work has been financed by Xunta de Galicia (PGIDT03PXIB30103PR).}

\section{EB12}

Bioaccumulation of reactive dyes by thermophilic cyanobacteria Sevgil Sadettin, Gönül Dönmez. Department of Biology, Faculty of Science, Ankara University 06100 Beşevler, Ankara, Turkey

Thermophilic cyanobacterial strains that could grow in the BG 11 media was isolated from hot springs and their reactive dye bioaccumulation was studied under thermophilic conditions in a batch system, in order to determine the optimal conditions required for the highest dye accumulation. In the experiments performed with newly isolated Synechocystis sp. and Phormidium sp., the optimum $\mathrm{pH}$ values at about $25 \mathrm{mg} \mathrm{l}^{-1}$ initial reactive dye concentrations was determined as 8.5 for Remazol Blue and Reactive Black B and 9.5 for Reactive Red RB. At the optimum pH values, dye bioaccumulation by thermophilic cyanobacteria was investigated at $10-78 \mathrm{mg} \mathrm{l}^{-1}$ initial dye concentrations under $40^{\circ} \mathrm{C}, 45^{\circ} \mathrm{C}$ and $50^{\circ} \mathrm{C}$ temperatures. At the end of the experiments, Phormidium sp. was shown high dye bioaccumulation than the Synechocystis sp., with maximum uptake yields ranging from 13 to $97 \%$ at all tested dye concentrations. In the experiments with Synechocystis sp., the highest specific dye uptake of measured were $56.25 \mathrm{mg} \mathrm{g}^{-1}$ for $78.3 \mathrm{mg} \mathrm{l}^{-1}$ Reactive Red RB, $80.22 \mathrm{mg} \mathrm{g}^{-1}$ for $72.4 \mathrm{mg} \mathrm{l}^{-1}$ Remazol Blue and $35.33 \mathrm{mg} \mathrm{g}^{-1}$ for $62.0 \mathrm{mg}^{-1}$ Reactive Black B under $40^{\circ} \mathrm{C}$ temperatures. When the high temperatures was used, Phormidium sp. was shown to have the maximum dry weight and bioaccumulation yield, which can be considered as satisfactory in terms of dye removal from subject wastewater effluents under thermophilic conditions.

\section{Acknowledgement}

This study was supported by Ankara University Biotechnology Institute.

\section{EB13}

Hexavalent chromium reduction by sulfate-reducing bacteria Demet Çetin ${ }^{1}$, Sedat Dönmez ${ }^{2}$, Gönül Dönmez ${ }^{1}:{ }^{1}$ Department of Biology, Faculty of Science, Ankara University, 06100 Beşevler, Ankara, Turkey; ${ }^{2}$ Department of Food Engineering, Faculty of Engineering, Ankara University, 06110 Dışkapı, Ankara, Turkey

Sulfate-reducing bacteria (SRB) that could grow on modified postgate $\mathrm{C}$ medium (PC) containing chromium(VI) were isolated from industrial wastewater and their chromium(VI) reduction capacities were investigated as a function of changes in the initial $\mathrm{pH}$ values, chromium, sulfate, $\mathrm{NaCl}$ concentrations and carbon source. The optimum $\mathrm{pH}$ value at $50 \mathrm{mg} \mathrm{l}^{-1}$ initial chromium(VI) concentrations was determined as 8 . Chromium(VI) reduction by SRB was investigated at 22.7-98.4 $\mathrm{mg} \mathrm{l}^{-1}$ initial chromium(VI) concentrations. At the end of the experiments, the mixed cultures of SRB were found to reduce more than $99 \%$ of the initial chromium(VI) levels which ranged from 22.7 to $74.9 \mathrm{mg}^{-1}$ within $2-6$ days period. The effects of initial $0-9.0 \mathrm{~g}^{-1}$ concentrations of sulfate and $0-6 \%(\mathrm{w} / \mathrm{v})$ concentrations of $\mathrm{NaCl}$ to chromium reduction were showed that, the lowest concentrations of sulfate and $\mathrm{NaCl}$, were the best for chromium reduction in the PC media including $50 \mathrm{mg} \mathrm{l}^{-1}$ chromium(VI). When the $25 \%$ whey was used as carbon source in the PC medium, $99.6 \%$ of the $65.9 \mathrm{mg} \mathrm{l}^{-1}$ initial chromium(VI) concentration was reduced within 2 days of incubation period. 


\section{Acknowledgement}

This study was supported by Ankara University Biotechnology Institute.

\section{EB14}

Biodesulfurization: A documental review J. Ferrer, Simon Bolivar University, Environmental Engineering Lab. Caracas, Venezuela

A documental review about larger interest aspects in biodesulfurization technique is showed. Especifically, the investigation is related to general framework and the justification of this technique, degradatives pathways elucidated up to now, involved microorganism, important elements in development of bacterial desulfurization and progress areas, and future tendency.

\section{EB15}

In situ bioremediation of a $p$-nitrophenol contaminated site and assessment of its community structure Debarati Paul, Gunjan Pandey, Sumeet Labana, Rakesh K. Jain Institute of Microbial Technology, Sector 39A, Chandigarh 160036, India. E-mail: rkj@imtech.res.in (R.K. Jain)

Biodegradation of $p$-nitrophenol (PNP), a priority pollutant, was studied as a model system for bioremediation of sites contaminated with nitroaromatic/organic compounds. Bioremediation studies were carried out in PNP-spiked soil in small plots under natural field conditions using Arthrobacter protophormiae RKJ100. Role of carrier material was examined by immobilizing the bacteria on corncob powder prior to adding them to soil. These studies demonstrated successful removal of PNP by immobilized cells that were able to deplete PNP completely in 5 days, whereas free cells were able to deplete $75 \%$ PNP in the same time period. Monitoring the fate of released bacteria revealed fairly stable population of the cells when they were immobilized on corncob powder throughout the period of study. On the other hand, there was a decrease of $2.7 \mathrm{log}$ units in colony forming units of free cells at the end of the study (30 days). Bacterial community structure and diversity was also studied for the pesticidecontaminated site wherein the effect of addition of an exogenous strain on the existing soil community structure and on soil functionality was determined using molecular techniques. As revealed by restriction fragment length polymorphism (RFLP) studies 45 different phylotypes could be identified on the basis of similar banding patterns. Sequencing of representative clones of each phylopyte showed that the community structure of the pesticide-contaminated soil mainly constituted of Proteobacteria and Actinomycetes. Terminal fragment length polymorphism (T-RFLP) analysis showed only subtle changes in community structure during the process of bioremediation.

\section{EB16}

Partial purification of peptidic Xanthomonas bacteriocins produced in Escherichia coli Jong Kook Moon ${ }^{1}$, Eun Jung Roh ${ }^{2}$, Ki Young Yang ${ }^{1}$, Byung Sik Yang ${ }^{1}$ Eun Pyo Moon ${ }^{2}$, Do Hyun Jo ${ }^{1}$, ${ }^{1}$ Department of Molecular Science and Technology, Ajou University, SuWon 442-749, South Korea; ${ }^{2}$ Department of Life Science, Ajou University, SuWon 442-749, South Korea. E-mail: dhj832@ajou.ac.kr (D.H. Jo)

Bacteriocins encompass an array of structurally different molecules produced by a number of phylogenetically distinct bacterial groups and trigger the killing of the same or closely related species. The recombined Escherichia coli strain harboring a bacterocin coding region of Xanthomonas campestris pv glycines 8ra was disrupted to obtain cell homogenate. Peptidic Xanthomonas bacteriocins $(\mathrm{PXB})$ were separated by lowering $\mathrm{pH}$ and adding salt. The resulting PXB's were partially purified using ion exchangers, gel filtration. Two final active fractions, $\mathrm{A}$ and $\mathrm{B}$, were obtained with a yield of $0.005 \%$ and $500-1000$-fold purification. The activity of $\mathrm{PXB}$ was stable at the $\mathrm{pH}$ ranging from 7.0 to 10.0 .

\section{EB17}

Decolorization and detoxification of different reactive dyes with a laccase Trametes versicolor Andreja Kresal, Vanja Kokol, Vera Golob Textile Department, University of Maribor, 2000 , Slovenia

Wastewater from textile dyeing industries is characterized by high chemical and biological oxygen demands (COD and BOD) and intense color due to the extensive use of synthetic dyes. As dyes of complex aromatic structures are resistant to removal by the typical microbial population and may be toxic to the microorganisms present in the treatment plants, discharge of the wastewater to the treatment plants may lead to its failure. Beside, direct discharge of these effluents into municipal wastewater plants and/or environment may cause the formation of toxic carcinogenic and/or unhealthy breakdown products. Different chemical and physical methods (adsorption, coagulation-flocculation, oxidation, filtration and electrochemical treatments) for color removal have been proposed, but due capital costs and slow operating speed as well as huge amounts of sludge creation there is still a great need to develop an economic and effective method. The use of lignin degrading white-rot fungi and their enzymes (laccase, lignin peroxidase, manganese peroxidase) has attracted increasing scientific attention due their ability to oxidative degrade a wide range of recalcitrant organic compounds. In the contribution, the decolorization efficacy of different commercial textile reactive dyes (anthraquinone, azo, triphenylmethane) will be investigated after the treatment by laccase from Trametes versicolor. In order to examine the reuse of enzymatically decolorized liquors, the ecological suitability and the toxicity of the degradation products after different time of enzyme exposure will be studied.

\section{Acknowledgement}

This work was carried out within the scope of research project E! 3100 CAWAB.

\section{EB18}

Influence of heavy metals on growth and extracellular enzyme production of a Trichoderma harzianum strain with biocon-

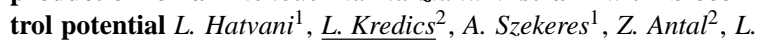
Manczinger ${ }^{1}, A . \mathrm{Nagy}^{3}$, C. Vágvölgyi ${ }^{1}{ }^{1}{ }^{1}$ Department of Microbiology, University of Szeged, P.O. Box 533, H-6701 Szeged, Hungary; ${ }^{2}$ Hungarian Academy of Sciences, University of Szeged, Microbiological Research Group, Hungary; ${ }^{3}$ Pilze-Nagy Ltd. Kecskemét, P.O. Box 407, Hungary. E-mail: kredics@bio.u-szeged.hu (L. Kredics)

Trichoderma species are common soil inhabiting asexual filamentous fungi with teleomorphs belonging to the Hypocreales order of the Ascomycota division. Besides the industrial and clinical importance of the genus, certain strains have been found to cause great losses in mushroom cultivation while other strains are well known to possess high antagonistic activity against several 
plant pathogenic fungi and therefore used as biocontrol agents. Important mechanisms of antagonism include competition and mycoparasitism, which - among others - can be related to the fast growth of Trichoderma strains and the production of several extracellular enzymes. The influence of certain, soil-occurring heavy metals on mycelial growth and the secretion of extracellular enzymes involved in competition and mycoparasitism was examined in this study regarding an effective, potential biocontrol isolate of Trichoderma harzianum. The metal ions zinc, manganese, copper, iron, lead and mercury were applied at the concentrations of 8,16 , $24,32,40,60$ and $80 \mu \mathrm{M}$, and dry mycelial weight as well as the activities of extracellular $\beta$-glycosidase, cellobiohydrolase, trypsinand chymotrypsin-like protease and $\mathrm{N}$-acetyl-glucosaminidase enzymes were determined. It was found that mercury totally blocked mycelial growth, while other metal ions exerted a much lower influence on growth. The presence of heavy metals did not have a significant effect on the activity of the examined extracellular enzymes with the exception of trypsin-like protease, which showed a four- to six-fold rise in activity in the presence of certain sublethal concentrations of copper. Based on these results, our further aim is to develop copper-resistant derivatives by mutagenesis from Trichoderma strains with biocontrol potential. Since proteases play an important role in mycoparasitism, these strains could be applied within the frames of integrated pest management in combination with copper-containing fungicides, resulting in an enhanced level of crop protection even with reduced amounts of fungicides.

\section{Acknowledgement}

This work was supported by grants F037663 of the Hungarian Scientific Research Fund and grant OMFB-00219/2002 of the Hungarian Ministry of Education.

\section{EB19}

An in vitro method for the characterization of biocontrol abilities B. Leitgeb ${ }^{1}$, L.Kredics $^{2}$, A. Szekeres $^{3}$, L. Manczinger $^{3}$, C. Vágvölgyi ${ }^{3}:{ }^{1}$ Institute of Biophysics, Biological Research Center of the Hungarian Academy of Sciences, Temesvári krt. 52, H6726 Szeged, Hungary; ${ }^{2}$ Microbiological Research Group, Hungarian Academy of Sciences, University of Szeged, P.O. Box 533, H-6701 Szeged, Hungary; ${ }^{3}$ Department of Microbiology, University of Szeged, P.O. Box 533, H-6701 Szeged, Hungary. E-mail: kredics@bio.u-szeged.hu (L. Kredics)

The significance of biocontrol agents (BCAs) is that some of them possess good antagonistic abilities against plant pathogenic fungi. A significant number of the most prominent fungi for the purposes of agricultural application belong to the genus Trichoderma. In previous studies, in vitro assays on agar plates were reported as the generally used method for the evaluation of antagonistic abilities, as the results of these assays are well transferable to the practical application. The aim of the present study was to develop an accurate, image analysis-based method for the evaluation of the biocontrol characters of BCAs. Randomly selected Trichoderma isolates were tested against Fusarium culmorum. In the currently developed method, the areas of the fungal colonies were calculated on Petri dishes by measuring the occupied surface of the medium on digital images. The inhibition effect was recorded as the value of biocontrol index (BCI), which was calculated from the ratio of the area of the Trichoderma colony and the total area occupied by the colonies of Trichoderma and the plant pathogen. The proposed method was tested for numerous parameters, and the results revealed that BCI proves to be capable for the accurate measurement and scale of the biocontrol abilities of fungal isolates.

\section{Acknowledgement}

This work was supported by grants F037663 of the Hungarian Scientific Research Fund and grant OMFB-00219/2002 of the Hungarian Ministry of Education.

\section{EB20}

The effect of advanced oxidation processes and recirculation on biodegradation of leachates from aerobic landfills Liliana Krzystek, Anna Zieleniewska-Jastrzebska, Stanisław Ledakowicz Department of Bioprocess Engineering, Technical University of Lodz, 90-924 Lodz, Poland

Modern landfills are built and operated in a way which allows us to treat them as a special type of bioreactor. Simulation of municipal waste biodegradation in lysimeters provides knowledge on basic processes that take place in an aerated landfill. The aim of aeration is to stabilise mainly biodegradable and nitrogen containing components and to reduce methanogenic potential. Stabilised leachates from old landfills contain big quantities of refractive carbon compounds that cannot be removed by biological methods. In such case most advantageous is to apply advanced oxidation processes (AOPs). The objective of this study is an experimental simulation of a landfill aerobic stabilisation and the impact of AOPs and recirculation of leachate on the reduction of organic load. The performance of the processes was monitored by the reduction in time of basic indices of organic load $\left(\mathrm{BOD}_{5}, \mathrm{COD}, \mathrm{TOC}, \mathrm{VFA}, \mathrm{TKN}, \mathrm{N}_{-} \mathrm{NH}_{4}{ }^{+}\right)$and changes in biogas composition. The simulation of aerobic landfill processes was carried out in lysimeters with a fixed bed of household solid waste stabilised during 8 months in anaerobic conditions. Leachates taken from the lysimeters were recirculated and subjected to advanced oxidation processes, i.e. ozonation and UV radiation with the addition of $\mathrm{H}_{2} \mathrm{O}_{2}$. Experimental studies showed that the aerobic waste stabilisation was a very quick process. During a month the bed was stabilised, reaching a significant reduction of organic load indices. Aeration of the lysimeters caused a quick reduction of mainly degradable organic substance (in terms of $\mathrm{BOD}_{5}$ ) and $\mathrm{N}^{-\mathrm{NH}_{4}}{ }^{+}$and VFA. The reduction of methanogenic potential of the landfill was even faster. The composition of gas at the outlet from the lysimeter changed and after one day already its content was similar to atmospheric air. A more frequent recirculation of leachates enhanced greatly the aerobic biodegradation. It was found that application of advanced oxidation processes (especially ozonation) contributed to a growing reduction of the organic load in the leachates from aerated lysimeters. The application of leachate ozonation resulted in a very high degree of reduction of organic compounds (up to $77 \%$ ).

\section{EB21}

Aerobic batch biodegradation of potato stillage-effect of temperature M. Krzywonos, E. Cibis, T. Miśkiewicz Department of Bioprocess Engineering, Wrocław University of Economics, Komandorska 118/120, 53-345 Wrocław, Poland. E-mail: malgorzata.krzywonos@ae.wroc.pl (M. Krzywonos)

The objective of the experimental study was to assess the effect of temperature on the extent of aerobic batch biodegradation of potato 
stillage with a mixed culture of bacteria of the genus Bacillus. The experiments were performed at 20,30,35, 40, 45, 50, 55, 60, 63 and $65^{\circ} \mathrm{C}$, at $\mathrm{pH} 7$, in five 11 working volume stirred tank reactor (STR) (Biostat ${ }^{\circledR} \mathrm{B}, \mathrm{B}$. Braun Biotech International). The duration of the process was $120 \mathrm{~h}$. Initial COD of the stillage amounted to $51.9 \mathrm{~g} \mathrm{O}_{2} / \mathrm{l}$, the main carbon sources being reducing substances $(18.7 \mathrm{~g} / \mathrm{l})$, organic acids (determined as their sum) $(12.2 \mathrm{~g} / \mathrm{l})$ and glycerol $(3 \mathrm{~g} / \mathrm{l})$. At $65^{\circ} \mathrm{C}$, no COD reduction or biomass increment was found to occur. At the other investigated temperatures, the reduction in COD measured after suspended solids (SS) separation varied from $77.6 \%\left(55^{\circ} \mathrm{C}\right)$ to $89.1 \%\left(35^{\circ} \mathrm{C}\right)$. Without SS separation, COD reduction ranged between $55.6 \%\left(20^{\circ} \mathrm{C}\right)$ and $75.1 \%\left(35^{\circ} \mathrm{C}\right)$. This indicates that, in terms of the extent of COD reduction, the optimal process temperature was $35^{\circ} \mathrm{C}$ and that there was a local optimum at about $63{ }^{\circ} \mathrm{C}$. According to the temperature applied, the content of reducing substances decreased by $84.3-96 \%$, that the organic acids by $91.7-99.6 \%$, and that of glycerol by $91.5-96 \%$. The experiments also produced the following two findings: (1) the rise in temperature brought about a decrease of biomass concentration in the STR (measured as SS and bacterial number), and (2) temperature was a factor affecting the demand for ammonia nitrogen $\left(\mathrm{N}-\mathrm{NH}_{4}\right)$, which was the highest at 20 and $60^{\circ} \mathrm{C}$. The high $\mathrm{N}-\mathrm{NH}_{4}$ demand observed both over the higher and lover ranges of the investigated temperature should be attributed to the release of $\mathrm{N}-\mathrm{NH}_{4}$ and to the large amounts of the biomass produced, respectively. The results obtained imply that the extent of potato stillage biodegradation with a mixed bacterial culture was high over a wide range of the investigated temperature.

\section{EB22}

Molecular analysis of dechlorinating gene in halorespirating bacterium and development of efficient bioremediation process R. Kurane, N. Tsukagoshi, T. Uenaka, N. Suzuki, S. Ezaki Biotechnology Research Center, Kubota Co. Ryugasaki, Ibaraki 301-0852, Japan. E-mail: rkurane@kubota.co.jp (R. Kurane)

Polychlorinated compounds such as tetrachloroethylene (PCE) have become serious environmental pollutants. Considerable attention has been paid to these organochlorine compounds. This paper describes the molecular analysis of dechlorinating gene in halorespirating bacterium and efficient bioremediation process. An anaerobic bacterium, that dechlorinates PCE to TCE, was isolated and identified as a species of the genus Desulfitobacterium. A novel PCE reductive dehalogenase (PrdA) gene from the Desulfitobacterium sp. strain KBC-1 was identified. These prd genes, including membrane anchor protein, were classified as a novel type of PCE reductive dehalogenase (approximately $40 \%$ homology with the general PCE dehalogenase). According to the substrate utility of this strain KBC1 and phylogenetic analysis of PrdA, the type of this microorganism may be expected to play the role of a primary degrader of PCE in the environment. High efficient bioremediation process so called the restricted aeration system which means microaerobic/aerobic reciprocal bioremediation process was developed.

\section{Acknowledgement}

This study was carried out as a part of the Project for analyzing and controlling the mechanism of biodegrading and processing entrusted by the New Energy and Industrial Technology Development Organization (NEDO).

\section{EB23}

Utilization of agroindustrial wastes for lipase production by Rhizopus oryzae Elena López, Francisco J. Deive, M. Angeles Sanromán, Maria A. Longo Department Chemical Engineering, University of Vigo, Campus Lagoas-Marcosende, 36310 Vigo, Spain. E-mail: mlongo@uvigo.es (M.A. Longo)

Lipases are extremely versatile enzymes, that catalyze both hydrolysis and synthesis reactions. They have a wide range of industrial applications, among which the manufacture of detergents, pharmaceuticals and fine chemicals are outstanding. The fungus Rhizopus oryzae has been reported to synthesize a number of commercially interesting enzymes. In this work, its ability to produce extracellular lipases when grown in solid state culture has been assessed. Cultures were carried out in Erlenmeyer flasks, using a complex medium and several supports, both synthetic (nylon sponge) and natural (barley bran, ground walnut or peanut). The latter appeared to be more suitable for lipase production. Since the best results were initially obtained with lipid-containing supports, barley bran cultures were supplemented with a vegetable oil, in an attempt to optimise lipase production and design an efficient procedure for reusing this agroindustrial waste. Surprisingly, this strategy did not improve enzyme synthesis. However, when a surfactant (Triton X-100) was added to the basal medium, a dramatic increase in extracellular activity was detected (up to 20 -fold). The results agreed with those previously obtained in submerged cultures of $R$. oryzae, in which addition of olive oil did not increase lipase production, while the presence of Triton X-100 had a remarkably beneficial effect. Also, enzyme concentration in solid state cultures was up to two-fold that of the submerged ones.

\section{Acknowledgements}

This work has been financed by Xunta de Galicia (PGIDT03PXIB30103PR).

\section{EB24}

Lead ions removal by yeasts biomass Olga G. Lozovaya, Taisiya P. Kasatkina, Valentin S. Podgorsky Institute of Microbiology and Virology National Academy of Sciences of Ukraine, Zabolotnogo Street, 154, Kiev 03143, Ukraine. E-mail: Lozovaya@serv.imv.kiev.ua (O.G. Lozovaya)

Lead ions are considered a high pollutant of different waters. In our previous work were selected seven potential sorbent-strains Rhodotorula mucilaginosa 1776, Rh. aurantiaca 1195, Rhodotorula sp. 4, Williopsis californica 248, Candida krusei 61т, Cryptococcus sp. WT of lead ions. It was determined their stability to high concentration (up to $750 \mathrm{mg} / \mathrm{l}$ ) of lead ions in medium. The $\mathrm{pH}$ changes and yeast physiologies of growth were studied in medium with these heavy metal ions. The influences of environmental factors such as $\mathrm{pH}$ of solution, age of microbial culture, biosorbent concentration in suspension, alive or living state of biosorbent, time of contact on sorption were investigated. The levels of maximal sorption ability and biomass affinity to heavy metal ions were established by experimentally received sorption isotherms with mathematical modeling of biosorption process separately for everyone researched yeast strain. The sorption isotherms obtained in these experiments for non-living yeast biomass showed that the maximal sorption capacity was 225 and $195 \times 10^{-6} \mathrm{~mol}$ (g sorbent) ${ }^{-1}$ for Rh. aurantiaca 1195 and $S$. cerevisiae 1968, respectively. In the case of living biomass, the high- 
est maximal sorption capacity was found for yeasts Cryptococcus sp. WT and Rh. aurantiaca 1195 . These cultures also demonstrated the high sorption affinity, which makes them especially efficient biosorbents at low concentrations of lead ions. The high efficiency of lead elution was shown with $0.1 \mathrm{~N}$ EDTA.

\section{EB25}

Isolation and identification of marine bacteria from deep-sea sediments Els Maas ${ }^{1}$, Cara Brosnahan ${ }^{1}$, Vicky Webb ${ }^{1}$, Helen Neil $^{2}$, Phil Sutton ${ }^{2}{ }^{1}$ Marine Biotechnology, National Institute for Water and Atmospheric Research Ltd., Kilbirnie, Wellington, New Zealand; ${ }^{2}$ Oceanography, National Institute for Water and Atmospheric Research Ltd., Kilbirnie, Wellington, New Zealand

Marine sediments were obtained using a piston corer with associated trigger core $(0.5 \mathrm{~m}, 0.06 \mathrm{~m}$ diameter). Cores were collected from depths of 270 to $3911 \mathrm{~m}$, along Norfolk Ridge and across Challenger Plateau. Sediments ranged from coarse carbonate sands in the north to sandy and silty hemipelagic mud with increasing depth and latitude. All samples sites underlie subtropical surface water masses associated with, and south of, the Tasman Front. Sediment samples were aseptically taken from the triggers cores upon recovery. Samples were stored in sterile tubes at $4{ }^{\circ} \mathrm{C}$ on board the vessel for 3-17days. The core samples were plated on several different agar types and incubated aerobically for 4 weeks at $16^{\circ} \mathrm{C}$. Individual colonies were sub-cultured and purified using standard microbiological techniques. Morphological and molecular taxonomy revealed that the bacteria isolated from the sediments were closely related to Novosphingomonas, Halomonas, Stappia, Glaciecola, Pseudoalteromonas and Leeuwenhoekiella. Phylogenetic trees constructed using 16S rRNA gene sequence data showed that two other isolates were unrelated to known genera. The bacterial isolates are currently being investigated for their biotechnological potential.

\section{EB26}

Biodegradation kinetics of olive oil mill wastewater by loofa immobilized Phanerochaete chrysosporium Mojtaba Ahmadi, Farzaneh Vahabzadeh, Babak Bonakdarpour Food Process Engineering and Biotechnology Research Center, Department of Chemical Engineering Amirkabir University of Technology Tehran, Iran. E-mail: far@aut.ac.ir (M. Ahmadi)

Olive oil mill wastewater (OMW) as the effluent of the concern of olive industry has high organic load. The conventional biological treatments despite of their simplicity and rather suitable performance are ineffective for the OMW treatment since phenolics possess antimicrobial activity. In order to carry out a proper treatment on OMW, use of microorganism able to degrade the phenolics thus, is necessary. The ability of Phanerochaete chrysosporium immobilized on loofa was studied. The basal mineral salt solution along with glucose, ammonium sulfate and yeast extract were used to dilute OMW properly. The fungus did not grow on the concentrated OMW. Therefore, OMW diluted by $20 \%$ was used thought this study. The extent of removal in this biotreatment, of total phenolics (TP) and COD were 90 and $50 \%$, respectively. While the color and aromatocity decreased by 60 and $95 \%$, respectively. The kinetic behavior of the loofa immobilized fungus was found to follow Monod equation. The maximum growth rate was $0.045 \mathrm{~h}^{-1}$ while the Monod constant based on the consumed TP and COD (mg/L) were 370 and 6900, respectively.

\section{EB27}

Removal of Remazol Brilliant Blue R (RBBR) from aqueous solutions by adsorption of polyelectrolyte complex Ruby Y. Salazar, Christina Guajardo, Luis J. Galán, Syed H. Imam, Katiushka Arévalo Istituto de Biotechnologia, F.C.B., UANL. Ciudad universitaria, San Nicolás de los Garza, N.L. Mx

The control of water pollution has become of increasing importance in recent years. The release of dyes into the environment constitutes only a small proportion of water pollution, but dyes are visible in small quantities due to their brilliance. Many dyes are difficult to decolourise due to their complex structure and synthetic origin. The adsorption of reactive dye Remazol Brilliant Blue R (RBBR) on polyelectrolyte complex (PEC) was studied in a batch systems. The adsorption parameters determined were: effect of the different values of $\mathrm{pH}$ on the adsorption of dye by PEC, and the effect of contact time on the amount of RBBR adsorbed (in $\mathrm{mg} \mathrm{g}^{-1}$ ). The data indicates that the adsorption capacity of RBBR by PEC is dependent on $\mathrm{pH}$. The maximum adsorption at $50 \mathrm{ppm}$ was $88.52 \%$ equal to $11.8 \mathrm{mg}$ of dye/g of polymer. The results show a tendency towards greater adsorption for reactive dyes ( $\mathrm{pH}$ range of 8-12). The effect of contact time was studied at initial concentration (50 ppm) of dye, the amount of RBBR adsorbed for these PEC increased and reached a constant value with the increase in contact time. The increase in the extent of removal of dye after 15 min of contact time is less and hence it is fixed as the optimum contact time. The PEC show their capacity to remove RBBR to aqueous solutions by adsorption.

\section{EB28}

Essential role of $\mathrm{Mg}^{2+}$ in flocculation of yeast Hiroshi Nishihara, Chihiro Onishi, Satoko Baba Department of Chemistry, Faculty of Education, Kagawa University, Takamatsu 760-8522, Japan. E-mail: nishihar@ed.kagawa-u.ac.jp (H. Nishihara)

Flocculation of Saccharomyces cerevisiae (diastaticus) IFO1958 was studied. Cells of IFO 1958 did not flocculate even in the stationary phase without $\mathrm{Mg}^{2+}$ (" $\mathrm{Mg}^{2+}$-deficient cells") although they began to flocculate strongly $18 \mathrm{~h}$ after inoculation in the presence of $\mathrm{Mg}^{2+}$ ("complete cells"). Cycloheximide completely inhibited induction of floc-forming ability of " $\mathrm{Mg}^{2+}$-deficient cells". Co-flocculation between "complete cells" and " $\mathrm{Mg}^{2+}$-deficient cells" was investigated by chemical modification. Treatment of " $\mathrm{Mg}^{2+}$-deficient cells" by proteolytic enzymes did not affect the co-flocculation with "complete cells". Photo-oxidation or mercaptoethanol-reduction of " $\mathrm{Mg}^{2+}$-deficient cells" failed to weaken the co-flocculation with "complete cells" while treatment of " $\mathrm{Mg}^{2+}$-deficient cells" by periodate brought about a significant loss of the co-flocculation. On the contrary, "complete cells" deflocculated by proteolysis or chemical modification of proteinaceous component failed to co-flocculate with " $\mathrm{Mg}^{2+}$-deficient cells". These findings suggest that " $\mathrm{Mg}^{2+}$-deficient cells" are non-flocculent because of lack of proteinaceous component essential for flocculation of cells of IFO 1958.

\section{EB29}

Evolution of the microbial community and its role in Toscano tobacco fermentation M. Paolino, D. Silvestro, M. Di Giacomo, D. Parente R\&D Biology Department, British American Tobacco Italia, Naples 80126, Italy

The industrial Toscano cigar production starts with the dark firecured Kentucky tobacco fermentation process. During this phase 
strong modifications take place, as ammonia production with a subsequent rise of the $\mathrm{pH}$ value and a rapid heat evolution leading to temperatures of up to $70^{\circ} \mathrm{C}$. Little is known about the microbial community in the Toscano cigar fermentation and its development as fermentation proceeds. The aim of this study is to investigate the microbial community composition, its dynamic and its influence on the Toscano cigar production process. Our results show that the fermentation could be divided into three different phases: initially yeasts are the predominant microorganisms while bacterial growth is partially inhibited; the middle phase is characterized by exponential growth of bacteria while yeasts disappear. In the final phase the microorganism population is mostly represented by sporigen microbial species. The occurrence of yeasts in the first phase could be attributed to their ability to grow at low temperature and low $\mathrm{pH}$ levels. The bacterial population flourishes after the yeast cells have reached a stationary phase and probably grows on residual nutrients and autolysing yeast cells. Yeasts and bacteria involved in the fermentation process were isolated and characterized. The microbial community was investigated by a combination of phenotypic and molecular approaches. The phenotypic characterization was based on both colony and cell morphology. The isolates were then identified by rRNA genes sequence analysis. Finally, in order to clarify the role of the identified microorganisms in the production process, a preliminary biochemical characterization was carried out.

\section{EB30}

Development of biosensors for benzene monitoring C. Alquati ${ }^{1}$, M. Papacchini ${ }^{2}$, G. Ludovisi ${ }^{2}$, G. Bestetti ${ }^{1}:{ }^{1}$ Department of Environmental Sciences, University of Milano-Bicocca, Piazza della Scienza, 120126 Milan, Italy; ${ }^{2}$ ISPESL, Department for Production Premises and Interaction with the Environment, Via Fontana Candida, 100040 Monteporzio Catone, Roma, Italy. E-mail: maddalena.p@tiscali.it (M. Papacchini)

Biosensors have undergone rapid development over the last few years; in particular, in environmental field many biosensors using microorganisms and purified enzymes as biological component, were recently studied. Benzene is present everywhere with high levels in the cities and sometimes, in petroleum processing plants. It is classified as carcinogenic compound of first class able to cause leukaemia. Because the evaluation of benzene requires complex instruments and quite long analysis times, it is required to study alternative systems for benzene detection simple, fast and highly sensitive, such as biosensors. From Pseudomonas putida MST, strain previously isolated in our laboratory and able to degrade benzene, we isolated genes encoding for benzene 1,2-dioxygenase and cis-1,2-dihydrodiolbenzene dehydrogenase to use in the development of two different hydrocarbon biosensors based on microorganisms and on purified enzymes. The genes isolated were cloned in pVLT33 and we developed three microbial systems carrying: (1) benzene dioxygenase, (2) dihydrodiol dehydrogenase and (3) benzene dioxygenase-dehydrogenase modified by PCR to obtain enzymes with histidine tag. The cloning was planned to construct recombinant strains able to overproduce the enzymes; the enzymatic activities will be evaluated both using whole cells and purified enzymes.

\section{EB31}

Study of operation condition of biofilter using fibril-form matrix for odor gas removal Don-Hee Park, Gwi-Taek Jeong, Kyoung-Min
Lee, Hyun-June Park School of Biological Science and Technology, Chonnam National University, 500-757 Gwangju, South Korea. Email: dhpark@chonnam.ac.kr (D.-H. Park)

This research was performed for developing of biological treatment process of odor gas such as MEK, $\mathrm{H}_{2} \mathrm{~S}$, and toluene, which is generated from the food waste recycling process. To establish the operational conditions of odor gas removal by small-scale biofiltration equipment, it was continuously operated by using toluene as a treating odor object. When the odor treating microorganisms were adhered to fibril form biofilter, high removal efficiency over $93 \%$ was obtained by biofilm formation. At $400 \mathrm{ppm}$ of inlet odor gas concentration and $10 \mathrm{~s}$ of retention time, the removal efficiency was $76 \%$ and $93 \%$ in first stage reactor and second stage reactor, respectively. However, the removal efficiency remained over $97 \%$ at the operational conditions above $15 \mathrm{~s}$ of retention time.

\section{EB32}

Synergistic antimicrobial effect of electrolyzed ozonated water Y.F. Hong ${ }^{1}$, Y.H. Park ${ }^{1}$, H.Y. Lee ${ }^{2}$, H.S. UHM ${ }^{2}$ : ${ }^{1}$ Laboratory of Applied Microbiology, Department of Molecular Science and Technology Ajou University, Suwon, South Korea 443-749; ${ }^{2}$ Laboratory of Energy Physics, Department of Molecular Science and Technology Ajou University, 443-749 Suwon, South Korea. E-mail: yhpark@ajou.ac.kr (Y.H. Park)

Ozonated water is produced using an ozone generator in a container filled with cold water. It is useful for sanitizing the surfaces of various products for which heat or chemical treatment is inappropriate, such as fresh food products. In this study, we investigated the antimicrobial effects of ozonated water and electrolyzed ozonated water against Escherichia coli, S. aureus, Bacillus subtilis and yeast, Saccharomyces cerevisiae for practical use in sanitizing various products. The results demonstrated that the electrolyzed ozone water was effective for the reduction of microbial population at relatively low concentration of ozone. Also, the electrolyzed and the ozonated water showed synergistic antimicrobial effects.

\section{EB33}

Involvement of glutathione $S$-transferases in xenobiotic detoxification in Saccharomyces cerevisiae M.D. Pereira, P.D.B. Adamis, A.D. Panek, E.C.A. Eleutherio Department of Bioquimica-IQ-UFRJ, Rio de Janeiro, Brazil. E-mail: marcosdp@iq.ufrj.br (M.D. Pereira)

Many xenobiotics can react spontaneously with thiol moieties of glutathione (GSH), forming GSH-conjugates, or via glutathione $S$-transferases (GST). These enzymes participate in detoxification of potentially harmful compounds from endo or xenobiotic origin. Using Saccharomyces cerevisiae as experimental model, we observed that cells mutated in the GTT1 or GTT2 genes showed twice as much cadmium absorption than the control strain. We proposed that the formation of the cadmium-glutathione complex is dependent on those transferases, since it was previously demonstrated that the cytoplasmic levels of this complex affect cadmium uptake. The addition of glutathione monoethyl ester (GME), a drug that mimics glutathione (GSH), to gttl $\Delta$ cells restored the levels of metal absorption to those of the control strain. However, with respect to $g t t 2 \Delta$ cells, addition of GME did not alter the capacity of removing cadmium from the medium. Taken together, these results suggest that Gtt1p and Gtt2p play different roles in the mechanism of cadmium detoxification. By analyzing the toxic effects of this 
metal, we verified that $g t t 2 \Delta$ and $g s h 1 \Delta$ cells showed, respectively higher and lower tolerance to cadmium stress than control cells, suggesting that although GSH plays a relevant role in cell protection, formation of the GSH-Cd ${ }^{2+}$ conjugate is deleterious to the mechanism of defense. Furthermore, analyzing the harmful effects of other xenobiotic, menadione (2-methyl-1,4-napthoquinone), we have also observed that Gtt1p and Gtt2p isoforms play distinct functions in the process of cell protection as well as in drug remove, since both strains showed lethal phenotypes after direct exposure to $20 \mathrm{mM}$ menadione. However, after adaptive treatments (mild-heat or exposure to a lower menadione concentration), cells acquired tolerance to menadione stress, although the gtt $2 \Delta$ mutant had still shown a higher sensitivity against drug toxicity. By analyzing the malondialdehyde (MDA) produced in response to menadione, we observed that $g t t 2 \Delta$ cells exhibited increased levels of lipid peroxidation, indicating that, during menadione exposure, GSH-conjugates are formed by the same transferase isoform, Gtt2p, involved in cadmium stress.

\section{Acknowledgement}

Financial support: STINT (Sweden), CNPq and FAPERJ (Brazil).

\section{EB34}

Degradation of anthracene by laccase of Coriolopsis rigida Diego Rodríguez-Solar, José Sieiro, Teresa Alcántara, María A. Longo, M. Angeles Sanromán Department Chemical Engineering, University of Vigo, Campus Lagoas-Marcosende, 36310 Vigo, Spain. E-mail: sanroman@uvigo.es (D. Rodriguez-Solar)

Polycyclic aromatic hydrocarbons (PAHs) are ubiquitous and persistent throughout the environment. They are generally distributed from both natural and industrial sources. Many PAHs can have a detrimental effect on the flora and fauna of affected habitats through uptake and accumulation in food chains, and in some instances, they induce serious health problems and/or genetic defects in humans. Many research efforts have been expended to find a suitable method for remediation of soil and water environments contaminated with PAHs. Amongst them, the use of ligninolytic fungi is particularly suitable for the development of such processes, since they produce extracellular lignin-degrading enzymes (MnP, LiP, laccase, ...) which degrade a wide range of organic pollutants. Coriolopsis rigida has been reported to produce extracellular laccase as the sole ligninolytic enzyme. This makes this fungus particularly suitable for the study of xenobiotics degradation by laccase. The purpose of this research was to obtain high laccase activities by $C$. rigida in solid state cultures and to determine their ability to degrade anthracene (typical PAH). Both in vivo and in vitro assays were performed. The former led to $60-80 \%$ degradation in 3 days depending on the culture conditions, whereas the latter showed a degradation percentage above $90 \%$ in 2 days when low mediator concentration (HBT) was added to the reaction mixture.

\section{Acknowledgements}

This work has been financed by the Spanish Ministry of Science and Technology and European FEDER (Project CTM2004-01539). The authors wish to thank Dra. M.J. Martínez (CIB, CSIC, Madrid, Spain) for providing Coriolopsis rigida.

\section{EB35}

Viable but nonculturable (VBNC) states of pathogenic bacteria is critical for public health A.A. Shabani, M.R. Akbari Eidgahi, A.H. Hajighorbani Centre for Biotechnology Research, Semnan University of Medical Sciences, Semnan, Iran. E-mail: shaebani@semums.ac.ir, aashaebani@yahoo.com (A.A. Shabani)

The phenomena, Viable but nonculturable(VBNC(states of various pathogenic bacteria such as Vibrio vulnificus, Vibrio cholerae O1, Salmonella enteritidis, Shigella dysenteriae 1, Enterococcus faecalis, Campylobacter jejeuni has been reported. They are viable, and can convert to culturable form. In addition Genetical Manipulation Microorganisms (GMMO s) had created too. Thus, the result of contamination with them will be infect. The detection of them need Molecular methods, because They had not distinguished by culture. Comparative analysis of PCR versus culture for detection of Shigella spp. in clinical samples were the aim of this research. The fecease of dysentria patients are cultured on Mac conkey agar and SS agar and follow up by other media. Some of genes that evaluaterd in this study were, virA, 16S rRNA, IpaH (Invasion plasmid antigen) and etc. Primers designed based on these genes and prepared from Danesh afza company, and then PCR performed on the DNA extracted from fecease of patients too. The results obtained from PCR and Culture were comparatively evaluated for their abilities to demonstrate Shigella species in fecal from shigellosis patients during a two year period in Semnan from Iran. The results demonstrated that PCR (especially IpaH gene) is preferable for detection of Shigella spp.

\section{EB36}

A low molecular weight protein fraction from human portal serum stimulates cell proliferation in immature $S$. mansoni Yehia M. Shaker ${ }^{1}$, M.M. Abdel Fattah ${ }^{2}$, E. Ashour ${ }^{1,2,3}$, H.M. Khattab ${ }^{3}$ H.M. Draz ${ }^{1}{ }^{1}$ Biochemistry Department, National Research Centre, Cairo, Egypt; ${ }^{2}$ Biochemistry Department, Ain Shams University, Cairo, Egypt; ${ }^{3}$ Pathology Department, Faculty of Medicine, Cairo University, Cairo, Egypt

The present work is a trial to study the portal serum factors which stimulate the cell proliferation of the schistosomules, aiming to find ways to block or inhibit their effects. Our previous studies showed that portal serum of human and hamster (highly susceptible hosts) and a $1-50 \mathrm{kD}$ fraction separated from human portal sera by ultrafiltration stimulate cell proliferation in immature schistosomules (20 days old) in vitro. For further identification of the portal serum factors in the range of $1-50 \mathrm{kD}$ that stimulate cell proliferation, schistosomules were incubated in vitro in medium containing $10 \%$ fetal calf serum, $10 \%$ portal human serum or $10 \%$ peripheral human serum or their fractions separated by native electrophoresis followed by electroelution, incubations were performed in presence of bromodeoxyuridine (BrdU) in order to measure differences in cell proliferation. The results showed that human portal sera enhanced cell proliferation of schistosomules compared to the peripheral serum. This stimulatory effect was substantially reproduced by fraction separated from human portal serum with molecular weight $20.8 \mathrm{kD}$. These results may help in designing a drug or antibody therapy to block the stimulating effect of the portal serum fraction and subsequently to disturb the life cycle of the parasite at early stage of development. 


\section{EB37}

Isolation and characterization of solid $\boldsymbol{n}$-alkane degrading bacteria Naoya Shinzato, Toru Matsui Center of Molecular Biosciences, University of the Ryukyus, Okinawa 903-0213, Japan. E-mail: naoya-s@comb.u-ryukyu.ac.jp (N. Shinzato)

Oil strage tank sludge, mainly composed of water and solid hydrocarbons (waxes) needs to be treated when harvesting the stored oil. Although the sludge treatment by microbial surfactant or microbial cracking are considered as the feasible method, microbial degradation of the waxes (ca. solid $n$-alkanes) have been reported in a very limited species such as Acinetobacter. In addition, long-chain $n$-alkanes (so called paraffin waxes) are one of the major components of oil, and their resistant properties to biological attack hold up the recovery of oil-polluted environments. In this report, we have screened $n$-tetracosane (C24) degrading bacteria from soils in Okinawan Island, an unique sub-tropical area in Japan, to know the bacterial diversity and their degrading mechanism.16SrDNA phylogenetic analysis of the isolates, totally ca 40 , showed they were not only Acinetobacter and Pseudomonas, but also other proteobacteria (Alcaligenes), actinomycetes (Gordonia, Nocardia, and Leifsonia), Bacillus, Staphylococcus, and unidentified ones. They also grew not only the solid $n$-alkanes but also iso-alkanes, mid-chain $n$-alkanes as the sole carbon source. Results for the biosurfactant production will also be shown.

\section{EB38}

Molecular investigation of environmental entorococci Domenico Pangallo $^{1}$, H. Drahovska ${ }^{2}$, P. Ferianc ${ }^{1}$, J. Turna ${ }^{2}$, J. Timko ${ }^{1}$ : ${ }^{1}$ Institute of Molecular Biology, SAV, Dubravska cesta 21, Bratislava 845 51, Slovakia; ${ }^{2}$ Department of Molecular Biology, Faculty of Natural Sciences, Comenius University, Bratislava, Slovakia. E-mail: turna@fns.uniba.sk (J. Turna)

The properties of 188 environmental enterococci were studied. The strains were isolated mainly from surface and waste waters and several strains from sheep manure were also included. Species identification was provided by combination of phenotypic (Micronaut System, Merlin) and molecular detection methods (automated ITSPCR, ddl-PCR). Several discrepancies were observed when comparing molecular and biochemical identification. Six enterococcal species were overall identified; E. faecium and E. hirae were the most abundant ones, almost $80 \%$ of isolates belonged to these two species. The distribution of selected genes conferring virulence to enterococci ( $c y l \mathrm{~A}$, gelE and $e s p)$ was investigated, the positive signal was obtained mainly for $E$. faecalis strains. The strains were also characterized for the possession of enterocin genes (ent $\mathrm{A}$, ent $\mathrm{B}$, ent $\mathrm{P}$, ent 31 , ent $\mathrm{L} 50 \mathrm{AB})$ and high frequency of enterocins was observed.

\section{EB39}

Biosorption of reactive dyes on Scenedesmus obliquus Sema Tan ${ }^{1}$, Hikmet Katırcıoğlu ${ }^{2}$, Zeki Öktem ${ }^{3}$, Aysun Ergene ${ }^{1}$ : ${ }^{1}$ KırıkkaleUniversity, Faculty of Science and Literature, Department of Biology, Yahsihan, Kırıkkale, Turkey; ${ }^{2}$ Gazi University, Faculty of Education, Department of Biology, Beşevler, Ankara, Turkey; ${ }^{3}$ KırıkkaleUniversity, Faculty of Science and Literature, Department of Chemistry, Yahsihan, Kırıkkale, Turkey

Biosorption of three different dyes (Reactive Black 5, Cibacron Brilliant Yellow, Cibacron Brilliant Red) onto immobilized Scenedesmus obliquus a microalga was investigated in a batch sys- tem. The immobilized alga exihibited the highest dye uptake capacity at the initial $\mathrm{pH}$ value of 2.0 for all dyes. The effect of temperature on equilibrium sorption capacity indicated that maximum was obtained at $25^{\circ} \mathrm{C}$ for RB5, CBY and CBR biosorption. The Freundlich, and Langmuir adsorption models were used for the mathematical description of the biosorption equilibrium and isotherm constants were evaluated.

\section{EB40}

Biocontrol properties of microbially-treated sugar beet wastes in presence of rock phosphate $N$. Vassilev, I. Nikolaeva, M. Vassileva Department of Chemical Engineering, Faculty of Sciences, University of Granada, c/Fuentenueva s/n, Granada-18071, Spain. E-mail: nbvass@yahoo.com (N. Vassilev)

The effect of soil application of sugar beet wastes (SB) treated with Aspergillus niger in the presence of rock phosphate (RP) on the control of fusarium wilt of tomato were studied. Two treatments and a control were used: inoculation with Glomus intraradices (AM), further inoculation with $A$. niger grown on $\mathrm{SB}+\mathrm{RP}$ medium, and the control (C). Application of the AM fungus increased plant growth, $\mathrm{P}$ and $\mathrm{N}$ uptake and reduced disease caused by Fusarium oxusporum f. sp. licopersici ( $F o l)$ as compared to non-mycorrhizal control plants. Soil amendment with $\mathrm{SB}+\mathrm{RP}+A$. niger resulted in $347 \%$ and $467 \%$ (versus $\mathrm{C}$ ) higher plant shoot biomass in plant-soil experiments contaminated or not with $\mathrm{Fol}$, respectively. In this case, disease severity and number of Fol CFU reached the lowest levels while soil phosphatase and beta-glucosidase activities increased compared to all other treatments. Fol negatively affected plant root mycorrhization determined in the AM treatment while the difference between the mycorrhization of plants grown in the presence and absence of F. oxysporum in $\mathrm{SB}+\mathrm{RP}+A$. niger-amended soil was insignificant ( $53 \%$ versus $59 \%$, respectively). In conclusion, the fermentation mixture containing mineralized organic matter, partially solubilized RP, and $A$. niger biomass could be efficiently used not only in improving plant growth, nutrient uptake and properties of degraded and polluted soils, as previously reported (Vassilev and Vassileva, 2003), but also in environmentally-mild management of fusarium wilt.

\section{Reference}

Vassilev, N., Vassileva, M., 2003. Appl. Microbiol. Biotechnol. 61, $435-440$.

\section{EB41}

Membrane bioreactors for removal of charged micropollutants from drinking water Svetlozar Velizarov, João G. Crespo, Maria A. Reis CQFB/REQUIMTE, Department of Chemistry, FCT, Universidade Nova de Lisboa, Caparica P-2829-516, Portugal. E-mail: velizarov@dq.fct.unl.pt (S. Velizarov)

Biological treatment processes allow for the effective elimination of charged inorganic micropollutants, e.g. a number of oxyanions, heavy metals, etc. from contaminated drinking water supplies. However, dedicated technologies have to be implemented in order to eliminate the target pollutants without changing the quality of treated water, avoiding its secondary pollution by cells, nutrients and metabolic by-products. Some innovative technologies, which combine the use of membranes with the bioconversion of charged micropollutants in order to deal with the secondary water contamination problem, will be presented and critically compared. A special 
focus will be given on pressure-driven membrane bioreactors, gastransfer membrane bioreactors and the novel ion exchange membrane bioreactor (IEMB). The latter concept has been developed and currently studied by our group. This process, based on integration of Donnan dialysis with bioconversion of one or more target pollutants to harmless products, has been modeled and experimentally verified for the removal of various charged inorganic pollutants such as nitrate, perchlorate and bromate by mixed microbial cultures under anoxic conditions. Tests of up to 3 months showed a very good operational stability. The essential role of the microbial membraneattached biofilm, which develops naturally in this type of systems, will be also demonstrated and discussed.

\section{EB42}

Development of compact bioreactor for nitrogen-removal by using poly(lactic acid) as electron donor Atsushi Watanabe, Hiroaki Uemoto, Masahiko Morita Environmental Science Research Laboratory, Central Research Institute of Electric Power Industry (CRIEPI), 1646 Abiko, Abiko-shi, Chiba 270-1194, Japan. E-mail: a-nabe@criepi.denken.or.jp (A. Watanabe)

Poly(lactic acid) (PLA), which is one of biodegradable plastics, is depolymerized by hydrolysis and releases soluble monomer or oligomer of lactic acid. Many bacteria can use the monomer and oligomer as an energy source or a carbon source. In this study, we applied PLA to an electron donor for denitrification process of the previously developed bioreactor, which could remove ammonia from wastewater by simultaneously carrying out two biological processes, aerobic nitrification and anaerobic denitrification. A bench-scale bioreactor was constructed with a gel-plate containing pure-cultured cells of Nitrosomonas europaea and Paracoccus denitrificans and a PLA-plate. The PLA-plate was prepared by mixing three kinds of PLAs with different molecular weight and tricalcium phosphate to keep the constant release of the electron donor for a long term. Batch treatment experiment with the bioreactor was repeated with an artificial wastewater containing ammonia for 100 days. The bioreactor could remove nitrogen from the artificial wastewater at nitrogenremoval rate of approximately $4 \mathrm{~g} \mathrm{~N}$ /day per square meter of gel-plate surface during the experiment period without an additional electron donor. The performance was equivalent to that obtained with our bioreactor using ethanol as electron donor for denitrification. The bioreactor using PLA dose not need an additional pump for serving an electron donor (e.g., ethanol) and a hollow space for serving. Therefore, the concept using solid electron donors like a PLA would be effective our bioreactor to compact and simplify, and would be possible to develop a portable or disposable bioreactor.

\section{EB43}

Leucosporidium antarcticum as a source of enzymes for biotechnology Arkadiusz Wojtasik ${ }^{1,2}$, Marianna Turkiewicz ${ }^{2}$, Jaroslaw Dziadek ${ }^{1}$, Pawel Parniewski ${ }^{1}:{ }^{1}$ Centre for Medical Biology PAS, 106 Lodowa Street, 93-232 Lodz, Poland; ${ }^{2}$ Faculty of Biotechnology and Food Sciences Technical University of Lodz, Stefanowskiego 4/10 Street, 90-924 Lodz, Poland. E-mail: awojtasik@cbm.pan.pl (A. Wojtasik)

Leucosporidium antarcticum is a psychrophilic yeast able to growth at low temperature. These microorganisms live in Antarctic marine waters and are endemic to that cold environment. Furthermore, L. antarcticum is also isolated from the digestive tract of
Antarctic krill Euphausia superba. Enzymes isolated from coldadapted microorganisms such as $L$. antarcticum having a specific activity at low temperatures ranging from 0 to $30^{\circ} \mathrm{C}$ are considered for utilization at biotechnological applications such as bioremediation, production of polyunsaturated fatty acids of dietary significance and might be a source of industrially useful enzymatic proteins. The main goal of this study was to construct a cDNA library of L. antarcticum. The partial cDNA library was obtained and some of the clones were analysed. The sequencing analyses allowed us to find an approximately 450 base pair nucleotide sequence which displayed a very high homology to disulfide bond chaperone belonging to the Hsp33 family from Psychrobacter sp. High similarity of that heat shock protein was found on an amino acid sequence level and was reaching nearly $85 \%$. The main object of our further research is to clone Hsp33 family protein gene and to obtain its expression in a mezophilic host strain. Also, further clones will be analysed to find other interesting genes encoding the psychrophilic proteins.

\section{Acknowledgement}

This work was partially funded by the KBN grant I29/205/05.

\section{EB44}

In vitro micropropagation and cultivation of endemic and endangered geophytes of Turkey $\underline{S . \ddot{O} z c a n^{1}}, \dot{I}$. Parmaksiz ${ }^{2}, S$. Mirici $^{3}$, S. Çöçü ${ }^{1}$, C. Sancak ${ }^{1}$, S. Uranbey ${ }^{1}$, E.O. Sarıhan ${ }^{1}$, A. Ipek ${ }^{1}$, D. Kaya $^{1}$, B. Gürbüz ${ }^{1}$, N. Arslan ${ }^{1}$ Department of Field Crops, Faculty of Agriculture, University of Ankara, 06110 Dışkapı, Ankara, Turkey; ${ }^{2}$ Department of Biology, Faculty of Science and Arts, University of Gaziosmanpaşa, Tokat, Turkey; ${ }^{3}$ Faculty of Education, University of Akdeniz, Antalya, Turkey

Out of 9000 plant species found in the flora of Turkey, about 3000 are endemic. Beautiful flowering (geophytes) bulbous plants form an important part of this rich biodiversity. Besides use as ornamental plants, these have great potential in perfume and pharmaceutical industry. Genera of Fritillaria, Ornithogalum, Muscari, Bellevalia, Tulipa, Galanthus, Sternbergia, Crocus, Arum and Biarum have important and critically endangered species with high export potential that enters into this group. Most of these are endangered and their collection from wild and export has been banned to conserve them. Large scale production and conservation of these species could also be achieved by in vitro techniques. Therefore bulb scale and immature embryo explants of Sternbergia candida, S. fischeriana, Muscari muscarimi, Fritillaria imperialis and $F$. persica were cultured on different nutrient media supplemented with various concentrations of plant growth regulators using different culture applications. Large numbers of bulblets were produced (over 100 bulblets/explants) from single immature embryos on nutrient media in most species tested after 12 months of culture initiation. Regenerated bulblets were kept at $5{ }^{\circ} \mathrm{C}$ for 5 weeks and then transplanted to soil successfully. To our knowledge the present study is the first report for in vitro bulblet production from immature embryos of geophytes. The procedure described here provides a prolific bulblet production system that may form the basis of bioreactor culture and conservation of endemic and endangered geophytes.

\section{EB45}

Bacterial degradation of fluorinated compounds $\underline{\text { M.I.M. Ferreira, }}$, D.B. Janssen Department of Biochemistry, GBB, Univer- 
sity of Groningen, Nijenborgh 4, 9747 AG, Groningen, The Netherlands

The commercial use of organofluorine compounds in industrial, pharmaceutical and pest-control applications has dramatically increased over the past few years, resulting in the introduction of numerous new organic compounds into the environment. Organofluorine compounds are chemically very stable and are assumed to be resistant to biological degradation. Given the chemical inertness of fluorinated organics, their bioactivity, and their potential for accumulation in the environment, it is important to understand their environmental fate and the mechanisms by which they might be degraded. Examples of the biodegradation of fluorinated compounds in literature are scarce, being fluorobenzoic acids the most commonly reported. Information on the cleavage of carbon-fluoride bonds in synthetic compounds is limited to fluoroacetate dehydrogenase. In this project we try to obtain more insight in the defluorination mechanisms by investigating the diversity of degradation routes for these compounds in several soil bacteria by making use of modern genetic tools. A Gram-positive strain capable of aerobic biodegradation of 4-fluorophenol (4-FP) as the sole source of carbon and energy was isolated by selective enrichment from soil samples collected near an industrial site. Batch cultures were set up and substrate consumption, accumulation of intermediates and product formation were monitored. The consortium was able to use 4-FP up to concentrations of $448.4 \mathrm{mg} \mathrm{l}^{-1}$ and was able to utilize a range of other organic compounds. Stoichiometric release of fluoride ions was measured in batch cultures suggesting that there is no formation of dead-end products during 4-FP metabolism.

\section{EB46}

Biobleaching of kraft cellulose pulp by Poliporus versicolor Aysun Ergene $^{1}$, Nazif Kolankaya ${ }^{2}{ }^{1}$ Kirlkkale University, Faculty of Science and Literature, Department of Biology, Yahsihan, Kırıkale, Turkey; ${ }^{2}$ Hacettepe University, Faculty of Science, Department of Biology, Beytepe, Ankara, Turkey

The suitability of culture supernatant from Poliporus versicolor for use in the biobleaching of kraft cellulose pulp was investigated. P. versicolor was found to grow on mycological broth (1\% soytone, $4 \%$ D-glucose and $0.5 \%$ cellulose pulp). Maximal extracellular ligninase production was detected after 7 days (7 nkat). The optimum biobleaching conditions are $30^{\circ} \mathrm{C}$ and $\mathrm{pH} 4.8$, with 10 days. In this condition $P$. versicolor decreased the Kapa number from 38.55 to 19.42 and increased brightness from 28 to 32.7 in 10-day treatment.

\section{EB47}

Genotypical differences affecting the response of Pisum sativum to differing boron/iron applications E.E. Hakki, U. Zeynep, M. Hamurcu, A. Tamkoc, M.B. Babaoglu, S. Gezgin Department of Field Crops, Faculty of Agriculture, Selcuk University, Kampus, Konya 42079, Turkey. E-mail: eehakki@ selcuk.edu.tr (E.E. Hakki)

Boron and iron are among the microelements required for the proper development of the vegetative and generative tissues of plants. Though iron is present in high amounts in almost all soil types, its bioavailability to crops is extremely reduced, hence most of the plants face an iron defficiency problem and while on one side crop productions effected, on the other hand the nutrition problems come up to human through contagious nutritional chains. Boron is also among the most problematic micronutrients of the major crop plan- tation areas of Turkey. Both defficiency and toxicity problems exist in a total of about $50 \%$ of the Central Anatolian soil where pea is among the legumes cultivated. Application of varying levels of boron and iron combinations in greenhouse and the analysis of plant acquisition via ICP-AES as well as determination of the effects of the element combinations both in morphological and molecular levels are the aim of our studies. The genetic bases of the response differences of plant genotypes to $\mathrm{B}$ and/or Fe, were investigated through the applications of molecular marker techniques. Considerable growth rate and stem size differences were detected within the parents (wild-type versus cultivar) and the F9 plants. The presence of efficient genotypes to high micronutrient levels are expected to help us increase the cultivation of the crop in problematic areas as well as in exploring the molecular bases of the microelement uptake mechanisms.

\section{EB48}

Biological treatment of butachlor by activated sludge method $M$. Borgheiy ${ }^{1}$, A. Hasani ${ }^{2}$, H. Dinkoo ${ }^{3}{ }^{1}$ Chemical Engineering Department, Sharif University, Tehran, Iran; ${ }^{2}$ Enviroumental Engineering Department, Science and Research Campus, Islamic Azad University, Tehran, Iran, $1477893855 ;{ }^{3}$ Chemical Engineering Department, Science and Research Campus, Islamic Azad University, Tehran, Iran

Butachlor is one of the selective systemic herbicide toxins that are act by inhibition of protein synthesis. This toxin is used exclusively in the rice, barely, cotton and wheat farmlands. Butachlor is belonging to chloroacetanilide herbicide group, which are consisting of butachlor, alachlor, acetochlor, metolachlor and poropachlor. In the view of bioenvironmental, butachlor is degraded in the soil by microbial activity. Its stability is about $6-10$ weeks. It is converted to the water-soluble derivatives in soil or water, with a slow evolution of carbon dioxide. Because of butachlor is one of the herbicide toxin, it is inhibitor factor against growth of bacteria and microorganisms. Microorganisms can be continuing their activities in the limited concentration of butachlor. Therefore treatment of industrial wastewater consist of concentrated butachlor by the biological treatment is impossible and it is necessary chemical or physico-chemical treatment are used. In this research, biological treatment methods are used. In the biological treatment, an activated sludge system with volume of 6.51 is used. In this method, butachlor with concentration of $5 \mathrm{mg} / \mathrm{l}$ are treated. Removal percent of butachlor for concentration of 2.5 and $3 \mathrm{mg} / \mathrm{l}$ are calculated to $41.20 \%$ and $41.67 \%$, respectively. Removal percent of COD is also calculated to $86 \%$.

\section{EB49}

Biodegradation kinetics of olive oil mill wastewater by loofa immobilized Phanerochaete chrysosporium Mojtaba Ahmadi, Farzaneh Vahabzadeh, Babak Bonakdarpour Food Process Engineering and Biotechnology Research Center, Department of Chemical Engineering, Amirkabir University of Technology, Tehran, Iran. E-mail: far@aut.ac.ir (M. Ahmadi)

Olive oil mill wastewater (OMW) as the effluent of the concern of olive industry has high organic load. The conventional biological treatments despite of their simplicity and rather suitable performance are ineffective for the OMW treatment since phenolics possess antimicrobial activity. In order to carry out a proper treatment on OMW, use of microorganism able to degrade the phenolics thus, is necessary. The ability of Phanerochaete chrysosporium immobilized 
on loofa was studied. The basal mineral salt solution along with glucose, ammonium sulfate and yeast extract were used to dilute OMW properly. The fungus did not grow on the concentrated OMW. Therefore, OMW diluted by $20 \%$ was used thought this study. The extent of removal in this biotreatment, of total phenolics (TP) and COD were 90 and $50 \%$, respectively. While the color and aromaticity decreased by 60 and $95 \%$, respectively. The kinetic behavior of the loofa immobilized fungus was found to follow Monod equation. The maximum growth rate was $0.045 \mathrm{~h}^{-1}$ while the Monod constant based on the consumed TP and COD (mg/L) were 370 and 6900, respectively.

\section{EB50}

Advanced start-up strategy of an anaerobic three-phase turbulent bed reactor treating winery wastewaters $\underline{R}$. Cresson, $H$. Carrère, N. Bernet, J.P. Delgenès Laboratoire de Biotechnologie de l'Environnement, Institut National de la Recherche Agronomique (INRA), Avenue des Etangs, 11100 Narbonne, France. E-mail: cresson@ensam.inra.fr (R. Cresson)

The objective of our study was to compare two start-up strategies for an anaerobic biofilm process, to create an effective biofilm and increase the organic loading rate (OLR) as quickly as possible. Two methanogenic three-phase biofilm reactors have been started, using the same operational parameters (solid hold-up ratio, gas velocity of $1 \mathrm{~mm} \mathrm{~s}^{-1}$ ), in order to test two different strategies:

- Maximal load strategy (reactor A): The OLR is increased as long as the global amount of removed COD (biogas production) increased.

- Maximal removal strategy (reactor B): The OLR is increased stepwise as soon as the COD removal rate reaches $80 \%$.

Both reactors have been operated for 90 days, until a volumetric OLR of $20 \mathrm{~g}_{\mathrm{COD}} 1^{-1} \mathrm{j}^{-1}$, with more than $90 \%$ of carbon removal. The total amount of COD removed and methane produced were higher in reactor B (19.6 and 32.2\%, respectively). In both reactors, the short hydraulic retention time (HRT) applied during all the experiment caused a rapid wash-out of planktonic bacteria and an exclusive use of the substrate by the attached micro-organisms, which accelerates the biofilm growth. The lag-phase was reduced to approximately 7 days. The reactor submitted to repetitive disturbance by the maximal removal strategy appeared to be more robust when confronted to perturbation like organic overload or nutritional deficiency. Experiments have demonstrated capability and the efficiency of the aggressive strategy for controlling anaerobic bioreactor start-up.

\section{EB51}

Production of bioplastics and hydrogen from sugar cane molasses fermentation M.G.E. Albuquerque, L.S. Serafim, M.A.M. Reis REQUIMTE-Departamento de Química, CQFB, FCT/UNL, Portugal, 2829-516 Caparica. E-mail: graca.albuquerque@dq.fct.unl.pt (M.G.E. Albuquerque)

Sustainability is the generally accepted paradigm for future industrial development. The re-integration of waste products into production processes is a major aspect of environmental sustainability. In this study the use of sugar cane molasses is being investigated for the production of bioplastics by mixed microbial cultures, with the added possibility of parallel biohydrogen production. Polyhydroxyalkanoates (PHAs) are polyesters synthesized by bacteria and accumulated as granules in the cytoplasm. Studies conducted by this group have shown that mixed microbial cultures subjected to dynamic feeding conditions may accumulate PHAs up to $80 \%$ cell dry weight, a value close to that obtained for pure cultures. Volatile fatty acids are good substrates for the production of PHAs by mixed cultures. On the other hand, sugar molasses, with a very high sugar content (about $50 \%$ dry weight), can produce organic acids by fermentation. The two-stage process being implemented in this study includes a molasses fermentation step, in which the high sugar content of the molasses is converted into volatile fatty acids (VFAs), and a PHA production step, in which the VFAs serve as the precursors to the formation of PHAs under dynamic feeding conditions. Moreover, hydrogen can be produced by anaerobic bacteria from carbohydrate-rich substrates giving organic fermentation end products, $\mathrm{H}_{2}$ and $\mathrm{CO}_{2}$. To optimize the production of both high-value products, design of experiments (DOE) is being used to elaborate a set of experiments to study the effect of $\mathrm{pH}$, hydraulic retention time and organic loading on both the organic acids distribution (which will serve as precursors for PHA production in the second step) and $\mathrm{H}_{2}$ production in the acidogenic fermentation reactor (a $11 \mathrm{CSTR}$ ). Preliminary results show that the effluent of the acidogenic reactor fed with $10 \mathrm{~g} / \mathrm{l}$ total sugars and operated at $\mathrm{pH} 7$ and $D=0.1 \mathrm{~h}^{-1}$ (composed mainly of acetate and propionate) can be successfully fed to a polymer-accumulating mixed culture. Under these conditions, the $\mathrm{H}_{2}$ production yield has been estimated at $3.9 \mathrm{~mol} \mathrm{H}_{2} / \mathrm{mol}$ sucrose.

\section{EB52}

Vanillin production by resting cells of Escherichia coli JM109/pBB1 from synthetic ferulic acid solutions: Effect of initial substrate and biomass concentration $\underline{P}_{\text {. Torre }}{ }^{1}$, D. De Faveri ${ }^{1}$, B. Rivas $^{2}$, P. Perego ${ }^{1}$, J.M. Domínguez ${ }^{2}$, A. Converti ${ }^{1}$ : ${ }^{1}$ Department of Chemical and Process Engineering, Genoa University, Via Opera Pia 15, 16145 Genoa, Italy; ${ }^{2}$ Department of Chemical Engineering, Vigo University (Campus of Ourense), Polytechnic Building, As Lagoas, 32004 Ourense, Spain. E-mail: Paolo.Torre@ dichep.unige.it (P. Torre)

Vanillin is a flavour compound used in food industry, fragrances and pharmaceutical preparations, which is nowadays mainly produced by chemical synthesis. The increased demand of natural products for the food industry as well as the high cost of natural vanillin extracted from vanilla pods has recently stimulated the research for alternatives to produce this compound by a natural way. The microbial transformation of ferulic acid, a phenolic compounds from lignin degradation, is recognized as being the most interesting alternative to produce natural vanillin. The combined effects of initial ferulic acid concentration $\left(S_{0}\right)$ and biomass concentration $\left(X_{0}\right)$ on vanillin production by resting cells of Escherichia coli strain were investigated using response surface methodology. E. coli JM109/pBB1 a recombinant strain producing key enzymes of ferulate catabolic pathway from P. fluorescens BF13 (feruloyl-CoA synthetase and feruloylCoA hydratase/aldolase) was utilized in this work. A $3^{2}$ full-factorial design was employed for experimental design. The results shown a possible inhibition phenomena at a vanillin concentration of about $0.1 \mathrm{~g} \mathrm{~L}^{-1}$ leading to the accumulation in the fermentation media of secondary compounds like vanillic acid and vanillin alcohol. 


\section{EB53}

Purification and downstream process of xylitol obtained biotechnologically from hemicellulosic hydrolyzate of corncobs

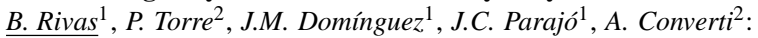
${ }^{1}$ Department of Chemical Engineering, Vigo University (Campus of Ourense), Polytechnic Building, As Lagoas, 32004 Ourense, Spain; ${ }^{2}$ Department of Chemical and Process Engineering, Genoa University, Via Opera Pia 15, 16145 Genoa, Italy. E-mail: brivas@uvigo.es (B. Rivas)

Biotechnological production of xylitol from lignocellulosic materials has been widely studied in the recent years with promising results that confirming the possible industrial application of this technology. Xylitol purification from fermented broth is the limiting stage of this process. Previous works suggest crystallization procedures in order to recovery xylitol from fermented synthetic solutions. The complexity of fermented hydrolyzate not allows direct crystallization. In this work, corncobs hydrolyzate obtained with autohydrolysis-posthydrolysis techniques, detoxificated with activated charcoal and concentrated was fermented to xylitol by $D$. hansenii. The fermented broth composition was $64.9 \%$ of xylitol $(68 \mathrm{~g} / \mathrm{L}), 13.3 \%$ of other sugars and $21.8 \%$ of other compounds that interferes in the crystallization process (as dry matter of the liquor). The fermented media was submitted to an absorption process with activated charcoal and concentrated until a xylitol concentration of $340 \mathrm{~g} / \mathrm{L}$. The liquor was then submitted to a second step of precipitation with ethanol, the best results achieved in this study were obtained with an ethanol/liquor ratio of 4 . In these conditions this treatment allows to remove a $56.9 \%$ of the impurities. The resulting solution was evaporated and crystallized containing $60 \%$ of ethanol and a xylitol concentration of $443 \mathrm{~g} / \mathrm{L}$. Crystallization was performed at $T=5{ }^{\circ} \mathrm{C}$ with slightly agitation. After $36 \mathrm{~h}$ were separated xylitol crystals with a recovery yield of $13 \%$ and a purity degree of $90 \%$.

\section{EB54}

Isolation and genomic amplification of single prokaryotic cells Thomas Kvist, Birgitte Ahring, Peter Westermann Environmental Microbiology and Biotechnology Group BioCentrum-DTU

Numerous publications have documented that only a minor number of the indigenous prokaryotic organisms found in complex environments such as the human intestine, biogas reactors, and soil are known, and probably only a fraction of this diversity can be accessed using traditional culturing techniques. Some of the reasons for this are the lack of knowledge of specific growth conditions, specific nutrients, and obligate coculture requirements. Also growth on a solid surface directly exposed to the atmosphere puts a very strong selective pressure on single cells supposed to develop into visible colonies. Therefore, the knowledge of these microorganisms is scarce and generally limited to the 16S rRNA genes that have been extracted from different environments and cloned for phylogenetic analyses. An obvious approach to circumvent these problems was the development of techniques based upon micromanipulation for isolation of single cells from complex mixtures. Continuous development of modern microscopes in combination with the precision of a servo-powered micromanipulator and the development of the modern microscopic micro injectors used in IVF techniques has further aided the manipulation of single cells. This technique, however, does not solve the problems of the non-culturable cells, and other approaches are needed to gain more information about these organisms. An approach to the non-culturable cells could be genomic analysis of isolated single cells without preceding cultivation. This PCR-based technique is widely used for genetic analysis of human cells, but due to the small amounts of DNA present in prokaryotic cells it has so far not been possible to produce identifiable amounts of DNA from single cell amplification using conventional polymerases. A promising alternative used for amplification of small amounts of DNA is the f29 DNA polymerase operating under isothermic conditions. Applying random hexamer primers, this polymerase carries out a multiple displacement amplification (MDA) of high molecular weight DNA template. In this study we demonstrate the successful application of MDA for selective amplification of genomic DNA from a single prokaryotic cell. The yield was $>20 \mathrm{mg}$ of amplified genomic DNA corresponding to about a 5 billion-fold amplification from a single cell. The technique was used to approach a large group of non-thermophilic Archaea found in agricultural soil. Our results show that combining MDA with fluorescent in situ hybridization and cell isolation by capillary micromanipulation enables an unprecedented ability to investigate new species without cultivation. Also this combination of techniques opens for studies of genetic heterogeneity within populations and processes such as horizontal gene transfer.

\section{EB55}

Precipitation of $\mathrm{Zn}^{2+}, \mathrm{Cu}^{2+}$ and $\mathrm{Pb}^{2+}$ at bench scale using biogenic hydrogen sulphide produced from the utilization of volatile fatty acids by sulphate reducing bacteria Maria Teresa Alvarez ${ }^{1,2}$, Carla Crespo ${ }^{2}$, Bo Mattiasson ${ }^{1}:{ }^{1}$ Department of Biotechnology, Center for Chemistry and Chemical Engineering, Lund University, P.O. Box 124, S-22100 Lund, Sweden; ${ }^{2}$ Instituto de Investigaciones Fármaco Bioquímicas, Universidad Mayor de San Andrés, La Paz, Bolivia

Biological production of hydrogen sulphide $\left(\mathrm{H}_{2} \mathrm{~S}\right)$ from sulphate using sulphate reducing bacteria (SRB) is popular within environmental biotechnology. SRB require absence of oxygen, presence of nutrients required for growth and oxidizable organic substrates (to supply hydrogen atoms for reduction of sulphate). Many organic wastes have been used as electron donors for the sulphate-reducers in the treatment of acid mine drainage (AMD) including straw, hay, sawdust, peat, spent mushroom compost and whey, however, other wastes such as municipal organic waste can be used. The aim of this work was to study the possibility of using SRB for the treatment of AMD at bench-scale. This process involved three stages: the volatile fatty acid (VFA) production by hydrolytic bacteria from the degradation of vegetables and fruits, the production of $\mathrm{H}_{2} \mathrm{~S}$ through the utilization of the produced VFAs by sulphate reducing bacteria and the precipitation of metals by using the biologically produced $\mathrm{H}_{2} \mathrm{~S}$. The substrates used for VFA production consisted of tomato, papaya, apple and banana. The $\mathrm{H}_{2} \mathrm{~S}$ produced from the degradation of VFAs was utilised for the precipitation of an artificial effluent simulating the heavy metal concentrations of a mine located at Bolivian Andean region, containing approximately $9 \mathrm{mg} / \mathrm{L}$ of $\mathrm{Zn}^{+2}, 8 \mathrm{mg} / \mathrm{L}$ of $\mathrm{Cu}^{+2}$ and $4 \mathrm{mg} / \mathrm{L}$ of $\mathrm{Pb}^{+2}$. The maximum concentration of hydrogen sulphide obtained was approximately $17 \mathrm{mM}$. Removal efficiencies of $97 \%, 98 \%$ and $100 \%$ for zinc, cooper, and lead, respectively, were achieved in the present work. 


\section{EB56}

Removal of dissolved nutrients from wastewater using a microalgae biofilter Line Christensen, Suvina Sooknandan, Jens Jorgen Lønsmann Iversen Department of Biochemistry and Molecular Biology, University of Southern Denmark, Odense, DK-5230, Denmark. E-mail: 1hm@bmb.sdu.dk (L. Christensen)

A microalgae biofilter can be used for treatment of wastewater from landbased fish farms in order to remove excess amounts of dissolved nutrients such as nitrate, ammonium and phosphate. A bubble column bioreactor has been developed for cultivation and characterization of microalgae. This type of bioreactor is equipped with a control system that enables online determination of the photosynthetic quotient and optimization of light intensity. Furthermore the bioreactor has a dualsparging system simultaneously allowing adequate mixing and high gas-liquid mass transfer coefficients. Different species of microalgae have been cultivated in batch and fed batch cultures to characterize growth and ability to take up the different dissolved nutrients. The specific growth rate and substrate uptake rate have been determined to compare and select the algal species most suited for use in a biofilter. Additionally the composition of lipid, protein and carbohydrates has been measured to determine the nutritional quality of the algae when used as animal feed.

\section{EB57}

Application of nitrogen-removal bioreactor capable of simultaneous nitrification and denitrification to wastewater treatment in power plants Masahiko Morita, Hiroaki Uemoto, Atsushi Watanabe Environmental Science Research Laboratory, Central Research Institute of Electric Power Industry (CRIEPI), 1646 Abiko, Abikoshi, Chiba 270-1194, Japan. E-mail: masahiko@ criepi.denken.or.jp (M. Morita)

At present, biological nitrogen-removal is mostly carried out through several complicated steps. To simplify the present systems for nitrogen-removal, we have investigated a new nitrogenremoval bioreactor using packed gel envelopes capable of simultaneous nitrification and denitrification. The envelope consists of two plate polymeric gels with a spacer in between. Ammonia oxidizer, Nitrosomonas europaea and denitrifier, Paracoccus denitrificans are co-immobilized in the plate gels. When the envelopes are exposed to wastewater containing ammonia, the immobilized $N$. europaea oxidizes ammonia to nitrite in the outer aerobic surfaces of envelopes. At the same time, as ethanol solution is injected into the internal anaerobic spaces of envelopes, the immobilized $P$. denitrificans reduces the nitrite to nitrogen gas using the ethanol solution as an electron donor for denitrification. In this way, the envelopes can remove ammonia from wastewater in a single step. We have already reported advantages of our bioreactor in laboratory-scale experiments. In this study, we show our large-scale bioreactor (water volume $1.8 \mathrm{~m}^{3}$ ) could treat three kinds of wastewater derived from coal power plants. Ammoniacontaining wastewater that occurred regularly in a coal power plant was continuously treated with the bioreactor using thirty envelopes for over a year. The bioreactor could remove more than $90 \%$ of total nitrogen at hydraulic retention time (HRT) of $24 \mathrm{~h}$. At HRT of $4 \mathrm{~h}$, the bioreactor accomplished a maximum rate (the transformation of $\mathrm{NH}_{4}{ }^{+}$to $\mathrm{N}_{2}$ ) of $6.0 \mathrm{~g} \mathrm{~N} /$ day $\mathrm{m}^{2}$ of the envelopes' surface. The performance was equivalent to that obtained in the laboratory-scale experiments. Furthermore, our bioreactor showed similar nitrogen- removal performances when it treated nitrate-containing wastewater occurring regularly and condensed ammonia-containing wastewater occurring at irregular intervals in coal power plants. These results show that our bioreactor can treat various wastewater containing nitrogen in coal power plants. Thus, our concept is effective to simplify the large-scale systems in coal power plants and the other plants.

\section{EB58}

Removal of metals from waste incinerator bottom ash by acidophilic microbes M.J.Moura, J. Sousa, M. CostaFerreira INETI/DB/UBB-Ed. F, Azinhaga dos Lameiros á Estrada do Paço do Lumiar, P-1649-038 Lisboa, Portugal. E-mail: joao.moura@ibqta.ineti.pt (M.J. Moura)

In order to establish an environmentally friendly process for the treatment of metal containing waste, in a Portuguese refinery a process involving sulphur oxidizing acidophilic microbes is being considered. Bioleaching of metal containing bottom ash, from fluidised bed incineration of sludge resulting from the refinery water treatment station, was performed using a sulphur oxidising acidophilic culture isolated from an acid pool resulting from the weathering of sulphur piles from the Claus plant. This sample served as inoculum for liquid medium cultures with $1 \%$ sterile sulphur flowers as source of energy. Application of Monod kinetics to adapted culture growth of free cells presented a value of $\mu=0.124 \mathrm{day}^{-1}$. Yield of sulphur conversion to sulfate after 17 days was $\eta=78 \%$. In the presence of bottom ash from the incineration of refinery sludges $\mu=0.141 \mathrm{day}^{-1}$ and the yield of sulphur conversion was $\eta=67.5 \%$. A $\eta_{\mathrm{Fe}}=90 \%$ removal of iron is obtained from the treated ash. X-ray fluorescence spectroscopy of the solid residue revealed a total removal of metals namely, $\mathrm{V}, \mathrm{Cu}$, $\mathrm{Ni}, \mathrm{Zn}$ and most of the Fe after 15 days of bioleaching.

\section{EB59}

Precipitation of $\mathrm{Cr}(\mathrm{III}), \mathrm{Ni}(\mathrm{II})$ and $\mathrm{Zn}$ (II) in solution by sulphate reducing bacteria G. Cabrera, J.M. Gómez, D. Cantero Biological and Enzymatic Reactors Research Group, Department of Chemical Engineering, Food Technology and Environmental Technologies, Faculty of Sciences, University of Cadiz (UCA), 11510 Puerto Real, Cádiz, Spain. E-mail: gema.cabrera@uca.es (G. Cabrera)

The present of heavy metals in the environment is a serious problem. They are commonly present in effluents from mining and industrial activities. Usually, chemical conventional methods are very expensive and they have limitations when heavy metals are in low concentrations. At the moment the interest increases for processes that involve microorganisms as alternative method. Some effluents present heavy metal sulphates which are soluble compounds. Sulphate-reducing bacteria (SRB), under anaerobic conditions, oxidize simple organic compounds (such as acetic acid and lactic acid) by utilizing sulphate as an electron acceptor and generate hydrogen sulphide. Hydrogen sulphide reacts with heavy metal ions to form insoluble metal sulphides that can be easily separated from a solution. The purpose of this work was to evaluate the ability of SRB to reduce $\mathrm{Cr}(\mathrm{III}), \mathrm{Ni}(\mathrm{II})$ and $\mathrm{Zn}$ (II) in artificial contaminated solution. Desulfovibrio vulgaris and Desulfovibrio sp. strains has been tested in this study. Batch cultures was carried out in $50 \mathrm{ml}$ sealed bottles with different concentrations of studied metals $(1-20 \mathrm{mg} / \mathrm{L})$, with $10 \%$ of inoculum bacterial and adapted to Postgate's medium C. A gaseous nitrogen current was employed to purge oxygen and obtain anaerobic conditions. The assays were incubated statically 
at $30^{\circ} \mathrm{C}$ during 14 days. Bacterial population was determined by counting in a Neubauer chamber with optical microscope. Sulfate concentration was measured by turbidity method and metal concentrations in the filtered supernatant were measured by ICP-AES. The first part of study consists of determine the maximum concentration of each metal at which D. vulgaris and Desulfovibrio sp. grow in similar way than control culture (without metal). Both cultures tolerate: $\mathrm{Cr}$ (III) $15 \mathrm{ppm}, \mathrm{Ni}$ (II) $8.5 \mathrm{ppm}, \mathrm{Zn}$ (II) $20 \mathrm{ppm}$. The maximum precipitation percentages were approximately: $25 \%$ (15 ppm $\mathrm{Cr}(\mathrm{III})$ ), $96 \%$ (8.5 ppm Ni(II)) and $99 \%$ (for D. vulgaris-10 ppm $\mathrm{Zn}(\mathrm{II})$ and Desulfovibrio sp.-15 ppm de $\mathrm{Zn}(\mathrm{II}))$. Time to reach the highest precipitation was minor for mixed culture (Desulfovibrio sp.) y all the cases. The next part was focused to study the precipitation percentage when metals are present in combination in the same metal levels (Cr(III)-Ni(II), Cr(III)-Zn(II), Ni(II)-Zn(II) and $\mathrm{Cr}(\mathrm{III})-\mathrm{Ni}(\mathrm{II})-\mathrm{Zn}(\mathrm{II}))$. The combination of metals does not affect significantly the bacterial growth and precipitation percentage of metals. This fact supposes an importance advantage so metals are commonly found together in the environment. Future experiments are focused in development of this process in continuous operation mode.

\section{EB60}

Kinetic modelling of the fungal biosolubilisation of coal B.O. Oboirien ${ }^{1}$, G. Searby ${ }^{1}$, D. Cowan ${ }^{2}$, S.T.L. Harrison ${ }^{1}:{ }^{1}$ Bioprocess Engineering Research Unit, Department of Chemical Engineering, University of Cape Town, Rondebosch, 7701, South Africa; ${ }^{2}$ Department of Biotechnology, University of the Western Cape, Belville, South Africa

Biosolubilisation and depolymerisation of coal has potential to produce a clean energy source or high value organic products from low rank coals such as lignite or sub-bituminous coal. These complex soluble phenolic compounds are of value as starting materials for biotransformation to value-added compounds such as antioxidants and flavourants. The bioprocess is carried out at ambient temperature and pressure and is perceived to be environmental benign. In the evaluation of coal solubilisation an important quantity for the assessment of process feasibility is the yield, i.e. the determination of the mass of product obtained per unit mass of coal solubilised. To date, results for coal biosolubilisation reported in the literature are qualitative or at best semi-quantitative, indicating trends with operating variables. The process kinetics has not been determined rigorously because measurement of fungal growth during coal solubilisation is hindered by the presence of the solid coal substrate. Knowledge of the profile of biomass growth is required for the rigorous determination of the kinetic parameters necessary for process design and optimisation. In this paper, the use of an indirect method for the estimation of the growth and metabolism of fungal biomass by measuring $\mathrm{CO}_{2}$ evolution and $\mathrm{O}_{2}$ consumption using an off-gas analyser is reported in the study of fungal coal solubilisation. Coal determined rigorously because measurement of fungal growth during coal solubilisation is hindered by the presence of the solid coal substrate. Knowledge of the profile of biomass growth is required for the rigorous determination of the kinetic parameters necessary for process design and optimisation. Biosolubilisation was carried out in a stirred tank slurry bioreactor with working volume of 1.01. Complete suspension of the coal particles of $650-800 \mu \mathrm{m}$ mean diameter was achieved at an agitation rate of $560 \mathrm{rpm}$. Growth yield coefficients based on coal and oxygen as well as maintenance coefficients were calculated from growth of the fungus under the same conditions using a non-coal carbon source such as glucose. These data were used to determine the stoichiometric coefficients for biomass growth, enabling the biomass production rate to be quantified in terms of $\mathrm{CO}_{2}$ production rate and $\mathrm{O}_{2}$ consumption rate.

\section{EB61}

A DNA-chip platform for parallel detection of microorganisms related to biofilm in industrial systems and drinking water systems Pernille Skouboe, Dorte Lauritsen, Kim Holmstrøm Bioneer A/S, Kogle Allé 2, DK-2970 Hørsholm, Denmark. E-mail: psk@bioneer.dk (P. Skouboe)

An oligonucleotide microarray for simultaneous detection and identification of pathogenic bacteria related to technical water systems as well as drinking water has been developed. The approach is based on the use of a tandem hybridization technique with two ribosomal 16S rDNA-PCR products, $1000 \mathrm{bp}$ and $500 \mathrm{bp}$ long, generated from two consensus PCR reactions using conserved ribosomal primers end-labeled with $\mathrm{Cy} 3$ and $\mathrm{Cy} 5$, respectively. The tandem hybridization technique implies an internally quality control for discrimination between target and non-target signals. The current prototype of the DNA-chip platform includes 20 oligonucleotide probes representing 11 different genera (and subgroups of species), e.g. Legionella, Mycobacterium, Aeromonas, Campylobacter, Vibrio and Enterococcus. The platform has been used for detection and identification of species from pure cultures, and initial experiments with water samples from industrial systems have been performed. The potential as well as the limitations of using a DNA-chip based detection format in its present form will be documented. Particularly, its potential application as a rapid method for initial screening of environmental or food samples will be addresses. The aim is to reduce and optimize the number of samples required for traditional microbiological identification tests.

\section{EB62}

Isolation and characterization of microorganisms for the biological inactivation of fumonisins W.-D. Moll ${ }^{1}, M$. Täubel ${ }^{1}$, E. Vekiru ${ }^{2}$, A. Frank ${ }^{2}$, A.P. Loibner ${ }^{3}$, R. Braun $^{3}$, G. Schatzmayr ${ }^{1}$ : ${ }^{1}$ Biomin $\mathrm{GmbH}$, Industriestraße 21, 3130 Herzogenburg, Austria; ${ }^{2}$ University of Natural Resources and Applied Life Sciences, Center for Analytical Chemistry, Vienna; ${ }^{3}$ Department for Agrobiotechnology, Institute for Environmental Biotechnology, Konrad Lorenz Straße 20, 3430 Tulln, Austria

In the course of a project for the development of a novel kind of a mycotoxin inactivating feed additive, the aim of this study was to isolate and characterize microorganisms with the specific ability to enzymatically break down and detoxify fumonisins, a group of structurally related fungal toxins, with fumonisin $\mathrm{B}_{1}\left(\mathrm{FB}_{1}\right)$ being the most abundant and - with respect to toxicology - also the most important representative of this group. These toxins are produced as secondary metabolites by some Fusarium species such as Fusarium verticillioides and $F$. proliferatum and are naturally occurring contaminants of cereal grains worldwide. They are found especially in maize and maize based products, and are known to be hazardous to human as well as to animal health. A natural feed additive, based on microorganisms and/or enzymes, should ensure the detoxification of 
fumonisins during feed uptake and digestion via microbial or enzymatic break down of these compounds, by that protecting the animal from the harmful effects of these mycotoxins. Besides an extensive screening of microbial strains derived from strain collections, various different natural habitats were investigated for the presence of $\mathrm{FB}_{1}$ degrading microbial activity, such as intestinal contents of pigs, soil samples, and naturally fumonisin contaminated maize. While testing of nearly 150 organisms from strain collections did not show positive results, fumonisin transforming activity could be detected in one soil sample and a number of maize samples. Trials in order to isolate the respective fumonisin degrading microorganisms resulted in a number of strains, whose $\mathrm{FB}_{1}$ degrading activity could be proven. The most promising bacterial and yeast strains were further characterized with regard to a general taxonomic description, and to different aspects of their toxin degradation behaviour. Approaching a more relevant in vivo situation, $\mathrm{FB}_{1}$ degradation trials in food- and feed-stuffs were conducted. Further on, the applicability of the respective organisms as stabilized lyophilisates was investigated.

\section{EB63}

Identification of different loci involved in arsenic resistance in one strain of Ochrobactrum tritici Rita Branco ${ }^{1}$, Ana Paula Chung $^{1}$, Paula Morais ${ }^{1,2}{ }^{1}$ Instituto do Ambiente e Vida, Depto de Zoologia 3004-517 Coimbra, Portugal; ${ }^{2}$ Departamento de Bioquímica, Faculdade de Ciências e Tecnologia da Universidade de Coimbra, Apartado 3126, 3001-401 Coimbra, Portugal. E-mail: rbranco@portugalmail.pt (R. Branco)

Arsenic is one of the most important global environmental pollutants and the toxicological effects are related to its chemical form and oxidation state. Arsenite [As(III)] is reported to be on average 100 times more toxic than arsenate[As(IV)]. This work shows the ability of one strain of the species Ochrobactrum tritici to grow in presence of several metals including arsenite, arsenate, selenite, selenate, tellurite and antimonite. Its arsenite MIC was determined as $50 \mathrm{mM}$, whereas for arsenate, this bacterium could resist to concentrations upper than $200 \mathrm{mM}$. We report the identification of two loci involved in high-level arsenic resistance. Sequencing of the first locus identified four complete genes in the following order: arsR, $\operatorname{arsD}$, arsA, arsB. The second locus containing genes for arsenic resistance was also characterized. Each sequence has been compared with nucleotide and protein databank (BLAST programs) and significant homology with known ORFs coding for arsenic resistance has been found. It is also possible that the phenomenon of high-level arsenic resistance in $O$. tritici could evolve other genes or loci. The ability of the $\alpha$-proteobacterium $O$. tritici to tolerate high levels of arsenic in addition to other oxyanions has considerable potential for detoxification and bioremediation of contaminated environments.

\section{EB64}

Mathematical modeling of biological thermophilic systems Libor Babak, Mojmir Rychtera Institute of Food Chemistry and Biotechnology, Brno University of Technology, 61200 Brno, Czech Republic

This work is based on the mathematical modeling of kinetics of a thermophilic bacteria cultivation system. The cultivation proceeded by way of batch and continuous on the synthetic medium with the main carbon source-lactose. This medium simulated an industrial waste approximately. Mixed thermophilic aerobic bacteria popula- tion, applied to the wastewater treatment (sludge V\&K Bystrice pod Hostynem), was used to the inoculation. The cultivation system consisted of the laboratory fermentor Biostat B (B. Braun Biotech) with working volume $2 \mathrm{~L}$ and the control unit connected with a computer. It is possible the temperature, $\mathrm{pH}$, aeration, stirring and foaming regulation. Physical and chemical cultivation conditions were optimized. The chemical oxygen demand (COD), generally expressive the impurity level, was selected as the main parameter for the cultivations run classification. But into the mathematical model also the kinetics of biomass growth, lactose consumption, production of choice metabolites (acetate, lactate, succinate) and dissolved oxygen concentration was included. The modeling was located to two head distinguishable growth phases of the microorganisms. An optimization and identification of mathematical model parameters was practised in the software language PSI/c. The difference between simulated curves and experimental data is not statistically significant on the relevancy level 0.05 ( $F$-test). It took place the COD degradation at $74.0 \%$ with the average yield coefficient $Y_{\mathrm{CHSK} / \mathrm{X}} 3.8 \mathrm{~g}$ COD/g biomass in the batch process with the air aeration only. There is a better way to the COD elimination $(>90.0 \%)$ - the aeration of air enriched by the pure oxygen. In experiments with the continuous system was obtained the $69.0 \%$ COD decrease after the steady state stabilization.

\section{Acknowledgement}

This work was supported by project MSM 0021630501.

\section{EB65}

Effect of temperature on the bacterial community and performance of biofilm reactors Hamid Zilouei, B. Guieyssem, B. Mattiasson Department of Biotechnology, Lund University, P.O. Box 124, 22100 Lund, Sweden. E-mail: hamid.zilouei@biotek.lu.se (H. Zilouei)

Fluorescence in situ hybridization (FISH) of whole cells using oligonucleotide probes was applied to study the influence of low temperature and temperature reduction on the bacterial community of biofilm reactors for the removal of chlorophenols (CPs). Two packed bed reactors were set up for degradation of a mixture of 2CP, 4-CP, 2,4-diCP, and 2,4,6-triCP as sole source of carbon and energy at $14{ }^{\circ} \mathrm{C}(\mathrm{RA})$ and $24^{\circ} \mathrm{C}(\mathrm{RB})$ and were inoculated with bacterial consortia adapted to these respective initial temperatures. The performance of the reactors was studied under different conditions of pollutant loading, aeration rate, and hydraulic retention times over 7 months. Total chlorophenol removal capacities of 1240 and $1420 \mathrm{mg}^{-1}$ day $^{-1}$ were achieved in the bioreactors RA and RB, respectively, under a total pollutant load of $1440 \mathrm{mg}^{-1} \mathrm{day}^{-1}$. The population of $\beta$-proteobacteria was the major bacterial community of the biofilm (35-47\%) followed by the $\gamma$-proteobacteria (12-6.5\%). Two bacteria with the ability to mineralize $50 \mathrm{mg}$ chlorophenols $\mathrm{1}^{-1}$ were isolated from the bioreactors and characterized as Ralstonia basilensis and Alcaligenes sp., both belonging to $\beta$-proteobacteria. Decreasing the temperature by $10^{\circ} \mathrm{C}$ (in two steps of $5^{\circ} \mathrm{C}$ each) resulted in an increase in the population of $\gamma$-proteobacteria and a decrease in the population of $\beta$-proteobacteria in both reactors. Application of genus specific probes showed an increase in the Pseudomonas population from $25 \%$ of the $\gamma$-proteobacteria at $14{ }^{\circ} \mathrm{C}$ to $59 \%$ at $4{ }^{\circ} \mathrm{C}$. The pollutant removal capacity decreased to 548 and $833 \mathrm{mg} \mathrm{l}^{-1} \mathrm{day}^{-1}$ in RA $\left(4^{\circ} \mathrm{C}\right)$ and RB $\left(14^{\circ} \mathrm{C}\right)$, respectively. The $\alpha$ and $\delta$-proteobacteria, Cytophaga-Flavobacteria and Actinobacteria 
represented very low portion (less than 4-5\%) of the total bacterial community at all temperatures tested.

\section{EB66}

Effect of portal sera of highly and poorly susceptible hosts and the Schistosomal antibody(ies) on cell proliferation of $S$. mansoni Schistosomules Y.M. Shaker ${ }^{1}$, M.M. Abdel Fattah ${ }^{2}$, E. Ashour ${ }^{1,2,3}$, H.M. Khattab ${ }^{3}$, H.M. Draz ${ }^{1}$ : ${ }^{1}$ Biochemistry Department, National Research Centre, Cairo, Egypt; ${ }^{2}$ Biochemistry Department, Ain Shams University, Cairo, Egypt; ${ }^{3}$ Pathology Department, Faculty of Medicine, Cairo University, Cairo, Egypt

Schistosomules of Schistosoma mansoni (20 days old) were incubated in RPMI 1640 medium containing 10\% fetal calf serum, 10\% hamster portal venous or $10 \%$ hamster peripheral venous serum (highly susceptible host) or $10 \%$ rat portal venous or $10 \%$ rat peripheral venous serum (poorly susceptible host) in presence of bromodeoxyuridine (BrdU) in order to measure differences in cell proliferation. Also the rate of cell proliferation of $S$. mansoni were assessed in vivo in hamster to study the cell proliferation in the natural ontogeny of the organism. The rates of cell proliferation as expressed by BrdU labeling indices (BLIs) were determined as a function of time of incubation by immunohistochemistry using monoclonal antibody to BrdU. Compared to Schistosomules cultured in presence of RPMI plus $10 \%$ fetal calf serum, BLIs were increased by $41 \%$ in the presence of hamster portal, but not in peripheral serum. While in case of rat, no significant changes were observed in the BLIs in both portal and peripheral sera. The experiment was repeated using hamster portal and peripheral sera containing different schistosomal IgG antibody titres. The results showed decreased values of BLIs compared to sera which did not contain the schistosomal antibody(ies). The in vivo results revealed that there was no cell proliferation of S. mansoni Schistosomules (6 days old) in the lungs. Cell proliferation was detected in Schistosomules of 17 days old and the results revealed a significant decrement in the BrdU labeling indices (BLIs) with the increase of the age of Schistosomules in vivo. The results indicated that hamster portal venous serum (highly susceptible host) could have stimulating factor(s) for schistosomule cell proliferation which is not found in rat (poorly susceptible host) and the presence of antibody(ies) greatly inhibit the cell proliferation. This could be due to the blocking of some portal serum factors, which stimulate the cell proliferation by the antibody(ies).

\section{EB69}

Removal of Remazol Brilliant Blue R (RBBR) from aqueous solutions by adsorption of polyelectrolyte complex Ruby Y. Salazar, Christina Guajardo, Luis J. Galán, Syed H. Imam, Katiushka Arévalo Instituto de Biotecnologia, F.C.B., UANL, Ciudad universitaria, San Nicolás de los Garza, N.L.Mx. E-mail: karevalo@ccr.dsi.uanl (K. Arevalo)

The release of dyes into the environment constitutes only a small proportion of water pollution, but dyes are visible in small quantities due to their brilliance. Many dyes are difficult to decolourise due to their complex structure and synthetic origin. The adsorption of reactive dye Remazol Brilliant Blue R (RBBR) on polyelectrolyte complex (PEC) was studied in a batch systems. The adsorption parameters determined were: effect of the different values of $\mathrm{pH}$ on the adsorption of dye by PEC, and the effect of contact time on the amount of RBBR adsorbed (in $\mathrm{mg} \mathrm{g}^{-1}$ ). The data indicates that the adsorption capacity of RBBR by PEC is dependent on $\mathrm{pH}$, The maximum adsorption at $50 \mathrm{ppm}$ was $88.52 \%$ equal to $11.8 \mathrm{mg}$ dye $/ \mathrm{g}$ of polymer. The results show a tendency towards greater adsorption for reactive dyes ( $\mathrm{pH}$ range of 8-12). The effect of contact time was studied at initial concentration ( $50 \mathrm{ppm})$ of dye, the amount of RBBR adsorbed for these PEC increased and reached a constant value with the increase in contact time. The increase in the extent of removal of dye after $15 \mathrm{~min}$ of contact time is less and hence it is fixed as the optimum contact time. The PEC show their capacity to remove RBBR to aqueous solutions by adsorption.

\section{Workshops}

\section{Public Perception}

Bringing life sciences to the public Susanne Schneider-Voss, $M$. Bachler, K. Garber, M. Tumpej, B. Streicher dialog<>gentechnik, Campus Vienna Biocenter 6/1, 1030 Vienna, Austria

Compared to other European nations, the Austrian population shows a low level of knowledge in biosciences and a strong denial of gene technology (1). The Austrian non-profit organisation dialog<>gentechnik, a scientific society, organizes various activities to raise awareness for the "hot topics" in the life sciences. According to its principle of independence, all activities are funded publicly. Projects on behalf of the Austrian authorities and international cooperations demonstrate credibility and trust in dialog<>gentechnik (2). A few examples will be presented. Dialogue with the public: On the occasion of the first anniversary that the GMO labelling rules became effective, the action "Gene technology on my plate" is performed Austrian wide in shopping centres. Here, consumers are informed about health and labelling aspects of GM food. Two days of open discussions were organized in the context of the Austrian Genome Research Program GEN-AU (3): topics were "Gene Diagnosis" (2002) and "Genome Research-what is in it for me?" (2004). An open lab is currently set up in Vienna to offer "hands on" experience in life sciences for everybody. Motivating students: In the very successful GEN-AU summer school (3), high school students spend 3-4 weeks in the lab and work with scientists. The best documentations are awarded. In an innovative project, student groups (age 16-18) work on the topics stem cells and cloning and develop units of an eLearning course which will be accessible for all Austrian schools in the near future. Engaging stakeholders: dialog $<>$ gentechnik manages interdisciplinary wor_king groups that develop leaflets, brochures and questionnaires on various aspects of gene diagnosis. Four products are currently distributed to the public and to health services. (1) European Commission. Eurobarometer 55.2, December 2001; (2) www.dialog-gentechnik.at; (3) www.genau.at.

\section{Professionals, Urban Consumers and Biotechnology in Turkey}

The aim of this paper is to compare the attitudes of the urban consumers with professionals towards to new technologies, especially to biotechnology. It was tried to find out in which area, medical, agriculture or industry, people can accept biotechnological developments and in which area not accept. 
The Survey was carried out in urban and rural areas of two cities (Ankara and Isparta). This paper is only analysing urban people by excluding villagers. Urban sample is consisted of 400 urban consumers and 200 professionals. Professionals were selected amongst pharmacists, doctors, agricultural engineering's and industrialists that is thought are affective in the process of developing new technologies and also the development of biotechnology in the society. Basic data was gathered by a questionnaire including both structured and open-ended questions besides deep interviewed.

\section{Biotechnology Workforce Development}

Workforce development for life sciences-The Scottish experience Carol Booth Scottish Entreprise, UK

The presentation will look at, the background and definition of Workforce Development for Scottish Enterprise, examine information available to support Scottish Enterprises economic intervention and where and when to intervene. Conclusions emerging from the evidence base will be used to outline Scottish Enterprises approach to workforce development and look at which actions might be required to address identified issues. Moving on to reasons for integrating Workforce Development into business development and how the Life Sciences cluster team at Scottish Enterprise, stakeholders and partners in Scotland have approached their current and future contributions to Workforce Development for Life Sciences using a variety of projects.

- Beacon Industrial Placements

- Pre Bio

- SCBC Tailored Courses

- Intern Studentships

- Management Development Programme

- Connect 360 and Masterclass

- RSE Fellowships

- Scottish People Management

- Leadership Programme

Research and training for bioprocessing, an Irish proposal Kurt Naujoks Director for Bioprocessing, Engineering Building, Ireland

The National Institute for Bioprocessing Research and Training $(N I B R T)$ in Ireland is a proposal that will be a state-of-the-art training, research and pilot plant service facility that brings together institutions with complementary expertise and state-of-the-art research technology, and industry partners. These include University College Dublin, Trinity College Dublin, Institute of Technology, Sligo and Dublin City University. NIBRT is an innovative collaboration between academic institutions at the forefront of biotechnology, cell biology, engineering and pharmacy and industry. For training, two separate training labs, for upstream and downstream training, in addition to 5 research labs are planted. It will also include a state-of-the-art pilot plant fermentation facility for fermentation optimisation, fermentation scale-up, product separation and purification, regulatory aspects and automation. By aligning with industrial demands, the new institute will tailor its training programmes while remaining on the cutting edge of biotechnology research and technologies. The fermentation facility will offer hands-on training workshops and educational modules for outside researchers and companies. These workshops cover the fundamentals of small-scale fermenta- tion, scale-up considerations, and fermentor design and set-up. The training and educational philosophy underpinning the NIBRT will focus on the needs of industry with an emphasis on providing training for accreditation of existing industry staff and prepare technicians and graduates for the technical, business, regulatory and professional aspects of the industry. The strategy is to provide specialised modules in NIBRT in support of courses established in the higher education institutions, which will provide the certificates, diplomas and degrees. Modules will be offered for all categories of students and will be given credits respected by other third level institutions in Ireland.

The role of professional graduate degrees in meeting current and future biotechnology industry workforce needs A. Stephen Dahms San Diego State University, USA

The presentation will review the status of new graduate training models designed to meet the unique needs of the biotechnology industry as it transitions to commercialization. Emphasis will be on professional master's degree programs in biotechnology and their various versions, with a focus on operational and funding strategies and industry acceptance. Discussion will also centre upon the creation and operation of industry-validated, specialized and highly targeted professional masters degrees in various refined aspects of the drug development process, including Regulatory Affairs, Biomedical Quality Systems, Clinical Affairs, Management of Drug Development, Management of Reimbursement Affairs, Bioinformatics, etc. The Eurodoctorate in Biotechnology, new combined MBA/PhD combined degrees in the molecular life sciences, the U.S. Professional Doctorate in Chemistry and the proposed U.S. Professional Doctorate in Biotechnology will be also discussed. Data will also be presented on the current workforce and the industry's projected needs.

\section{Global Responsibilities in Biotechnology}

Global human family - global health risks - global responsibilities Lars Bolund University of Aarhus, Denmark

Genetic studies show that mankind is a rapidly expanded population of closely related individuals with very similar disease sensitivity. Bad nutrition and infections dominate among the main health problems in the world. Apart from malnutrition, the overeating habits of the developed world are now creating problems in the developing world as well. Infectious diseases are also a global problem since new contagious agents like HIV, SARS and avian flew do not recognize borders. Thus, the global responsibilities of modern health care are obvious.

Educating and supporting research scientists from and in the developing world David McConnell Trinity College, Ireland

Many research scientists from and in developing countries find it nearly impossible to use their talents for the benefit of their own countries. Some struggle to develop research and education programmes with poor facilities, some leave science completely, and others migrate to more developed countries. The talents of such people are either being wasted or lost completely to their home countries just at the time they are most needed to combat the great humanitarian challenges of hunger, illness and lack of knowledge. Europe must strengthen programmes which allow third world scientists to work to their full potential in their home countries or regions. 
Europe and global life sciences: Pride and expectation Huanming Yang Beijing Genomics Institute, Chine Academy of Sciences, Beijing, China

Europe has all the reasons to be proud of being the cradle of modern science and of its achievements and resources in life sciences. As a model of having solved many of the problems that many other counties are now facing, Europe is expected by the whole world to make its further contribution to a better future of mankind and to play a more important role in the international community of life sciences.

\section{Biotechnology at the Canary Islands}

Tropical diseases and public health Basilio Valladares Director of the University Institute of Tropical Diseases and, Public Health, University of La Laguna, 38200 La Laguna, Tenerife, Spain. E-mail: bvallada@ull.es

The presentation will look at the main research interests of the University Institute of Tropical Diseases. These are the following:

1. Immunology and molecular biology of parasites. We express and purify recombinant proteins from Leishmania sp. which have been shown to act as immunomodulators and protect against disease such as L25, HSP70, HSP83. The study of Acanthamoeba pathogenic factors has also resulted in the isolation and silencing of extracellular proteins related to their pathogenecity, which has a great potential in the development of novel chemotherapeutics.

2. Diagnosis of parasites. The immunological diagnosis of leishmaniasis has been one of the main research interests in our laboratory for several years. As a result, we have identified peptides which could be used to develop kits for the immunological diagnosis of leishmaniasis such as HSP70 C-end, L25 N-end, etc. We have also developed some DNA based methods for the identification of Acanthamoeba species from biological and environmental sources.

3. Water quality. Biological parameters. Our water research group has the expertise to identify and characterise bacterial, viral and parasitic indicators of faecal contamination in diverse water sources including tap water, rivers, reservoirs, sea, etc. This research area has been developed in collaboration with the local sewage treatment plant and reservoir managing authorities. Currently, we are establishing a conjoined project with the Center for Disease Control and Prevention (CDC) in Atlanta, USA for the identification of water-borne emerging pathogens.

4. Development and formulation of chemotherapeutic antiparasitic agents. In this field, we evaluate the leishmanicidal activity both in vivo and in vitro of natural and synthetic drugs and synthetic peptides. In a later stage, the drugs which have shown the highest antiparasitic activity have been subjected to cytotoxicity assays and their molecular targets dissected. Some of the drugs tested in the last few years have been submitted to patent due to their outstanding activity. Finally, in order to allow the commercialization of these drugs, both in vivo and in vitro assays are being carried out to predict their chemical stability and degradation pathways. This will be followed by the use of liofilization and controlled crystallisation strategies for the development of efficient and safe treatments.
5. Human and population genetics. Tachykinins and their receptors in different tissues and groups of patients and their association with molecular polymorphisms is another one of our research interests. The knowledge of ligand and receptor sequences and their similarities will allow the rational development of drugs with specific activity against these receptors. Furthermore, we are also interested in the interspecific variation along the evolutionary scale of these markers.

6. Nitrate assimilation group. Research in our group is focused on understanding nitrate assimilation in the yeast Hansenula polymorpha. Several biotechnological companies use this yeast to produce heterologous proteins (hepatitis B vaccine). Genetic manipulation techniques for $H$. polymorpha are available in our laboratory.

Determination of lipid content in microalgae Héctor Mendoza Head of the Department of Biotechnology, Technological Institute of Canary Islands, Pozo Izquierdo 35119 Santa Lucía, Las Palmas, Spain

Single cell analysis by flow cytometry has proved to be a tool to perform simultaneous and rapid measurements related to cell morphology and physiological state. Previous studies showed the possibility of quantifying neutral and polar lipids spectrofluorometrically using a lipid specific fluorescent dye, Nile Red (NR), however the existence of inter and intraspecific variations in the fluorescent response had not been clearly established. In this work, two strains of marine microalgae: Crypthecodinium cohnii and Tetraselmis suecica, characterized both by high contents of polyunsaturated long chain fatty acids (DHA and EPA, respectively) and an hypersaline microalgae: Dunaliella salina, characterized by a high $\beta$-carotene production, were grown under different conditions and collected at different growth phases to be used for in vivo lipid quantification with NR by flow cytometry. Our results showed a high correlation between the mean fluorescence signal of NR stained cells and the neutral and polar lipid content measured by gravimetry for each strain. In this respect, these data make feasible the development of a rapid method for lipid quantification in monoalgal cultures. However, differences in the dye uptake related to specificity were detected. In this communication we also assess the possibility of use this cytometric technique to select microalgal strains with high lipid and polyunsaturated long chain fatty acids content from mixed samples. Performance of such technique would be a good alternative to the time-consuming traditional screening protocols based on gravimetry and gas chromatography and would optimise the search of new commercial strains of microalgae.

Production of recombinant proteins-food biotechnology Félix Claverie-Martín Head of Research Unit, Biomedical Research Institute, Hospital Universitario, de N.S. de Candelaria, Santa Cruz de Tenerife, Spain

Our group is involved in the cloning and production of proteins of interest to the food and pharmaceutical industry. We have recently expressed in yeast the cDNA that encodes the precursor of caprine chymosin. Chymosin is the enzyme responsible for the coagulation of milk in the abomasum of unweaned calves. This enzyme is secreted by gastric mucosa cells as an inactive precursor, known as prochymosin. In the acidic conditions of the lumen, prochymosin is converted into the active form by autocatalytic cleavage of the 
$\mathrm{N}$-terminal prosequence. Chymosin is used extensively in cheese production because it cleaves $\mathrm{K}$-casein in a specific manner with low proteolytic activity. Several biotechnology companies are producing the bovine recombinant enzyme for commercial use in the process of cheese making. We are interested in the caprine chymosin as an alternative because in the Canary Islands cheese has traditionally been made using goats milk with extract from the abomasum of newborn goats as coagulating factor. It is well known that the activity of these types of extracts varies depending on the age of the animal and the type of food ingested. These difficulties should be overcome using a recombinant caprine chymosin. The caprine mRNA used for the synthesis of the cDNA was obtained from the abomasum of milkfed kid goats. The cDNA fragment encoding the mature portion of caprine prochymosin was fused in frame to a signal sequence in yeast expression vectors. Culture supernatants of yeast cells transformed with the recombinant plasmids showed milk-clotting activity after activation at acid $\mathrm{pH}$. Proteolytic activity assayed toward casein fractions indicated that the recombinant caprine chymosin specifically hydrolysed $\kappa$-casein (Patent 200402025). The recombinant caprine chymosin could be an alternative milk coagulant in cheese making. Work is underway to optimise the expression of the new recombinant prochymosin for further purification and characterization.

Smart molecules for health Victor Martín Head of Research, University Institute of Bio-Organics "Antonio González", Avda. Astrofísico Francisco Sánchez 2, 38206 La Laguna, Tenerife, Spain

The Instituto Universitario de Bio-Orgánica "Antonio González" (IUBO) is a multidisciplinary research centre that belongs to the University of La Laguna. The IUBO is located at the town of La Laguna, inscribed on UNESCO's World Heritage List in 1999, and former capital of the Canary island of Tenerife. The geographical location in addition to its mild climate has made the Canary islands to posses a variety of ecosystems with unique plants and animals. The institute was started up in the 1960s with the need to study the natural products and secondary metabolites produced by those marine and terrestrial organisms, thus providing a new source of bioactive products. The main research lines that are being developed at IUBO are summarised in the following paragraphs:

Anticancer agents from natural sources: Several natural products and their semisynthetic derivatives are produced at the IUBO in diverse joint projects for the development of new antitumour drugs with novel mechanisms of action. As an example we can mention natural products from the mevalonic, shikimic or polyketide pathways. Some products have recently shown in vitro reversion of the resistance in multidrug resistant (MDR) tumour cell lines.

Genetic engineering: In vitro cultures of the plants Atropa baetica, Maytenus amazonica and M. macrocarpa are developed in order to manipulate their biosynthetic pathways and induce the production in large scale of the secondary metabolites for diverse applications, including arthritis, rheumatism, and back pain.

Marine organisms and toxins: Dinoflagellates are marine organisms responsible for the red tides and food poisoning episodes. Among others, okadaic acid and yessotoxin are the most common toxins present in European shellfish. The isolation of these products is best done from the microorganism cultures, since they are present in very low amounts in the natural sources. At the IUBO we develop culture systems to provide us with amounts of toxins large enough to perform biological, metabolic and bioactive studies. Insecticide and repellents: Natural products are being isolated for their use against plagues, specially those affecting agriculture. These projects are run in collaboration with a number of public institutions and agrochemical companies throughout Europe and Latin America.

Fine chemicals and pharmachemicals: Our institute possesses large expertise in the field of organic synthesis devoted to the synthesis of medicinal substances, with special focus on asymmetric processes. Of particular interest is the development of new methodologies for the total synthesis of biologically active substances like polyether toxins, (un)natural amino acids, sphingosine analogs, alkaloids, etc.

With an annual source of 100s of new compounds, the fine chemicals and medicinal chemistry branch at IUBO have recently started and anticancer screening program in collaboration with the Biomedical Research Unit at the Hospital Universitario N.S. de La Candelaria. The program is committed to the discovery of novel drugs for application in cancer treatment. The outcome of this project in its first year has been outstanding, leading to the finding of several leads that form the basis for current and future projects.

Research in aquaculture María Soledad Izquierdo Technological Institute of Canary Islands, Biotechnological Department, Playa de Pozo Izquierdo s/n, 35119 Santa Lucía, Gran Canaria, Spain

The presentation will look at the main research interests of the Biotechnological Department of the Technological Institute of Canary Islands. These are the following:

Nutrition and Feeding in Aquaculture: We conduct studies on digestion, absorption, transport, and utilization of the different nutrients applying also different techniques such as histology, enzymology, genetic or immunology among others. Aquaculture feeding is the main important cost in fish farms, being higher that the prize of fries, personnel cost or energetic costs. Thus, studies conducted on the improvement of diet formulation and the use of different dietary ingredients is one of our main research lines (such as vegetable oils and meals to be used as alternative to fish meal and oil, or carotenoid sources to improve fish colour). Not only the use of the different ingredient is studied, but also the effect of these ingredients on fish health, flesh quality, flesh healthy aspects related with human consumption, are being studied. Different formulae are being developed and patents of different diets are being obtained. Studies on nutritional requirements are also conducted allowing us to patent different formulae in larvae studies, developing microdiets to substitute the high-cost processes associated with live prey in larval nutrition. Development of immunostimulants, anti-stress diets, immune techniques to be applied as bio-indicators of fish health and welfare, as well as use of dietary ingredients derived from bio-reactors are other research lines in our group.

Genetics: Genetic techniques applied to aquaculture are being an important tool. Microsatellites are being used to determine genealogy of the fish, allowing to decreases important problems in aquaculture such as fish deformities. Genetic techniques such as micro-arrays and gene expression are being applied to obtain indicators of stress and health in fish. These technologies also permit to make different selective breeding programs, increasing the accuracy to estimate genetic parameters and evaluating brood- 
stock. Furthermore, this technology allows to obtain a procedure to increase the productivity and quality of fish hatcheries.

New Species for Aquaculture. Development of New Culture Techniques: The diversification of species cultured is one of the main objectives of European aquaculture, since nowadays only four marine species are commercially produced: gilthead sea bream. European sea bass, turbot and salmon. We have been developed rearing techniques for new species such as red porgy or Canarian abalone and also we are conducting studies on different new species, such as different sparids species, octopus or yellowtail.

New Rearing Technology: The election of adequate systems for fish growth for each species and site of production and the localization of more appropriate sites for farms, using GIS technology are also of special importance in our research team. Besides, new larval rearing techniques such as semi-extensive hatchery (mesocoms) were development and nowadays is being used to increase fry quality (survival, no-deformities, better growth). This technology is being exported to other countries in order to offer new technology easy to manage to be implanted in developing countries.
Biotechnology and taxation: Canary Islands Special Zone (ZEC) Alejandro Cañeque Project Officer, Canary Islands Special Zone, Ministry of Finance, C/Leon y Castillo $431-4^{a}$ planta, Edificio Urbis, 35007 Las Palmas, Spain. E-mail: acaneque@zec.org

The Canary Islands Special Zone is the newest tax instrument within the Canary Islands Economic and Fiscal Regime (REF). It offers a reduced tax rate of between 1 and $5 \%$ of Corporate Income Tax for companies setting up a business. The companies must cover the following minimum requirements: create employment and make a minimum investment. The ZEC offers other tax advantages such as the exemption from paying Transfer Tax and Stamp Duty and the Canary Islands General Indirect Tax (IGIC). This tax scheme particularly fosters the biotechnology and pharmaceutical sectors. 